\title{
Measurement of particulate matter size, concentration and mass emissions from in-use heavy duty vehicles
}

Richard Jason Byers
West Virginia University

Follow this and additional works at: https://researchrepository.wvu.edu/etd

\section{Recommended Citation}

Byers, Richard Jason, "Measurement of particulate matter size, concentration and mass emissions from in-use heavy duty vehicles" (1999). Graduate Theses, Dissertations, and Problem Reports. 1043.

https://researchrepository.wvu.edu/etd/1043

This Thesis is protected by copyright and/or related rights. It has been brought to you by the The Research Repository @ WVU with permission from the rights-holder(s). You are free to use this Thesis in any way that is permitted by the copyright and related rights legislation that applies to your use. For other uses you must obtain permission from the rights-holder(s) directly, unless additional rights are indicated by a Creative Commons license in the record and/ or on the work itself. This Thesis has been accepted for inclusion in WVU Graduate Theses, Dissertations, and Problem Reports collection by an authorized administrator of The Research Repository @ WVU. For more information, please contact researchrepository@mail.wvu.edu. 


\section{Measurement of Particulate Matter Size, Concentration And MASS EMISSIONS FROM IN-USE HEAVY DUTY VEHICLES}

\section{RICHARD J. BYERS}

THESIS SUBMITTED TO THE

College OF ENGINEERING AND Mineral RESOURCES

AT

WEST VIRGINIA UNIVERSITY

In PARTial Fulfillment of THE ReQuirements

FOR THE DEGREE OF

MASTER OF SCIENCE

IN

MECHANiCAL ENGINEERING

Donald Lyons, Ph.D. Chair

Mridul Gautam, Ph.D.

Nigel Clark, Ph.D.

GARY MORRIS, PH.D.

SANDEEP MEHTA

DEPARTMENT OF MECHANiCAL AND AEROSPACE EngineERING

MORgAnTOWN, WeSt ViRginia

1999

Keywords: Particulate Matter, Diesel, Natural Gas, Mossgas, SMPS, MOUDI, CYCLONES 


\title{
Abstract \\ Measurement of Particulate Matter Size, Concentration and Mass EMISSIONS FROM IN-USE HEAVY DUTY VEHICLES
}

\author{
RICHARD J. BYERS
}

As technological advancements lower heavy duty vehicle particulate matter (PM) mass emission rates, there is concern that these improvements are increasing the toxicity of the PM by virtue of a subsequent reduction in particle size. These very fine particles are able to better penetrate the alveolar region where they can cause serious lung disorders. Alternative fuels such as compressed and liquefied natural gas (CNG and LNG) and the synthetic diesel fuel Mossgas are attractive in that they reduce the levels of total PM. However, use of natural gas in internal combustion engines may produce a larger number of smaller particles than commercial diesel fuel, and little particle size information is available on Mossgas combustion.

The objective of this study was to design a measurement system that could resolve the particle emissions from heavy duty vehicles powered by all these fuels in terms of mass and size. This system employed four instruments, namely, the Scanning Mobility Particle Sizer (SMPS), the Micro-orifice Uniform Deposit Impactor (MOUDI) and the PM2.5 and PM10 cyclone. Three series of tests were performed with West Virginia University's Transportable Heavy Duty Vehicle Emissions Testing Laboratory on transit fleet buses from Pittsburgh, Pennsylvania's Port Authority Transit (PAT), the Regional Transit District (RTD) fleet of Boulder, Colorado and the Dallas Area Rapid Transit (DART) vehicles of Dallas, Texas.

The tests showed the effectiveness of the measurement system while returning mass and size data for the various fleets. While Mossgas lowered the mass emission rates of TPM, it had little effect on the mass median aerodynamic diameter (MMAD) as compared to diesel $\# 2$, with both fuels giving MMADs of approximately 0.11 to $0.12 \mu \mathrm{m}$. The SMPS data showed that diesel \#2 also yielded higher particle concentrations than Mossgas. Both fuels follow the same pattern in that the number of particles increases proportionally with an increase in diameter, reaching maxima in the $100 \mathrm{~nm}$ to $200 \mathrm{~nm}$ range. The natural gas vehicle (NGV) data shows that particle concentrations peak at approximately $30 \mathrm{~nm}$ in mobility diameter and produce close to $90 \%$ less TPM. The particulate measurement system allowed this full description of particle emissions to be performed quantitatively rather than mathematically. The formulaic conversion from particle number to mass and vice versa can lead to gross errors. This numerical approximation can thus be avoided with the step-process method using the four instruments, as this system can enable accurate and direct measurement of both particle parameters. 


\section{ACKNOWLEDGEMENTS}

In my end is my beginning, said T. S. Eliot. It is the end of nearly two decades of school, the beginning of an infinite number of possible futures. My last look at Morgantown is from the rooftop of the Engineering Sciences Building; sunrise and heat lightning, early fog through the valley, South Park waking somewhere in the mist behind the smoke stack. Before I take my leave of this perch, this school, this city and this state, I need to express my deep appreciation to all the people who have made possible this end and this beginning.

Somehow, through school and life my family has always been there to celebrate my successes, pull me up after my failures and allow me a place to come to when the distinction between the two was lost. With words of wisdom and encouragement, home cooked meals just through the always open doors and an appreciation of my too often strange tales, they showed me strength when mine had wavered, let me rest when I was restless and let me laugh at myself. Not everyone made it to see me through, but the spirit still lingers. Maybe it isn't just the coffee that keeps me working through the long night. So Mother, Rob, Stacey (Ruckle) and baby Grace, thank you for it all.

Without Dr. Gautam as my advisor and my friend, I would not be here today or going where I'm going tomorrow. Your unwavering faith in me as a student and as a person has made me work harder at being both. There were times that I questioned these over the last two years, but you never did. So, if John Colebank ever asks after me again, tell him that he's doing fine. Tell him that his advisor saw to that. 
I must express my sincere gratitude to Sandeep Mehta. You took on a Herculean task when you decided to teach me how to be a graduate student. I now know why the engine works, why the computer doesn't, why not to pet the dogs in Boulder and why I would never want your job. You are underappreciated, overworked and underpaid. Somehow, you took the time to teach me everything that the textbooks didn't. I wouldn't be going to Columbus without your help.

Before I roll through the litany of people that helped in my research, I want to thank the people that saw me through life. First, there is Amy. There are few constants in life, but through it all, you were always there or just a phone call away; whether for a long night spent with our fears and joys or a half-hour discussion on the problems of time travel. You never let me down. I want to thank Scott, my roommate for half of these six years, for the rides when my car either wouldn't run or couldn't leave, the late night explanations to an endless stream of study questions, for letting me in the door when I 'locked myself out' and especially for sitting beside me at the bar. I also want to thank Shelley for more reasons than this book could ever hold. To those that have gone unmentioned by name, my appreciation is no less, but the list too long.

Lastly, I wish to acknowledge the people that made this work possible: the crew of the mobile lab; Leo for the MOUDI program; the secretaries in room 357 - your adopted son is finally leaving the nest; Ryan and Wes for a good game of frisbee and football; Dan for inspiring me to finish my thesis; Drs. Clark and Morris for serving on my committee.

With this, my six years at West Virginia University come to a close. Through them all, I can now say that I have seen the "garlic and sapphires in the mud." 


\section{TABLe OF CONTENTS}

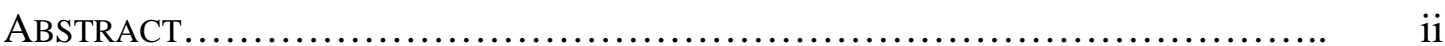

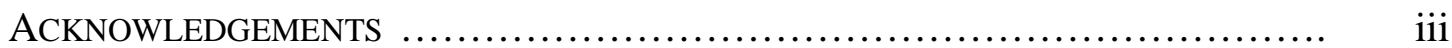

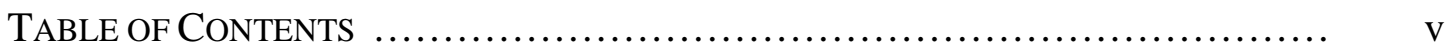

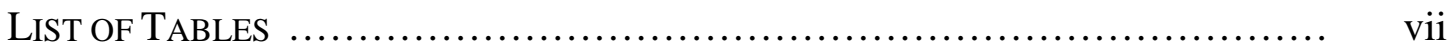

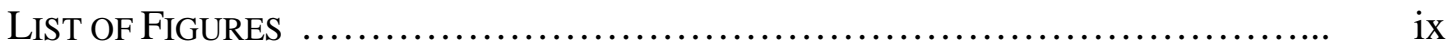

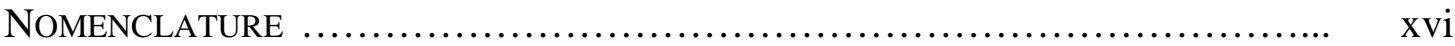

CHAPTER

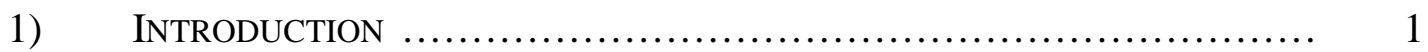

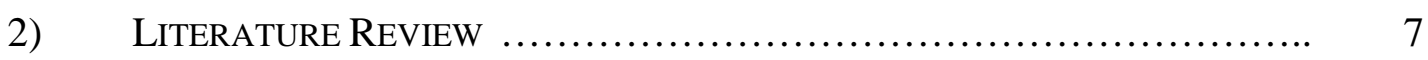

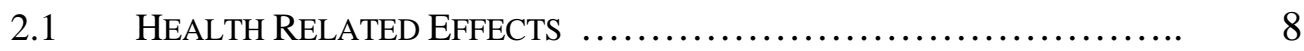

2.2 PARTICLE BEHAVIOR AND FoRMATION PROCESSES ................. 13

2.3 PARTICLE - TO - PARTICLE PROCESSES ........................... 17

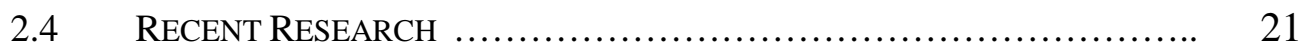

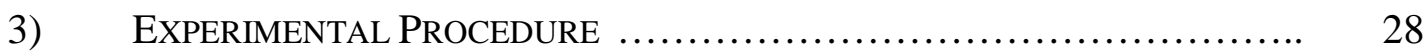

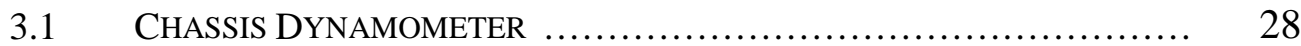

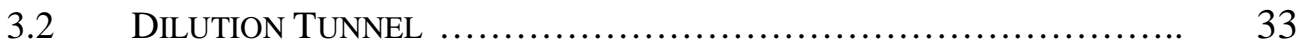

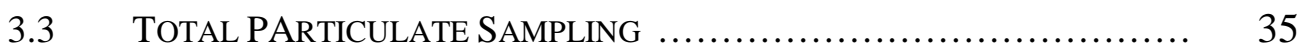

3.4 SYSTEM MODIFICATIONS ...................................... 37

3.4.1 PARTICULATE SAMPLING: MOUDI ...................... 41

3.4.1.1 MOUDI DESCRIPTION AND THEORY ............... 47

3.4.1.2 MOUDI OPERATION .............................. 50

3.4.2 PARTICULATE SAMPLING: CYClONES ....................... 53

3.4.2.1 CYCLONE DESCRIPTION AND THEORY ............ 57

3.4.2.2 CYCLONE OPERATION ........................... 59

3.4.3 SMPS DESCRIPTION AND THEORY ....................... 61

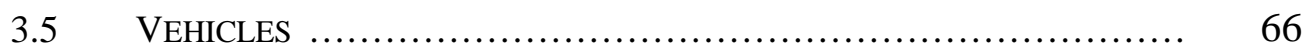

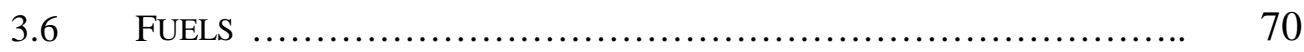

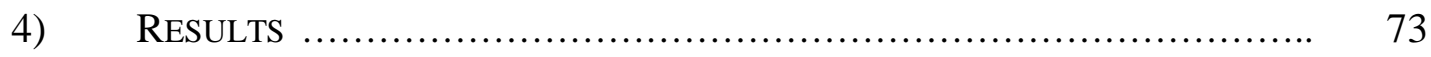

4.1 MOUDI DATA REDUCTION TECHNIQUES $\ldots \ldots \ldots \ldots \ldots \ldots \ldots \ldots \ldots . \ldots \ldots$

4.2 CyClONE DATA REDUCTION TECHNIQUES ......................... 75

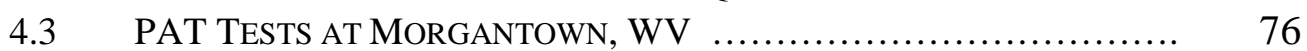

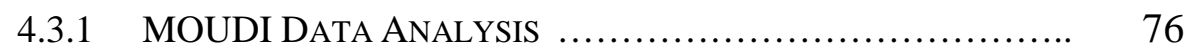

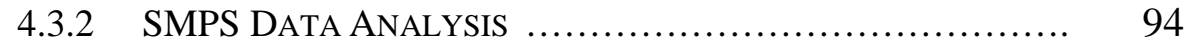

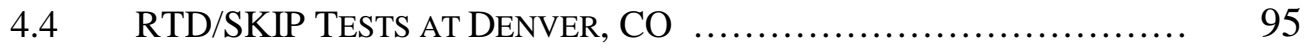

4.4.1 CyCLONE DATA ANALYSIS ................................ 95 


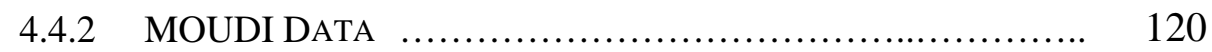

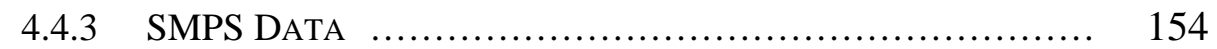

$4.5 \quad$ DART TESTS AT DALLAS, TX .................................. 175

4.5.1 CYCLONE DATA ........................................... 175

4.5.2 MOUDI DATA ............................................ 197

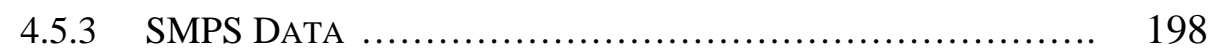

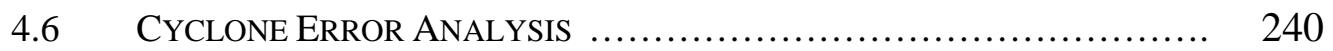

4.6.1 EQUATIONS FOR ANISOKINETIC SAMPLING ERROR ........... 241

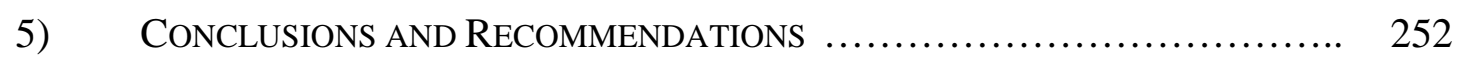

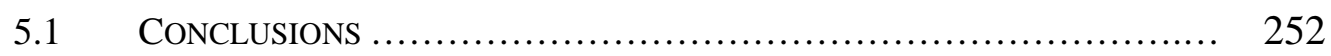

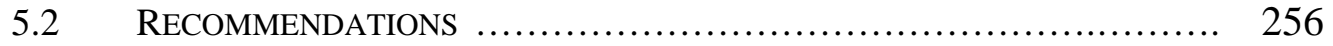

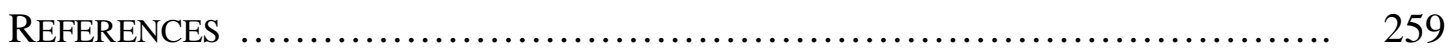

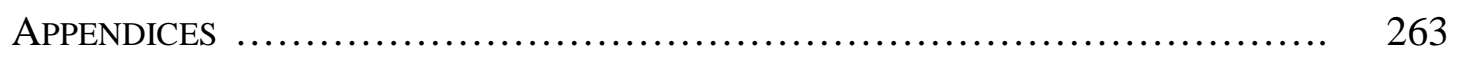

Appendix A: Cyclone Data Reduction Program ................. 264

APPENDiX B: CyClOnE DAtA SHEETS ........................... 270 


\section{LIST OF TABLES}

TABLE

PAGE

3.1 PM10 Cyclone Mass Flow Controller Calibration .............. 55

3.2 PM 2.5 Cyclone Mass Flow Controller CAlibration ............ 56

3.3 GENERAL SPECIFICATIONS OF GO BOULDER SKIP BusES .............. 67

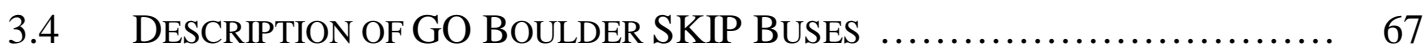

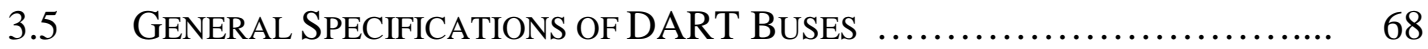

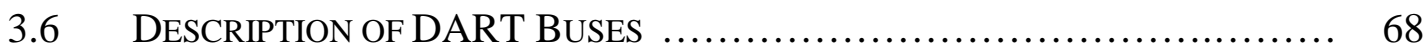

3.7 GENERAL SPECIFICATIONS OF PAT BusES ......................... 69

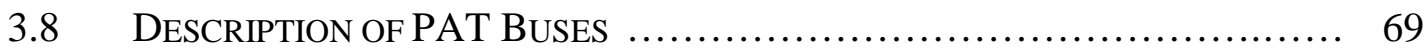

3.9 LHV COMPARISON OF D2, LNG AND CNG $\ldots \ldots \ldots \ldots \ldots \ldots \ldots \ldots \ldots \ldots \ldots$

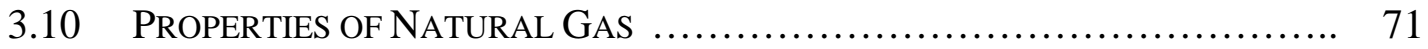

3.11 Properties of Federal Diesel And Mossgas Fuels $\ldots \ldots \ldots \ldots \ldots \ldots \ldots . . \ldots 2$

4.1 Mass Median Diameters ANd GeOMETRIC StANDARD DEVIATIONS FOR DDC 6V92TA PITTSBURgh PORT AUTHORITY TRANSIT Buses ..... 93

4.2 RTD/SKIP Bus 1012 CyCLONE DATA ............................ 117

4.3 COMPARISON OF Bus 1015 MOUDI AFTER-FILTER AND STAGE \#10 .... 138

4.4 LIST OF MOUDI DATA FROM CENTRAL BUSINESS DISTRICT CYCLES FOR RTD/SKIP CNG BUS 1015 ..................................... 145

4.5 COMPARISON OF MOUDI TEFLO AFTER-FILTER WEIGHTS FOR RTD/SKIP CNG BUSES 1015 AND 1013 vS. D2 BUS 1012 .............. 146

4.6 COMPARISON OF MOUDI STAGE \#4 WEIGHTS FOR RTD/SKIP CNG BusEs 1015 AND 1013 vs. D2 Bus 1012 .......................... 147

4.7 Mass Median AERodynamic Diameters AND GEOMETRIC STANDARD DEVIATIONS FOR DIESEL AND CNG RTD/SKIP BUSES 
4.8 MOUDI DATA FOR RTD/SKIP CNG BusES 1013 AND 1015 .......... 150

4.9 Statistical ANALYSIS OF DiESEL FuELED DART

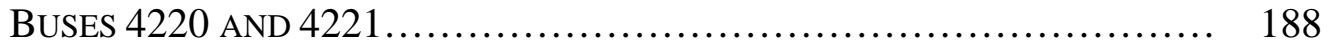

4.10 Statistical ANALYSIS OF LNG FUELED DART

BusES 4320, 4321, 4322 AND 4323 ............................ 190

4.11 Statistical ANALYSIS OF LNG FuELED DART

BusES 4324, 4325, 4328 AND 4329 ............................... 191

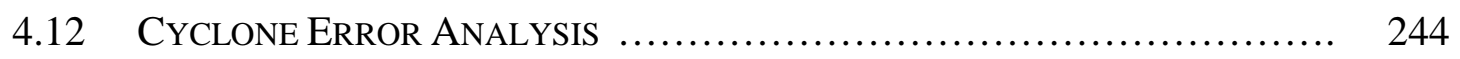




\section{LiST OF FigureS}

FIGURE

PAGE

3.1 RTD/SKIP BUS 1014 ON THE THDVETL CHASSIS DYNAMOMETER ....... 31

3.2 THE THDVETL INSTRUMENTATION TRAILER ....................... 31

3.3 SAMPLING LOCATIONS WITH CYCLONES ............................. 39

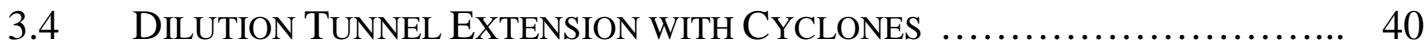

3.5 Dilution Tunnel Extension with MOUDI $\ldots \ldots \ldots \ldots \ldots \ldots \ldots \ldots \ldots . \ldots 2$

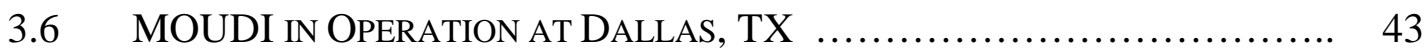

3.7 General Cyclone SetuP ....................................... 54

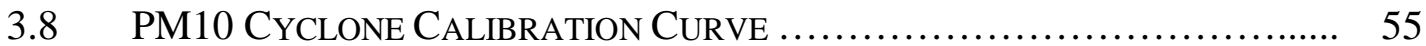

3.9 PM2.5 Cyclone Calibration Curve ............................. 56

4.1 MOUDI SizE Distribution FOR DDC 6V92TA Bus \#1 D2 Run\#1 ..... 78

4.2 MOUDI Size Distribution FOR DDC 6V92TA Bus \#1 D2 Run \#2 $\ldots . .79$

4.3 MOUDI SizE Distribution FOR DDC 6V92TA Bus \#1 D2 Run \#3 ..... 80

4.4 MOUDI SizE DistribUtion FOR DDC 6V92TA Bus \#1 MG RUN \#1 ..... 81

4.5 MOUDI Size Distribution FOR DDC 6V92TA Bus \#1 BLEND Run \#1... 82

4.6 MOUDI SizE DistribUtion FOR DDC 6V92TA Bus \#1 BLEND Run \#2... 83

4.7 MOUDI SizE Distribution FOR DDC 6V92TA Bus \#2 MG Run \#1 ..... 85

4.8 MOUDI SizE Distribution FOR DDC 6V92TA Bus \#3 MG Run \#1 ..... 86

4.9 MOUDI SizE Distribution FOR DDC 6V92TA Bus \#3 MG RUN \#2 ..... 87

4.10 MOUDI Size Distribution FOR DDC 6V92TA Bus \#3 D2 Run \#1 ..... 88

4.11 MOUDI SizE Distribution FOR DDC 6V92TA Bus \#4 D2 RUN \#1 ..... 89 
4.12 MOUDI SizE DisTRIBUTION FOR DDC 6V92TA Bus \#4 D2 RUN \#2 ...... 90

4.13 MOUDI Size DisTRIBUTION FOR DDC 6V92TA Bus \#4 MG RUN \#1 ...... 91

4.14 MOUDI Size DistRIBUTION FOR DDC 6V92TA Bus \#4 MG RUN \#2 ..... 92

4.15 15 NM SMPS PARTICLE CONCENTRATION FOR DDC 6V92TA BUS \#3

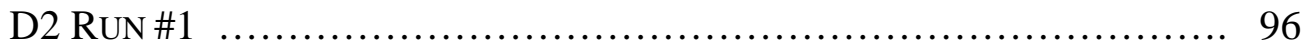

4.16 30 NM SMPS PARTICLE CONCENTRATION FOR DDC 6V92TA BUS \#3

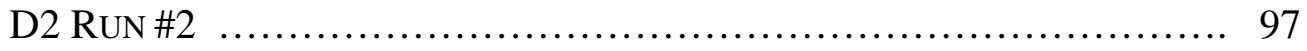

4.1750 NM SMPS PARTICLE CONCENTRATION FOR DDC 6V92TA BUS \#3 D2 RUN\#3 ...................................................... 98

4.18 100 NM SMPS PARTICLE CONCENTRATION FOR DDC 6V92TA Bus \#3

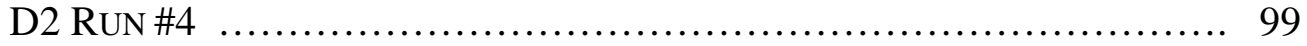

4.19 200 NM SMPS PARTICLE CONCENTRATION FOR DDC 6V92TA BUS \#3

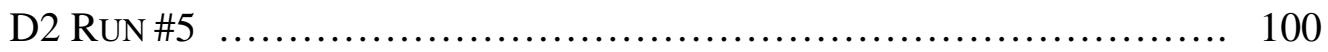

4.20 15 NM SMPS PARTICLE CONCENTRATION FOR DDC 6V92TA BUS \#3

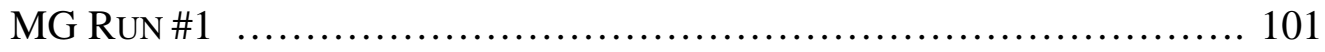

4.21 30 NM SMPS PARTICLE CONCENTRATION FOR DDC 6V92TA BUS \#3

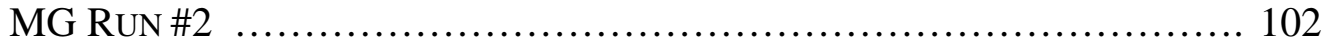

4.22 50 NM SMPS PARTICLE CONCENTRATION FOR DDC 6V92TA BUS \#3

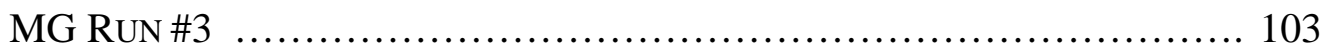

4.23 100 NM SMPS PARTICLE CONCENTRATION FOR DDC 6V92TA BUS \#3 MG RUN\#4 ..................................................... 104

4.24200 NM SMPS PARTICLE CONCENTRATION FOR DDC 6V92TA Bus \#3

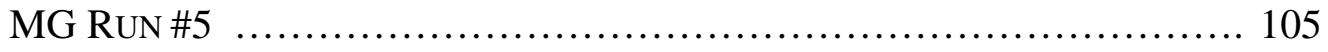

4.25 15 NM SMPS PARTICLE CONCENTRATION FOR DDC 6V92TA BuS \#4 D2 RUN\#1 ....................................................... 106

4.26 30 NM SMPS PARTICLE CONCENTRATION FOR DDC 6V92TA BUS \#4

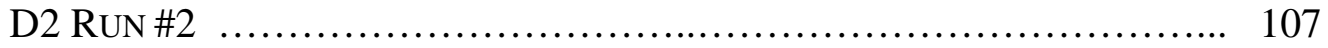

4.2750 NM SMPS PARTICLE CONCENTRATION FOR DDC 6V92TA BUS \#4

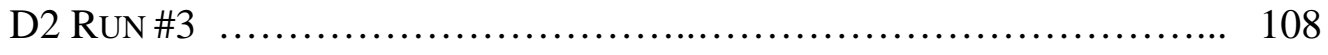


4.28 100 NM SMPS PARTICLE CONCENTRATION FOR DDC 6V92TA Bus \#4

D2 RUN \#4

4.29 200 NM SMPS PARTICLE CONCENTRATION FOR DDC 6V92TA Bus \#4 D2 RUN \#5

4.30 15 NM SMPS PARTICLE CONCENTRATION FOR DDC 6V92TA Bus \#4

MG RUN\#1

4.31 30 NM SMPS PARTICLE CONCENTRATION FOR DDC 6V92TA BUS \#4

MG RUN \#2

4.32 50 NM SMPS PARTICLE CONCENTRATION FOR DDC 6V92TA BUS \#4 MG RUN\#3

4.33 100 NM SMPS PARTICLE CONCENTRATION FOR DDC 6V92TA Bus \#4

MG RUN \#4

4.34200 NM SMPS PARTICLE CONCENTRATION FOR DDC 6V92TA Bus \#4

MG RUN \#5

4.35 URG FlOW CHART FOR PM10 CYCLONE .......................... 118

4.36 URG FlOW CHART FOR PM2.5 CYCLONE ........................... 119

4.37 MOUDI Size Distribution FOR B5.9 Bus 1010 D2 Run \#2 ......... 122

4.38 MOUDI SizE Distribution FOr B5.9 Bus 1010 D2 RUn \#3 ......... 123

4.39 MOUDI Size Distribution FOR B5.9 Bus 1011 D2 RUn \#1 ......... 124

4.40 MOUDI SizE DistRIBUTION FOR B5.9 BUS 1011 D2 RUN \#3 ......... 125

4.41 MOUDI SizE Distribution FOR B5.9 Bus 1012 D2 SS Run\#1 ......... 127

4.42 MOUDI SizE Distribution FOR B5.9 Bus 1012 D2 Run \#1 ........ 128

4.43 MOUDI Size Distribution FOR B5.9 Bus 1012 D2 RUn \#3 ......... 129

4.44 MOUDI Size Distribution fOR B5.9 Bus 1012 D2 Run \#5 ......... 130

4.45 MOUDI Size DistribUtion FOR B5.9 Bus 1012 D2 RUn \#6 ......... 131

4.46 MOUDI Size Distribution FOR B5.9 Bus 1012 D2 Run \#7 ......... 132

4.47 MOUDI SizE Distribution FOR B5.9 Bus 1012 D2 Run \#9 ......... 133 
4.48 MOUdi Size Distribution For B5.9 Bus 1012 D2 Run \#10 .......... 134

4.49 MOUDI Size Distribution For B5.9 Bus 1013 CNG Run \#1 .......... 136

4.50 MOUDI Size DistRIBUTION FOR B5.9 Bus 1013 CNG RUn \#3 _......... 137

4.51 MOUDI Size Distribution FOR B5.9 Bus 1015 CNG Run \#2 …...... 139

4.52 MOUDI SizE DistRIBUTION FOR B5.9 Bus 1015 CNG RUn \#3 …...... 140

4.53 MOUDI Size Distribution For B5.9 Bus 1015 CNG Run \#4 .......... 141

4.54 MOUDI Size DistRIBUTION FOR B5.9 Bus 1015 CNG Run \#5 …...... 142

4.55 MOUDI Size DistribUtion For B5.9 Bus 1015 CNG Run \#6 .......... 143

4.56 MOUDI Size Distribution FOR B5.9 Bus 1015 CNG Run \#7 .......... 144

4.57 COMPARISON OF MOUDI StAGE \#4 FOR RTD/SKIP BUSES ............... 148

4.58 COMPARISON OF MOUDI AFTER-FILTER FOR RTD/SKIP BUSES $\ldots \ldots \ldots . . .148$

4.59 20 NM SMPS PARTICLE CONCENTRATION FOR B5.9 Bus 1010 D2 ….... 156

4.60 50 NM SMPS PARTICLE CONCENTRATION FOR B5.9 BUS 1010 D2 ….... 157

4.61 100 NM SMPS PARTICLE CONCENTRATION FOR B5.9 BUS 1010 D2 …... 158

4.62 150 NM SMPS PARTICLE CONCENTRATION FOR B5.9 Bus 1010 D2 ........ 159

4.63 200 NM SMPS PARTICLE CONCENTRATION FOR B5.9 BUS 1010 D2 ….... 160

4.64 10 NM SMPS PARTICLE CONCENTRATION FOR B5.9 Bus 1011 D2 ….... 161

4.65 20 NM SMPS PARTICLE CONCENTRATION FOR B5.9 BUS 1011 D2 ….... 162

4.66 50 NM SMPS PARTICLE CONCENTRATION FOR B5.9 BUS 1011 D2 …..... 163

4.67 100 NM SMPS PARTICLE CONCENTRATION FOR B5.9 BUS 1011 D2 _....... 164

4.68 150 NM SMPS PARTICLE CONCENTRATION FOR B5.9 Bus 1011 D2 …..... 165

4.69 200 NM SMPS PARTICLE CONCENTRATION FOR B5.9 Bus 1011 D2 …..... 166

4.70 20 NM SMPS PARTICLE CONCENTRATION FOR B5.9 BUS 1012 D2 …...... 168 
4.71 60 NM SMPS PARTICLE CONCENTRATION FOR B5.9 BUS 1012 D2 …....... 169

4.72 100 NM SMPS PARTICLE CONCENTRATION FOR B5.9 Bus 1012 D2 ….... 170

4.73 150 NM SMPS PARTICLE CONCENTRATION FOR B5.9 BUS 1012 D2 ….......171

4.7460 NM SMPS PARTICLE CONCENTRATION FOR B5.9 Bus 1012 D2 …...... 172

4.75 100 NM SMPS PARTICLE CONCENTRATION FOR B5.9 BUS 1012 D2 …..... 173

4.76100 NM SMPS PARTICLE CONCENTRATION FOR B5.9 BUS 1012 D2 …...... 174

4.77 15 NM SMPS PARTICLE CONCENTRATION FOR B5.9 BUS 1013 CNG ........ 176

4.78 25 NM SMPS PARTICLE CONCENTRATION FOR B5.9 BUS 1013 CNG ....... 177

4.79 35 NM SMPS PARTICLE CONCENTRATION FOR B5.9 Bus 1013 CNG ….... 178

4.80 45 NM SMPS PARTICLE CONCENTRATION FOR B5.9 BUS 1013 CNG ........ 179

4.81 55 NM SMPS PARTICLE CONCENTRATION FOR B5.9 BUS 1013 CNG ........ 180

4.82 15 NM SMPS PARTICLE CONCENTRATION FOR B5.9 BUS 1015 CNG ........ 181

4.8331 NM SMPS PARTICLE CONCENTRATION FOR B5.9 Bus 1015 CNG ........ 182

4.8415 NM SMPS PARTICLE CONCENTRATION FOR B5.9 Bus 1015 CNG ........ 183

4.85 31 NM SMPS PARTICLE CONCENTRATION FOR B5.9 Bus 1015 CNG ........ 184

4.8650 NM SMPS PARTICLE CONCENTRATION FOR B5.9 BUS 1015 CNG ….... 185

4.87 PM10 CyClone Flow RATES For LNG DART Buses 4323 AND 4329 ... 193

4.88 PM2.5 CyClONE Flow RATE FOR LNG DART BUSES 4323 AND 4329... 194

4.89 PM10 AND PM2.5 CyClone Flow RATE FOR LNG DART Bus 4328_.... 195

4.90 15 NM SMPS PARTICLE CONCENTRATION FOR M11 D2 Bus $4220 \ldots \ldots \ldots . .199$

4.9135 NM SMPS PARTICLE CONCENTRATION FOR M11 D2 Bus $4220 \quad \ldots \ldots \ldots . .200$

4.92 50 NM SMPS PARTICLE CONCENTRATION FOR M11 D2 Bus $4220 \ldots \ldots \ldots . .201$

4.9380 NM SMPS PARTICLE CONCENTRATION FOR M11 D2 BUS $4220 \ldots \ldots \ldots . .202$ 
4.94150 NM SMPS PARTICLE CONCENTRATION FOR M11 D2 BUS $4220 \ldots \ldots \ldots .203$

4.95 10 NM SMPS Particle ConCENTRATION FoR M11 D2 Bus $4221 \ldots \ldots \ldots .204$

4.9620 NM SMPS PARTICLE CONCENTRATION FOR M11 D2 Bus $4221 \ldots \ldots \ldots . \quad 205$

4.97 33 NM SMPS PARTICLE CONCENTRATION FOR M11 D2 BUS $4221 \ldots \ldots \ldots .206$

4.98 55 NM SMPS PARTICLE CONCENTRATION FOR M11 D2 Bus $4221 \ldots \ldots \ldots . .207$

4.99150 NM SMPS PARTICLE CONCENTRATION FOR M11 D2 BUS $4221 \ldots \ldots . .208$

4.100 15 NM SMPS PARTICLE CONCENTRATION FOR L10 LNG BUS 4321 ........ 210

4.101 20 NM SMPS PARTICLE CONCENTRATION FOR L10 LNG BUS 4321 ........ 211

4.10230 NM SMPS PARTICLE CONCENTRATION FOR L10 LNG BUS 4321 ........ 212

4.103 40 NM SMPS PARTICLE CONCENTRATION FOR L10 LNG BUS 4321 ........ 213

4.104 60 NM SMPS PARTICLE CONCENTRATION FOR L10 LNG BUS 4321 ........ 214

4.105 15 NM SMPS PARTICLE CONCENTRATION FOR L10 LNG BUS 4329 ......... 215

4.106 25 NM SMPS PARTICLE CONCENTRATION FOR L10 LNG BUS 4329 ........ 216

4.107 33 NM SMPS PARTICLE CONCENTRATION FOR L10 LNG BUS 4329 ........ 217

4.108 43 NM SMPS PARTICLE CONCENTRATION FOR L10 LNG BUS 4329 ......... 218

4.109 55 NM SMPS PARTICLE CONCENTRATION FOR L10 LNG BUS 4329 ........ 219

4.110 20 NM SMPS PARTICLE CONCENTRATION FOR L10 LNG BUS 4320 ......... 221

4.111 30 NM SMPS PARTICLE CONCENTRATION FOR L10 LNG BUS 4320 ........ 222

4.11240 NM SMPS PARTICLE CONCENTRATION FOR L10 LNG BUS 4320 ........ 223

4.113 50 NM SMPS PARTICLE CONCENTRATION FOR L10 LNG BUS 4320 ......... 224

4.114 10 NM SMPS PARTICLE CONCENTRATION FOR L10 LNG BUS 4323 ......... 225

4.11520 NM SMPS PARTICLE CONCENTRATION FOR L10 LNG BUS 4323 ......... 226

4.116 30 NM SMPS PARTICLE CONCENTRATION FOR L10 LNG BUS 4323 ........ 227 
4.11740 NM SMPS PARTICLE CONCENTRATION FOR L10 LNG BuS 4323 ....... 228

4.118 50 NM SMPS PARTICLE CONCENTRATION FOR L10 LNG Bus 4323 ........ 229

4.119 10 NM SMPS PARTICLE CONCENTRATION FOR L10 LNG BuS 4325 ....... 230

4.120 15 NM SMPS PARTICLE CONCENTRATION FOR L10 LNG BuS 4325 ........ 231

4.121 20 NM SMPS PARTICLE CONCENTRATION FOR L10 LNG BuS 4325 ........ 232

4.122 30 NM SMPS PARTICLE CONCENTRATION FOR L10 LNG Bus 4325 ........ 233

4.12345 NM SMPS PARTICLE CONCENTRATION FOR L10 LNG BuS 4325 ........ 234

4.124 20 NM SMPS PARTICLE CONCENTRATION FOR L10 LNG Bus 4328 ........ 235

4.125 25 NM SMPS PARTICLE CONCENTRATION FOR L10 LNG Bus 4328 ........ 236

4.126 33 NM SMPS PARTICLE CONCENTRATION FOR L10 LNG Bus 4328 ........ 237

4.127 45 NM SMPS PARTICLE CONCENTRATION FOR L10 LNG BuS 4328 ........ 238

4.12860 NM SMPS PARTICLE CONCENTRATION FOR L10 LNG Bus 4328 ........ 239 


\section{NOMENCLATURE}

$\begin{array}{ll}\text { CNG } & \text { Compressed Natural Gas } \\ \text { LNG } & \text { Liquefied Natural Gas } \\ \text { SMPS } & \text { Scanning Mobility Particle Sizer } \\ \text { MOUDI } & \text { Micro-orifice Uniform Deposit Impactor } \\ \text { RTD } & \text { Rapid Transit District } \\ \text { PAT } & \text { Port Authority Transit } \\ \text { DART } & \text { Dallas Area Rapid Transit } \\ \text { PM2.5 } & \text { Cyclone with 2.5 } \mu \text { m particle diameter cut-point } \\ \text { PM10 } & \text { Cyclone with 10 } \mu \text { m particle diameter cut-point } \\ \text { MMAD } & \text { Mass Median Aerodynamic Diameter } \\ \text { GSD } & \text { Geometric Standard Deviation } \\ \text { NGV } & \text { Natural Gas Vehicle } \\ \text { TSP } & \text { Total Suspended Particulate } \\ \text { EPA } & \text { Environmental Protection Agency } \\ \text { PM } & \text { Particulate Matter } \\ \text { TPM } & \text { Total Particulate Matter } \\ \text { CPC } & \text { Condensation Particle Counter } \\ \text { THDVETL } & \text { Transportable Heavy Duty Vehicle Emissions Testing Laboratory } \\ \text { D2 } & \text { Diesel Fuel No. } 2 \\ \text { MG } & \text { Mossgas } \\ \text { IARC } & \text { International Agency for Research on Cancer } \\ \end{array}$




\begin{tabular}{|c|c|}
\hline NIOSH & National Institute for Occupational Safety and Health \\
\hline WHO & World Health Organization \\
\hline HEI & Health Effects Institute \\
\hline SOF & Soluble Organic Fraction \\
\hline CONCAWE & Conservation of Clean Air and Water in Europe \\
\hline $\mathrm{CNC}$ & Condensation Nucleus Counter \\
\hline$d N / d \log \left(d_{p}\right)$ & Number Weighted Distribution Over Logarithmic Particle Range \\
\hline $\mathrm{dm} / \mathrm{d} \log \left(\mathrm{d}_{\mathrm{p}}\right)$ & Mass Weighted Distribution Over Logarithmic Particle Range \\
\hline$d_{b}$ & Mobility Equivalent Diameter \\
\hline $\mathrm{d}_{\mathrm{m}}$ & Mass Equivalent Diameter \\
\hline $\mathrm{C}$ & Cunningham Slip Correction Factor \\
\hline$\chi$ & Dynamic Shape Factor \\
\hline$\rho_{\mathrm{p}}$ & Inherent Material Density \\
\hline$\rho_{\mathrm{e}}$ & Effective Density \\
\hline$d_{p, \max }$ & Maximum Particle Diameter \\
\hline $\mathrm{d}_{\mathrm{p}, \min }$ & Minimum Particle Diameter \\
\hline $\operatorname{Pr}$ & Road Load Power \\
\hline $\mathrm{V}$ & Vehicle Speed \\
\hline$\rho_{\mathrm{a}}$ & Density of Air \\
\hline$C_{D}$ & Drag Coefficient \\
\hline A & Frontal Area of Vehicle \\
\hline $\mathrm{C}_{\mathrm{r}}$ & Coefficient of Rolling Resistance \\
\hline M & Vehicle Mass \\
\hline
\end{tabular}




\begin{tabular}{|c|c|}
\hline $\mathrm{g}$ & Gravitational Force \\
\hline $40 \mathrm{CFR}$ & 40 Code of Federal Regulations \\
\hline CFV & Critical Flow Venturi \\
\hline CVS & Constant Volume Sampler \\
\hline Q & Venturi Flow Rate \\
\hline $\mathrm{K}_{\mathrm{v}}$ & Venturi Calibration Constant \\
\hline $\mathrm{P}$ & Actual Absolute Pressure \\
\hline $\mathrm{T}$ & Actual Absolute Temperature \\
\hline $\operatorname{Re}$ & Reynolds Number \\
\hline$\rho$ & Fluid Density \\
\hline $\mathrm{V}_{\mathrm{o}}$ & Mean Velocity at the Nozzle Throat \\
\hline $\mathrm{W}$ & Nozzle Diameter \\
\hline$\mu$ & Air Viscosity \\
\hline Stk & Stokes Number \\
\hline$\rho_{\mathrm{p}}$ & Particle Density \\
\hline $50 d_{a e}$ & Value of Particle Diameter Collected with $50 \%$ Efficiency \\
\hline Stk $_{50}$ & Critical Stokes Number \\
\hline $\mathrm{n}$ & Number of Jets \\
\hline $\mathrm{F}$ & Centrifugal Force \\
\hline $\mathrm{r}$ & Radius of Curvature of the Particle Trajectory \\
\hline $\mathrm{t}_{\mathrm{r}}$ & Retention Time \\
\hline $\mathrm{u}_{\mathrm{c}}$ & Inlet Velocity \\
\hline $\mathrm{u}_{\mathrm{t}}$ & Tangential Particle Velocity \\
\hline
\end{tabular}




\begin{tabular}{|c|c|}
\hline $\mathrm{R}$ & Radius of Cyclone \\
\hline $\mathrm{N}_{\mathrm{t}}$ & Number of Revolutions \\
\hline$\tau$ & Relaxation Time \\
\hline $\mathrm{x}$ & Distance of Air Stream \\
\hline $\mathrm{B}_{\mathrm{c}}$ & Time to Travel Distance of Air Stream \\
\hline$\tau_{100}$ & Relaxation Time of the Smallest Particles that can be Collected \\
\hline$\varepsilon$ & Efficiency of the Cyclone \\
\hline DMA & Differential Mobility Analyzer \\
\hline$Z_{p}$ & Electrical Mobility \\
\hline $\mathrm{n}$ & Number of Elementary Charges on the Particle \\
\hline e & Elementary Charge \\
\hline $\mathrm{q}_{\mathrm{sh}}$ & Sheath Air Flow Rate \\
\hline $\mathrm{q}_{\mathrm{a}}$ & Aerosol Flow Rate \\
\hline$r_{1}$ & Inner Radius of Annular Space \\
\hline$r_{2}$ & Outer Radius of Annular Space \\
\hline $\mathrm{V}$ & Average Voltage of Collector Rod \\
\hline $\mathrm{L}$ & Characteristic Length \\
\hline S & Saturation Ratio \\
\hline M & Molecular Weight \\
\hline $\mathrm{R}$ & Universal Gas Constant \\
\hline$d^{*}$ & Kelvin Diameter \\
\hline LEV & Low Emitting Vehicle \\
\hline $\mathrm{hp}$ & Horsepower \\
\hline
\end{tabular}




\begin{tabular}{|c|c|}
\hline rpm & Revolutions Per Minute \\
\hline LHV & Lower Heating Value \\
\hline HHV & Higher Heating Value \\
\hline Stoich. & Stoichiometric \\
\hline ECD & Equivalent Cut Diameter \\
\hline$f(d)$ & Frequency Function of Lognormal Curve \\
\hline $\exp$ & Exponential \\
\hline$\sigma g$ & Geometric Standard Deviation \\
\hline $\mathrm{E}$ & Error \\
\hline DF & Dilution Factor \\
\hline $\mathrm{M}_{\text {actual }}$ & Actual Mass \\
\hline$\%$ Diff & Percent Difference \\
\hline CBD & Central Business District \\
\hline DDC & Detroit Diesel Corporation \\
\hline $1 \mathrm{pm}$ & Liters Per Minute \\
\hline slpm & Standard Liters Per Minute \\
\hline $\mathrm{V}_{\text {actual }}$ & Actual Volume \\
\hline $\mathrm{T}_{\mathrm{stp}}$ & Temperature at Standard Conditions \\
\hline $\mathrm{P}_{\mathrm{stp}}$ & Pressure at Standard Conditions \\
\hline $\mathrm{V}_{\mathrm{stp}}$ & Volume at Standard Conditions \\
\hline $\mathrm{AF}$ & MOUDI After-Filter \\
\hline S10 & MOUDI Stage \#10 \\
\hline $\mathrm{CV}$ & Coefficient of Variance \\
\hline
\end{tabular}




$\begin{array}{ll}\mathrm{u} & \text { Dilution Tunnel Stream Velocity } \\ \mathrm{R} & \text { Sampling Tube Radius } \\ \mathrm{T}_{\mathrm{o}} & \text { Standard Absolute Temperature } \\ \mathrm{P}_{\mathrm{o}} & \text { Standard Absolute Temperature } \\ \mu_{\mathrm{o}} & \text { Viscosity at Standard Absolute Temperature } \\ \lambda & \text { Mean Free Path } \\ \lambda_{\mathrm{o}} & \text { Mean Free Path at Standard Pressure and Temperature } \\ \alpha & \text { Inertial Fraction } \\ \mathrm{c} & \text { Sample Concentration } \\ \mathrm{c}_{\mathrm{o}} & \text { Stream Concentration } \\ \mathrm{u} & \text { Sampling Tube Inlet Velocity } \\ \mathrm{RH} & \text { Relative Humidity } \\ \Phi_{\mathrm{dil}} & \text { Flow Rate of Constant Volume Sampler } \\ & \end{array}$




\section{ChAPTER 1 - INTROdUCTION}

Born from necessity, the past two decades have been witness to studies, experiments and inventions in order to understand and limit the amount of particulate matter exhausted from automobiles. A multitude of epidemiological studies have been conducted to determine the respiratory health-related effects attributed to total suspended particles and size distinct finer fractions [Monn et al., 1995]. As associations between particulate matter and respiratory disorders are evidenced more clearly, the need to fully understand and quantify vehicle particle emissions becomes crucial.

Common sense tells us that breathing polluted air will be detrimental to our health. Science found the reason. Several of the leading safety and health institutes have addressed scientifically what logic perforce infers. The National Institute for Occupational Safety and Health (NIOSH), the International Agency for Research on Cancer (IARC), the Health Effects Institute (HEI), the World Health Organization (WHO) - all these have detailed and concluded the existence of potential health effects from exposure to diesel exhaust [Kittelson et al., 1998].

Total suspended particulate (TSP) has long been believed to cause lung and other respiratory disorders. The main focus of early research was on the associated health effects caused by industry and their plumes of thick black smoke. Coal dust and steel mill pollution are obvious. It wasn't until 1977 that regulations were passed in Congress on the less obvious, though more ubiquitous vehicle particulate emissions. TSP became, and still is, the Environmental Protection Agency's (EPA) standard for regulatory codes. Since the initial investigations and subsequent restrictions, vehicles have become 
"cleaner," that is, they produce less total particulate matter (TPM) emissions. However, recent research has focused less on the total mass emitted. The trend is now to study the number of particles, as this is seen as a more critical factor in determining the adverse health effects attributed to particulate matter (PM).

Ultrafine PM is classified as particles having a diameter of $0.1 \mu \mathrm{m}$ or less. Ultrafine particles are more toxic than fine or coarse particles because they are able to reach the deep lung. Studies have shown that the toxicity level of a particle increases as its size decreases. This is due to the fact that smaller particles penetrate tissue barriers easier, have greater surface area per unit mass and are more soluble than larger particles [Mauderly et al., 1998]. Concurrently, for the same mass a shift in particle diameter from $1.0 \mu \mathrm{m}$ to $0.1 \mu \mathrm{m}$ increases the number of particles by approximately 1000 . Clearly, particles substantially less than $0.1 \mu \mathrm{m}$ in aerodynamic diameter will significantly increase the total number of particulates.

Reductions in total particulates have occurred ever since initial regulations took effect. One method in which this has been accomplished is increased injection pressures for diesel engines. The fuel spray is made more homogeneous which therefore reduces locally rich sites in the combustion chamber. While increasing efficiency and decreasing total particulate mass, the size of the particles may decrease due to the finer droplets [Johnson et al., 1994]. This in turn could lead to higher number concentrations in the exhaust aerosol. Another course for decreasing total particulate mass is using an alternative fuel source - natural gas. Compressed and liquefied natural gas fueled engines have significantly reduced total mass emission rates, but at the same time, a larger portion 
of this total is ultrafine. The drive toward lowered total mass output may lead to an increase in the toxicity of the ambient air.

Diesel is the common fuel choice for heavy duty vehicles due to its thermal efficiency, availability and cost. However, diesel fuel combustion has shown to produce the largest amount of PM mass (compared to gasoline and alternative fuels). As mentioned, natural gas fueled vehicles have much lower PM mass emissions than those powered by diesel. Synthetic fuels, such as those refined by the Fischer-Tropsch process are gaining acceptance as a viable alternative as well, but little research has been done on PM emissions. The obvious question is why not use one of these "cleaner" alternative fuels? Beside the supply, cost, availability, and other distribution-related issues, the toxicity of emissions from vehicles powered by these fuels remains a largely unanswered question. The thrust of this study is to investigate the PM mass and size emissions attained from all these fuels.

As associations between particulate matter and respiratory disorders become clearer and grow stronger, classification of particles becomes more vital. While the focus has shifted to particle size and number, mass must not be overlooked. These three properties are all necessary for a clear and comprehensive analysis of PM vehicle emissions. Investigations that solely focus on number do not portray the entire spectrum; similarly, those that concentrate only on mass elide an increasingly important aspect in fully understanding particulate matter.

There are several instruments commercially available for measuring particles with respect to size and mass. Four were chosen to give a comprehensive view of diesel and alternative fuel exhaust aerosols - PM2.5 and PM10 cyclones, a Micro-orifice Uniform 
Deposit Impactor (MOUDI) and a Scanning Mobility Particle Sizer (SMPS), which consists of an electrostatic classifier in conjunction with a Condensation Particle Counter (CPC). These instruments have inherently different theoretical operations and classification techniques, but when combined, can fully describe the size and mass of particles.

A cyclone is an inertial particle separator which uses centrifugal forces to remove heavier particles from a gas stream. The sample radially enters the cyclone, where it spirals downward along the tapered cylinder casing in solid body rotation. While making these revolutions, the particles are accelerated outward to the walls where they swirl down to a hopper at the bottom. The motion of the gases consists of an inner vortex moving toward the exit and subsequently to a filter, and an outer vortex moving in the opposite direction, which contains the heavier particles.

A vacuum pump was used to draw the required flow through the cyclone body and in-line filter. The URG cyclones employed in this study operated at 16.7 liters per minute (for the PM2.5 cyclone) and 28.3 liters per minute (for the PM10). Two filters were used to capture the smaller particles which bypassed the collection hopper at the bottom of the cyclone due to their lower inertia. These particles remain in the air stream and are subsequently taken into the inner vortex where they then exit the cyclone. The weight of the filters yielded the mass of particles less than $10 \mu \mathrm{m} / 2.5 \mu \mathrm{m}$, respectively. Cyclones were chosen for this $50 \mathrm{~d}_{\mathrm{ae}}$ (aerodynamic diameter at $50 \%$ efficiency) gravimetric method, rather than a purely total particulate matter (TPM) measurement system. The filters yielded size selective mass ranges of particles less than $2.5 \mu \mathrm{m}$ and $2.5 \mu \mathrm{m}<\mathrm{d}_{\mathrm{p}}<10 \mu \mathrm{m}$ through the difference in the two cyclones. 
The MOUDI is a ten-stage cascade impactor that classifies particles by their aerodynamic diameter in the range of $18 \mu \mathrm{m}$ to $0.056 \mu \mathrm{m}$. The advantage the MOUDI has over other cascade type impactors is its collection of small particles with a moderate pressure drop and uniform deposit. With these features, it is well suited to measure diluted engine exhaust particulate matter. The MOUDI was chosen because it, like the cyclones, can separate particles in prescribed size ranges with the final result being a mass based description with respect to size. It provides a necessary junction between the cyclones and the SMPS.

The SMPS determines particle size distributions by classifying particles according to their electrical mobilities. The electrical mobility equivalent diameter measured is the diameter of a sphere that has the same dynamic mobility as the particle of concern. The aerosol is charged according to the Boltzmann distribution of charge. The recorded signal during a scan corresponds to the actual number-size distribution as calculated by the total particulate flux. A narrow range of the electrical mobilities of positive ions passes through the Differential Mobility Analyzer and exits as a monodisperse aerosol [Greenwood et al., 1996]. This aerosol then enters the CPC where each particle is counted. This system allows for precise size selective number distributions of submicron particles, which in combination with the MOUDI and cyclones, encompasses the entire spectrum of particulate matter size and mass.

A series of emissions measurements were conducted over a broad range of fuels to investigate the changing nature of particles. Since most current research has focused on diesel fueled engines and is fairly well documented, tests performed on diesel vehicles in the laboratories can be used as a validation of the techniques employed in this study. 
This base data was also used as a reference for all the variations in test conditions, such as fuel type, sampling method, sampling location and exhaust conditioning. Testing was performed on Detroit Diesel 6V92TA Port Authority Transit (PAT) buses with the Transportable Heavy Duty Vehicle Emissions Testing Laboratory (THDVETL). These tests comprised three fuel types (Diesel \#2, Mossgas, 50\%Diesel \#2 /50\% Mossgas) with particulate measurements made using the MOUDI and SMPS. Also with THDVETL, Cummins ISB5.9-175 and B5.9-G Rapid Transit District (RTD) buses were tested on Diesel \#2 (D2) and Compressed Natural Gas (CNG). For these, the MOUDI, SMPS, PM2.5 cyclone and PM10 cyclone were used to determine the effects on PM by the different fuels. The final tests were conducted on Cummins L10-G and M11 Dallas Area Rapid Transit (DART) buses with the transportable laboratory using the SMPS, PM2.5 cyclone and PM10 cyclone on D2 and Liquefied Natural Gas (LNG).

Chapter 2 will discuss previous research performed in the area of particle size and concentration, and mass emissions measurement and analysis. This includes healthrelated effects associated with particles, formation characteristics and recent studies conducted with the SMPS and MOUDI. Chapter 3 details the experimental procedures used as well as the theoretical operation of the instrumentation used in this study. Also detailed in this section is the laboratory setup and operation, including the vehicles and fuels tested. Chapter 4 is a presentation of the data and discussion of all findings. Also analyzed are sources of error and uncertainty. Chapter 5 presents the conclusions reached and proffers recommendations for modifications to testing procedure for any future research performed. 


\section{Chapter 2 - Literature ReVIEW}

Air pollution has become one of the largest dilemmas facing the world as we enter the twenty-first century. Urban skylines are often obscured by thick, brown smog; national parks are following suit and eastern states are polluted from sites hundreds of miles away. Vehicles, spewing every major air pollutant, are a large contributor to this ever increasing problem. One of the most pernicious is particulate matter, as it not only affects the respiratory system, but also acts as a transport for other toxic chemicals which condense upon this substrate. The Environmental Protection Agency (EPA) has recognized PM as a dangerous element abounding in the ambient air and continues to strengthen regulations on vehicle emission levels of PM.

The presence of post-combustion particles has long been known and research has been conducted over the years in order to find the means to accurately measure PM. As recent health studies indicate [IARC, 1989; HEI, 1995; NIOSH, 1988], those measurements must also include the size of the particles, not only the mass. Several researchers have taken this one step further and have studied the processes a particle undergoes once it enters the atmosphere. This is necessary to understanding the size/mass properties of particles and crucial to proper measurement. Several instruments have been developed to perform these measurements and have either been widely tested and/or gaining acceptance.

This chapter details a selection of research studies that have addressed these issues. Specifically, the focus is on health issues, post-combustion particle formation and experimentation conducted. 


\subsection{Health Related Effects}

Particle deposition in the lung is difficult to accurately predict due to the myriad of variables which must be accounted for. These include air intake and exhalation rates, inhalation method (nose or mouth), geometry of the airways and relative humidity levels seen by the particle. Clearly, these parameters will vary between individuals, but generalized models have been developed to best describe the path of a particle and the effect size has upon this course (the term size refers to the aerodynamic diameter of a particle).

It has been determined that particles which deposit at any site in the respiratory system can initiate or exacerbate a biological response. This response is classified as a lung disease [Lippmann et al., 1983]. The single most dominant parameter for predicting whether a particle will deposit in the lung is the aerodynamic equivalent diameter $50 \mathrm{~d}_{\mathrm{ae}}$, which is defined as the diameter of a smooth, unit density sphere that has the same settling velocity as the particle. For certain size ranges of inhaled particles, the deposition sites have been classified.

Large, coarse particles (those greater than $10 \mu \mathrm{m}$ in diameter) do not enter the respiratory tract beyond the entranceways. While nasal passages collect particles more effectively than oral, particles of this size and larger are prevented from further penetration regardless of the inhalation mechanism [Lippmann et al., 1983].

Fine particles are defined as those that have an aerodynamic diameter larger than 0.1

$\mu \mathrm{m}$ but less than $2.5 \mu \mathrm{m}$. However, Swift (1983) states that there is neither a 
physiological basis nor any epidemiological studies which point toward $2.5 \mu \mathrm{m}$ being of any greater importance than say $2.0 \mu \mathrm{m}$ or $3.0 \mu \mathrm{m}$. This size was chosen strictly on the basis of outdoor aerosol chemistry. Lippmann, Gurman and Schlesinger (1983) found $3.0 \mu \mathrm{m}$ to be a more representative diameter for the fine definition. They show that particles above this size have less pulmonary deposition due to diminished penetration. Particles in this approximate size range predominantly deposit due to impaction in the tracheobronchial region and more specifically, large bronchial airways [Martonen and Lowe, 1983]. By their size and according mass, these particles are small enough to bypass collection but have sufficient momentum to escape the streamlines and therefore impact. This type of deposition also accounts for particle behavior in the larynx. Therefore, the upper respiratory tract is most affected by fine particles because of the inertias of these heavier particles. Prolonged exposure to particles of this size has been shown to cause bronchial cancers [Lippmann et al., 1983]. Martonen and Lowe (1983) also found that the efficiency of particle deposition in the tracheobronchial region correlates with the frequency of involvement as areas of bronchial carcinoma.

For the bronchial and upper respiratory tract, there are three diseases that have been associated with particulate matter - asthma, bronchitis and cancer [Stern et al., 1973]. Asthma is caused by an immune system response in which the airways narrow due to a swelling of tissue and constriction of muscles. This response occurs when the presence of hydrocarbons is detected. As particles deposit in the airways, the hydrocarbons that have condensed upon the particle surface begin to solubilize, causing the immune system to induce a thinning of the air passages in order to restrict the amount of harmful hydrocarbons that can enter the respiratory system. Not only can the inhalation of 
particulate matter exacerbate a preexisting condition, but it can also initiate asthma problems.

Bronchitis has been shown to occur as an irritation response to deposited PM [Stern et al., 1973]. The natural reaction for this area of the respiratory system is to produce mucus when particles enter the bronchial region. The mucus is then cleared to the esophagus and trachea, where it can be expelled. If this response is repeated due to prolonged exposure, the airways of the bronchi begin to fill as the mucus is produced in excess. PM has not been proven to instigate bronchitis, but it does worsen preexisting conditions [Stern et al., 1973].

The last and most serious disease linked to PM is cancer [Stern et al., 1973]. Cells can be penetrated by particles, and the condensate which adheres to the surface of the particles can solubilize. As cells attempt to repair the damage incurred, some of the responses may be prone to error. These injured cells divide and can possibly mutate due to a quickened division. This can lead to DNA errors that cause the cells to perform improperly. At localized sites, these damaged cells function with the mutated DNA and develop into tumors.

In all three of these lung disorders, it is the body's response to the foreign particle that leads to the most severe disorders. This shows that short-term exposure may not influence these responses to the point of affliction, but long-term exposure may degrade the body's natural defense mechanisms to the point where these reactions begin to harm rather than protect.

As particle size decreases, the potential for penetration increases; this is due to the fact that finer particles can more readily enter cells and compromise tissue barriers. Once 
penetration has occurred, these small particles have inherent properties which heighten toxicity. Per unit mass, finer particles have more surface area than larger ones, and they also dissolve more rapidly [Mauderly et al., 1998]. Gases that condense upon the outer surface can thus more easily enter the system. This will enhance the bioavailability of solubilized agents [Mauderly et al., 1998].

For particles in the size range of $0.5 \mu \mathrm{m}$ to approximately $2.0 \mu \mathrm{m}$, sedimentation is the mode of deposition dominant in the tracheobronchial region [Lippmann et al., 1983]. Particles that fall within this size range have lower inertia, so they will move farther through the region. They also remain in the stream rather than impacting against a surface. However, the particles in the aerosol reach their terminal settling velocity and can no longer remain airborne [Cadle, 1975]. At this point, they will fall and deposit in the pulmonary region. Translocation may then occur, which will effectively disperse some of the sediment throughout the entire system through mucus clearance [Lippmann et al., 1983]. Particles below this prescribed lower limit cannot be as easily modeled since they do not reach their terminal settling velocity and their motion is governed by diffusion.

For particles less than $0.1 \mu \mathrm{m}$, Brownian motion is the major transport mechanism responsible. Approximately $99 \%$ of all deposition in the lung of these ultrafine particles occurs via diffusion. These particles are continually bombarded by air molecules and therefore are subject to Brownian motion [Yu and Hu, 1983]. When the particle diameter approaches the mean free path of air molecules, the medium in which it is contained can no longer be classified as a perfect continuum. The collisions between air molecules and particles will cause random translational motion [Reist, 1993]. At this point, the crucial 
parameter for particle deposition probability classification is the thermodynamic diameter, as it will determine the terminal settling velocity [Stern et al., 1973]. Particles of this size are able to reach the deep lung because they do not impact or plate upon the ciliated surfaces of the upper respiratory tract. The particles in the aerosol diffuse to the alveolar regions because they remain continually suspended in the medium until molecular bombardment causes a directional force great enough to move them toward a surface.

Deposition in the alveoli may not only lead to diseases of the lung, but also in several other areas of the body. Macrophages in the alveolar region digest foreign bodies and are cleared approximately once every two months [Stern et al., 1973]. Continual deposition in the alveoli will lessen the efficiency of the macrophages to dissolve the particles. If this occurs, the toxic particles will remain in the deep lung for months and clearance may contain undissolved particles which will be transferred to the liver, spleen, kidneys, heart and brain through the blood [Stern et al., 1973].

Much particulate matter research has been concentrated on the effects of particle size and the associated health disorders. Even though a majority of studies have shifted focus from mass to size, it is important not to overlook mass as a viable quantity for relating PM to morbidity and mortality. Knutson and Lioy (1995) have done studies in an attempt to relate PM mass concentrations to health related effects. They found similarities in the mass-health relationship in several geographic locations even though their climates and pollutant compositions differed. This has implications to the effect that mass may yet be a useful, unifying measure of PM. While these experiments were conducted on the ambient, they have a direct correlation to vehicle particulate emissions. 
The number, size and total mass of particulate matter inhaled and retained have shown in epidemiological studies [Braun et al., 1992; Pope and Dockery, 1992; Dockery et al., 1993] to be associated with respiratory health effects: these associated effects include lung function parameters, respiratory symptoms and even mortality [Monn et al., 1995]. The World Health Organization (1996), the Health Effects Institute (1995), the International Agency for Research on Cancer (1989), and the National Institute for Occupational Safety and Health (1988) have all reached similar conclusions [Kittelson $e t$ al., 1998]. It is evident that the three major parameters defining particulate (mass, size and number) need to be measured and evaluated cohesively, rather than each being studied independently.

\subsection{Particle Behavior and Formation Processes}

Particulates begin to change in size and nature immediately upon exiting the combustion chamber. They may grow by condensation, coagulation and adsorption, shrink by evaporation and desorption, or form from gases by nucleation. Once the particles have been exhausted from the tailpipe to the ambient air of a dilution tunnel, these processes may all occur, thereby altering the original state of the PM emissions. Temperature, saturation ratios, engine design and residence time all effect particle size. Therefore, a thorough examination is needed to fully describe the size, mass and number of particulate emissions. The three regimes within which a particle may exist or pass through are nuclei, accumulation and coarse. 
Kittelson et al., (1998) found that a majority of particulate mass from diesel engines exists in the accumulation mode in the size range of 0.05 to $1.0 \mu \mathrm{m}$. The nuclei mode generally consists of 5 to $50 \mathrm{~nm}$ mobility equivalent diameter particles. This mode contains only 1 to $20 \%$ of the total particle mass, but more than $90 \%$ of the number of particles. The coarse mode is defined as everything beyond the accumulation mode and contains 5 to $20 \%$ of the particle mass. In order to understand the entire process of particle behavior, the nature of initial particle formation must be analyzed.

For diesel engines, the exhaust aerosol is largely made up of agglomerated solid carbonaceous material and ash, as well as volatile organics and sulfur compounds [Kittelson et al., 1998]. While the quantities of these organic species vary with fuel sulfur content and additives, the formation process remains largely unchanged. The solid carbon, generally referred to as soot, is formed during the combustion process in areas where the fuel spray is not leaned out until considerable cooling of the gases takes place during expansion [Ferguson, 1986]. The carbon matrix core is an amorphous and microcrystalline mixture, where the texture is dependent upon such factors as engine geometry, fuel mixture and combustion chamber temperature [Greenwood et al., 1996]. The organic content develops from any process that causes hydrocarbon formation and their partial oxidation products. A portion of the fuel and lube oil does not undergo complete oxidation and appears as volatile or soluble organic compounds [Kittelson et al., 1998]. Another form of particulate precursor is the hydrocarbon droplet. This consists of hydrocarbons and semivolatile species and forms after the exhaust manifold as temperatures begin to fall [Greenwood et al., 1996]. These two types of particulate nuclei constitute the nucleation mode. 
Nucleation, when new particles form from gaseous organic and inorganic species, is the mechanism for formation of measured nuclei mode particles in exhaust aerosols. These particles are comprised primarily of volatile materials and are greatly affected by saturation conditions. If the saturation ratio, defined as the partial pressure of the gaseous organic species divided by the saturation pressure of the species, is sufficiently high, the new particles will form [Kittelson et al., 1998]. As these newly developed nuclei pass through the exhaust stream, they begin to grow once the temperature drops below $300^{\circ} \mathrm{C}$, provided the species remains saturated [Greenwood et al., 1996]. If the saturation conditions drop, the volatiles will evaporate back into their previous gaseous state.

There are two types of volatile organic nucleation that may occur in vehicle exhaust - homogeneous and heterogeneous [Reist, 1993]. Homogeneous nucleation, also termed self-nucleation, occurs when vapor molecules form molecular clusters which can develop and disintegrate even for unsaturated species [Kittelson et al., 1998]. Assuming the vapor is supersaturated, the chains or clusters will grow large enough to permit condensation. Nuclei produced by homogeneous nucleation are transferred to larger particles by interactions with other like-sized particles [NRC, 1979].

Heterogeneous nucleation can occur if other nuclei are already present in the exhaust aerosol. These available nuclei are dissimilar in size and do not form molecular clusters as in homogeneous nucleation [Reist, 1993]. The saturation levels necessary for this nucleated condensation to occur are much lower than those for self-nucleation since the nuclei are in the aerosol and do not need to be formed from gaseous vapors. For both forms of nucleation, saturation is necessary for condensation of the volatile organics to occur. 
Nucleation and adsorption are inversely related gas-to-particle conversion processes [Kittelson et al., 1998]. Adsorption retards nucleation. The large surface area available on carbonaceous agglomerates provides a substrate for adsoprtion to occur. For older engines, the soluble organic fraction (SOF) in the exhaust will adsorb on the additional surface area of the carbon cores created during combustion. This will prevent the saturation levels from reaching the point where nucleation can occur [Kreso et al., 1998]. Conversely, with improved fuel atomization by high pressure injectors in newer engines, these carbon cores are decreased in size and surface area. The surface area available may not be sufficient to adsorb the organic species; therefore, nucleation will occur and the number of nuclei-mode particles will increase. These two particle growth processes are crucial in determining which mode (nuclei or accumulation) will dominate. As mentioned, newer technology in engine components is shifting the particulate toward the nuclei-mode and larger numbers of smaller particles.

Where nuclei-mode particles generally consist of sulfates and hydrocarbons, accumulation-mode particles are typically agglomerates of carbonaceous soot [Kittelson et al., 1998]. Most of the particulate mass can be found in this range, regardless of formation processes. The amount of adsorption of SOF on these particles will determine their size, but they generally will be in the submicron range. Particles having a diameter greater than this are said to be in the coarse-mode. Typically, particles will not grow to this size by adsorption. In order to exceed the upper limit of the accumulation mode, the particles are thought to undergo an additional process. High concentration levels may cause coagulation of particles in the accumulation mode and/or to a lesser extent, particles may be temporarily lost on the cylinder walls or exhaust system surfaces then 
reentrained causing the increased size [Kittelson et al., 1998]. Due to the nature of these particles, relatively few are necessary to skew the mass/size relationship toward the larger sizes. However, they are the least representative of actual engine particulate matter emissions.

\subsection{Particle-to-Particle Processes}

An understanding of gas-particle interactions is imperative to accurately describe the particulate emissions. However, there are several particle-particle and particle-wall processes that may occur which will alter measurements. More specifically, the measurements will report under sampling because these interactions will cause losses in the system. The processes that particles may undergo as they move through a sampling system are inertial impaction, thermophoresis, diffusion, coagulation and electrostatic deposition. Kittelson, Watts and Arnold (1998) have performed a thorough analysis of these sampling system losses, and it is largely their work that is presented here.

Inertial impaction will occur when particle inertias are large enough that the particles are unable to remain in the streamlines. This occurrence may result in over or under sampling. More importantly, particles may be lost to wall surfaces and randomly reentrain into the exhaust stream. Sampling tube inlets may also be sites of deposition [Reist, 1993]. It is of special importance for large particles because of their relatively higher mass. A small loss due to impaction may translate into a large decrease in total mass measured. The unpredictable reentrainment may lead to even greater sampling errors as particles collected may not be representative of actual particles emitted. This is 
the method by which coarse-mode particles are believed to appear; these may alter the mass based size distributions recorded by instruments such as the MOUDI.

Thermophoresis is a process in which temperature gradients in sampling systems cause particle motion toward wall surfaces. Particles may move from the higher temperature air stream to the cooler walls. This assymetric force is weak, but exhaust particulate has a great enough mobility to be path affected. Thermophoresis is of greater importance for smaller particles, because the thermal force generated is more pronounced in the free molecular region (Knudsen number $>>1$ ). The momentum transferred to one side of the particle will be greater than that to the other, so a net thermal force results from the impulse in the direction of the gradient imparted to the particle by the impinging gas molecules [Reist, 1993; Cadle, 1975]. This phenomenon needs to be as limited as possible to insure representative sampling. If thermophoresis does occur, a layer of particles may develop on the sampling system surfaces due to this gradient as the cooler walls will, in effect, draw the PM out of the exhaust stream. High tunnel velocities can create large shear forces that peel the deposited soot from the tunnel walls which will return them to the air stream; similarly, sampling lines may also be subjected to reentrainment. Kittelson et al., (1990) claimed that the measured PM mass may be comprised of as much as 20 to $45 \%$ of these reentrained particles.

Diffusion is another method for particle loss and is also of more importance for smaller particles $(<0.05 \mu \mathrm{m}$ in diameter). Similar to thermophoresis, diffusion can occur due to a concentration gradient, i.e., the particles move from areas of high concentration to areas of low concentrations, which occur at boundary layers. The particles may also be subject to diffusion by Brownian motion; both causes will lead to particulate sampling 
losses as particles adhere to wall surfaces. For particles near the molecular regime, their diffusion coefficients increase inversely with a decrease in size. Highly turbulent flows and their subsequent thin boundary layers allow for high mass transfer coefficients and therefore faster diffusive properties [Kittelson et al., 1990]. In turbulent deposition, a uniform aerosol concentration is established in the viscous sublayer. The particles then reach the wall surface by molecular diffusion [Reist, 1993]. As with all particles lost to the walls, reentrainment may occur and distort measurements.

Collision of particles may result in size distribution distortions. Small particles which collide and agglomerate with larger or similar sized particles are then lost in measurements. The total mass of particulates collected will not be altered, but a portion of the fine particles will go to increasing diameters. As polydispersity of the exhaust aerosol increases, the coagulation coefficient increases. Coagulation is governed by the rate of thermal diffusion for particles subject to Brownian motion and their subsequent high diffusion coefficients enhance the coagulation process [Hinds, 1982]. This will tend to reduce the polydisperse aerosol into a more monodisperse one, where the smallest particles become forever lost to measurement and cease to exist as an exhaust species in the more uniform sample [Reist, 1993].

Electrostatic deposition is often used to remove particles from a dirty gas stream; however, this is very counterproductive when the task is to measure all the emitted particles [Baron and Willeke, 1993]. This type of loss is generally only relevant for larger particles in sampling lines. Transfer tubes may impart static charges to particles, which will draw them toward wall surfaces and/or cause particle-particle attractions. This may also lead to post-collection measurement errors if the filter or substrate is 
statically charged. As the particular collection medium discharges, mass recordings will drop. Therefore, care must be taken to have neutral (elementary unit charge) collection mechanisms and transport lines.

Post-combustion particle behavior and formation are complex in nature and difficult to control. While dilution tunnels are designed to simulate ambient conditions, it is not always possible to match those criteria. A further question is which particles are most representative of actual vehicle particulate emissions. Since the particles begin to change in form and size immediately upon exiting the combustion chamber, it is difficult to assign the moniker 'actual particulate matter emissions.' Nucleation and adsorption are competing processes and will largely determine and effect measurements. At this point, no standards have been set to regulate dilution levels. This does not imply a limited importance of these issues, but rather portrays the inherent complexities of particulate matter measurements. Clearly, the processes need to be fully understood so future associations between current measurement techniques and measured quantities can be made.

While formation processes do not lend to simple control, sampling with respect to particle behavior can be standardized to minimize measurement errors. Limiting temperature and concentration gradients will reduce sampling errors greatly, as thermophoresis and diffusion account for a large portion of measurement inaccuracies. There are relatively simple techniques that can be employed to lessen these effects as well as for electrostatic deposition. Thermophoresis can be limited by insulating the dilution tunnel and the sampling lines. This will limit surface cooling and therefore minimize any thermal gradients. Decreasing particle concentrations will lessen 
coagulation, which can be done by further diluting the exhaust sample. Electrostatic deposition can be avoided by using stainless steel or Tygon (not Teflon) sampling lines and removing static charge from any filter media via a neutralizer. Careful measurement methodologies can make negligible possible errors associated with particle behavior.

\section{$2.4 \quad$ Recent Research}

Much of current particulate matter research has focused on particle size and number, as this is now being viewed as a more important classification than mass and concentration. This has led to instruments such as the SMPS becoming the particulate sampler of choice and cyclones losing favor. This poses a problem however, because converting particle number to mass is an inherently flawed practice. The assumption that fossil fuel combustion produces spherical, unit density particulates has been shown to be vastly misguided [CONCAWE, 1998; Maricq et al., 1999; Kelly and McMurry, 1992].

Due to this recent trend, there is little current research being conducted using cyclones. Predominantly, the SMPS and occasionally the MOUDI are being used. This section will review two studies performed on particulate matter. The first is from work done at Ford Motor Company and the second by the Conservation of Clean Air and Water in Europe (CONCAWE, the oil companies' European organisation for environment, health and safety).

Maricq et al., (1999) recently conducted a study on the effects of using a dilution tunnel on gasoline and diesel particulate matter measurements. The goal was to monitor differences, if any, in dilution tunnel particle size distributions and those from the 
tailpipe. An ejector pump was used to remove a portion of the tailpipe exhaust prior to the dilution tunnel for the comparative study. Ejector systems have gained attention as alternatives to full flow dilution tunnels for measuring particulate matter due to the effects dilution may have on particles. For both, a model 3934 SMPS and model 3010-S Condensation Nucleus Counter (CNC) were used. These experiments were carried out on three gasoline vehicles and one diesel. While the study centered on high speed testing to show dissimilarities, it is the lower speed analyses that were plangent: the size distributions were similar at both the tailpipe and dilution tunnel. Again, this study's focus was not on speeds below $50 \mathrm{mph}$, but some evidence can be extrapolated to this effect. Also, mass accumulation was performed theoretically rather than empirically. It is these two aspects that are of importance here.

As mentioned, Maricq et al., 1999 used two SMPS systems with $577 \mathrm{~nm} 50$ dae cutpoint impactors. For the classifier flow rates used ( $0.4 \mathrm{lpm}$ aerosol, $4.0 \mathrm{lpm}$ sheath), the size resolution range is given as $\pm 0.5 \mathrm{~nm}$ at $15 \mathrm{~nm}$ to $\pm 30 \mathrm{~nm}$ at $500 \mathrm{~nm}$. Two minute scans were used and were conducted at 3 to 5 minute intervals.

The vehicles tested and fuels used are shown below.

\begin{tabular}{c|ll} 
Vehicle Name & \multicolumn{1}{|c}{ Description } & \multicolumn{1}{|c}{ Fuel } \\
\hline US4 & $\begin{array}{l}\text { 1997 4 cylinder passenger car w/ 3-way } \\
\text { catalyst } \\
19974 \text { cylinder passenger car w/ 3-way } \\
\text { catalyst } \\
\text { E4 }\end{array}$ & $\begin{array}{l}\text { California Phase II summer } \\
\text { European certification fuel }\end{array}$ \\
US8 & $\begin{array}{l}\text { 1996 cylinder passenger car } \\
\text { w/ oxidation catalyst }\end{array}$ & $\begin{array}{l}\text { California Phase II summer } \\
\text { premium grade }\end{array}$ \\
DV & $\begin{array}{l}\text { European certification fuel } \\
\text { premium \#2 diesel }\end{array}$
\end{tabular}


The gasoline vehicles were tested with an uninsulated transfer line. For vehicle US8, Maricq et al., (1999) found good agreement between the dilution tunnel and ejector system data. Vehicle US4 showed particle number emissions 1.5 times higher in the tunnel at $50 \mathrm{mph}$ and twice as high at $60 \mathrm{mph}$, with similar size distribution shapes. This trend was continued with vehicle E4 at 31, 44 and $63 \mathrm{mph}$. At higher speeds the temperature increased, thus the difference in particle number between the two methods.

This trend continued for the case of an insulated transfer line. US8 was in agreement within $\sim 20 \%$ at the two lower speeds, and increased greatly at the highest speed. An accumulation mode peak was observed for US4 and E4 for the lower two speeds (50 and $60 \mathrm{mph}, 31$ and $44 \mathrm{mph}$, respectively). However, the transfer line temperature ranged from $\sim 160^{\circ} \mathrm{C}$ to $>250^{\circ} \mathrm{C}$. Also, these peaks were not observed during all test sequences.

The last parameter for the gasoline vehicles was the use of a silicone rubber coupler for the transfer line. Of note, below $60 \mathrm{mph}$, the dilution tunnel and ejector pump particle size distributions were in agreement. At the highest speed, an ultrafine mode developed in the tunnel measurements.

For the one diesel vehicle tested, DV, the speed variation effects were very obvious. An accumulation mode centered at approximately $100 \mathrm{~nm}$ was observed at all speeds and both sampling locations. At the two higher speeds, the distribution became bimodal with a large ultrafine mode peak occurring at approximately $18 \mathrm{~nm}$ for $60 \mathrm{mph}$, and increasing fivefold in size and shifting to approximately $35 \mathrm{~nm}$.

The number weighted particle size distributions were recorded as $d N / d \log \left(D_{p}\right)$. This was plotted over the width of the channel, which was logarithmically normalized. 
From these particle number measurements, a mass weighted distribution was found by multiplying by the particle density and the cube of the particle diameter, $\mathrm{D}_{\mathrm{p}}$. These were both further converted to emission rates by multiplying by the exhaust flow rate and integrating over particle diameter. There were, however, large errors associated with this particle number to mass calculation. Maricq et al., (1999) give the mass emission rate error, $2 \sigma$, as $\pm 50 \%$.

The errors that arise from this type of conversion are due to the assumptions that particle density is equal to one gram per cubic centimeter and that the particles are spherical. Kelly and McMurry (1992) performed a study on particle density to correlate aerodynamic diameter with mobility equivalent diameter. They found that shape and porosity can affect measured effective densities. The effective density, $\rho_{\mathrm{e}}$, is given by

$$
\rho_{e}=\left(\frac{C\left(d_{m}\right)}{C\left(d_{b}\right) \chi}\right)^{3} \rho_{p}
$$

where:

$$
\begin{array}{lll}
\mathrm{d}_{\mathrm{b}} & = & \text { mobility equivalent diameter } \\
\mathrm{d}_{\mathrm{m}} & = & \text { mass equivalent diameter } \\
\mathrm{C} & = & \text { associated slip correction factor } \\
\chi & = & \text { dynamic shape factor } \\
\rho_{\mathrm{p}} & = & \text { inherent material density }
\end{array}
$$

In order to infer the inherent material density, the dynamic shape factor must be known as well as the porosity of the particle. This implies that density and shape are conjoined, 
with the presence of non-spherical shapes affecting density calculations. Clearly, particle density may vary from the value of unity assumed by most researchers. Also, exhaust particulates cannot be considered spheres, as they are generally chains of very small particle-like units [Reist, 1993; CONCAWE, 1998].

Maricq et al., (1999) apparently assumed the particles are perfect cubes. For spherical particles, the number weighted distribution would need to be multiplied by $\pi \mathrm{D}_{\mathrm{p}}{ }^{3} / 6$; this $\pi / 6$ term appeared to be neglected. Regardless, either method will return mass values that cannot be taken as real. There is in fact no reliable, accurate, simple method of converting number to mass for exhaust particulates. Even though the basis for equivalent diameter measurements (electrical mobility, aerodynamic) assumes this, these classifications only relate the actual particle to an ideal, spherical particle. For example, aerodynamic equivalent diameters liken the terminal settling velocities, and mobility equivalent diameters, the electrical mobilities. The direct number to mass/volume conversion does not do this. In other words, mass and volume cannot be equated to any other physical properties.

The other study which demonstrates the inherent difficulties in converting number to mass is the CONCAWE (1998) research on particle size, number and mass from diesel and gasoline vehicles. The size and number of particles were found using an SMPS and the mass by a Berner low pressure impactor and gravimetric filtration. It is clear from the data that extracting mass weighted size distributions from number based measurements is not plausible. The core focus of the CONCAWE study was to compare particle mass and size between diesel and gasoline fueled vehicles. As a result of comparing actual mass 
measurements to those given by converting SMPS data, the difference is elucidated. The equation used was

$$
\frac{d M}{d \log d_{p}}=\frac{d N}{d \log d_{p}} \times \frac{\pi}{6 \times 10^{18}} \times \frac{1}{3 \log _{e} 10} \times \frac{d_{p, \text { max }}^{3}-d_{p, \text { min }}^{3}}{\log d_{p, \text { max }}-\log d_{p, \text { min }}}
$$

under the assumption of spherical, unit density particles with diameters uniformly distributed on a logarithmic scale. The results yielded the following for a vehicle run with diesel \#1 and diesel \#3.

\begin{tabular}{c|cc} 
Instrument & $\begin{array}{c}\text { Mass Emission Rate, D1 } \\
\mathbf{~ m g ~ / ~ k m ~}\end{array}$ & $\begin{array}{c}\text { Mass Emission Rate, D3 } \\
\mathbf{~ m g ~ / ~ k m ~}\end{array}$ \\
\hline SMPS & 157.0 & 156.5 \\
Impactor & 47.2 & 20.7 \\
Filter & 70.0 & 29.9
\end{tabular}

At best, the conversion method returned mass emission rates more than double; at worst, greater than 7.5 times. This occurred even though the SMPS scans a much more narrow band of sizes than the other techniques. This is likely due to the fact that the long chains, consisting of agglomerates of small units, are better described as fractals than spheres. The diameter of these particles will be greater than a spherical particle of the same mass, and therefore will lead to an overestimation of mass when the conversion from number is made. The CONCAWE (1998) report did not report the values obtained by this method due to the large errors associated with the conversion. 
As should be made abundantly clear, the connection between particle number and mass is erroneous for vehicle exhaust particulates. The CONCAWE (1998) study gives impetus to the notion that in order to relate the two properties, mass needs to be measured by devices such as an impactor and/or methods such as filter collection. Cyclones are an appealing mass measuring instrument in that they possess some size selective ability. Used in conjunction with an impactor and gravimetric filter, they form a useful connection between the two, as the MOUDI does with the SMPS and particulate filter collection. 


\section{Chapter 3 - Experimental Procedure}

All experiments were conducted using the Transportable Heavy Duty Vehicle Emissions Testing Laboratory (THDVETL). The laboratory was modified to accommodate the particulate measurement systems. This chapter details the laboratory design, the modifications made, the instrumentation used, and the vehicles tested as well as the fuels used.

\subsection{Chassis Dynamometer}

The Transportable Heavy Duty Vehicle Emissions Testing Laboratory (THDVETL) is comprised of a chassis dynamometer built into a semi-trailer and an instrumentation trailer that houses the data acquisition and emissions measuring equipment [Gautam et al., 1991]. Vehicle inertia is simulated by a series of rotating flywheels which provide a load on the engine and also simulate coast down conditions. Eddy current dynamometers are used to simulate aerodynamic drag and rolling resistance of the vehicle. Exhaust flow from the vehicle is ducted into a full flow, double dilution tunnel for analysis.

The maximum design speed for the dynamometer is $560 \mathrm{rpm}$. This is based on driving cycles which had maximum speeds of $55 \mathrm{mph}(92 \mathrm{kph})$. A range of heavy duty vehicle tire sizes (diameters) was estimated to be $3.25 \mathrm{ft}$ to $4.0 \mathrm{ft}$. These correspond to a rotational speed of $483 \mathrm{rpm}$ for a $3.25 \mathrm{ft}(1 \mathrm{~m})$ diameter tire and $392 \mathrm{rpm}$ for a $4 \mathrm{ft}(1.22$ m) tire. The tires rest on 12.6 inch $(32 \mathrm{~cm})$ diameter rollers set with their axes along the 
length of the trailer. Four rollers support the single or forward rear axle assembly. Each roller is built around a 2.4 inch $(6 \mathrm{~cm})$ diameter shaft running through spherical roller flange bearings, and each pair of rollers is connected via a flexible coupling so tire speed will be the same on each side of the test vehicle.

Hub adapters for both demountable and spoked style rims have been fabricated for power transfer. The engine power is absorbed through the rear wheels by the adapters as opposed to the rollers because the slippage between the tires and the rollers and the heat generated during this process give rise to inaccuracies in the test results. The hub adapters are $1.8 \mathrm{ft}(55 \mathrm{~cm})$ diameter, 0.5 inch $(13 \mathrm{~mm})$ thick aluminum rims that drive a Spicer 1810 series coupled assembly. These transmit power to the absorbers and flywheels, which are situated symmetrically on each side of the test vehicle. Spline travel in the coupled assembly permits testing of vehicles with outside tire tracks of 88 to 96 inches $(223.5$ to $243.8 \mathrm{~cm})$. The coupled assemblies can withstand torque up to 16,415 ft-lb (22256 N-m) on each side.

The coupled assembly drives a 24 inch $(61 \mathrm{~cm})$ diameter shaft running in a pillow block. This shaft, through a spline and companion flange, drives a 16,665 ft-lb (22595 $\mathrm{N}-\mathrm{m}$ ) Lebow speed and torque transducer which provides the data logging computer with time-varying output torque during each test at a rate of $10 \mathrm{~Hz}$ [Ferguson, 1993]. This transducer drives a second shaft by a companion flange that transfers power to the rightangle speed increasing drive then to the flywheels. The shaft passes through the differential with the spider gear carrier bored out to permit passage of the shaft that is splined to lock the two side gears in the assembly at the same speed. The shaft is supported by a bearing on the axle housing flange and the spider gears themselves. 
Extending from the far side of the flywheel differential, the shaft drives a 1610 series double universal joint and a second shaft which turns a similar differential (ratio 1:5:73) used to drive the power absorbers.

The flywheel assemblies are designed to simulate a 40,000 lb (18144 kg) load at the maximum anticipated tire diameter and can simulate over $66,000 \mathrm{lb}(30,000 \mathrm{~kg})$ load with $3.25 \mathrm{ft}$ diameter tires. Since the flywheel inertia must simulate different vehicle weights, flywheels in the assembly can be selectively engaged and disengaged. Each flywheel assembly has four drive rotors on a driven shaft running in two pillow blocks and eight flywheels of different size resting on bearings on the shaft. By engaging the flywheels to the drive rotors, vehicle mass can be simulated in $250 \mathrm{lb}(113 \mathrm{~kg})$ increments.

The power absorbers are air-cooled, eddy current Mustang model CC300s that measure $1.3 \mathrm{ft}(40 \mathrm{~cm})$ in length by $1.5 \mathrm{ft}(45 \mathrm{~cm})$ in diameter. They can absorb $300 \mathrm{hp}$ $(224 \mathrm{~kW})$ continuously and over $1000 \mathrm{hp}(745.7 \mathrm{~kW})$ peak. Dynamometer load at any specific speed is controlled by the direct current supplied to the coils, while the energy is dissipated in the disk rotors, which is cooled by centrifugal air flow. The absorbers are mounted on two bearings with a torque arm force transducer to measure the absorbed torque. The absorber unit is housed in an expanded metal mesh cage to maintain adequate cooling yet operate safely. Figures 3.1 and 3.2 show THDVETL in operation.

Speed and torque are both controlled accurately as a function of time. While the driver is responsible for speed control, the torque is controlled automatically. The load due to rolling resistance and wind load simulated by the eddy current dynamometers is maintained by separate Dyne-Systems Dyn-Loc IV closed loop Proportional Integral 


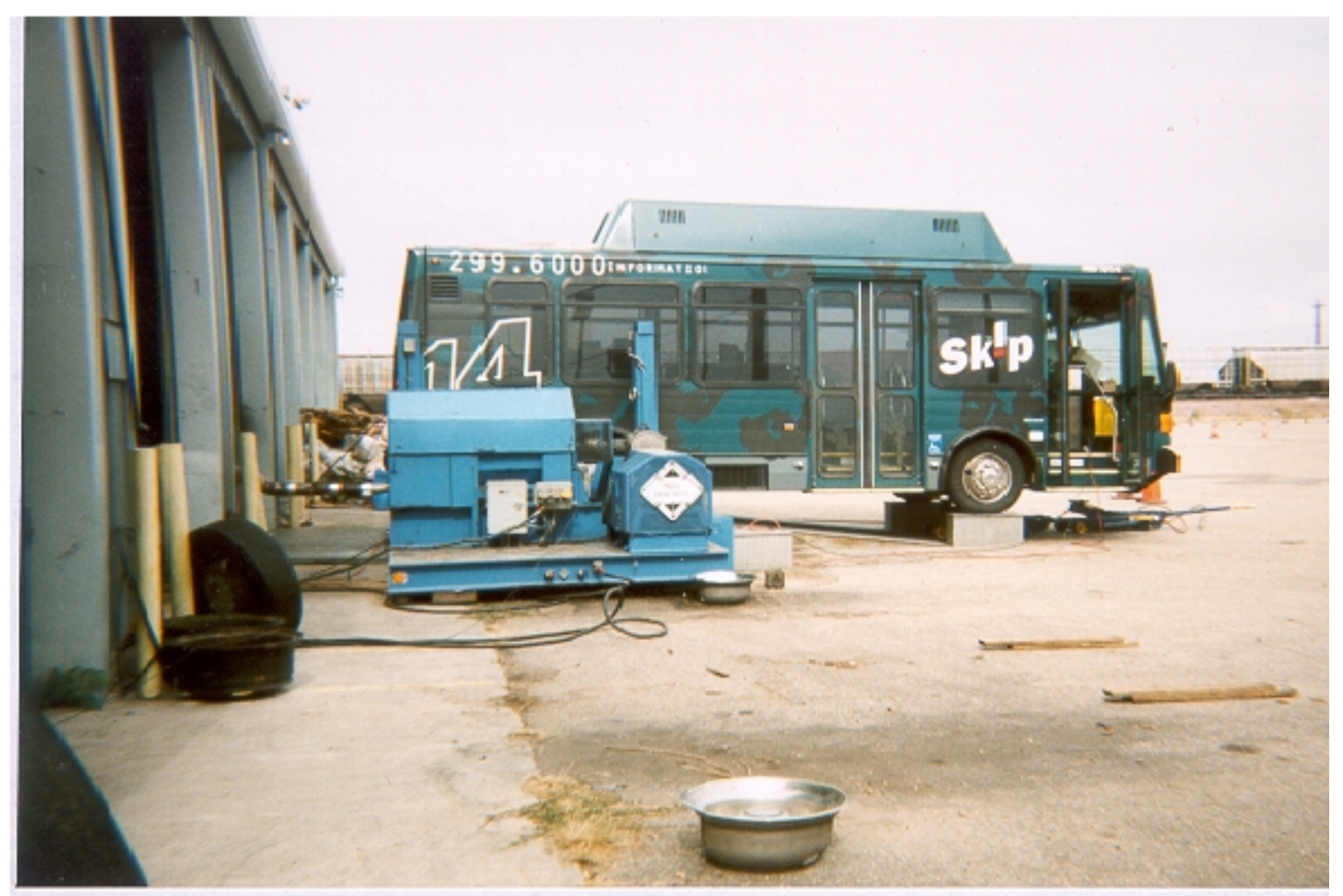

Figure 3.1 - RTD/SKIP Bus 1014 on the THDVETL Chassis Dynamometer

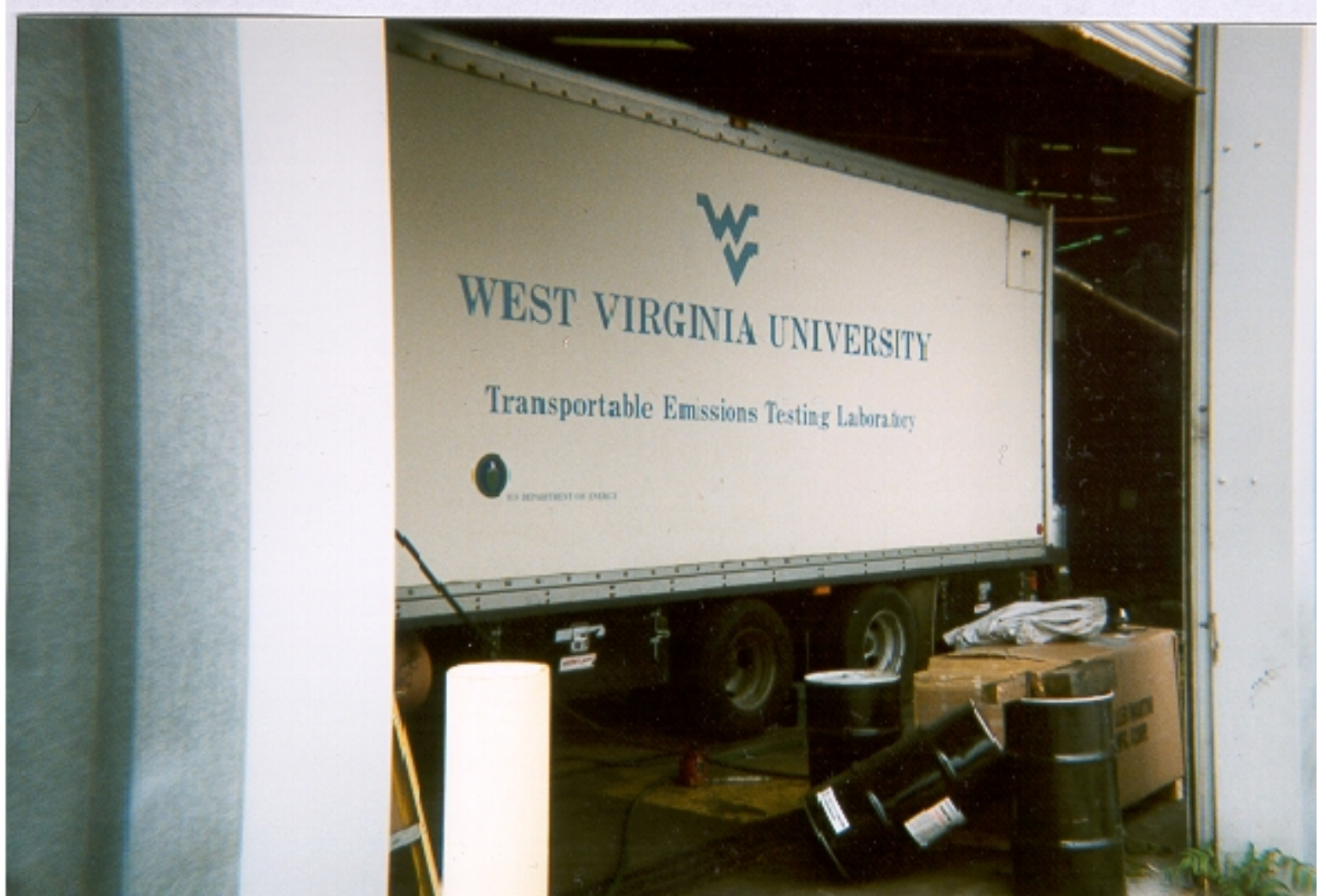

Figure 3.2 - THEDVETL Instrumentation Trailer During RTD/SKIP Testing 
Differential controllers [Ferguson, 1993]. During testing, the controllers receive the torque set point from the control computer and relay the set point to the power absorbers [Ferguson, 1993]. The set point, updated every 100 milliseconds, is equal to the road load and is calculated by using the following equation:

$$
P_{r}=V\left(\frac{\rho_{a} C_{D} A V^{2}}{2}+C_{r} M g\right)
$$

where:

$$
\begin{array}{lll}
\mathrm{P}_{\mathrm{r}} & = & \text { road load power } \\
\mathrm{V} & = & \text { vehicle speed } \\
\rho_{\mathrm{a}} & = & \text { density of air } \\
\mathrm{C}_{\mathrm{D}} & = & \text { drag coefficient } \\
\mathrm{A} & = & \text { frontal area of vehicle } \\
\mathrm{C}_{\mathrm{r}} & = & \text { coefficient of rolling resistance } \\
\mathrm{M} & = & \text { vehicle mass } \\
\mathrm{g} & = & \text { gravitational force }
\end{array}
$$

Speed and hub torque are logged at a frequency of $10 \mathrm{~Hz}$ throughout a test for verification regression analysis. The torque transducer is calibrated before testing is performed [Ferguson, 1993]. 


\subsection{Dilution Tunnel}

Dilution tunnels are designed to simulate ambient conditions and to allow measurement of exhaust constituents including those in gaseous and particulate form. As previously detailed, natural, ambient dilution greatly affects the makeup of particles as well as vapor phase hydrocarbons. Thus, great care must be taken to control dilution levels for proper analysis of these and other species. The system employed by WVU is the critical flow venturi - constant volume sampler in accordance with the requirements of the Code of Federal Regulations 40 (40 CFR, Part 86, Subpart N).

The 40 CFR states that the dilution tunnel shall be constructed such that the exhaust gas and dilution air are fully mixed without lowering or adding additional backpressure on the engine. Since the tunnel flow must be turbulent (Re greater than 4000), the diameter must be kept small and the length sufficient to allow complete mixing (ten diameters) prior to any sampling probes. The tunnel must be fabricated of a grounded, electrically conductive material which will not react with any exhaust constituents. Another requirement is that water condensation must not be allowed to occur within the tunnel. Thus, the diluted exhaust temperature must be kept sufficiently high. To meet these criteria, WVU designed and built the following dilution tunnel for use with the transportable vehicle emissions testing laboratory [Gautam et al., 1991].

The dilution tunnel is constructed of 22 gage 304 stainless steel and is $20 \mathrm{ft}(6 \mathrm{~m})$ in length and 18 inches $(45 \mathrm{~cm})$ in diameter; therefore, all sampling is done after $15 \mathrm{ft}$ (4.5m) to insure fully mixed flow [Ferguson, 1993]. The stainless steel was chosen because it is corrosion resistant, electrically conductive and could be grounded to the 
instrument trailer on which it resides. This last requirement will prevent particle loss to the walls via the electrical attraction between particles and the tunnel surface. To further enable turbulent fully mixed flow, an 8 inch orifice plate [Smith and Gautam, 1992] was installed.

A $75 \mathrm{hp}$ blower is used to draw the necessary dilution air. This flow is regulated using a critical flow venturi (CFV) capable of controlling a flow rate of 1000, 1500, 2000 scfm under choked conditions. The equation used for determining the throat diameters for choked flow is given by:

$$
A^{*}=\frac{m}{C_{D}} \frac{\sqrt{T_{s}}}{P_{s}} \sqrt{\frac{R}{\gamma}\left(\frac{\gamma+1}{2}\right)^{\frac{\gamma+1}{\gamma-1}}}
$$

where

$$
\begin{array}{lll}
\mathrm{A}^{*} & =\text { throat area } \\
\mathrm{m} & =\text { mass flow rate } \\
\mathrm{C}_{\mathrm{D}} & = & \text { discharge coefficient } \\
\mathrm{T}_{\mathrm{S}} & = & \text { standard temperature } \\
\mathrm{P}_{\mathrm{S}} & = & \text { standard pressure } \\
\mathrm{R} & = & \text { gas constant } \\
\gamma & = & \text { specific weight }
\end{array}
$$

A differential pressure gage monitors the drop at the throat to insure choked flow in the venturi. Though the flow is continually choked, the constant volume sampler cannot actually maintain a constant volume. This is due to nonstandard conditions which may 
and often do occur at the venturi. The $40 \mathrm{CFR}$ gives the equation for measuring actual flow rate, $\mathrm{Q}$, as

$$
Q=\frac{K_{v} P}{\sqrt{T}}
$$

where:

$$
\begin{array}{lll}
\mathrm{K}_{\mathrm{v}} & = & \text { calibration coefficient } \\
\mathrm{P} & = & \text { actual pressure } \\
\mathrm{T} & = & \text { actual temperature }
\end{array}
$$

\subsection{Total Particulate Sampling}

Gravimetric particulate matter emissions measurements are conducted by transferring a portion of the exhaust from the primary dilution tunnel to a secondary dilution tunnel. It is there further diluted with air and cooled to a temperature not exceeding $125^{\circ} \mathrm{F}\left(50^{\circ} \mathrm{C}\right)$. This allows a representative sample of the particulate matter to be obtained in the dilute sample by filtration, where weighing will determine the mass collected on the Pallflex T60A20 70mm glass fiber filters. These filters are more than 98\% efficient when sampling particles down to $0.1 \mu \mathrm{m}$ in diameter.

The particulate sampling system was designed to perform in accordance with the 40 CFR. The secondary tunnel maintains the double-diluted sample at a temperature of $125^{\circ} \mathrm{F}\left(50^{\circ} \mathrm{C}\right)$ or lower immediately before the primary particulate filter. This is necessary because vapor phase organics condense out above this temperature. Particulate matter collected in a dilution tunnel is influenced by the conditions at which the tunnel is operated, most significant being the dilution ratio and temperature [Abbass et al., 1989]. 
The exhaust sample is drawn through a 0.5 inch $(1.3 \mathrm{~cm})$ diameter transfer tube located at the sampling zone in the primary dilution tunnel. The inlet faces upstream and is approximately 7 inches $(17.8 \mathrm{~cm})$ in length before the $90^{\circ}$ bend. Total flow and extra secondary dilution air flow through the secondary tunnel are controlled by two Sierra 740 series mass flow controllers in line with two Gast series 1023 rotary vane pumps. The total flow ranges from $0-7 \mathrm{scfm}(0-198 \mathrm{lpm})$ while secondary dilution air flow ranges from 0 - 4 scfm $(0-113 \mathrm{lpm})$. The sample flow rate through the secondary tunnel varies proportionally with the flow through the primary dilution tunnel. The mass flow controllers were calibrated using a Meriam Instruments laminar flow element (LFE) model 50MW20 rated at $0-23 \mathrm{scfm}(0-652 \mathrm{slpm})$. This unit measures flow accurately by monitoring the pressure drop across the element.

The secondary dilution tunnel is 3.0 inches $(7.62 \mathrm{~cm})$ in diameter and 30 inches $(76.2$ $\mathrm{cm}$ ) in length. This provides sufficient residence time for the exhaust sample to be mixed with the dilution air and to maintain a temperature below $125^{\circ} \mathrm{F}\left(50^{\circ} \mathrm{C}\right)$. At the end of the secondary tunnel, a $70 \mathrm{~mm}$ filter holder is placed which contains the primary and secondary filters. The filter holder is constructed of stainless steel to prevent reactions with the corrosive exhaust sample and is designed to allow easy access to both filters. The filters are conditioned for 12 hours at $50 \%$ relative humidity and $70^{\circ} \mathrm{F}$ in an Envirotronics model SH8 environmental chamber before and after a test. This removes the effects a hygroscopic media may present. Conditioning the filters at the same relative humidity will insure that equal amounts of water are trapped in the filter before and after testing, thereby taring bound water mass during the weighing process. Two reference filters are placed in the environmental chamber to monitor the humidity control function 
of the chamber. The reference filters are weighed before and after a test to compare gravimetric changes. In accordance with the 40 CFR specifications, if the average weight of the reference filter varies between weighings by greater than $\pm 5 \%$ of the nominal filter loading (a recommended minimum of $5.3 \mathrm{mg}$ ), then all test filters being conditioned are discarded and the emissions tests repeated. If the average weight of the reference filters decreases by more than $1 \%$ but less than $5 \%$ of the nominal filter loading, the tests may either be repeated or the amount of weight loss added to the net weight of the sample. If the difference in reference filter weights increases by more than $1 \%$ but less than $5 \%$ the test may be repeated or the values accepted [Ferguson, 1993].

The particulate filters are stored in glass petri dishes during environmental conditioning. These dishes are covered but not sealed to prevent mass gain due to gravitational settling on the filter while still allowing water vapor exchange.

Since the dilution air is not filtered, background particulate samples are taken. The total particulate mass is determined from weighing the filters before and after the test (post-conditioning). A Cahn 32 microbalance, with a 1.25 gram weighing capacity and a sensitivity of $0.1 \mu \mathrm{g}$, is used for weighing the filters. The difference in before and after filter weight minus the background mass is equal to the total particulate mass.

\subsection{System Modifications}

Since the design of the transportable emissions testing laboratory did not incorporate additional particulate sampling devices beyond those stated in the 40 CFR, the dilution tunnel needed to be extended, and modifications to the plumbing and data 
logging devices were necessary. In order for the laboratory to remain a mobile facility, limitations were placed on the design of the tunnel extension, namely location, removability and length. Also, new lines needed to be laid, computer program control reworked and the instantaneous cyclone flow rate feedback response logged. As noted, with the existing infrastructure mostly fixed, the necessary modifications had to be conducted around the current laboratory design rather than in conjunction with the laboratory.

A 54 inch $(1.37 \mathrm{~m})$ long and 12 inch $(30.5 \mathrm{~cm})$ diameter extension, constructed of 16 gage 304 stainless steel, was built to accommodate the additional sampling probes required for the MOUDI and cyclones. Three sealable ports with removable 2 inch blank flanges were welded to the extension to facilitate the samplers. These three ports were located approximately 42 inches from the tunnel connection in a triangular pattern; the cyclone probes are diametrically opposed, with the MOUDI probe $90^{\circ}$ from each cyclone probe. Each sampler extends approximately 4 inches from the wall surface in order to negate boundary layer effects while not disturbing the inlets of other probes. Since there is only a slight difference between sampling velocities of the three instruments (13 lpm maximum) and their position posterior to all other gas and particle sampling probes, any adverse flow effects were deemed negligible. Figure 3.3 shows a schematic of the sampling probe location and Figure 3.4 shows the system during operation. For installation of the SMPS sampling system, a 3/4 inch inlet probe was placed in the transfer line which connected the vehicle tailpipe to the dilution tunnel. This stainless steel sampling probe drew raw exhaust from the vehicle tailpipe to a mobile, mini-dilution tunnel. With this system, dilution levels were controlled via mass flow controllers. 


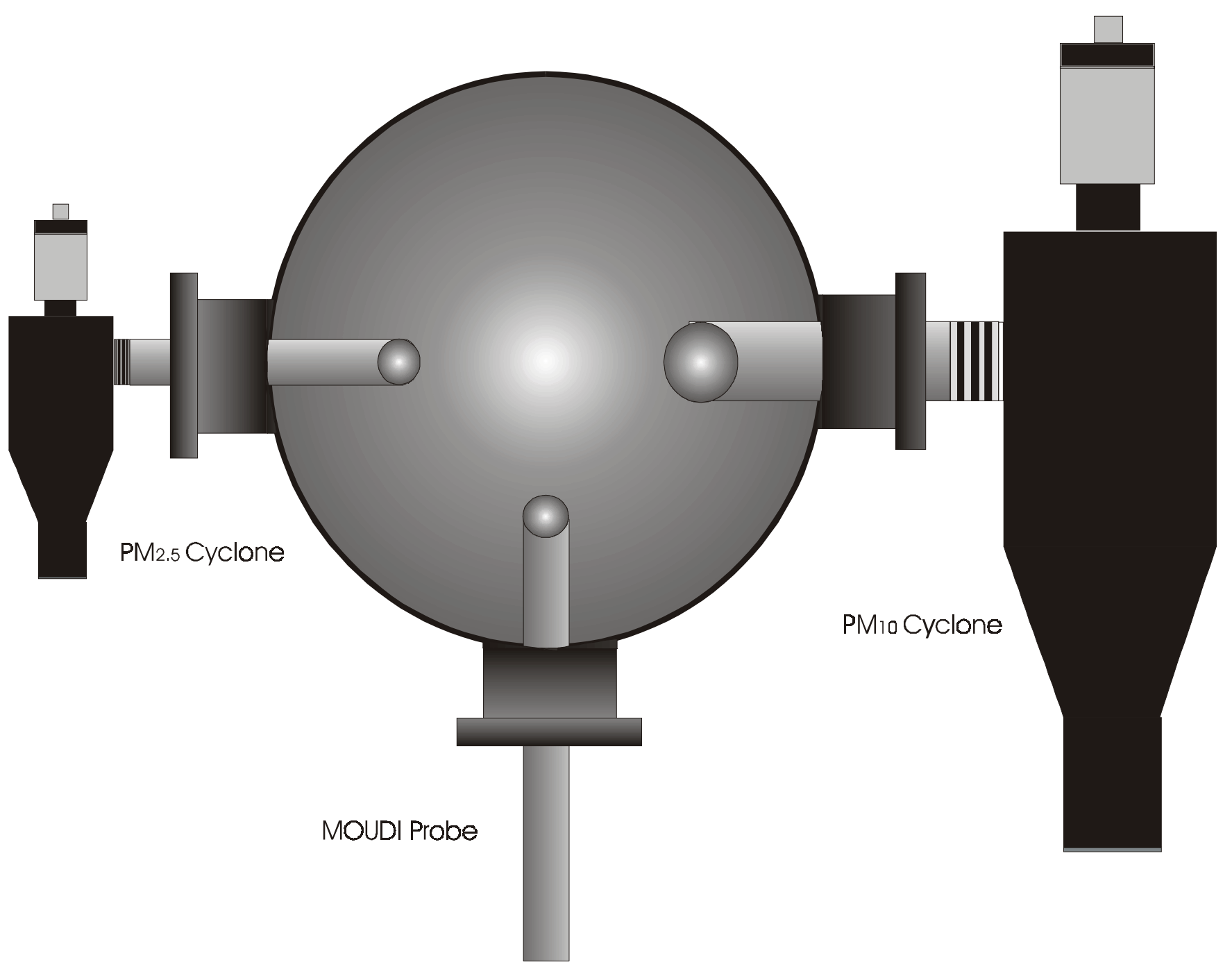

Figure 3.3: Sampling Locations with Cyclones (Not to Scale) 


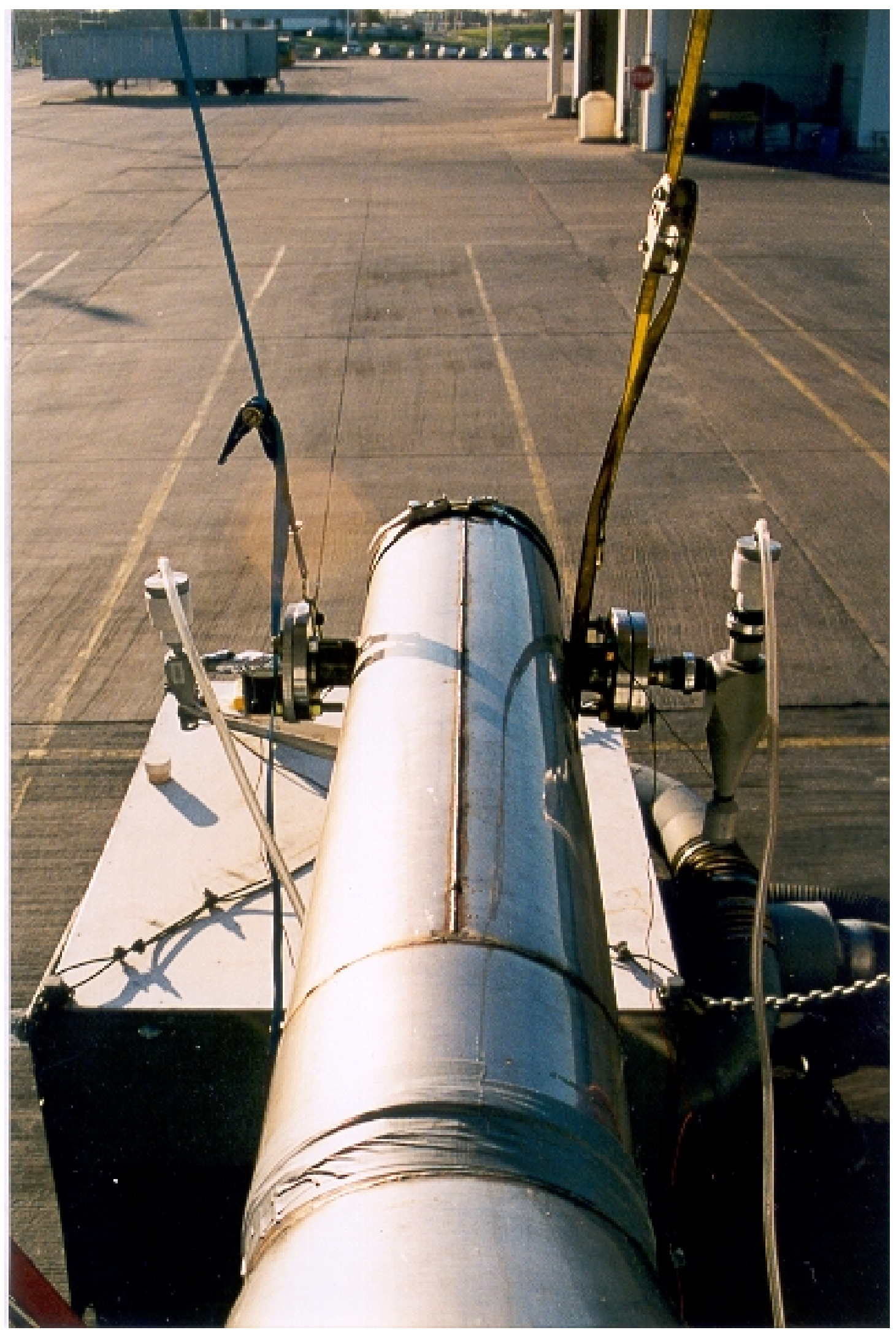

Figure 3.4 - Cyclones and Dilution Tunnel Extension in Use During DART Testing 
A complete description of this mini-dilution system is not presented here. The effect of dilution ratios is beyond the scope of this study. A comprehensive analysis of the theory and operation of this device can be found in Bugarski's (1999) study.

\subsubsection{Particulate Sampling: MOUDI}

The MOUDI requires a flow rate of $301 \mathrm{pm}(\sim 1 \mathrm{cfm})$ which is controlled through the pressure drop through the stages. The transfer tube used to draw a sample from the tunnel was $3 / 4$ inch in diameter, with a $90^{\circ}$ bend 4 inches from the inlet. Due to limitations imposed by the existing laboratory setup, the stainless steel sampling tube was long and extended from the ports on the primary dilution tunnel extension to the MOUDI. Since the MOUDI requires constant attention during testing and extensive preparation between tests, it has to be easily accessible to the operator, thus necessitating its placement on a workbench. This requirement placed additional constraints on the length of the PM sampling line. The transfer tube was insulated to minimize any thermophoretic processes that may occur. Figure 3.5 shows a schematic of the MOUDI sampling location and Figure 3.6 shows the MOUDI in operation.

Samples from the MOUDI were collected on eleven aluminum substrates and one $37 \mathrm{~mm}$ Gelman Sciences Teflo filter with a pore size of $2 \mu \mathrm{m}$. This thin teflon membrane filter with a polyolefin ring collects particles with aerodynamic diameters less than 52 nanometers. For particles greater than $35 \mathrm{~nm}$ at a face velocity of $23 \mathrm{~cm} / \mathrm{s}$, these filters have a collection efficiency of 99.98\% [Liu et al., 1983]. A further discussion of these afterfilters follows in the description of MOUDI operation. 


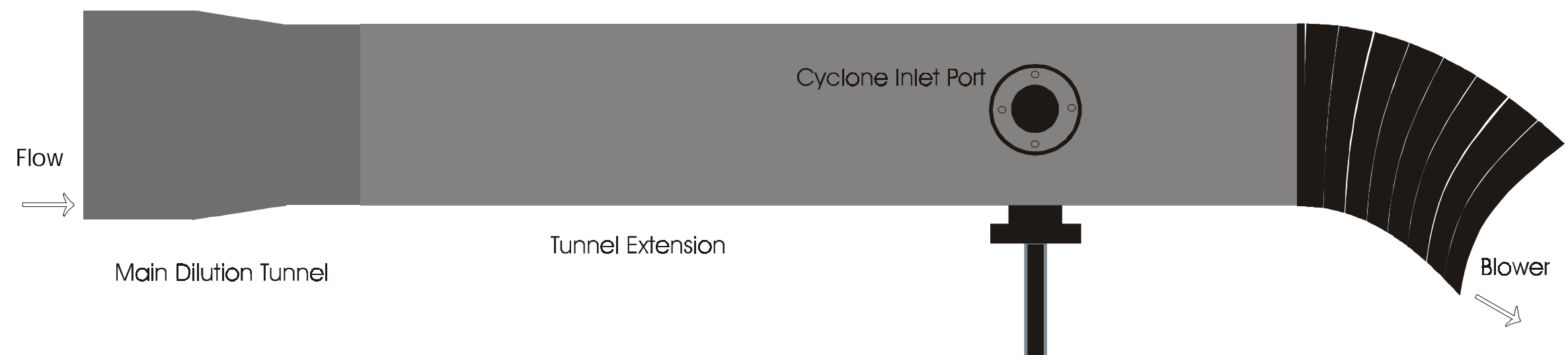

N

Insulated Sample Probe

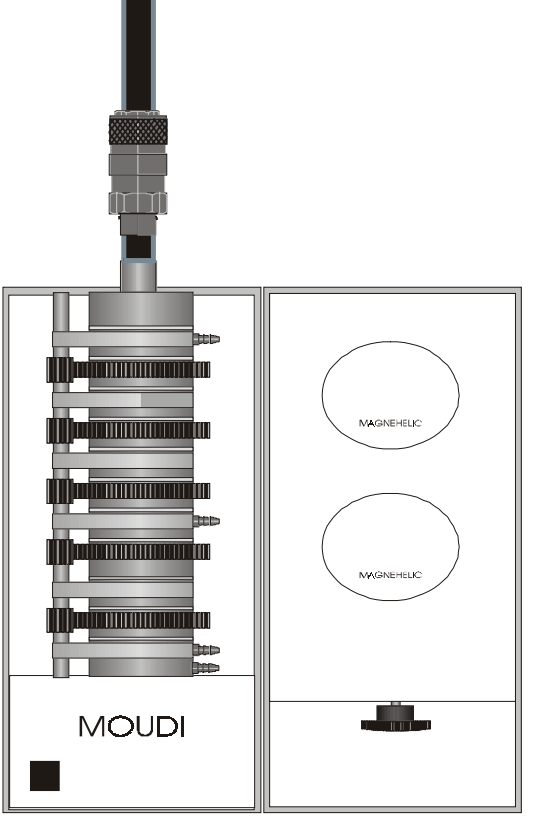

Figure 3.5 - Tunnel Extension with MOUDI (Not Drawn to Scale) 


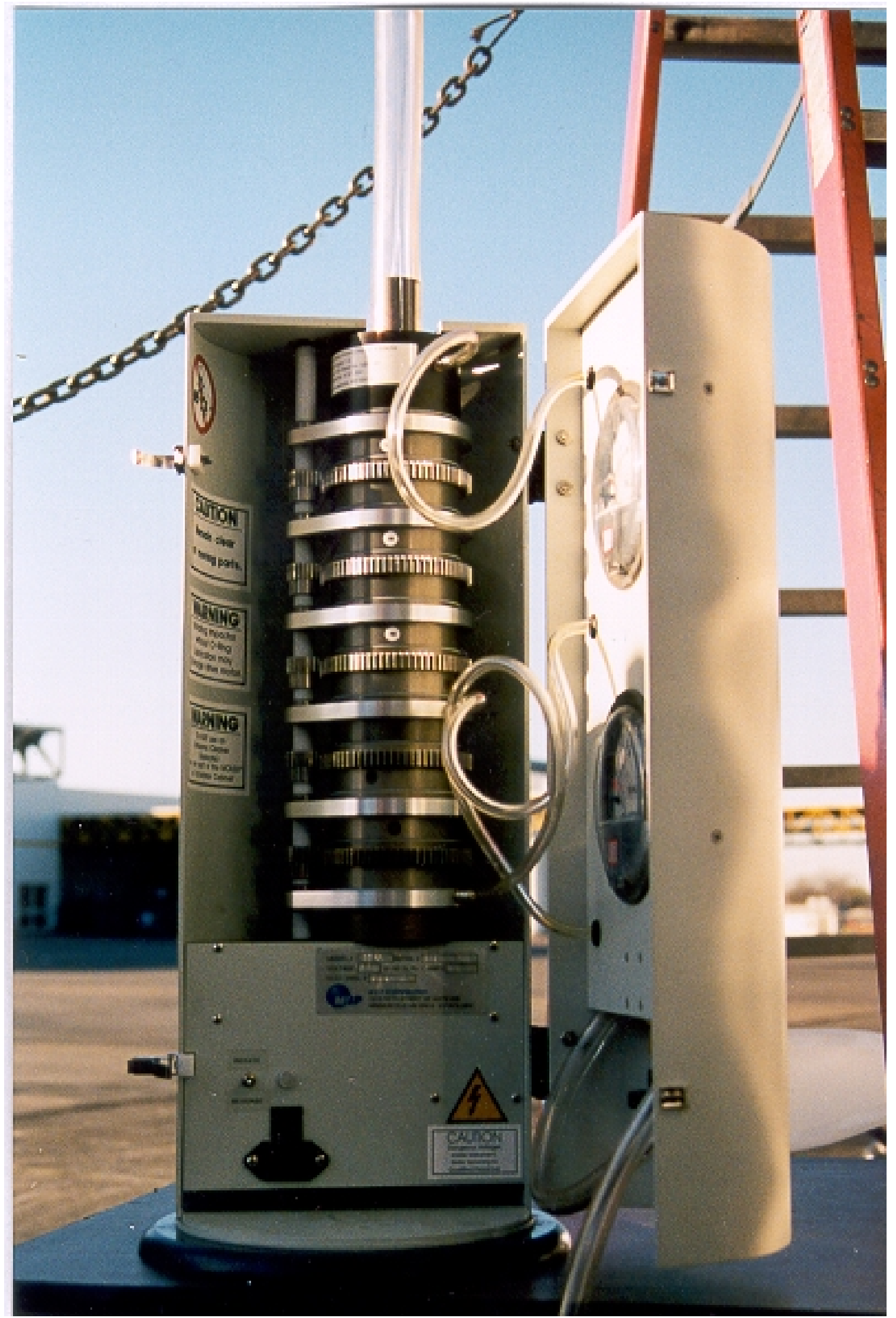

Figure 3.6 - The MOUDI in Operation During DART Fleet Testing 
The aluminum substrates were treated similarly to glass fiber filters in terms of environmental conditioning and handling. The only difference was the use of plastic petri dishes instead of glass and the heightened importance of discharging static buildup. Gelman Sciences $47 \mathrm{~mm}$ Analyslides were used for transport and storage of the substrates rather than glass dishes for two reasons. The first is the sheer number of substrates (12) needed per test. Since space and weight limitations had to be considered there would simply not be room for the transportation of nearly 600 petri dishes needed for the 48 tests. The second reason the Anaylslides were opted for was to maintain surface integrity of the substrates. Since the aluminum substrates have a low tare weight ( $\sim 70 \mathrm{mg})$, they tend to shift if not secured in their holders. If this happens, particulates may be lost to the inside of the dish.

Static charge builds up readily on the aluminum substrates. If this charge is not removed prior to weighing, the gravimetric values recorded are essentially worthless. If the substrates are allowed to discharge during the weighing process, the weight will drop continually and no value can be accurately assigned. To reduce the chance for static buildup long sleeves should not be worn during weighing. The close proximity of some fibers can cause static charges to accumulate. Static still may build on the substrate during testing as well as storage. Therefore, it is necessary to neutralize the charge before all weighing is performed. This is done by passing the substrate over (approximately one inch above) a Nucleospot Local Air Ionizer model P-2042, manufactured by NRD, Inc. The polonium isotope in the Nucleospot generates an equal number of positive and negative ions as it emits alpha particles. This will effectively neutralize the static charge; however, some charges cannot be completely removed. This 
generally can be detected during initial weighing, so the substrate is simply not used for testing.

Several researchers have espoused the necessity of applying a thin layer of grease to the substrate surface in order to minimize particle bounce [Marple and Liu, 1974; Baron and Willeke, 1993; Marple et al., 1991]. This layer will increase particle adhesion to the impaction surface. Upon impact, some of the particles may bounce off the substrate and get reentrained in the sample stream where they will pass to successive stages. This will distort the size distribution toward the smaller diameter regions with no definitive method to predict or correct for this phenomenon. However, in this study, the MOUDI substrates were not greased. There were four reasons for using ungreased substrates. The most important of these was the fact that of all trials with greased substrates, the post-test gravimetric analysis yielded at least one sample that had lost mass. The grease applied migrated to the outer rings of the filter holders and was thus lost during sampling.

Another problem was the application of the grease. It was applied by spraying the collecting portion of the substrate, but the layer rapidly seeped under the protecting mask toward the area of the substrate that was clamped in the holder. Also, the surface was no longer flat due to non-uniform spray patterns. This may lead to increased particle bounce and/or caking of particulates. If the impaction surface is not flat, the particles may collide at angles with the uneven surface, accelerating particles toward the edges of the substrate. Another possibility is that ridges in the impaction surface will not allow uniform deposition; this will negate one of the primary functions of the MOUDI (Microorifice Uniform Deposit Impactor). In order to combat this, a thin layer of grease 
was applied with a brush. While a noticeably more accurate way of dispensing the surface layer, this lead to the third reason for forgoing the grease.

Brushing the grease on a substrate is a delicate task. It demands fine detail and considerable time. Preparing one test, eleven substrates, without grease is a time consuming job in itself. It has been estimated through repeated testing that to perform one experiment, two hours are needed. Applying grease by brushing will add approximately an additional half an hour per test. If this process were accurate (i.e. no mass loss due to grease migration) then this additional work would be an acceptable added procedure. However, since no method of application yielded a test without negative sample weights, the theory could not justify the practice.

The final reason for not greasing the substrates was an empirical one learned through a multitude of testing. Particle bounce was visibly noticeable on the upper stages (0 and 1). These very large particulates appear not to be passing to succeeding impaction plates as hypothesized, but they are left upon the stages themselves. That is, the particles on these stages have inertias that are too great to even be reentrained by the sample stream. These particles were often lost in terms of total mass measurements, but they were also the least representative of actual engine exhaust particulates. These particles were largely agglomerates of several reentrained particles that have grown over several tests. In terms of size, these coarse mode particulates are greater than approximately 10 $\mu \mathrm{m}$, and it is well recognized that particles of this size are not products of combustion, are not respirable and are usually neglected in air quality studies. For this litany of reasons the MOUDI substrates were left ungreased. 
One of the major benefits the MOUDI offers is a uniformly deposited sample. As mentioned, this prevents caking of particulates on the substrates. Without this attribute, sampling times would need to be greatly reduced to avoid the problems associated with particle stacking and similar sampling woes.

\subsubsection{MOUDI Description and Theory}

The Microorifice Uniform Deposit Impactor (MOUDI) was developed at the University of Minnesota by Virgil A. Marple, Kenneth L. Rubow, and Steven M. Behm. This 10-stage cascade impactor classifies particles by their aerodynamic diameter in the range of $18 \mu \mathrm{m}$ to $0.056 \mu \mathrm{m}$. The advantage the MOUDI has over other cascade type impactors is its ability to collect ultrafine particles with a moderate pressure drop and uniform deposit. With these features, the MOUDI is well suited to measure diluted engine exhaust particulate matter.

By rotating the impaction plates, a uniform deposit can be obtained upon the MOUDI substrates. The nozzles are placed at calculated distances from the center of rotation to allow a concentric deposit. This reduces the chance for particle stacking and caking, which could lead to greater bounce and uneven surface impaction. The stages are rotated relative to those above and below. While the impaction plate is rotated in conjunction with the upper nozzle plate, the nozzle plate is rotated in relation to the lower impaction plate. This concept creates a system where each nozzle plate rotates relative to its corresponding impaction plate. 
This unique version of the cascade impactor allows for the measurement of a wide range of particle sizes, while limiting, and in some cases eliminating the seemingly inherent problems associated with impactors.

Two criteria need to be met for the MOUDI to perform ideally. The first requirement is that the axial (y) component of the fluid velocity in the region between the jet exit plane and the impaction plate must be a function of y only, where y is parallel to the centerline of the nozzle. The second is that the particle velocity at the jet exit plane is uniform across the entire jet. If the fluid velocity is equal to that of the particles, then only the first requirement is needed for ideal behavior. In general, this condition is met, but the boundary layer at the nozzle wall alters the particle velocity. This will cause differing relative velocities between the particles and the fluid, therefore necessitating both criteria [Marple and Willeke, 1979].

The velocity flow field is a function of the impactor configuration and the Reynolds (Re) number of the nozzle flow, with Re given by

$$
\operatorname{Re}=\frac{\rho V_{o} W}{\mu}
$$

where:

$$
\begin{aligned}
& \rho=\text { fluid density } \\
& \mathrm{V}_{\mathrm{o}}=\text { mean velocity at the nozzle throat } \\
& \mathrm{W}=\text { nozzle diameter } \\
& \mu=\text { air viscosity }
\end{aligned}
$$


While the physical parameters and Reynolds number describe the flow field, the particles' equation of motion will determine the trajectory of the particles in the flow field [Marple and Liu, 1974]. The solution of the motion equation is a function of the particle Stokes number, which is the ratio of the aerodynamic particle stopping distance to the radius of the nozzle throat [Marple and Willeke, 1979].

Therefore, the governing equation for the MOUDI is

$$
S t k=\frac{\rho_{p} C V_{o} D_{p}^{2}}{9 \mu W}
$$

where:

$$
\begin{aligned}
& \text { Stk }=\text { Stokes number } \\
& \rho_{p}=\text { particle density } \\
& C \quad=\text { Cunningham slip correction factor } \\
& D_{p}=\text { particle diameter }
\end{aligned}
$$

The Stokes number predicts whether a particle will impact or follow the air stream, where it will remain suspended until it eventually loses the momentum to stay within the streamlines and impacts. It can also be expressed as the ratio of the particle relaxation time to the transit time of the fluid flow through the impaction region, where the relaxation time is defined as the time for a particle initially at rest to accelerate within 1/e of the velocity of the airstream [Hering, 1995]. A more useful form of this equation is used to define particle size: 


$$
50 d_{a e}=\sqrt{\frac{9 \mu \pi n W^{3}}{4 \rho_{p} Q C}} \sqrt{S t k_{50}}
$$

where:

$$
\begin{aligned}
& 50 \mathrm{~d}_{\mathrm{ae}} \quad=\text { value of particle diameter collected with } 50 \% \text { efficiency } \\
& \mathrm{Stk}_{50} \quad=\text { critical Stokes number } \\
& \mathrm{n} \quad=\text { number of jets per stage } \\
& \mathrm{Q} \quad=\text { volumetric flow rate }
\end{aligned}
$$

By manipulating the slip factor (C) and nozzle diameter (W), the operating pressure drops for the MOUDI can be kept relatively low and small particles collected. The particle slip factor, a function of the ratio between the particle aerodynamic diameter and the mean free molecular path of the surrounding medium, is increased as the pressure decreases. Similarly, a large number of small nozzles will allow collection at low pressures. This is also important in limiting particle bounce of the large particles due to their high inertia.

\subsubsection{MOUDI Operation}

The actual testing procedure of the MOUDI is one that must be learned through practice and repeated trials. There is but one critical parameter that must be met to assure proper operation; the flowrate through the stages. This seemingly simple requirement can be greatly affected by a number of factors, each of which involves system leaks. Only after a series of controlled error tests can the MOUDI data be considered accurate.

One source of leaks is the body stage and orifice plate o-rings. These rings must be free of any debris and must always be properly greased. In-field testing is rarely 
performed under clean conditions (at least those to satisfy successful MOUDI operation). Relatively small debris can cause an improper seal between the stage bodies, which will cause a flowrate of less than the necessary $30 \mathrm{lpm}$. Similarly, if these o-rings begin to desiccate, leaks may occur through the stages. If they do become dry, the MOUDI may not rotate properly: this could cause catastrophic failure to the motor and/or gear assembly.

As can be seen, small variations can have adverse effects upon the MOUDI. Another seemingly insignificant but quite vital parameter is the cleanliness of the body stage washers. Once again, small debris that may easily adhere to the PTFE washers that fit between the stages can cause relatively large leaks during operation. In contrast to the o-rings, the washers must be free of grease. If grease accumulates, the seal may not be air tight due to slipping as the stages rotate and/or air pockets being created where the vacuum may draw in outside air. Due to the close proximity to the o-rings, grease quickly migrates to the washers as the substrates are removed and reloaded during testing. Regular and routine cleaning was performed to maintain these required conditions.

The greatest source of leaks in the MOUDI occurs at the afterfilter. It is crucial that the filter and holder are secured tightly; however, appearances can be deceiving. What may seem to be a proper seal can actually leak. The 37-mm Teflo filter has an outer ring which is necessary to withstand the clamping by the holder with out damaging the filter face. Unfortunately, this ring is often the site of leaks. Depending upon the desired collection efficiency, afterfilters with pore size of $1 \mu \mathrm{m}$ or $2 \mu \mathrm{m}$ can be used. The $2 \mu \mathrm{m}$ filter has an outer ring which has a malleability that allows for a snug fit in the 
holder; the $1 \mu \mathrm{m}$ filter does not and cannot create an air tight seal. It should be noted that the only brand of these filters used was Gelman Sciences Teflo, so other brands may be suitable for MOUDI regardless of pore size.

One final cause of leaks lies within the nozzle plates. If the orifices become clogged (only the lower four stages can be thus affected) the air stream is diverted around the plates, causing deterioration of these o-rings. As more nozzles become plugged by particulates, the pressure builds through the lower stages (7-10) and increases the perturbations in the air flow, thereby adding more sample to the o-rings and bypassing the substrates. A simple check of the lower pressure gage will alert the user to this possible scenario. The orifices should be cleaned by a solution that will not in anyway affect them. Sonication is not recommended because the nozzles may be damaged. Liquinox or isopropyl alcohol should be sufficient to dislodge and remove any accumulated particulates in the stages. The stages should be cleaned before any problem is noticed to avoid having to use harsher chemicals such as methanol, methyl chloride, or toluene. Repeated use of these chemical cleansers may begin to abrade the nozzles and cause diameter distortion.

If all these criteria are met, along with a careful loading of the substrates and holders, the MOUDI will operate properly. The necessary maintenance should be developed into an unvarying routine and performed periodically, depending on working conditions and the sample particulate matter material. 


\subsubsection{Particulate Sampling: Cyclones}

Two opposed cyclone probes are used to draw diluted exhaust samples of 16.7 lpm for the PM2.5 cyclone and 28.3 lpm for the PM10. Both cyclones were operated in a similar manner. A rotary vane vacuum pump, which was connected to a flow controller, drew the respective sample flow through the cyclone. It was necessary to rework the system in order to maintain a constant volumetric flow rate. Temperatue was monitored at the cyclone inlet and integrated into the feedback flow control system to calculate and continuously update the actual flow rate. Figure 3.7 shows the cyclone sampling system.

The existing laboratory setup was altered in order to accommodate the addition of the cyclones. The laboratory computer controlled operation of the PM10 and PM2.5 sampling systems. The system was initiated for a preset duration for both steady state and transient tests. A Gast 1023 vacuum pump (10 cfm at a pressure of $10 \mathrm{psig})$ and a Sierra Instruments Inc. Model 740 mass flow controller $(0-7 \mathrm{scfm})$ were used for the PM10 cyclone. A Gast rotary vane vacuum pump, Model no. 0523 and Sierra Instruments Inc. Model 840 mass flow controller were installed to sample through the PM2.5 cyclone. The pump was designed for $4.50 \mathrm{cfm}$ at a pressure of $10 \mathrm{psig}$ and the mass flow controller was rated for 0 to $20 \mathrm{slpm}$. Flexible Tygon tubing was used for both the PM2.5 and PM10 cyclone sampling systems. Both mass flow controllers were calibrated for volumetric control using a Gillian Bubble Flow Meter. Atmospheric pressure and ambient temperature were measured using an absolute pressure gage and a 


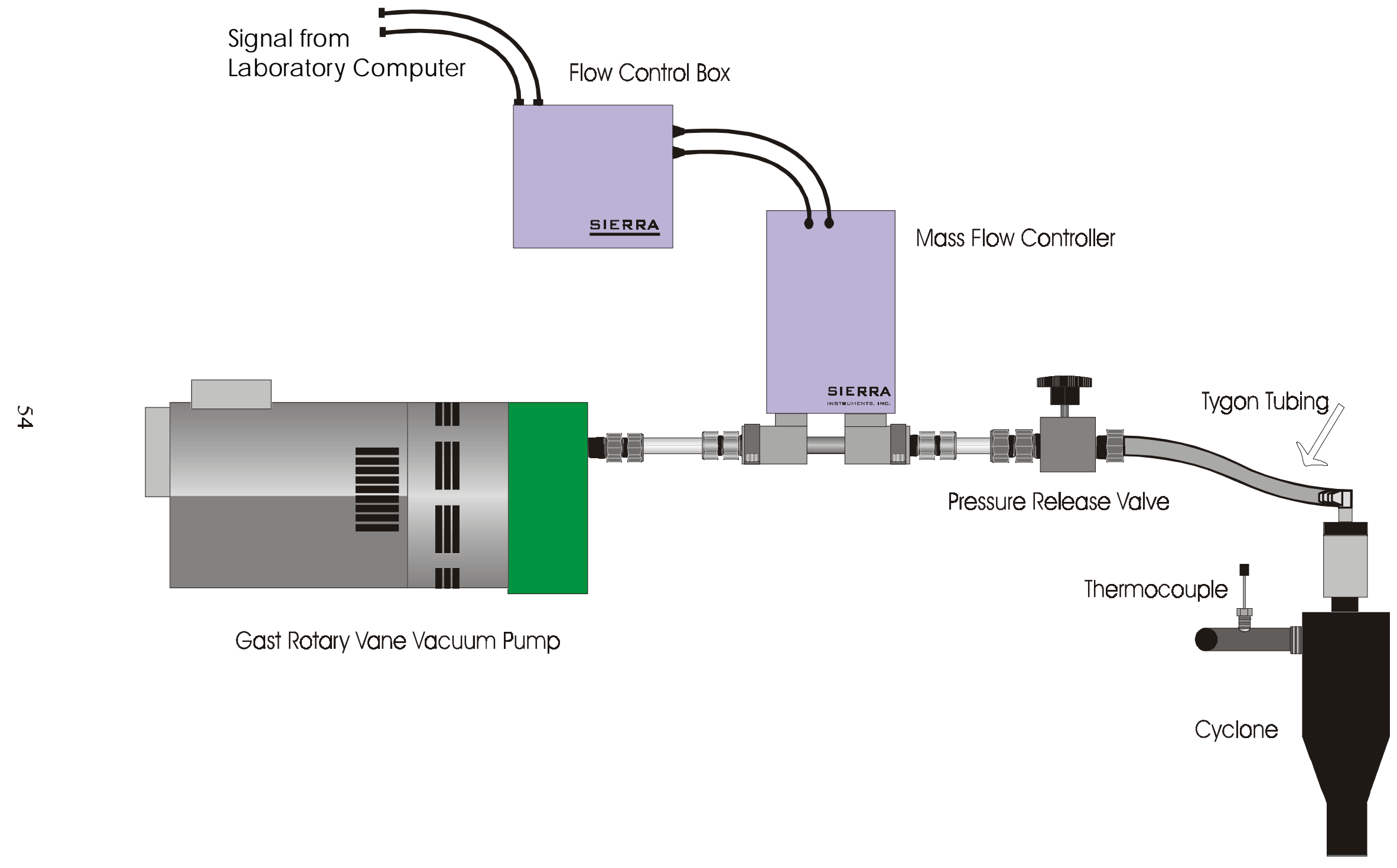

Figure 3.7 - General Cyclone Setup (Not Drawn to Scale) 
J-type thermocouple, respectively. For each set point, ten measurements were made and then averaged. A least-squares straight-line was then determined to fit the flow rate with a corresponding signal. The laboratory computer software was modified to allow operation of the cyclone sampling system for both steady state and transient operations.

Tables 3.1 and 3.2 and Figures 3.8 and 3.9 show the calibration of the PM10 and PM2.5 flow controllers.

Table 3.1 - PM10 Cyclone Modified Flow Controller Calibration

$\begin{array}{ccccccc}\text { Input Voltage } & \text { Actual Flow Rate } & \text { Temperature } & \text { Pressure } & \begin{array}{c}\text { Standard Flow } \\ \text { Rate } \\ \text { slpm }\end{array} & \text { Fit } & \text { Difference } \\ \text { Volts } & \text { lpm } & \text { K } & \text { in Hg } & & & \% \\ 1 & 17.1 & 286 & 29.3 & 17.2 & 0.99318 & -0.68 \\ 1.5 & 24.3 & 286 & 29.3 & 24.5 & 1.51423 & 0.95 \\ 2 & 31.2 & 288 & 29.3 & 31.2 & 1.99257 & -0.37\end{array}$

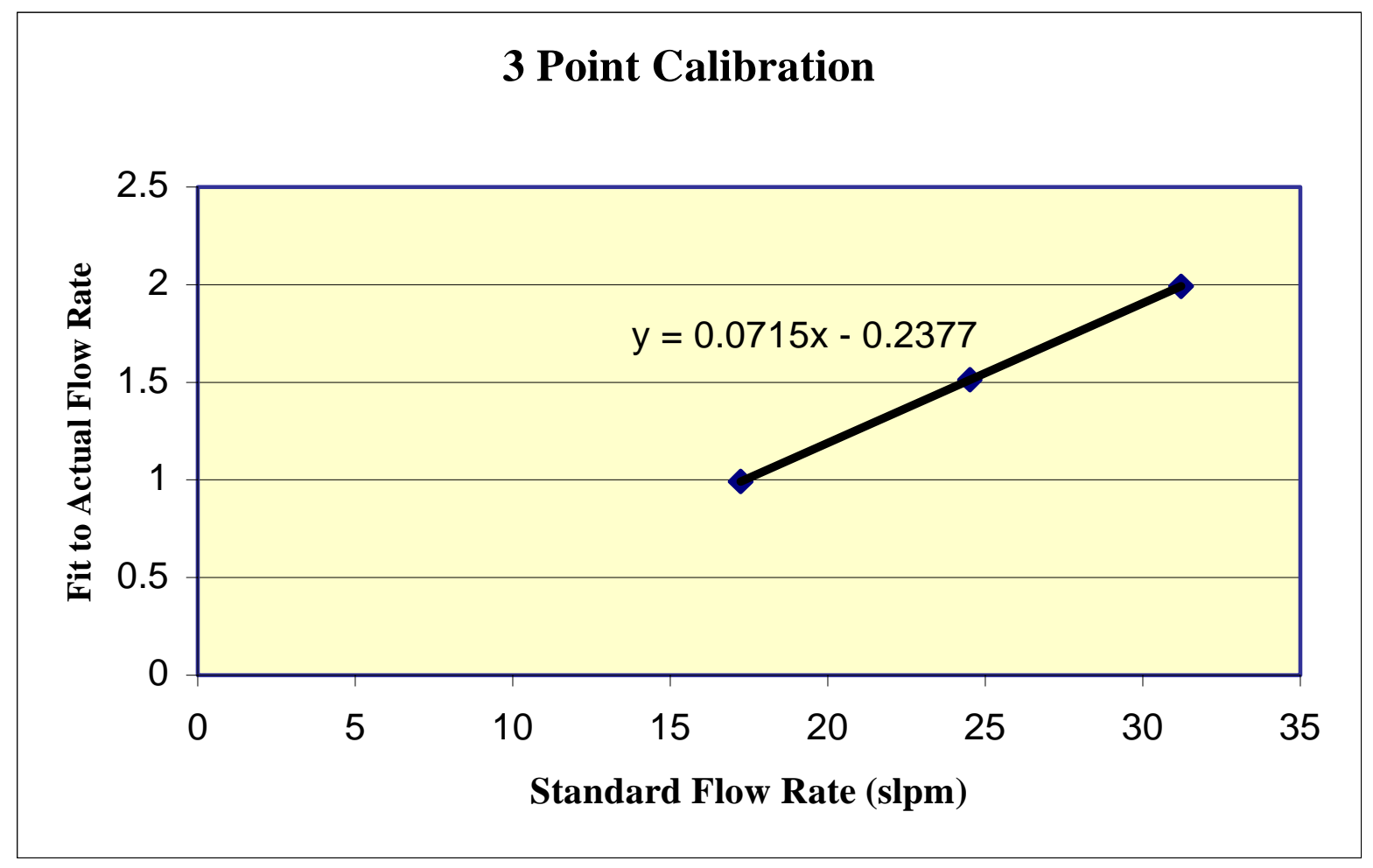

Figure 3.8 - Modified Volumetric Flow Control Calibration (PM10 Cyclone) 
Table 3.2 - PM2.5 Cyclone Modified Flow

Controller Calibration

\begin{tabular}{|c|c|c|c|c|c|c|}
\hline $\begin{array}{c}\text { Input Voltage } \\
\text { Volts }\end{array}$ & $\begin{array}{c}\text { Actual Flow } \\
\text { Rate } \\
\text { Ipm }\end{array}$ & $\begin{array}{c}\text { Temperature } \\
\text { K }\end{array}$ & $\begin{array}{c}\text { Pressure } \\
\text { in } \mathrm{Hg}\end{array}$ & $\begin{array}{c}\text { Standard Flow Rate } \\
\text { slpm }\end{array}$ & Fit & Difference \\
\hline 3 & 13.1 & 302 & 29.1 & 12.4 & 3.06059 & 2.02 \\
\hline 3.5 & 15.0 & 302 & 29.1 & 14.2 & 3.50351 & 0.1 \\
\hline 4 & 16.9 & 303 & 29.1 & 16.0 & 3.92322 & -1.92 \\
\hline 4.5 & 19.2 & 303 & 29.1 & 18.1 & 4.4385 & .1 .37 \\
\hline 5 & 22.0 & 303 & 29.1 & 20.7 & 5.07422 & 1.48 \\
\hline
\end{tabular}

\section{Point Calibration}

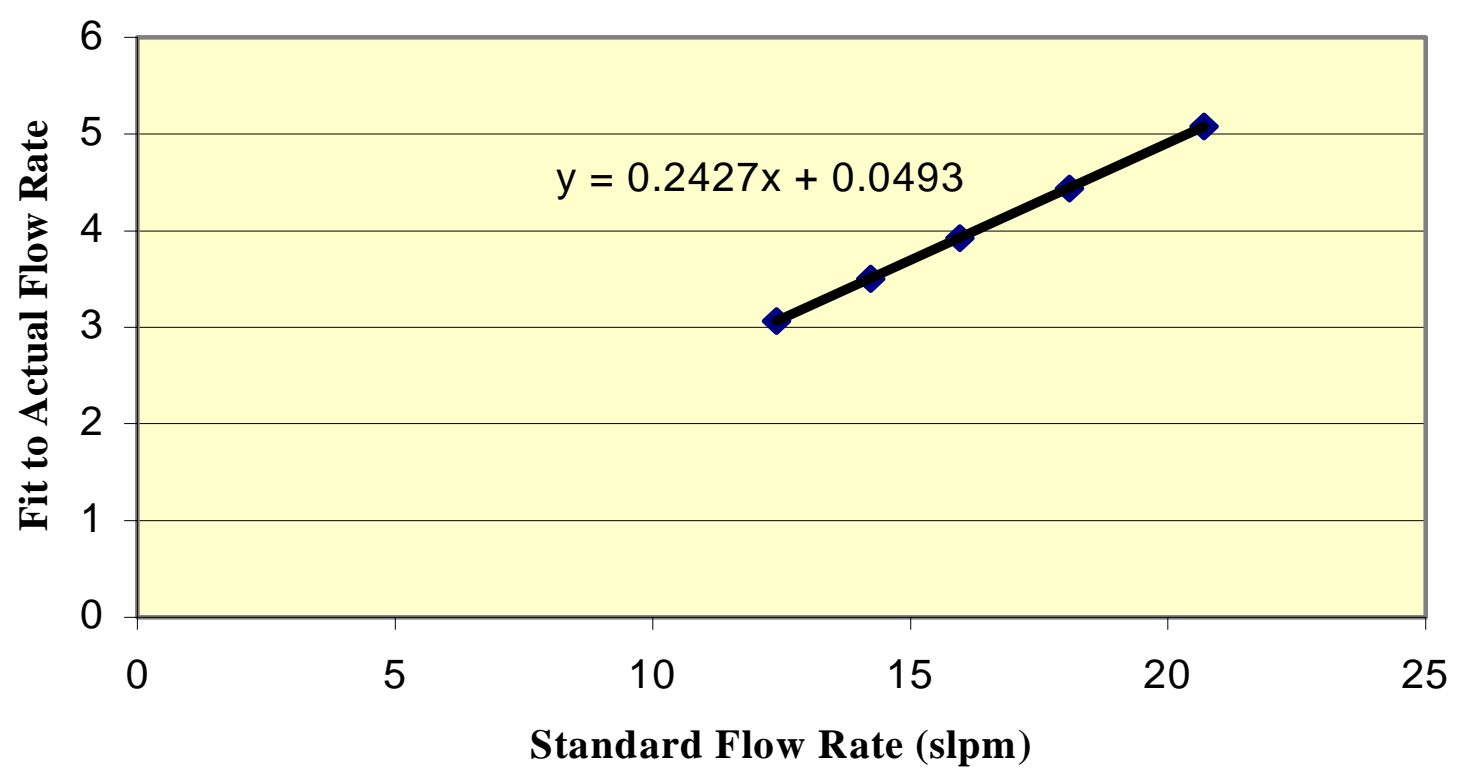

Figure 3.9 - Modified Volumetric Flow Control Calibration (PM2.5 Cyclone)

In conjunction with this system, an Omega J-type 6620SS thermocouple was tapped and welded into each cyclone inlet. The laboratory data acquisition system measured and archived these values at $1 \mathrm{~Hz}$ during a test. The PM2.5 and PM10 mass 
flow controllers were controlled on a continuous basis to maintain actual flow rates of $16.7 \mathrm{lpm}$ and $28.3 \mathrm{lpm}$, respectively.

\subsubsection{Cyclone Description and Theory}

PM10 and PM2.5 cyclones are inertial particle separators which use centrifugal forces to remove heavier particles from a gas stream ( $>10 \mu \mathrm{m}$ and $>2.5 \mu \mathrm{m}$, respectively). The diluted exhaust sample enters the cyclone tangentially, where it spirals downward along the tapered cylinder casing in solid body rotation. Through the course of these revolutions, heavier particles are accelerated outward to the walls where they swirl down to a hopper at the bottom, while the smaller particles remain in the swirling streamlines. The double vortex flow in the cyclone consists of a forced inner vortex moving toward the exit and subsequent filter which contains those particles that do not have the inertia to move to the walls, and a forced outer vortex moving in the opposite direction which contains the heavier particles. The controlling centrifugal force, $\mathrm{F}$, is

$$
F=\frac{\pi d_{p}^{3} \rho u_{t}^{2}}{6 r}
$$

where:

$$
\begin{array}{lll}
\mathrm{u}_{\mathrm{t}} & =\quad \text { tangential particle velocity } \\
\mathrm{r} & =\quad \text { radius of curvature of the particle trajectory } \\
\mathrm{d}_{\mathrm{p}} & =\quad \text { particle diameter. }
\end{array}
$$

The retention time, $\mathrm{t}_{\mathrm{r}}$, for an element of the rigid air stream within the cyclone is

$$
t_{r}=\frac{N_{t}(2 \pi R)}{u_{c}}
$$


where:

$$
\begin{array}{lll}
\mathrm{N}_{\mathrm{t}} & = & \text { number of revolutions } \\
\mathrm{R} & = & \text { radius of cyclone } \\
\mathrm{u}_{\mathrm{c}} & = & \text { inlet velocity. }
\end{array}
$$

Both these equations can be used to determine the efficiency of the cyclone. From the centrifugal force relation, it can be seen that the removal efficiency increases greatly with the particle diameter and the velocity, and inversely with the radius of the particle trajectory. The retention time equation can be expanded to give a more rigorous definition of the collection efficiency. Since the particle radial velocity is

$$
u_{r}=\frac{u_{c}^{2} \tau}{R}
$$

where $\tau$ is the relaxation time of the particle, the time to travel a distance, $x$, across the air stream is

$$
t_{x}=\frac{x R}{u_{c}^{2}} \tau
$$

and the time to travel the entire distance, $\mathrm{B}_{\mathrm{c}}$, of the air stream is

$$
t=\frac{B_{c} R}{u_{c}^{2} \tau}
$$

By equating this time with the transit time through the cyclone, the relaxation time of the smallest particles that can theoretically be collected with $100 \%$ efficiency can be found: 


$$
\tau_{100}=\frac{B_{c}}{2 \pi u_{c} N_{t}}
$$

Furthermore, this can be rewritten in terms of the particle diameter

$$
d=\sqrt{\frac{9 B_{c} \mu}{\pi \rho u_{c} N_{t}}}
$$

The efficiency, $\varepsilon$, is found from the ratio of $\mathrm{x} / \mathrm{Bc}$

$$
\varepsilon=\frac{2 \pi N_{t} u_{c} \tau}{B_{c}}
$$

Simplifying these several expressions using the Stokes Number,

$$
S t k=\frac{2 u_{c} \tau}{B_{c}}
$$

we finally arrive at the cyclone efficiency

$$
\varepsilon=\pi N_{t} S t k
$$

From this form of the equation, it can be seen that the efficiency of the cyclone is dependent upon the number of revolutions and the dimensionless Stokes Number. By applying this theory, high efficiency cyclones have been developed which can capture respirable particles.

\subsubsection{Cyclone Operation}

As can be seen by the governing equations for the cyclone, the inlet flow is a vital parameter in operation. This is the only controllable variable during use of the cyclone, as construction obviously cannot be modified. A vacuum pump is used to draw the 
required flow through the cyclone body and in-line filter. For the PM2.5 cyclone the necessary flow rate is $16.7 \mathrm{lpm}$; for the PM10 cyclone, $28.3 \mathrm{lpm}$. Maintenance of this exact flow can be difficult if the conditions at which the sample is being collected are not near standard. As described in the previous section, cyclone use in a dilution tunnel requires a feedback control system to adjust the flow to account for variations in temperature and pressure. This signal was used to control a mass flow controller that will be responsible for maintaining the required constant flow.

Primary and secondary T60A20 Pallflex glass fiber filters are used to capture the smaller particles that get carried out through the vortex finder. Filter loading will generally not effect the vacuum flow since the pressure drop within the cyclone itself is minimal. Particles that remain in the air stream are subsequently taken into the inner vortex where they then exit the cyclone. The weight of the filters yielded the mass of particles less than $10 \mu \mathrm{m}$ and $2.5 \mu \mathrm{m}$, assuming a rigid cut-off diameter. This is, of course, not possible due to the very nature of the $50 \mathrm{~d}_{\mathrm{ae}}$ method of measuring particle size: the cyclone will only be $50 \%$ efficient for particles $10 / 2.5 \mu \mathrm{m}$ and smaller, respectively.

Cyclones do circumvent theory to a degree since it is not trivial to completely describe the inner and outer vortex flow fields due to complexities stemming from possible turbulent flow behavior and deviations from solid body rotation. Although these discrepancies are inherent in cyclones, they are still used to collect particles based on size because they do give a substantial removal efficiency of large particles. Recent refinements by companies such as URG have advanced cyclone technology by redesigning the body shape and inlet. These have led to cyclones that can capture particles smaller than most thought possible five years ago. 


\subsubsection{SMPS Description and Theory}

The Scanning Mobility Particle Sizer (SMPS) by TSI, Inc. consists of two main subsystems: an Electrostatic Classifier or Differential Mobility Analyzer and a Condensation Particle Counter. The Differential Mobility Analyzer (DMA) removes a known size fraction of submicron particles from an inlet sample stream. Once the mobility equivalent diameters are measured, the particles pass to the Condensation Particle Counter (CPC) which measures the concentration of the particles. The system provides software that can be used to scan and record a range of particle sizes or one distinct size.

The aerosol to be analyzed first enters an impactor which removes particles larger than a known size. This size selective removal mechanism's cut-point diameter (50dae) is a function of the nozzle diameter and flow rate. These two values are used in tandem with the Stokes number to calculate the 50dae. These larger particles must be removed because they contribute negatively, that is increase the number of multiply charged particles. Once the aerosol passes through the impactor, it enters the DMA.

The DMA is used to remove a size-selective fraction of submicron particles from the polydisperse aerosol. To accomplish this, the sample stream first enters a $\mathrm{Kr}-85$ Bipolar Charger where the aerosol particles are subjected to high concentrations of bipolar ions. The random thermal motion of the ions causes frequent particle-ion collisions, which impart a bipolar charge distribution to the particles as they reach equilibrium. The DMA contains two concentric cylinders. Between these cylinders flow 
the polydisperse aerosol as well as sheath air. The charged aerosol surrounds the inner core of sheath air as both flow (laminar) down the annulus without mixing. The inner cylinder is the collector rod and is maintained at a negative voltage while the outer cylinder is grounded. This voltage potential creates an electric field between the cylinders, which in part determines the electrical mobility of the particles.

In the presence of an electric field, the collector rod attracts the positively charged particles through the sheath air. Particles are precipitated along the collector rod, with the location of them being determined by their electrical mobility. Particles with low electrical mobility are precipitated along the lower portion of the rod. As particle mobility increases, the precipitation takes place at increasing distances on the collector rod. Only the particles within a narrow band of electrical mobilities exit along with the monodisperse air. These pass through a small slit at the bottom of the collector rod. In order to find which particles are allowed to exit, the electrical mobilities must first be determined.

A particle within an electric field is subjected to an electric force that causes it to move quickly through the suspending gas. The resulting drag force is expressed by the Stokes law as the particle reaches its terminal settling velocity. By equating the electrical force to the Stokes law, the electrical mobility can be calculated. The combined equation for this is

$$
Z_{p}=\frac{n e C}{3 \pi \mu D_{p}}
$$

where: 


$$
\begin{array}{lll}
\mathrm{n} & = & \text { number of elementary charges on the particle } \\
\mathrm{e} & = & \text { elementary charge }\left(1.6 * 10^{-19} \text { Coulombs }\right) \\
\mathrm{C} & = & \text { Cunningham slip correction } \\
\mu & = & \text { gas viscosity } \\
\mathrm{D}_{\mathrm{p}} & = & \text { particle diameter }
\end{array}
$$

The range of particle sizes removed by the DMA depends on this mobility as well as the aerosol flow rate and the geometry of the concentric cylinders. Knudson (1975) found these parameters could be given by

$$
Z_{p}=\frac{q_{s h}}{2 \pi V L} \ln \left(\frac{r_{2}}{r}\right)
$$

where:

$$
\begin{array}{lll}
\mathrm{q}_{\mathrm{sh}} & = & \text { sheath air flow rate } \\
\mathrm{r}_{1} & = & \text { inner radius of annular space } \\
\mathrm{r}_{2} & = & \text { outer radius of annular space } \\
\mathrm{V} & = & \text { average voltage of collector rod } \\
\mathrm{L} & = & \text { characteristic length }
\end{array}
$$

By combining these two equations, the diameter of particles that exit the DMA can be determined: 


$$
D_{p}=\frac{2 n e \bar{V} L C}{3 \mu q_{s h} \ln \left(\frac{r_{2}}{r_{1}}\right)}
$$

The two terms that can be manipulated are the collector rod voltage and the sheath air flow rate. Increasing the voltage will increase the size of particles exiting the DMA; conversely, increasing the sheath air flow rate will decrease the size. The only term that is variable but uncontrolled is the number of charges. Assuming a Boltzmann charge distribution, multiple charges, up to six per particle, are corrected for using software tools.

Once the aerosol exits the DMA, it enters the CPC. The CPC counts the particles optically, but must first make them large enough to be measured. It does this by condensing alcohol onto each particle, then passing them through a light source. Hence, particle concentrations are measured on a real-time basis.

Upon entering the CPC, the aerosol is passed over a heated pool of butanol where it is saturated. This saturated aerosol then flows into a condenser where it is cooled by thermal diffusion. This process allows the butanol to condense onto the particle surface, wherein they grow into droplets. This heterogeneous condensation is sustained through the presence of condensation nuclei in a saturated state. The saturation ratio, the partial vapor pressure divided by the saturation pressure, will decide the range of detectable particles, as seen by the Kelvin equation: 


$$
S=\exp \left[\frac{4 \sigma M}{\rho R T d^{*}}\right]
$$

where:

$$
\begin{array}{lll}
\mathrm{S} & = & \text { saturation ratio } \\
\sigma & = & \text { surface tension } \\
\mathrm{M} & = & \text { molecular weight } \\
\rho & = & \text { density of butanol } \\
\mathrm{R} & = & \text { universal gas constant } \\
\mathrm{T} & = & \text { absolute temperature } \\
\mathrm{d}^{*} & = & \text { Kelvin diameter }
\end{array}
$$

The Kelvin diameter is defined as the diameter of a droplet that has reached equilibrium, such that it will neither evaporate nor grow for a given saturation ratio. Solving the equation for the Kelvin diameter, the droplet size can be found:

$$
d^{*}=\left(\frac{4 \sigma M}{\rho R T}\right) * \frac{1}{\ln S}
$$

At this point, the particles are large enough to be detected optically. As they pass through a light beam, they cause the light to scatter. This scattering is projected onto a photodetector which counts the total number of pulses induced by the particle laden aerosol. This is the method for counting the particles if they are at concentrations lower than 10,000 particles $/ \mathrm{cm}^{3}$. Errors begin to propagate at the higher end of this range due 
to coincidence, as more than one particle may occupy the sensing volume at the same time. CPC models 3022A and 3025A use real-time counting as concentrations approach the upper limit to automatically take coincidence into account. However, statistical errors limit the accuracy of readings at lower concentrations as well. The uncertainty increases as the total number of particles counted decreases. Ideally, concentrations should remain above $10 \%$ of the low end and below $10 \%$ of the high end to reduce the statistical uncertainty and coincidence.

The software supplied by the manufacturer runs the SMPS during operation, though several values need to be inputed. For a full scan of all detectable particle sizes, the software requires the scan times and flow rates for the aerosol and sheath air. For single particle size measurements, the only data necessary is the size to be tracked, the flow rates and the models of the DMA and CPC being used. The rest is computer controlled and requires no additional attention throughout the duration of a test.

The DMA draws sample from the tunnel through a short section of carbon impregnated tubing. This tubing type is also used to connect the CPC to the DMA.

\subsection{Vehicles}

The three sites where these tests were conducted were Denver, Colorado, Morgantown, West Virginia, and Dallas, Texas. The tests in Denver were performed from September 26 to October 6, 1998. Of the six buses tested, three were powered by 1997 ISB 5.9 liter Cummins diesel engines and three by 1997 B5.9 liter Cummins natural gas engines. The latter three were Low Emitting Vehicle (LEV) certified. These natural 
gas fueled vehicles had lean-burn, spark ignited engines. The Regional Transportation District (RTD) in conjunction with GO Boulder supplied all of the buses. The vehicle specifications are shown below in Table 3.3 and the descriptions in Table 3.4 [Clark, et al., 1999a].

\section{Table 3.3 - General Specifications of GO Boulder SKIP Buses}

\begin{tabular}{ll}
\hline Bus & World Trans 3000 \\
Manufacturer & World Trans of Newton, Kansas \\
\hline Gross Vehicle Weight & $18,780 \mathrm{lb}$ \\
Engines & Cummins ISB5.9 and B5.9 \\
Configuration & In-line 6 cylinder \\
Displacement & 5.9 liters \\
Intake & Turbocharged /Air to air after-cooled \\
Horsepower & 175 hp diesel \\
Horsepower & 195 hp natural gas \\
Exhaust Conditioning & Nelson Integrated Muffler/Catalyst \\
\hline
\end{tabular}

Table 3.4 - Description of GO Boulder SKIP Buses

\begin{tabular}{c|cccccc} 
Vehicle ID & $\mathbf{1 0 1 0}$ & $\mathbf{1 0 1 1}$ & $\mathbf{1 0 1 2}$ & $\mathbf{1 0 1 3}$ & $\mathbf{1 0 1 4}$ & $\mathbf{1 0 1 5}$ \\
\hline Fuel & Diesel & Diesel & Diesel & CNG & CNG & CNG \\
Engine & ISB5.9 & ISB5.9 & ISB5.9 & B5.9 & B5.9 & B5.9 \\
Year & 1997 & 1997 & 1997 & 1997 & 1997 & 1997 \\
Catalyst & Yes & Yes & Yes & Yes & Yes & Yes*
\end{tabular}

* Bus 1015 had catalyst removed prior to testing, though LEV certified. 
The DART fleet was tested from February 13 through March 10, 1999. These tests were performed on two vehicle types. The diesel buses were 1998 6-cylinder Cummins M11-280E+ (10.8 liter) and the LNG were 1998 6-cylinder Cummins L10-280G (10 liter). Both the vehicles had 5 speed automatic transmissions and were manufactured by Nova. These buses were similarly equipped with Nelson Integrated Muffler/Catalysts. Table 3.5 gives the vehicle specifications and Table 3.6 gives the descriptions.

\section{Table 3.5 - General Specifications of DART Buses}

\begin{tabular}{ll}
\hline Bus & DART \\
Manufacturer & Nova \\
\hline Engine (diesel) & Cummins M11-280E+ \\
Engines (natural gas) & Cummins L10-280G \\
Configuration & In-line 6 cylinder \\
Displacement (diesel) & 10.8 liters \\
Displacement (natural gas) & 10 liters \\
Intake & Turbocharged /After-cooled \\
Horsepower & 280 hp @ 2100 rpm \\
Exhaust Conditioning & Nelson Integrated Muffler/Catalyst \\
\hline
\end{tabular}

Table 3.6 - Description of DART Buses

\begin{tabular}{c|cccccccccc} 
Vehicle ID & $\mathbf{4 2 2 0}$ & $\mathbf{4 2 2 1}$ & $\mathbf{4 3 2 0}$ & $\mathbf{4 3 2 1}$ & $\mathbf{4 3 2 2}$ & $\mathbf{4 3 2 3}$ & $\mathbf{4 3 2 4}$ & $\mathbf{4 3 2 5}$ & $\mathbf{4 3 2 8}$ & $\mathbf{4 3 2 9}$ \\
\hline Fuel & D2 & D2 & LNG & LNG & LNG & LNG & LNG & LNG & LNG & LNG \\
Engine & M11 & M11 & L10 & L10 & L10 & L10 & L10 & L10 & L10 & L10 \\
Year & 1998 & 1998 & 1998 & 1998 & 1998 & 1998 & 1998 & 1998 & 1998 & 1998 \\
Catalyst & Yes & Yes & Yes & Yes & Yes & Yes & Yes & Yes & Yes & Yes
\end{tabular}


The tests conducted on the PAT fleet were over a 6-week period, beginning December 12, 1998 and lasting through January 29, 1999. The buses were all equipped with Detroit Diesel 6V92 turbocharged/aftercooled, 9 liter, 6 cylinder engines. Table 3.7 gives the vehicle specifications and Table 3.8 gives the descriptions.

\section{Table 3.7 - General Specifications of PAT Buses}

\begin{tabular}{ll}
\hline Bus & PAT \\
Manufacturer & Orion Bus Industries \\
\hline Engines & 2 Stroke DDC 6V92 \\
Configuration & 6 cylinder V \\
Displacement & 9.05 liters \\
Intake & Turbocharged /After-cooled \\
Horsepower & 253 hp @ 2100 rpm \\
Torque & $880 \mathrm{ft}-1 \mathrm{~b} @ 1200$ rpm \\
Exhaust Conditioning & Oxidation Catalytic Converter \\
\hline
\end{tabular}

Table 3.8 - Description of PAT Buses

\begin{tabular}{c|cccc} 
Vehicle ID & $\mathbf{2 0 2 5}$ & $\mathbf{2 0 2 9}$ & $\mathbf{2 0 4 8}$ & $\mathbf{2 0 3 0}$ \\
\hline Fuel & D2/MG & D2/MG & D2/MG & D2/MG \\
Engine & DDC 6V92TA & DDC 6V92TA & DDC 6V92TA & DDC 6V92TA \\
Year & 1991 & 1991 & 1991 & 1991 \\
Catalyst & Yes & No & Yes & No
\end{tabular}




\subsection{Fuels}

Four fuels were used over the course of the testing of these transit buses: diesel \#2 (D2), compressed natural gas (CNG), liquefied natural gas (LNG) and Mossgas. The latter three are considered alternative fuels, as diesel and gasoline are the standard fuels.

CNG and LNG are similar except in form, so they can be categorized together. Natural gas is composed primarily of methane $\left(\mathrm{CH}_{4}\right)$, but may also contain ethane, propane and heavier hydrocarbons. Trace quantities of nitrogen, oxygen, carbon dioxide, sulfur compounds and water may also be present. The liquefaction process can remove the trace elements and compounds, with the exception of nitrogen. While CNG is pressurized natural gas, LNG is cooled natural gas. To form LNG, natural gas is cooled to approximately $-260^{\circ} \mathrm{F}$ at atmospheric pressure. At these conditions, it condenses to a liquid. For both forms, natural gas is delivered to an engine as a low-pressure vapor.

Vehicles fueled with natural gas have been shown to reduce mass emission rates of PM, carbon monoxide and oxides of nitrogen. Hence, it may be gaining acceptance as a fuel for transit bus fleets which have been seen as major sources of urban pollution.

In comparison, both forms of natural gas have lesser Lower Heating Values than diesel fuel and are also less efficient on a per gallon basis. The Lower Heating Value (LHV) is the total heat obtained from combustion minus the latent heat of vaporization of the water formed by combustion. The LHV comparison values are shown in Table 3.9. below and general properties of natural gas follow in Table 3.10. 
Table 3.9 - Lower Heating Value Comparison of Fuels

\begin{tabular}{c|cc} 
Fuel & LHV & $\begin{array}{c}\text { Gallons needed for same } \\
\text { mileage as gasoline }\end{array}$ \\
\hline Diesel & $129,000 \mathrm{Btu} /$ gallon & 0.89 \\
LNG & $76,000 \mathrm{Btu} /$ gallon & 1.50 \\
CNG & 114,000 Btu/equiv. gallon & 1.0
\end{tabular}

Table 3.10 - General Properties of Natural Gas

\begin{tabular}{l|c|c}
\multicolumn{1}{c|}{$\begin{array}{c}\text { Fuel } \\
\text { Property }\end{array}$} & Units & Value for Natural Gas \\
\hline Molecular Weight & & $\sim 18$ \\
Specific Gravity & $\mathrm{kg} / \mathrm{m}^{3}$ & $\sim 0.79$ \\
Specific Heat (vapor) & $\mathrm{kJ} / \mathrm{kg} \cdot \mathrm{K}$ & $\sim 2.0$ \\
$\mathrm{HHV}$ & $\mathrm{MJ} / \mathrm{kg}$ & 50 \\
$\mathrm{LHV}$ & $\mathrm{MJ} / \mathrm{kg}$ & 45 \\
LHV of Stoich. Mixture & $\mathrm{MJ} / \mathrm{kg}$ & 2.9 \\
(A/F) @ Stoich. & & 14.5
\end{tabular}

The last fuel used during a portion of this study was a synthetic form of diesel. Mossgas is a type of fuel that uses the Fischer-Tropsch process. Natural gas is first converted into synthesis gas, which is a mixture of carbon monoxide and hydrogen, by high temperature reforming or partial oxidation. This synthesis gas is then converted into hydrocarbons by the Fischer-Tropsch reaction:

$$
\mathrm{CO}+2 \mathrm{H}_{2} \rightarrow-\mathrm{CH}_{2}-+\mathrm{H}_{2} \mathrm{O}
$$

After completion of the Fischer-Tropsch process, the light olefins that remained, such as propene, butene, pentene and hexene were used to synthesize the fuel used in this study. 
This conversion of olefins to distillate (COD) had no detectable sulfur, an aromatic content of $10 \%$ by volume and a cetane number of approximately 50 . The lubricity improver Paradyne 655 was added to attain proper lubricity levels [Clark et al., 1999]. The properties of this form of automotive fuel and Federal no. 2 diesel fuel are shown in Table 3.11 below [Clark et al., 1999].

Table 3.11

\begin{tabular}{|c|c|c|c|c|c|}
\hline $\begin{array}{c}\text { Fuel } \\
\text { Analysis }\end{array}$ & ASTM & Units & $\begin{array}{c}100 \% \text { MG } \\
\text { (Mossgas Data) }\end{array}$ & $\begin{array}{c}100 \% \text { MG } \\
\text { (SWRI Data) }\end{array}$ & $\begin{array}{c}\text { Federal } \\
\text { Diesel Fuel }\end{array}$ \\
\hline Flash Point & D93 & ${ }^{\circ} \mathrm{C}$ & 100 & 97 & \\
\hline Cloud Point & D2550 & ${ }^{\circ} \mathrm{C}$ & & $<-60$ & \\
\hline Water Content & D1744 & vol \% & 0.01 & & \\
\hline Ash & D482 & wt $\%$ & $<0.01$ & & \\
\hline $\begin{array}{l}\text { Distillation } \\
\text { IBP } \\
10 \% \\
50 \% \\
90 \% \\
\text { FBP }\end{array}$ & D86 & ${ }^{\circ} \mathrm{C}$ & $\begin{array}{l}321.1 \\
360.8\end{array}$ & $\begin{array}{l}229.9 \\
235.3 \\
254.7 \\
323.7 \\
361.2\end{array}$ & $\begin{array}{l}188 \\
212 \\
256 \\
307 \\
331\end{array}$ \\
\hline Kinematic Viscosity & $\mathrm{D} 445$ & $\mathrm{cSt} @ 40^{\circ} \mathrm{C}$ & 2.974 & 2.98 & \\
\hline Sulfur & D2622 & mass $\%$ & $<0.001$ & & \\
\hline Sulfur & D5453 & $\mathrm{ppm}$ & & $<5$ & \\
\hline Sulfur & D4294 & wt $\%$ & & & 0.02 \\
\hline Cetane Index & D4737 & & & & 48.7 \\
\hline Cetane Number & D613 & & 51.4 & 48.9 & \\
\hline Density @ 20ㄷ & $\mathrm{D} 4052$ & & 0.8007 & 0.8042 & 0.8366 \\
\hline API Gravity @ $15.6^{\circ} \mathrm{C}$ & D287 & ${ }^{\circ} \mathrm{API}$ & & 44.0 & 37.4 \\
\hline $\begin{array}{l}\text { Total Aromatics } \\
\text { PNA }\end{array}$ & D5186 & mass $\%$ & & $\begin{array}{l}9.18 \\
0.21\end{array}$ & \\
\hline $\begin{array}{l}\text { FIA } \\
\text { Aromatic } \\
\text { Olefins } \\
\text { Saturate }\end{array}$ & D1319 & vol \% & & & $\begin{array}{c}24.7 \\
1.5 \\
73.8\end{array}$ \\
\hline Aromatics & IP391 & vol \% & 10.1 & & \\
\hline $\begin{array}{l}\text { Lubricity SLBOCLE } \\
\text { Neat Fuel } \\
\text { With Paradyne Additive }\end{array}$ & D6078 & grams & & $\begin{array}{l}1950 \\
3800\end{array}$ & \\
\hline $\begin{array}{l}\text { Carbon/Hydrogen } \\
\text { Carbon } \\
\text { Hydrogen } \\
\text { Nitrogen } \\
\text { Residual } \\
\text { Oxygen (by diff) } \\
\end{array}$ & D2274 & mass $\%$ & & $\begin{array}{l}83.98 \\
14.43 \\
\\
1.59 \\
\end{array}$ & $\begin{array}{l}86.11 \\
13.37 \\
<0.03\end{array}$ \\
\hline $\begin{array}{l}\text { Heat of Combustion } \\
\text { Gross } \\
\text { Net }\end{array}$ & $\overline{\mathrm{D} 240}$ & Btu/gal & & $\begin{array}{l}134,712 \\
125,878\end{array}$ & $\begin{array}{l}137,609 \\
129,147\end{array}$ \\
\hline
\end{tabular}




\section{Chapter 4 - Results}

This chapter presents the data gathered from the three test sites. It also describes the data reduction methods used for the MOUDI and the cyclones and an error analysis.

\subsection{MOUDI Data Reduction Techniques}

The MOUDI data is recorded as a mass-weighted particle size distribution. In order to arrive at this, some reduction techniques are needed to convert the mass collected on each stage to an appropriate weighted distribution. Through calibration of the MOUDI by the manufacturer, MSP Corporation, the effective cut diameters (ECD) were established. These are the aerodynamic particle diameters collected at 50\% efficiency for each stage. From these values, a midpoint diameter, $\mathrm{d}_{\mathrm{p}}$, can be found using the following equation:

$$
d_{p}=\operatorname{anti} \log \left(\frac{\log E C D_{i}+\log E C D_{i+1}}{2}\right)
$$

where the subscript $i$ refers to the stage in question and $i+1$ to the proceeding stage. The logarithmic values are used because it is assumed that the distribution will be lognormal in form. The normalized frequency is then determined, which is the mass collected per stage divided by the total mass and the logarithmic width of each stage. This is necessary 
to spread out the smaller size ranges and compress the larger ones. This unitless value, $\mathrm{dm} / \mathrm{d} \log \left(\mathrm{d}_{\mathrm{p}}\right)$, is the ordinate with the abscissa being the logarithm of the particle diameter. In order to find the mass median aerodynamic diameter $\left(\mathrm{d}_{\mathrm{MM}}\right)$ and the geometric standard deviation (GSD or $\sigma_{\mathrm{g}}$ ) of the distribution, a lognormal curve is generated for a range of all possible particle diameters. The functional form of this equation is

$$
f(d)=\frac{\exp \left[-0.5\left(\frac{\ln d_{p}-\ln d_{M M}}{\ln \sigma_{g}^{2}}\right)\right]}{\sqrt{2 \pi} \log \sigma_{g}}
$$

Obviously, the values for $\mathrm{d}_{\mathrm{MM}}$ and $\sigma_{\mathrm{g}}$ cannot be found directly as they are variables in the equation. Therefore, an iterative scheme is necessary to solve the equation. This is done by simultaneously solving for the sum of the square of the error between the values generated for the fit equation and the frequency. When the error is minimized, the iterations, n, return the mass median aerodynamic diameter and the geometric standard deviation for the distribution. In mathematical terms

$$
\begin{aligned}
& E_{i}=f\left(d_{p}\right)-f\left(d_{i}\right) \\
& E_{\text {min }}=\sum_{i=1}^{n} E_{i}^{2}
\end{aligned}
$$

The minimum error is generally not zero, so the values are not exact. However, this deviation is very small and is reduced further by recording the accuracy of the two values to only three significant digits. 


\subsection{Cyclone Data Reduction Techniques}

The cyclone filters (primary and secondary) were weighed and the mass values were reduced to yield actual grams of particulate matter. A Qbasic program was written to determine these values as well as those from the $70 \mathrm{~mm}$ gravimetric filtration method employed as a standard for the laboratory. This program compared the final weights of the cyclone filters with each other and the secondary dilution tunnel calculated filter weights. This program can be found in Appendix A.

In order to reduce the data using raw mass values from the filters to actual data, the measured mass is first divided by the total volume sampled by the cyclone. This is found by summing the instantaneous flow data recorded by the system computer. A further explanation of this method for the cyclones is detailed in a later section (see §4.4.1). Also needed are the total volume of the diluted exhaust through the primary dilution tunnel and the dilution factor, DF. The functional form of this equation is

$M_{\text {actual }}=\left[\frac{\text { Filter Weight }}{\text { Sample Volume }}-\left\{\frac{\text { Background Filter Weight }}{\text { Background Sample Volume }} \times\left(1-\frac{1}{D F}\right)\right\}\right] \times$ Tunnel Volume

As shown, the background data must be subtracted from the sample data since the intake dilution air is unfiltered. The percent difference, \%Diff, is then calculated by the equation: 


$$
\% \text { Diff }=\frac{M_{\text {actual }, a}-M_{\text {actual }, b}}{\left(\frac{M_{\text {actual }, a}+M_{\text {actual }, b}}{2}\right)} \times 100
$$

where $a$ and $b$ represent the PM10 and PM2.5 cyclones.

\subsection{PAT Tests at Morgantown, WV}

\subsubsection{MOUDI Data Analysis}

Four Detroit Diesel Corporation 6V92 turbocharged/aftercooled (DDC 6V92TA) buses were tested on the mobile emissions testing laboratory's chassis dynamometer in Morgantown, West Virginia, with the MOUDI. Three test fuels were used - diesel \#2, Mossgas and a $50 \%$ blend of both fuels. Since the MOUDI may not be capable of measuring total mass as mentioned, the data only represents a proportionality of the mass weighted size distribution.

The first bus placed on the chassis dynamometer was tested with all three fuel types on the Central Business District (CBD) cycle. It was first thought reasonable to sample throughout the entire fourteen ramps of the cycles, but this soon proved itself unwise. The Pittsburgh Port Authority Transit (PAT) buses, powered by DDC 6V92TA engines, produced high concentrations of PM, regardless of fuel type. High mass loadings on the MOUDI substrates are not desired for two reasons. The first is that for high mass collecting stages, the particulates are no longer deposited on a flat impaction surface. The particles accumulate to such a degree that the substrate surface is 
completely covered, leading to particle loss. The second and more important reason for limiting PM concentrations is that the nozzle plate orifices clog very quickly. Repeated trials at high concentrations can cause the lower stages (7-10) to accumulate the soot along the perimeter of the orifices, which in turn heightens the chance for particles inexorably to lodge in the narrowed openings. This will affect cut off efficiencies and flow rates. Since it is not practical to clean the nozzle plates after each test, the PM concentrations must be kept relatively low. Also, repeated cleaning will abrade the tiny nozzles, thus affecting performance. It was determined that sampling four ramps (11-14) instead of all fourteen would yield sufficient mass to portray the representative size distribution.

Bus No.2025 was equipped with a catalyst. This vehicle was run on D2 for three CBDs, one full and two partial. As can be seen from Figures 4.1 - 4.6, the mass median aerodynamic diameters (MMAD) were all $0.12 \mu \mathrm{m}$. The one CBD run on Mossgas had an MMAD of $0.11 \mu \mathrm{m}$, and the two runs on the 50/50 blend of D2 and Mossgas had MMADs of 0.12 and $0.11 \mu \mathrm{m}$. The statistics for all the buses can be found in Table 4.1.

The first test on all fuels was conducted over the entire fourteen ramps of the CBD cycle, with the other tests for only the final four ramps. From the three diesel runs, it was noted that the number of ramps sampled was adequate in that the MMAD did not vary and the geometric standard deviations (GSD) were similar. Sampling for fewer than four ramps did lead to a high GSD. One test was inadvertently cut short after two ramps were sampled due to a power outage. The resulting GSD was 3.97 with an MMAD of $0.14 \mu \mathrm{m}$. From this, it was determined that a minimum of four ramps was required. This was also deemed sufficient for the other fuels. 
Figure 4.1 - MOUDI Size Distribution for DDC 6V92TA Bus \#2025 CBD Run \#1 Fuel: D2

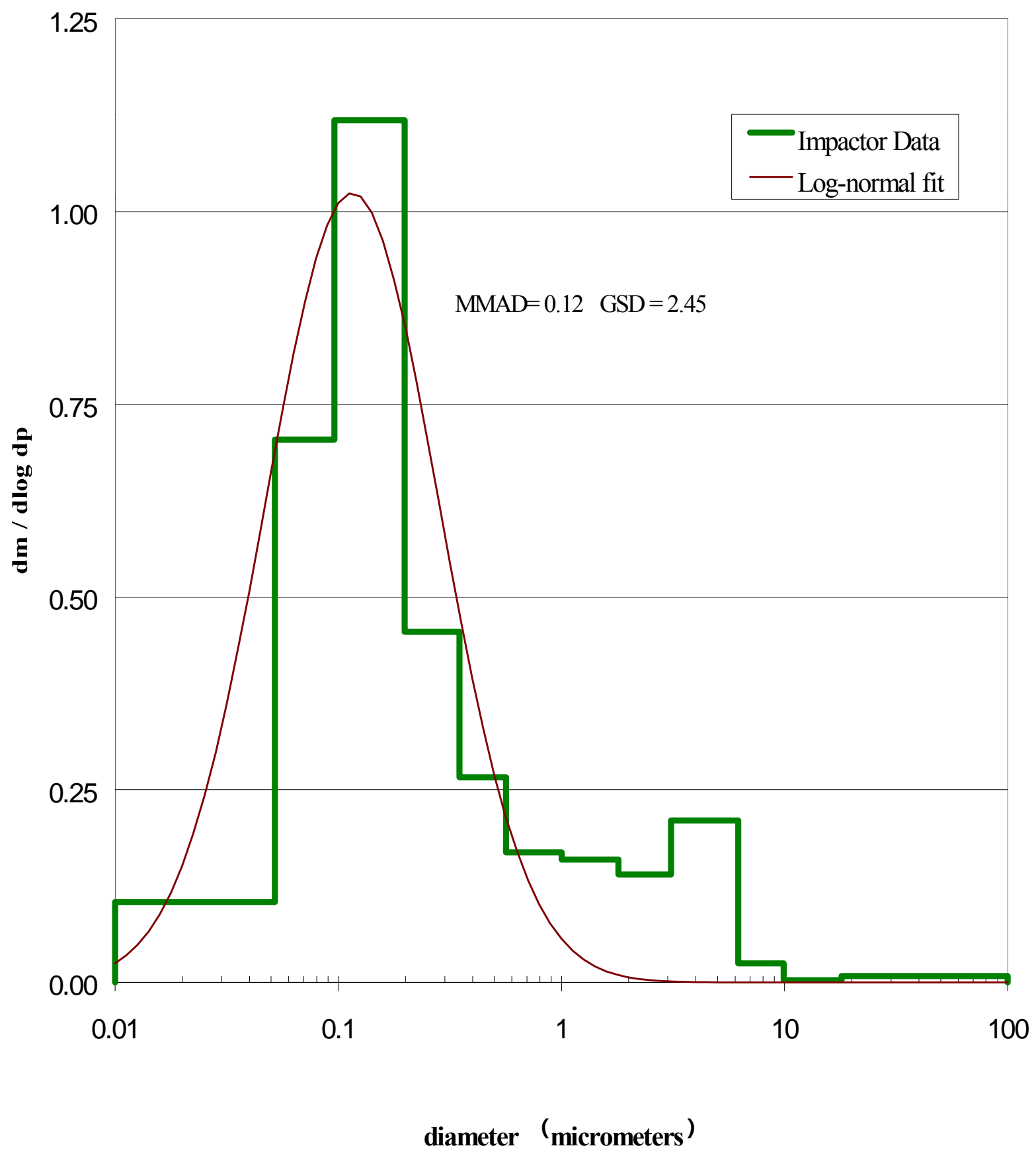


Figure 4.2 - MOUDI Size Distribution for

DDC 6V92TA Bus \#2025

4 Ramps of CBD Run \#2 Fuel: D2

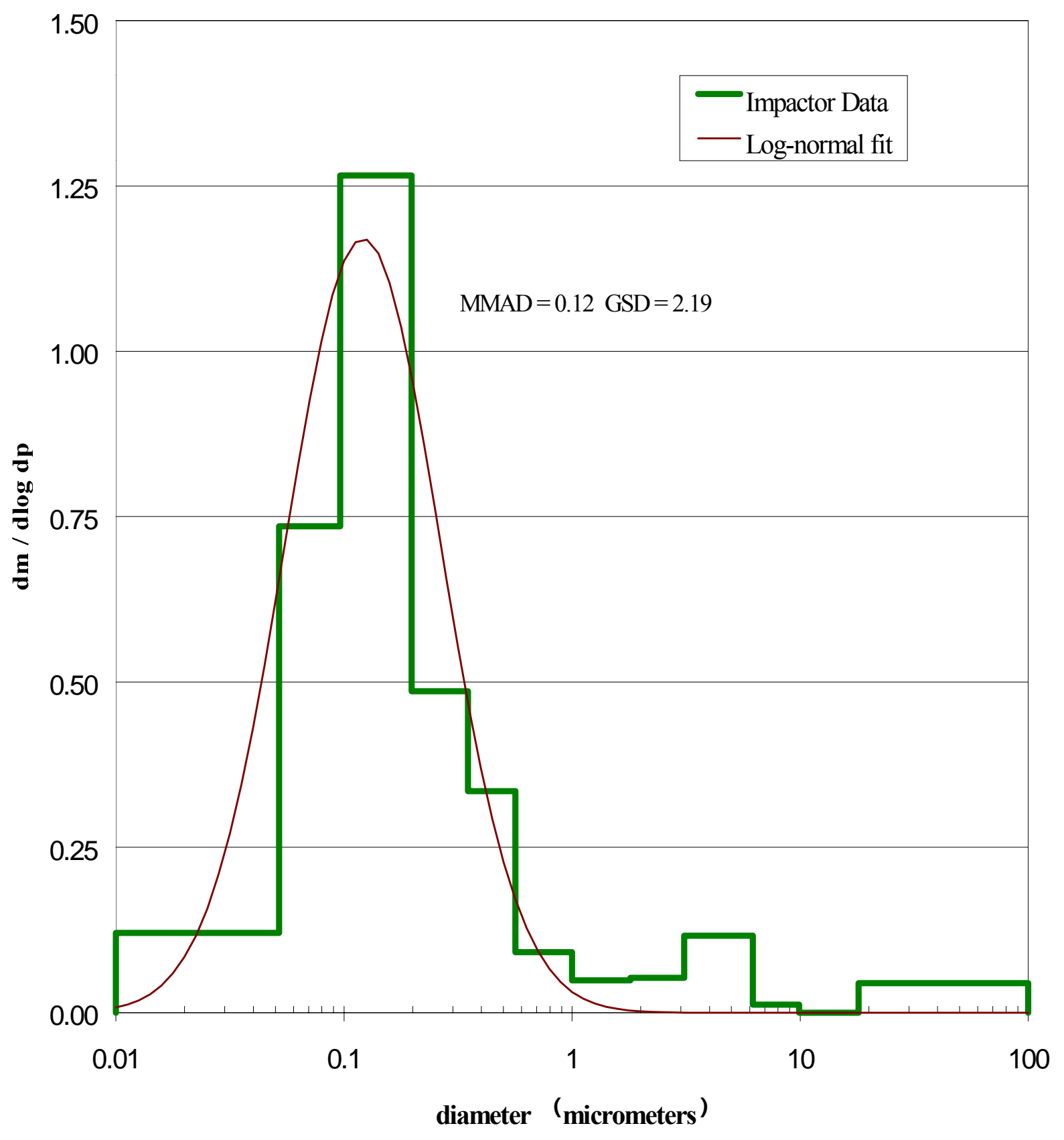


Figure 4.3 - MOUDI Size Distribution for

DDC6V92TA Bus \#2025

4 Ramps of CBD Run\#3 Fuel: D2

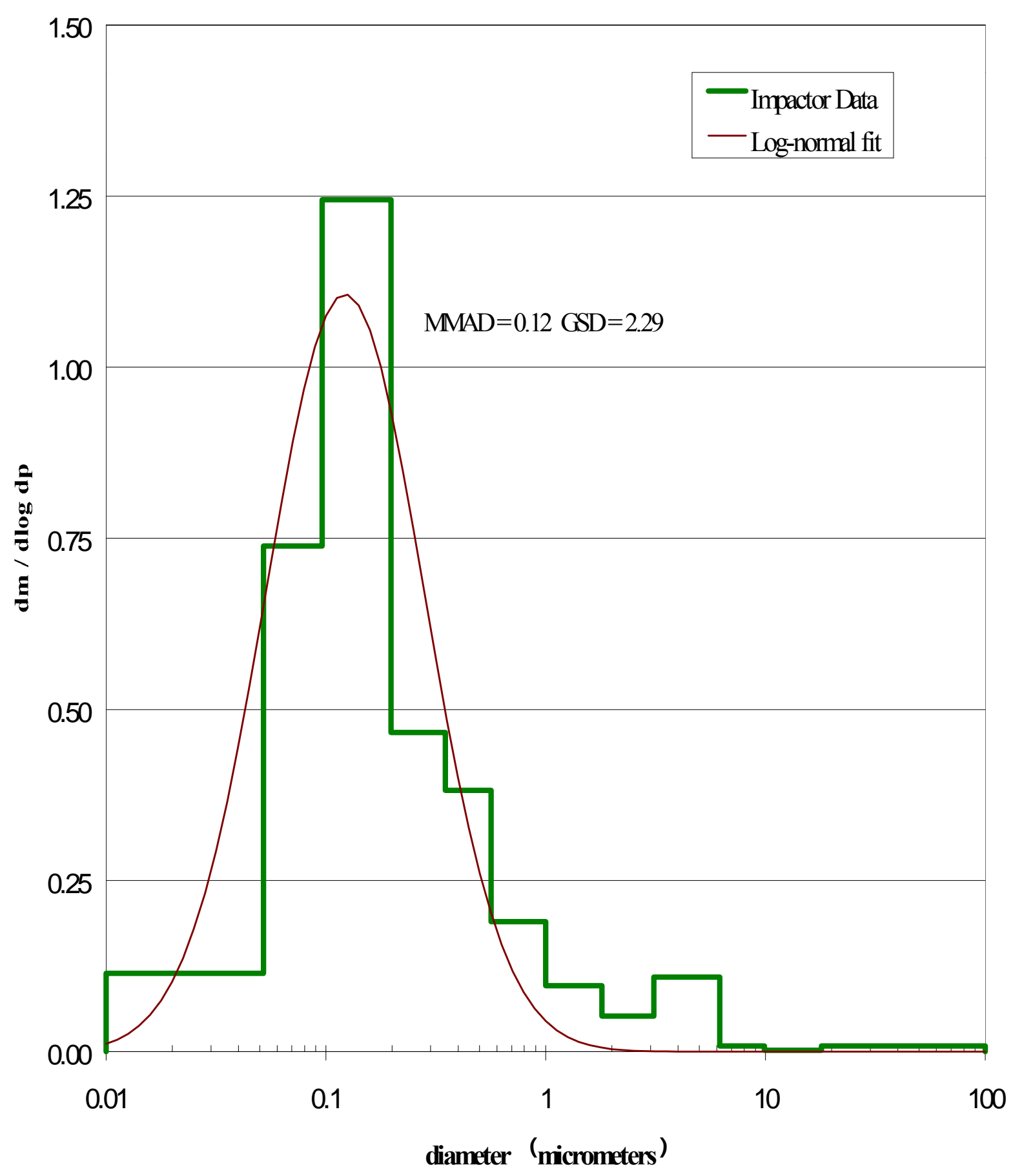


Figure 4.4 - MOUDI Size Distribution for

DDC 6V92TA Bus \#2025

CBD Run \#1 Fuel: MG

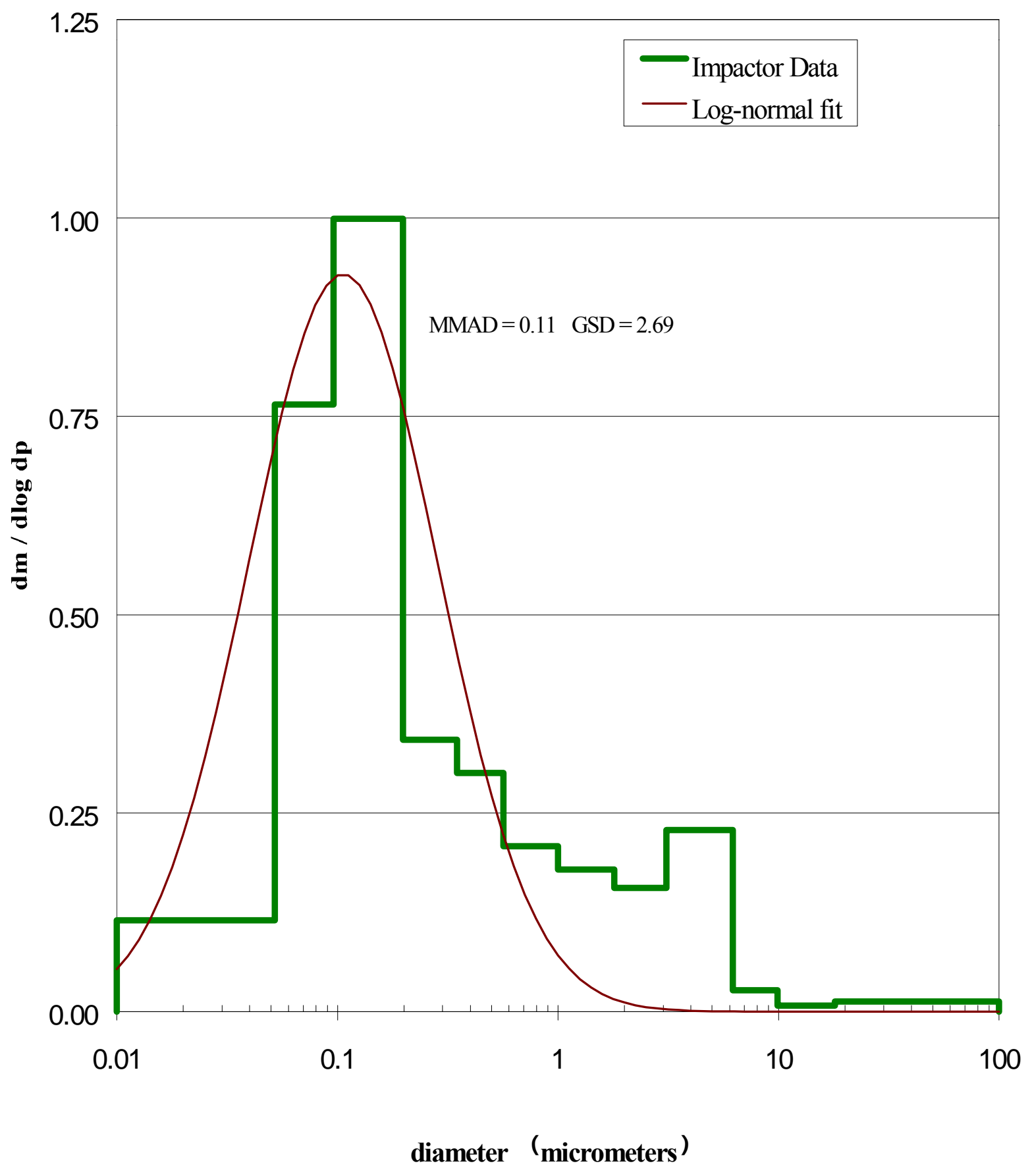


Figure 4.5 - MOUDI Size Distribution for

DDC 6V92TA Bus \#2025

CBD Rum\#1 Fuel: 50\%D2/50\%MG

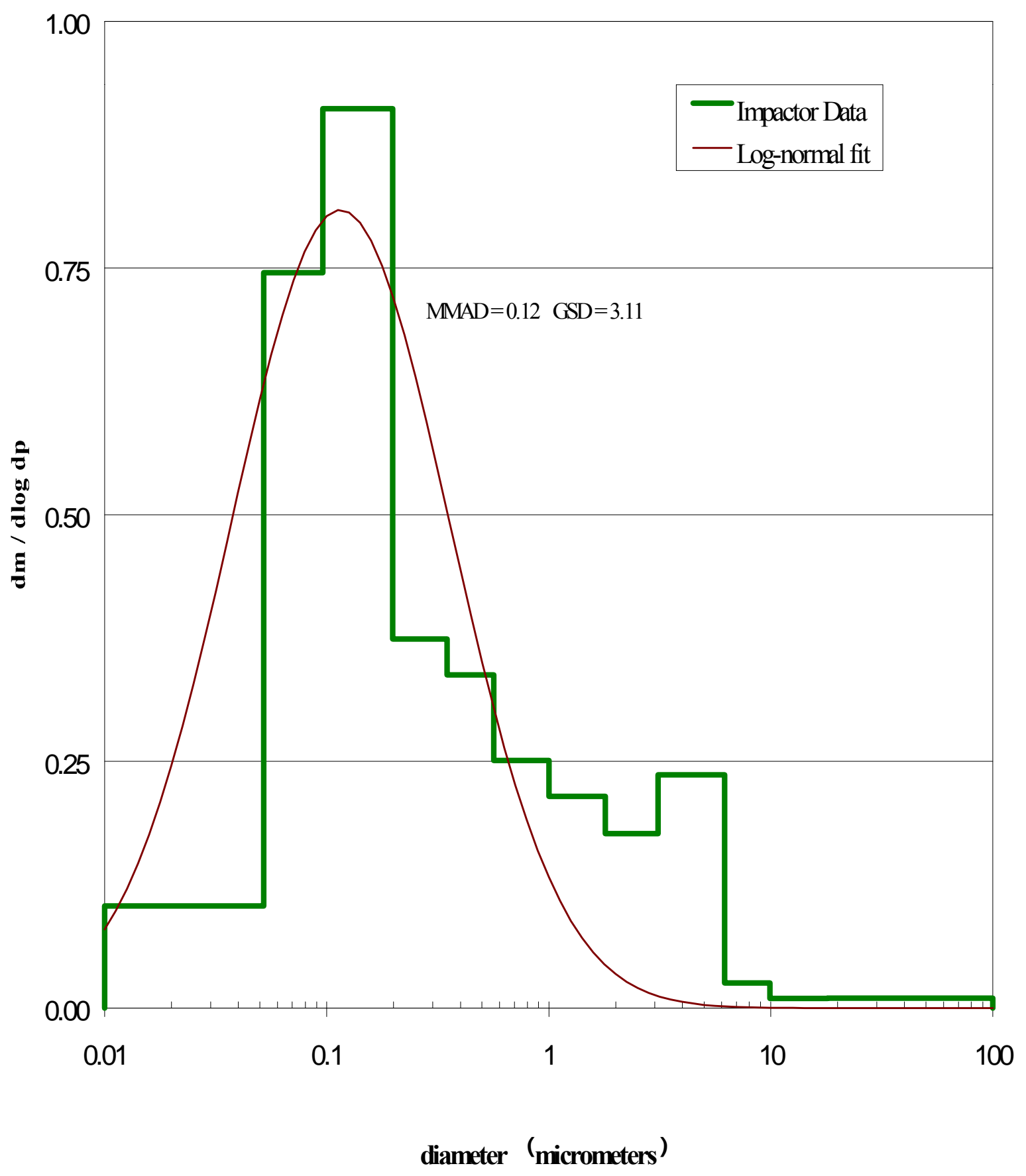


Figure 4.6 - MOUDI Size Distribution for DDC6V92TA Bus \#2025 4 Ramps of CBDRum \#2 Fuel: 50\%D2 / 50\%MG

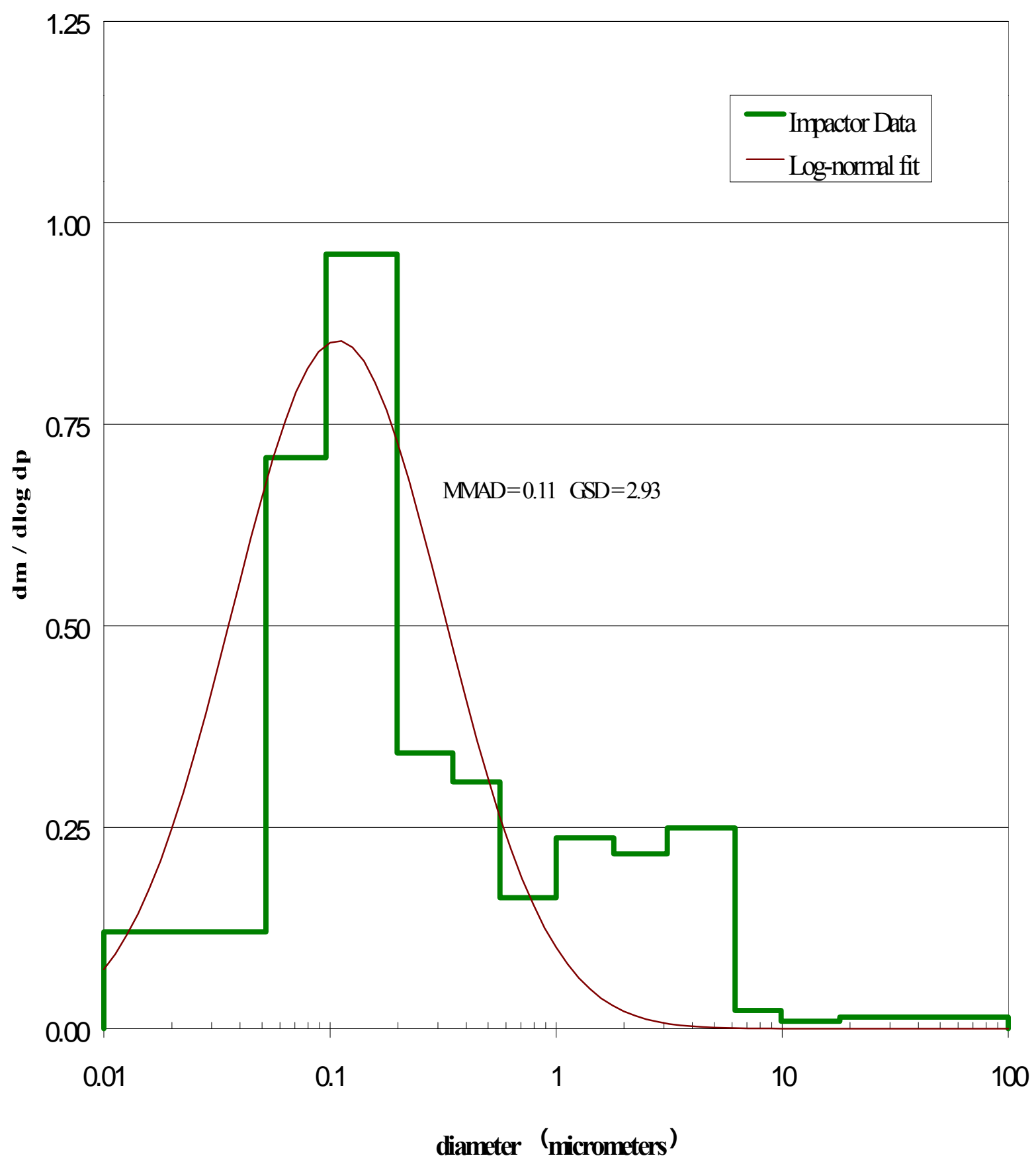


PAT Bus No.2029 was not equipped with a catalyst, was only tested once with Mossgas. While this limited experimentation was insufficient to draw any conclusions or make direct comparisons for this bus, there was ample diesel data available from the other buses. This one test (Figure 4.7) yielded an MMAD of $0.12 \mu \mathrm{m}$, and was included with the other runs for the comparative analysis.

The data showed little, if any, deviation between D2 and Mossgas. It was therefore concluded that the blend would provide no new or unexpected results and could be eliminated from the testing procedure.

The one diesel test (see Figure 4.8) for Bus No.2048 (catalyst equipped) gave an MMAD of $0.12 \mu \mathrm{m}$, and the two Mossgas runs yielded 0.11 and $0.12 \mu \mathrm{m}$ (Figures $4.9 \&$ 4.10). These tests were conducted on four ramps of the CBD. Bus \#2030 was not fitted with a catalyst. This bus yielded similar results with the MMADs of $0.12 \mu \mathrm{m}$ for both of the D2 and both of the Mossgas tests (Figures $4.11-4.14$ ). There appeared to be little noticeable difference in the two buses without a catalyst and the two with a catalyst.

For the fourteen $\mathrm{CBD}$ tests, the mass median aerodynamic diameters varied by ten nanometers from 0.11 to $0.12 \mu \mathrm{m}$ over the entire span of vehicles and fuels. As shown in the table and by the histograms, the mass weighted size distributions do not vary. The geometric standard deviations were similar as well, all showing a single accumulation mode peak with two thirds of the particle mass within the approximate size range of 0.05 $\mu \mathrm{m}$ to $3.0 \mu \mathrm{m}$. 
Figure 4.7 - MOUDI Size Distribution for DDC 6V92TA Bus \#2029

4 Ramps of CBD Run \#1 Fuel: MG

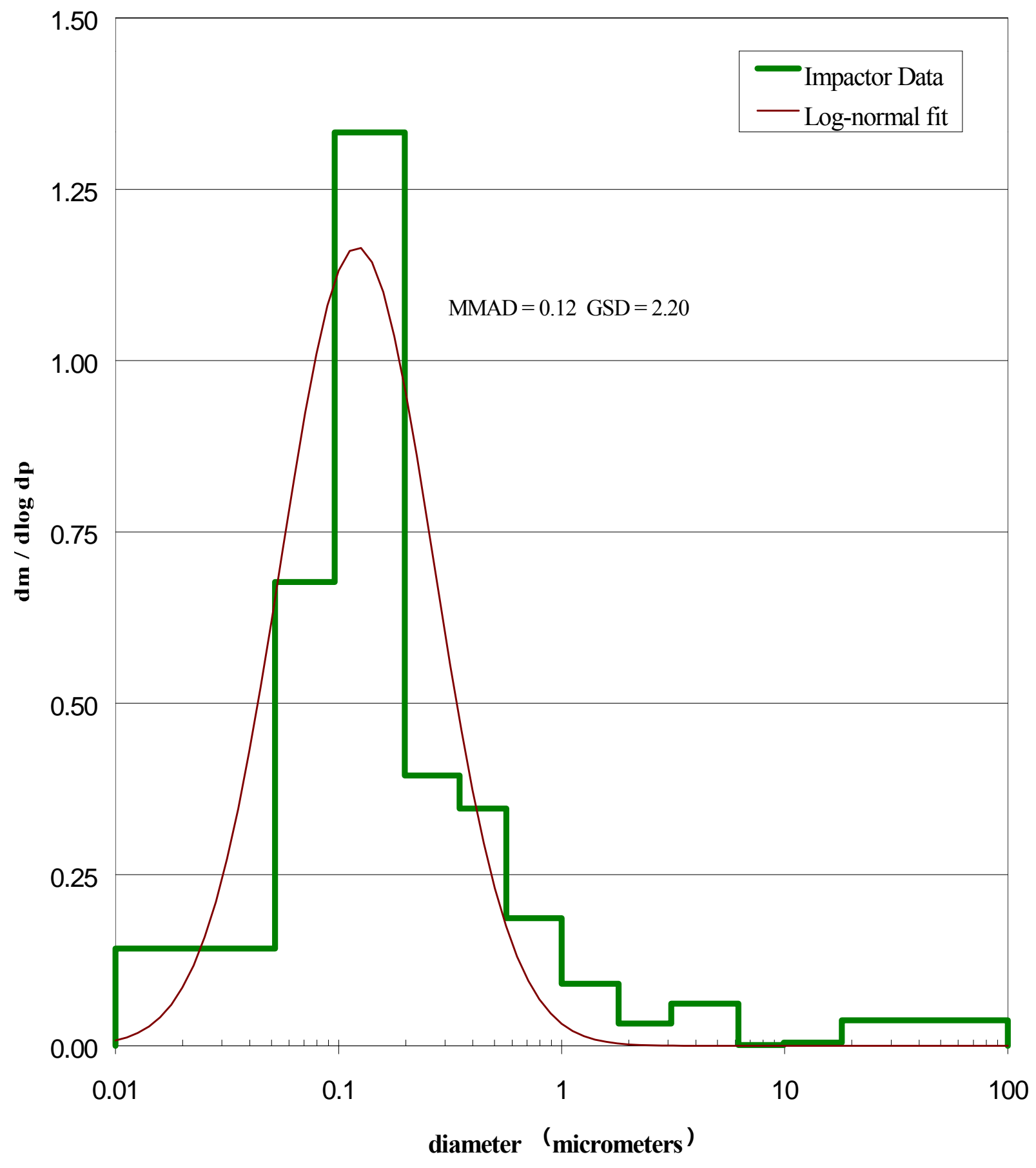


Figure 4.8 - MOUDI Size Distribution for

DDC 6V92TA Bus \#2048

4 Ramps of CBD Run \#1 Fuel: MG

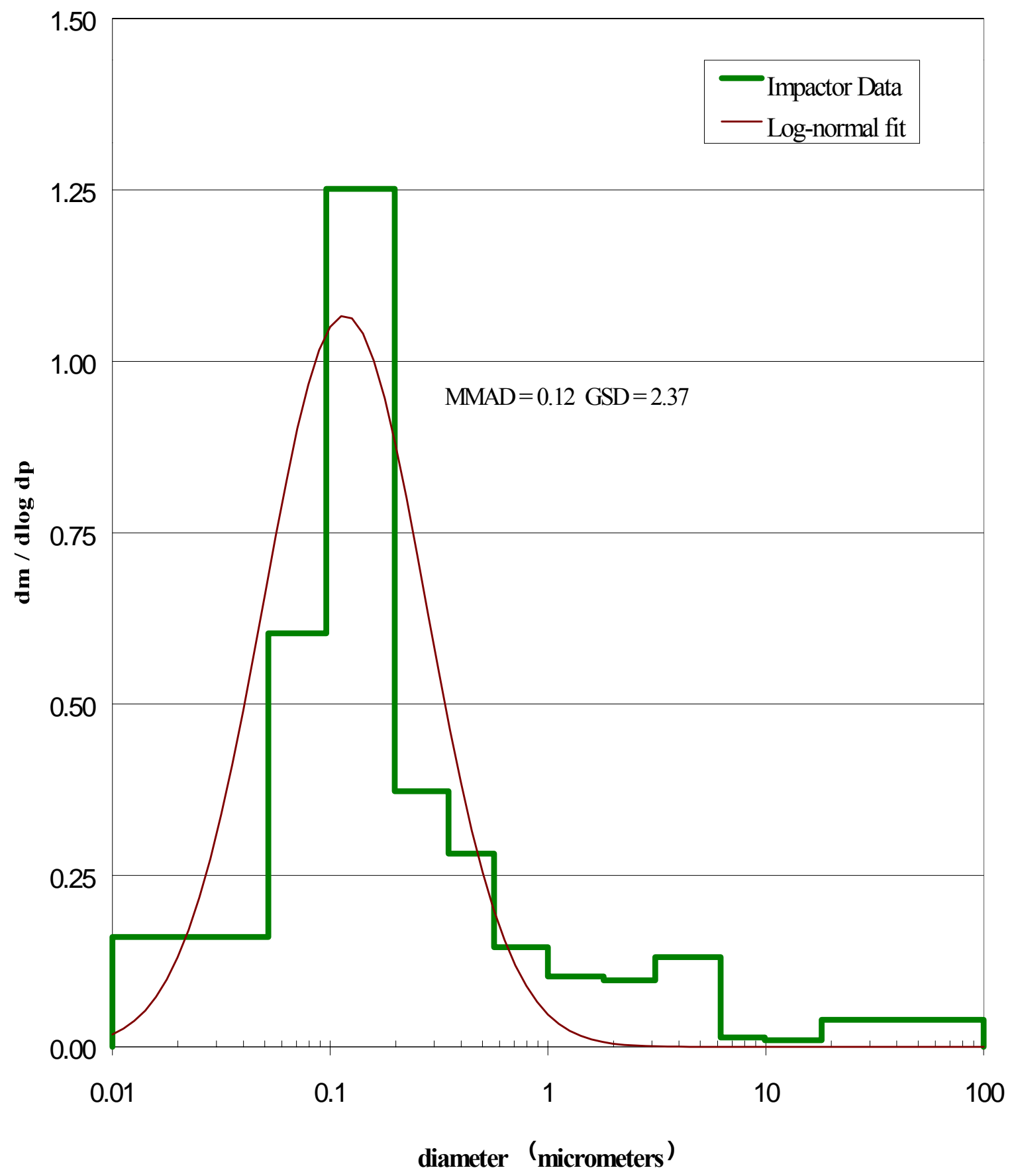


Figure 4.9 - MOUDI Size Distribution for DDC 6V92TA Bus \#2048 4 Ramps of CBD Run \#2 Fuel: MG

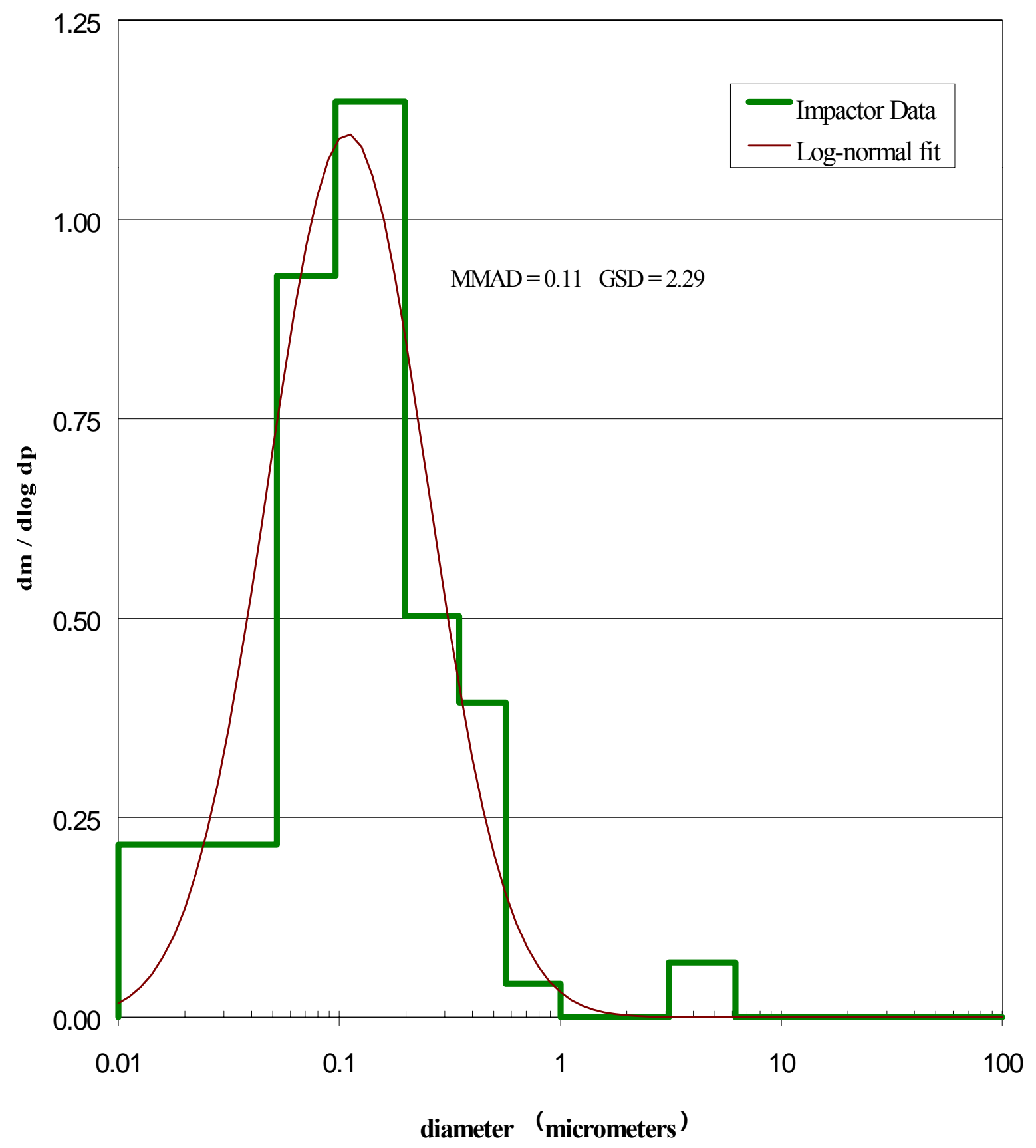


Figure 4.10 - MOUDI Size Distribution for

DDC 6V92TA Bus \#2048

4 Ramps of CBD Run \#1 Fuel: D2

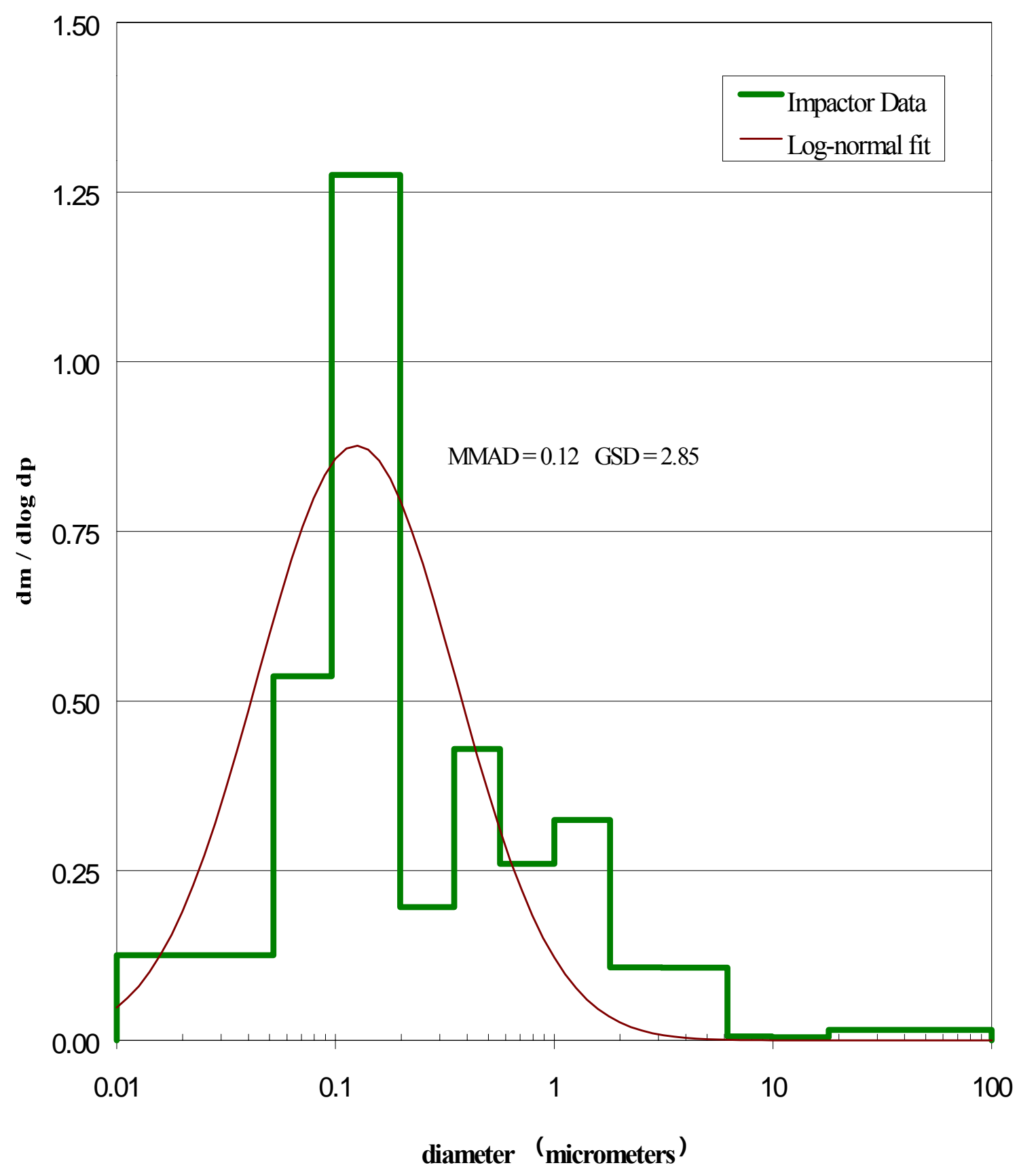


Figure 4.11 - MOUDI Size Distribution for DDC 6V92TA Bus \#2030

4 Ramps of CBD Run \#1 Fuel: D2

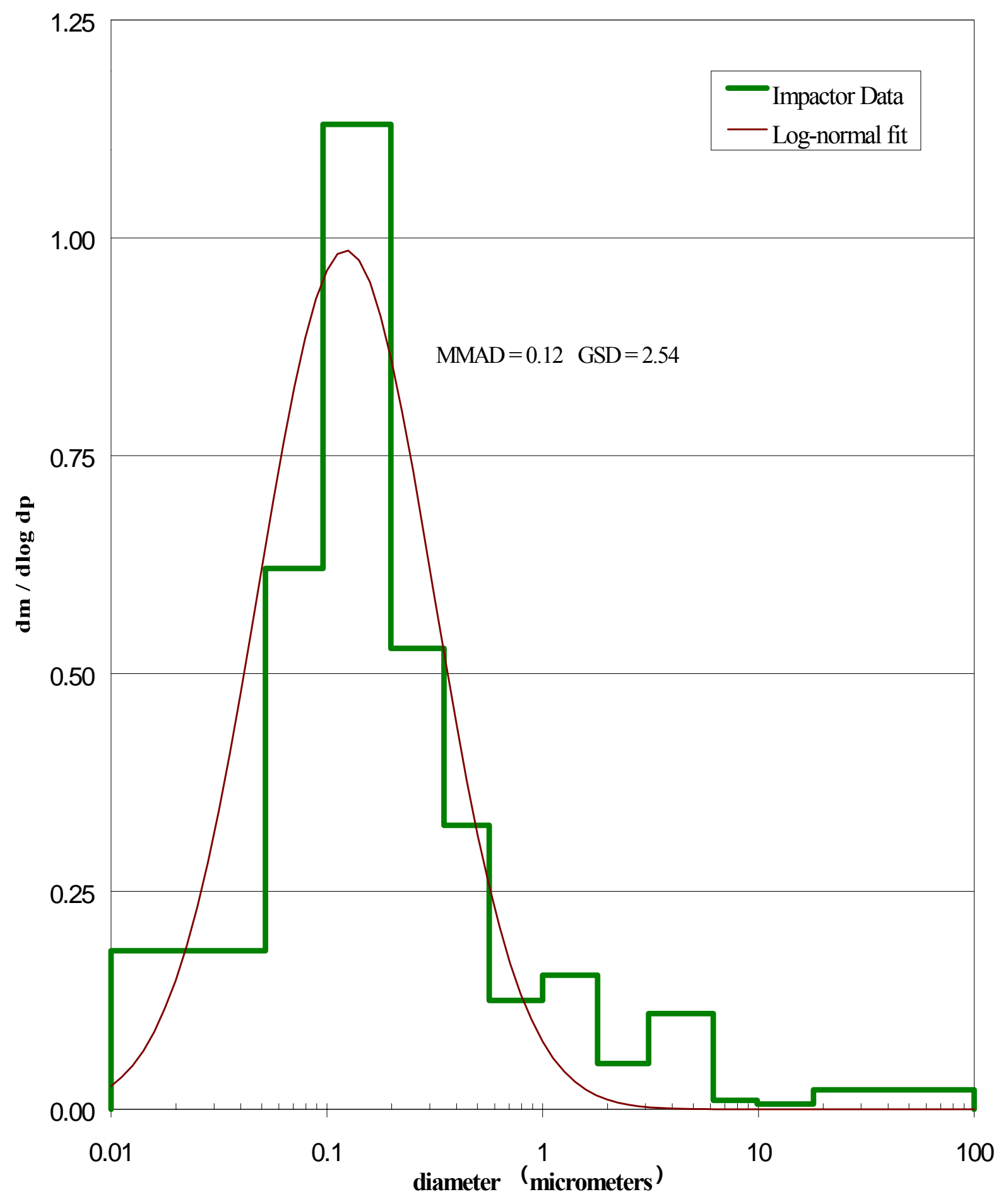


Figure 4.12 - MOUDI Size Distribution for DDC 6V92TA Bus \#2030 4 Ramps of CBD Run\#2 Fuel: D2

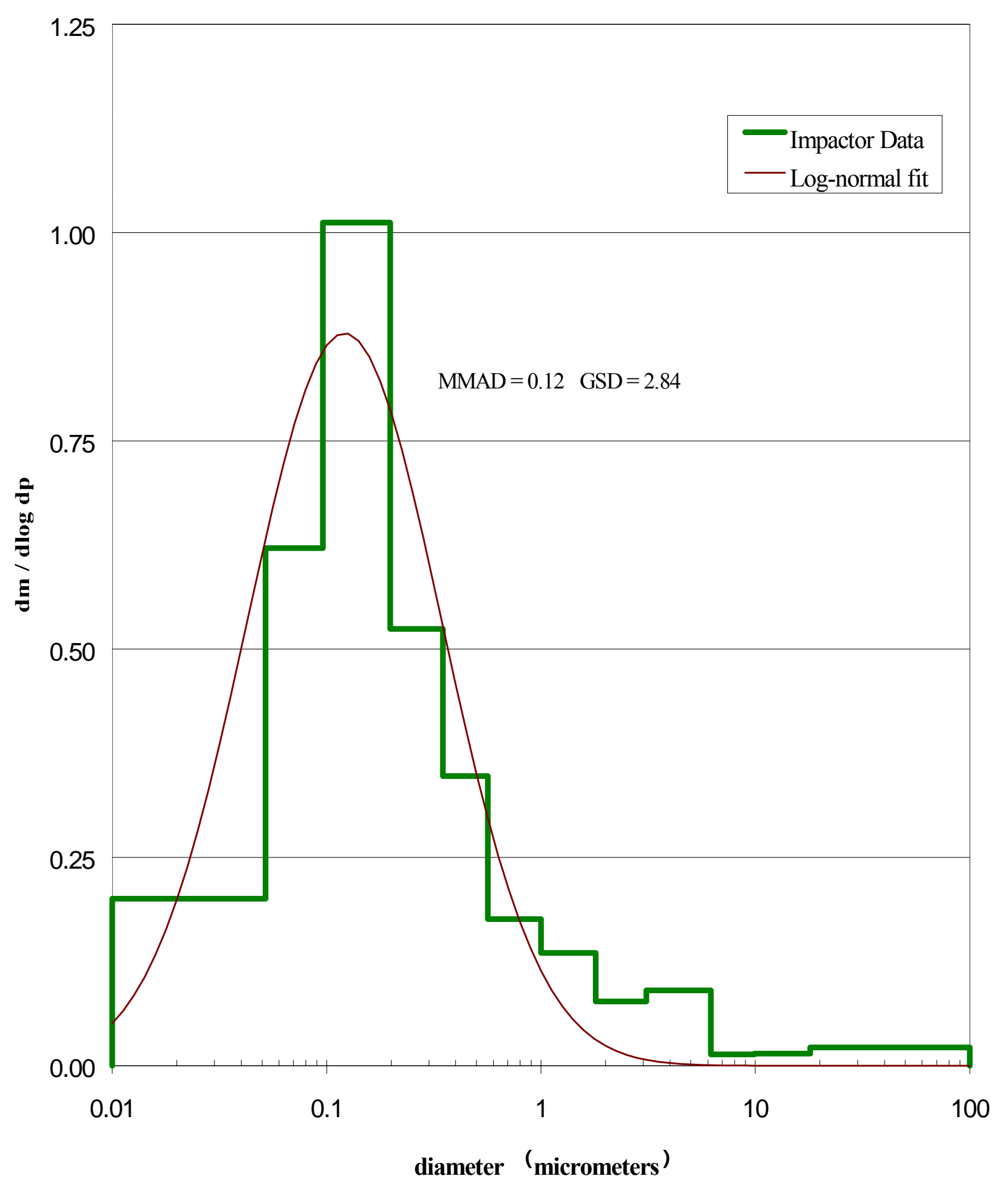


Figure 4.13 - MOUDI Size Distribution for

DDC 6V92TA Bus \#2030

4 Ramps of CBD Run \#1 Fuel: MG

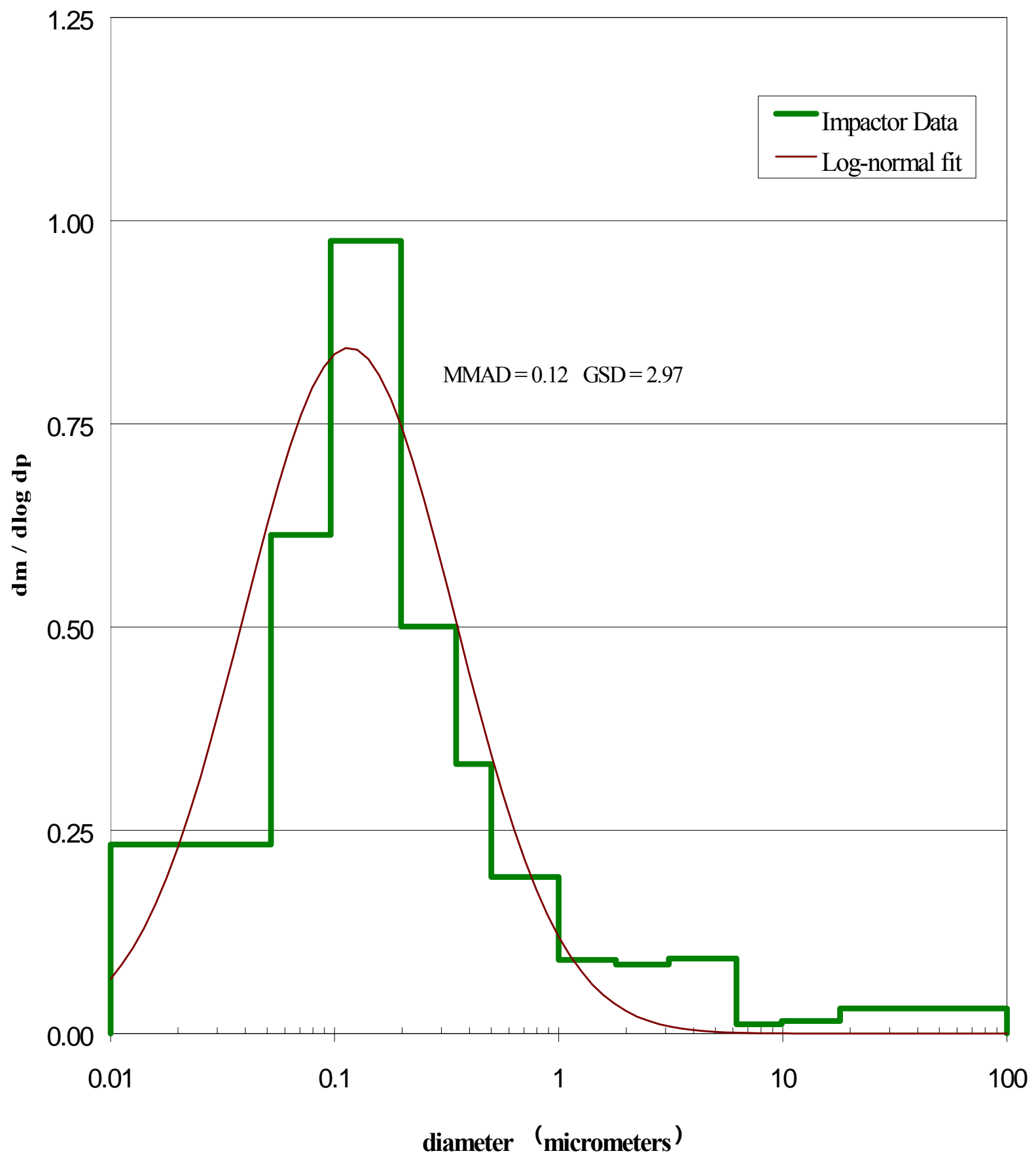


Figure 4.14 - MOUDI Size Distribution for DDC 6V92TA Bus \#2030

4 Ramps of CBD Run\#2 Fuel: MG

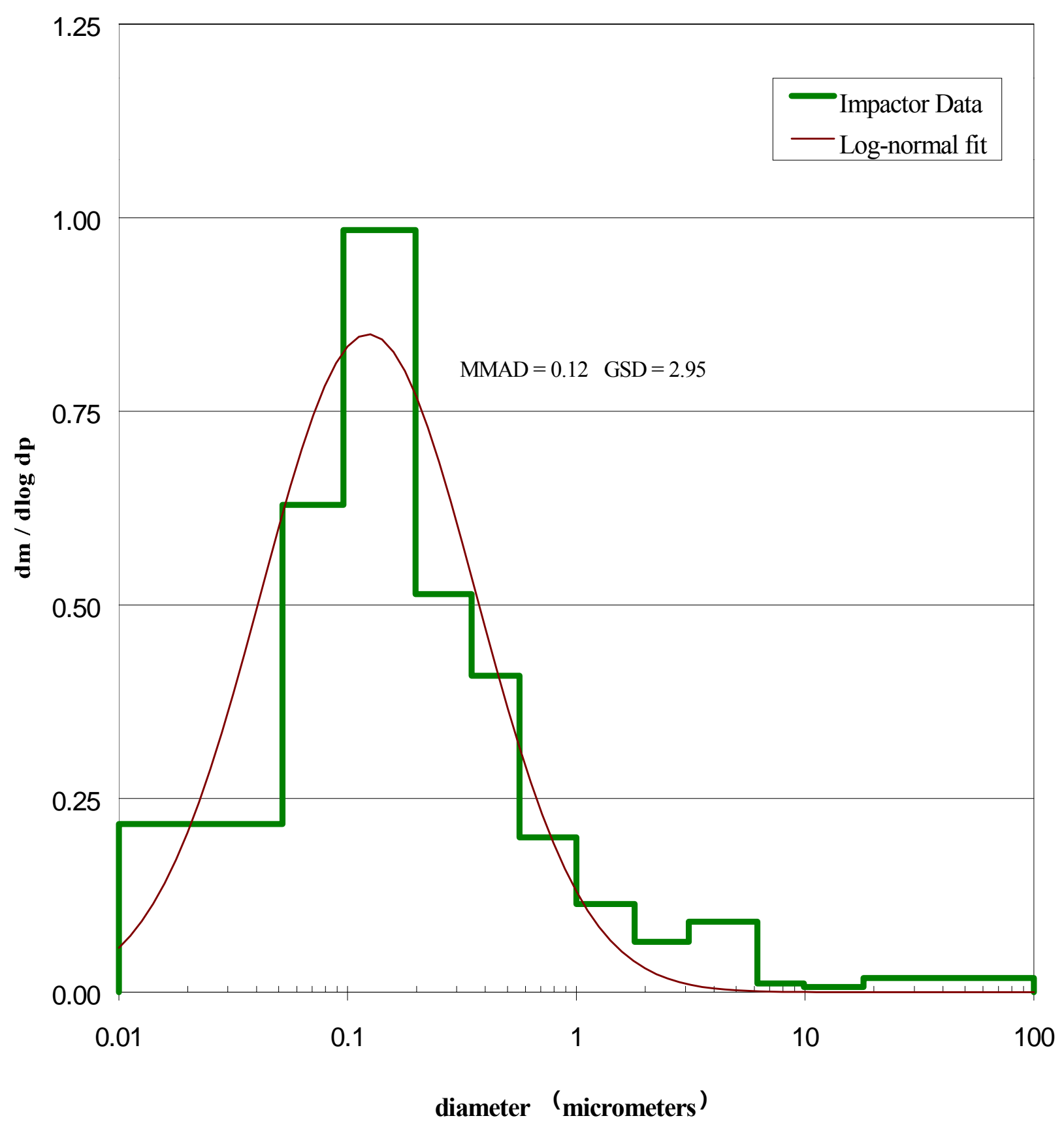


Table 4.1 - Mass Median Diameters and Geometric Standard Deviations for DDC 6V92TA Pittsburgh Port Authority Transit Buses

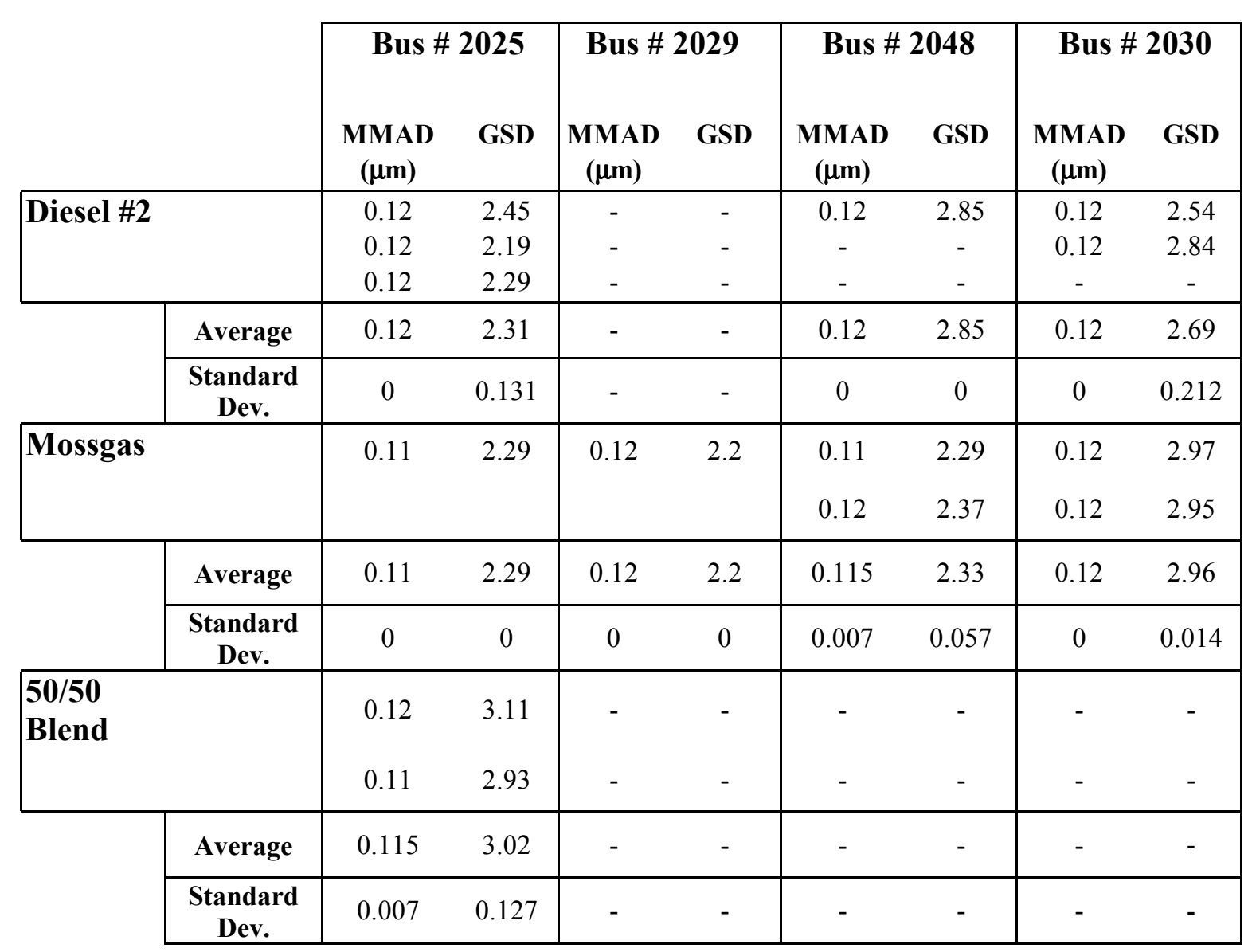

The striking similarity between the mass-median aerodynamic diamters for the fuels was somewhat unexpected. While the total mass of particulate matter has been shown to be lower for vehicles powered by Fischer-Tropsch process fuels, the size distribution wasn't necessarily altered. Olefins are unsaturated and are often considered precursors of soot formation, and aromatics can acquire $\mathrm{CH}_{2}$ chains [Heywood, 1988]. It is possible that the olefins the Mossgas was distilled from permitted similar particle growth as the aromatics found in diesel. Therefore, though Mossgas contained less aromatics than diesel, the olefins it did contain may have allowed similar particle formation to occur. 
Fuel sulfur is known to increase total particulate matter (TPM). Conversion rates for sulfur in fuel to sulfates on particulates have been found to be 1 to $3 \%$ [Baranescu, 1988]. Fuel sulfur is converted to sulfur dioxide during combustion. The portion of the sulfur dioxide that is not exhausted is further oxidized into $\mathrm{SO}_{3}$, sulfur trioxide. This then combines with water vapor to form sulfuric acid, which can condense or adsorb onto particulate surfaces or undergo homogeneous nucleation, depending on the saturation ratios. This is only a fraction of the total sulfur found in fuels such as diesel \#2. Therefore, sulfur-free Mossgas may lower TPM without greatly affecting the mass median aerodynamic diameter determined by the MOUDI.

The Federal Diesel No. 2 Fuel used in this study contained $0.02 \%$ sulfur by weight. This is considerably lower than typical diesel fuel, which generally contains 0.03 to $0.035 \%$ sulfur by weight. However, the mass-based size distribution for these diesel

fuel tests were very similar with respect to the location of the mass median to those obtained in previous and later testing. Therefore, the effects of fuel sulfur on the MOUDI particle MMAD were limited if not undetectable. This could further explain the similarities found between diesel and Mossgas.

\subsubsection{SMPS Data Analysis}

The SMPS was operated for PAT Buses 2048 and 2030 on the CBD cycle using the mini-dilution tunnel mentioned previously. Bus \#2048 was tested with D2 and Mossgas using the Model 3022A CPC, with a DMA sheath flow of $18 \mathrm{lpm}$ and an aerosol flow of $1.8 \mathrm{lpm}$. Bus 2030 was tested with the Model 3025A CPC for the D2 runs and 
Model 3022A for the Mossgas runs. The sheath and aerosol flow rates remained the same.

The transient tests yielded data (see Figures 4.15 - 4.34) that corresponded well with that returned by the MOUDI. The mass-weighted size distributions determined from the MOUDI data showed the MMAD to be in the 0.11 to $0.12 \mu \mathrm{m}$ range, and the total mass emission rates were shown to be greater for the buses powered by D2. These trends were also found in the SMPS particle concentration data. For the particle diameters tracked $(15,30,50,100$ and $200 \mathrm{~nm})$, it was seen that particle concentrations increased for both fuels with each successive size increment, with the highest concentrations occurring in the $100-200 \mathrm{~nm}$ range. Every test on both buses followed this pattern of increasing particle number, and the D2 particle concentrations were higher than the Mossgas concentrations for each particle size measured. This supported the findings from the total mass emission measurements as well as the MOUDI.

\subsection{RTD/SKIP Tests at Denver, CO}

\subsubsection{Cyclone Data Analysis}

Both PM10 and PM2.5 cyclones were operated on the RTD-SKIP Cummins B5.9 diesel and natural gas buses. Approximately forty-five tests were conducted on the six buses. This batch of tests was the preliminary trial for cyclone sampling. The main goal 
Figure 4.15 - 15 nm SMPS Particle Concentration for DDC 6V92TA Bus \#2048

\section{CBD Run \#1 Fuel: D2}

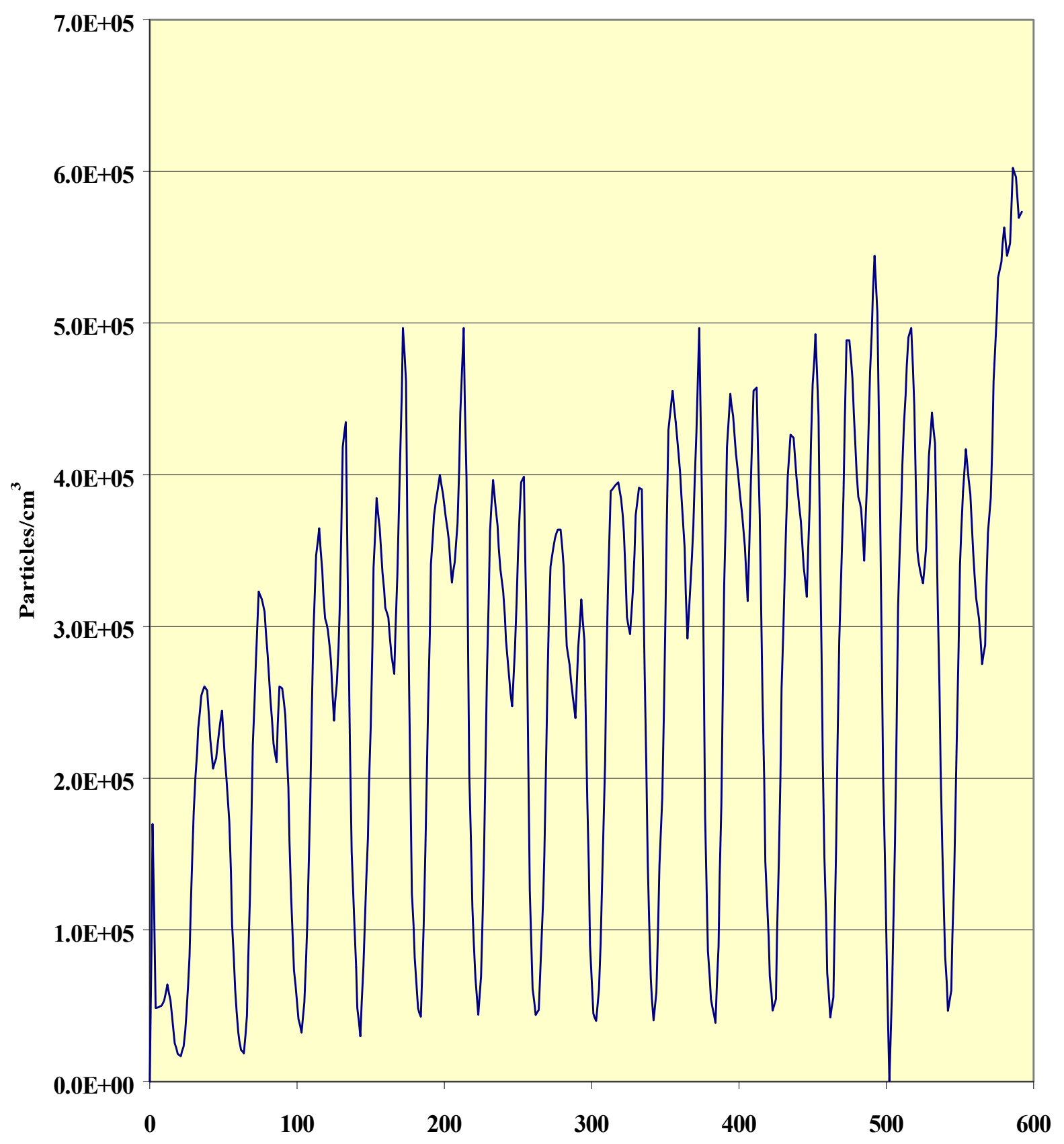

Time (seconds) 
Figure 4.24 - 200 nm SMPS Particle Concentration for DDC 6V92TA Bus \#2048 CBD Run \#5 Fuel: MG

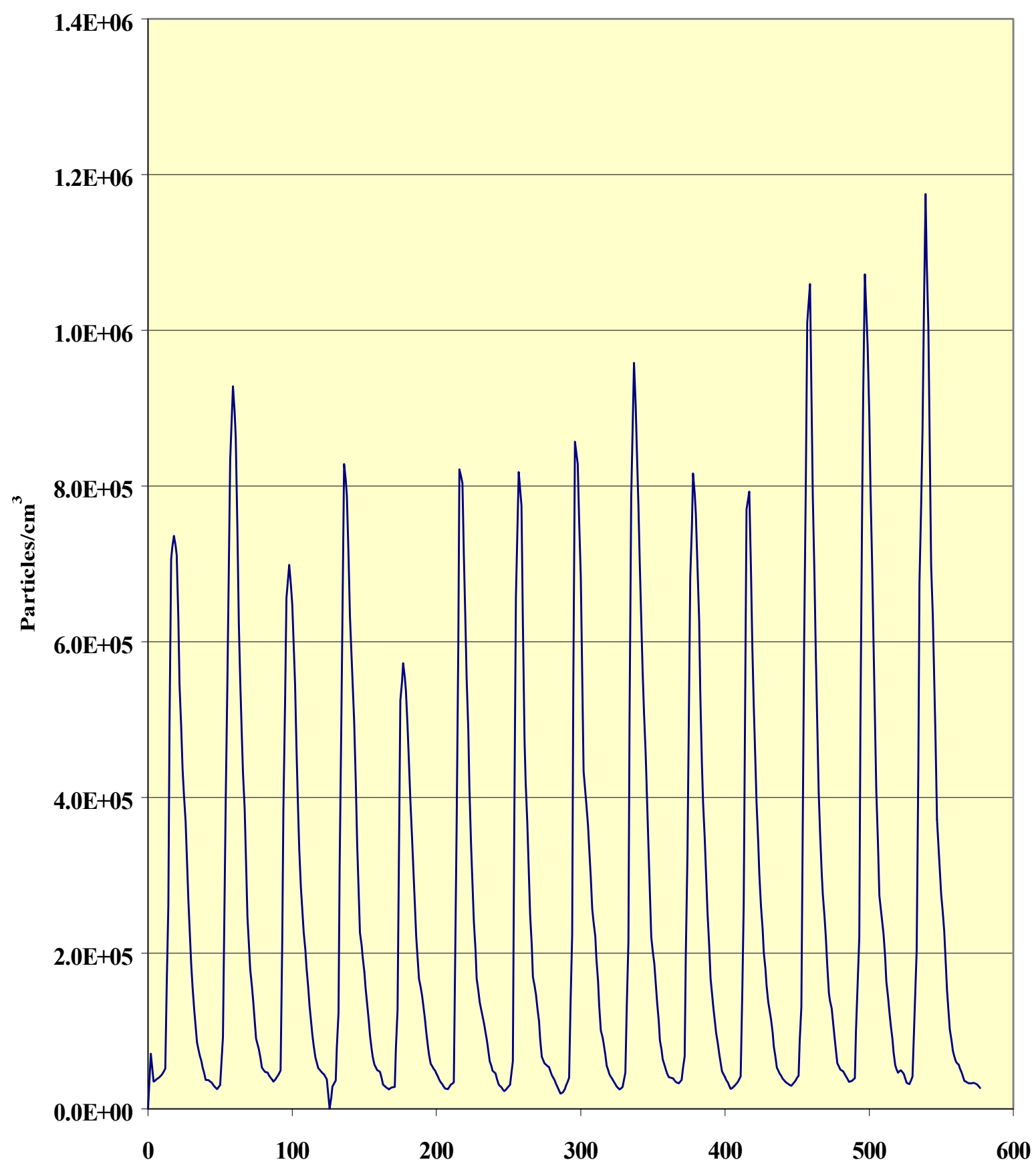

Time (seconds) 
Figure 4.17 - 50 nm SMPS Particle Concentration for DDC 6V92TA Bus \#2048 CBD Run \#3 Fuel: D2

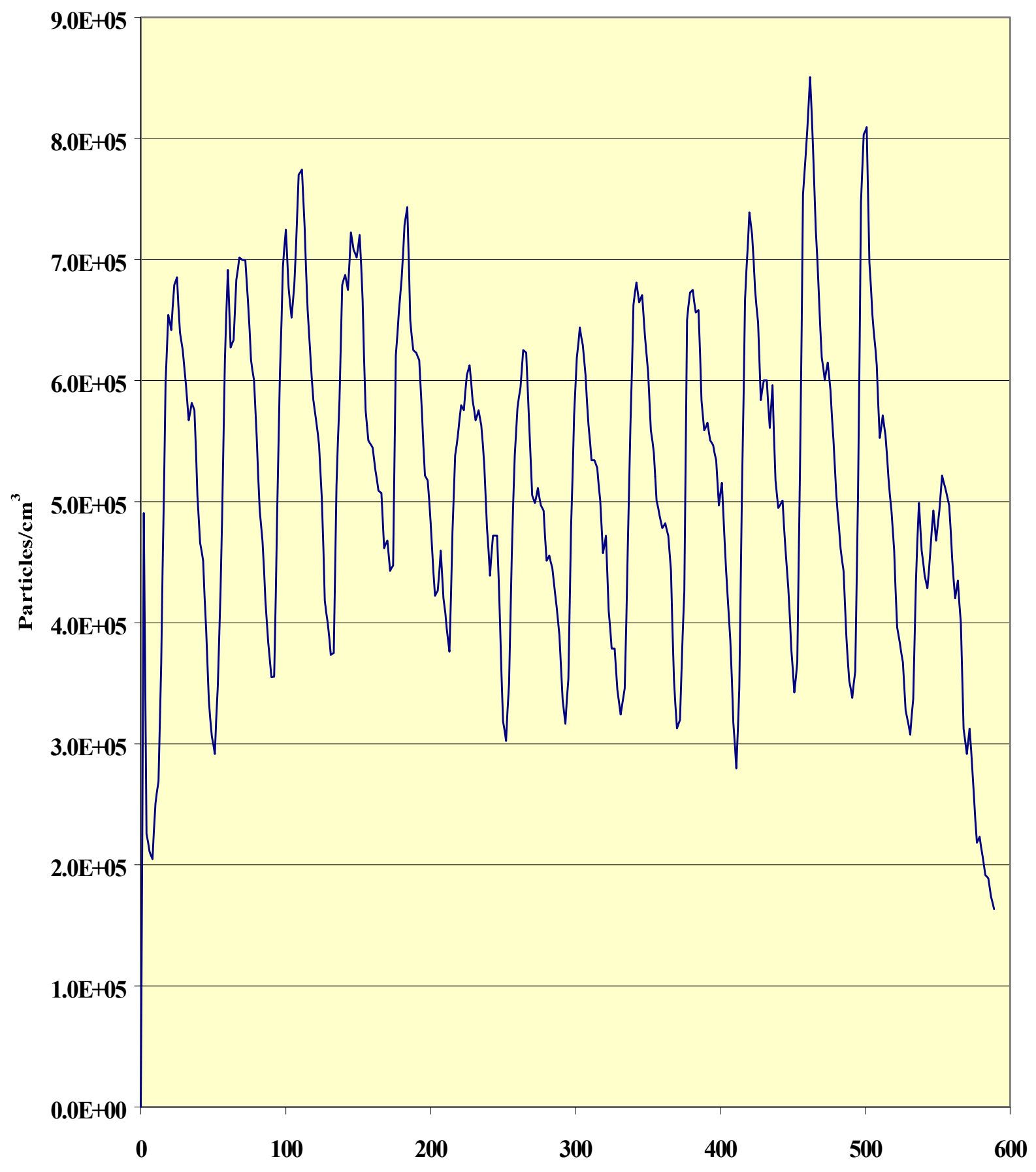

Time (seconds) 
Figure 4.18 - 100 nm SMPS Particle Concentration for DDC 6V92TA Bus \#2048 CBD Run \#4 Fuel: D2

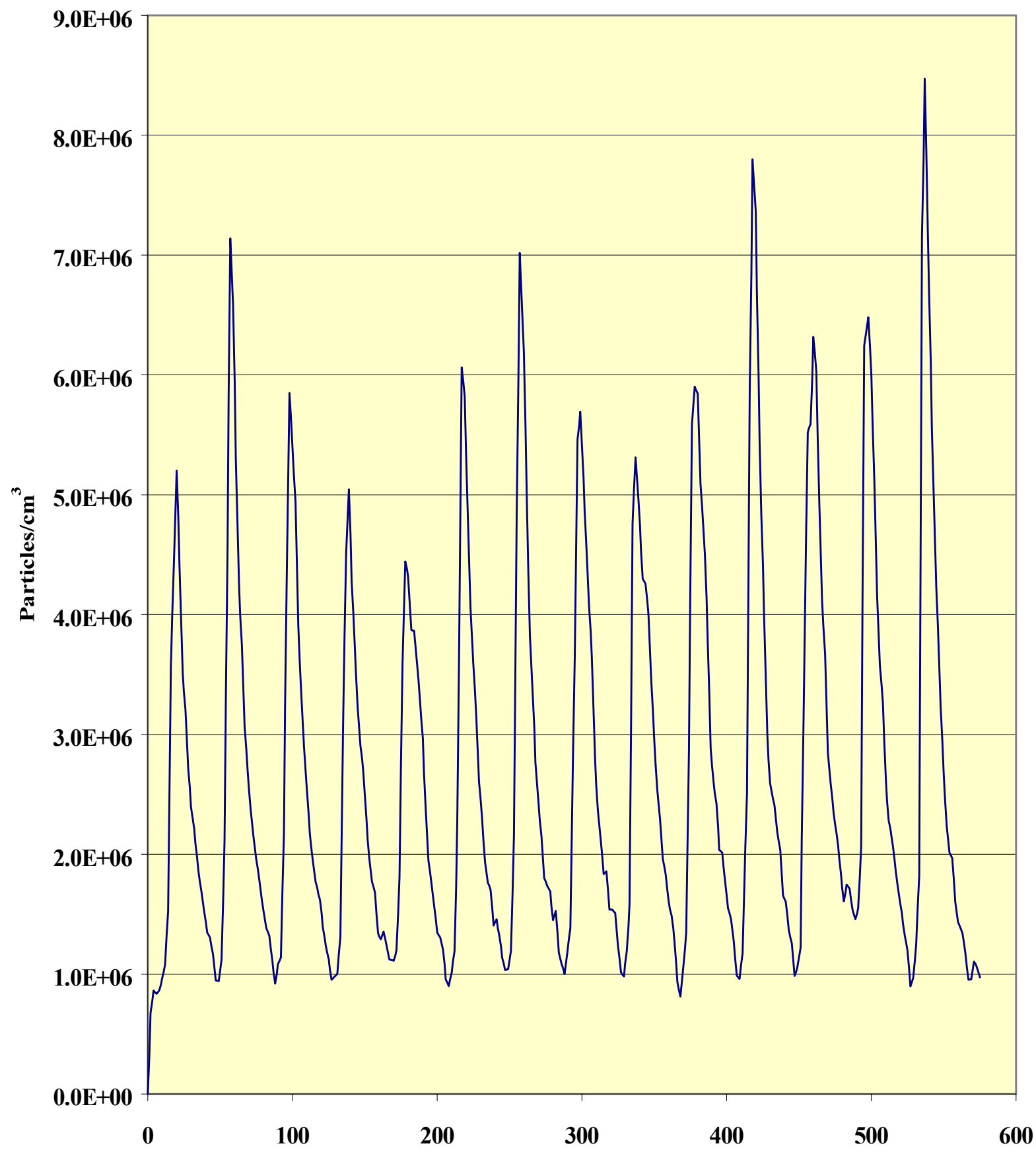

Time (seconds) 
Figure 4.19 - 200 nm SMPS Particle Concentration for DDC 6V92TA Bus \#2048 CBD Run \#5 Fuel: D2

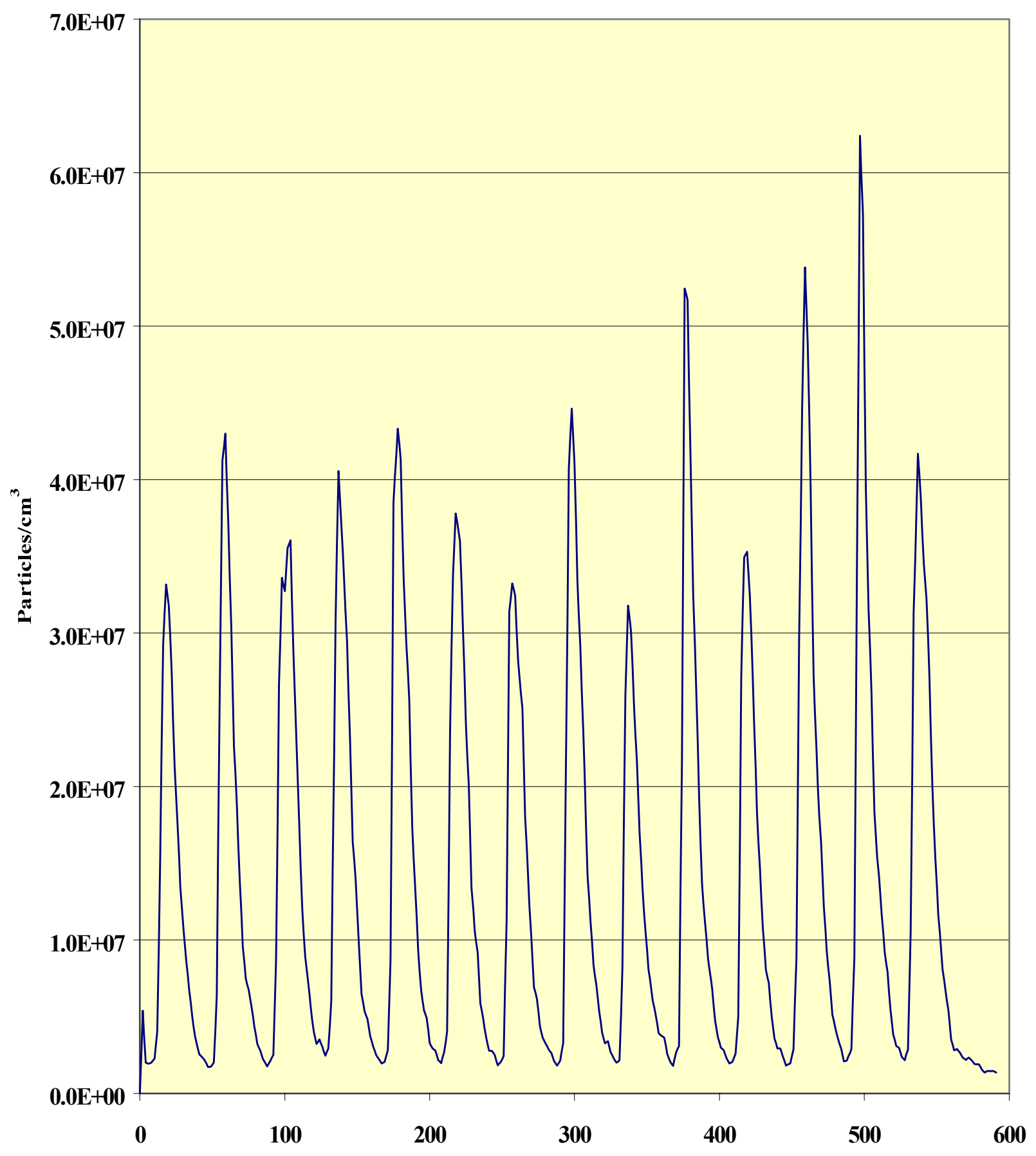

Time (seconds) 
Figure 4.20 - 15 nm SMPS Particle Concentration for DDC 6V92TA Bus \#2048 CBD Run \#1 Fuel: MG

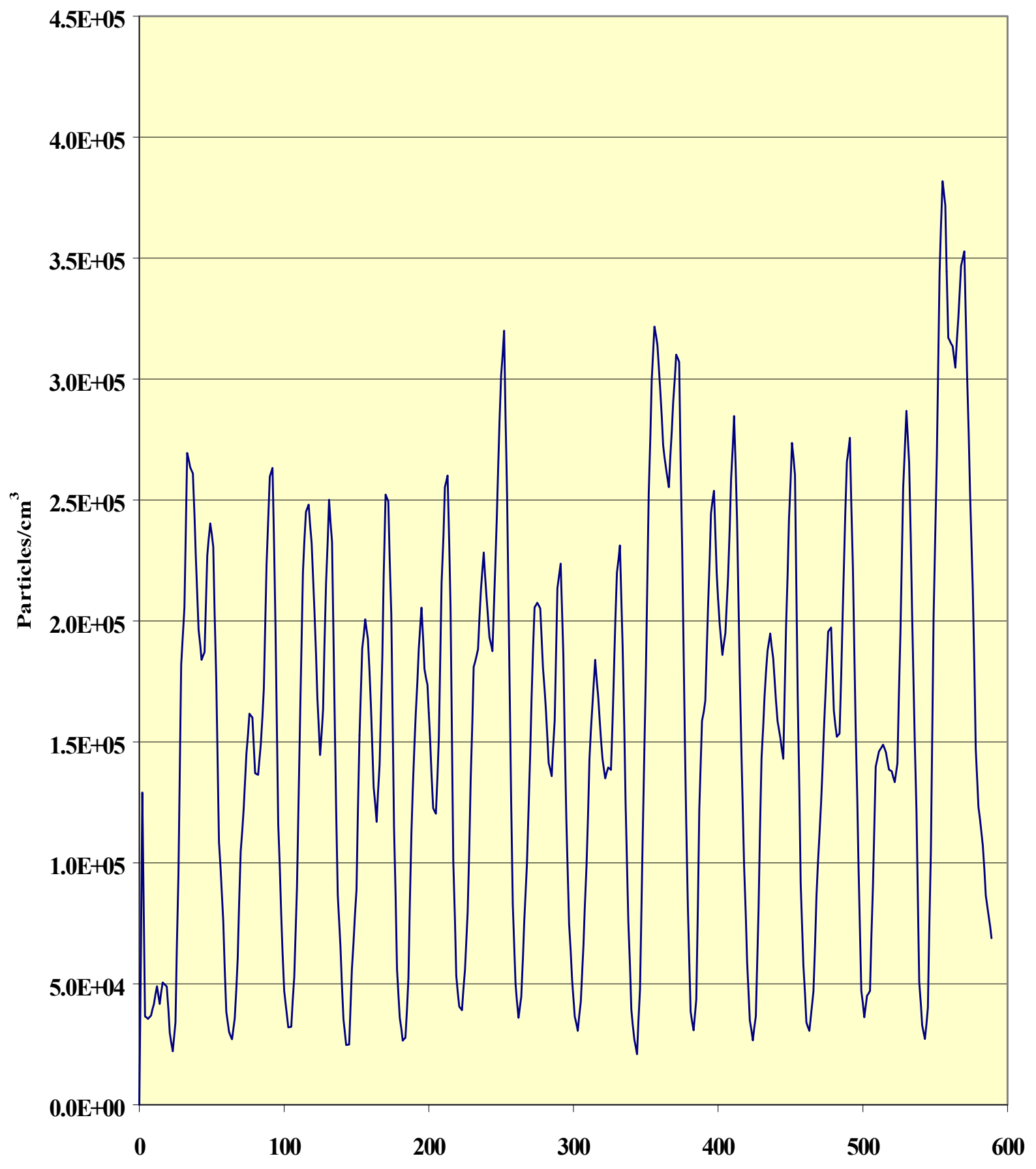

Time (seconds) 
Figure 4.21 - $30 \mathrm{~nm}$ SMPS Particle Concentration for DDC 6V92TA Bus \#2048 CBD Run \#2 Fuel: MG

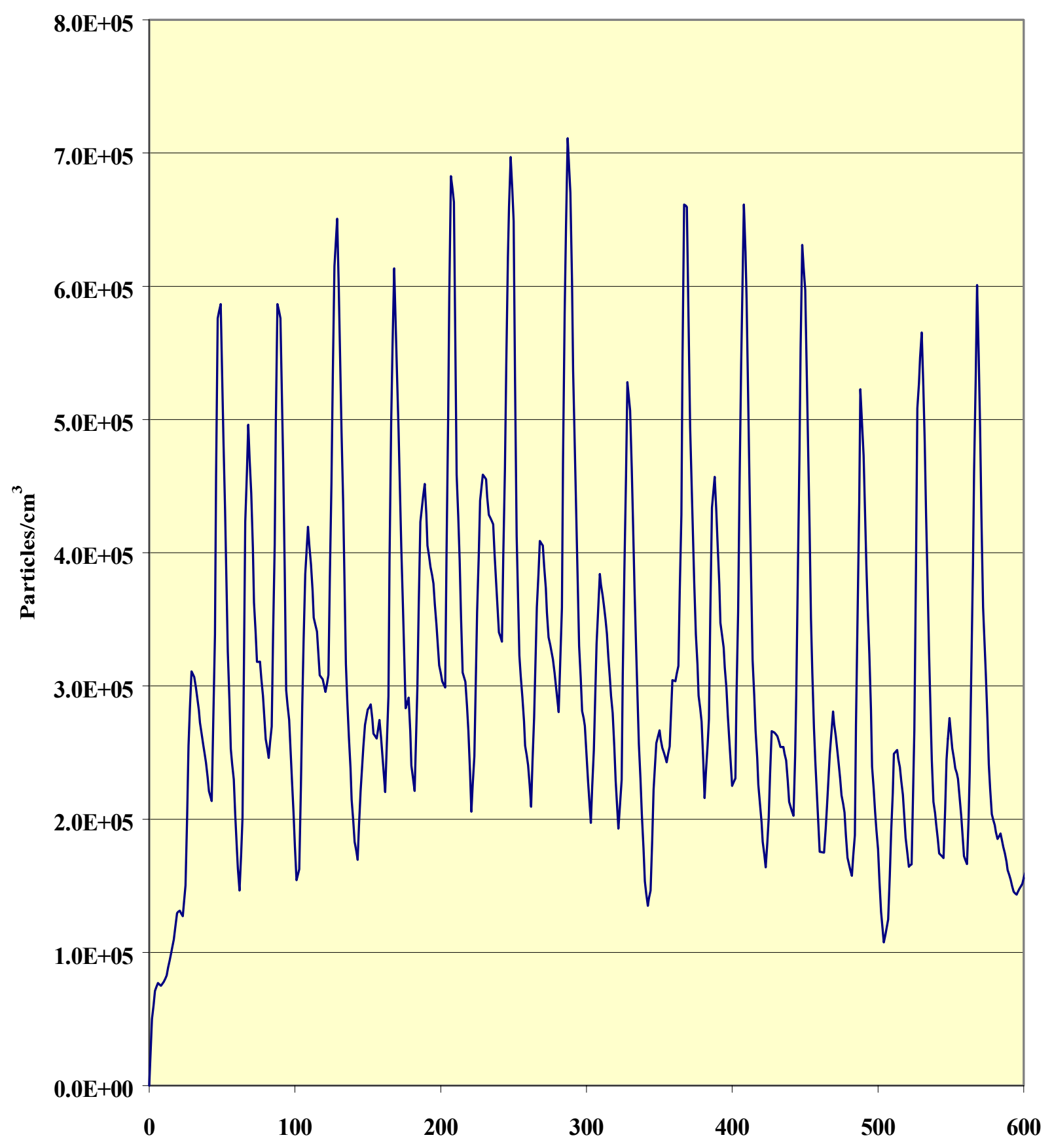

Time (seconds) 
Figure 4.22 - 50 nm SMPS Particle Concentration for DDC 6V92TA Bus \#2048 CBD Run \#3 Fuel: MG

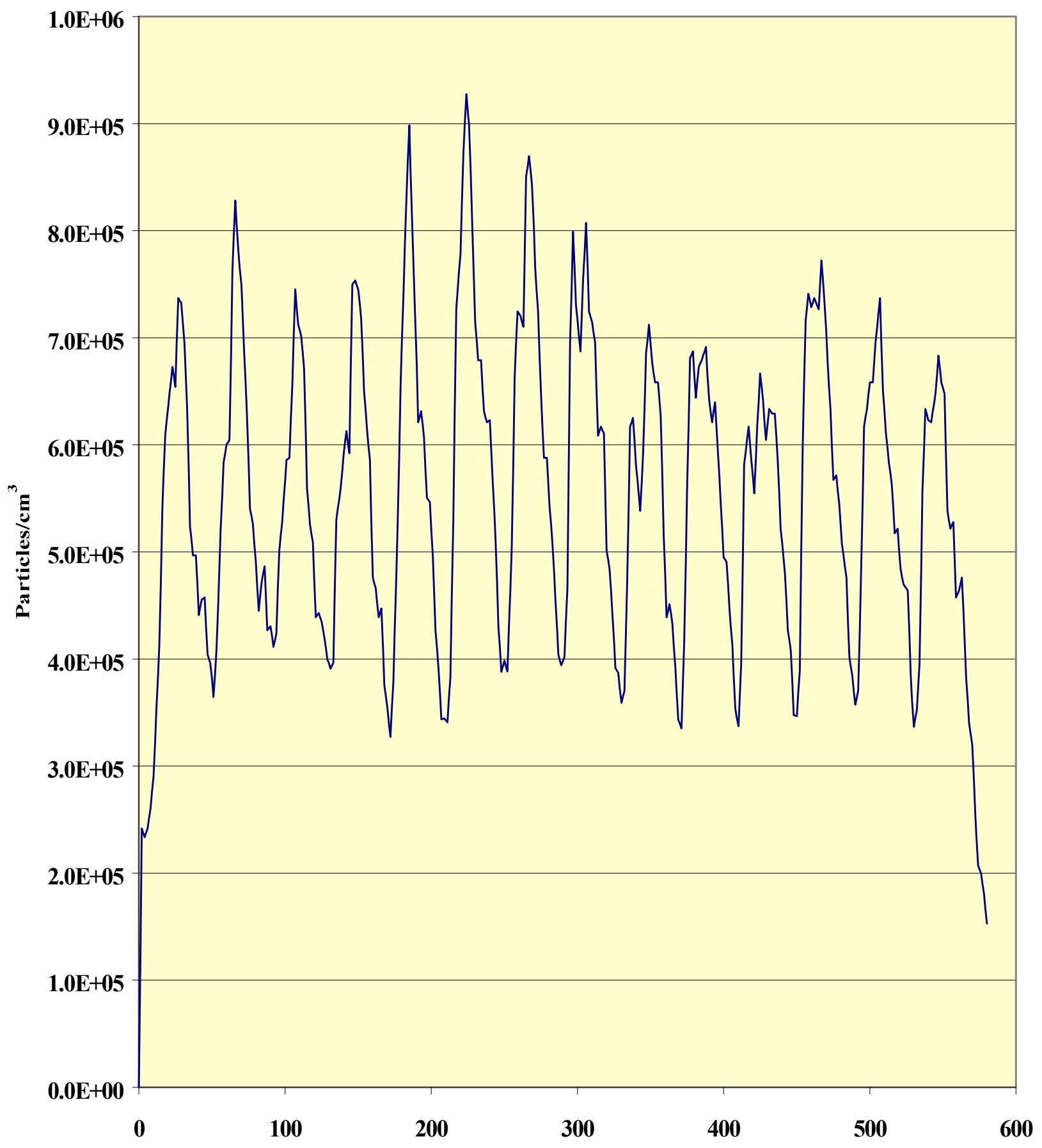

Time (seconds) 
Figure 4.23 - $100 \mathrm{~nm}$ SMPS Particle Concentration for DDC 6V92TA Bus \#2048 CBD Run \#4 Fuel: MG

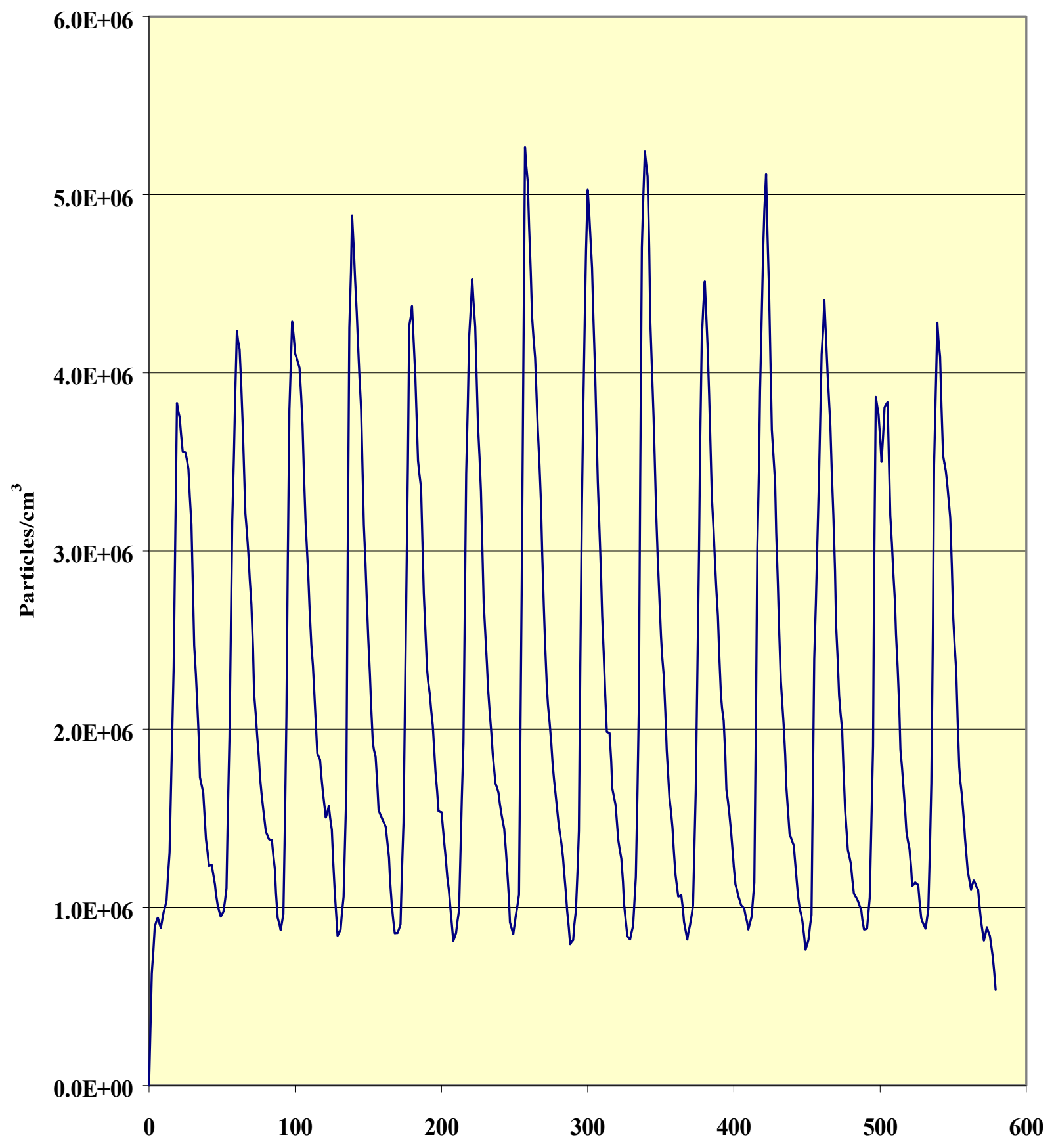

Time (seconds) 
Figure 4.24 - 200 nm SMPS Particle Concentration for DDC 6V92TA Bus \#2048 CBD Run \#5 Fuel: MG

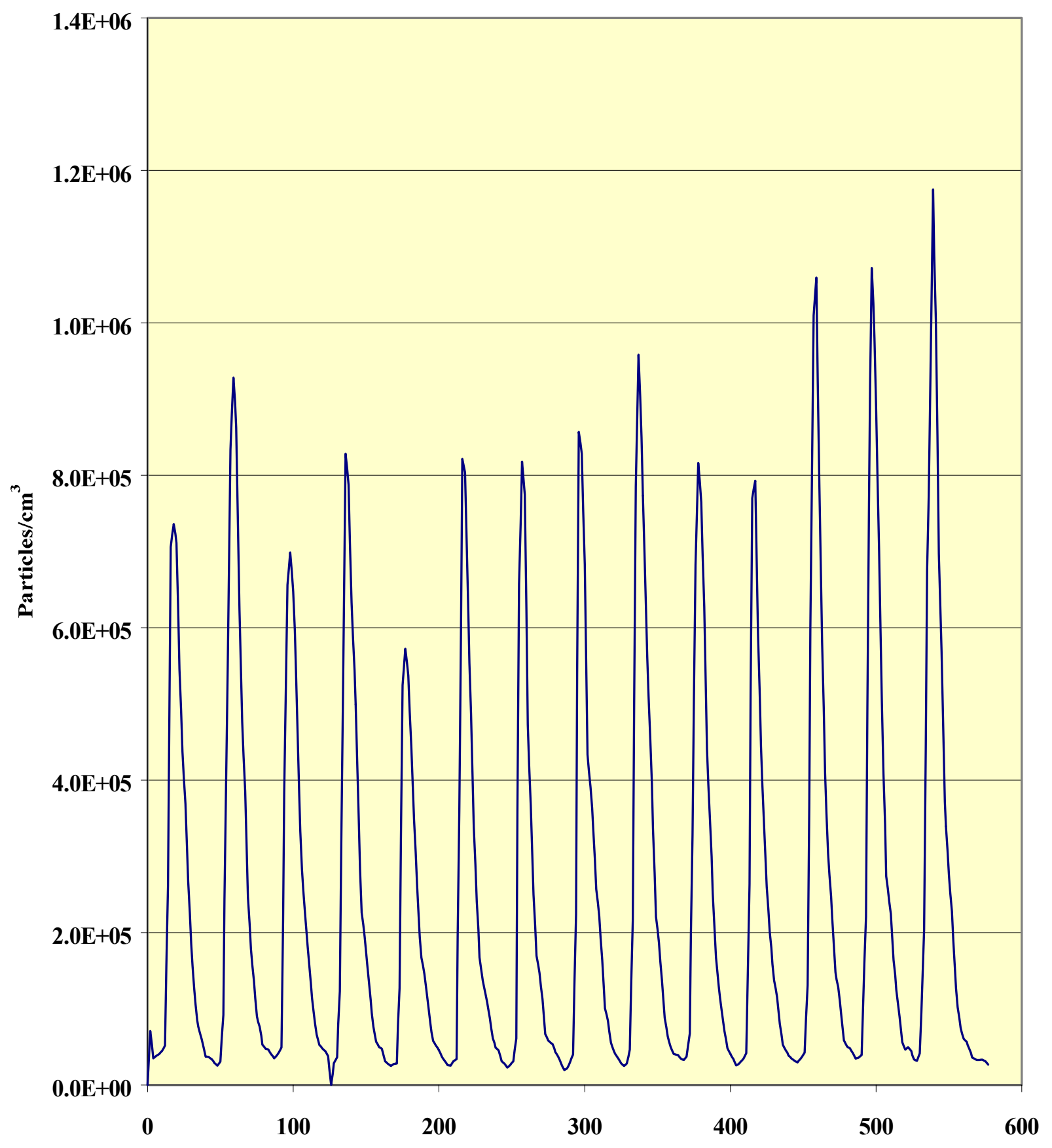

Time (seconds) 
Figure 4.25 - $15 \mathrm{~nm}$ SMPS Particle Concentration for DDC 6V92TA Bus \#2030 CBD Run \#1 Fuel: D2

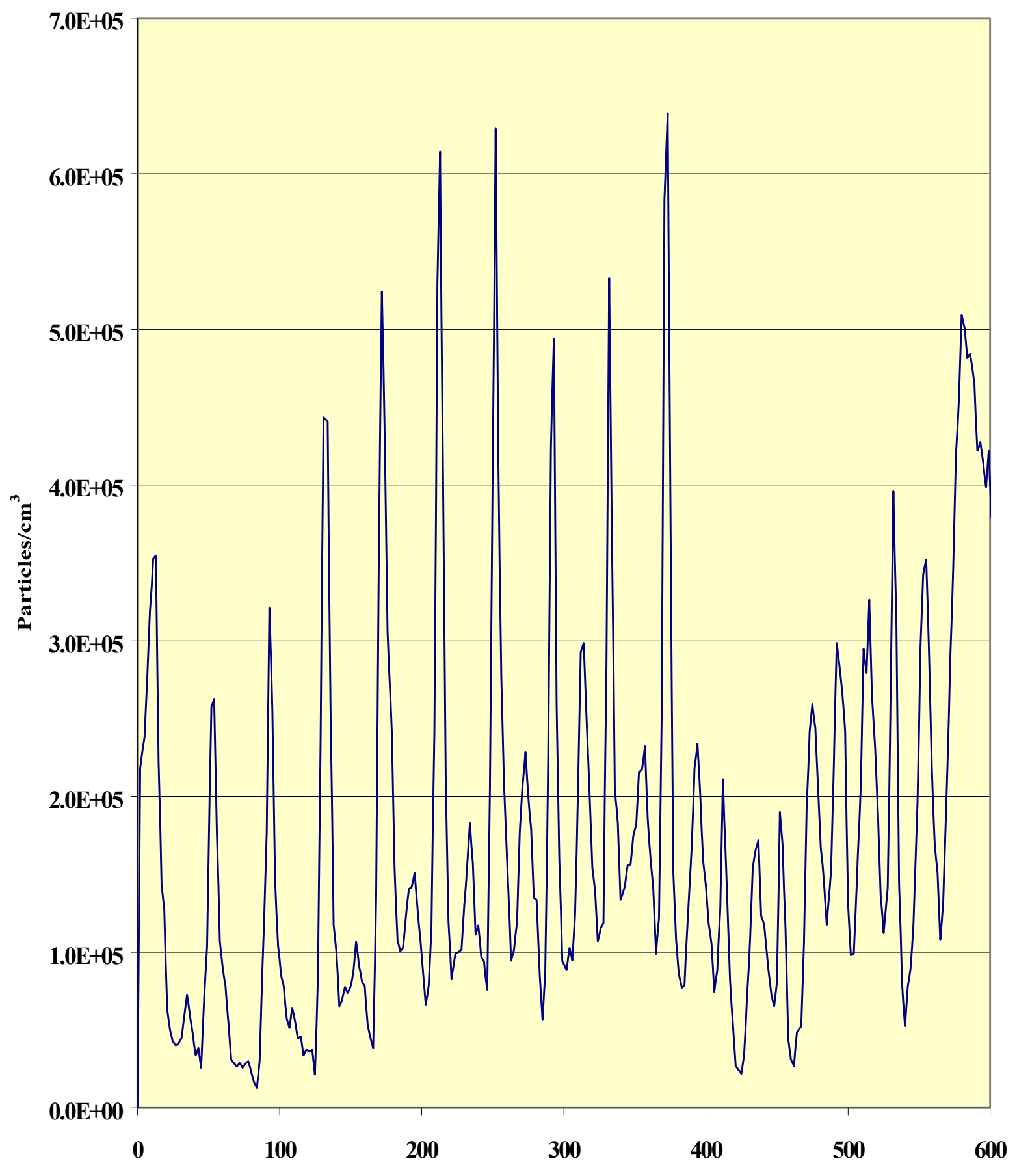

Time (seconds) 
Figure 4.26 - 30 nm SMPS Particle Concentration for DDC 6V92TA Bus \#2030 CBD Run \#2 Fuel: D2

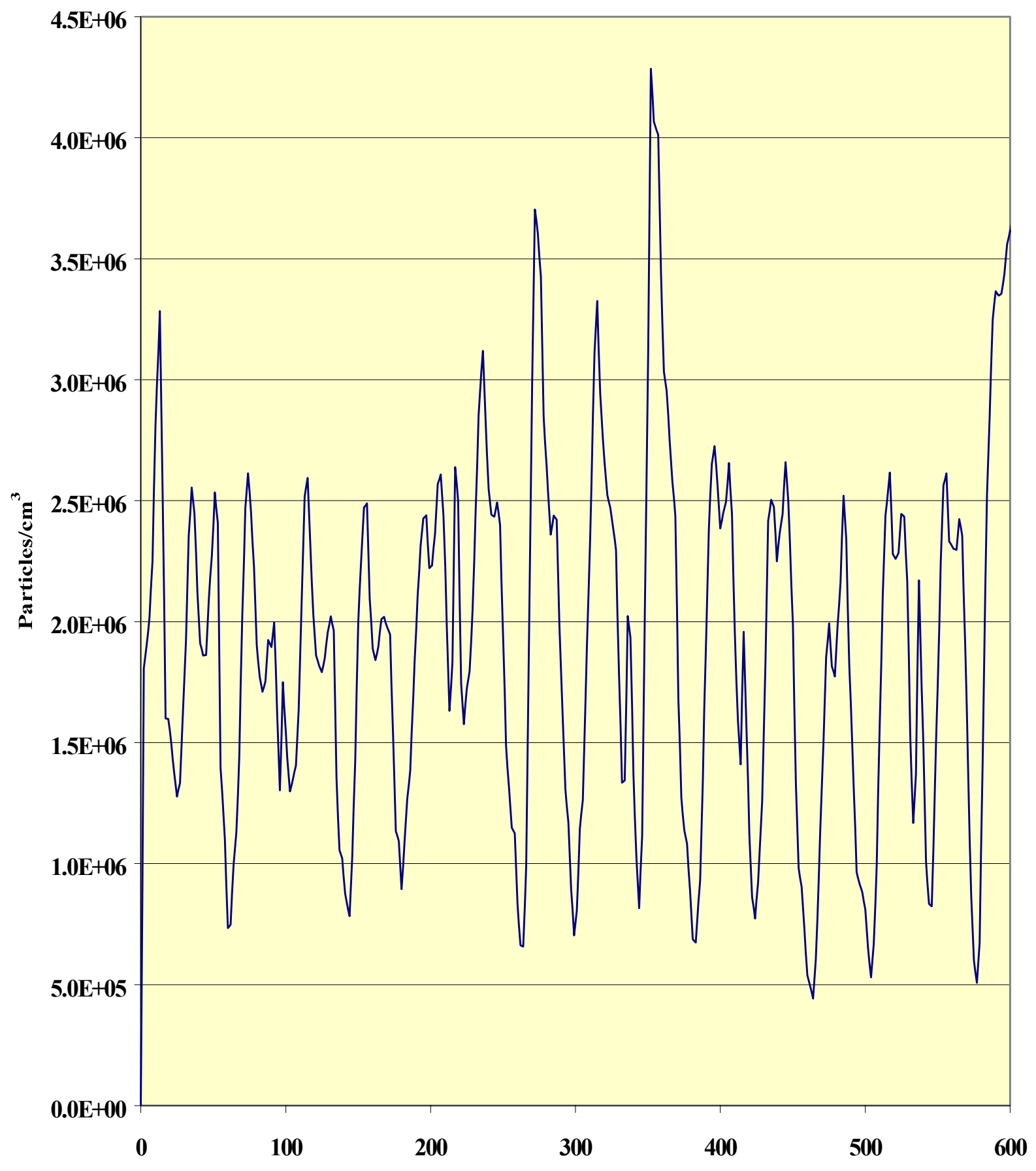

Time (seconds) 
Figure 4.27 - $50 \mathrm{~nm}$ SMPS Particle Concentration for DDC 6V92TA Bus \#2030 CBD Run \#3 Fuel: D2

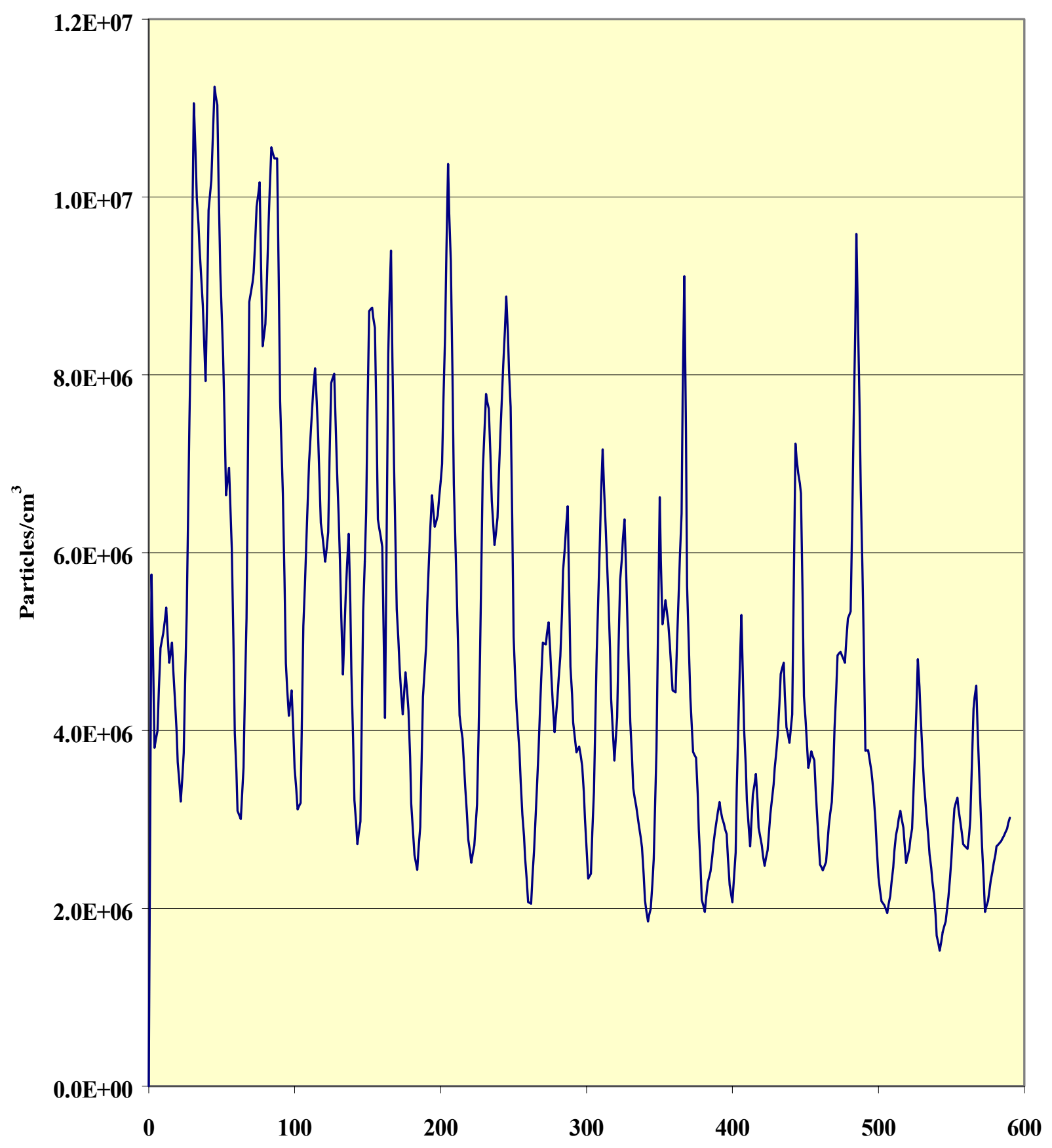

Time (seconds) 
Figure 4.28 - 100 nm SMPS Particle Concentration for DDC 6V92TA Bus \#2030 CBD Run \#4 Fuel: D2

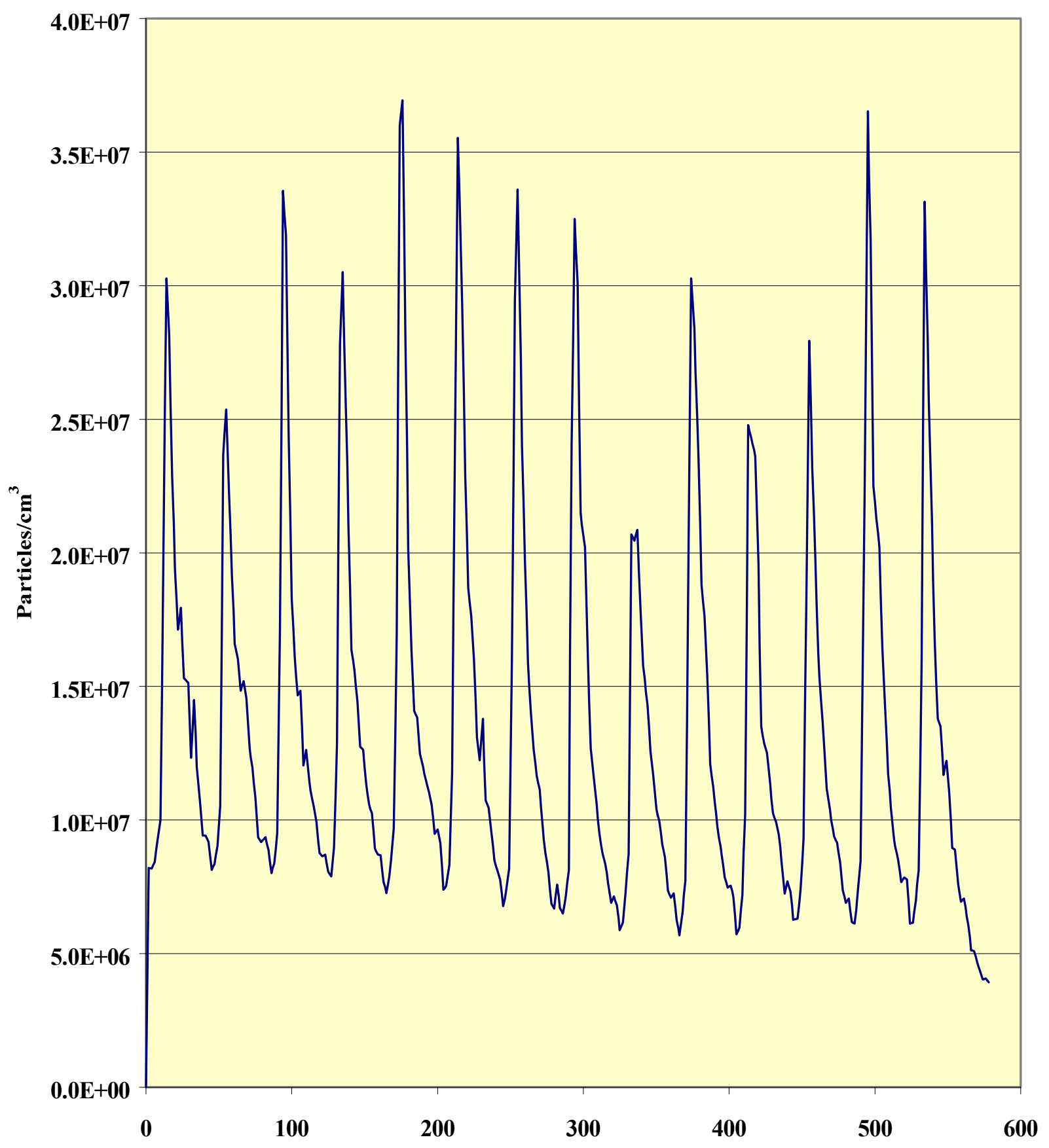

Time (seconds) 
Figure 4.29 - 200 nm SMPS Particle Concentration for DDC 6V92TA Bus \#2030 CBD Run \#5 Fuel: D2

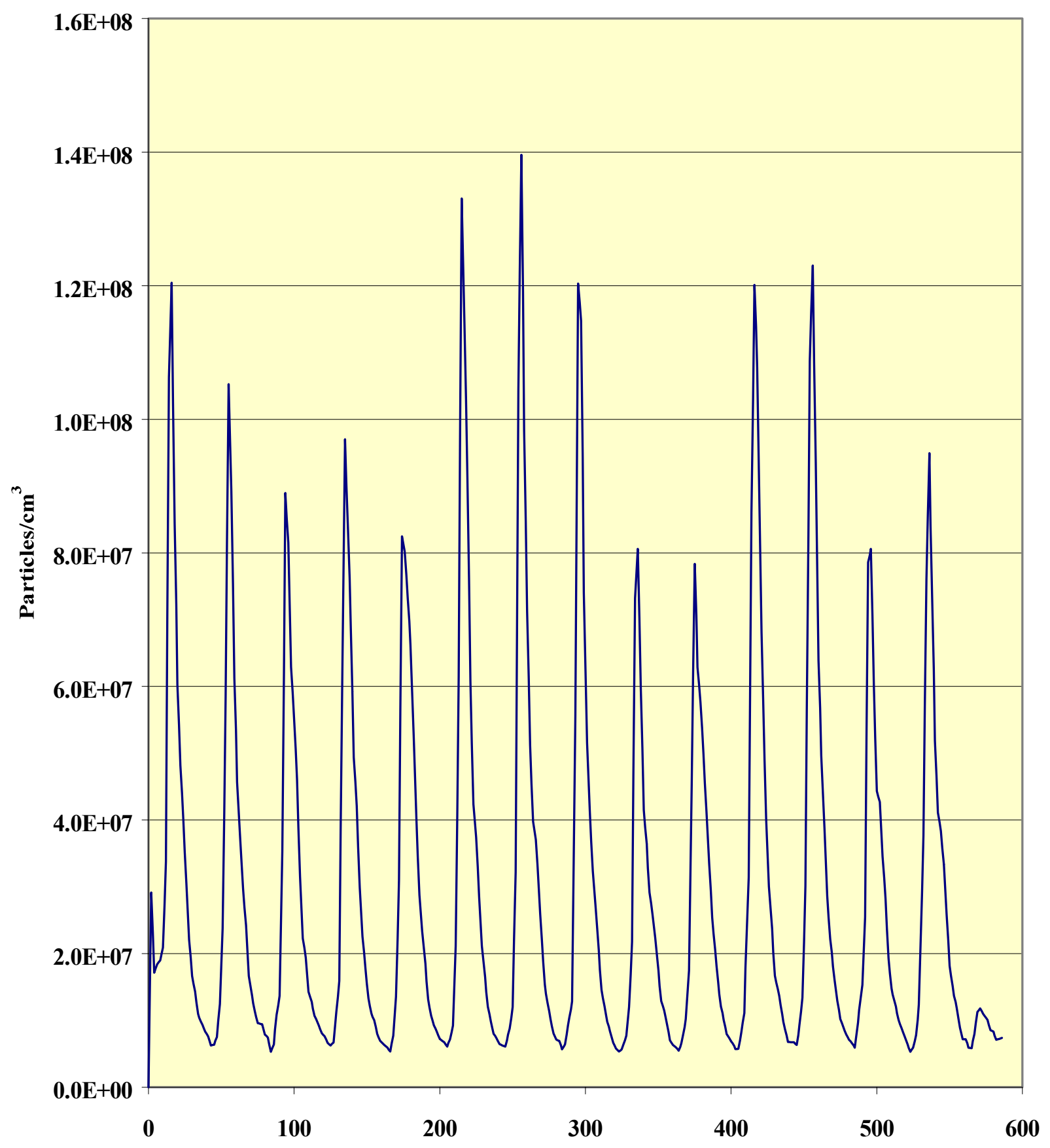

Time (seconds) 
Figure 4.30 - 15 nm SMPS Particle Concentration for DDC 6V92TA Bus \#2030 CBD Run \#1 Fuel: MG

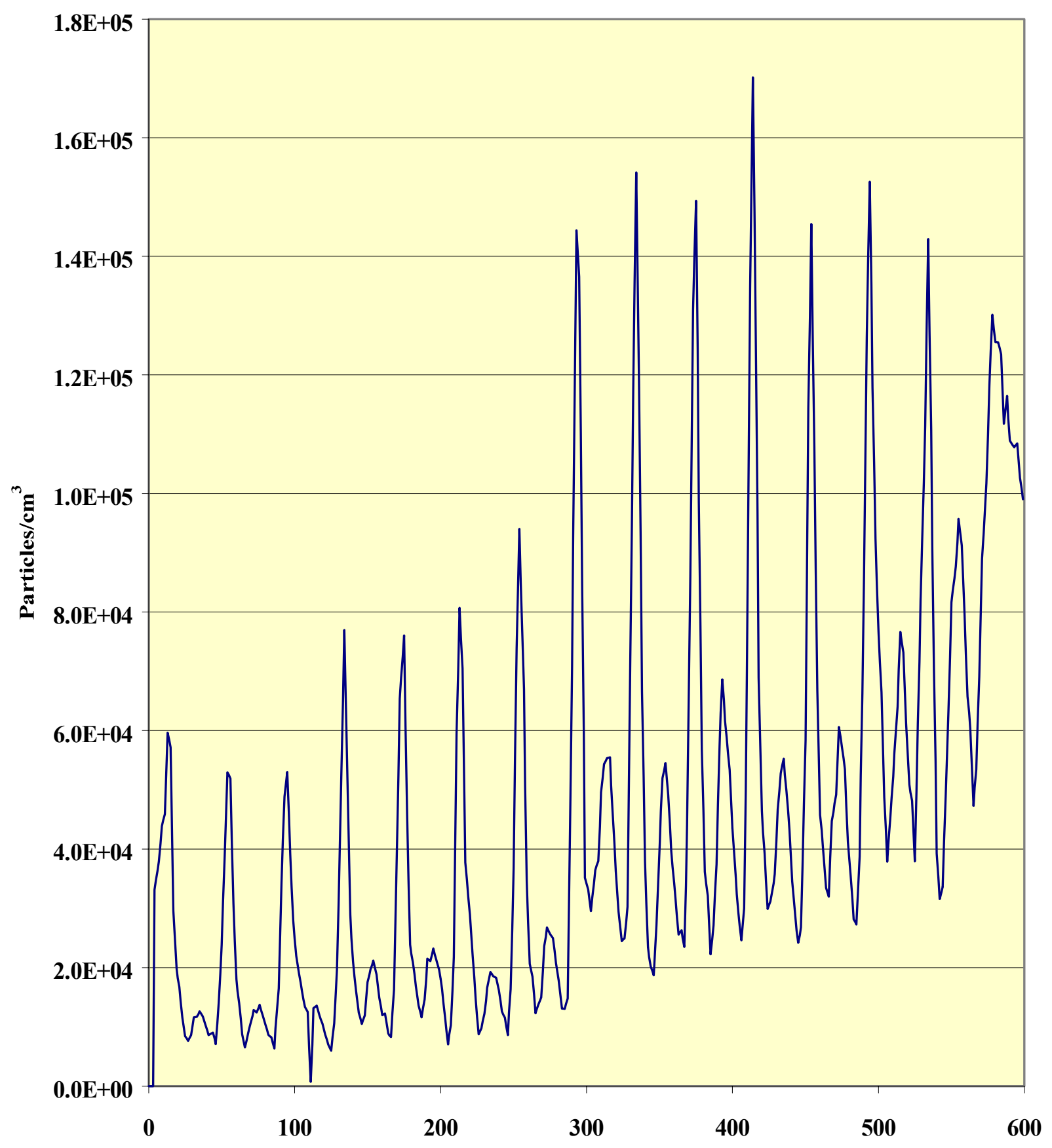

Time (seconds) 
Figure 4.31 - 30 nm SMPS Particle Concentration for DDC 6V92TA Bus \#2030 CBD Run \#2 Fuel: MG

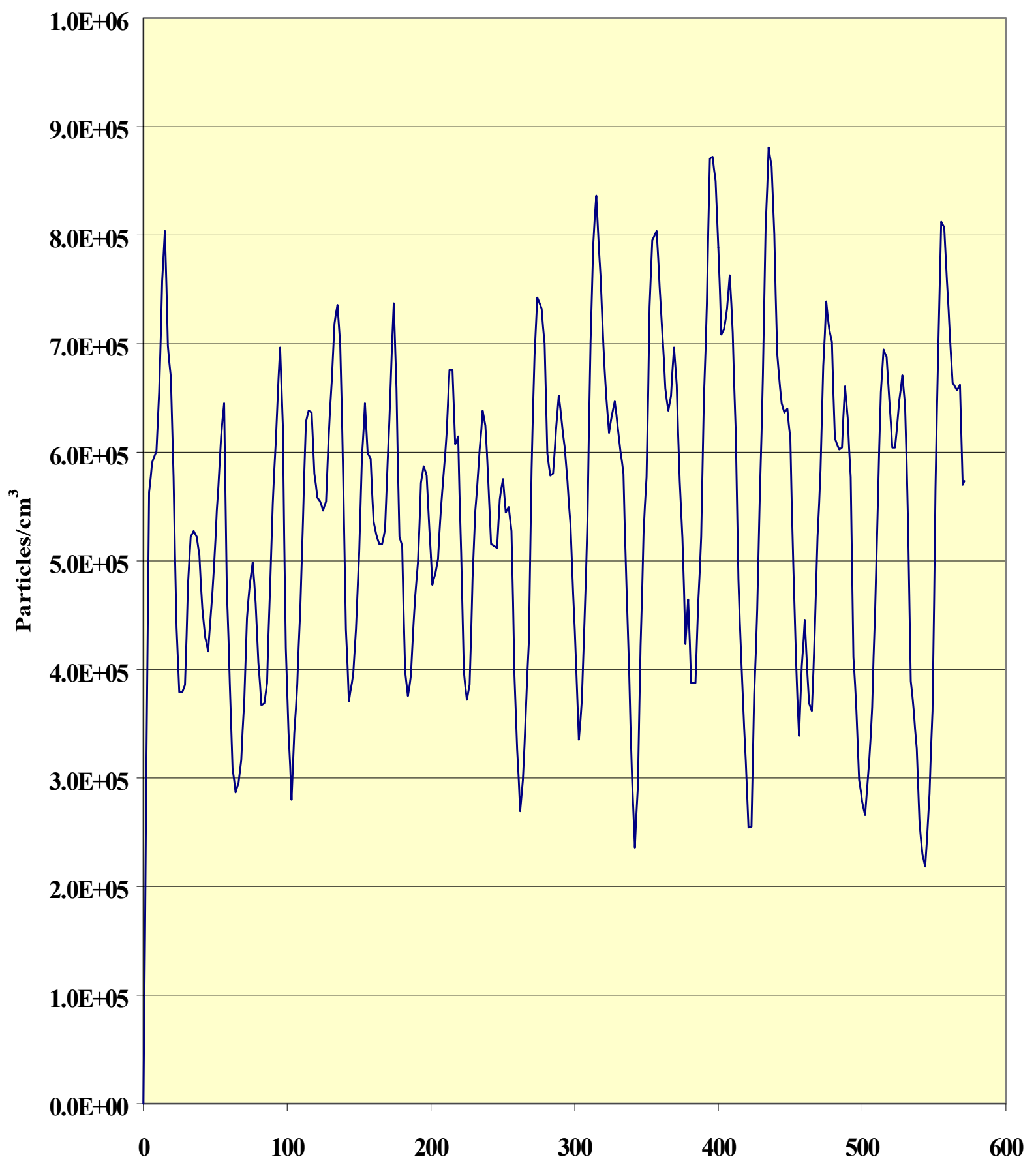

Time (seconds) 
Figure 4.32 - 50 nm SMPS Particle Concentration for DDC 6V92TA Bus \#2030 CBD Run \#3 Fuel: MG

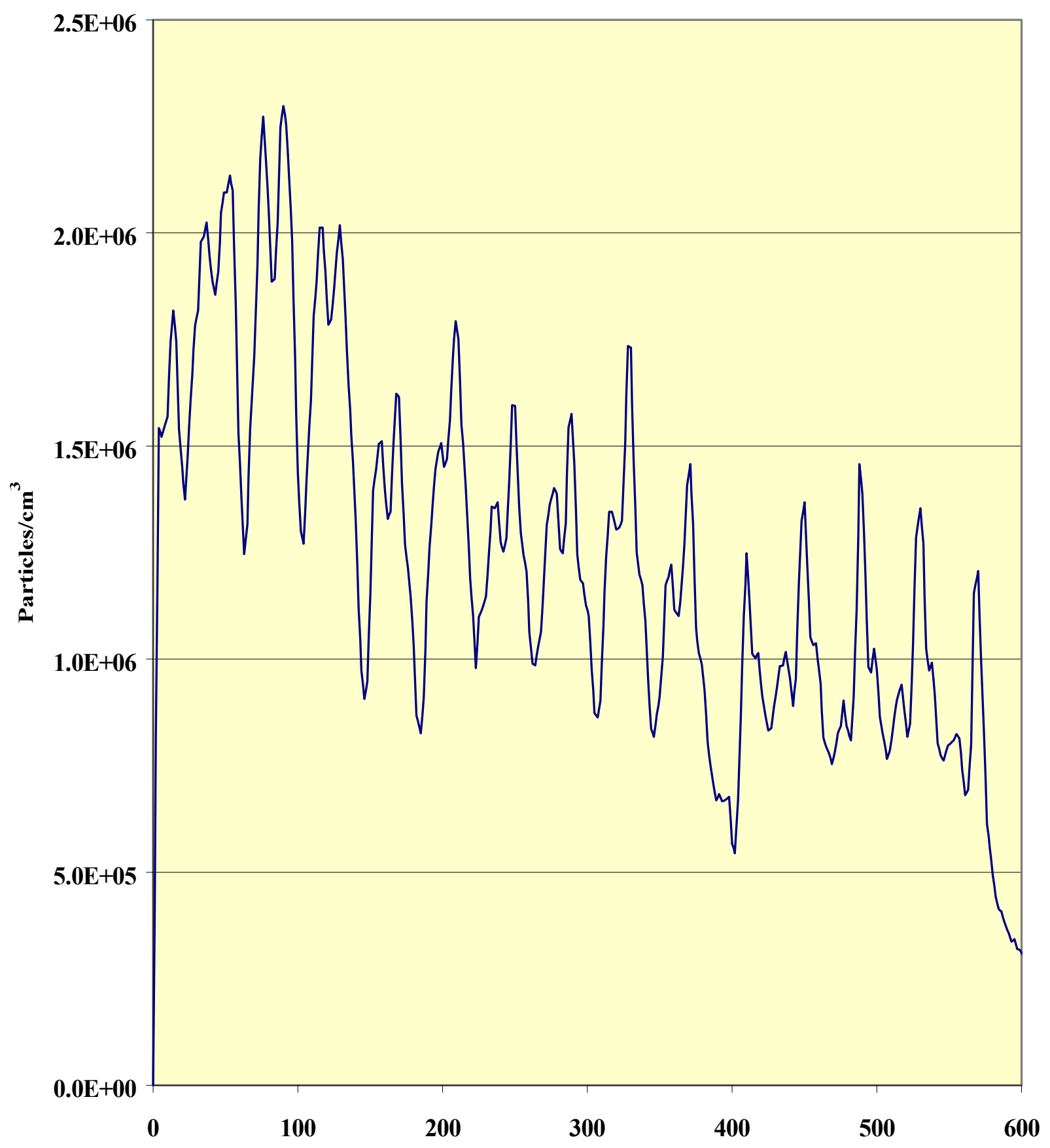

Time (seconds) 
Figure 4.33 - $100 \mathrm{~nm}$ SMPS Particle Concentration for DDC 6V92TA Bus \#2030 CBD Run \#4 Fuel: MG

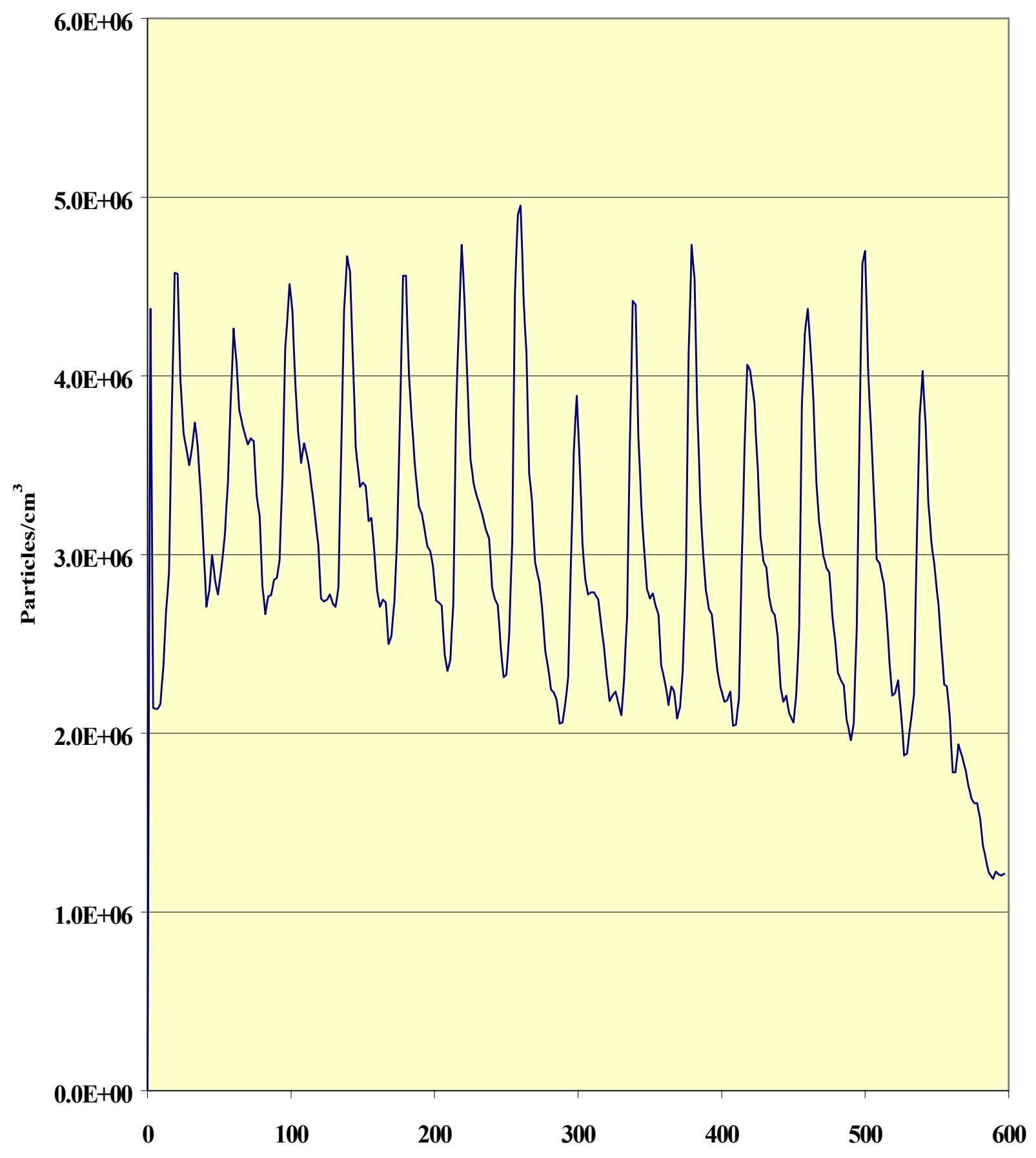

Time (seconds) 
Figure 4.34 - 200 nm SMPS Particle Concentration for DDC 6V92TA Bus \#2030 CBD Run \#5 Fuel: MG

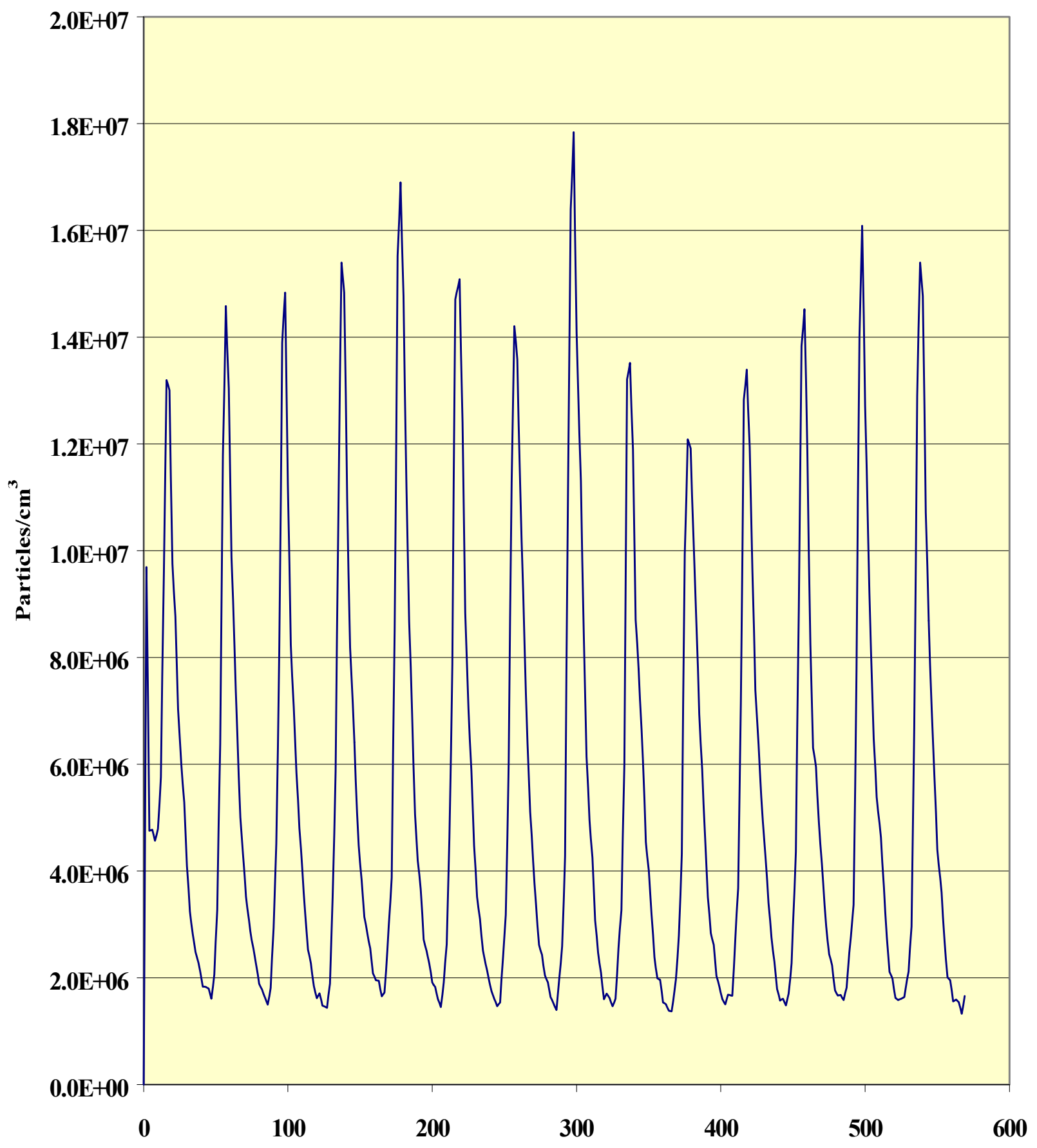

Time (seconds) 
of these experiments was to devise an accurate protocol for future research, not to obtain precise data. These tests resulted in one conclusion that greatly aided subsequent experimentation: volumetric flow measurements must be used as a means of sampling rather than mass flow. Several tests returned erroneous data in that the PM2.5 cyclone yielded higher total mass than the PM10. The data from Bus 1012 is shown below in Table 4.2. The far right block column shows the percent difference between the final mass values. A positive value means that the PM2.5 mass was greater. As can be seen, the PM2.5 cyclone collected more mass than the PM10 cyclone for approximately onehalf of the runs.

The PM10 cyclone's flow rate was controlled using the pump and mass flow controller described earlier. However, this system did not contain the thermocouple and feedback controls, thus giving a near constant, standard flow of $28.3 \mathrm{lpm}$. The PM2.5 cyclone flow was controlled by the same pump inline with a Dwyer rotameter $(0-20$ slpm) and Sierra mass flow meter (0 - 300 slpm). Both measurement devices are precise to $\pm 0.5 \mathrm{slpm}$. The mass flow meter was calibrated by a previously calibrated mass flow controller. It is reasonable to assume that both systems would maintain the required flow with the same level of accuracy since both cyclone probes were subjected to the same conditions.

While the ambient temperature over the course of testing was generally close to standard, the exhaust stream was not. Though not measured, the dilution tunnel temperature can reach $120^{\circ} \mathrm{F}$ at the sampling site, as shown in later tests. Since the flow rates are crucial for properly functioning cyclones, these temperature conditions could greatly affect the cut sizes. Another unforeseen source of error is the lower atmospheric 
Table 4.2 - RTD/SKIP Bus 1012 Cyclone Data

\begin{tabular}{|c|c|c|c|c|c|c|}
\hline Bus 1012 - D2 & Filter Wt. & Time & Flow rate & Cyclone & $\begin{array}{c}\text { Cyclone - } \\
\text { TPM }\end{array}$ & $\begin{array}{c}\% \\
\text { difference }\end{array}$ \\
\hline Test ID & grams & $\sec$ & $L / \min$ & liters & grams & \\
\hline 1245-1 (ss-20mph) PM2.5 & $6.9 \mathrm{E}-05$ & 180 & 16.7 & $5.01 \mathrm{E}+01$ & $2.06 \mathrm{E}-01$ & $79 \%$ \\
\hline 1245-1 (ss-20mph) PM10 & $5.1 \mathrm{E}-05$ & 180 & 28.3 & $8.49 \mathrm{E}+01$ & 8.99E-02 & \\
\hline 1245-2 (ss-20mph) PM2.5 & 3.7E-05 & 180 & 16.7 & $5.01 \mathrm{E}+01$ & $9.46 \mathrm{E}-02$ & $1 \%$ \\
\hline 1245-2 (ss-20mph) PM10 & $6.2 \mathrm{E}-05$ & 180 & 28.3 & $8.49 \mathrm{E}+01$ & $9.36 \mathrm{E}-02$ & \\
\hline 1246-1 (CBD) PM2.5 & 0.000239 & 567.9 & 16.7 & $1.58 \mathrm{E}+02$ & 4.89E-01 & $0 \%$ \\
\hline 1246-1 (CBD) PM10 & 0.000405 & 567.9 & 28.3 & $2.68 \mathrm{E}+02$ & $4.89 \mathrm{E}-01$ & \\
\hline 1246-2 (CBD) PM2.5 & 0.000245 & 567.9 & 16.7 & $1.58 \mathrm{E}+02$ & $5.01 \mathrm{E}-01$ & $-1 \%$ \\
\hline 1246-2 (CBD) PM10 & 0.000419 & 567.9 & 28.3 & $2.68 \mathrm{E}+02$ & $5.06 \mathrm{E}-01$ & \\
\hline $1246-3$ (CBD) PM2.5 & 0.000338 & 567.9 & 16.7 & $1.58 \mathrm{E}+02$ & $6.90 \mathrm{E}-01$ & $-3 \%$ \\
\hline $1246-3$ (CBD) PM10 & 0.000591 & 567.9 & 28.3 & $2.68 \mathrm{E}+02$ & 7.12E-01 & \\
\hline $1246-5$ (CBD) PM2.5 & 0.000299 & 567.9 & 16.7 & $1.58 \mathrm{E}+02$ & $6.09 \mathrm{E}-01$ & $0 \%$ \\
\hline $1246-5$ (CBD) PM10 & 0.000508 & 567.9 & 28.3 & $2.68 \mathrm{E}+02$ & $6.11 \mathrm{E}-01$ & \\
\hline $1246-6$ (CBD) PM2.5 & 0.000306 & 567.9 & 16.7 & $1.58 \mathrm{E}+02$ & $6.22 \mathrm{E}-01$ & $-7 \%$ \\
\hline $1246-6(C B D) P M 10$ & 0.000558 & 567.9 & 28.3 & $2.68 \mathrm{E}+02$ & $6.70 \mathrm{E}-01$ & \\
\hline $1250(s s-25 m p h) P M 2.5$ & $8.5 \mathrm{E}-05$ & 180 & 16.7 & $5.01 \mathrm{E}+01$ & $5.51 \mathrm{E}-01$ & $4 \%$ \\
\hline 1250 (ss-25mph) PM10 & 0.000138 & 180 & 28.3 & $8.49 \mathrm{E}+01$ & $5.28 \mathrm{E}-01$ & \\
\hline 1251 (ss-30mph) PM2.5 & 0.000161 & 567.9 & 16.7 & $1.58 \mathrm{E}+02$ & $3.29 \mathrm{E}-01$ & $-3 \%$ \\
\hline 1251 (ss-30mph) PM10 & 0.000281 & 567.9 & 28.3 & $2.68 \mathrm{E}+02$ & $3.39 \mathrm{E}-01$ & \\
\hline 1263-1 (CBD) PM2.5 & 0.000265 & 567.9 & 16.7 & $1.58 \mathrm{E}+02$ & $5.49 \mathrm{E}-01$ & $6 \%$ \\
\hline 1263-1 (CBD) PM10 & 0.000421 & 567.9 & 28.3 & $2.68 \mathrm{E}+02$ & $5.15 \mathrm{E}-01$ & \\
\hline 1264-2 (CBD) PM2.5 & 0.000235 & 567.9 & 16.7 & $1.58 \mathrm{E}+02$ & 4.39E-01 & $2 \%$ \\
\hline $1264-2(C B D) P M 10$ & 0.000389 & 567.9 & 28.3 & $2.68 \mathrm{E}+02$ & 4.29E-01 & \\
\hline 1265 (CBD) PM2.5 & 0.000227 & 567.9 & 16.7 & $1.58 \mathrm{E}+02$ & 4.69E-01 & $-8 \%$ \\
\hline $1265(C B D) P M 10$ & 0.000415 & 567.9 & 28.3 & $2.68 \mathrm{E}+02$ & $5.06 \mathrm{E}-01$ & \\
\hline 1266 (CBD) PM2.5 & 0.000291 & 567.9 & 16.7 & $1.58 \mathrm{E}+02$ & $6.04 \mathrm{E}-01$ & $-13 \%$ \\
\hline $1266(C B D) P M 10$ & 0.000559 & 567.9 & 28.3 & $2.68 \mathrm{E}+02$ & $6.84 \mathrm{E}-01$ & \\
\hline 1267 (CBD) PM2.5 & 0.000448 & 567.9 & 16.7 & $1.58 \mathrm{E}+02$ & $9.21 \mathrm{E}-01$ & $4 \%$ \\
\hline $1267(C B D) P M 10$ & 0.000731 & 567.9 & 28.3 & $2.68 \mathrm{E}+02$ & $8.86 \mathrm{E}-01$ & \\
\hline
\end{tabular}


pressure at one mile above sea level. From the equation of state, deviations from standard conditions are shown to have a large impact.

$$
V_{\text {actual }}=V_{s t p} \times\left[\left(\frac{T_{\text {actual }}}{T_{s t p}}\right)\left(\frac{P_{s p p}}{P_{a c t u a l}}\right)\right]
$$

If the temperature did approach $120^{\circ} \mathrm{F}$ during a cycle, the actual volume would be 1.7 times greater than the standard (neglecting pressure). The effects of higher flow will be much larger for the PM10 cyclone. The cut point diameter will drop as flow increases, as can be seen in the flow charts (Figures $4.35 \& 4.36$ ) provided by the manufacturer, URG, and reprinted by permission.

Flow Chart for URG-200-30EA, 28.3Lpm 10 $\mu$ m cut Cyclone

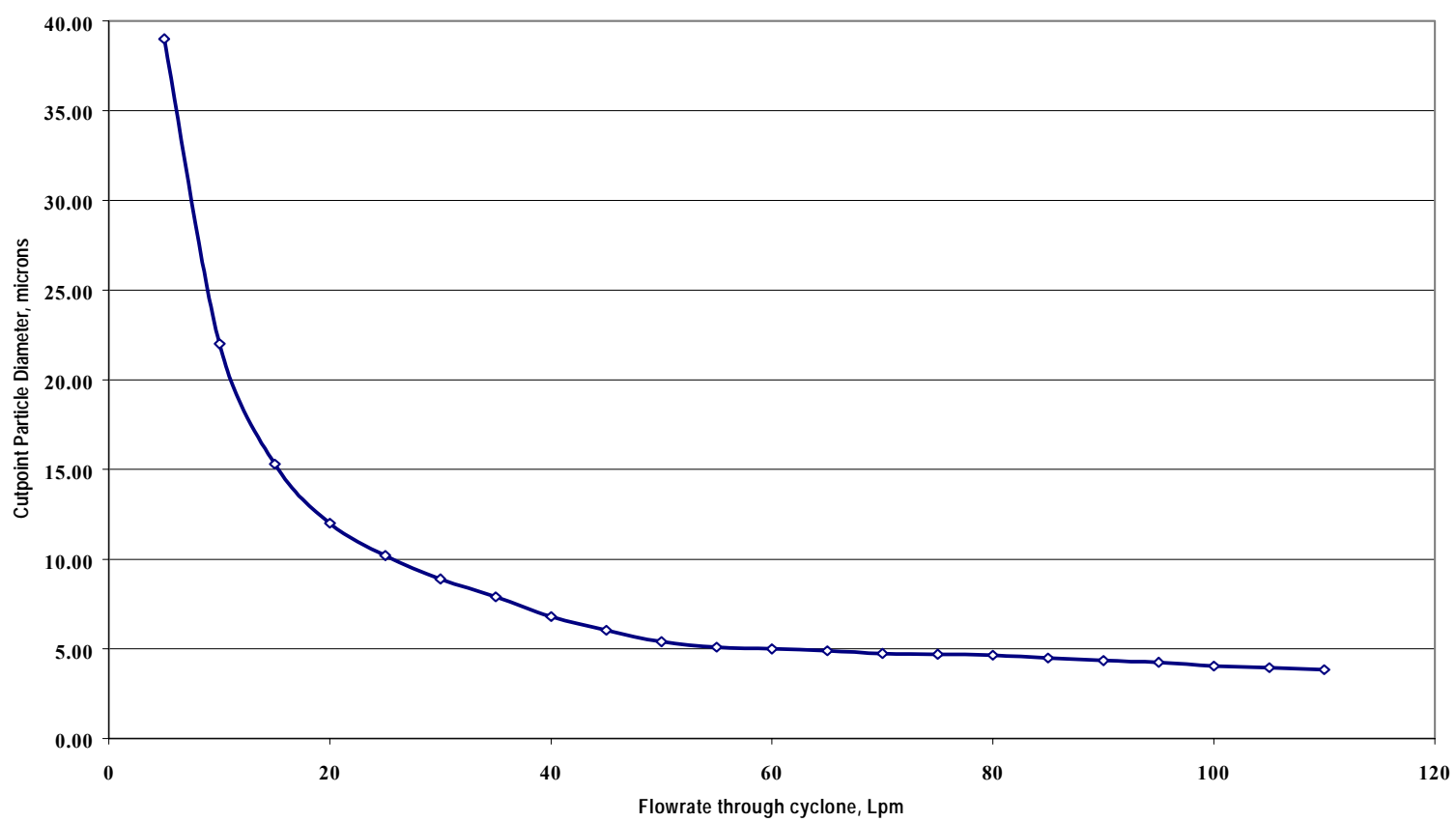

Figure 4.35 (Reprinted from URG by Permission) 


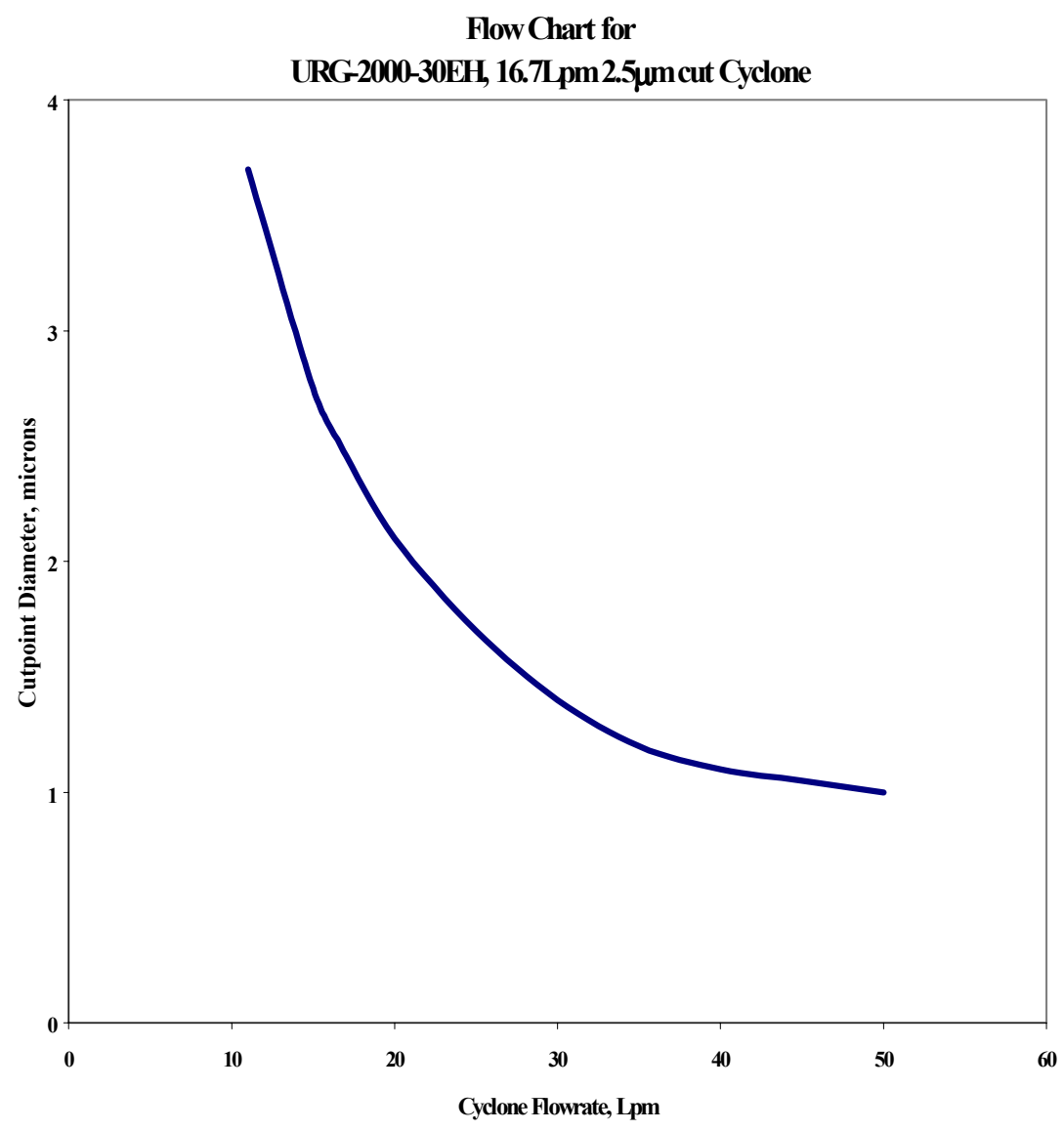

Figure 4.36 (Reprinted from URG by Permission)

Using the $120^{\circ} \mathrm{F}$ level as a worst case, the cut point diameter would be lowered to approximately $1.75 \mu \mathrm{m}$ for the PM2.5 cyclone and approximately $6 \mu \mathrm{m}$ for the PM10. The lost particles associated with this cut point diameter drop will affect the PM10 cyclone more since the particles in this broad spectrum $(6-10 \mu \mathrm{m})$ will generally weigh more than the narrow range of $1.75-2.5 \mu \mathrm{m}$.

By this reasoning alone, the PM10 cyclone should still have captured a higher quantity of total mass. However, this could occur if the mass flow meter system did not maintain the 16.7 slpm flow. The flow for the PM2.5 cyclone was manually controlled during testing by restricting or opening the rotameter inlet and monitoring the digital display on the flow meter. Since a positive displacement meter was not used, the total 
flow could not be recorded. Errors could abound in this system, possibly leading to the higher mass values for the PM2.5 cyclone. The randomness of the mass values, with respect to the cyclone comparison, shows that the error was not constant, which lends support to this assumption. Needless to say, the sampling measurement system was completely reworked for the next fleet testing, as described in an earlier section.

\subsubsection{MOUDI Data}

The RTD/SKIP buses were also tested with the MOUDI. In contrast to the cyclones, the MOUDI performed well and returned a wealth of data. Since this was the first substantial time the MOUDI was used, a base of diesel data was needed for guidelines for operation and fuel data. From these tests, an unwavering procedure was developed for the MOUDI, the bulk of diesel size distributions became the standard check for accurate sampling and a comparison was made between natural gas and diesel.

The first three buses tested on the chassis dynamometer, 1010, 1011 and 1012 were diesel fueled. The vehicles were tested under both steady state and transient conditions (CBD). Due to time constraints, the steady state tests run at $20 \mathrm{mph}$ were conducted for three minutes. From the relatively low speed and short time, the "clean" buses did not emit enough particulate matter for the MOUDI to allow size distribution data. With such limited mass collected on the substrates, analysis could not be made. Substrate weights for these steady state tests were very low, encouraging large deviations by small discrepancies. Evidence to this notion can be found in the final steady state test run at $30 \mathrm{mph}$ rather than $20 \mathrm{mph}$ and for 568 seconds rather than 180 seconds. The 
increased duration and speed seemed to partially remedy the problem, with this being the only steady state test for which a size distribution could be determined (see Figure 4.41). However, the mass median aerodynamic diameter was lower, $0.10 \mu \mathrm{m}$, than all but one other diesel test.

Bus 1010 had this transient diesel test with the lowest MMAD, as shown in Figure 4.37. With the understanding that this was the very first cycle tested, at the site, along with the MOUDI, this lower value was likely due to human error. Only one other transient test (Figure 4.38) was performed for this bus, giving an MMAD of $0.12 \mu \mathrm{m}$ with a GSD of 2.02. As for the DDC 6V92TA tests conducted in Morgantown, the duration of the CBD sample time had to be scaled back in order to avoid clogging of the MOUDI stages.

For Bus 1011, two CBD tests were conducted, one for four ramps, the other for nine. Both runs resulted in MMADs of $0.11 \mu \mathrm{m}$ with GSDs of 2.66 and 2.21, respectively (see Figures $4.39 \& 4.40$ ). The effect of the five extra ramps sampled appears to be only detectable in the GSD. The lower the GSD, the more prominent the mass peak. The more important factor, MMAD, was similarly unaffected.

The final diesel bus, 1012, was tested over two nonconsecutive days. The first CBD was sampled for only four ramps and did suffer from the reduced test time (see Figure 4.42). While the distribution appeared similar to the others, the GSD was 3.10. The proceeding two tests (see Figures $4.43 \& 4.44$ ) were for nine ramps and have lower GSD values as well as MMADs. These two runs were nearly identical and match extremely well with the following test, which was conducted for all fourteen 
Figure 4.37 - MOUDI Size Distribution for Cummins B5.9

Bus 10104 Ramps of CBD Run \#2 Fuel: D2

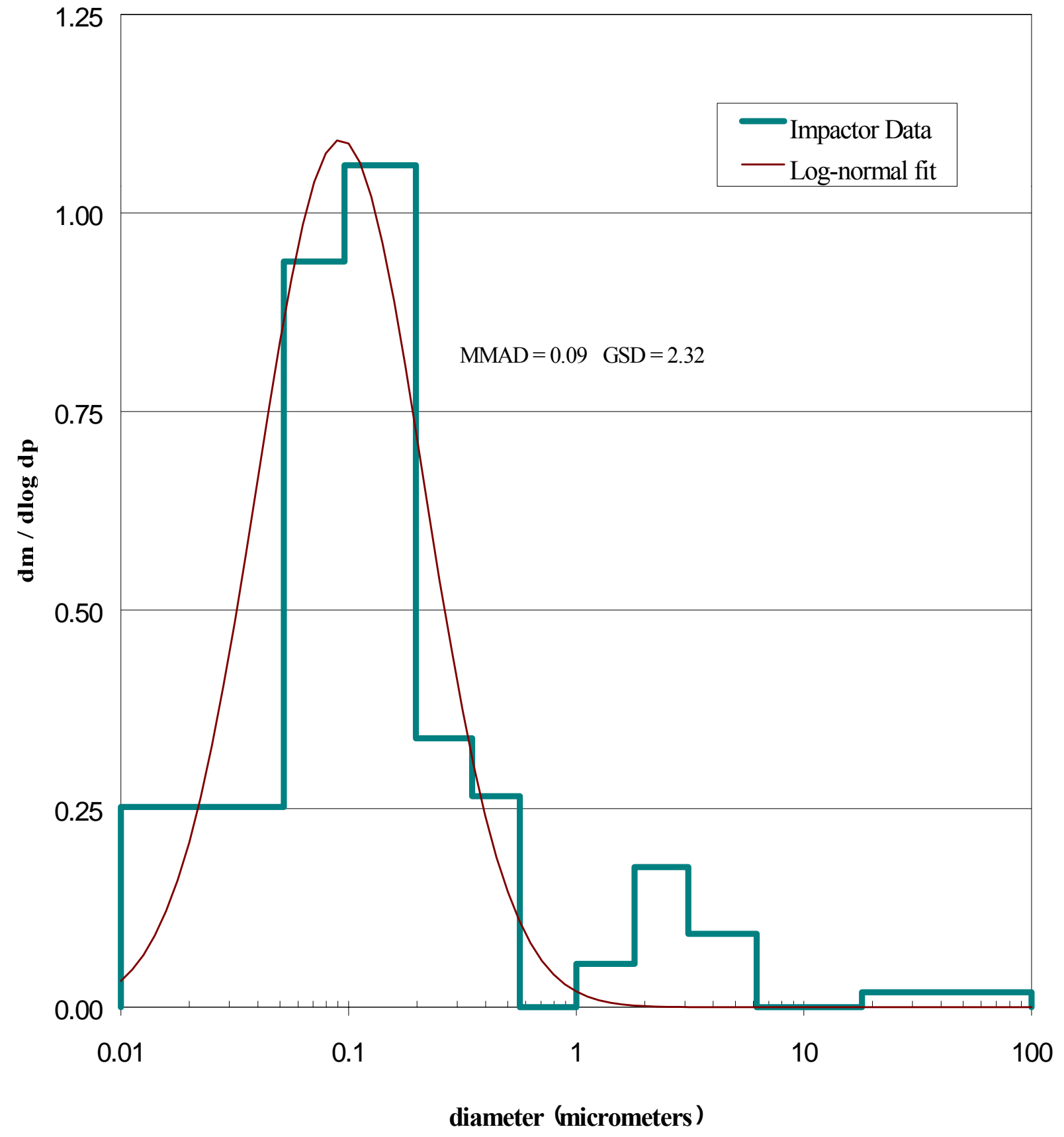


Figure 4.38 - MOUDI Size Distribution for Cummins B5.9 Bus 1010 9 Ramps of CBD Run \#3 Fuel: D2

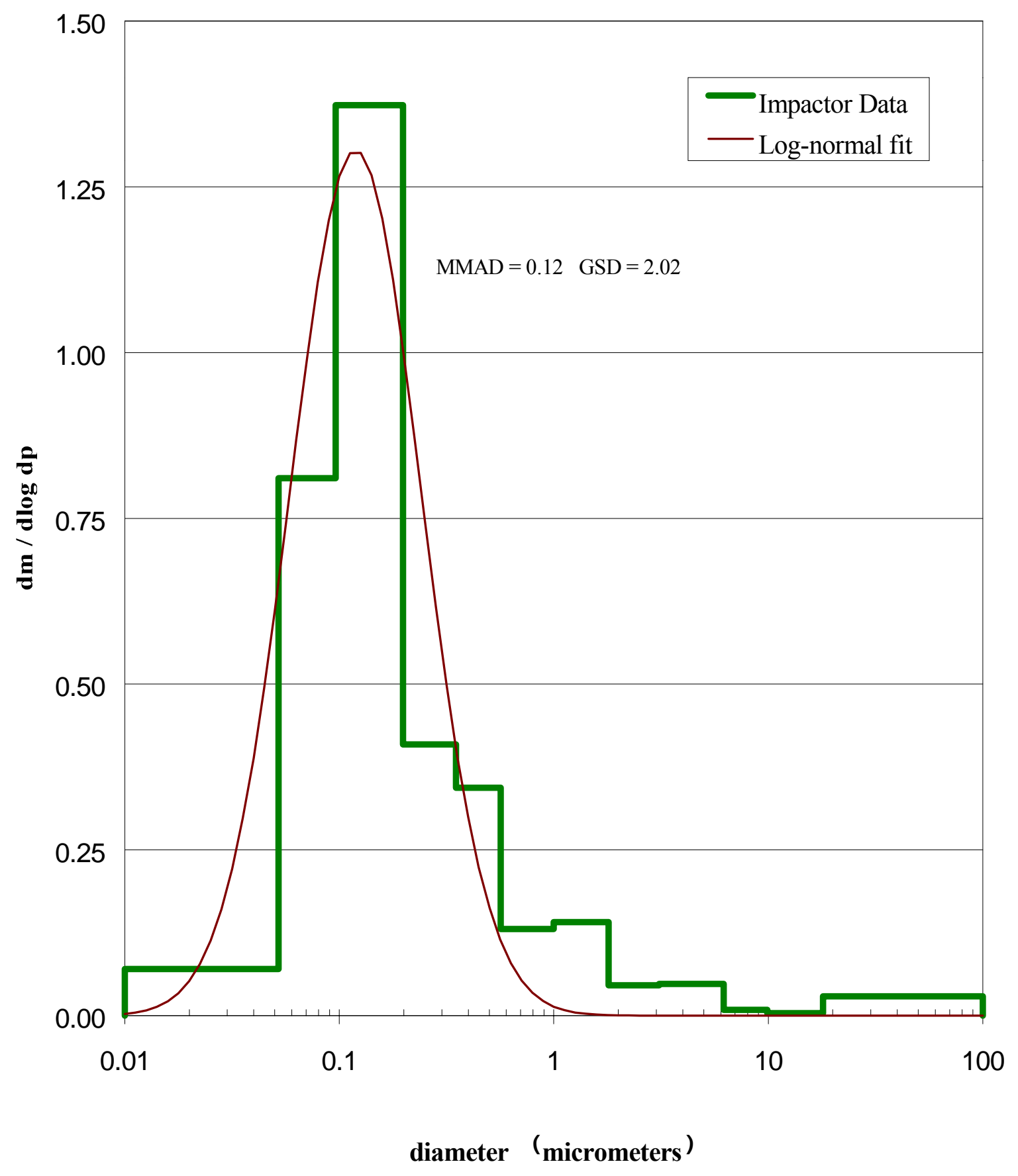


Figure 4.39 - MOUDI Size Distribution for Cummins B5.9 Bus 1011 4 Ramps of CBD Run \#1 Fuel: D2

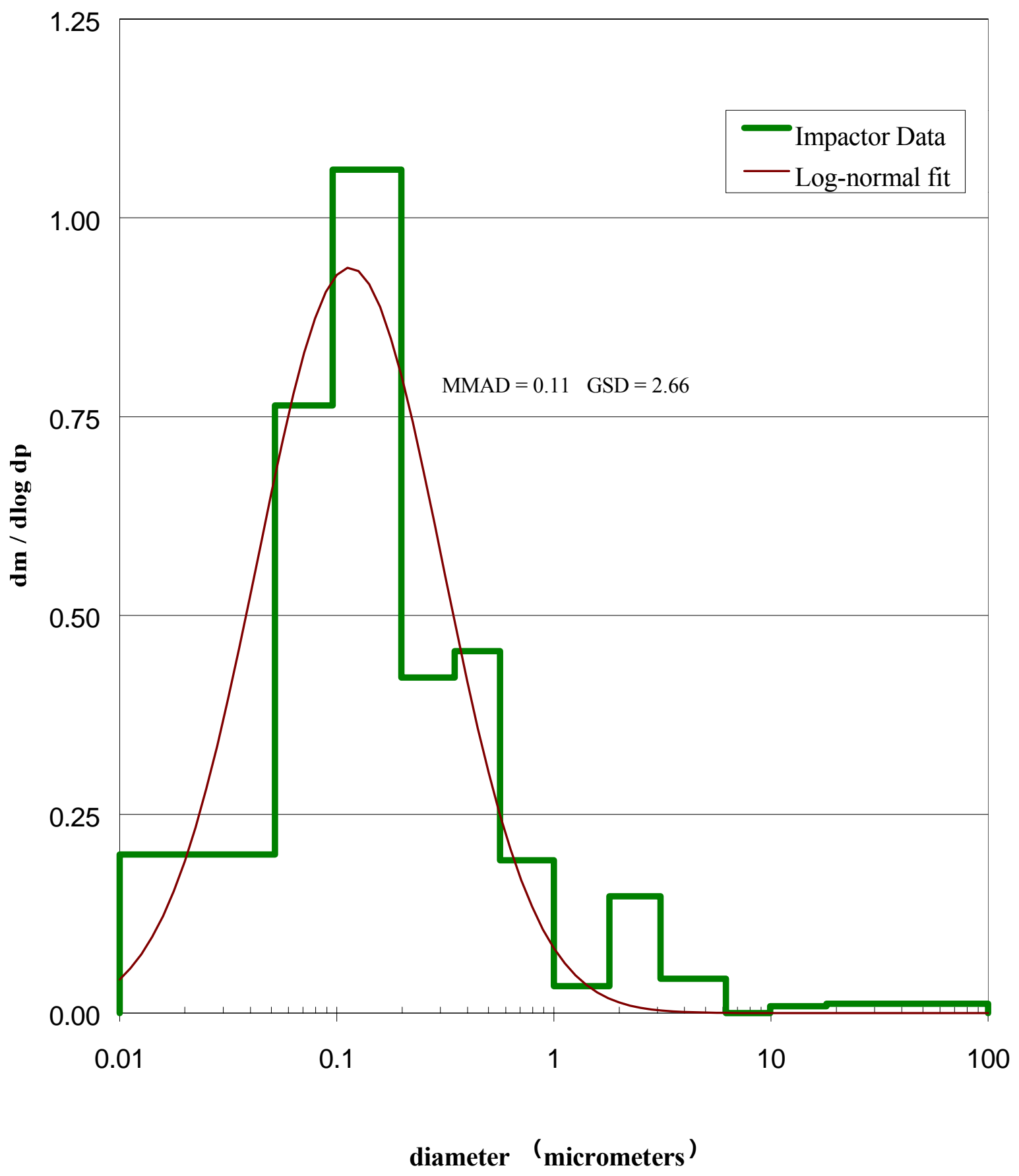


Figure 4.40 - MOUDI Size Distribution for Cummins B5.9

Bus 10119 Ramps of CBD Run \#3 Fuel: D2

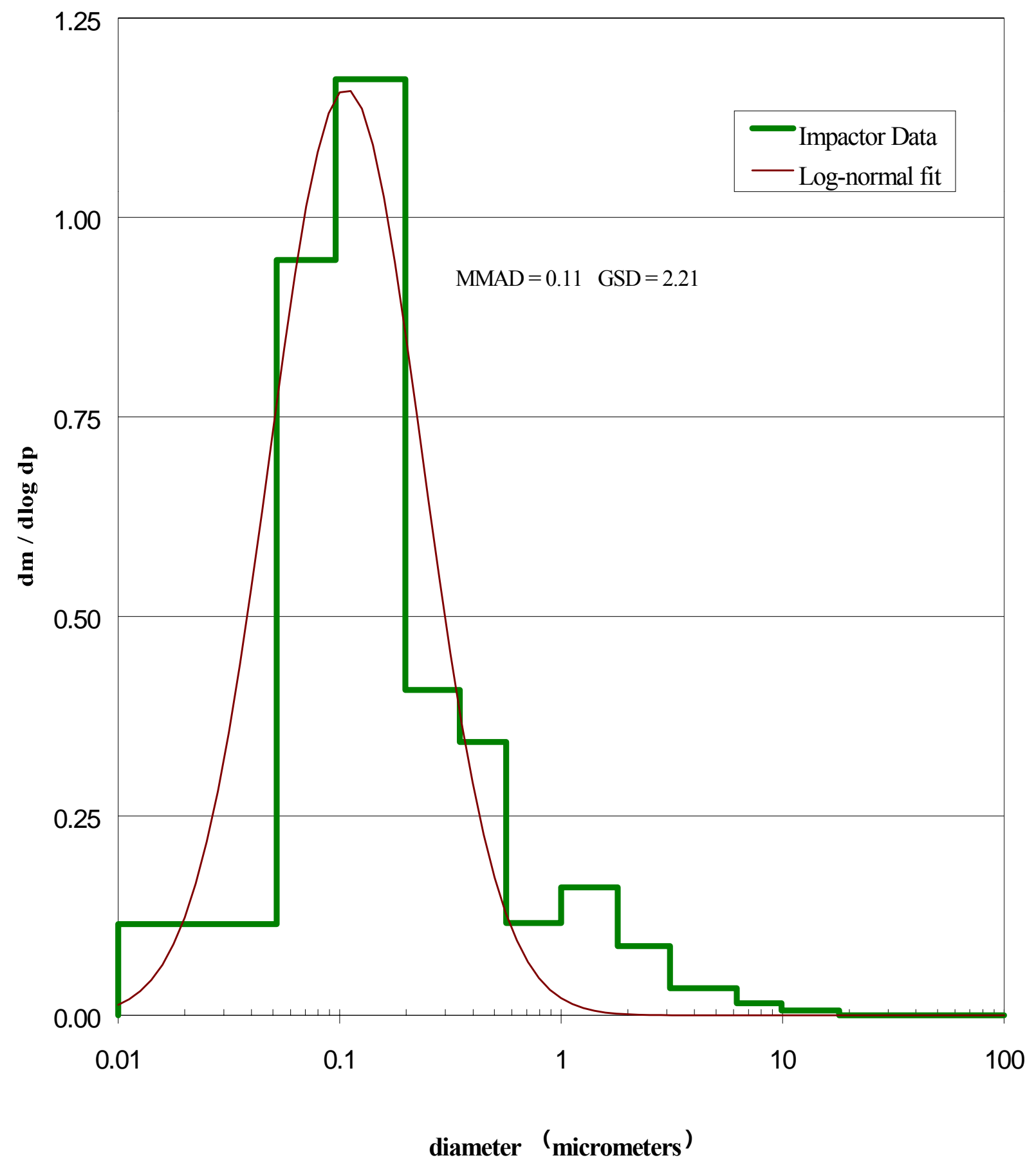


ramps (see Figure 4.45). The next batch of tests was conducted for the entire cycle and yielded distributions that were very similar for all the runs.

The final three transient tests all had MMADs of $0.12 \mu \mathrm{m}$ (see Figures 4.46 4.48). Considering this bus had given $0.11 \mu \mathrm{m}$ for three of the previous four tests, the ambient conditions were checked to see if relative humidity levels may have played a part in the $0.01 \mu \mathrm{m}$ increase in the median diameter. Contrary to what was expected, the relative humidity levels were higher for the first day than the second. Day one had levels between $35-40 \%$, for the second day, $24-28 \%$. There seems to be no quantifiable explanation for the slight increase. The average MMAD for the Bus 1012 was $0.115 \mu \mathrm{m}$ (neglecting the first 4-ramp test), which is only $\pm 5 \mathrm{~nm}$ from every test.

The first CNG bus tested, 1013, was similarly run on 20 mph steady state modes and $\mathrm{CBD}$ cycles. As with the diesel, the natural gas particulate emissions were too low for the steady state runs to give a reliable size distribution, though these tests were conducted for 568 seconds. The two CBD cycles did allow for a mass based distribution. These two tests show the distribution to be bimodal in form. As Figures 4.49 and 4.50 show, run \#1 had peaks at $0.03 \mu \mathrm{m}$ and $2.81 \mu \mathrm{m}$, while the second run had them at 0.02 $\mu \mathrm{m}$ and $1.92 \mu \mathrm{m}$. The MOUDI has no resolution below $52 \mathrm{~nm}$, hence, determining a precise median is not possible.

The second mode is of more interest than the first. The bimodal distribution for Bus 1013 (see Figures $4.49 \& 4.50$ ) shows a particularly unique feature. The second mode is not this prominent for any of the other buses, and varies greatly from run to run. This leads to the assumption that this mass mode does not consist primarily of combustion formed particles. In the absence of a chemical analysis, its source is more 
Figure 4.41 - MOUDI Size Distribution for Cummins B5.9

Bus 1012 SS 30mph Run \#1 Fuel: D2

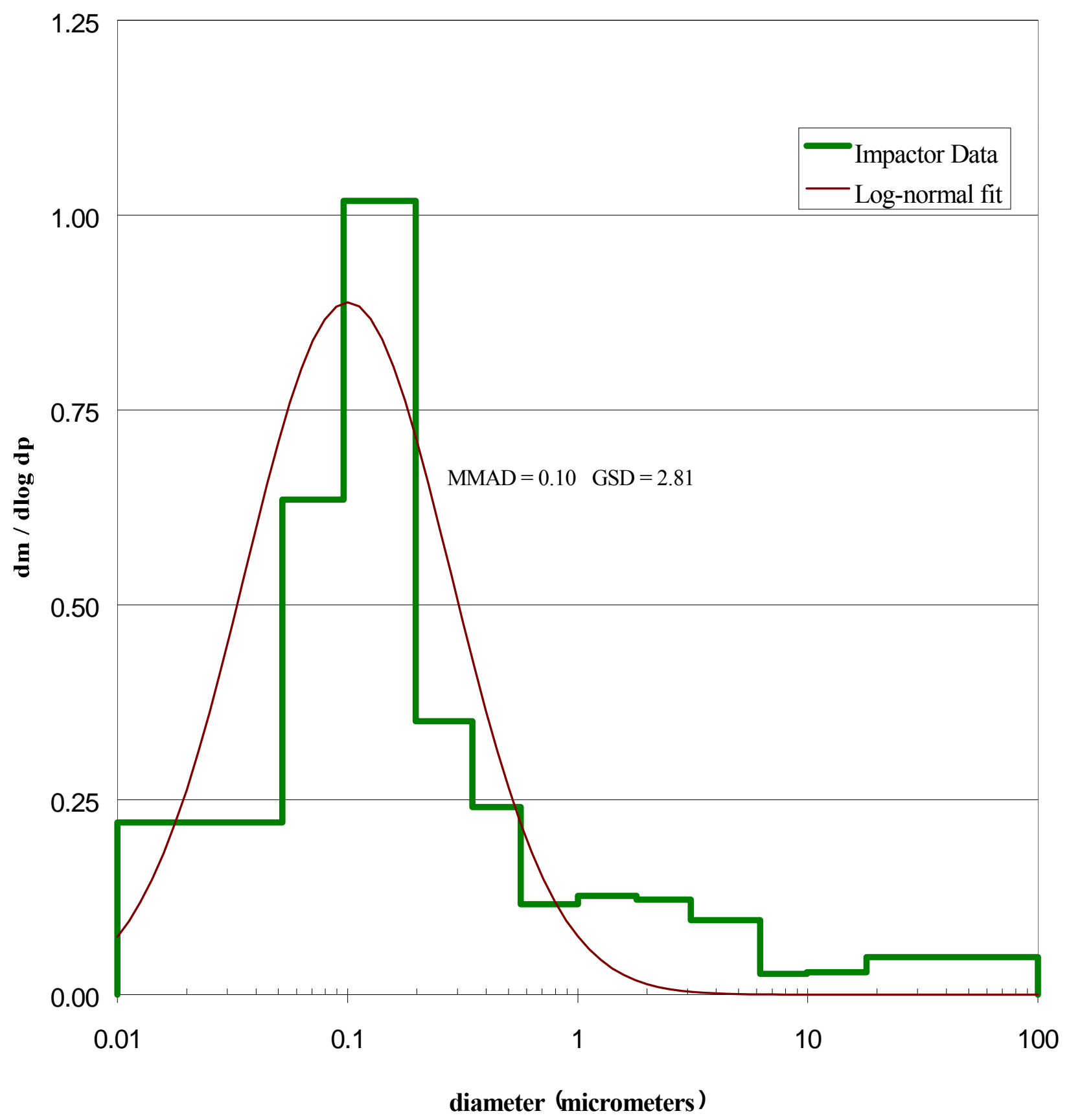




\section{Figure 4.42 - MOUDI Size Distribution for Cummins B5.9 Bus 10124 Ramps of CBD Run \#1 Fuel: D2}

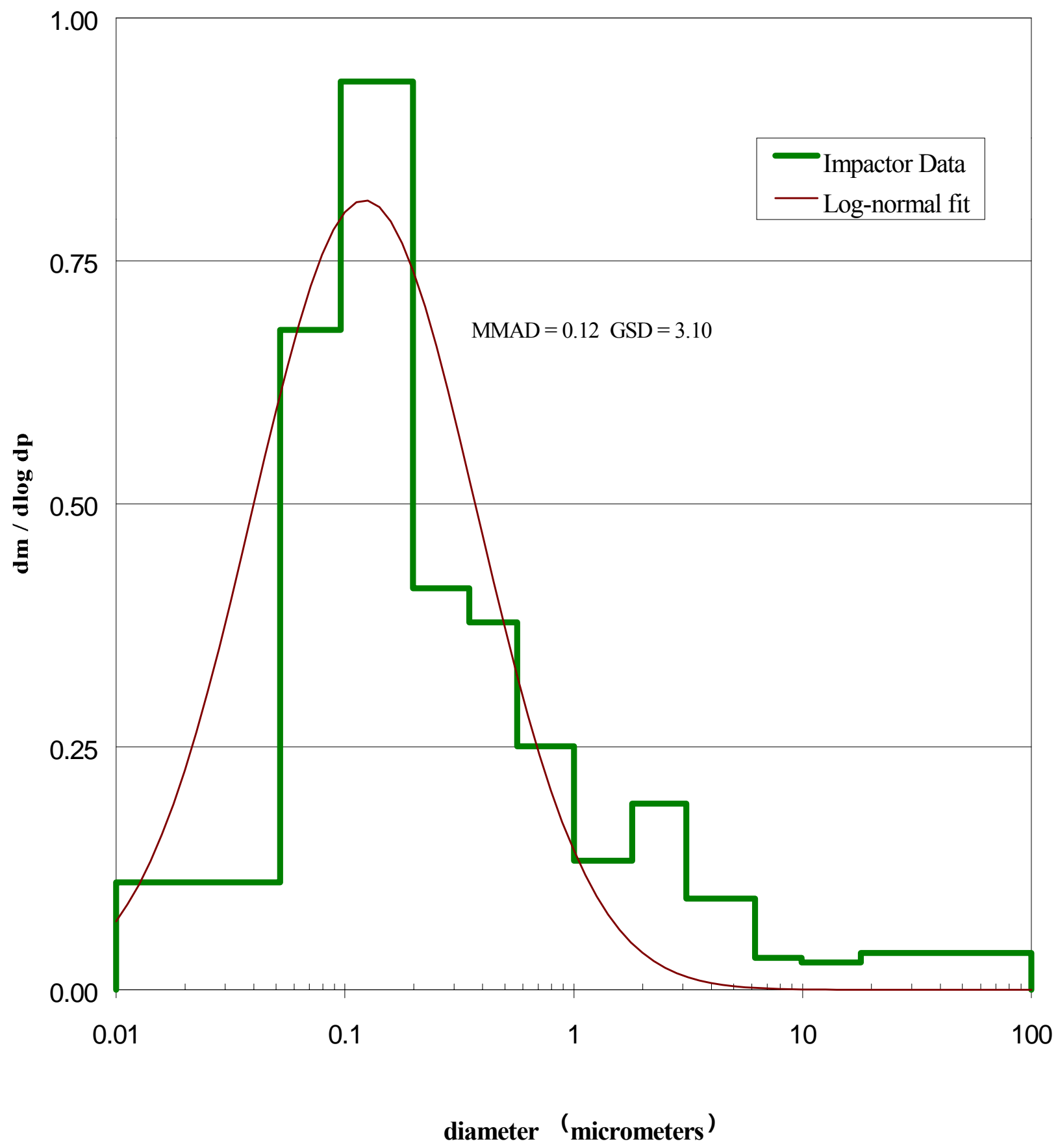


Figure 4.43 - MOUDI Size Distribution for Cummins B5.9

Bus 10129 Ramps of CBD Run \#3 Fuel: D2

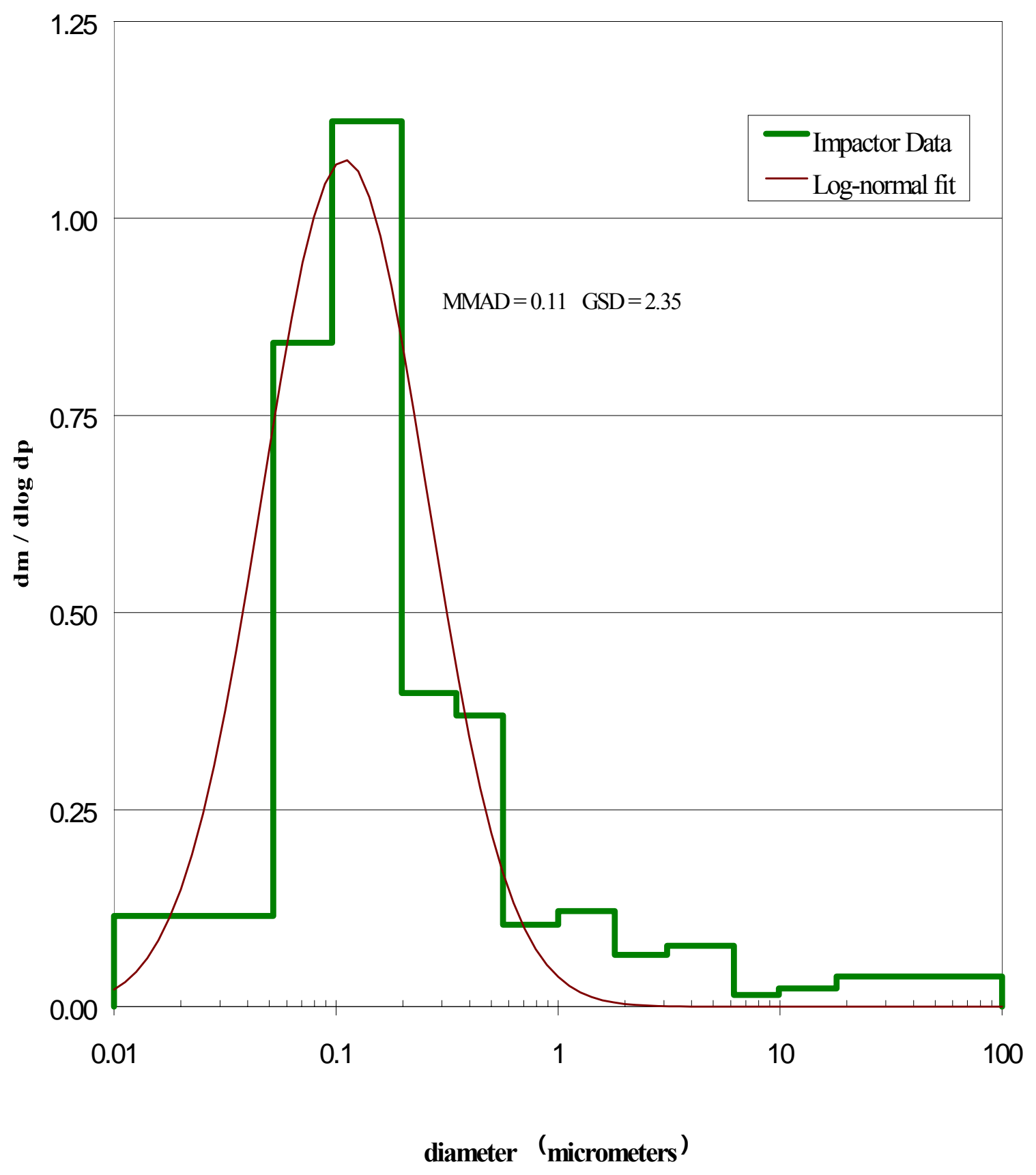




\section{Figure 4.44 - MOUDI Size Distribution for Cummins B5.9 Bus 10129 Ramps of CBD Run \#5 Fuel: D2}

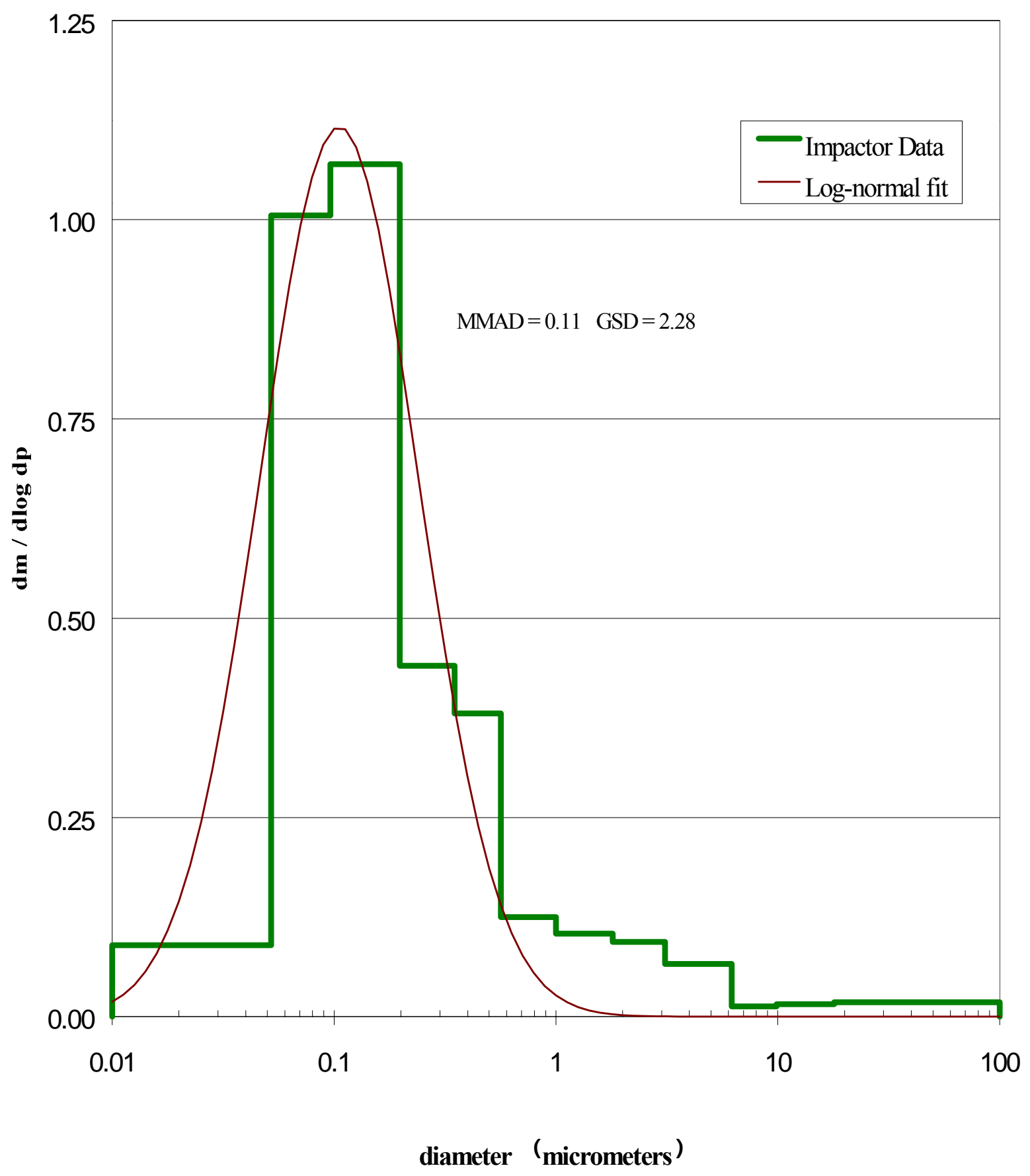




\section{Figure 4.45 - MOUDI Size Distribution for Cummins B5.9 Bus 1012 CBD Run \#6 Fuel: D2}

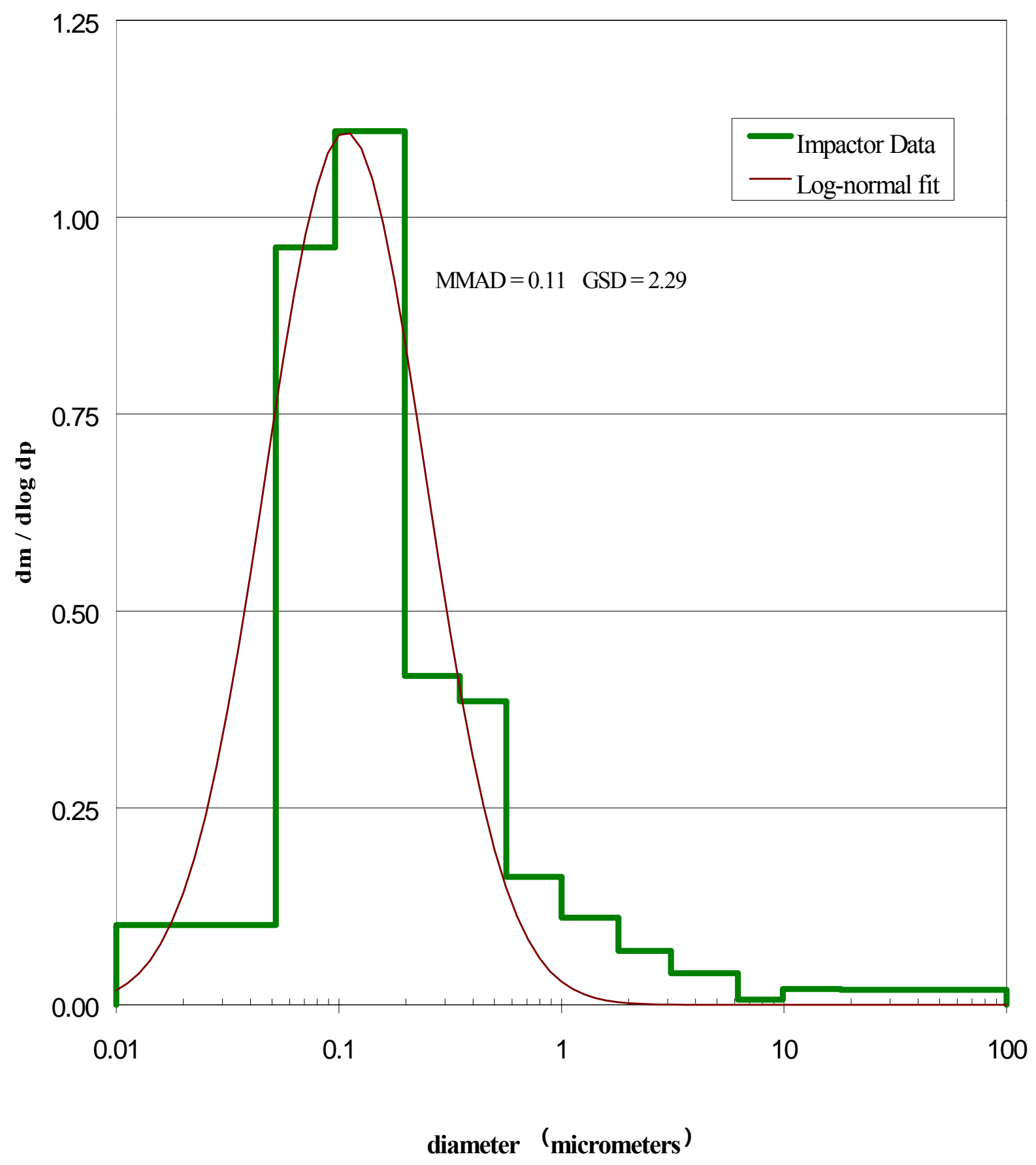


Figure 4.46 - MOUDI Size Distribution for Cummins B5.9 Bus 1012 CBD Run \#7 Fuel: D2

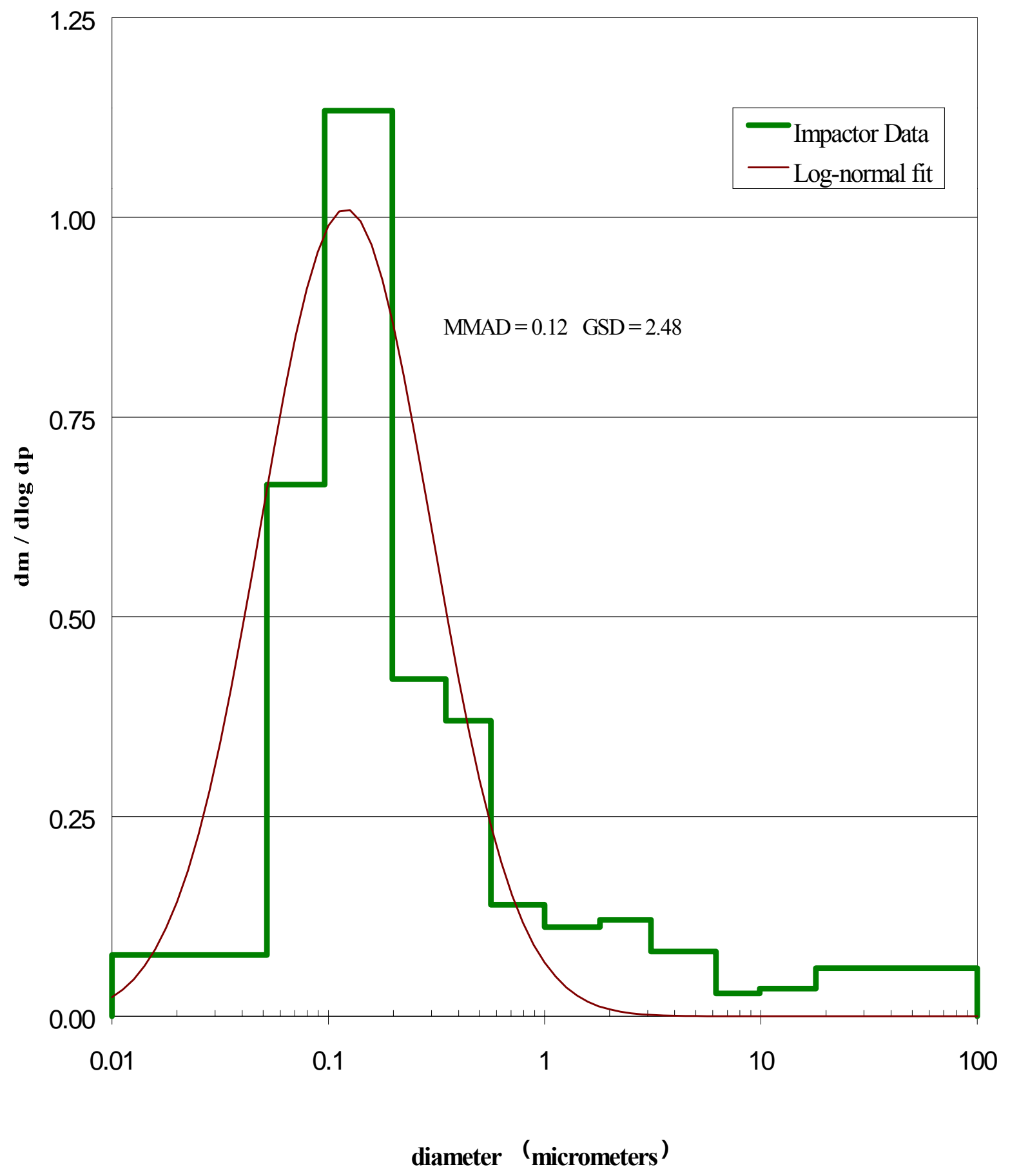




\section{Figure 4.47 - MOUDI Size Distribution for Cummins B5.9 Bus 1012 CBD Run \#9 Fuel: D2}

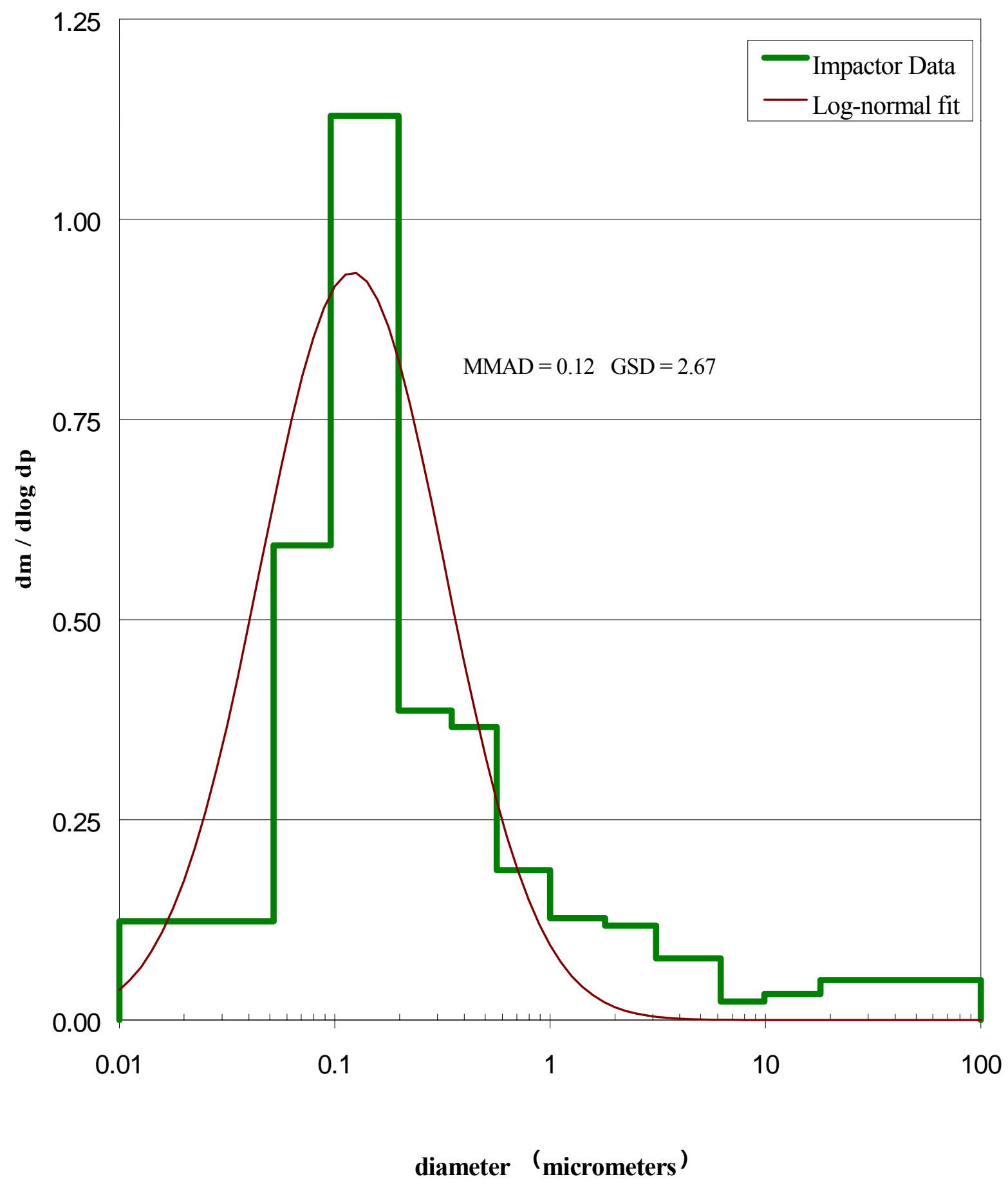




\section{Figure 4.48 - MOUDI Size Distribution for Cummins B5.9 Bus 1012 CBD Run \#10 Fuel: D2}

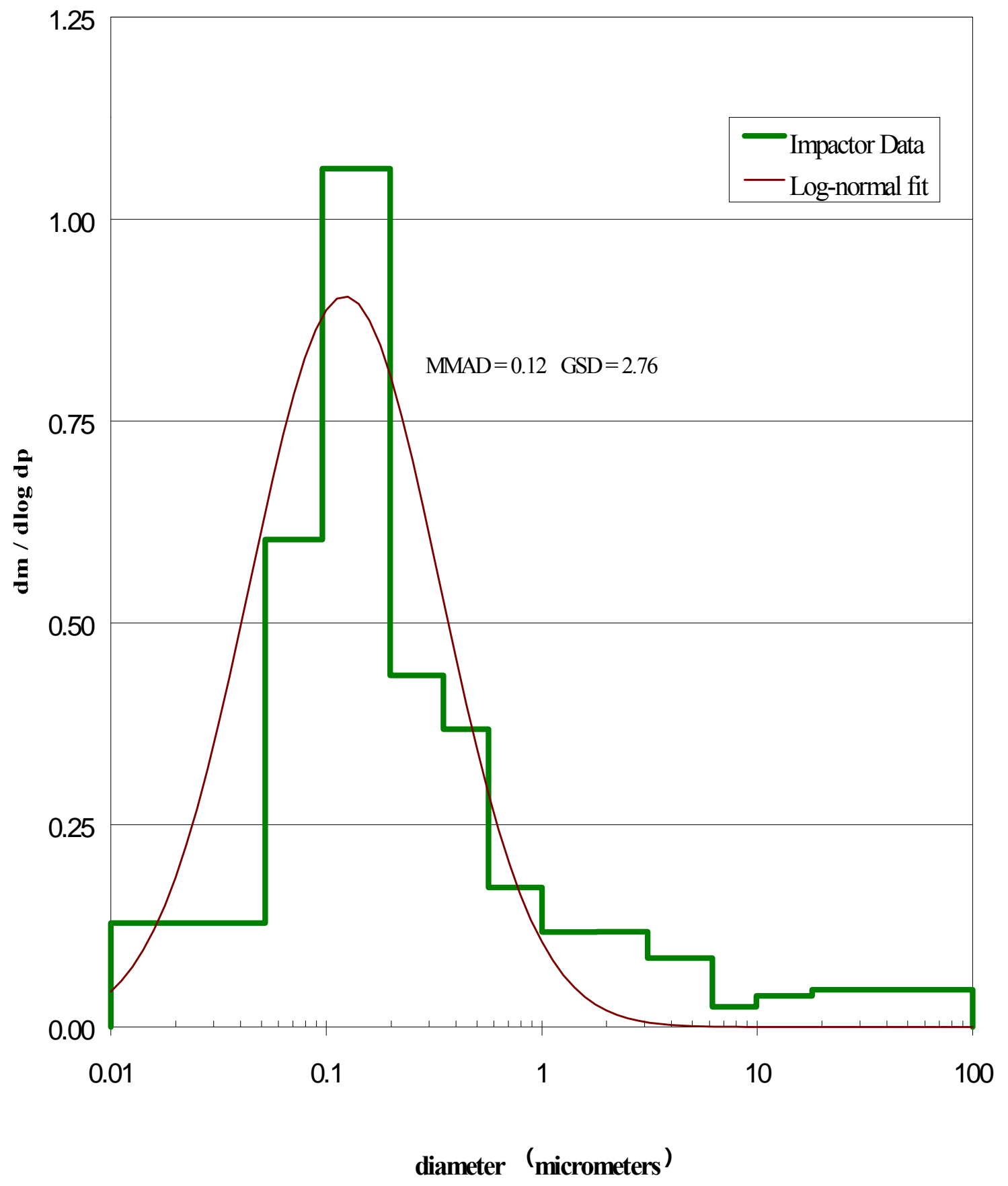


likely engine cylinder metal, lube oil and/or a variety of others not fuel derived. The mode appears to be random in amplitude and median, though constant for this particular vehicle. Empirically, the mass found on these stages was metallic in appearance, suggesting engine cylinder wear to be the main source. This mode was not found in the rest of the vehicles, but is believed to appear in other natural gas buses, which will be discussed later.

Bus 1014 emitted very little particulate matter. As such, no real data was obtained. This highlights one of the limitations of the MOUDI. One of the subsequent factors of testing a low emitting vehicle is the possibility of water condensing in the dilution tunnel and sample lines. Dilution levels must be kept relatively low to allow exhaust specie analysis, which increases the likelihood of water condensation. Also, natural gas contains a higher quantity of water than diesel fuel. This increases the chance for condensation. During testing, water did condense upon the MOUDI substrates and the sampling line walls. This further degraded the MOUDI's ability to collect an accurate sample that could be analyzed to construct a size distribution.

Bus 1015 was tested over two non-consecutive days. For the first day, two CBD cycles were run yielding MMADs of 0.04 and $0.05 \mu \mathrm{m}$ with respective GSDs of 2.47 and 3.51 (see Figures $4.31 \& 4.32$ ). These two tests resulted in an unexpected high concentration on the tenth stage $(0.096 \mu \mathrm{m}$ cut diameter, $0.0707 \mu \mathrm{m}$ midpoint diameter). While the collected particulate mass was the highest on the after-filter, as was the case for all the natural gas vehicles, the normalized value was more than twice that of the final stage for the first test and one and a half times for the second. Only after the bus was tested a second time could an explanation for this anomaly be found. 
Figure 4.49 - MOUDI Size Distribution for Cummins B5.9 Bus 1013 CBD Run \#1 Fuel: CNG

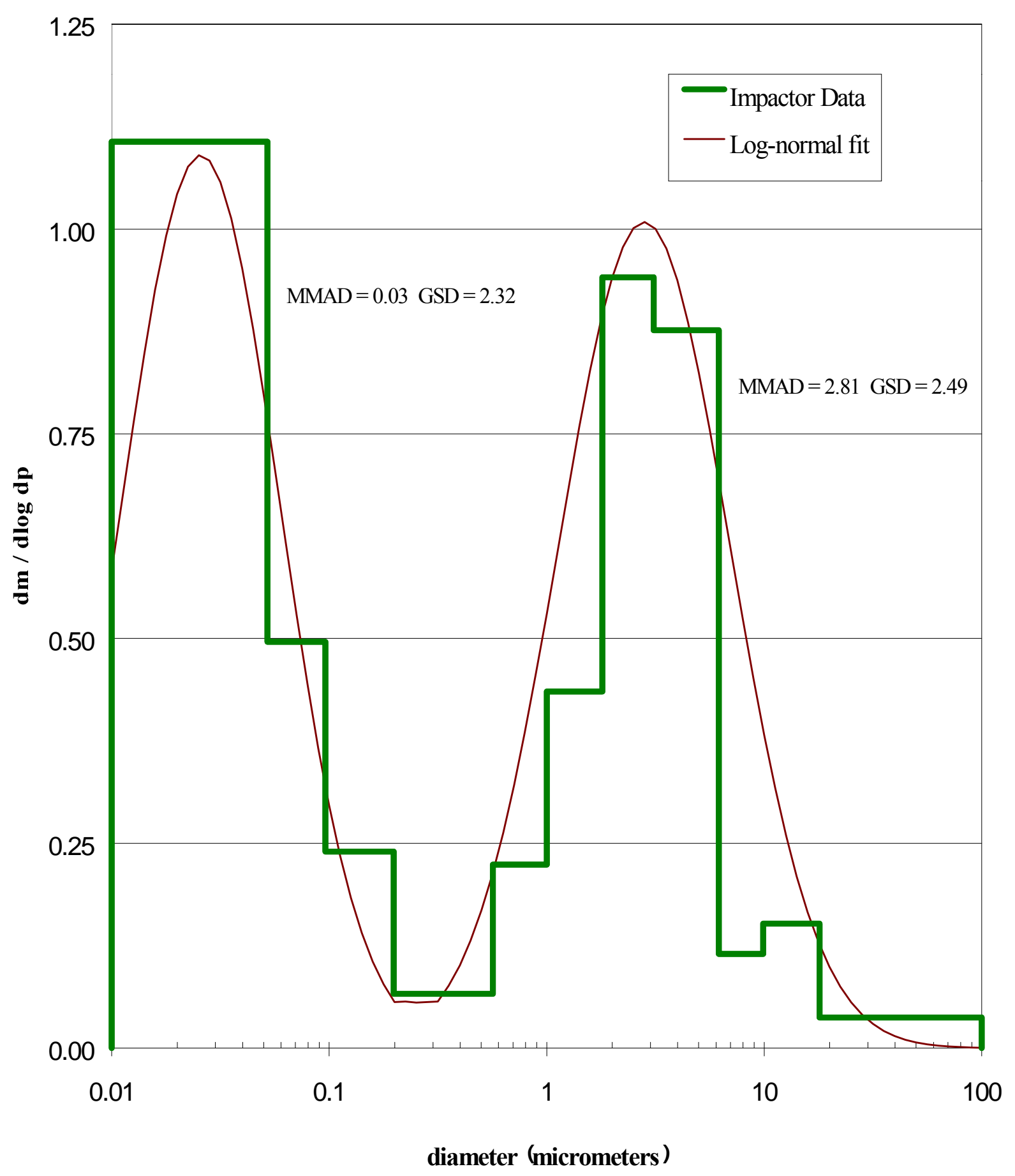




\section{Figure 4.50 - MOUDI Size Distribution for Cummins B5.9 Bus 1013 CBD Run \#3 Fuel: CNG}

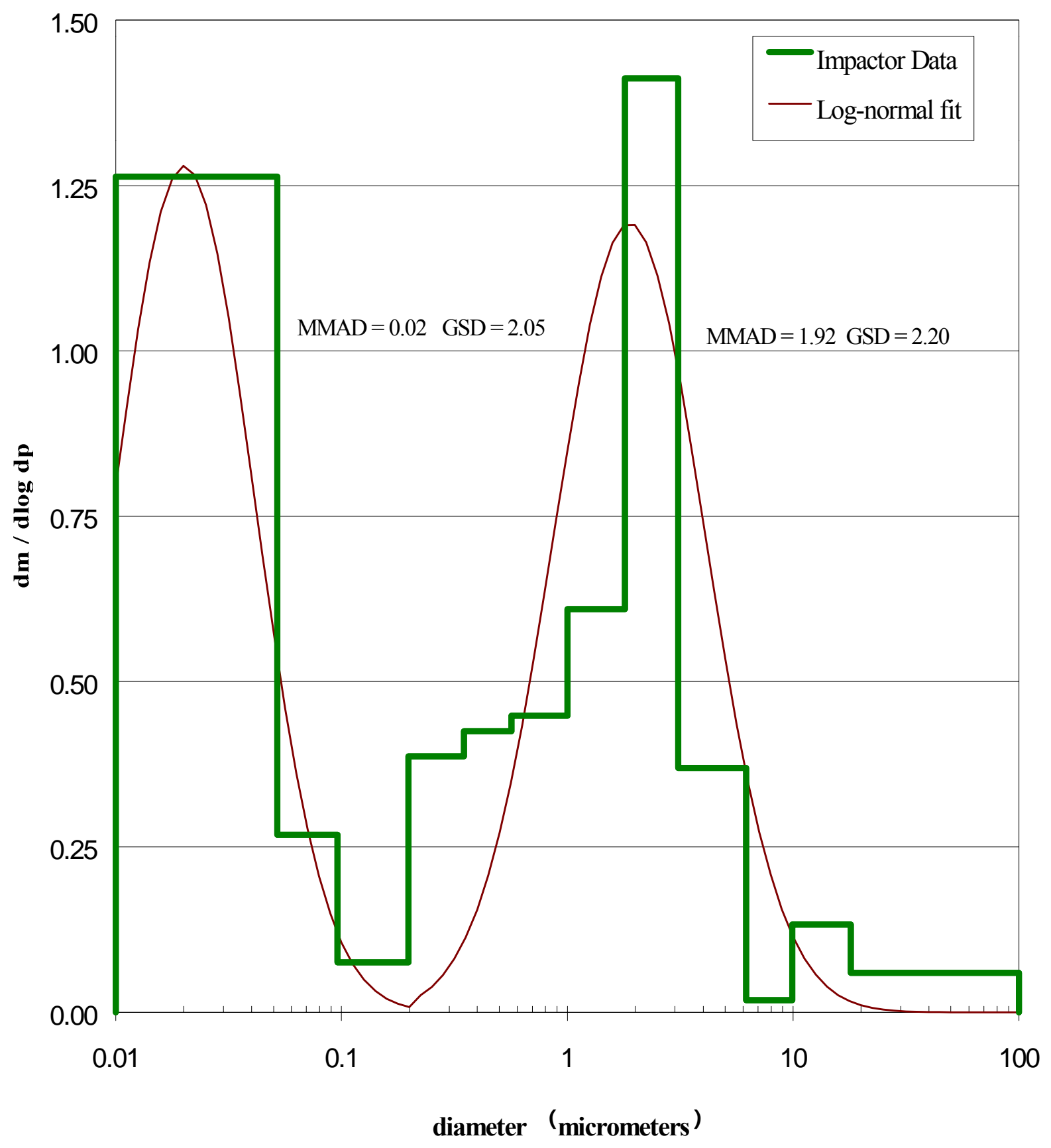


Four CBD tests were run on the second day. All four yielded distributions that were quite similar, as shown in Figures 4.53 - 4.56. However, no MMAD could be found without a subsequently large GSD (>5.6). A value that high generally means that the distribution is minimally lognormal and the confidence level in the median is weak. This did raise questions about the previous day's tests. For the same bus, how could the distributions be so vastly different? The ambient background data was checked, but held no answers. Subtracting the background from the MOUDI substrate weights had no effect on this or any other test. As it turns out, the Bus 1015 from day one had been altered prior to testing on day two - the catalyst had been removed. As can be seen, the total mass values are all higher for the non-catalyst version of the bus. The TPM data from the secondary dilution filter system also showed this to be the highest emitting CNG vehicle. While the connection is not perfectly clear, the removal of the catalyst did affect the size distribution. Comparing the data from stage ten and the after-filter shows how the particle size was altered. This comparison is portrayed in the Table 4.3 below and all the stages for Bus 1015 in Table 4.4. A comparison was also made for the after-filters and Stage \#4 substrates of Buses 1013 and 1015 versus those from Bus 1012 in Tables 4.5 and 4.6, respectively and graphically in Figures 4.57 and 4.58. The statistics for all the vehicles are shown in Table 4.7 and the entire mass spectrum is detailed in Table 4.8.

Table 4.3 - Comparison of Bus 1015 MOUDI After-Filter and Stage \#10

\begin{tabular}{l|cc|cccc|}
\cline { 2 - 7 } Mass (mg) & Run \#2 & Run \#3 & Run \#2 & Run \#3 & Run \#4 & Run \#5 \\
\hline AfterFilter & 0.058 & 0.066 & 0.081 & 0.070 & 0.090 & 0.096 \\
Stage 10 & 0.045 & 0.037 & 0.024 & 0.024 & 0.027 & 0.029 \\
\cline { 2 - 7 } Total Mass & 0.1790 & 0.1685 & 0.2120 & 0.1630 & 0.1950 & 0.2120 \\
\cline { 2 - 7 } Avg. AF & 0.062 & & 0.084 & & & \\
Avg. S10 & 0.041 & & 0.026 & & &
\end{tabular}




\section{Figure 4.51 - MOUDI Size Distribution for Cummins B5.9 Bus 1015 CBD Run \#2 Day \#1 Fuel: CNG}

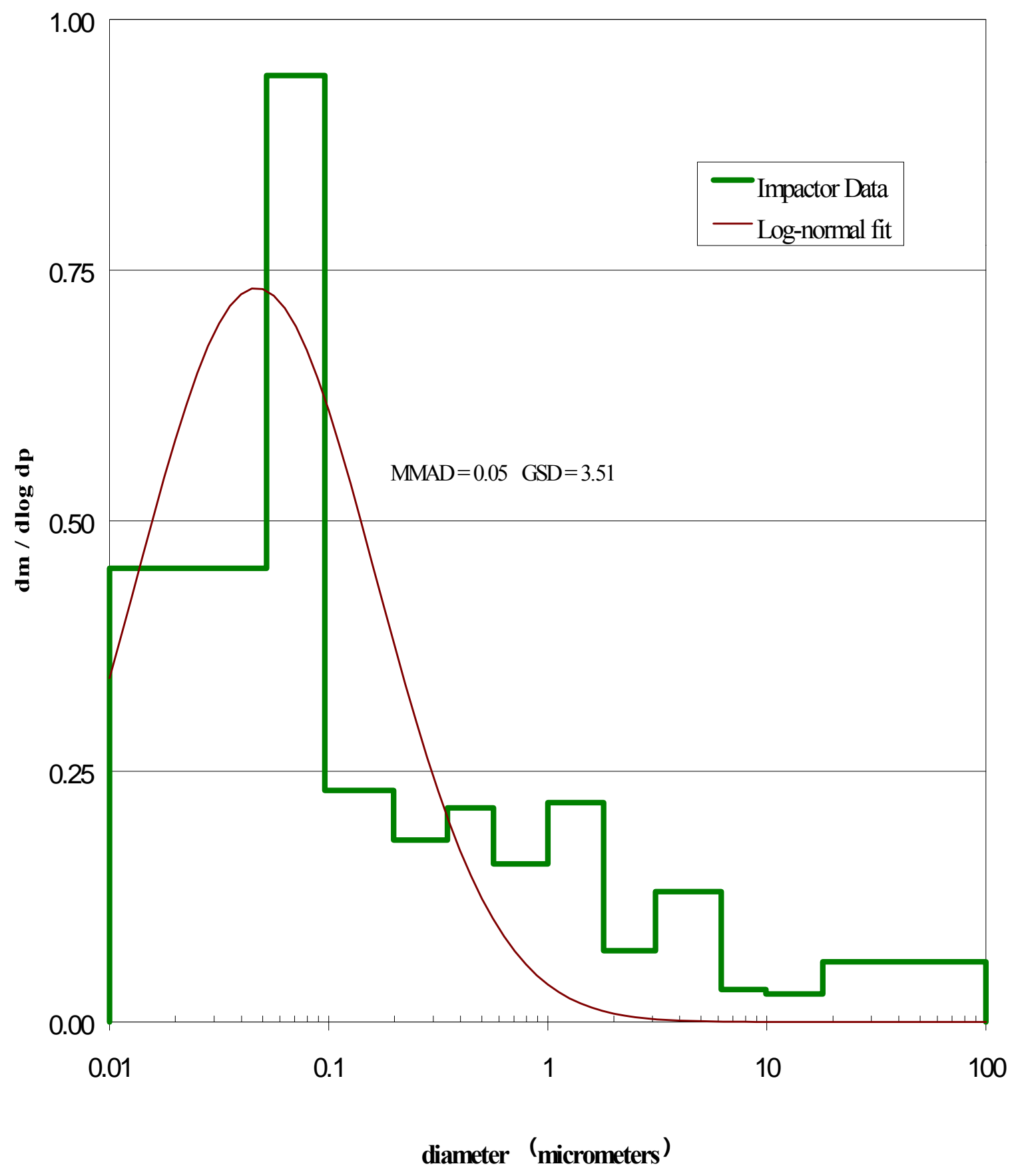


Figure 4.52 - MOUDI Size Distribution for Cummins B5.9 Bus 1015 CBD Run \#3 Day \#1 Fuel: CNG

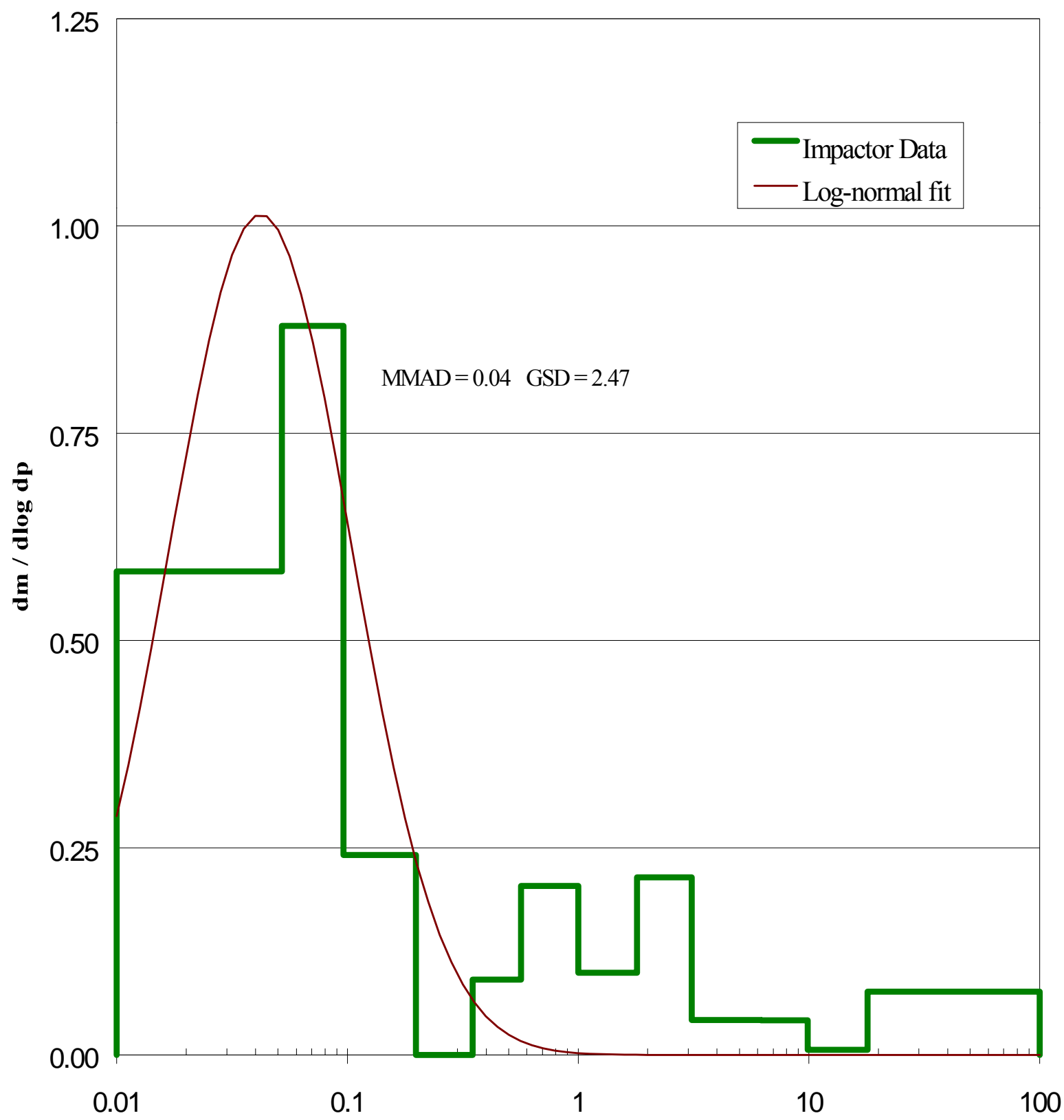

diameter (micrometers) 


\section{Figure 4.53 - MOUDI Size Distribution for Cummins B5.9 Bus 1015 CBD Run \#2 Day \#2 Fuel: CNG}

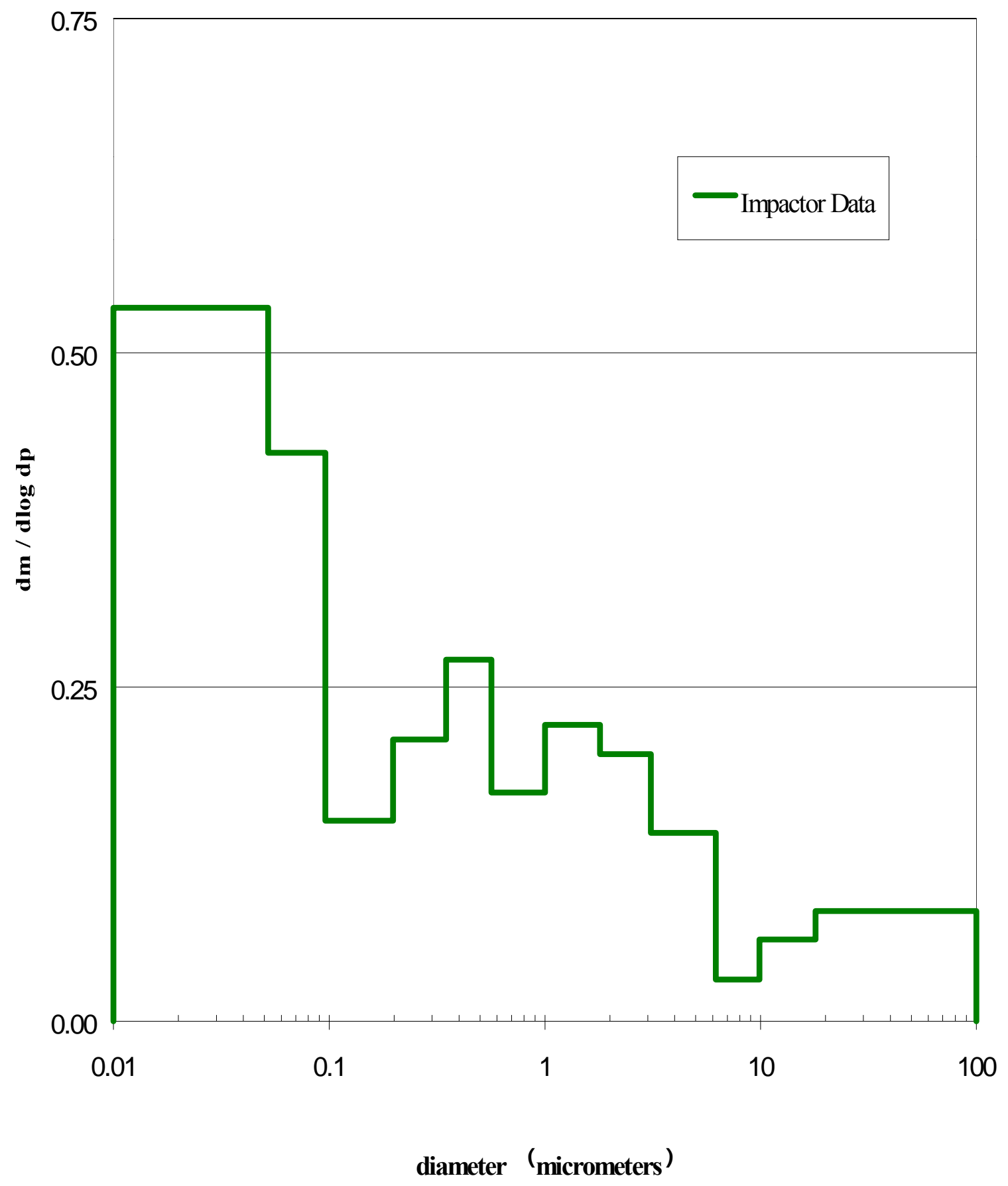


Figure 4.54 - MOUDI Size Distribution for Cummins B5.9

Bus 1015 CBD Run \#3 Day \#2 Fuel: CNG

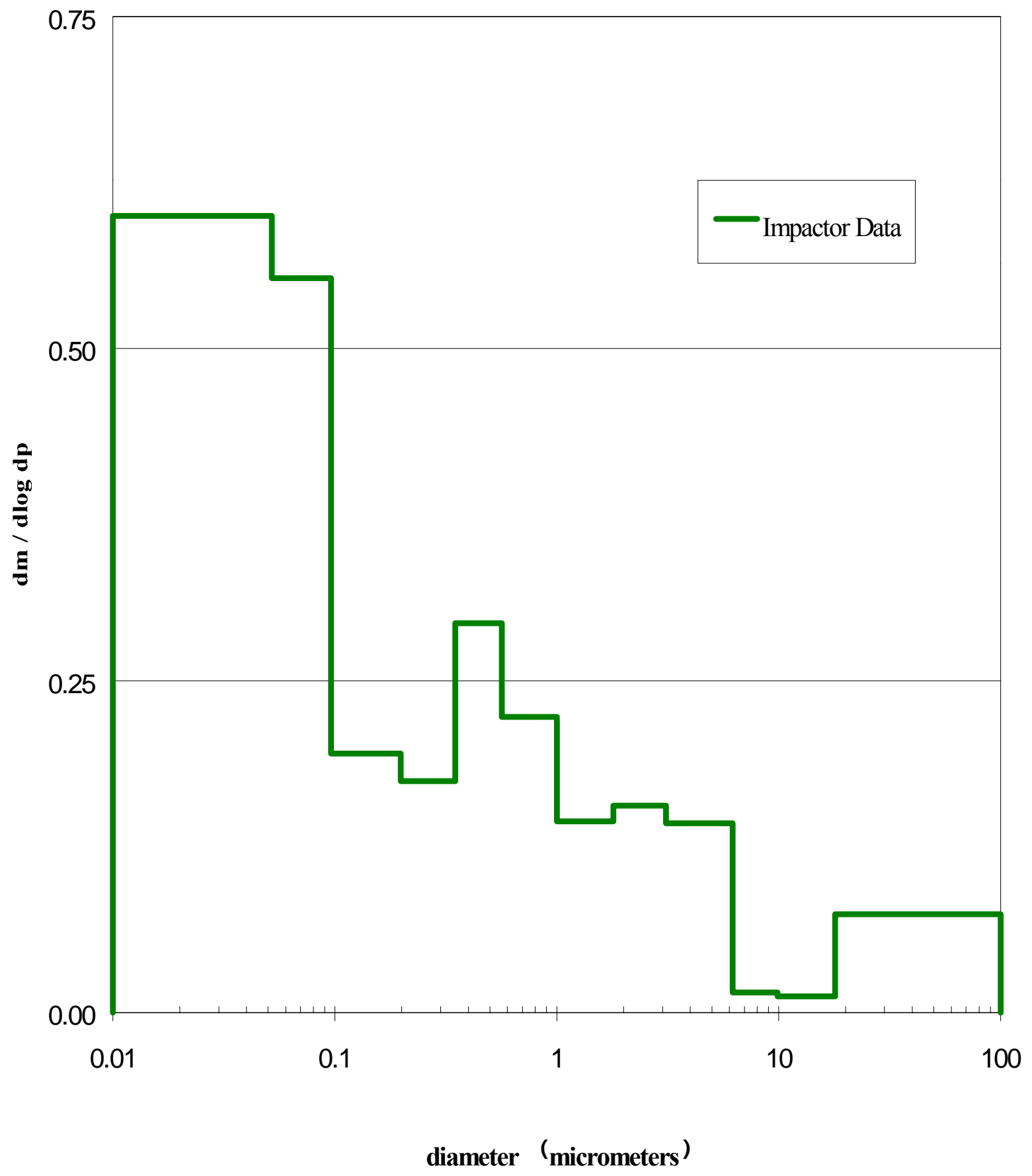


Figure 4.55 - MOUDI Size Distribution for Cummins B5.9

Bus 1015 CBD Run \#4 Day \#2 Fuel: CNG

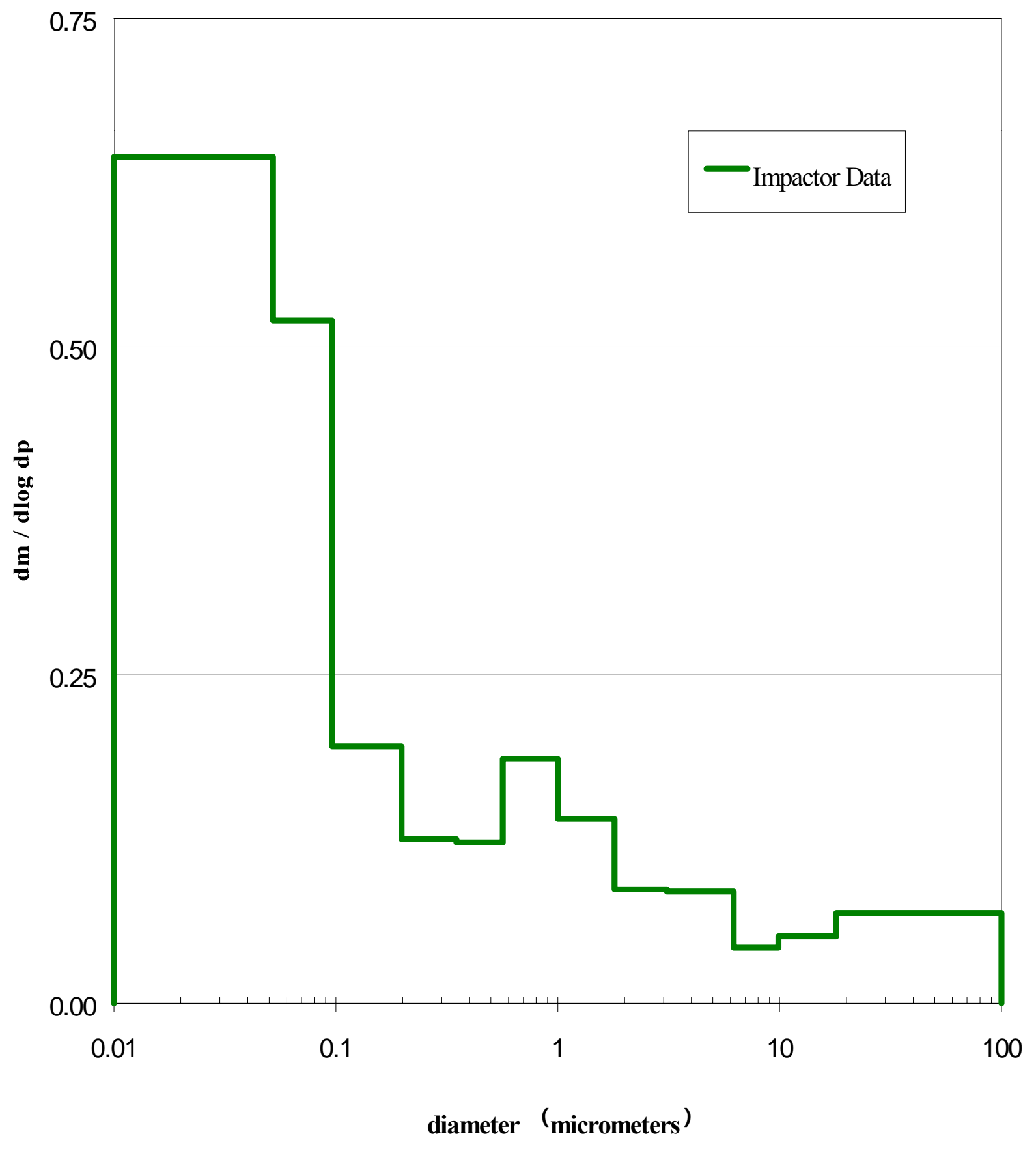


Figure 4.56 - MOUDI Size Distribution for Cummins B5.9

Bus 1015 CBD Run \#5 Day \#2 Fuel: CNG

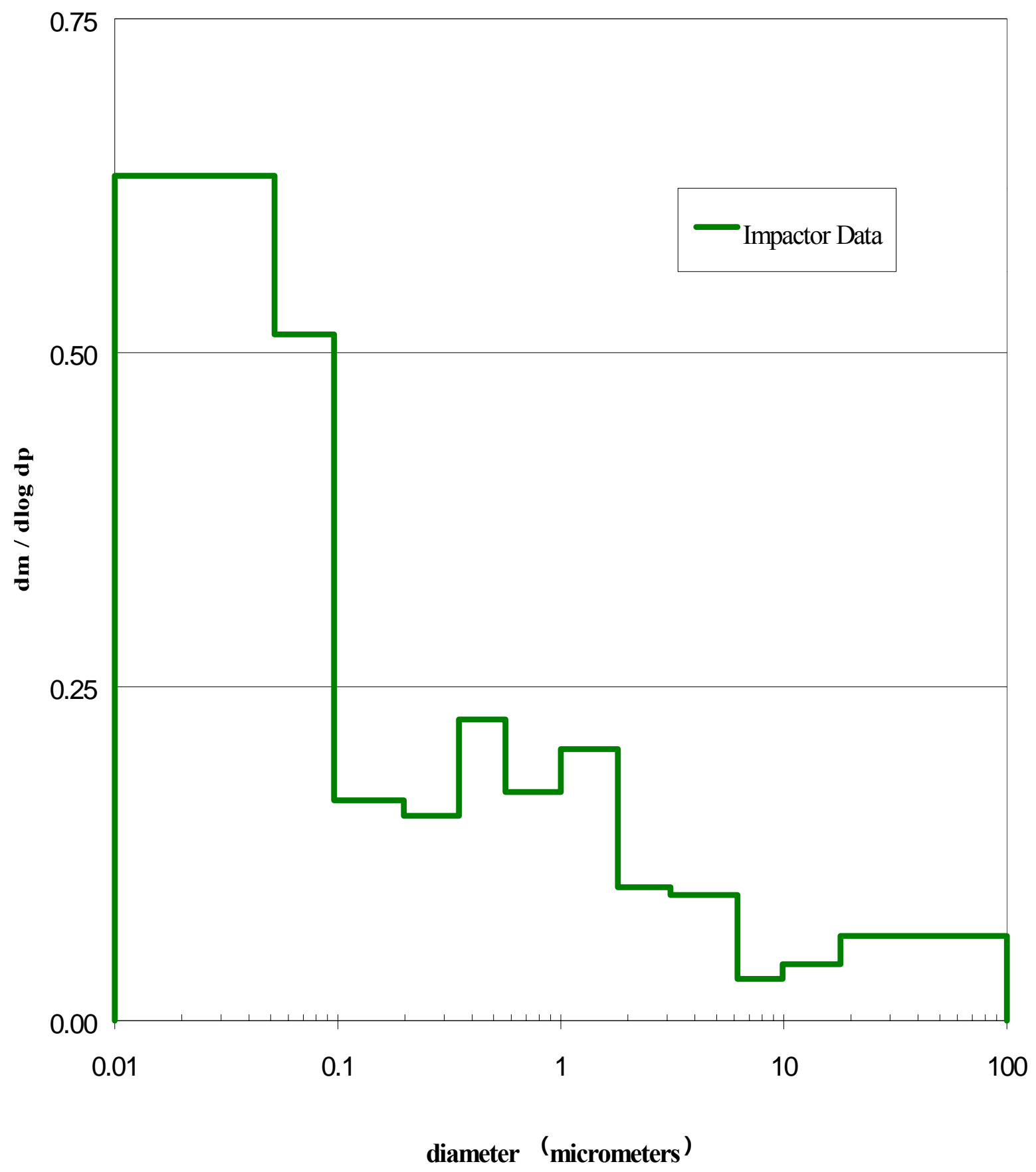


Table 4.4 - List of MOUDI Data from Central Business District Cycles for RTD/SKIP CNG Bus 1015

\begin{tabular}{|c|c|c|c|c|c|c|c|c|}
\hline \multicolumn{3}{|c|}{ With Catalyst } & \multicolumn{6}{|c|}{ Without Catalyst } \\
\hline$C B D \# 2$ & & & $C B D \# 2$ & & & $C B D \# 4$ & & \\
\hline Stage & $\begin{array}{l}\text { Mass } \\
(\mathrm{mg})\end{array}$ & $\begin{array}{l}\% \text { of total } \\
\text { mass }\end{array}$ & Stage & $\begin{array}{l}\text { Mass } \\
\text { (mg) }\end{array}$ & $\begin{array}{l}\% \text { of total } \\
\text { mass }\end{array}$ & Stage & $\begin{array}{l}\text { Mass } \\
\text { (mg) }\end{array}$ & $\begin{array}{l}\% \text { of total } \\
\text { mass }\end{array}$ \\
\hline $\mathbf{F}$ & 0.058 & $32 \%$ & $\mathbf{F}$ & 0.081 & $38 \%$ & $\mathbf{F}$ & 0.09 & $46 \%$ \\
\hline 10 & 0.045 & $25 \%$ & 10 & 0.024 & $11 \%$ & 10 & 0.027 & $14 \%$ \\
\hline 9 & 0.013 & $7 \%$ & 9 & 0.01 & $5 \%$ & 9 & 0.012 & $6 \%$ \\
\hline 8 & 0.008 & $4 \%$ & 8 & 0.011 & $5 \%$ & 8 & 0.006 & $3 \%$ \\
\hline 7 & 0.008 & $4 \%$ & 7 & 0.012 & $6 \%$ & 7 & 0.005 & $3 \%$ \\
\hline 6 & 0.007 & $4 \%$ & 6 & 0.009 & $4 \%$ & 6 & 0.009 & $5 \%$ \\
\hline 5 & 0.01 & $6 \%$ & 5 & 0.012 & $6 \%$ & 5 & 0.007 & $4 \%$ \\
\hline 4 & 0.003 & $2 \%$ & 4 & 0.01 & $5 \%$ & 4 & 0.004 & $2 \%$ \\
\hline 3 & 0.007 & $4 \%$ & 3 & 0.009 & $4 \%$ & 3 & 0.005 & $3 \%$ \\
\hline 2 & 0.007 & $4 \%$ & 2 & 0.008 & $4 \%$ & 2 & 0.01 & $5 \%$ \\
\hline 1 & 0.005 & $3 \%$ & 1 & 0.013 & $6 \%$ & 1 & 0.01 & $5 \%$ \\
\hline $\mathbf{0}$ & 0.008 & $4 \%$ & $\mathbf{0}$ & 0.013 & $6 \%$ & $\mathbf{0}$ & 0.01 & $5 \%$ \\
\hline$C B D \# 3$ & & & $C B D \# 3$ & & & $C B D \# 5$ & & \\
\hline Stage & $\begin{array}{c}\text { Mass } \\
\text { (mg) }\end{array}$ & $\begin{array}{l}\% \text { of total } \\
\text { mass }\end{array}$ & Stage & $\begin{array}{l}\text { Mass } \\
\text { (mg) }\end{array}$ & $\begin{array}{l}\% \text { of total } \\
\text { mass }\end{array}$ & Stage & $\begin{array}{l}\text { Mass } \\
\text { (mg) }\end{array}$ & $\begin{array}{l}\% \text { of total } \\
\text { mass }\end{array}$ \\
\hline $\mathbf{F}$ & 0.066 & $42 \%$ & $\mathbf{F}$ & 0.07 & $43 \%$ & $\mathbf{F}$ & 0.096 & $45 \%$ \\
\hline 10 & 0.037 & $23 \%$ & 10 & 0.024 & $15 \%$ & 10 & 0.029 & $14 \%$ \\
\hline 9 & 0.012 & $8 \%$ & 9 & 0.01 & $6 \%$ & 9 & 0.011 & $5 \%$ \\
\hline 8 & 0 & $0 \%$ & 8 & 0.007 & $4 \%$ & 8 & 0.008 & $4 \%$ \\
\hline 7 & 0.003 & $2 \%$ & 7 & 0.01 & $6 \%$ & 7 & 0.01 & $5 \%$ \\
\hline 6 & 0.008 & $5 \%$ & 6 & 0.009 & $6 \%$ & 6 & 0.009 & $4 \%$ \\
\hline 5 & 0.004 & $3 \%$ & 5 & 0.006 & $4 \%$ & 5 & 0.011 & $5 \%$ \\
\hline 4 & 0.008 & $5 \%$ & 4 & 0.006 & $4 \%$ & 4 & 0.005 & $2 \%$ \\
\hline 3 & 0.002 & $1 \%$ & 3 & 0.007 & $4 \%$ & 3 & 0.006 & $3 \%$ \\
\hline 2 & 0.008 & $5 \%$ & 2 & 0.003 & $2 \%$ & 2 & 0.008 & $4 \%$ \\
\hline 1 & 0.001 & $1 \%$ & 1 & 0.002 & $1 \%$ & 1 & 0.009 & $4 \%$ \\
\hline 0 & 0.009 & $6 \%$ & 0 & 0.009 & $6 \%$ & 0 & 0.01 & $5 \%$ \\
\hline
\end{tabular}


Table 4.5 - Comparison of MOUDI Teflo After-Filter Weights for RTD/SKIP CNG Buses 1015 and 1013 vs. D2 Bus 1012

CNG Bus 1015

\begin{tabular}{|c|c|c|c|c|c|}
\hline \multirow{2}{*}{$\begin{array}{c}1262-2 \\
\text { Stage }\end{array}$} & \multicolumn{5}{|c|}{ 1271-3 } \\
\hline & Mass (mg) & $\%$ of total mass & Stage & Mass (mg) & $\%$ of total mass \\
\hline $\mathbf{F}$ & $0.058^{\circ}$ & $32 \%$ & $\mathbf{F}$ & 0.07 & $43 \%$ \\
\hline \multicolumn{3}{|l|}{$1262-3$} & \multicolumn{3}{|l|}{$1271-4$} \\
\hline Stage & Mass (mg) & $\%$ of total mass & Stage & Mass (mg) & $\%$ of total mass \\
\hline $\mathbf{F}$ & $0.066^{\circ}$ & $42 \%$ & $\mathbf{F}$ & 0.09 & $46 \%$ \\
\hline \multicolumn{3}{|l|}{ 1271-2 } & \multicolumn{3}{|l|}{ 1271-5 } \\
\hline Stage & Mass (mg) & $\%$ of total mass & Stage & Mass (mg) & $\%$ of total mass \\
\hline $\mathbf{F}$ & 0.081 & $38 \%$ & $\mathbf{F}$ & 0.096 & $45 \%$ \\
\hline \multicolumn{6}{|c|}{ CNG Bus 1013} \\
\hline $1256-1$ & \multicolumn{5}{|c|}{$1256-3$} \\
\hline Stage & Mass (mg) & $\%$ of total mass & Stage & Mass (mg) & $\%$ of total mass \\
\hline $\mathbf{F}$ & 0.042 & $32 \%$ & $\mathbf{F}$ & $0.038^{\circ}$ & $42 \%$ \\
\hline \multicolumn{6}{|c|}{ D2 Bus 1012} \\
\hline $1246-5$ & \multicolumn{5}{|c|}{ 1263-1 } \\
\hline Stage & Mass (mg) & $\%$ of total mass & Stage & Mass (mg) & $\%$ of total mass \\
\hline $\mathbf{F}$ & 0.029 & $6 \%$ & $\mathbf{F}$ & 0.027 & $5 \%$ \\
\hline $1246-6$ & \multicolumn{5}{|c|}{$1264-2$} \\
\hline Stage & Mass (mg) & $\%$ of total mass & Stage & Mass (mg) & $\%$ of total mass \\
\hline $\mathbf{F}$ & 0.036 & $7 \%$ & $\mathbf{F}$ & 0.038 & $9 \%$ \\
\hline
\end{tabular}

Each CNG test compared to each D2 test.

Positive value represents \% higher CNG after-filter than D2.

\begin{tabular}{|c|cccccc|cc|}
\cline { 2 - 8 } \multicolumn{1}{l|}{} & \multicolumn{5}{c|}{ Bus 1015 } & \multicolumn{2}{c|}{ Bus 1013 } \\
\multicolumn{1}{l|}{} & $\mathbf{1 2 7 1 - 2}$ & $\mathbf{1 2 7 1 - 3}$ & $\mathbf{1 2 7 1 - 4}$ & $\mathbf{1 2 7 1 - 5}$ & $\mathbf{1 2 6 2 - 2}$ & $\mathbf{1 2 6 2 - 3}$ & $\mathbf{1 2 5 6 - 1}$ & $\mathbf{1 2 5 6 - 3}$ \\
\hline $\mathbf{1 2 4 6 - 5}$ & $95 \%$ & $83 \%$ & $103 \%$ & $107 \%$ & $67 \%$ & $78 \%$ & $37 \%$ & $27 \%$ \\
$\mathbf{1 2 4 6 - 6}$ & $77 \%$ & $64 \%$ & $86 \%$ & $91 \%$ & $47 \%$ & $59 \%$ & $15 \%$ & $5 \%$ \\
$\mathbf{1 2 6 3 - 1}$ & $100 \%$ & $89 \%$ & $108 \%$ & $112 \%$ & $73 \%$ & $84 \%$ & $43 \%$ & $34 \%$ \\
$\mathbf{1 2 6 4 - 2}$ & $100 \%$ & $59 \%$ & $81 \%$ & $87 \%$ & $42 \%$ & $54 \%$ & $10 \%$ & $0 \%$ \\
\hline
\end{tabular}


Table 4.6 - Comparison of MOUDI Stage \#4* Weights for RTD/SKIP CNG Buses 1015 and 1013 vs. D2 Bus 1012

$$
\text { * Stage \#4 ECD }=1.8 \mu \mathrm{m}, \mathrm{Dp}=2.4 \mu \mathrm{m}
$$

CNG Bus 1015

\begin{tabular}{|c|c|c|c|c|c|}
\hline $1262-2$ & & & $1271-3$ & & \\
\hline Stage & Mass (mg) & $\%$ of total mass & Stage & Mass (mg) & $\%$ of total mass \\
\hline 4 & 0.003 & $2 \%$ & 4 & 0.006 & $4 \%$ \\
\hline $1262-3$ & & & $1271-4$ & & \\
\hline Stage & Mass (mg) & $\%$ of total mass & Stage & Mass (mg) & $\%$ of total mass \\
\hline 4 & 0.008 & $5 \%$ & 4 & 0.004 & $2 \%$ \\
\hline 1271-2 & & & $1271-5$ & & \\
\hline Stage & Mass (mg) & $\%$ of total mass & Stage & Mass (mg) & $\%$ of total mass \\
\hline 4 & 0.01 & $5 \%$ & 4 & 0.005 & $2 \%$ \\
\hline CNG B & Is 1013 & & & & \\
\hline $1256-1$ & & & $1256-3$ & & \\
\hline Stage & Mass (mg) & $\%$ of total mass & Stage & Mass (mg) & $\%$ of total mass \\
\hline 4 & 0.016 & $12 \%$ & 4 & 0.015 & $16 \%$ \\
\hline D2 Bus & 1012 & & & & \\
\hline $1246-5$ & & & $1263-1$ & & \\
\hline Stage & Mass (mg) & $\%$ of total mass & Stage & Mass (mg) & $\%$ of total mass \\
\hline 4 & 0.01 & $2 \%$ & 4 & 0.014 & $3 \%$ \\
\hline $1246-6$ & & & $1264-2$ & & \\
\hline Stage & Mass (mg) & $\%$ of total mass & Stage & Mass (mg) & $\%$ of total mass \\
\hline 4 & 0.008 & $2 \%$ & 4 & 0.012 & $3 \%$ \\
\hline
\end{tabular}

Each CNG test compared to each D2 test.

Positive value represents \% higher CNG after-filter than D2.

\begin{tabular}{|c|cccccc|cc|}
\cline { 2 - 8 } \multicolumn{1}{c|}{} & \multicolumn{7}{c|}{ Bus 1015 } & \multicolumn{2}{c|}{ Bus 1013 } \\
\hline $\mathbf{1 2 4 6 - 5}$ & $0 \%$ & $-50 \%$ & $-86 \%$ & $-67 \%$ & $-108 \%$ & $-22 \%$ & $46 \%$ & $40 \%$ \\
$\mathbf{1 2 4 6 - 6}$ & $22 \%$ & $-29 \%$ & $-67 \%$ & $-46 \%$ & $-91 \%$ & $0 \%$ & $67 \%$ & $61 \%$ \\
$\mathbf{1 2 6 3 - 1}$ & $-33 \%$ & $-80 \%$ & $-111 \%$ & $-95 \%$ & $-129 \%$ & $-55 \%$ & $13 \%$ & $7 \%$ \\
$\mathbf{1 2 6 4 - 2}$ & $-18 \%$ & $-67 \%$ & $-100 \%$ & $-82 \%$ & $-120 \%$ & $-40 \%$ & $29 \%$ & $22 \%$ \\
\hline
\end{tabular}


Figure 4.57 - Comparison of MOUDI Stage \#4 Substrates for RTD/SKIP Cummins B5.9 Buses

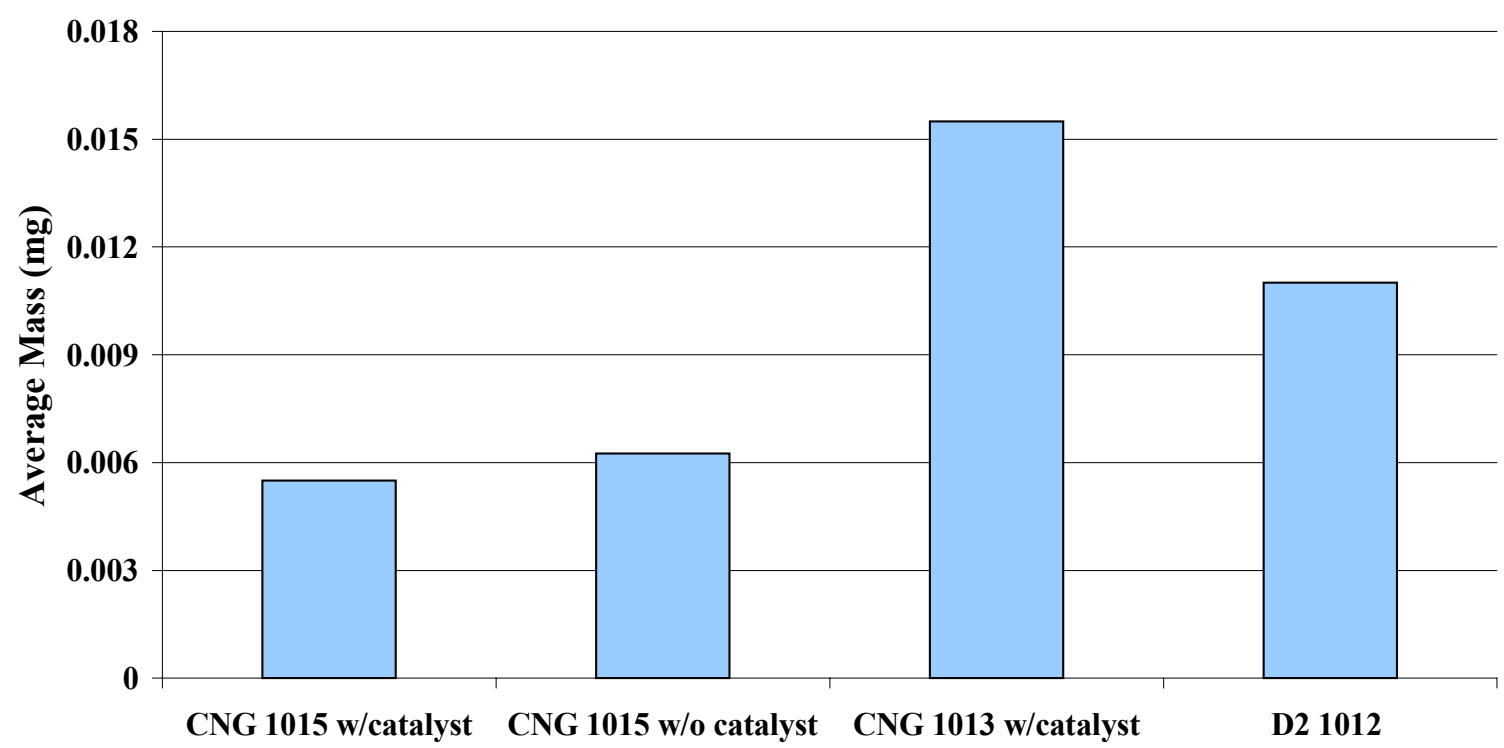

Bus Identification

Figure 4.58 - Comparison of MOUDI Teflo After-Filters for RTD/SKIP Cummins B5.9 Buses

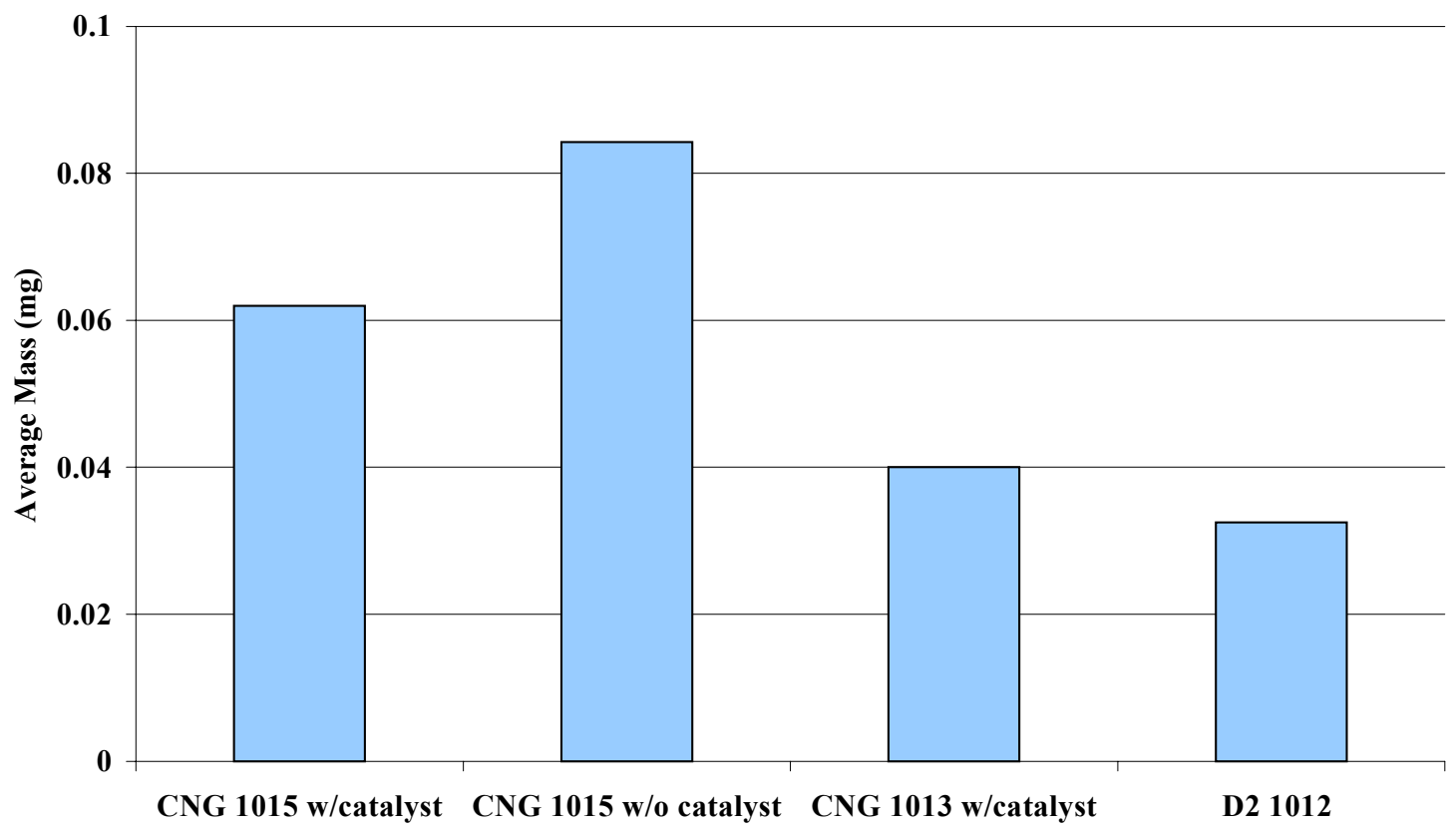

Bus Identification 
Table 4.7 - Mass Median Diameters and Geometric Standard Deviations for Diesel and CNG RTD/SKIP Buses

\begin{tabular}{|c|c|c|c|c|c|c|}
\hline & \multicolumn{2}{|c|}{ Bus \#1010 } & \multicolumn{2}{|c|}{ Bus \#1011 } & \multicolumn{2}{|c|}{ Bus \#1012 } \\
\hline & $\begin{array}{c}\text { MMAD } \\
(\mu \mathrm{m})\end{array}$ & GSD & $\begin{array}{c}\text { MMAD } \\
(\mu \mathrm{m})\end{array}$ & GSD & $\begin{array}{c}\text { MMAD } \\
(\mu \mathrm{m})\end{array}$ & GSD \\
\hline & $\begin{array}{l}0.09 \\
0.12\end{array}$ & $\begin{array}{l}2.32 \\
2.02\end{array}$ & $\begin{array}{l}0.11 \\
0.11\end{array}$ & $\begin{array}{l}2.66 \\
2.21\end{array}$ & $\begin{array}{l}0.12 \\
0.11 \\
0.11 \\
0.11 \\
0.12 \\
0.12 \\
0.12\end{array}$ & $\begin{array}{c}3.1 \\
2.35 \\
2.28 \\
2.29 \\
2.48 \\
2.67 \\
2.76 \\
\end{array}$ \\
\hline Average & 0.105 & 2.17 & 0.11 & 2.44 & 0.116 & 2.56 \\
\hline $\begin{array}{c}\text { Standard } \\
\text { Dev. }\end{array}$ & 0.021 & 0.212 & 0.000 & 0.318 & 0.005 & 0.302 \\
\hline
\end{tabular}

\begin{tabular}{|c|cc|cc|cc|}
\cline { 2 - 7 } \multicolumn{1}{c|}{} & \multicolumn{2}{|c|}{ Bus \#1013 } & \multicolumn{2}{c|}{ Bus \#1014 } & \multicolumn{2}{c|}{ Bus \#1015 } \\
& $\begin{array}{c}\text { MMAD } \\
(\mu \mathrm{m})\end{array}$ & GSD & $\begin{array}{c}\text { MMAD } \\
(\mu \mathrm{m})\end{array}$ & GSD & $\begin{array}{c}\text { MMAD } \\
(\boldsymbol{\mu m})\end{array}$ & GSD \\
\cline { 2 - 7 } & 0.09 & 2.32 & 0.11 & 2.66 & 0.12 & 3.1 \\
& 0.12 & 2.02 & 0.11 & 2.21 & 0.11 & 2.35 \\
& & & & & 0.11 & 2.28 \\
& & & & & 0.11 & 2.29 \\
\hline $\begin{array}{c}\text { Average } \\
\text { Standard } \\
\text { Dev. }\end{array}$ & 0.105 & 2.17 & 0.11 & 2.44 & 0.112 & 2.48 \\
\hline
\end{tabular}


Table 4.8 - MOUDI Data for RTD/SKIP CNG Buses 1013 and 1015

Test ID: Bus 1013

CBD \#1

\begin{tabular}{|c|c|c|c|c|c|c|c|c|}
\hline \multicolumn{9}{|c|}{$\begin{array}{l}\text { Impactor } \\
\text { Data }\end{array}$} \\
\hline $\begin{array}{c}E C A D \\
(\mu \mathrm{m})\end{array}$ & $\begin{array}{c}\text { Mass } \\
\text { mg }\end{array}$ & $\begin{array}{c}\text { frequency } \\
\text { (normalized) }\end{array}$ & $\begin{array}{l}\text { mid } d p \\
(\mu \mathrm{m})\end{array}$ & stage & $\begin{array}{c}\text { net mass } \\
\text { mg }\end{array}$ & $\begin{array}{c}\text { cumulative } \\
\text { mass } \\
\text { mg }\end{array}$ & $\begin{array}{c}\text { cumulative \% } \\
\text { < ECAD }\end{array}$ & $\begin{array}{c}E C A D \\
(\mu \mathrm{m})\end{array}$ \\
\hline 0.01 & 0.0420 & 0.448 & 0.0228 & $\mathrm{f}$ & 0.0420 & 0.004 & $97 \%$ & 0.01 \\
\hline 0.052 & 0.0070 & 0.201 & 0.0707 & 10 & 0.0070 & 0.049 & $63 \%$ & 0.052 \\
\hline 0.096 & 0.0040 & 0.097 & 0.1379 & 9 & 0.0040 & 0.053 & $60 \%$ & 0.096 \\
\hline 0.198 & 0.0010 & 0.031 & 0.2629 & 8 & 0.0010 & 0.054 & $59 \%$ & 0.198 \\
\hline 0.349 & 0.0010 & 0.036 & 0.4441 & 7 & 0.0010 & 0.055 & $58 \%$ & 0.349 \\
\hline 0.565 & 0.0040 & 0.123 & 0.7517 & 6 & 0.0040 & 0.059 & $55 \%$ & 0.565 \\
\hline 1 & 0.0080 & 0.239 & 1.3416 & 5 & 0.0080 & 0.067 & $49 \%$ & 1 \\
\hline 1.8 & 0.0160 & 0.517 & 2.3622 & 4 & 0.0160 & 0.083 & $37 \%$ & 1.8 \\
\hline 3.1 & 0.0190 & 0.482 & 4.3841 & 3 & 0.0190 & 0.102 & $22 \%$ & 3.1 \\
\hline 6.2 & 0.0100 & 0.376 & 7.8345 & 2 & 0.0100 & 0.112 & $15 \%$ & 6.2 \\
\hline 9.9 & 0.0110 & 0.323 & 13.3492 & 1 & 0.0110 & 0.123 & $6 \%$ & 9.9 \\
\hline 18 & 0.0080 & 0.082 & 42.4264 & 0 & 0.0080 & 0.131 & $0 \%$ & 18 \\
\hline 100 & & & & $\begin{array}{l}\text { Total } \\
\text { mass }\end{array}$ & 0.1310 & & & \\
\hline
\end{tabular}

Test ID: Bus 1013

CBD \#3

Impactor

Data

\begin{tabular}{|c|c|c|c|c|c|c|c|c|}
\hline $\begin{array}{r}E C A D \\
(\mu \mathrm{m}) \\
\end{array}$ & $\begin{array}{c}\text { Mass } \\
\text { mg } \\
\end{array}$ & $\begin{array}{c}\text { frequency } \\
\text { (normalized) }\end{array}$ & $\begin{array}{c}\operatorname{mid} d p \\
(\mu \mathrm{m})\end{array}$ & $\overline{\text { stage }}$ & $\begin{array}{c}\text { net mass } \\
\text { mg } \\
\end{array}$ & $\begin{array}{c}\text { cumulative } \\
\text { mass } \\
\text { mg }\end{array}$ & $\begin{array}{c}\text { cumulative \% } \\
\text { < ECAD }\end{array}$ & $\begin{array}{r}E C A D \\
(\mu \mathrm{m}) \\
\end{array}$ \\
\hline 0.01 & 0.038 & 0.583 & 0.0228 & $\mathrm{f}$ & 0.038 & 0.004 & $96 \%$ & 0.01 \\
\hline 0.052 & 0.003 & 0.124 & 0.0707 & 10 & 0.003 & 0.041 & $55 \%$ & 0.052 \\
\hline 0.096 & 0.001 & 0.035 & 0.1379 & 9 & 0.001 & 0.042 & $54 \%$ & 0.096 \\
\hline 0.198 & 0.004 & 0.179 & 0.2629 & 8 & 0.004 & 0.046 & $49 \%$ & 0.198 \\
\hline 0.349 & 0.004 & 0.210 & 0.4441 & 7 & 0.004 & 0.05 & $45 \%$ & 0.349 \\
\hline 0.565 & 0.005 & 0.222 & 0.7517 & 6 & 0.005 & 0.055 & $40 \%$ & 0.565 \\
\hline 1 & 0.007 & 0.301 & 1.3416 & 5 & 0.007 & 0.062 & $32 \%$ & 1 \\
\hline 1.8 & 0.015 & 0.698 & 2.3622 & 4 & 0.015 & 0.077 & $15 \%$ & 1.8 \\
\hline 3.1 & 0.005 & 0.183 & 4.3841 & 3 & 0.005 & 0.082 & $10 \%$ & 3.1 \\
\hline 6.2 & 0.001 & 0.009 & 7.8345 & 2 & 0.001 & 0.083 & $9 \%$ & 6.2 \\
\hline 9.9 & 0.006 & 0.066 & 13.3492 & 1 & 0.006 & 0.089 & $2 \%$ & 9.9 \\
\hline \multirow{2}{*}{$\begin{array}{c}18 \\
100\end{array}$} & 0.002 & 0.030 & 42.4264 & 0 & 0.002 & 0.091 & $0 \%$ & 18 \\
\hline & & & & $\begin{array}{l}\text { total } \\
\text { mass }\end{array}$ & 0.0910 & & & \\
\hline
\end{tabular}

Sum $\quad 0.1$ 
Table 4.8 cont' $^{\prime} d$

Test ID: Bus 1015

CBD \#2

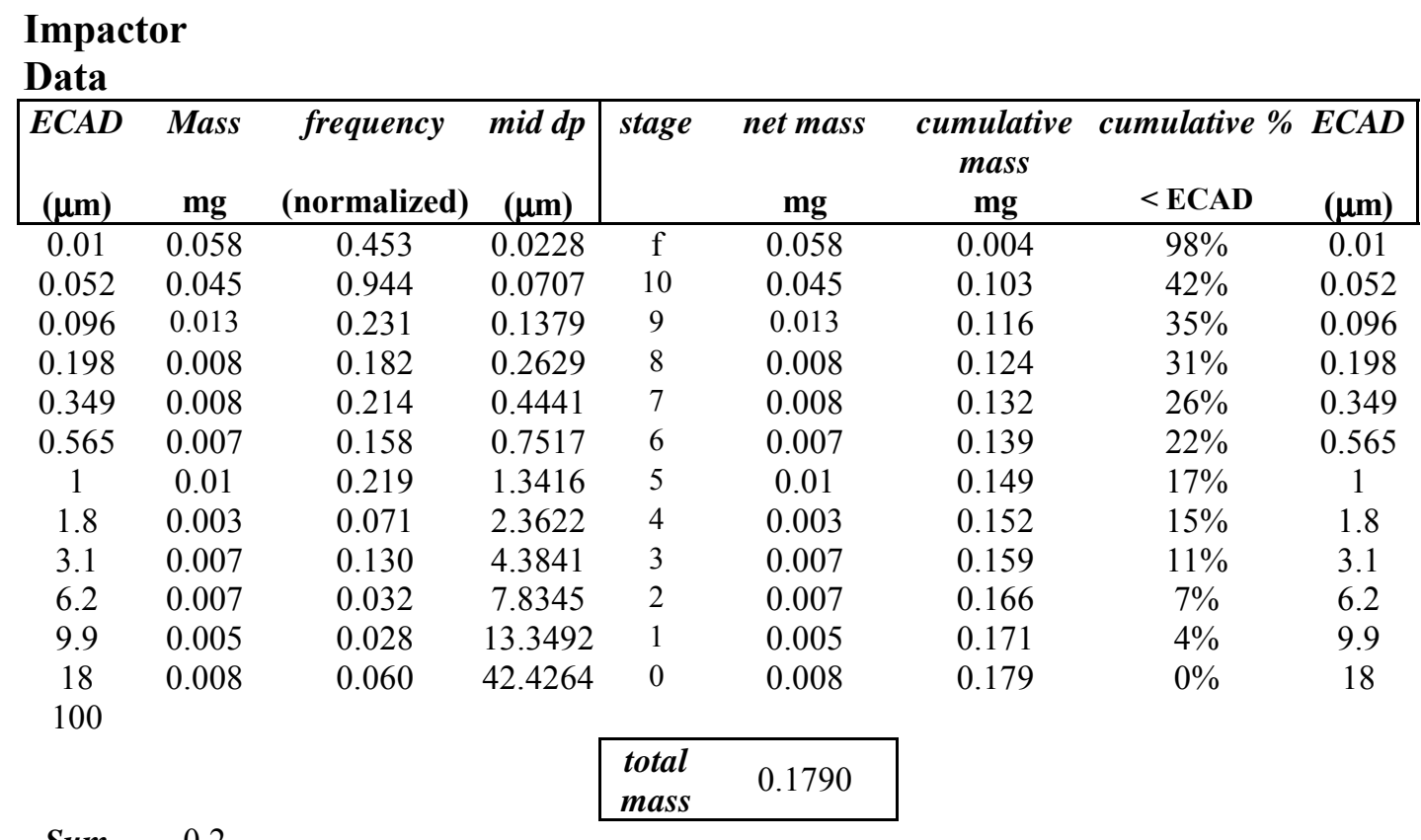

Test ID: Bus 1015

CBD \#3

Impactor

Data

\begin{tabular}{|c|c|c|c|c|c|c|c|c|}
\hline $\begin{array}{r}E C A D \\
(\mu \mathrm{m}) \\
\end{array}$ & $\begin{array}{c}\text { Mass } \\
\text { mg } \\
\end{array}$ & $\begin{array}{c}\text { frequency } \\
\text { (normalized) }\end{array}$ & $\begin{array}{c}\text { mid } d p \\
(\mu \mathrm{m})\end{array}$ & stage & $\begin{array}{c}\text { net mass } \\
\text { mg } \\
\end{array}$ & $\begin{array}{c}\text { cumulative } \\
\text { mass } \\
\text { mg }\end{array}$ & $\begin{array}{c}\text { cumulative \% } \\
\text { < ECAD }\end{array}$ & $\begin{array}{r}E C A D \\
(\mu \mathrm{m}) \\
\end{array}$ \\
\hline 0.01 & 0.066 & 0.583 & 0.0228 & $\mathrm{f}$ & 0.066 & 0.004 & $97 \%$ & 0.01 \\
\hline 0.052 & 0.037 & 0.879 & 0.0707 & 10 & 0.037 & 0.103 & $35 \%$ & 0.052 \\
\hline 0.096 & 0.012 & 0.242 & 0.1379 & 9 & 0.012 & 0.115 & $27 \%$ & 0.096 \\
\hline 0.198 & 0 & 0.000 & 0.2629 & 8 & 0 & 0.115 & $27 \%$ & 0.198 \\
\hline 0.349 & 0.003 & 0.091 & 0.4441 & 7 & 0.003 & 0.118 & $25 \%$ & 0.349 \\
\hline 0.565 & 0.008 & 0.204 & 0.7517 & 6 & 0.008 & 0.126 & $20 \%$ & 0.565 \\
\hline 1 & 0.004 & 0.099 & 1.3416 & 5 & 0.004 & 0.13 & $18 \%$ & 1 \\
\hline 1.8 & 0.008 & 0.214 & 2.3622 & 4 & 0.008 & 0.138 & $13 \%$ & 1.8 \\
\hline 3.1 & 0.002 & 0.042 & 4.3841 & 3 & 0.002 & 0.14 & $11 \%$ & 3.1 \\
\hline 6.2 & 0.008 & 0.042 & 7.8345 & 2 & 0.008 & 0.148 & $6 \%$ & 6.2 \\
\hline 9.9 & 0.001 & 0.006 & 13.3492 & 1 & 0.001 & 0.149 & $6 \%$ & 9.9 \\
\hline 18 & 0.009 & 0.076 & 42.4264 & 0 & 0.009 & 0.158 & $0 \%$ & 18 \\
\hline \multirow[b]{2}{*}{ Sum } & \multirow[b]{2}{*}{0.2} & & & $\begin{array}{l}\text { total } \\
\text { mass }\end{array}$ & 0.1580 & & & \\
\hline & & & & & & & & \\
\hline
\end{tabular}


Table 4.8 cont' $^{\prime} d$

Test ID: Bus 1015

CBD \#5

\begin{tabular}{|c|c|c|c|c|c|c|c|c|}
\hline \multicolumn{9}{|c|}{$\begin{array}{l}\text { Impactor } \\
\text { Data }\end{array}$} \\
\hline $\begin{array}{c}E C A D \\
(\mu \mathrm{m})\end{array}$ & $\begin{array}{c}\text { Mass } \\
\text { mg }\end{array}$ & $\begin{array}{c}\text { frequency } \\
\text { (normalized) }\end{array}$ & $\begin{array}{c}\text { mid } d p \\
(\mu \mathrm{m})\end{array}$ & stage & $\begin{array}{c}\text { net mass } \\
\text { mg }\end{array}$ & $\begin{array}{c}\text { cumulative } \\
\text { mass } \\
\mathrm{mg}\end{array}$ & $\begin{array}{c}\text { cumulative \% } \\
\text { < ECAD }\end{array}$ & $\begin{array}{l}E C A D \\
(\mu \mathrm{m})\end{array}$ \\
\hline 0.01 & 0.081 & 0.534 & 0.0228 & $\mathrm{f}$ & 0.081 & 0.004 & $98 \%$ & 0.01 \\
\hline 0.052 & 0.024 & 0.425 & 0.0707 & 10 & 0.024 & 0.105 & $50 \%$ & 0.052 \\
\hline 0.096 & 0.01 & 0.150 & 0.1379 & 9 & 0.01 & 0.115 & $46 \%$ & 0.096 \\
\hline 0.198 & 0.011 & 0.211 & 0.2629 & 8 & 0.011 & 0.126 & $41 \%$ & 0.198 \\
\hline 0.349 & 0.012 & 0.271 & 0.4441 & 7 & 0.012 & 0.138 & $35 \%$ & 0.349 \\
\hline 0.565 & 0.009 & 0.171 & 0.7517 & 6 & 0.009 & 0.147 & $31 \%$ & 0.565 \\
\hline 1 & 0.012 & 0.222 & 1.3416 & 5 & 0.012 & 0.159 & $25 \%$ & 1 \\
\hline 1.8 & 0.01 & 0.200 & 2.3622 & 4 & 0.01 & 0.169 & $20 \%$ & 1.8 \\
\hline 3.1 & 0.009 & 0.141 & 4.3841 & 3 & 0.009 & 0.178 & $16 \%$ & 3.1 \\
\hline 6.2 & 0.008 & 0.031 & 7.8345 & 2 & 0.008 & 0.186 & $12 \%$ & 6.2 \\
\hline 9.9 & 0.013 & 0.061 & 13.3492 & 1 & 0.013 & 0.199 & $6 \%$ & 9.9 \\
\hline 18 & 0.013 & 0.082 & 42.4264 & 0 & 0.013 & 0.212 & $0 \%$ & 18 \\
\hline 100 & & & & $\begin{array}{l}\text { total } \\
\text { mass }\end{array}$ & 0.2120 & & & \\
\hline
\end{tabular}

Test ID: Bus 1015

CBD \#6

Impactor

Data

\begin{tabular}{|c|c|c|c|c|c|c|c|c|}
\hline $\begin{array}{r}E C A D \\
(\mu \mathrm{m}) \\
\end{array}$ & $\begin{array}{c}\text { Mass } \\
\text { mg } \\
\end{array}$ & $\begin{array}{c}\text { frequency } \\
\text { (normalized) }\end{array}$ & $\begin{array}{c}\operatorname{mid} d p \\
(\mu \mathrm{m})\end{array}$ & stage & $\begin{array}{c}\text { net mass } \\
\text { mg }\end{array}$ & $\begin{array}{c}\text { cumulative } \\
\text { mass } \\
\text { mg }\end{array}$ & $\begin{array}{c}\text { cumulative \% } \\
\text { < ECAD }\end{array}$ & $\begin{array}{r}E C A D \\
(\mu \mathrm{m}) \\
\end{array}$ \\
\hline 0.01 & 0.07 & 0.600 & 0.0228 & $\mathrm{f}$ & 0.07 & 0.004 & $98 \%$ & 0.01 \\
\hline 0.052 & 0.024 & 0.553 & 0.0707 & 10 & 0.024 & 0.094 & $42 \%$ & 0.052 \\
\hline 0.096 & 0.01 & 0.195 & 0.1379 & 9 & 0.01 & 0.104 & $36 \%$ & 0.096 \\
\hline 0.198 & 0.007 & 0.174 & 0.2629 & 8 & 0.007 & 0.111 & $32 \%$ & 0.198 \\
\hline 0.349 & 0.01 & 0.293 & 0.4441 & 7 & 0.01 & 0.121 & $26 \%$ & 0.349 \\
\hline 0.565 & 0.009 & 0.223 & 0.7517 & 6 & 0.009 & 0.13 & $20 \%$ & 0.565 \\
\hline 1 & 0.006 & 0.144 & 1.3416 & 5 & 0.006 & 0.136 & $17 \%$ & 1 \\
\hline 1.8 & 0.006 & 0.156 & 2.3622 & 4 & 0.006 & 0.142 & $13 \%$ & 1.8 \\
\hline 3.1 & 0.007 & 0.143 & 4.3841 & 3 & 0.007 & 0.149 & $9 \%$ & 3.1 \\
\hline 6.2 & 0.003 & 0.015 & 7.8345 & 2 & 0.003 & 0.152 & $7 \%$ & 6.2 \\
\hline 9.9 & 0.002 & 0.012 & 13.3492 & 1 & 0.002 & 0.154 & $6 \%$ & 9.9 \\
\hline 18 & 0.009 & 0.074 & 42.4264 & 0 & 0.009 & 0.163 & $0 \%$ & 18 \\
\hline \multirow[b]{2}{*}{ Sum } & \multirow[b]{2}{*}{0.2} & & & $\begin{array}{l}\text { total } \\
\text { mass }\end{array}$ & 0.1630 & & & \\
\hline & & & & & & & & \\
\hline
\end{tabular}


Table 4.8 cont' $^{\prime} d$

Test ID: Bus 1015

CBD \#7

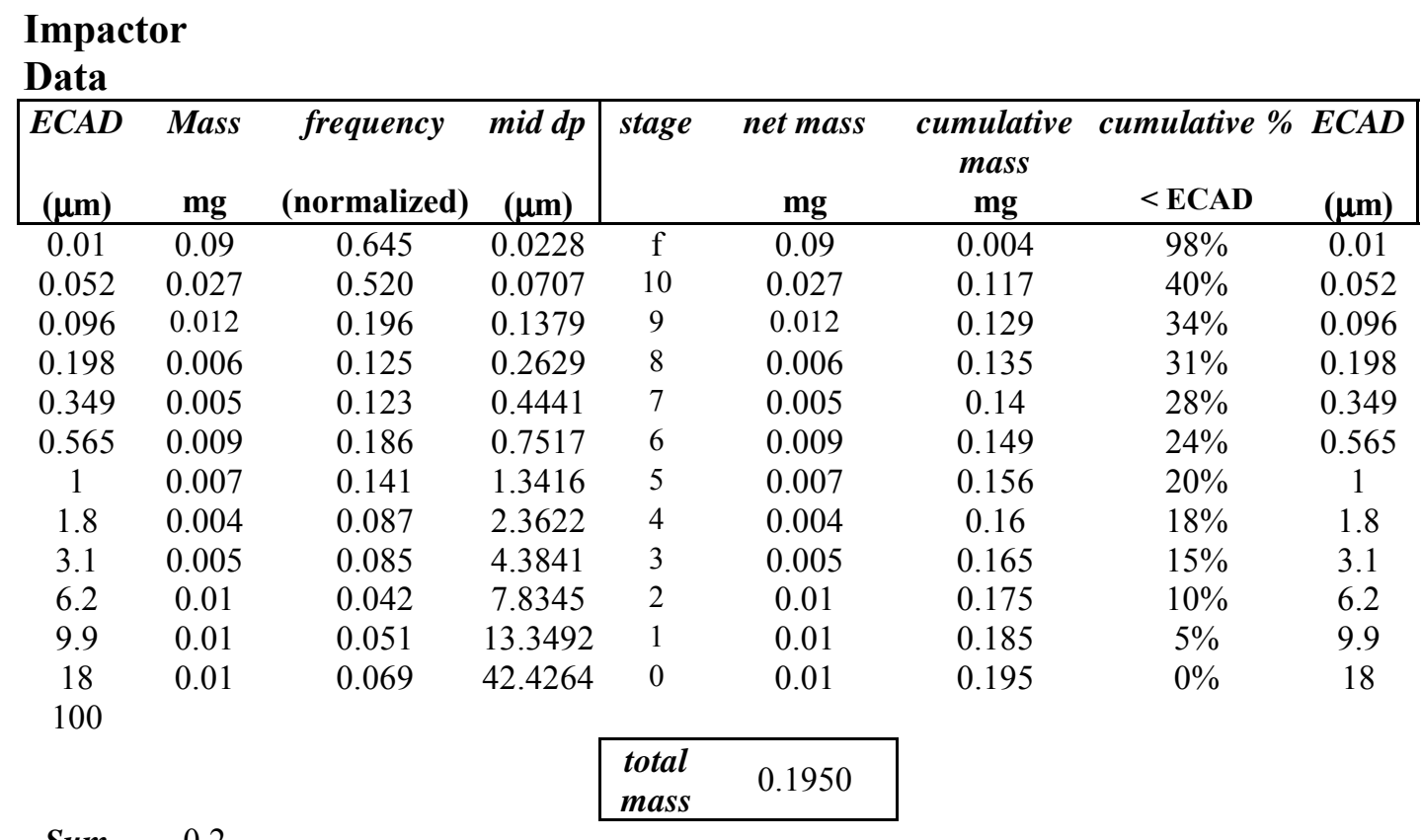

\section{Test ID: Bus 1015}

\section{CBD \#8}

\section{Impactor}

Data

\begin{tabular}{|c|c|c|c|c|c|c|c|c|}
\hline $\begin{array}{c}E C A D \\
(\mu \mathrm{m}) \\
\end{array}$ & $\begin{array}{c}\text { Mass } \\
\text { mg } \\
\end{array}$ & $\begin{array}{c}\text { frequency } \\
\text { (normalized) }\end{array}$ & $\begin{array}{c}\text { mid } d p \\
(\mu \mathrm{m}) \\
\end{array}$ & stage & $\begin{array}{c}\text { net mass } \\
\text { mg } \\
\end{array}$ & $\begin{array}{c}\text { cumulative } \\
\text { mass } \\
\text { mg }\end{array}$ & $\begin{array}{r}\text { cumulative } \\
<\text { ECAD }\end{array}$ & $E C A D$ \\
\hline 0.01 & 0.096 & 0.632 & 0.0228 & $f$ & 0.096 & 0.004 & $98 \%$ & 0.01 \\
\hline 0.052 & 0.029 & 0.514 & 0.0707 & 10 & 0.029 & 0.125 & $41 \%$ & 0.052 \\
\hline 0.096 & 0.011 & 0.165 & 0.1379 & 9 & 0.011 & 0.136 & $36 \%$ & 0.096 \\
\hline 0.198 & 0.008 & 0.153 & 0.2629 & 8 & 0.008 & 0.144 & $32 \%$ & 0.198 \\
\hline 0.349 & 0.01 & 0.225 & 0.4441 & 7 & 0.01 & 0.154 & $27 \%$ & 0.349 \\
\hline 0.565 & 0.009 & 0.171 & 0.7517 & 6 & 0.009 & 0.163 & $23 \%$ & 0.565 \\
\hline 1 & 0.011 & 0.203 & 1.3416 & 5 & 0.011 & 0.174 & $18 \%$ & 1 \\
\hline 1.8 & 0.005 & 0.100 & 2.3622 & 4 & 0.005 & 0.179 & $16 \%$ & 1.8 \\
\hline 3.1 & 0.006 & 0.094 & 4.3841 & 3 & 0.006 & 0.185 & $13 \%$ & 3.1 \\
\hline 6.2 & 0.008 & 0.031 & 7.8345 & 2 & 0.008 & 0.193 & $9 \%$ & 6.2 \\
\hline 9.9 & 0.009 & 0.042 & 13.3492 & 1 & 0.009 & 0.202 & $5 \%$ & 9.9 \\
\hline 18 & 0.01 & 0.063 & 42.4264 & 0 & 0.01 & 0.212 & $0 \%$ & 18 \\
\hline & & & & $\begin{array}{l}\text { total } \\
\text { mass }\end{array}$ & 0.2120 & & & \\
\hline
\end{tabular}

Sum 0.2 
While the non-catalyst version of the bus emitted considerably higher total mass, the mass on stage \#10 dropped as that less than $0.052 \mu \mathrm{m}$ rose. The catalyst appeared to lower the mass of the finest fraction of particles, while increasing those slightly larger. It should be noted that the percent mass values for the final and the tenth stage differ by an average of 6 and $7 \%$, respectively. It is considerable because these two stages contain approximately $60 \%$ of the total collected mass. It has been purported that natural gas combustion produces only water droplets in this ultrafine region. This cannot be accurate since the MOUDI after-filters are conditioned, which will remove water from the filter. In actuality, the CNG Buses 1013 and 1015 produced more particulate mass less than 52 $\mathrm{nm}$ in aerodynamic diameter than the D2 Bus 1012. A further discussion of these findings is presented in Chapter 5.

In Table 4.6 and Figure 4.57 a comparison was made of stage \#4 of the MOUDI to demonstrate the uniqueness of the bimodal size distribution determined for CNG Bus 1013. The average mass found on the fourth stage for Bus 1013 was more than double that of Bus 1015 and was even greater than the mass collected from the diesel Bus 1012. This highlighted the fact that the second mode was likely vehicle-specific and not formed as a result of combustion.

\subsubsection{SMPS Data}

As with the other instruments, the SMPS was operated on all six of the RTD/SKIP transit buses. The model 3025A CPC was used, and the DMA aerosol flow rate set at $2.0 \mathrm{lpm}$ and the sheath flow rate at $20 \mathrm{lpm}$. For the first three vehicles, the 
SMPS scanned for particles in the general size range of 10 to $200 \mathrm{~nm}$. This was intended to give a broad spectrum of diesel particulate matter, since it is well established that the mass median size is around the $100-120 \mathrm{~nm}$ range. The following graphs show the realtime particle trace on a number basis, giving the particle concentration of a determined size throughout the CBD cycle.

Bus 1010 gave data very indicative of a typical diesel vehicle (see Figures 4.59 4.63). The particle count gradually increased from the lowest diameter scanned, $20 \mathrm{~nm}$, until it reached a peak maximum at $100 \mathrm{~nm}$. At $150 \mathrm{~nm}$, nominal concentrations were around $5.0 \times 10^{5}$ particles per cubic centimeter, with spikes nearing $2.0 \times 10^{6}$ at the maximum. This trend was similar at $200 \mathrm{~nm}$, except that the nominal concentration was halved.

The results from Bus 1011, as shown in Figures 4.64 - 4.69 mimic those of Bus 1010 in the size pattern, though this bus did have higher overall concentrations. Again, the concentrations are the highest for the $100 \mathrm{~nm}$ diameter particles, and lowest for the smallest size scanned, this time $10 \mathrm{~nm}$. From Figure 4.64, it can be seen that the resolution at $10 \mathrm{~nm}$ is poor. This size is at the lower limit for the SMPS where statistical errors can magnify. Though the smallest size the 3025A CPC can detect is $3 \mathrm{~nm}$, measurements performed at the low end have limited accuracy. This uncertainty is more prominent for the $3025 \mathrm{~A}$ than other model CPCs in that it only samples a portion of the aerosol.

The 20 and $50 \mathrm{~nm}$ concentration data can be used to resolve the after-filter mass values found using the MOUDI. Since not every particle diameter can be traced, there are limits to the resolution of the after-filter data. Again, this can lead back to the easily 
Figure 4.59 - 20 nm SMPS Particle Concentration for Cummins B5.9 Bus 1010 CBD Run \#1 Fuel: D2

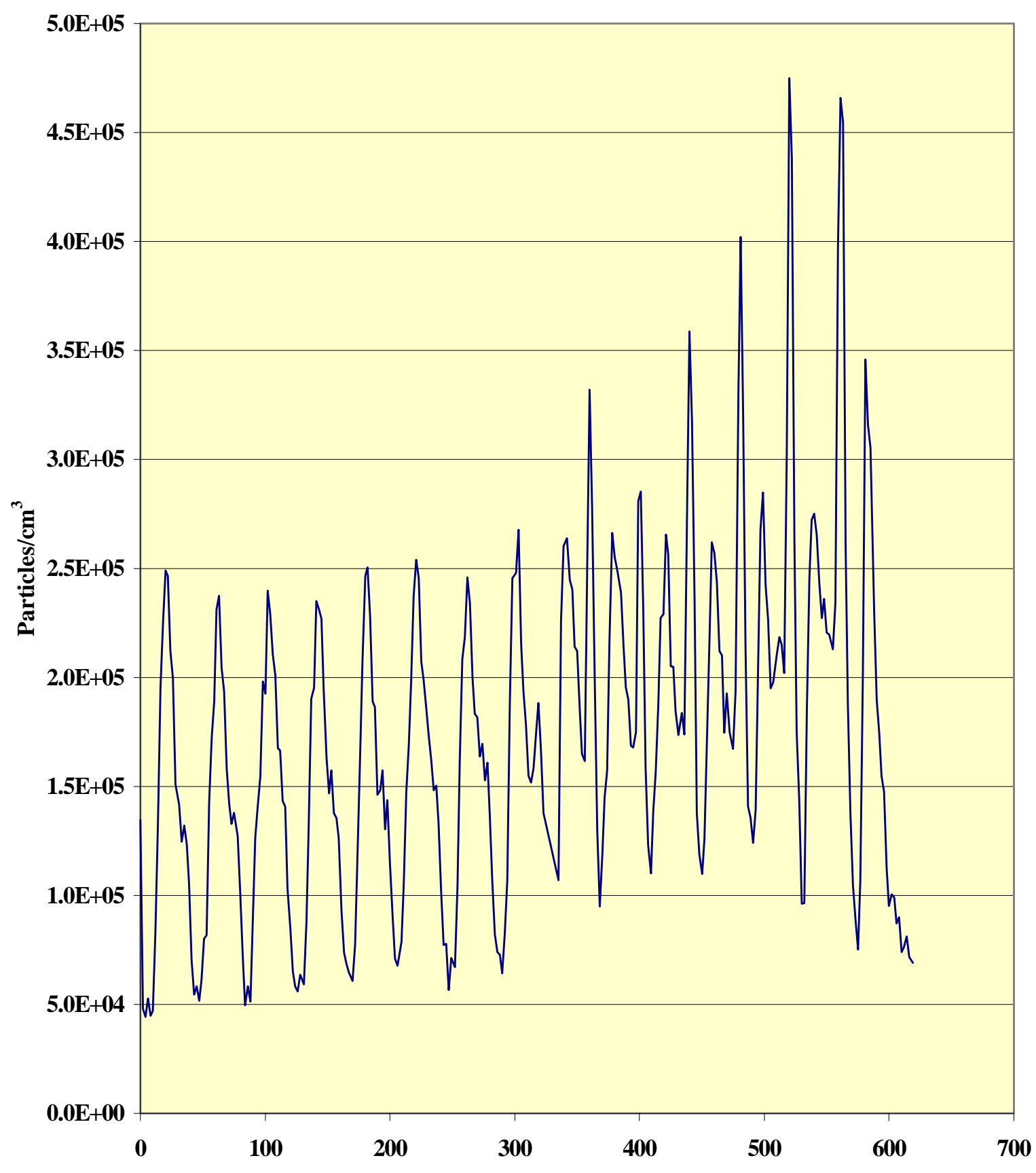

Time (seconds) 
Figure 4.60 - $50 \mathrm{~nm}$ SMPS Particle Concentration for Cummins B5.9 Bus 1010 CBD Run \#2 Fuel: D2

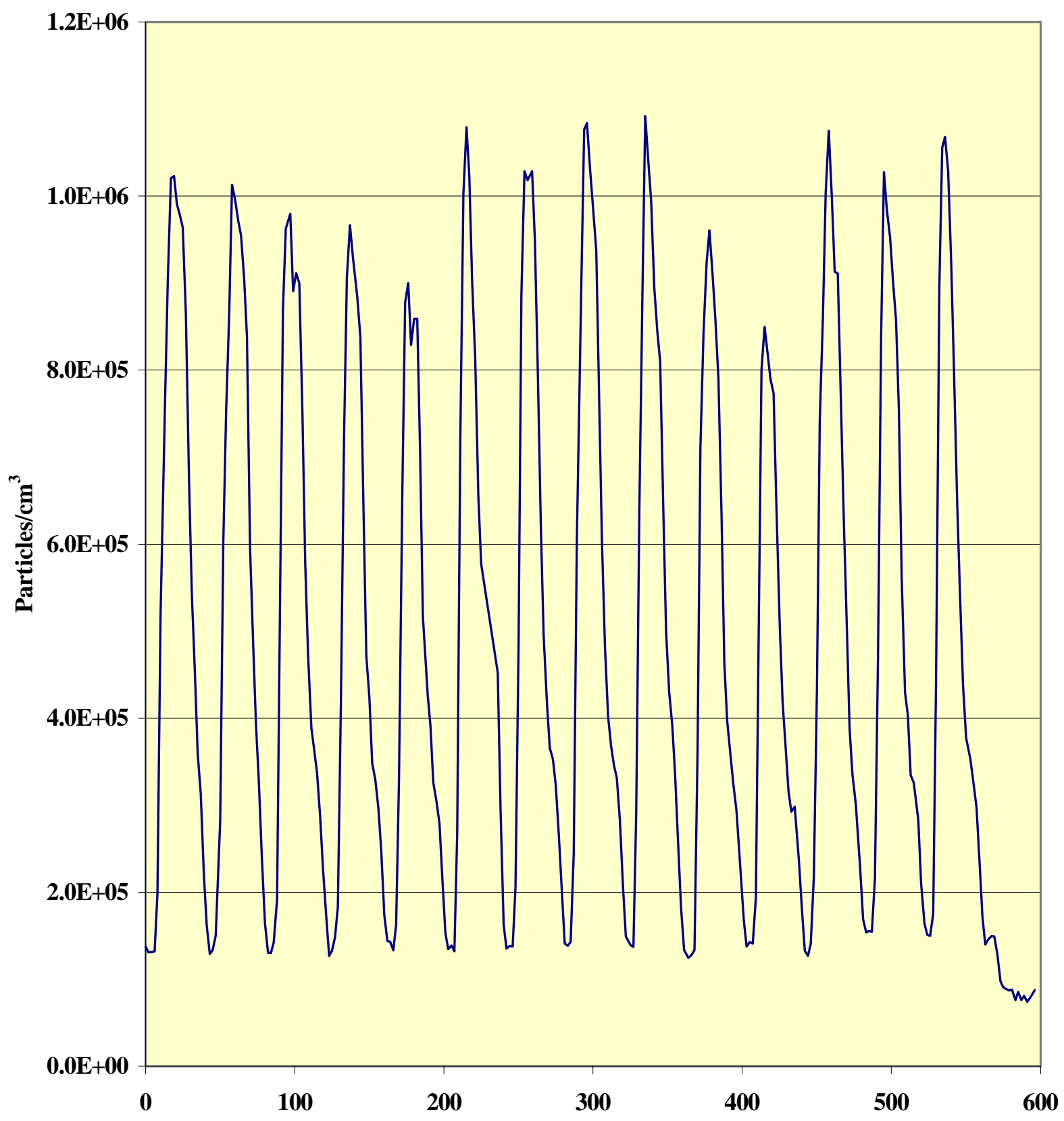

Time (seconds) 


\section{Figure 4.61 - 100 nmSMPS Particle Concentration for Cummins B5.9 Bus 1010 CBDRun\#3 Fuel: D2}

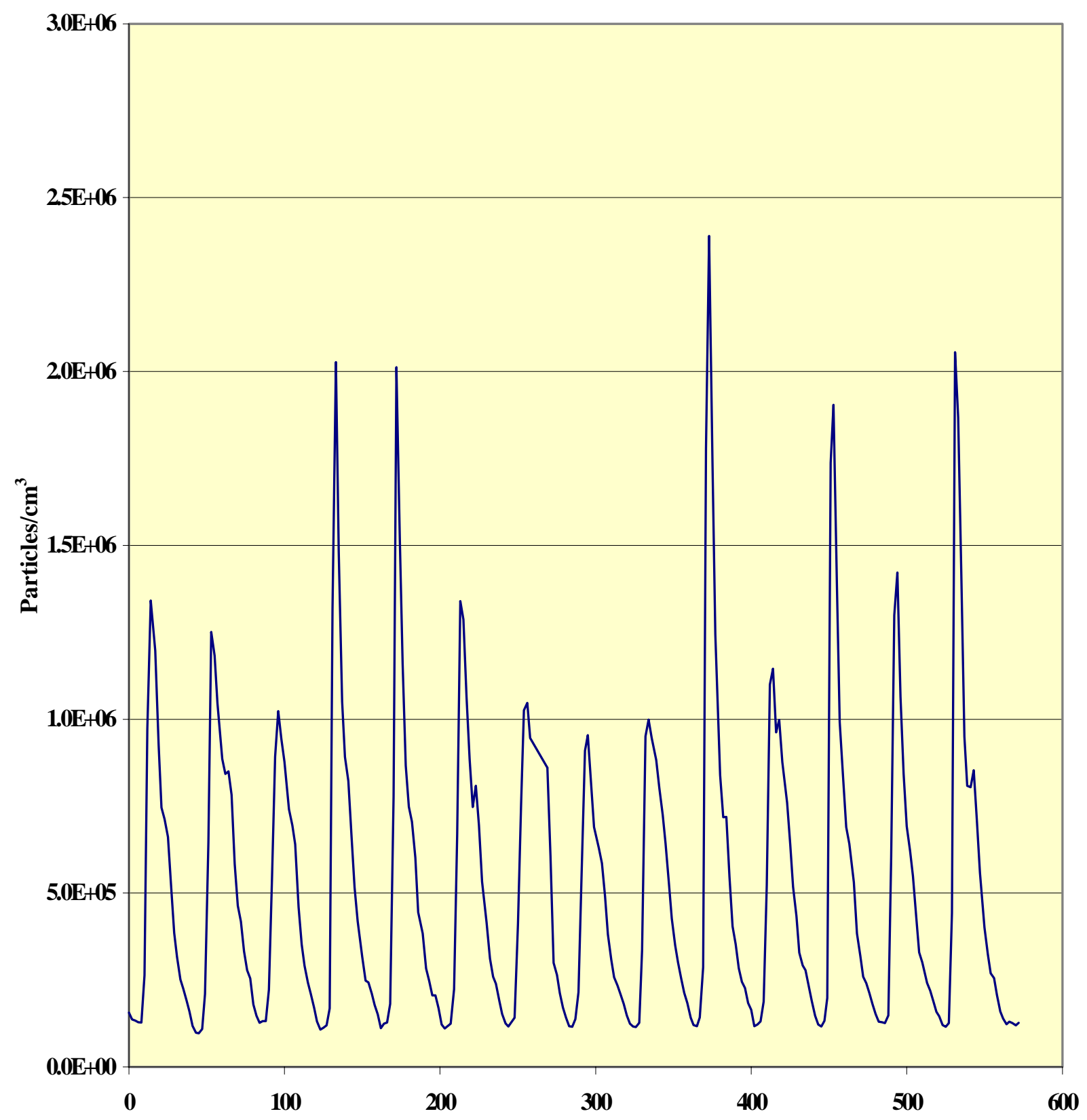

Time(seconds) 
Figure 4.62 - 150 nm SMPS Particle Concentration for Cummins B5.9 Bus 1010 CBD Run \#4 Fuel: D2

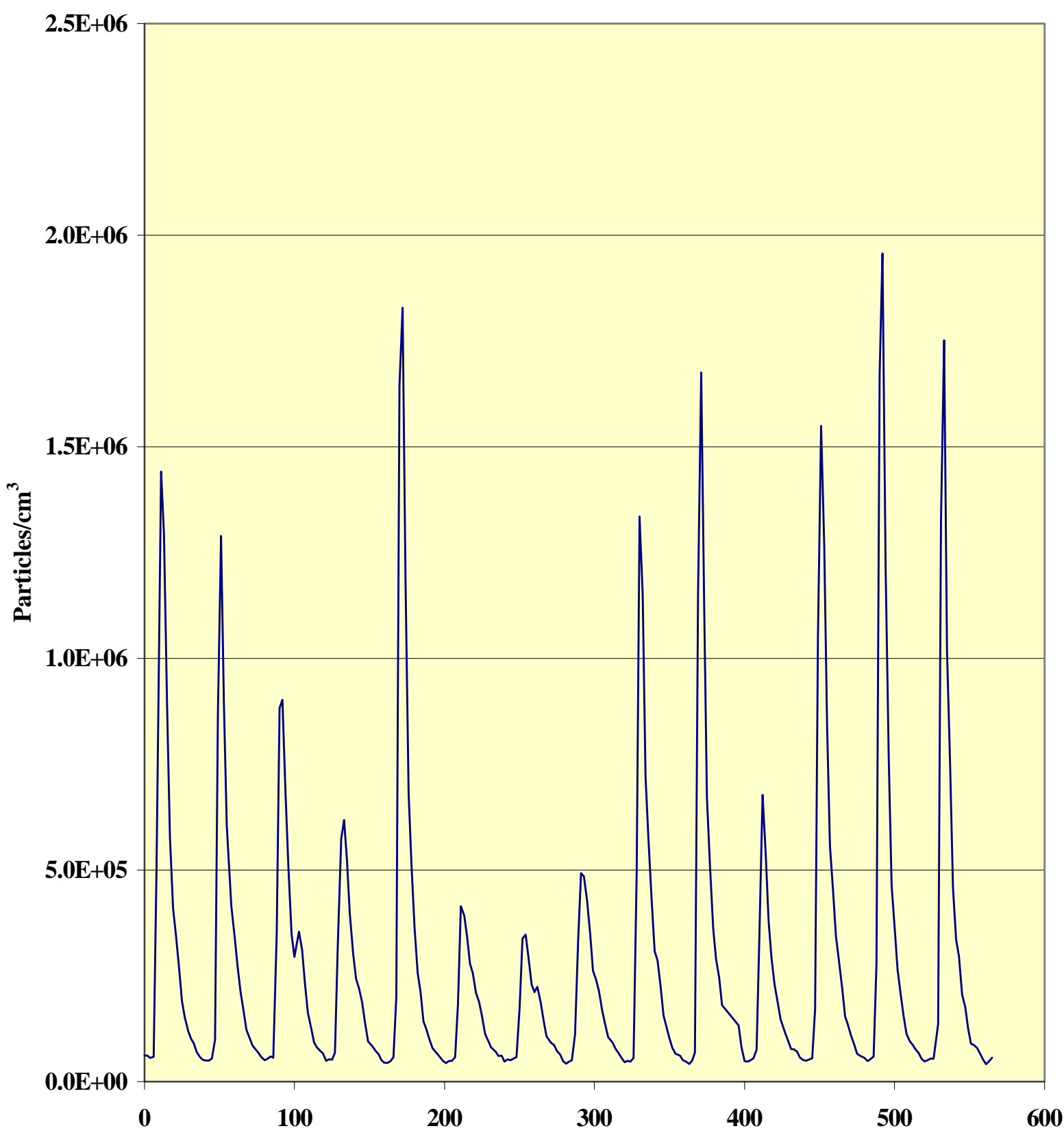

Time (seconds) 
Figure 4.63 - 200 nm SMPS Particle Concentration for Cummins B5.9 Bus 1010 CBD Run \#5 Fuel: D2

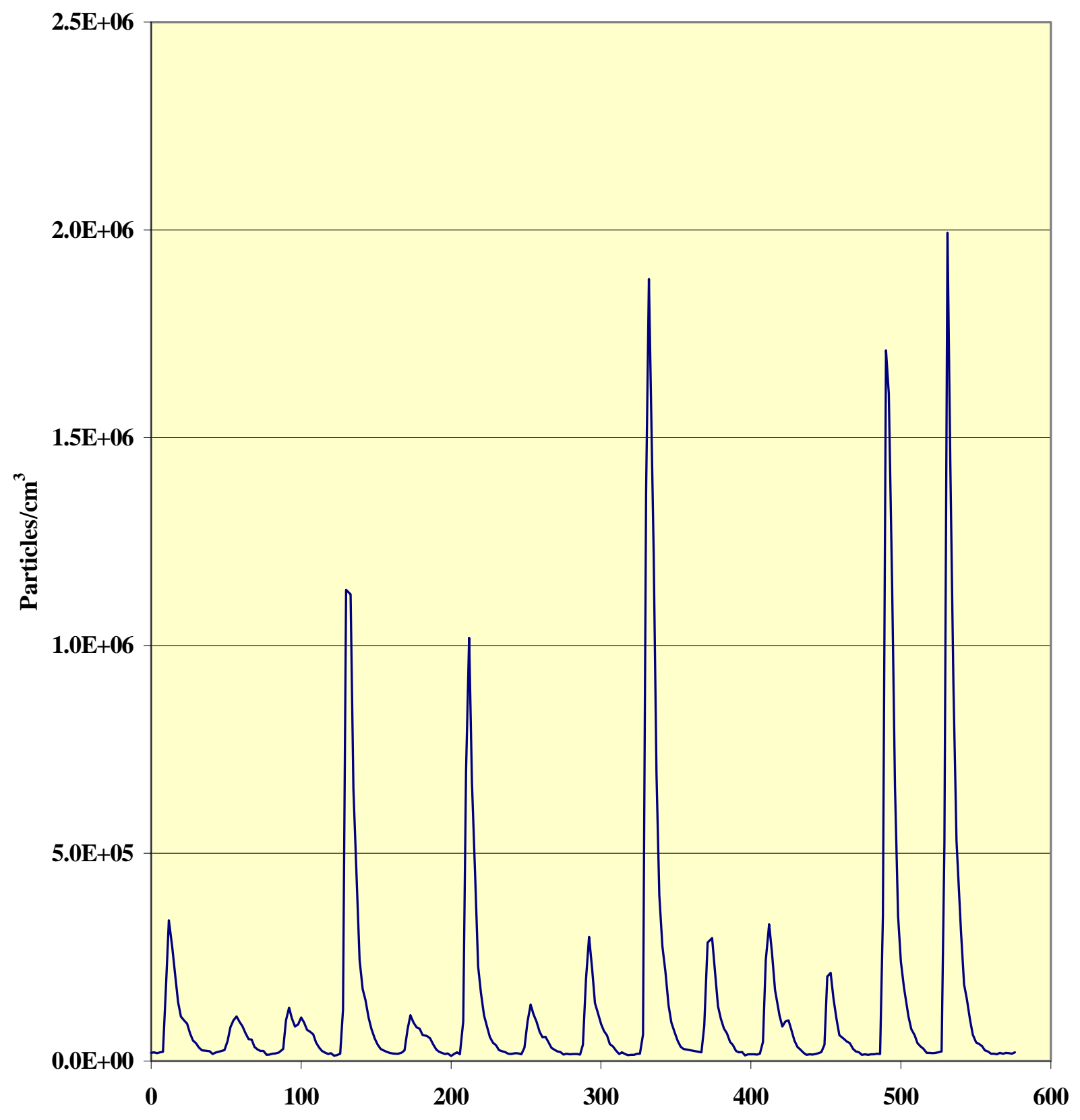

Time (seconds) 
Figure 4.64 - 10 nm SMPS Particle Concentration for Cummins B5.9 Bus 1011 CBD Run \#1 Fuel: D2

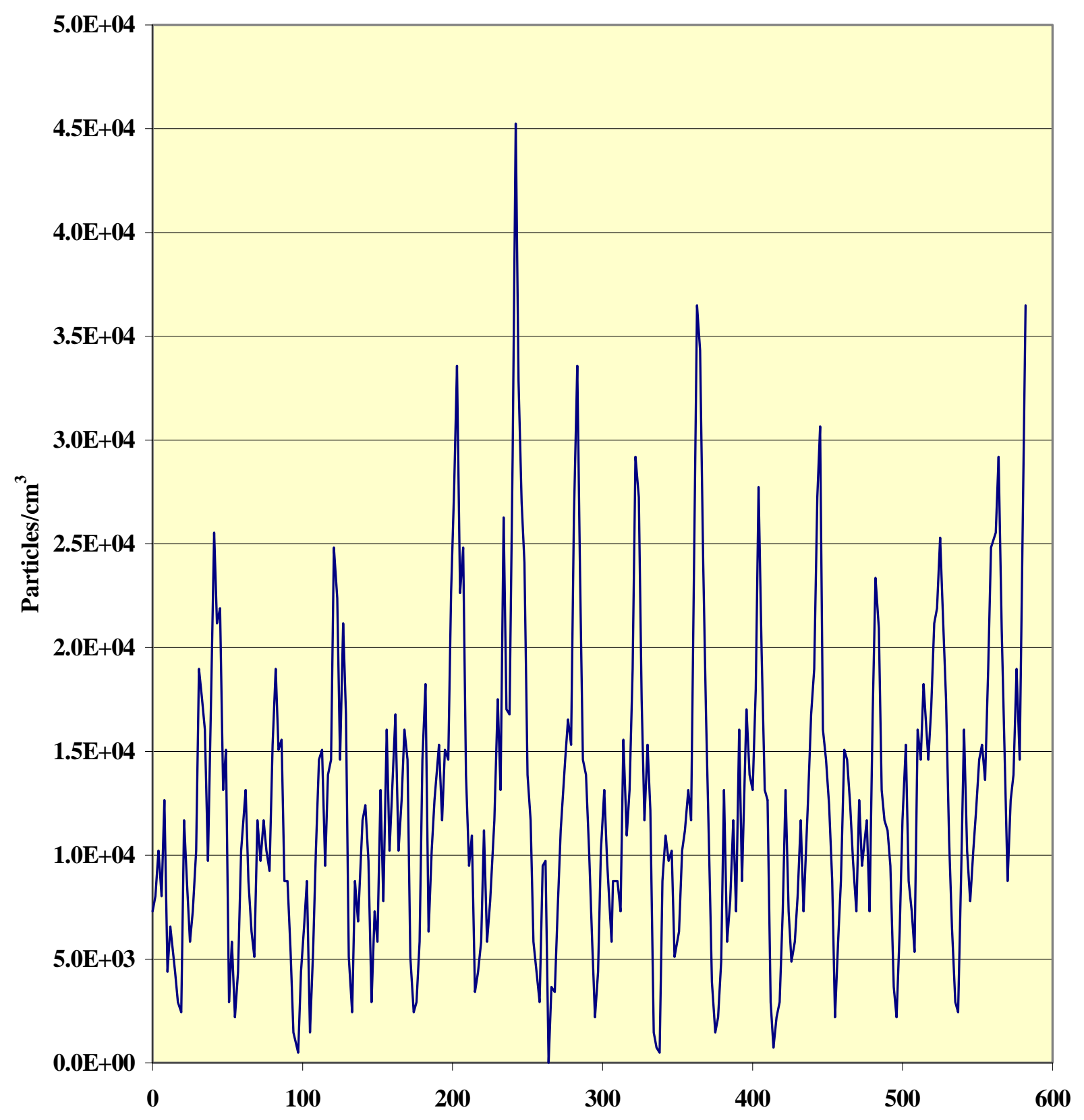

Time (seconds) 
Figure 4.65 - $20 \mathrm{~nm}$ SMPS Particle Concentration for Cummins B5.9 Bus 1011 CBD Run \#2 Fuel: D2

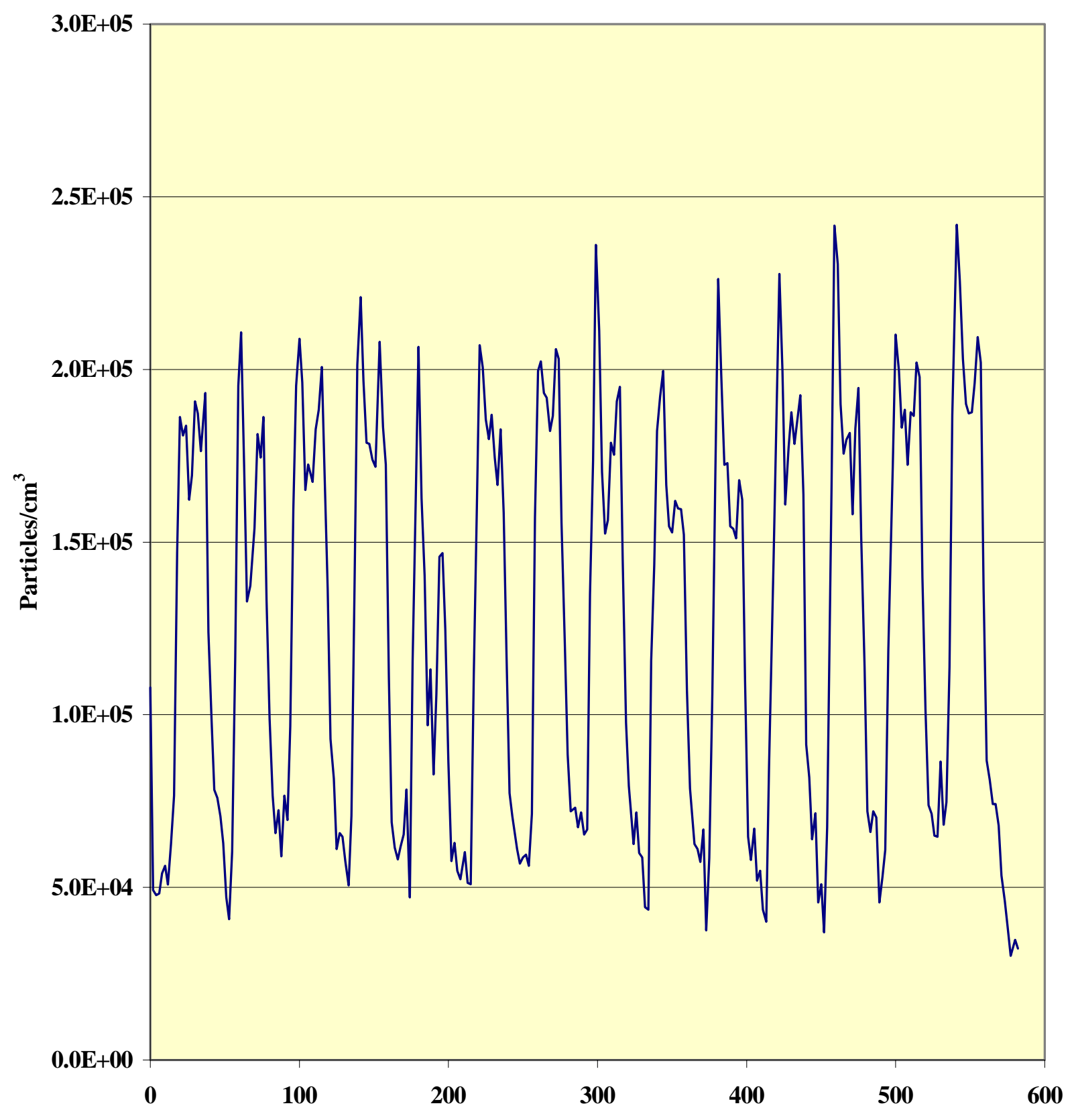

Time (seconds) 
Figure 4.66 - $50 \mathrm{~nm}$ SMPS Particle Concentration for Cummins B5.9 Bus 1011 CBD Run \#3 Fuel: D2

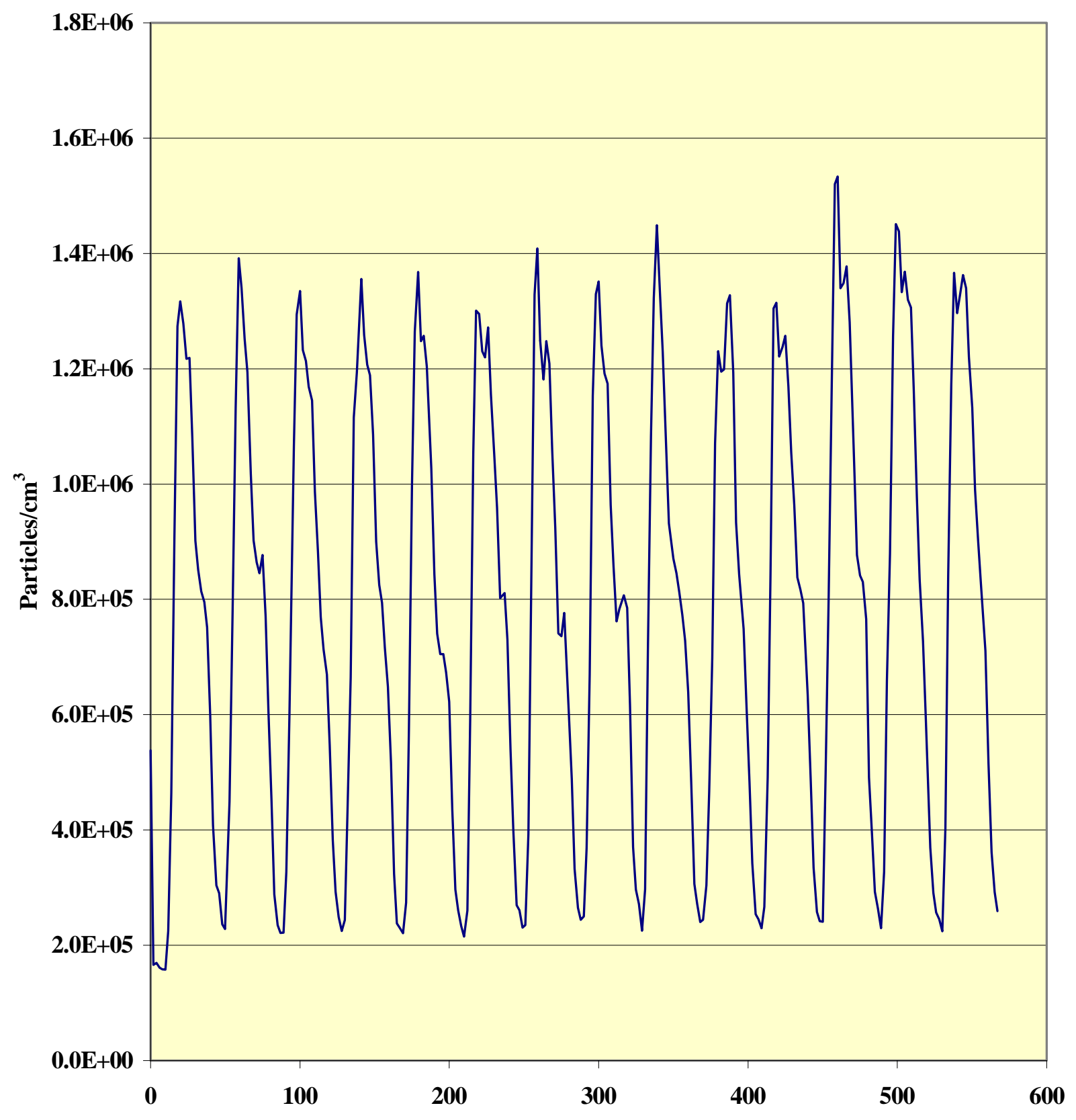

Time (seconds) 
Figure 4.67 - 100 nm SMPS Particle Concentration for Cummins B5.9 Bus 1011 CBD Run \#4 Fuel: D2

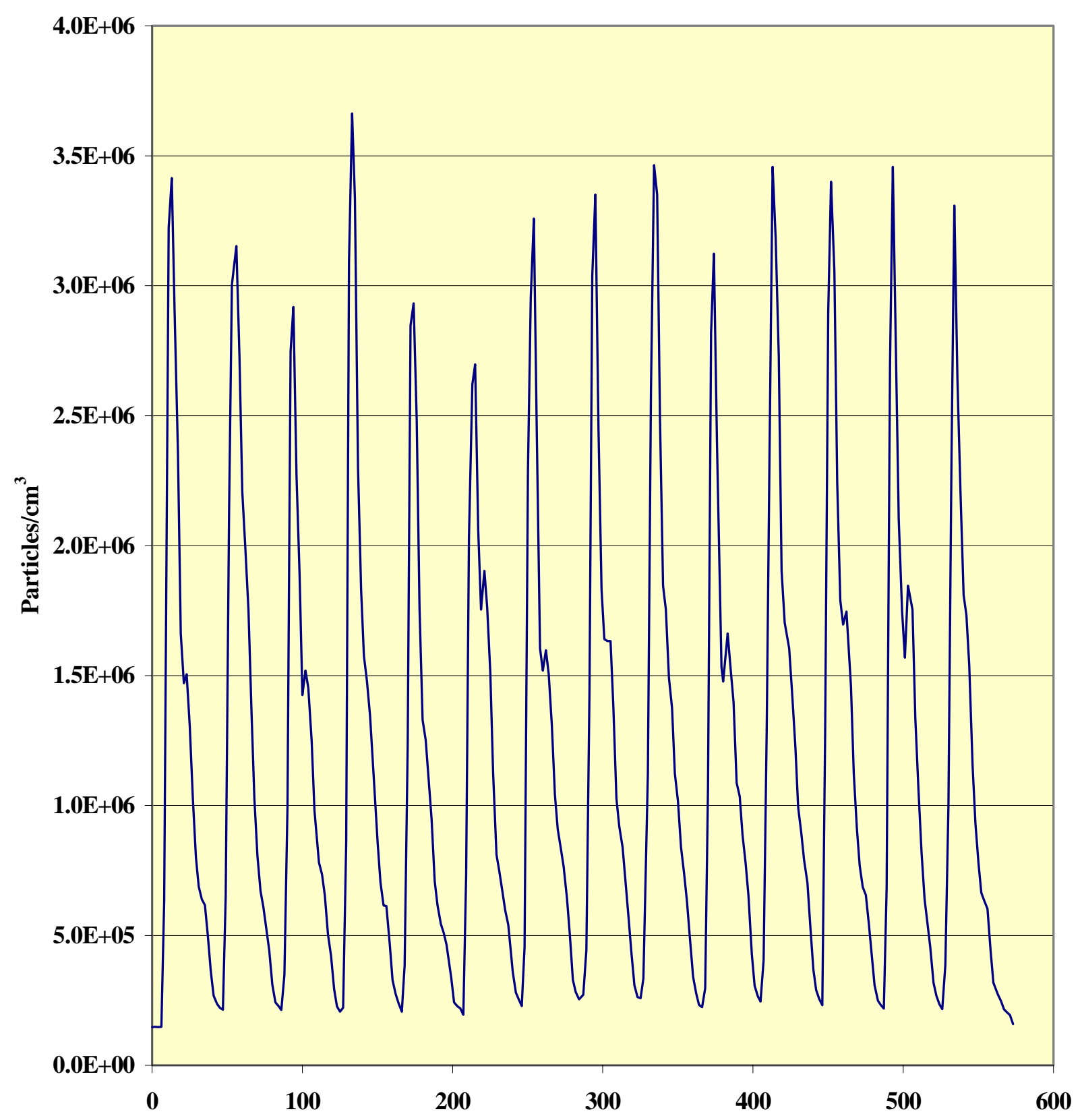

Time (seconds) 
Figure 4.68 - 150 nm SMPS Particle Concentration for Cummins B5.9 Bus 1011 CBD Run \#5 Fuel: D2

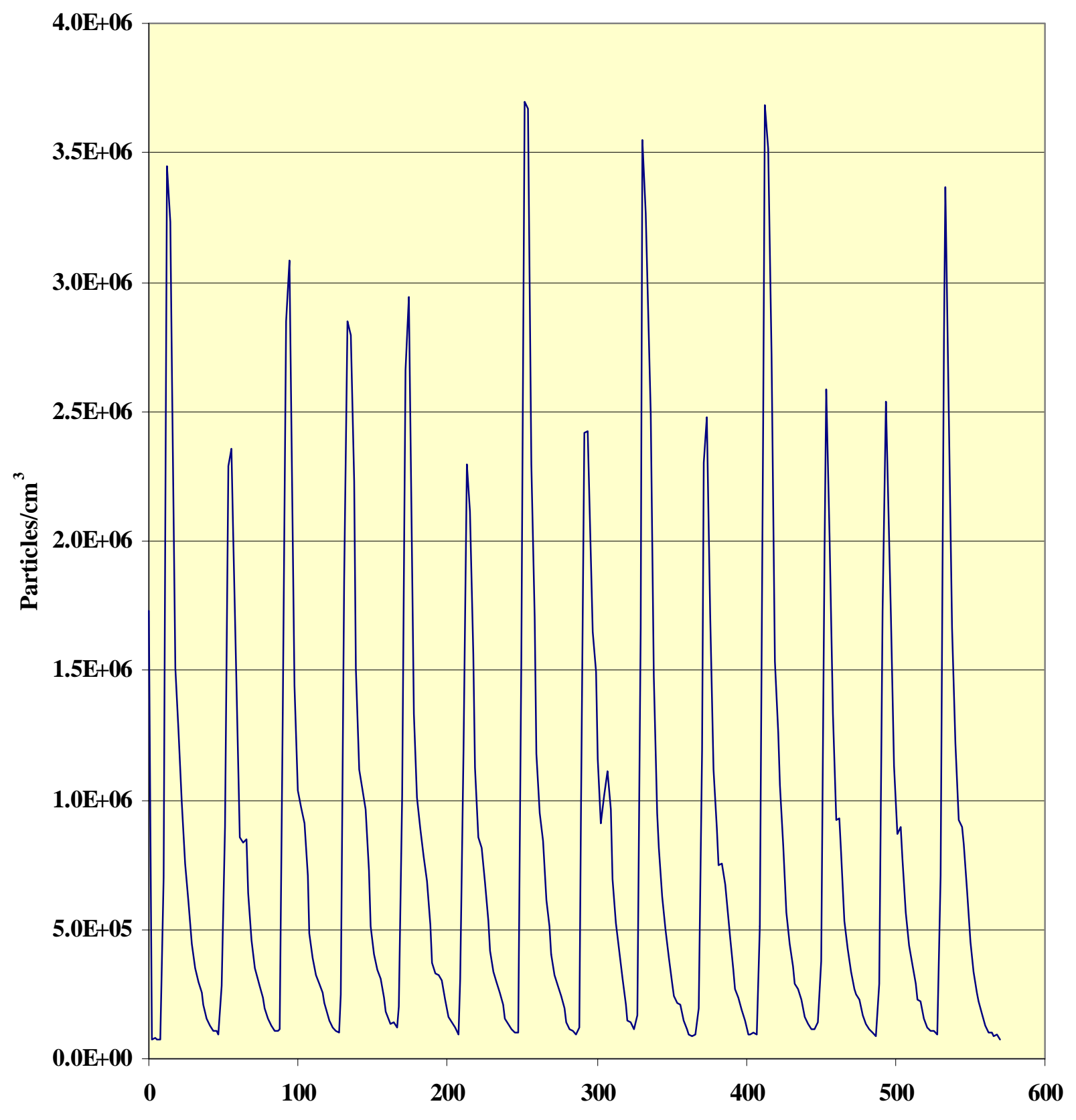

Time (seconds) 
Figure 4.69 - 200 nm SMPS Particle Concentration for Cummins B5.9 Bus 1011 CBD Run \#6 Fuel: D2

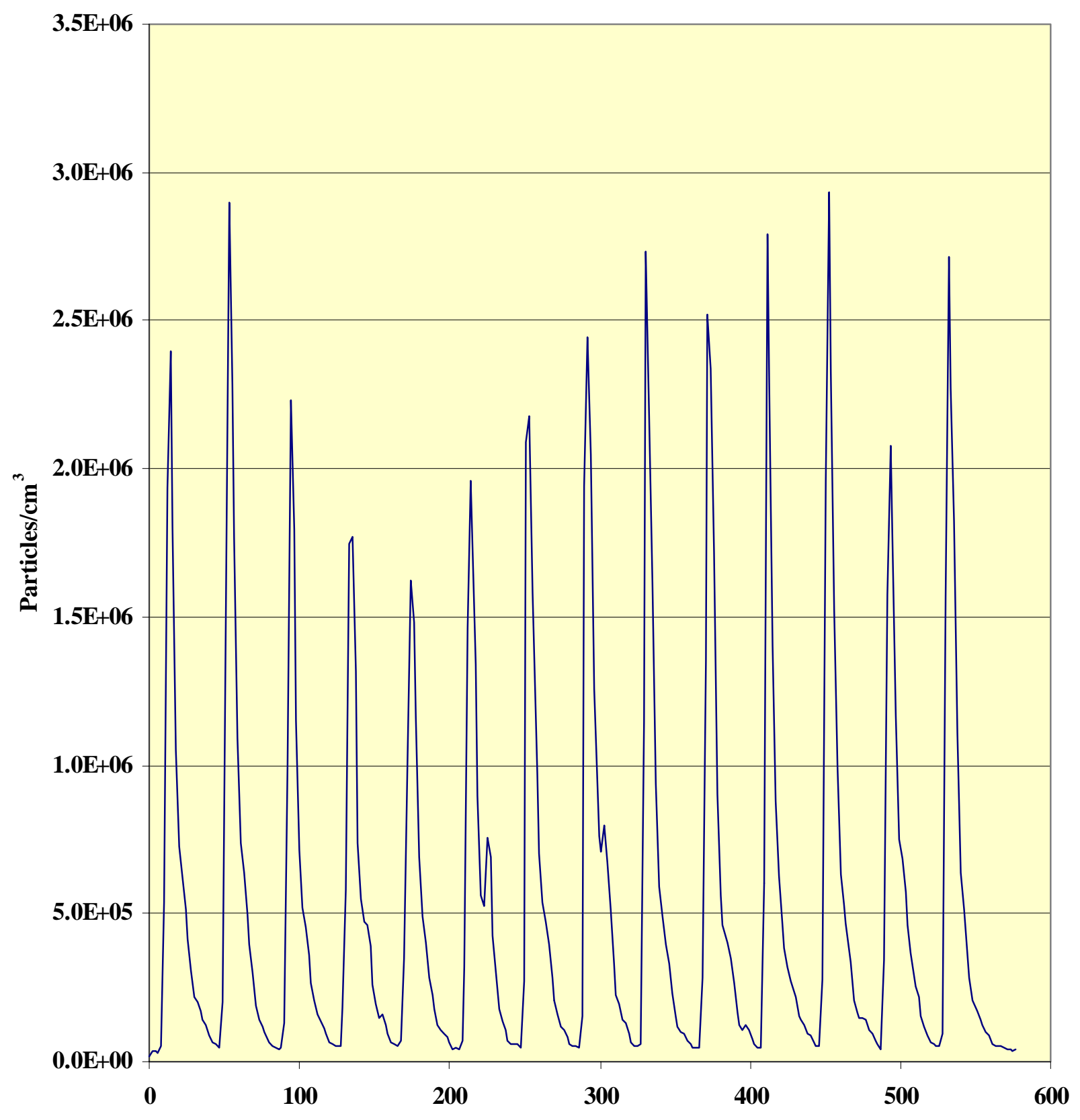

Time (seconds) 
misleading solution of simply converting mass to number and vice versa. Also, since the devices use different definitions of diameter, this conversion takes on more negative consequences. It is only of value to compare this data empirically and look for trends and patterns rather than facts and numbers. The pattern showed that the makeup of the smallest size-selected mass capable of being collected by the MOUDI for these two vehicles was largely concentrated at the upper levels $(\sim 50 \mathrm{~nm})$. As the mobility diameter decreased, so did the total number of particles. This implied that if the MOUDI had another stage in the range of $20-50 \mathrm{~nm}$, most of the particulate matter would be collected on it.

Bus 1012 showed similar results to the previous two buses. The notable aspect to these tests goes back to the data from the MOUDI. The MOUDI gave a mass median diameter of $0.11 \mu \mathrm{m}$ for day one and $0.12 \mu \mathrm{m}$ for day two. By comparing the particle concentration at $60 \mathrm{~nm}$ from the SMPS, it was seen that the first day of testing (see Figures $4.70 \& 4.73$ ) had many more particles at this size than the second. This could be the reason for the slight shift in the median found by the MOUDI. The larger quantity of particles at $60 \mathrm{~nm}$ could pull the distribution to the left and subsequent lesser diameters. However, from the $100 \mathrm{~nm}$ plots, it appeared that the concentrations may have varied significantly between tests (see Figures $4.72,4.75 \& 4.76$ ).

The natural gas Bus 1013 was scanned for particle size from 15 to $55 \mathrm{~nm}$ (see Figures 4.77 - 4.88). Comparing the 15 and $25 \mathrm{~nm}$ data, it was shown that a small change in diameter can lead to large changes in the number of particles. After the eighth ramp for run $\# 2$, the concentration levels more than double for the $25 \mathrm{~nm}$ particles. Comparatively, this size reaches a peak concentration of $8.0 \times 10^{6}$ while the $15 \mathrm{~nm}$ 
Figure 4.70 - 20 nm SMPS Particle Concentration for Cummins B5.9 Bus 1012 CBD Run \#1 Fuel: D2

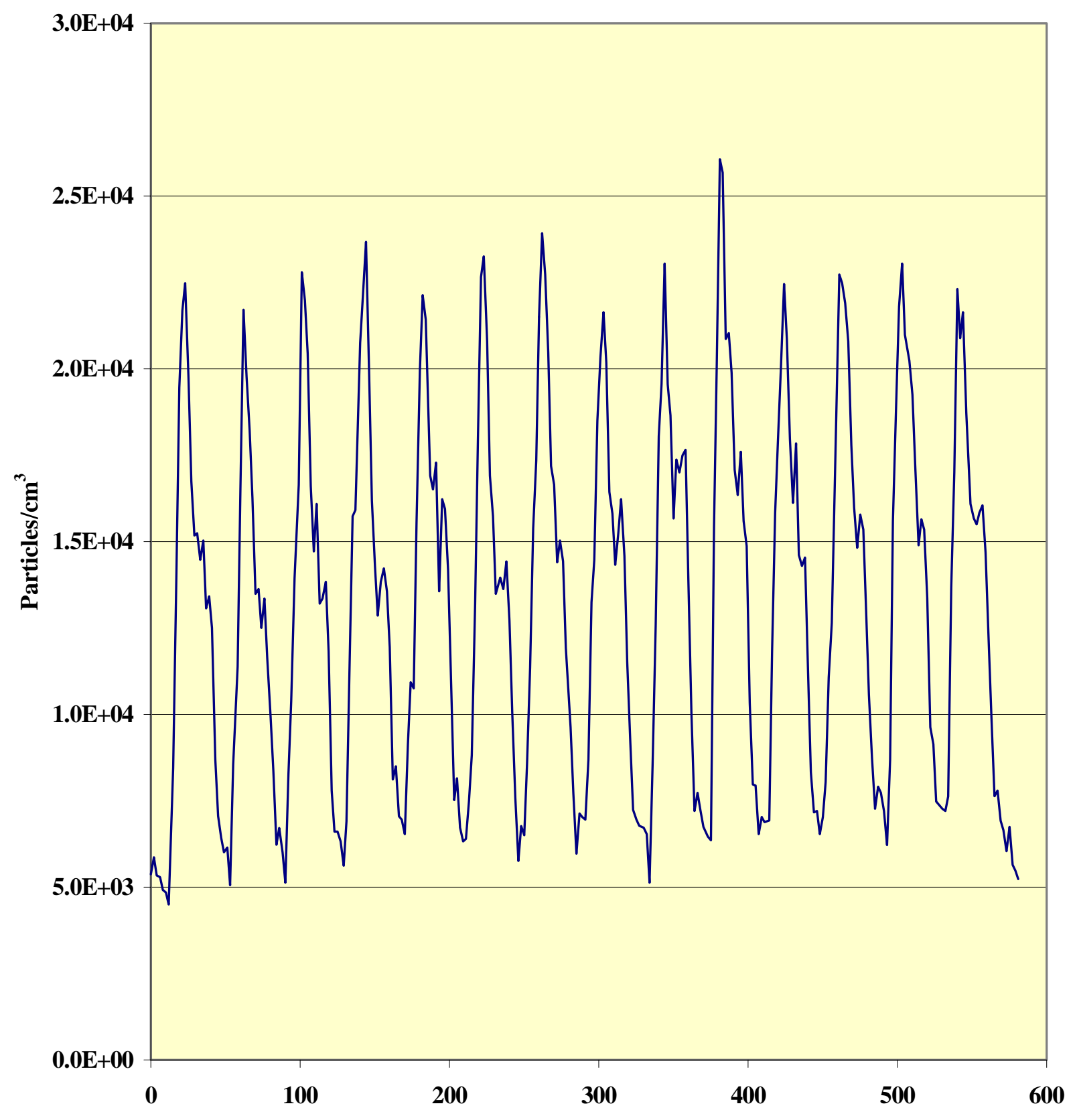

Time (seconds) 
Figure 4.71 - 60 nm SMPS Particle Concentration for Cummins B5.9 Bus 1012 CBD Run \#2 Fuel: D2

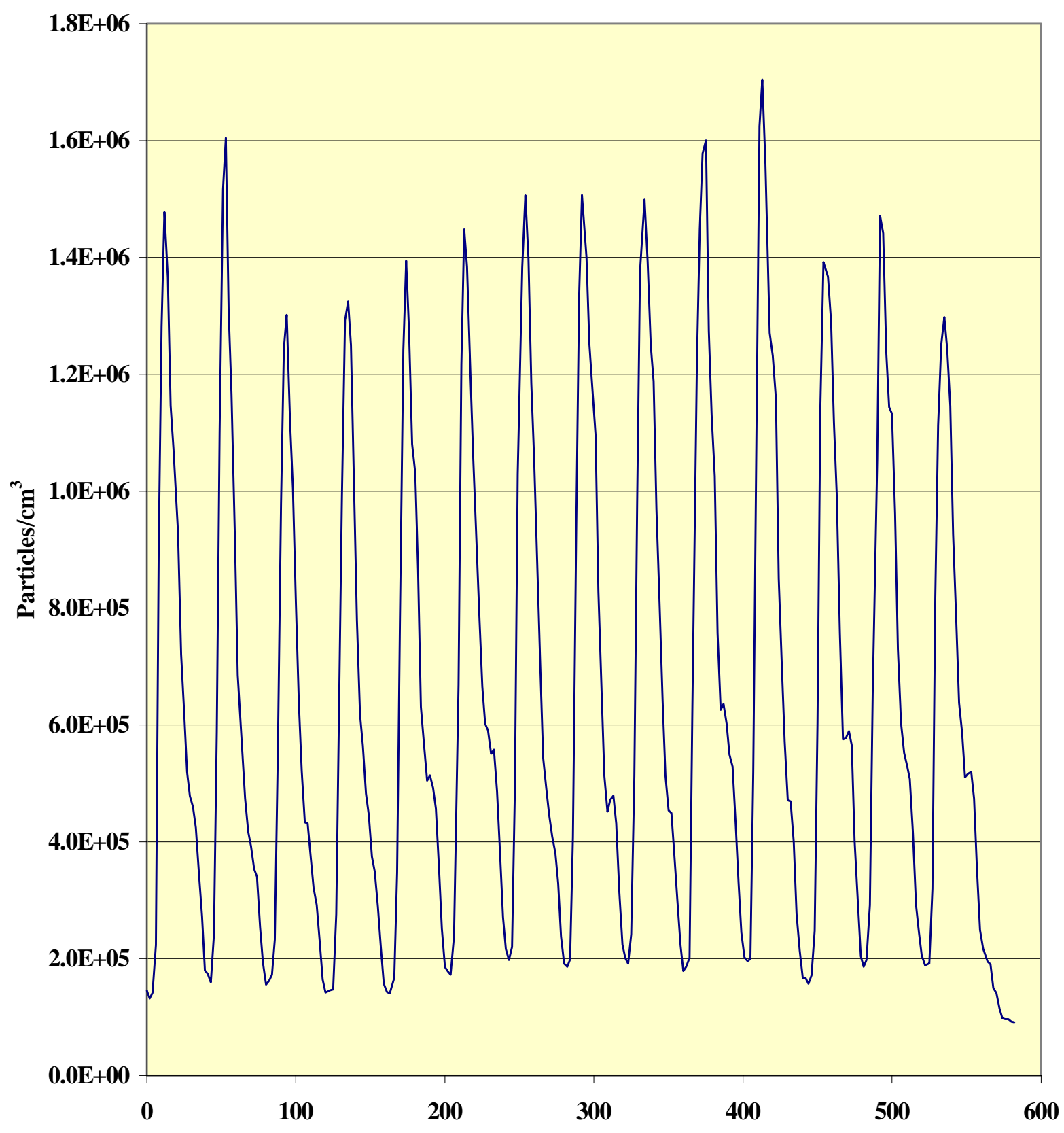

Time (seconds) 
Figure 4.72 - 100 nm SMPS Particle Concentration for Cummins B5.9 Bus 1012 CBD Run \#3 Fuel: D2

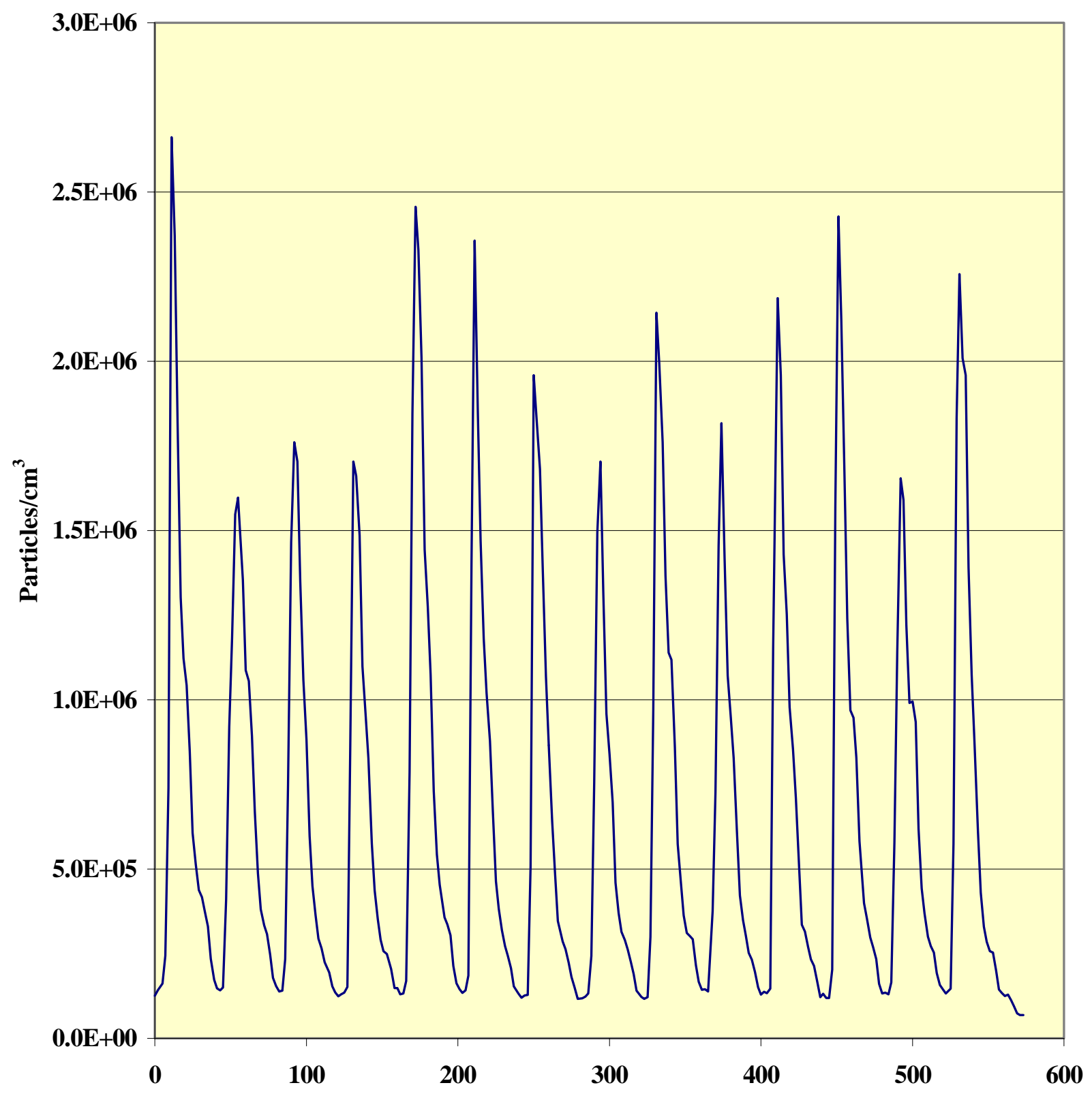

Time (seconds) 
Figure 4.73 - $150 \mathrm{~nm}$ SMPS Particle Concentration for Cummins B5.9 Bus 1012 CBD Run \# 4 Fuel: D2

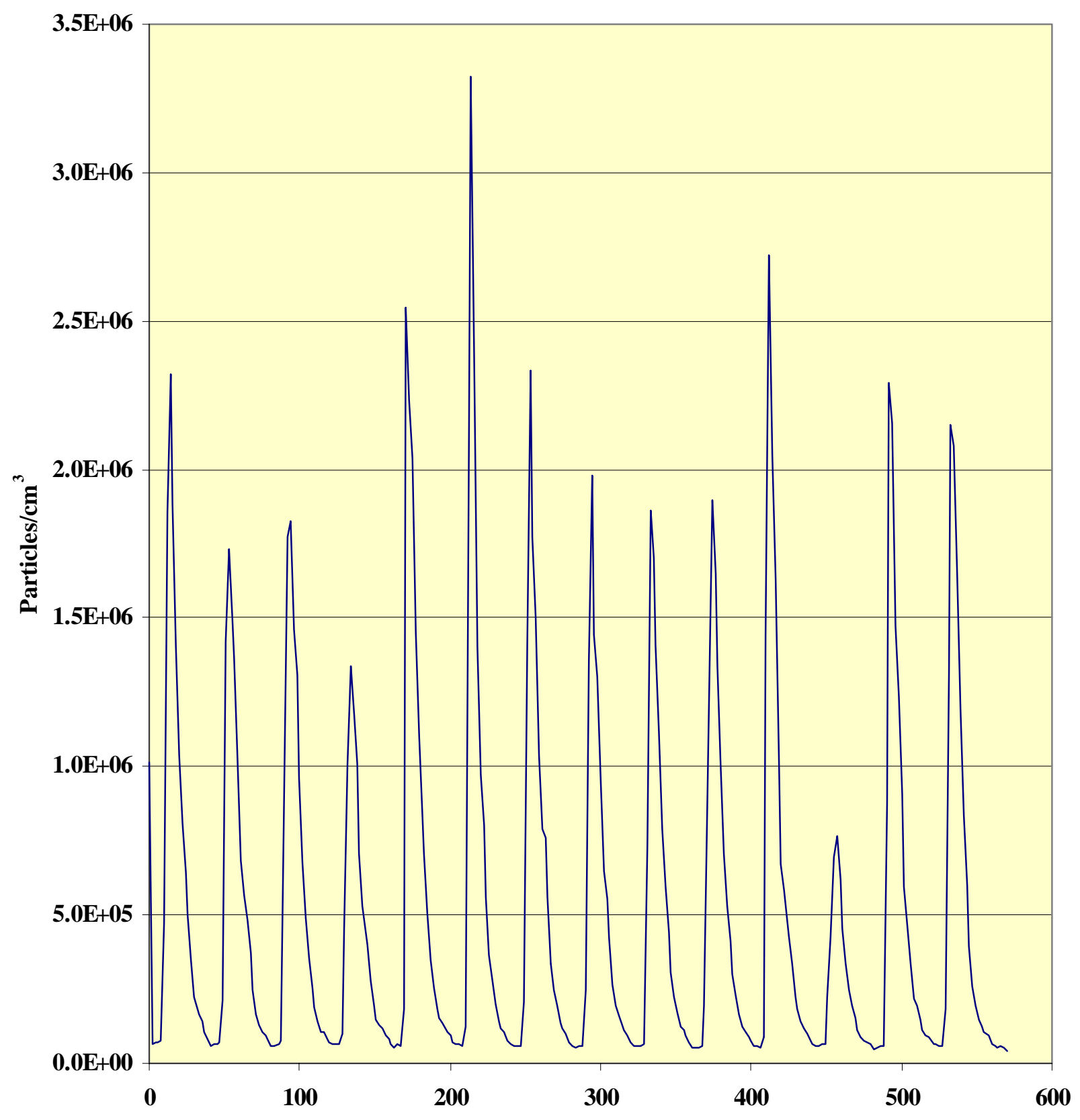

Time (seconds) 
Figure 4.74 - 60 nm SMPS Particle Concentration for Cummins B5.9 Bus 1012 CBD Run \#5 Fuel: D2

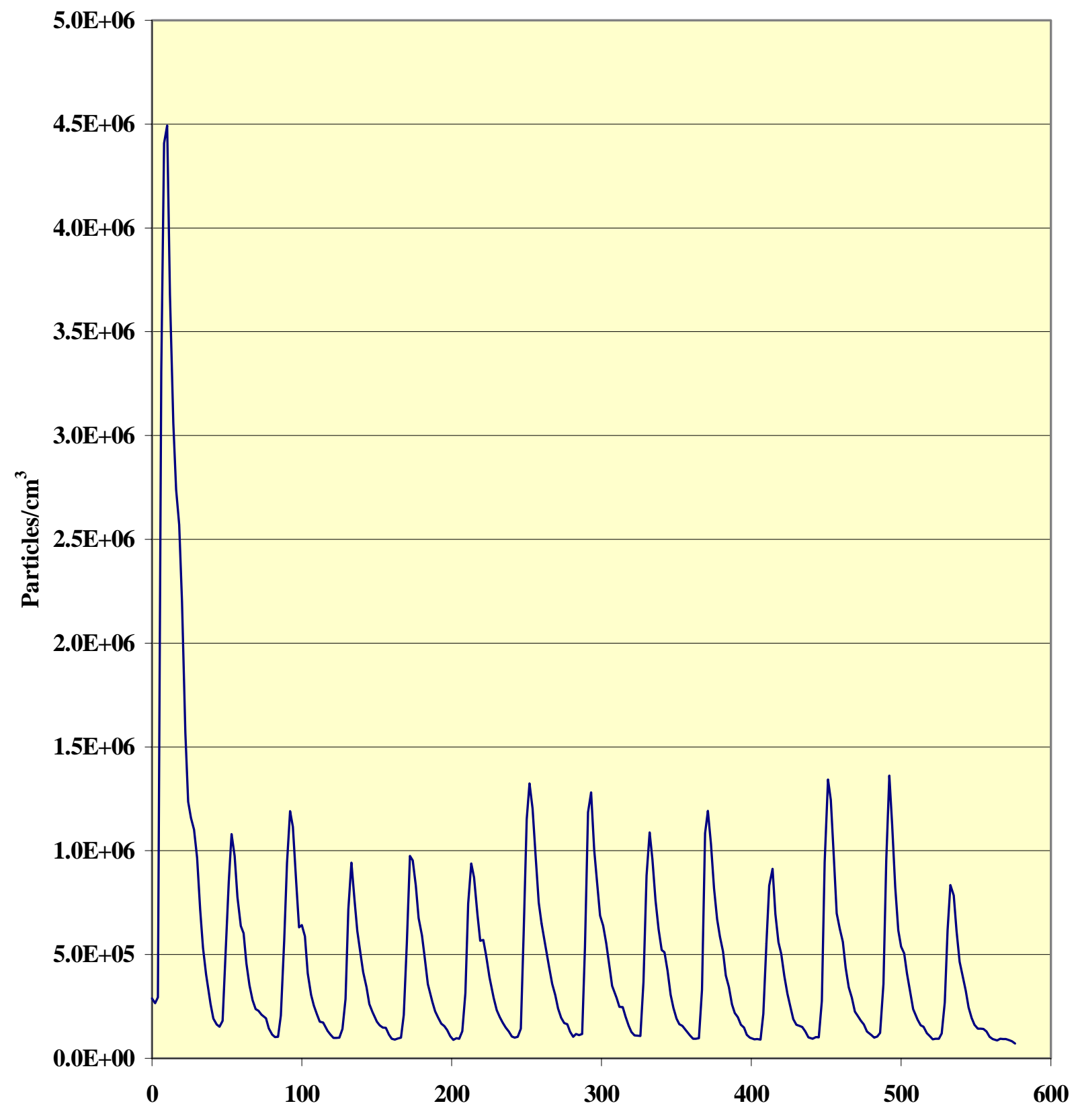

Time (seconds) 
Figure 4.75 - 100 nm SMPS Particle Concentration for Cummins B5.9 Bus 1012 CBD Run \#6 Fuel: D2

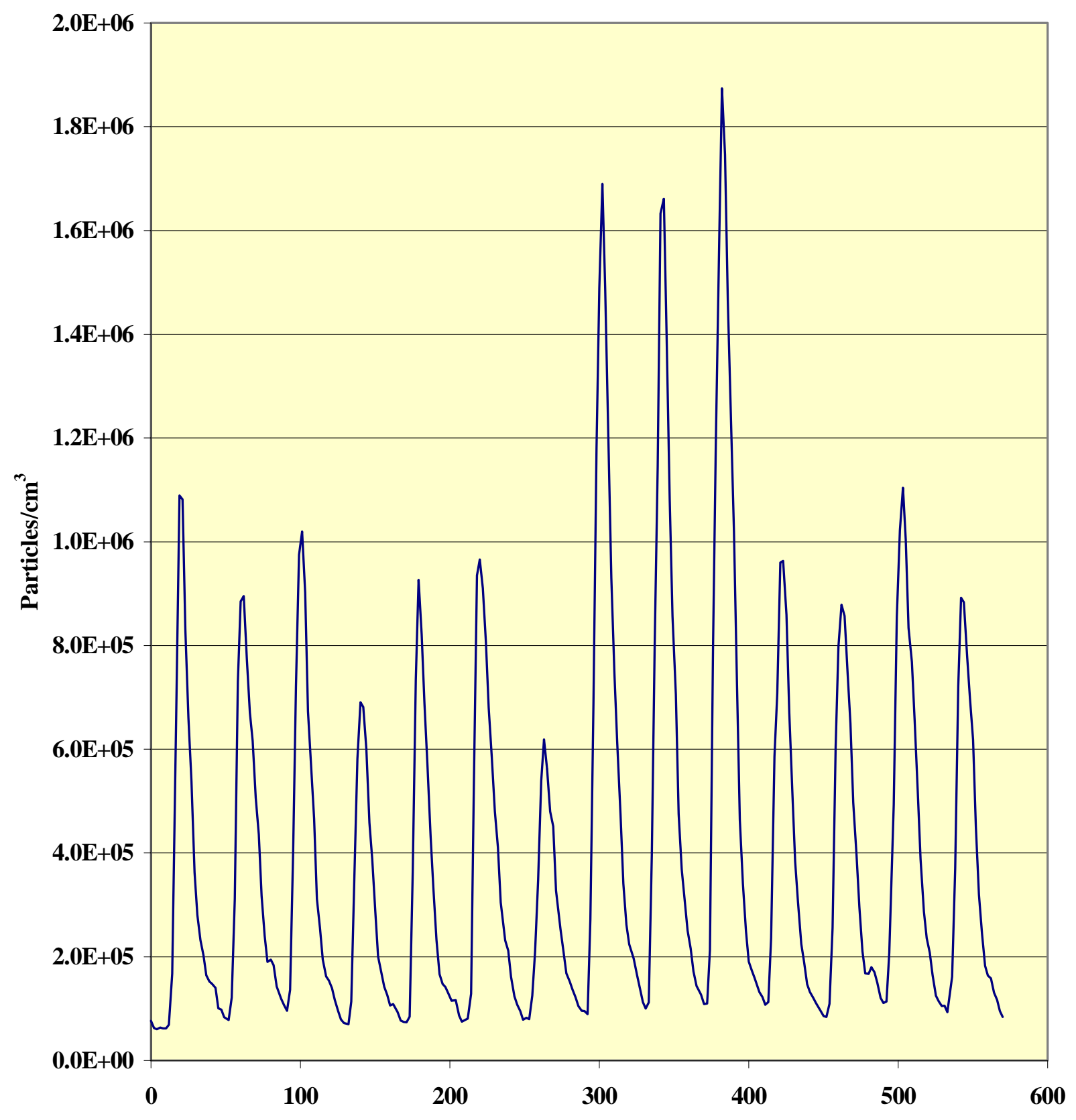

Time (seconds) 
Figure 4.76 - $100 \mathrm{~nm}$ SMPS Particle Concentration for Cummins B5.9 Bus 1012 CBD Run \#7 Fuel: D2

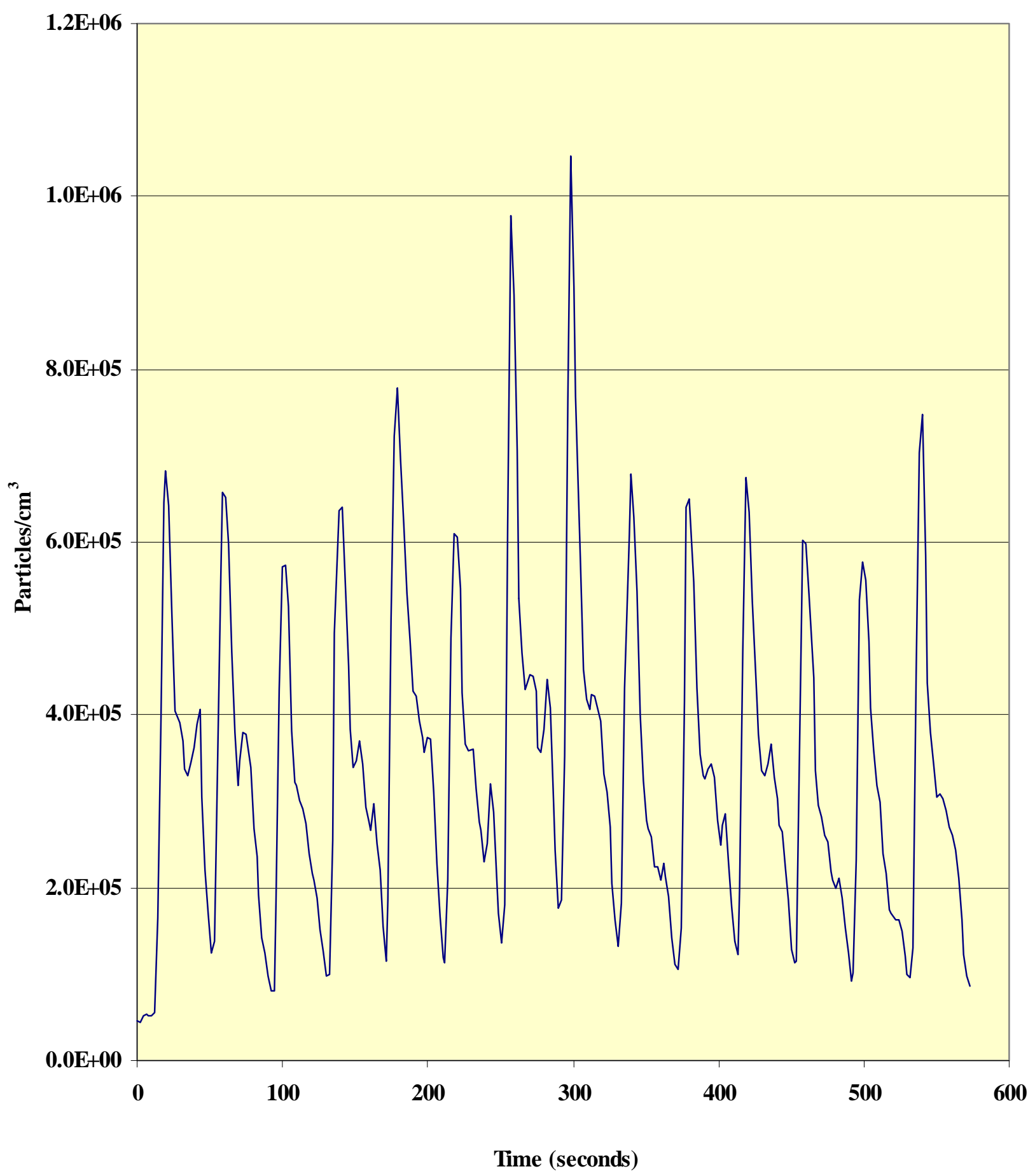


particles peak to $4.5 \times 10^{6}$. Continuing this analogy, the next three sizes traced showed large differences for each $10 \mathrm{~nm}$ increment change. Also, the majority of these particles did not appear until after half of the CBD ramps had been cycled; this was likely caused by water condensation. Exhaust temperatures rise throughout the duration of the cycle, thereby evaporating the water that is present at the outset. From the plots, it appears that the condensation was very high at the beginning of the test and greatly effected the SMPS and CPC.

Bus 1015, as mentioned earlier, was tested with and without a catalyst. There was a decided difference in the distribution between the two, and this was further examined with the SMPS data. By comparing the non-catalyst (NC) runs to those catalyst equipped (CE), the difference in the exhaust conditioning was highlighted. The CE particle concentrations at $15 \mathrm{~nm}$ are 150 to 200 times higher than the NC concentrations (see Figures $4.82 \& 4.84)$. Conversely, the $31 \mathrm{~nm}$ data showed that the NC concentrations were 5 times higher that the $\mathrm{CE}$ concentrations (see Figures $4.83 \& 4.85$ ). Figure 4.83 is a plot for one test; the SMPS was inadvertently paused twice during the course of the

cycle. The mechanism for this drastic change in particle size is uncertain, but can be attributed to the catalyst.

\subsection{DART Tests at Dallas, $T X$}

\subsubsection{Cyclone Data}

The Dallas Area Rapid Transit (DART) buses were tested with the transportable emissions laboratory in February and March of 1999. Of the vehicles tested with the 
Figure 4.77 - 15 nm SMPS Particle Concentration for Cummins B5.9 Bus 1013 CBD Run \#1 Fuel: CNG

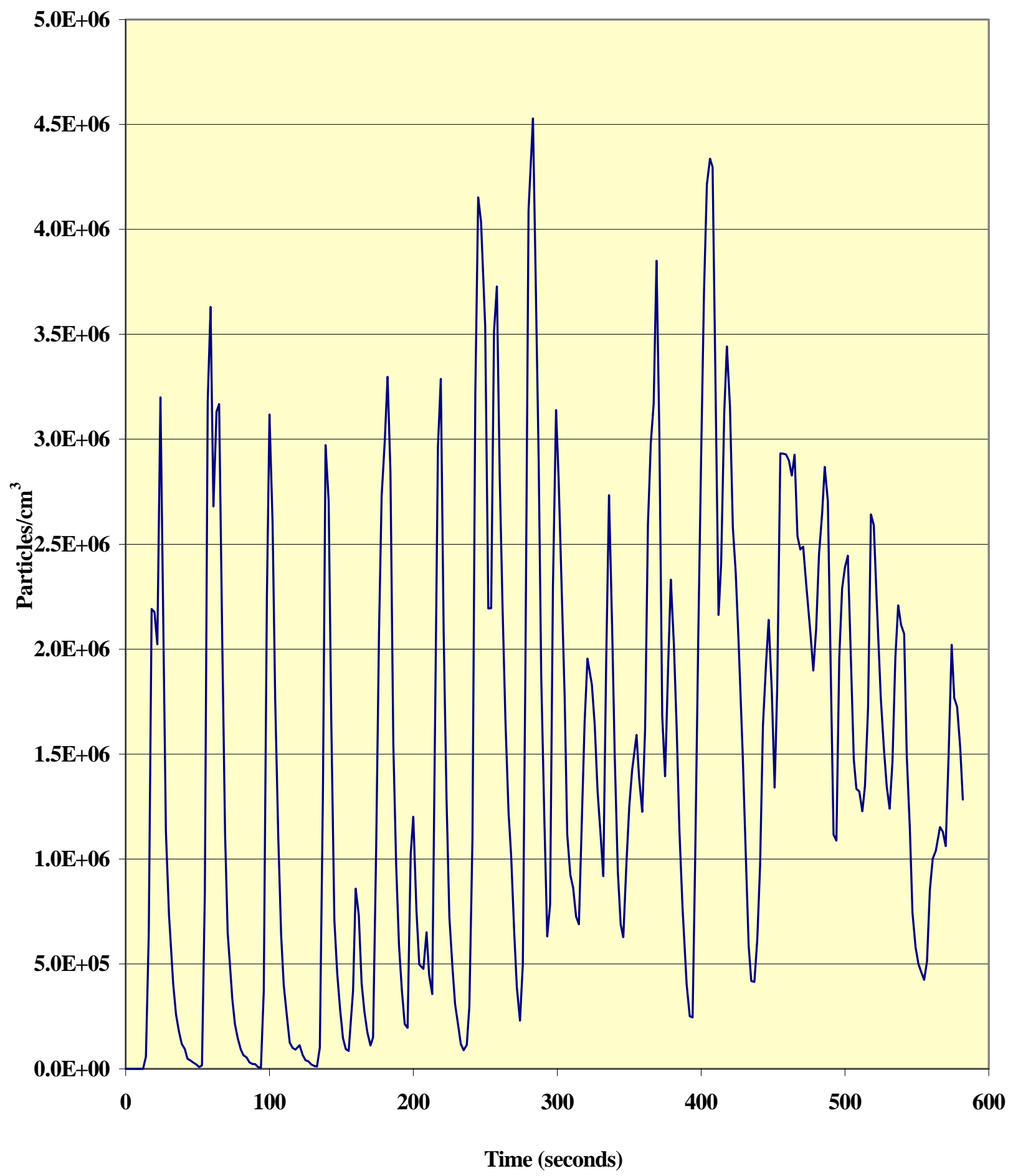


Figure 4.78 - 25 nmSMPS Particle Concentration for Cummins B5.9 Bus 1013 CBDRun\#2 Fuel: CNG

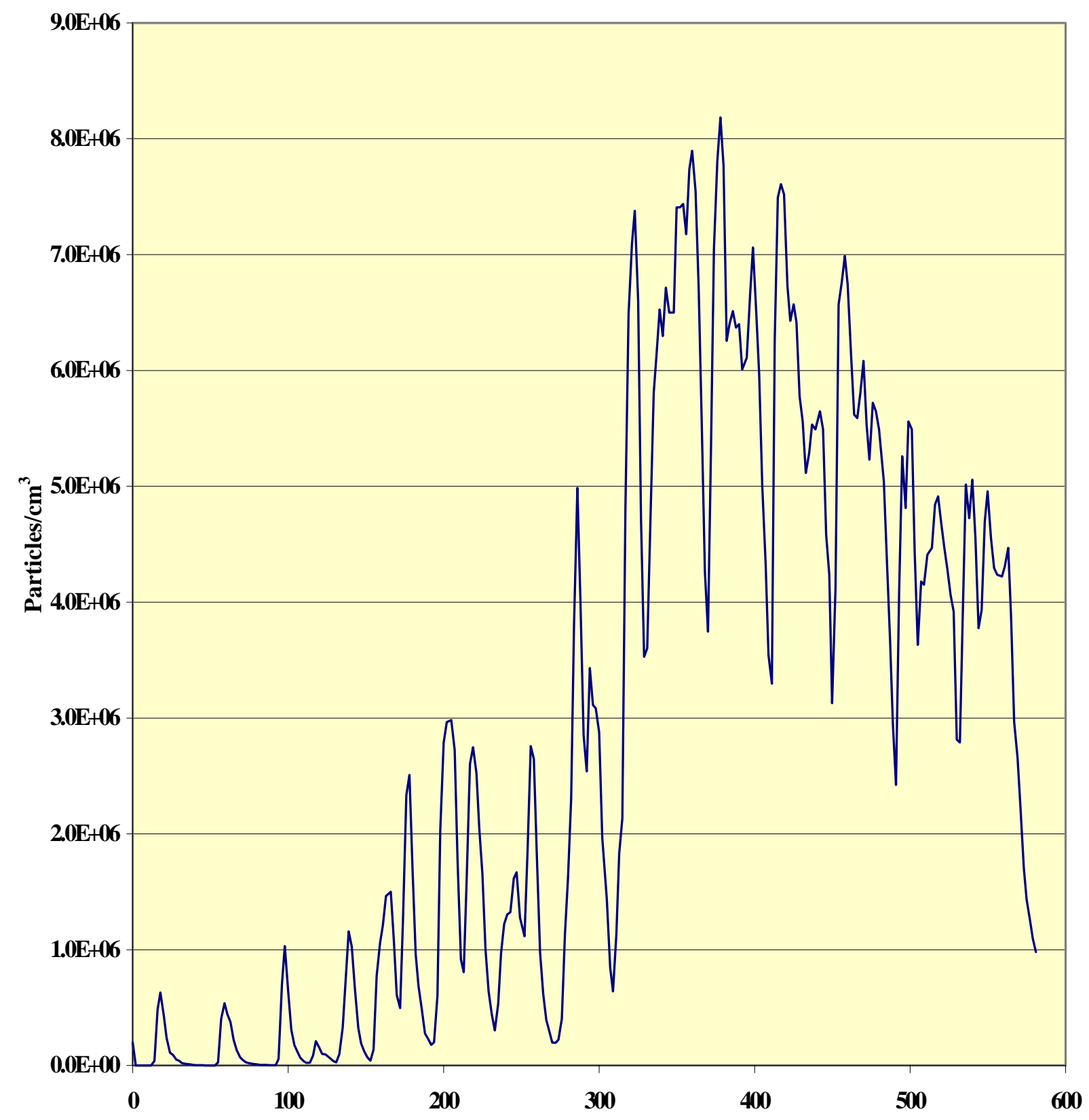

Time (seconds) 


\section{Figure 4.79 - 35 nm SMPS Particle Concentration for Cummins}

B5.9 Bus 1013 CBD Run \#3 Fuel: CNG

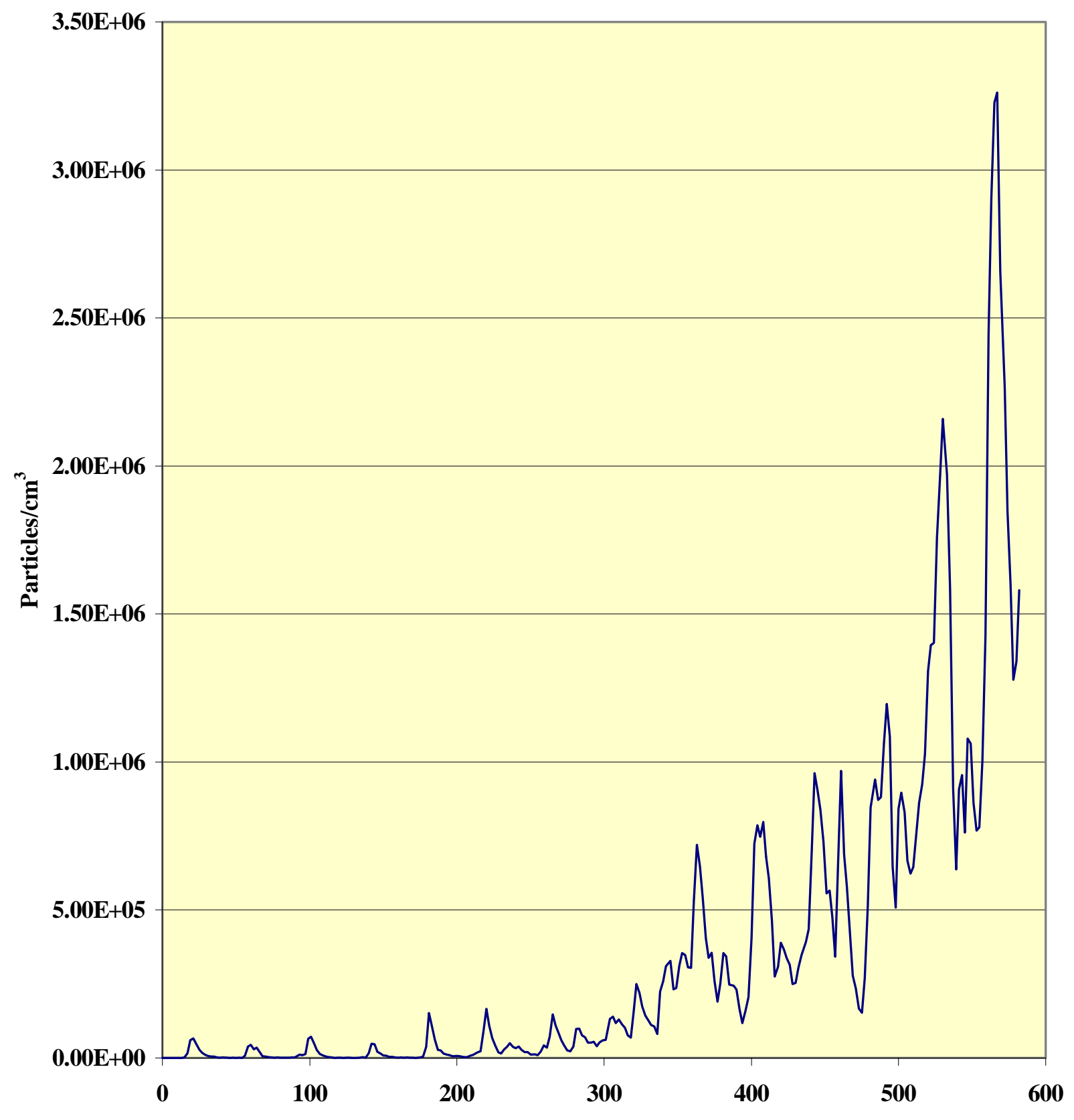

Time (seconds) 
Figure 4.80 - 45 nm SMPS Particle Concentration for Cummins B5.9 Bus 1013 CBD Run \#4 Fuel: CNG

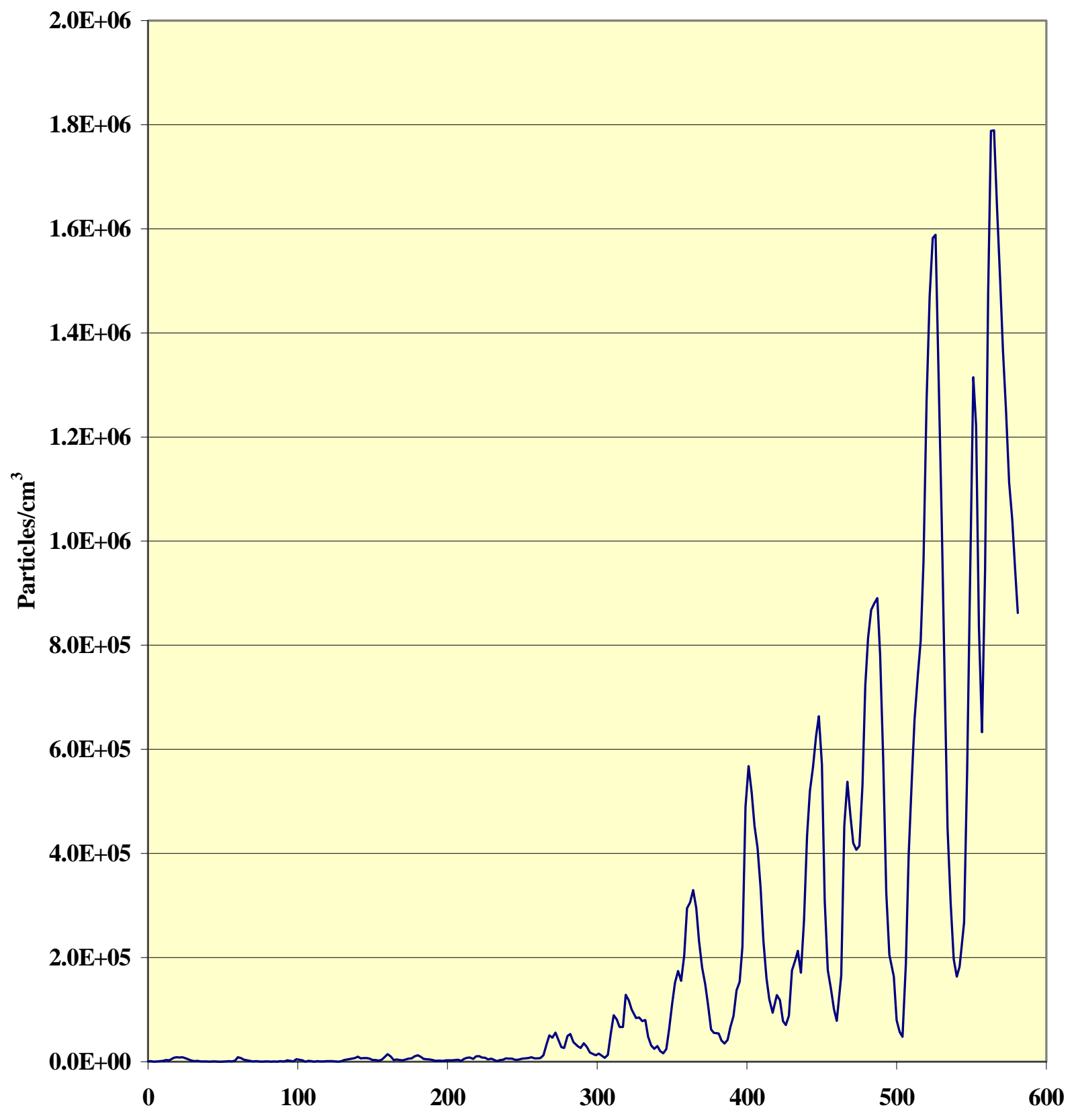

Time (seconds) 
Figure 4.81 - 55 nm SMPS Particle Concentration for Cummins B5.9 Bus 1013 CBD Run \#5 Fuel: CNG

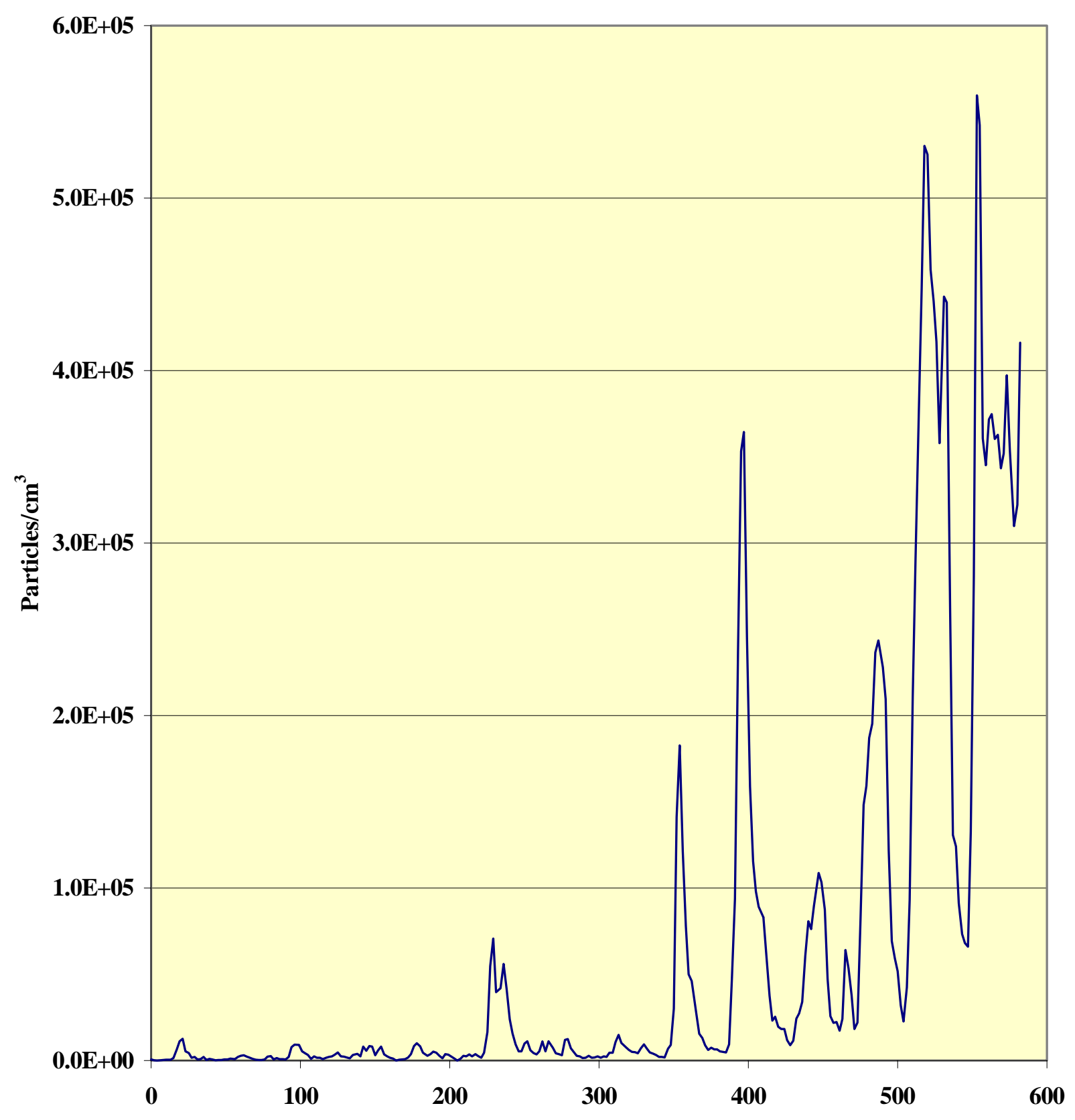

Time (seconds) 
Figure 4.82 - 15 nm SMPS Particle Concentration for Cummins B5.9 Bus 1015 CBD Run \#2 Fuel: CNG*

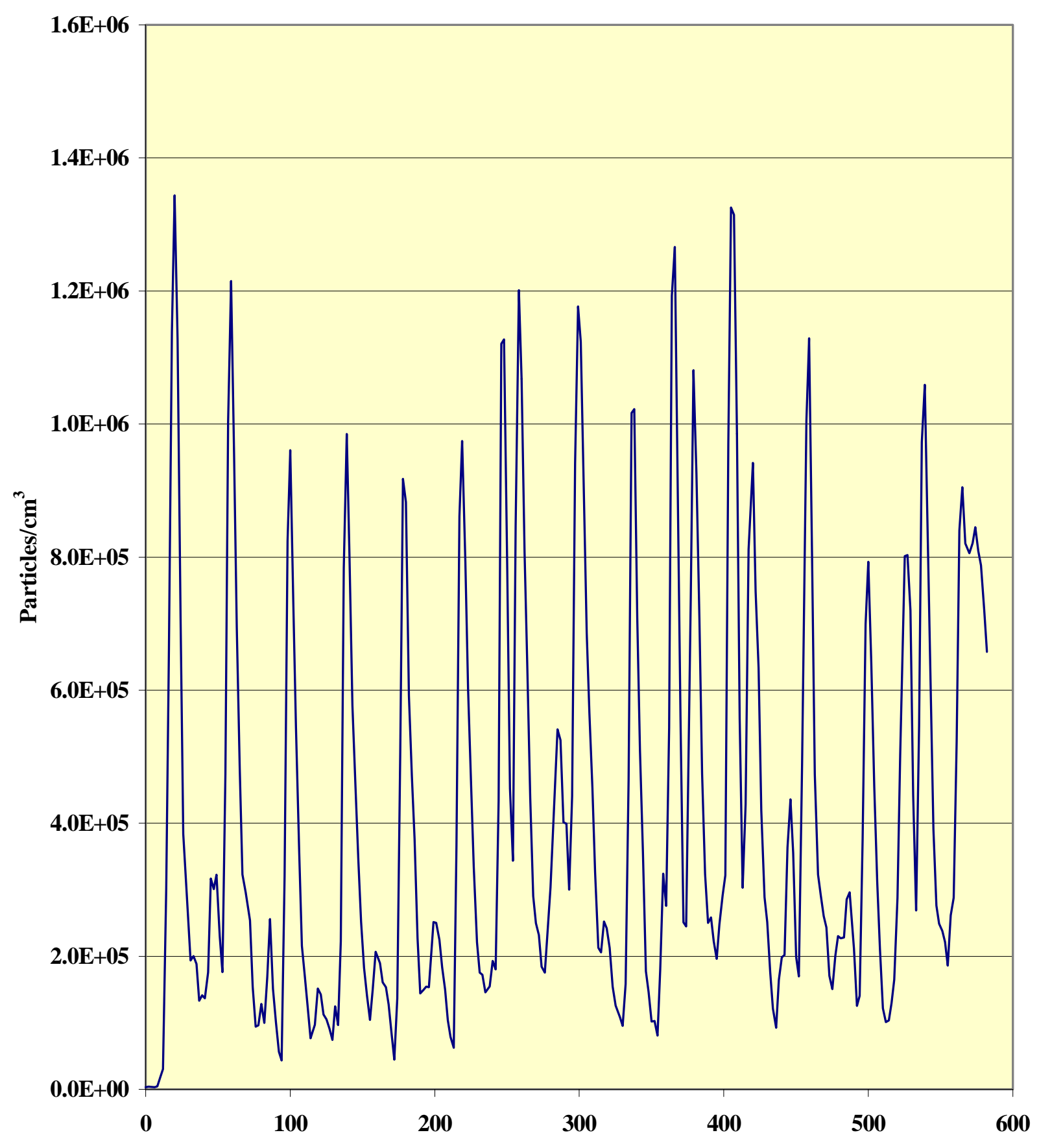

Time (seconds) 

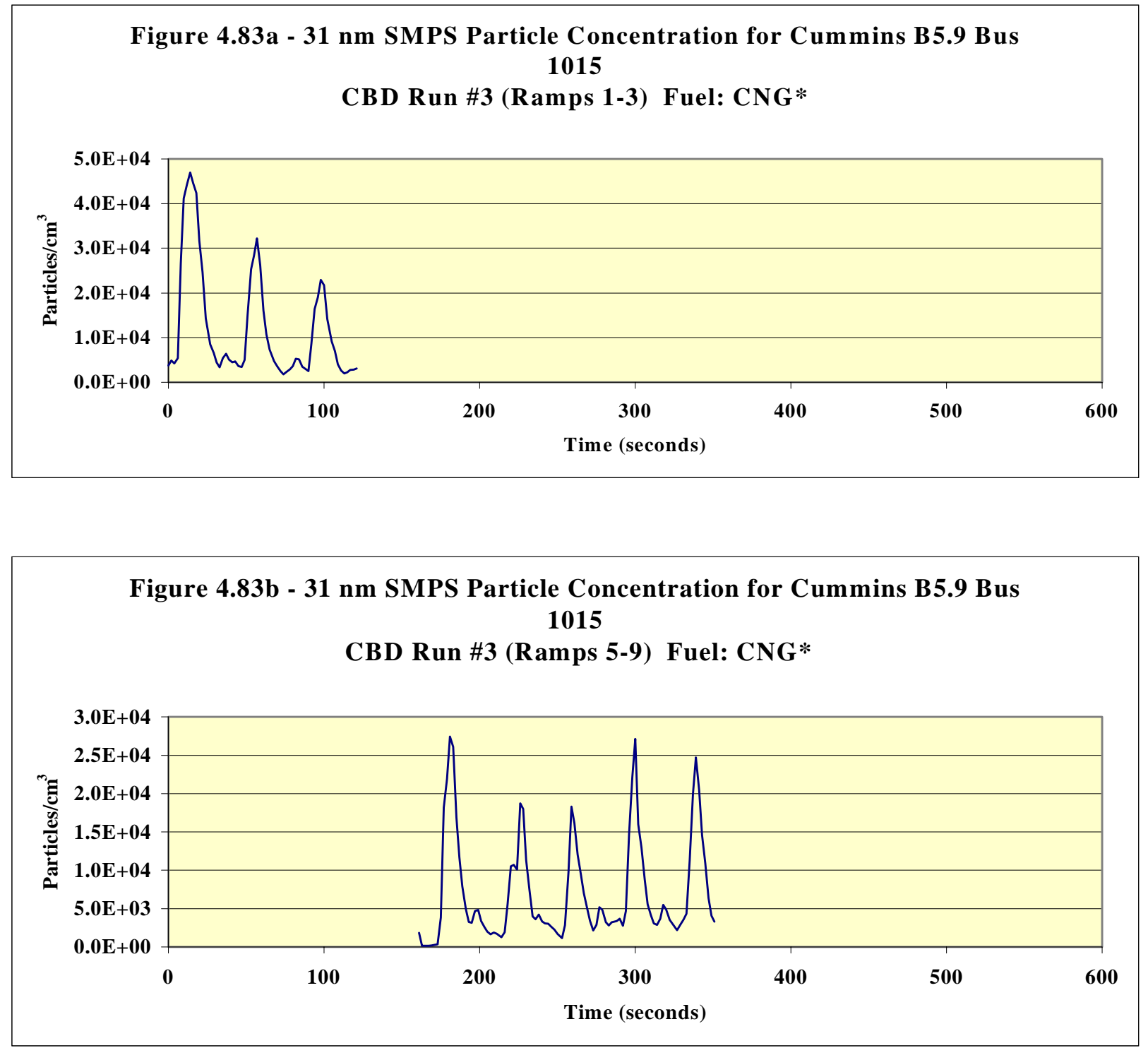

Figure 4.83c - $31 \mathrm{~nm}$ SMPS Particle Concentration for Cummins B5.9 Bus 1015

CBD Run \#3 (Ramps 11-14) Fuel: CNG*

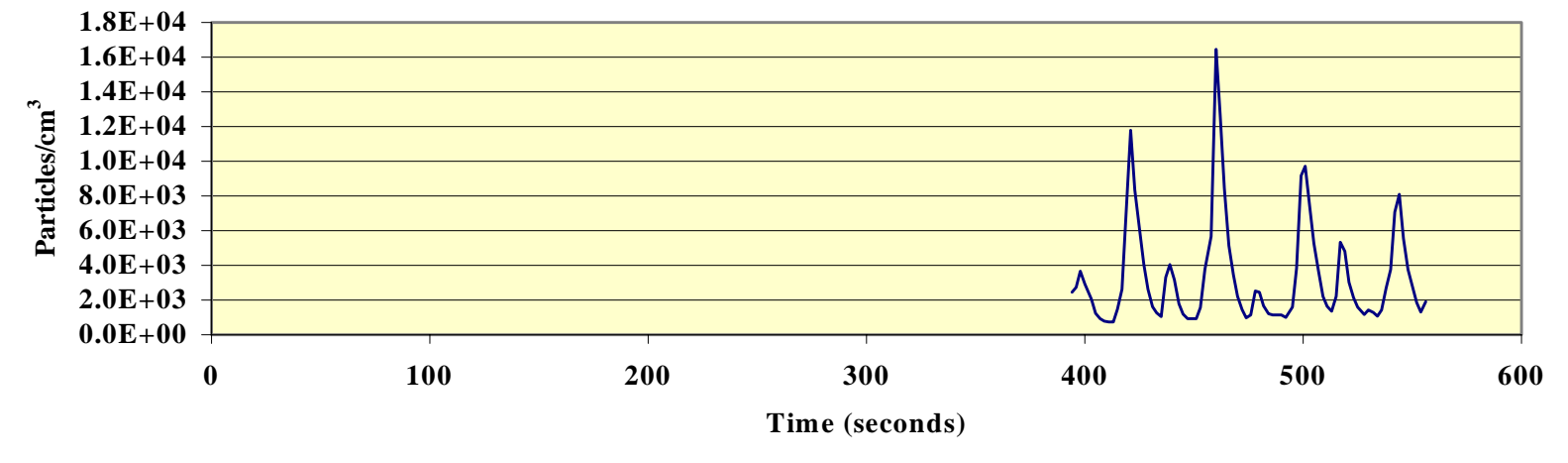


Figure 4.84 - 15 nm SMPS Particle Concentration for Cummins B5.9 Bus 1015 CBD Run \#4 Fuel: CNG*

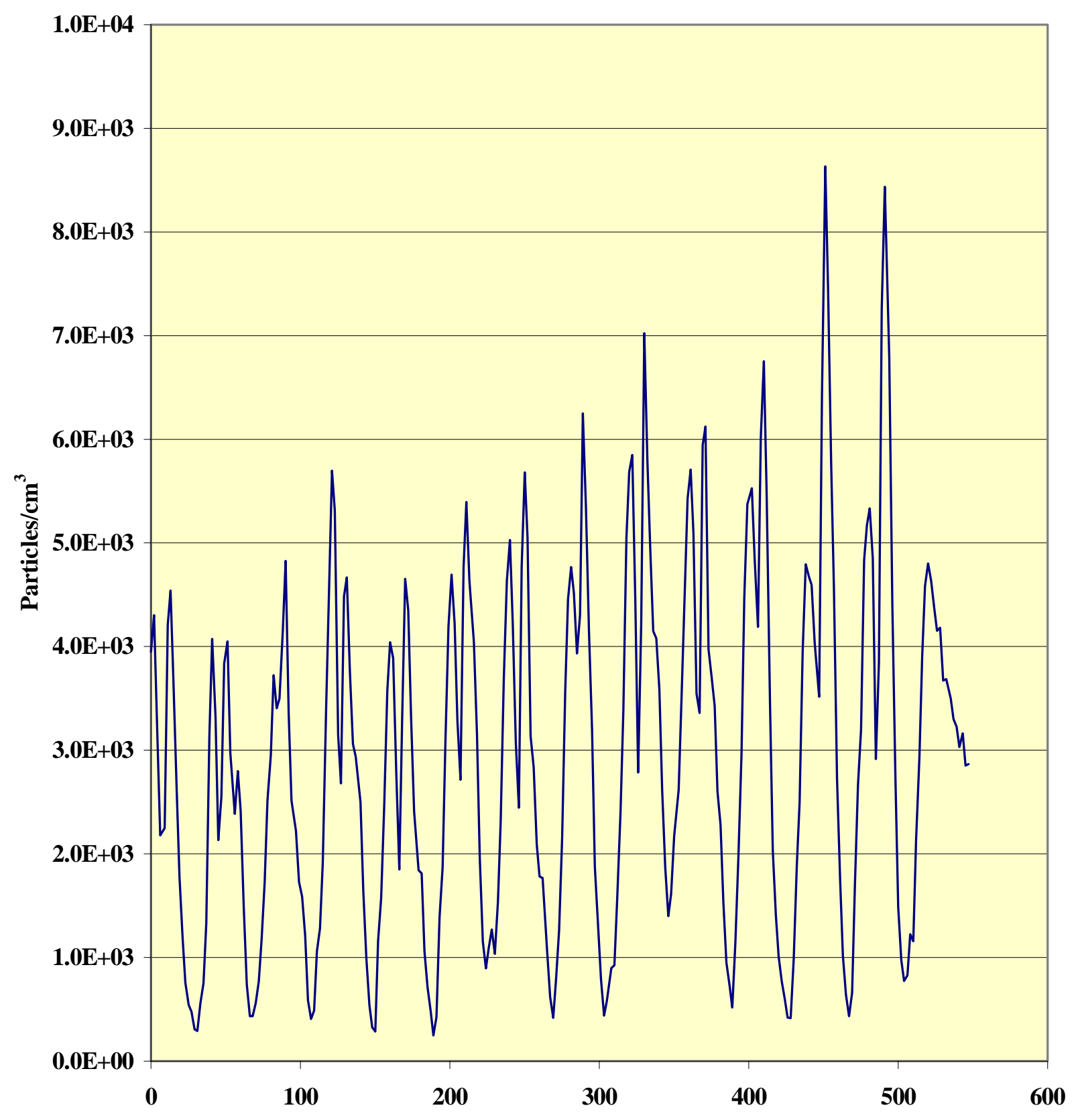

Time (seconds) 
Figure 4.85 - 31 nm SMPS Particle Concentration for Cummins B5.9 Bus 1015 CBD Run \#5 Fuel: CNG*

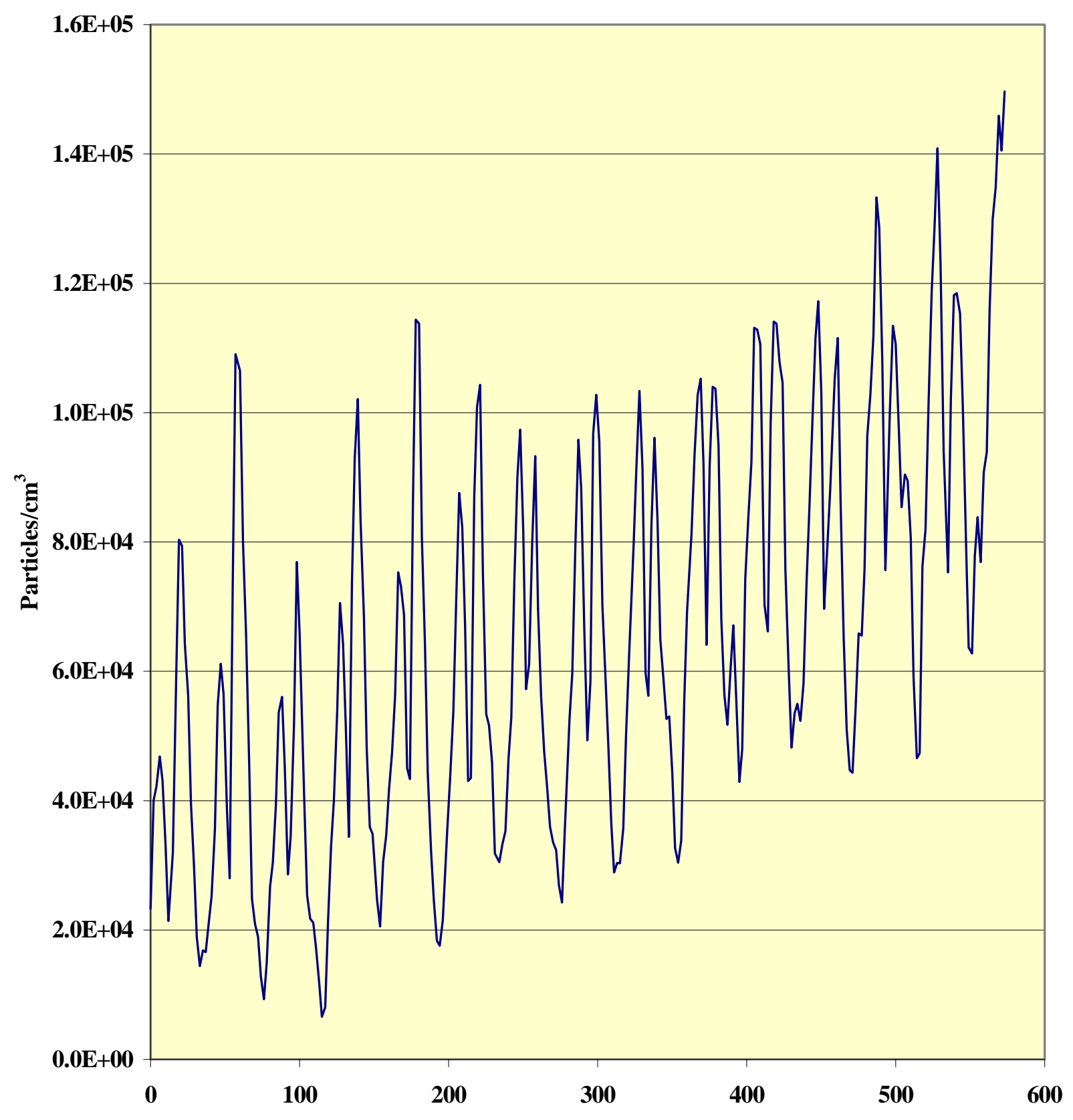

Time (seconds) 
Figure 4.86 - 50 nm SMPS Particle Concentration for Cummins B5.9 Bus 1015 CBD Run \#6 Fuel: CNG*

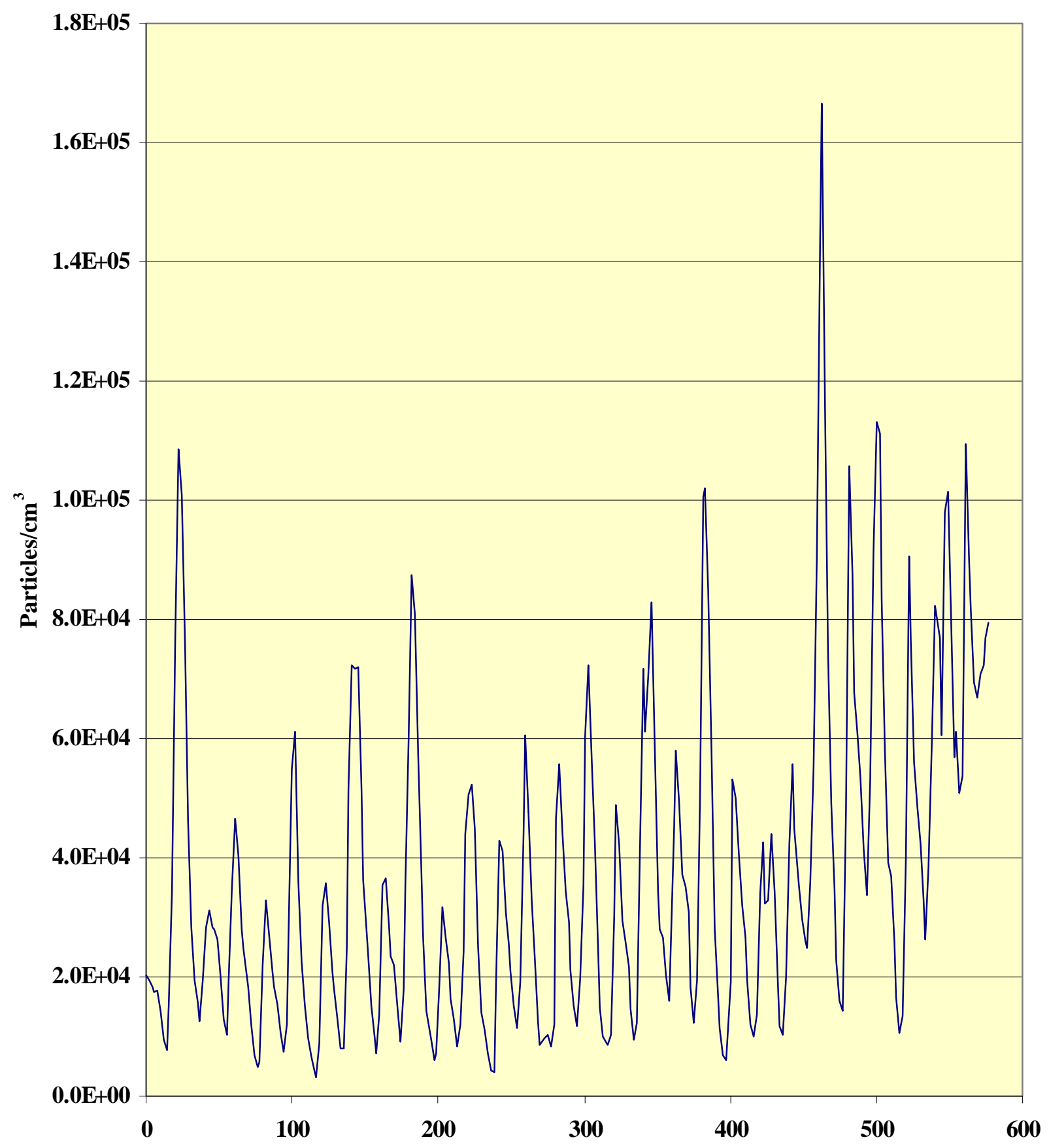

Time (seconds) 
cyclones, two were diesel fueled and eight were powered by liquefied natural gas. These buses were tested under steady state conditions at $20 \mathrm{mph}$ and the Central Business District (CBD) cycle. The cyclone data shows an interesting difference in the two vehicle types and a possible source of error in the operation of the particulate matter collection system used by the transportable laboratory.

The two diesel buses, 4220 and 4221, showed similar results statistically, though Bus 4220 had a greater mass emission rate. Based on the PM10 average mass values, Bus 4220 produced $40 \%$ more mass for the CBD cycle and $34 \%$ more for the steady state tests. Using the PM2.5 data, the differences were $47 \%$ for the CBD cycles and $50 \%$ for the steady states. This leads to the assumption that Bus 4220 produced considerably more particulate matter, with a large fraction of this less than $2.5 \mu \mathrm{m}$ in diameter.

As predicted, the tests run on the CBD cycle had much lower coefficients of variance than those at $20 \mathrm{mph}$ steady state. This can be attributed to difficulty in maintaining a constant vehicle speed for ten minutes. Test to test variability is expected due to speed fluctuations attributed to the driver. Also, the first test yielded the lowest mass values for the steady states for each bus. This was not expected. Though the engine was warmed up to a point where the exhaust gases stabilized before any data was taken for all the tests, more particulate was produced with each successive test. While this seems too systematic to be also deemed driver variability, there are reasons to believe that this was the cause of the anomaly. The steady state tests were conducted before the CBDs, thereby being the first time the driver operated the vehicle. For the first tenminute test, the driver may be more tentative and still learning the bus (i.e. where the automatic transmission shifts). With repeated trials, the driver tends to be more 
aggressive and may increase speed slightly to rise above shift points, which were generally between 18 and $21 \mathrm{mph}$ for these buses. Certainly, trying to maintain a speed while the bus is shifting is difficult, causing the driver to keep the speed above that point.

The CBD cycle tests gave very repeatable data, as can be seen from the low coefficients of variance $(\mathrm{CV})$ and standard deviations in the Tables $4.9-4.11$. One very plangent result was the fact that the mass values given by the secondary dilution tunnel system were lower than those from the PM10 cyclone. This should not occur since the secondary dilution gravimetric filter collection method has no size selective abilities. While the PM10 cyclone will remove those particles larger than $10 \mu \mathrm{m}$, the $70 \mathrm{~mm}$ filter will collect them. This effect was even more noticeable in the LNG data and clearly shows an error in either collection or the data reduction. This will be analyzed in depth after the presentation of all ten of the buses.

The two cyclones were compared to each other with respect to total, actual mass. These tables can be found in Appendix B. Bus 4220 showed that the cyclones collected nearly equivalent amounts of actual particulate matter, with the PM10 yielding less than $1 \%$ more mass than the PM2.5 for the steady state runs. This trend continues for all other diesel tests except that the percentage increases to a maximum of $25 \%$. This follows previous particle size data. Diesel fuel combustion generally produces particles less than $1.0 \mu \mathrm{m}$ in diameter. Kittelson et al. (1998) gives the mass percent above $1.0 \mu \mathrm{m}$ to be 5 to $20 \%$. The CONCAWE (1998) study found that $85 \%$ of the mass of diesel particulate emissions was less than $1.0 \mu \mathrm{m}$. The fact that the cyclone data followed this pattern was crucial. The serious errors in the RTD/SKIP cyclone testing were thus eliminated. Following the RTD/SKIP testing, the sampling and flow rate control systems were 
revamped. The new flow control systems were employed for the DART buses. Since several researchers have found similar diesel data, further tests were not performed. The diesel buses were used to verify the cyclone collection system.

Table 4.9 - Statistical Analysis of Diesel Fueled DART Buses 4220 and 4221

\begin{tabular}{|c|c|c|c|}
\hline & & $\begin{array}{c}\text { Bus } 4220 \\
\text { CBD }\end{array}$ & $\begin{array}{c}\text { Bus } 4221 \\
\text { CBD }\end{array}$ \\
\hline $70 \mathrm{~mm}$ & $\begin{array}{l}\text { Average Mass (grams) } \\
\text { Standard Deviation } \\
\text { Coefficient of Variance } \\
\end{array}$ & $\begin{array}{c}1.064 \\
0.078 \\
7 \%\end{array}$ & $\begin{array}{c}0.611 \\
0.010 \\
2 \%\end{array}$ \\
\hline PM2.5 & $\begin{array}{l}\text { Average Mass (grams) } \\
\text { Standard Deviation } \\
\text { Coefficient of Variance }\end{array}$ & $\begin{array}{c}1.348 \\
0.034 \\
3 \%\end{array}$ & $\begin{array}{c}0.831 \\
0.068 \\
8 \%\end{array}$ \\
\hline PM10 & $\begin{array}{l}\text { Average Mass (grams) } \\
\text { Standard Deviation } \\
\text { Coefficient of Variance }\end{array}$ & $\begin{array}{c}1.424 \\
0.084 \\
6 \%\end{array}$ & $\begin{array}{c}0.943 \\
0.097 \\
10 \%\end{array}$ \\
\hline
\end{tabular}

\begin{tabular}{|c|c|c|c|}
\hline & & $\begin{array}{l}\text { Bus 4220 } \\
\text { SS-20mph }\end{array}$ & $\begin{array}{l}\text { Bus 4221 } \\
\text { SS-20mph }\end{array}$ \\
\hline $70 \mathrm{~mm}$ & $\begin{array}{l}\text { Average Mass (grams) } \\
\text { Standard Deviation } \\
\text { Coefficient of Variance }\end{array}$ & $\begin{array}{c}0.747 \\
0.252 \\
34 \%\end{array}$ & $\begin{array}{c}0.379 \\
0.039 \\
10 \%\end{array}$ \\
\hline PM2.5 & $\begin{array}{l}\text { Average Mass (grams) } \\
\text { Standard Deviation } \\
\text { Coefficient of Variance }\end{array}$ & $\begin{array}{c}0.963 \\
0.298 \\
31 \%\end{array}$ & $\begin{array}{c}0.577 \\
0.070 \\
12 \%\end{array}$ \\
\hline PM10 & $\begin{array}{l}\text { Average Mass (grams) } \\
\text { Standard Deviation } \\
\text { Coefficient of Variance }\end{array}$ & $\begin{array}{c}0.953 \\
0.213 \\
22 \%\end{array}$ & $\begin{array}{c}0.678 \\
0.185 \\
27 \%\end{array}$ \\
\hline
\end{tabular}


While diesel particulate matter is mostly submicron is diameter, natural gas particulate is believed to be much smaller. However, the cyclones show that this may only be true for certain vehicles. Depending on the bus, the PM10 cyclone had higher mass values than the PM2.5 by anywhere from 0 - 5\% for Bus 4322 to $60-100 \%$ for Bus 4321. This would tend to support the idea that the particles in the range of at least 2.5 to $10 \mu \mathrm{m}$ are not formed by natural gas combustion but rather other factors such as cylinder wear, oil in the chamber, etc.

The steady state tests conducted resulted in similar percent differences between the cyclones, with Buses 4329 and 4321 being the exceptions. This will be detailed later. This higher mass value for the PM10 was generally within a range of $10-25 \%$. Unlike the CBD tests, the steady states did not deviate greatly. The effects of accelerations/decelerations appear to cause this discrepancy between the two modes. The diesel vehicles do not show this, adding support to the claim that engine wear and condition are possible causes of the large quantity of particulate collected in the $2.5-10$ $\mu \mathrm{m}$ range.

It should be noted that the PM mass emission rates from the LNG vehicles were extremely low and this fact increased the variability in the gravimetric analyses of cyclone filters. For example, Bus 4321 had a difference in the range of 60 to $100 \%$, 4322 , in the range of 0 to $5 \%$, and 4328 from 4 to $34 \%$. The results from Bus 4322 were the most expected as the particulate emissions are thought to be much smaller than the cyclone cut diameters. Buses 4321 and 4324 on the other hand do not follow this pattern. These were also the highest TPM emitters, more than twice the other buses. It appears that these two vehicles suffered the most from non-combustion particle emissions. 
Table 4.10 - Statistical Analysis of LNG Fueled DART

Buses 4320, 4321, 4322 and 4323

\begin{tabular}{|c|c|c|c|}
\hline & & $\begin{array}{c}\text { Bus 4320* } \\
\text { CBD }\end{array}$ & $\begin{array}{c}\text { Bus } 4321 \\
\text { CBD }\end{array}$ \\
\hline $70 \mathrm{~mm}$ & $\begin{array}{l}\text { Average Mass (grams) } \\
\text { Standard Deviation } \\
\text { Coefficient of Variance }\end{array}$ & $\begin{array}{l}- \\
- \\
-\end{array}$ & $\begin{array}{c}0.015 \\
0.005 \\
36 \%\end{array}$ \\
\hline PM2.5 & $\begin{array}{l}\text { Average Mass (grams) } \\
\text { Standard Deviation } \\
\text { Coefficient of Variance }\end{array}$ & $\begin{array}{c}0.080 \\
0.004 \\
5 \%\end{array}$ & $\begin{array}{c}0.093 \\
0.021 \\
23 \%\end{array}$ \\
\hline PM10 & $\begin{array}{l}\text { Average Mass (grams) } \\
\text { Standard Deviation } \\
\text { Coefficient of Variance }\end{array}$ & $\begin{array}{c}0.092 \\
0.001 \\
1 \%\end{array}$ & $\begin{array}{c}0.212 \\
0.045 \\
21 \%\end{array}$ \\
\hline
\end{tabular}

\begin{tabular}{|c|c|c|c|}
\hline & & $\begin{array}{c}\text { Bus } 4322 \\
\text { CBD }\end{array}$ & $\begin{array}{c}\text { Bus } 4323 \\
\text { CBD }\end{array}$ \\
\hline $70 \mathrm{~mm}$ & $\begin{array}{l}\text { Average Mass (grams) } \\
\text { Standard Deviation } \\
\text { Coefficient of Variance }\end{array}$ & $\begin{array}{c}0.059 \\
0.007 \\
13 \%\end{array}$ & $\begin{array}{l}- \\
-\end{array}$ \\
\hline PM2.5 & $\begin{array}{l}\text { Average Mass (grams) } \\
\text { Standard Deviation } \\
\text { Coefficient of Variance }\end{array}$ & $\begin{array}{c}0.090 \\
0.013 \\
14 \%\end{array}$ & $\begin{array}{c}0.048 \\
0.003 \\
7 \%\end{array}$ \\
\hline PM10 & $\begin{array}{l}\text { Average Mass (grams) } \\
\text { Standard Deviation } \\
\text { Coefficient of Variance }\end{array}$ & $\begin{array}{c}0.093 \\
0.015 \\
16 \%\end{array}$ & $\begin{array}{c}0.062 \\
0.006 \\
10 \%\end{array}$ \\
\hline
\end{tabular}

* Two tests yielded higher mass values for the PM2.5 cyclone than the PM10.

These were omitted from the analysis. 
Table 4.11 - Statistical Analysis of LNG Fueled DART

Buses 4324, 4325, 4328 and 4329

\begin{tabular}{|c|c|c|c|}
\hline & & $\begin{array}{c}\text { Bus } 4324 * \\
\text { CBD }\end{array}$ & $\begin{array}{c}\text { Bus } 4325 \\
\text { CBD }\end{array}$ \\
\hline $70 \mathrm{~mm}$ & $\begin{array}{l}\text { Average Mass (grams) } \\
\text { Standard Deviation } \\
\text { Coefficient of Variance }\end{array}$ & $\begin{array}{c}0.143 \\
0.010 \\
7 \%\end{array}$ & $\begin{array}{c}0.031 \\
0.001 \\
4 \%\end{array}$ \\
\hline PM2.5 & $\begin{array}{l}\text { Average Mass (grams) } \\
\text { Standard Deviation } \\
\text { Coefficient of Variance }\end{array}$ & $\begin{array}{c}0.184 \\
0.046 \\
25 \%\end{array}$ & $\begin{array}{c}0.045 \\
0.025 \\
56 \%\end{array}$ \\
\hline PM10 & $\begin{array}{l}\text { Average Mass (grams) } \\
\text { Standard Deviation } \\
\text { Coefficient of Variance }\end{array}$ & $\begin{array}{c}0.248 \\
0.017 \\
7 \%\end{array}$ & $\begin{array}{c}0.071 \\
0.013 \\
18 \%\end{array}$ \\
\hline
\end{tabular}

\begin{tabular}{|c|c|c|c|}
\hline & & $\begin{array}{c}\text { Bus } 4328 * \\
\text { CBD }\end{array}$ & $\begin{array}{c}\text { Bus } 4329 * * \\
\text { CBD }\end{array}$ \\
\hline $70 \mathrm{~mm}$ & $\begin{array}{l}\text { Average Mass (grams) } \\
\text { Standard Deviation } \\
\text { Coefficient of Variance }\end{array}$ & $\begin{array}{l}- \\
- \\
-\end{array}$ & $\begin{array}{c}0.052 \\
0.013 \\
26 \%\end{array}$ \\
\hline PM2.5 & $\begin{array}{l}\text { Average Mass (grams) } \\
\text { Standard Deviation } \\
\text { Coefficient of Variance }\end{array}$ & $\begin{array}{c}0.083 \\
0.011 \\
14 \%\end{array}$ & $\begin{array}{c}0.100 \\
0.010 \\
10 \%\end{array}$ \\
\hline PM10 & $\begin{array}{l}\text { Average Mass (grams) } \\
\text { Standard Deviation } \\
\text { Coefficient of Variance }\end{array}$ & $\begin{array}{c}0.095 \\
0.005 \\
5 \%\end{array}$ & $\begin{array}{c}0.150 \\
0.040 \\
27 \%\end{array}$ \\
\hline
\end{tabular}

* First test was omitted from analysis.

** Three tests yielded higher mass values for the PM2.5 cyclone than the PM10.

These were omitted from the analysis. 
Though it should be noted that the highest TPM values from the LNG vehicles are still more than two and a half times lower than the best diesel. Bus 4329 resulted in three of five CBD tests with higher PM2.5 mass values than PM10. This was caused by water condensation in the sampling lines and on the filters themselves. After a test was completed, the lines had to be drained because there was so much water in them. The effects on the sampling system can be seen by analyzing the instantaneous flow rates for the cyclones. They were highly unstable compared to flows from the other buses. Figures $4.87-4.89$ below show several flow rates comparing tests on steady state and CBD modes, and the effects of water condensation on the CBD cycle. The flow distortion was also believed to be a major cause of the cyclone errors in the RTD/SKIP testing. Water condensation was also a factor for those natural gas vehicles and has shown to decrease the collection efficiency of the PM10 cyclone dramatically.

One of the other possible rewards of using the PM10 cyclone is that a comparison can be made between the cyclone data and the secondary dilution tunnel based TPM collection method to find those particles that are greater than $10 \mu \mathrm{m}$. However, as the data clearly shows, this was not possible. For every test on every bus, regardless of fuel, the $70 \mathrm{~mm}$ filter weights returned values less than those from the PM10 cyclone. In fact, for the LNG steady states, six of seven yielded negative masses. Natural gas burns clean, but not that clean! For the LNG CBD tests, the $70 \mathrm{~mm}$ filter collected generally $50 \%$ less particulate matter than the PM10 cyclone; Bus 4321 had the $70 \mathrm{~mm}$ values between 150\% to $185 \%$ lower than the cyclone. Even the diesel data showed similar results. Taking the negative mass values into account, it seems likely that it is the 

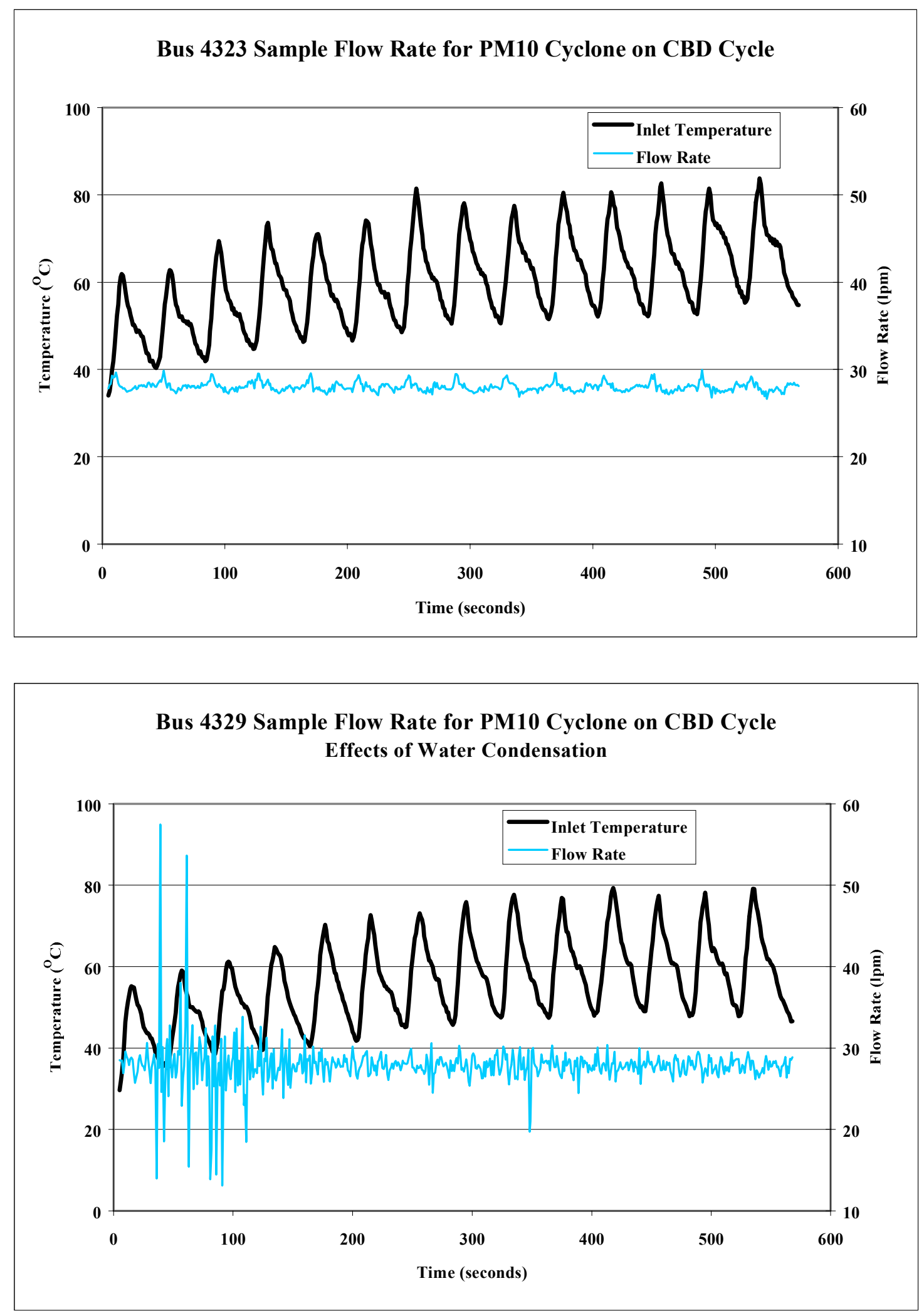

Figure 4.87 - PM10 Cyclone Sample Flow Rates 

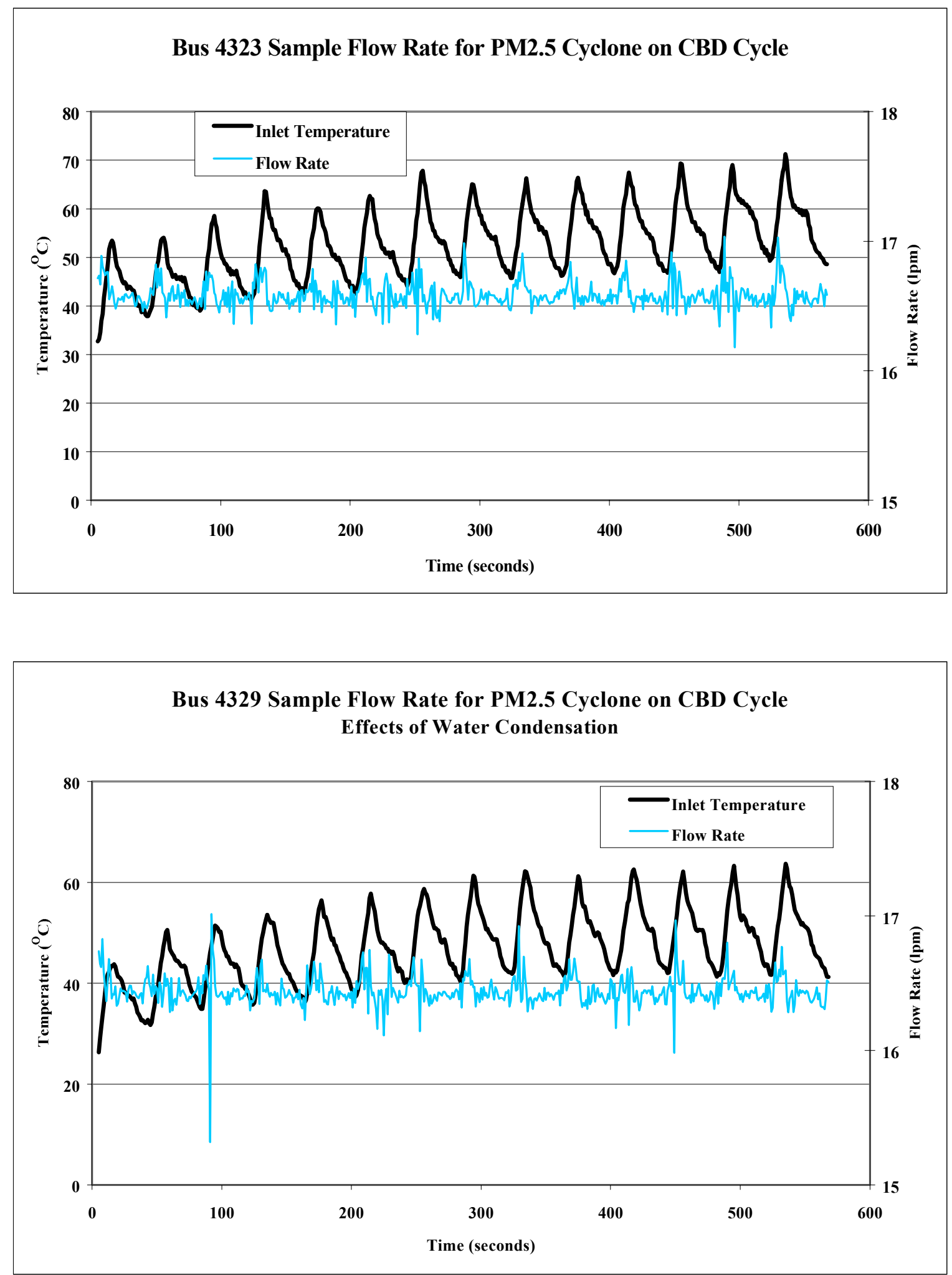

Figure 4.88 - PM2.5 Cyclone Sample Flow Rates 

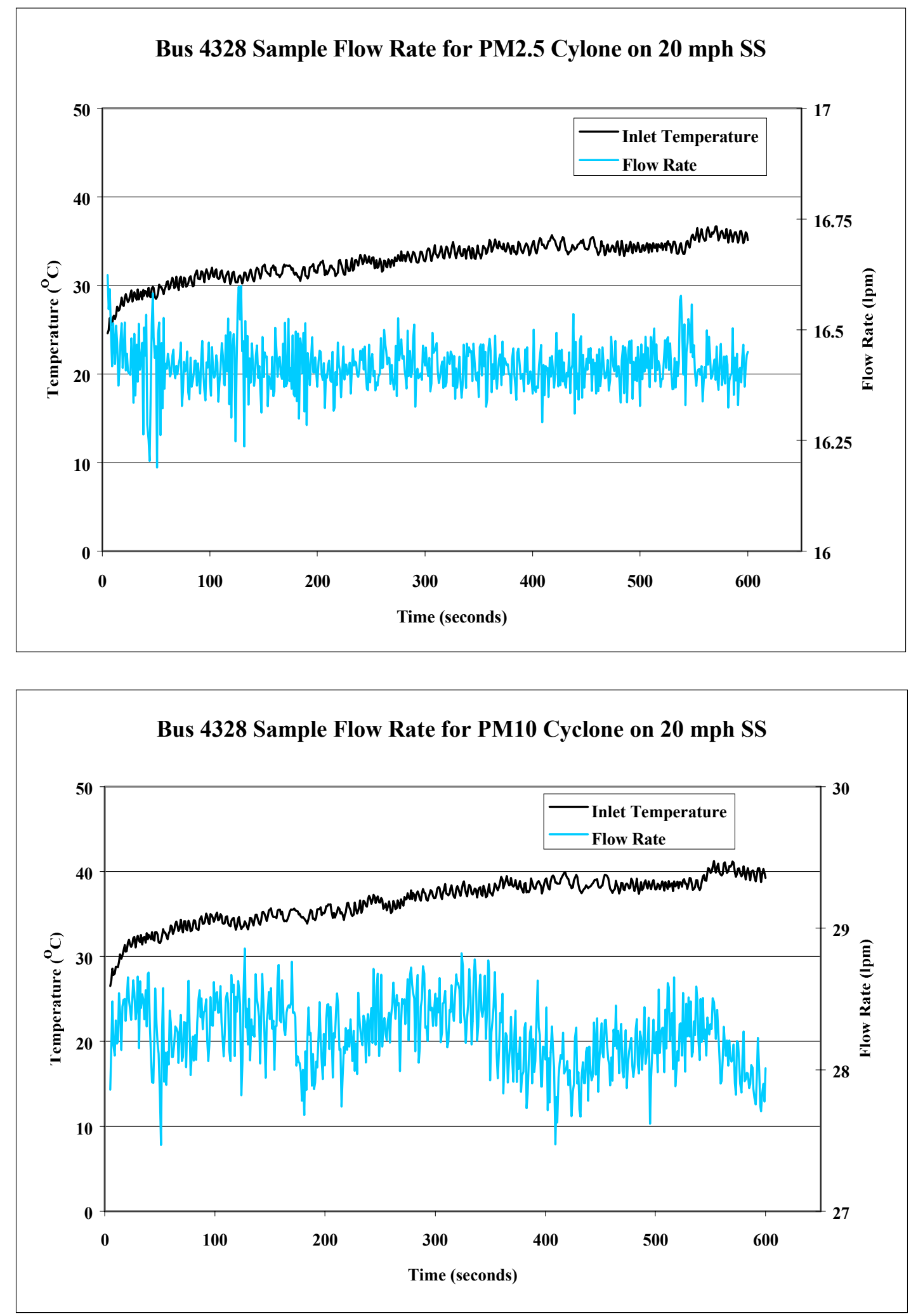

Figure 4.89 - Steady State Sample Flow Rates for Cyclones 
secondary dilution tunnel collection method that is incorrect, not the cyclone. Actually, it is not believed to be the method itself, but the background removal that is the cause.

The cyclones and the $70 \mathrm{~mm}$ filter system used the same method of background removal, made necessary since the dilution air was unfiltered. At the end of a testing day, the ambient air was sampled by the dilution tunnel for a period long enough to allow sufficient mass to accumulate on the filters. This value was then divided by the total volume sampled. It should be noted that this quantity of particles pervaded the ambient during the entire day and was not related to the vehicle being tested. The questions that remain are "For the $70 \mathrm{~mm}$ filters, how can the background plus the vehicle exhaust particulate be less than the background alone?" and "Why weren't the cyclones equally affected?."

The answer to the first question can be found in the answer to the second: the cyclones are size selective. Neither large particles that may be present in the ambient nor agglomerated particles sheared from the tunnel wall surfaces are collected. Relatively few particles larger than $10 \mu \mathrm{m}$ will cause a significant increase in the mass collected. This effect will be made more prominent when the vehicles are clean, such as those run on natural gas. The $70 \mathrm{~mm}$ filter captures all particles indiscriminately. These may or may not have been in the air at the time of sampling. More likely, the particulates collected during the background sample are large agglomerations $(>10 \mu \mathrm{m})$ that were reentrained from the tunnel wall. Without the hot exhaust being inducted into the dilution tunnel, the temperature of the air stream will be close if not equal to the ambient temperature. The thermophoretic effects that drew particles to the wall surfaces during the temperature gradient dissipate as the tunnel wall is no longer cooler than the air 
flowing through it. This may allow those agglomerated particles to be removed more easily than during the course of testing. Again, the cyclones will not be similarly affected because they are size selective and will not collect these large particles. This was supported by the fact that the diesel data shows the comparison between the two systems to be less divergent than the LNG data. Higher filter mass collection during the course of a test will limit the adverse effects of a non-representative background sample. Also, the background mass found by the cyclones was minimal, with filter loadings on the order of $10 \mu \mathrm{g}$.

\subsubsection{MOUDI Data}

The MOUDI was operated for several of the LNG vehicles. Unfortunately, little data resulted and one conclusion followed: the MOUDI requires long sampling times to effectively measure low mass emitting vehicles. So little appreciable mass was collected on the substrates that no size distribution could be formed. This shows one of the subtleties of the MOUDI. While the DDC 6V92TA and Cummins B5.9 test times had to be scaled back to not overload the stages, the Nova bus tests needed to be run much longer than the $\mathrm{CBD}$ or a reasonable steady state test will allow.

Any significant mass that was collected by the MOUDI was on the after-filter. For LNG Bus 4328, the two $20 \mathrm{mph}$ steady state runs gave $27 \mu \mathrm{g}$ and $39 \mu \mathrm{g}$, and the CBDs, $48 \mu \mathrm{g}$ for both. Making an approximate comparison between the PM2.5 cyclone and the MOUDI after-filter showed the cyclone mass to be $2-\sim 2.5 \%$ times greater for the two CBDs and 2.7 times for the one steady state. This suggests that roughly half of 
the mass collected by the PM2.5 cyclone was less than $0.052 \mu \mathrm{m}$. Though using the MOUDI for total mass values is generally avoided, the after-filter can be assumed an accurate mass measure. It should be noted that only the upper stages of the MOUDI were affected by particle bounce. It is also not possible for most particles that may be reentrained in the stream as a result of bounce to reach the last stage. Though the direct correlation between particle size and mass has been derided previously, the smaller particles $(<0.052 \mu \mathrm{m})$ will weigh less than the larger ones. This makes the relatively high mass collected on the after-filter more meaningful. Approximately one-third to one-half of the total mass below $2.5 \mu \mathrm{m}$ was less than $52 \mathrm{~nm}$ in diameter for these three tests.

\subsubsection{SMPS Data}

The SMPS was used to sample the same transit buses as the cyclones. All the tests were conducted with the model 3010 CPC except for those performed on Buses 4323, 4325, 4220 and 4221. The CPC malfunctioned during testing of Bus 4322 and was replaced by the $3025 \mathrm{~A}$. The sheath air flow rate was $18 \mathrm{lpm}$ and the aerosol flow rate was $1.8 \mathrm{lpm}$ for all of the runs.

Diesel Bus 4220 produced 40 - 50\% more total particulate matter than the other diesel bus, 4221. The cyclone data lead to the conclusion that the heavier polluting bus produced this extra mass largely at diameters less than $2.5 \mu \mathrm{m}$. This is supported by the SMPS data (see Figures 4.90 - 4.99). While Bus 4221 produced ten times more $15 \mathrm{~nm}$ particles and similar quantities as Bus 4220 for the other sizes tracked, Bus 4220 emitted approximately five times more particles at $150 \mathrm{~nm}$. Once again, while no quantitative 


\section{Figure 4.90 - 15 nm SMPS Particle Concentration for Cummins M11 Bus 4220 CBD Run \#1 Fuel: D2}

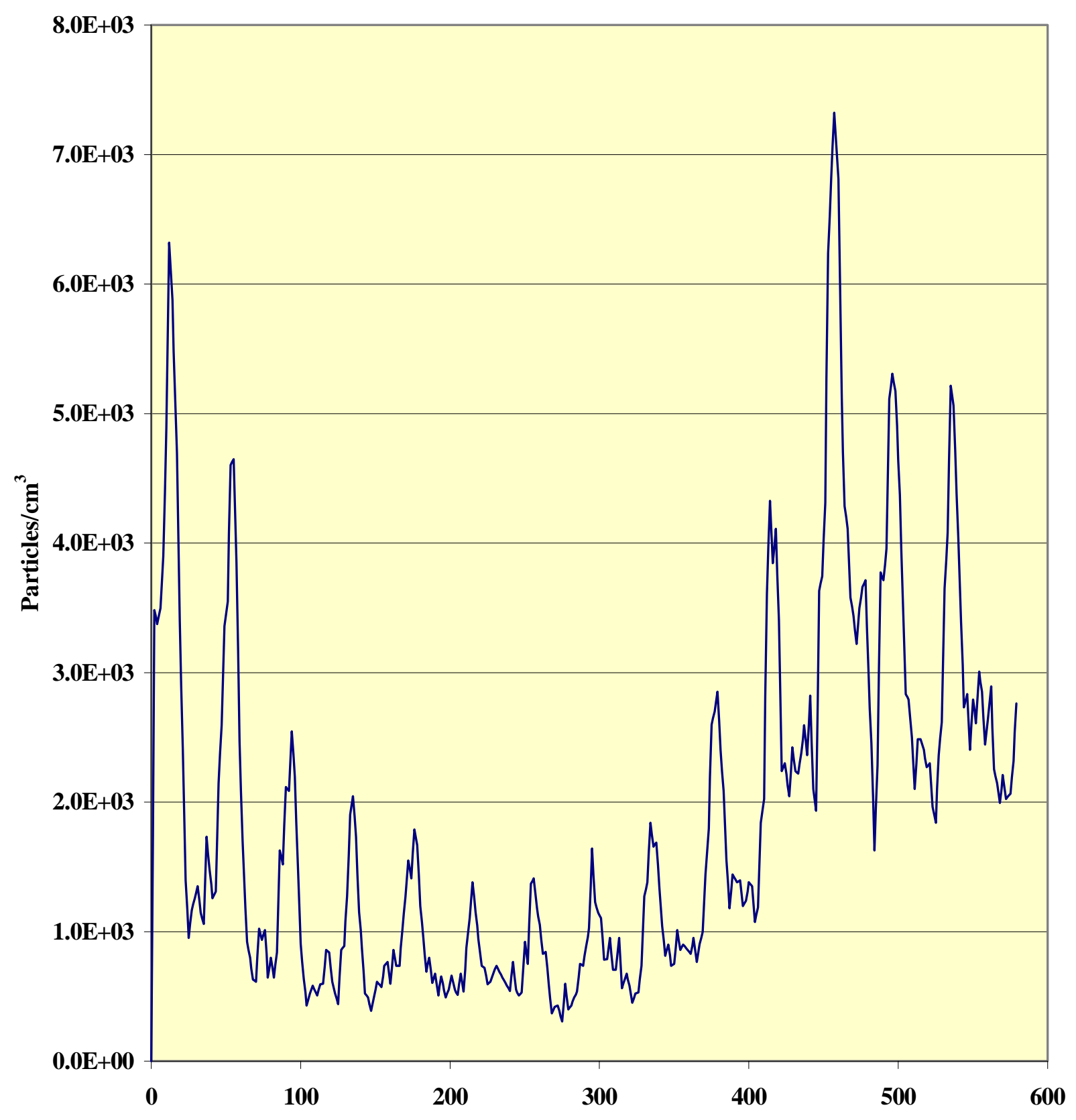

Time (seconds) 
Figure 4.91 - 35 nm SMPS Particle Concentration for Cummins M11 Bus 4220 CBD Run \#2 Fuel: D2

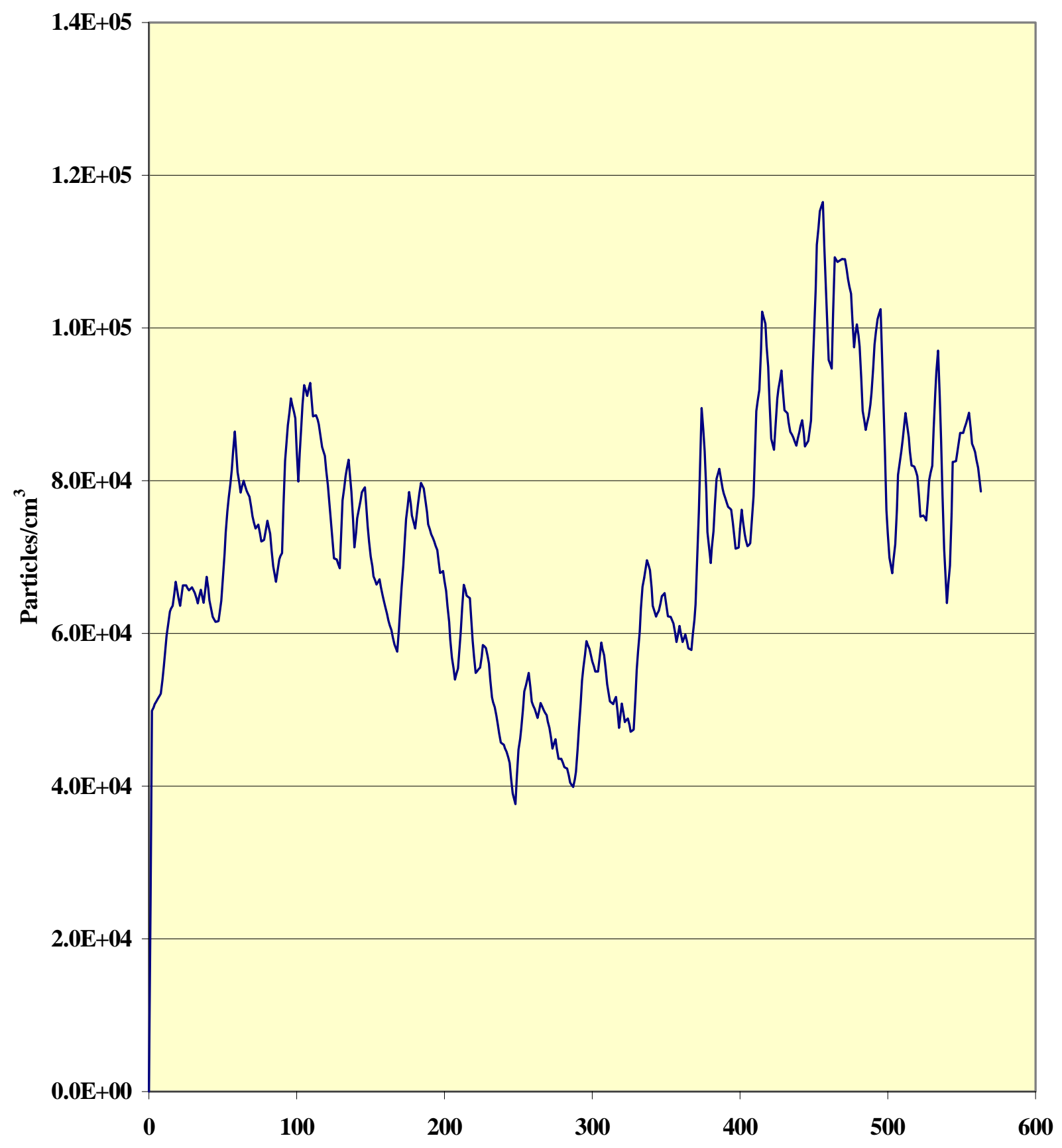

Time (seconds) 
Figure 4.92 - 50 nm SMPS Particle Concentration for Cummins M11 Bus 4220 CBD Run \#3 Fuel: D2

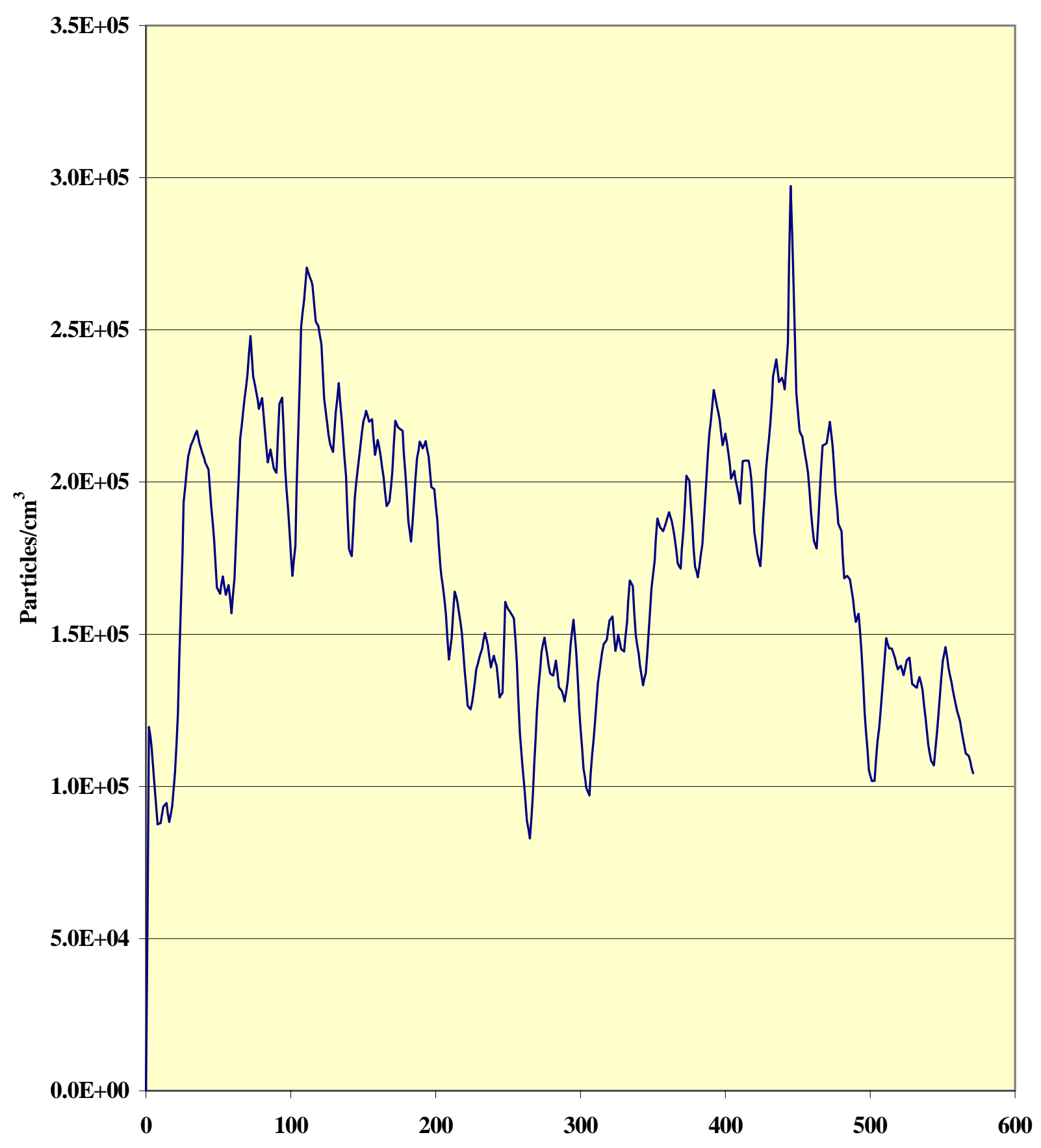

Time (seconds) 
Figure 4.93 - 80 nm SMPS Particle Concentration for Cummins M11 Bus 4220 CBD Run \#4 Fuel: D2

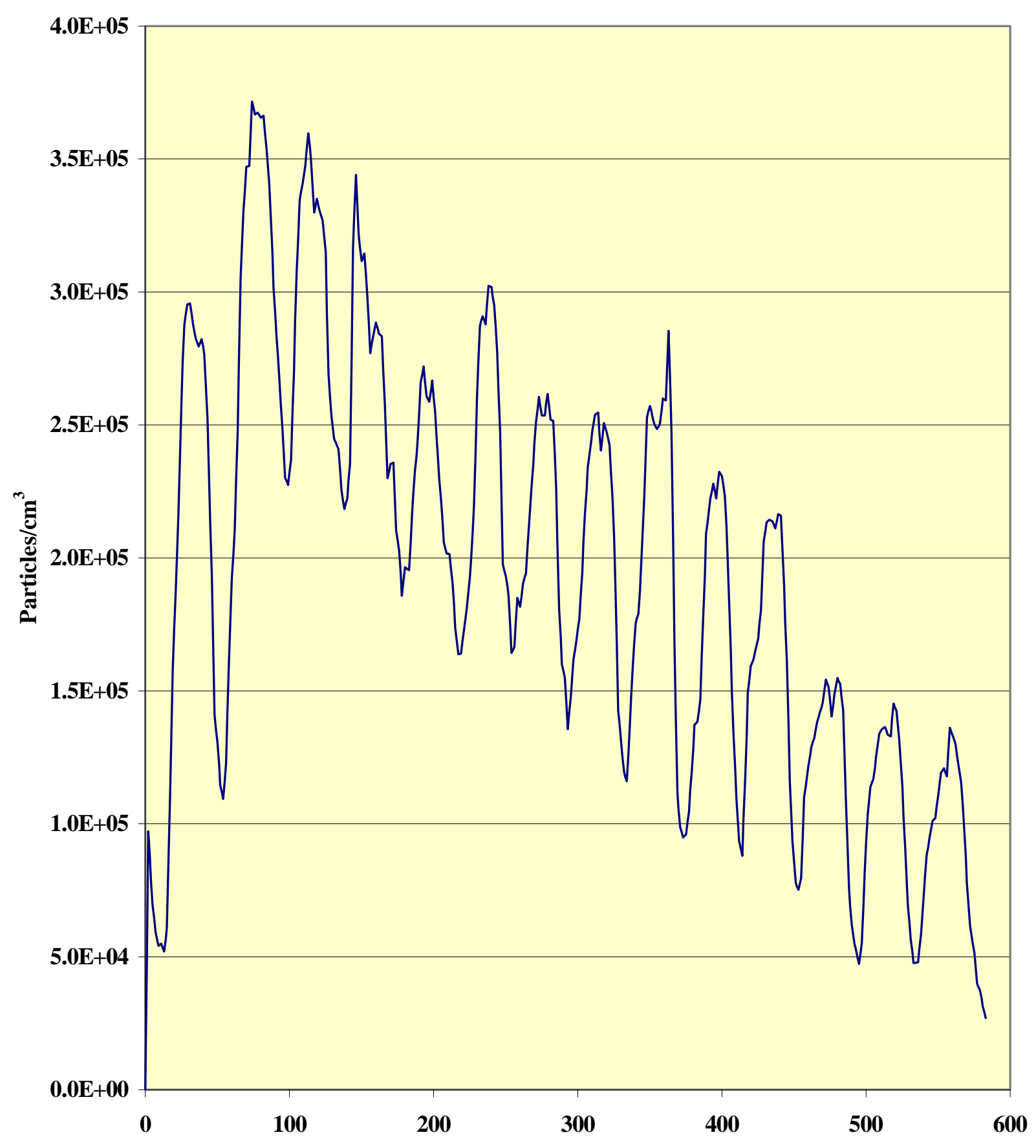

Time (seconds) 
Figure 4.94 - 150 nm SMPS Particle Concentration for Cummins M11 Bus 4220 CBD Run \#5 Fuel: D2

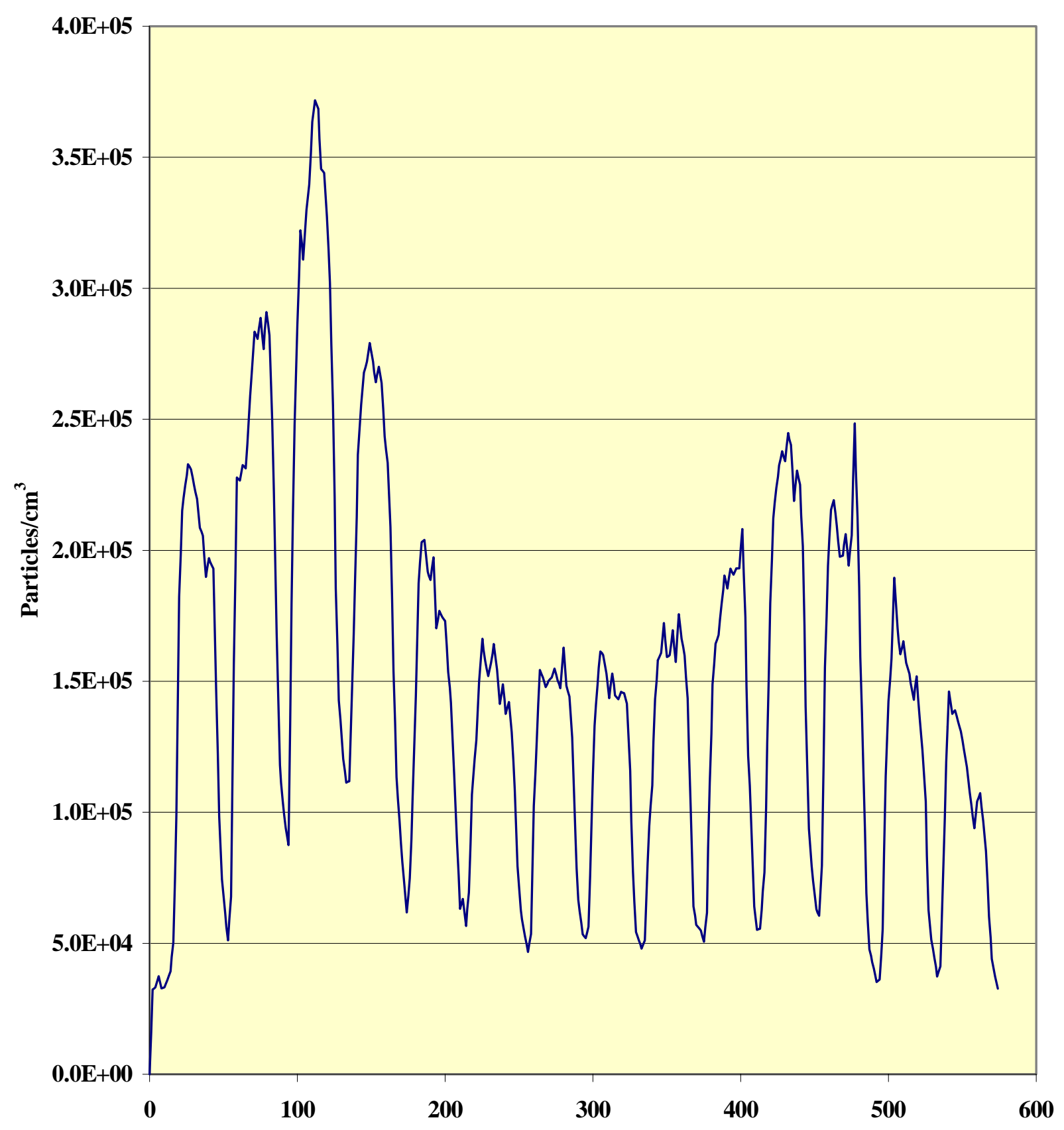

Time (seconds) 


\section{Figure 4.95 - 10 nm SMPS Particle Concentration for Cummins}

M11 Bus 4221 CBD Run \#1 Fuel: D2

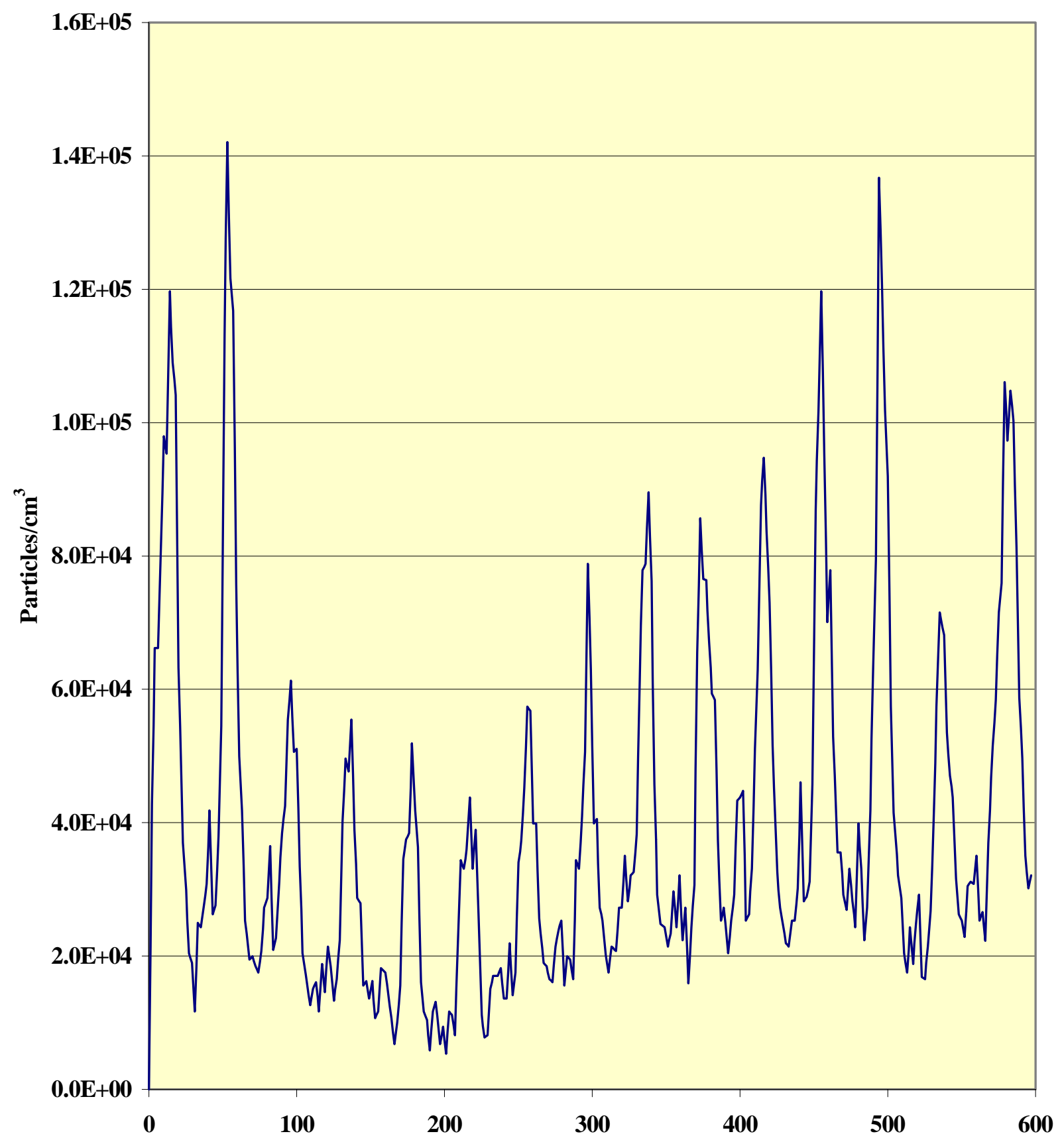

Time (seconds) 
Figure 4.96 - 20 nm SMPS Particle Concentration for Cummins M11

Bus 4221 CBD Run \#2 Fuel: D2

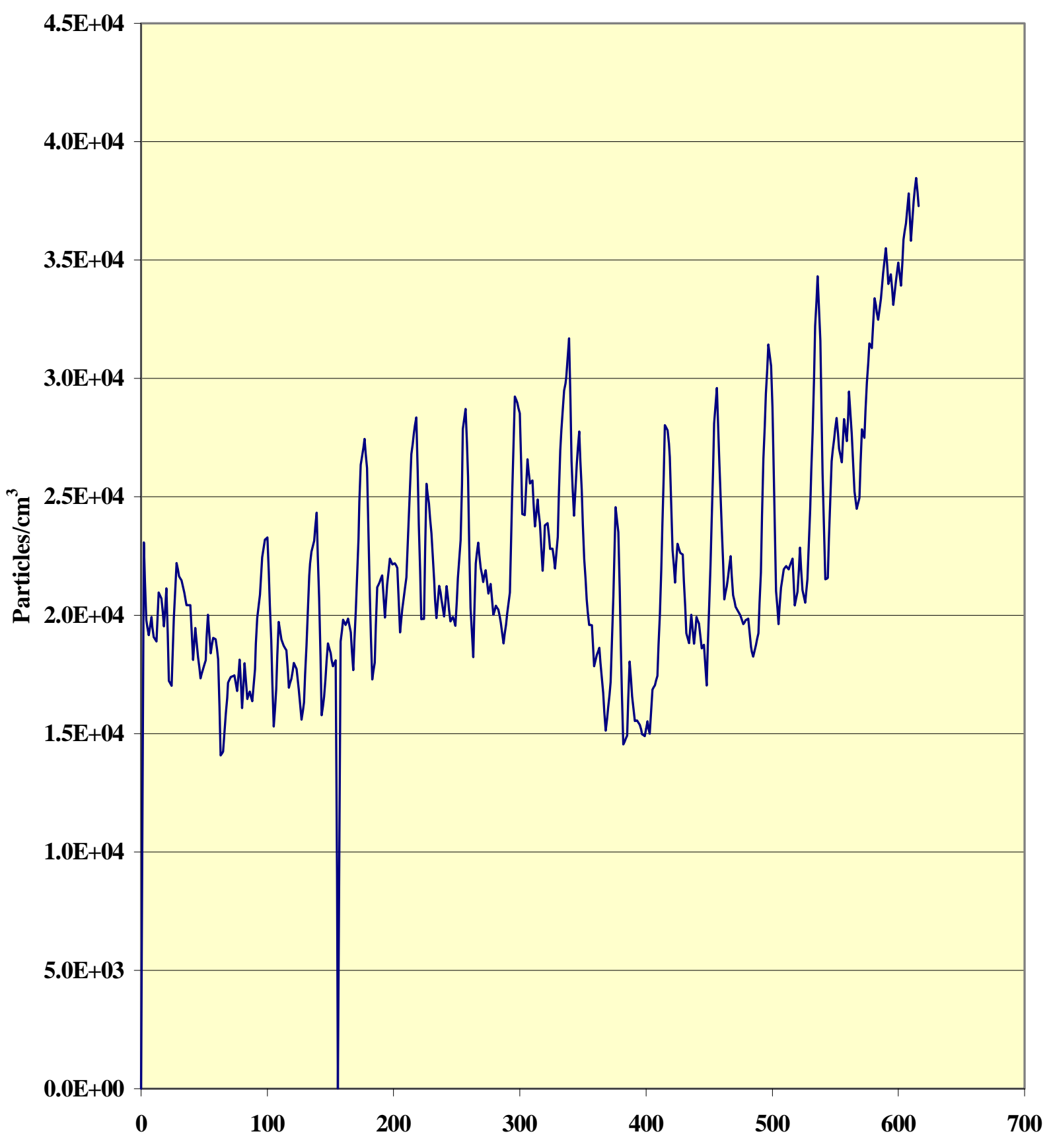

Time (seconds) 
Figure 4.97 - 33 nm SMPS Particle Concentration for Cummins

M11 Bus 4221 CBD Run \#3 Fuel: D2

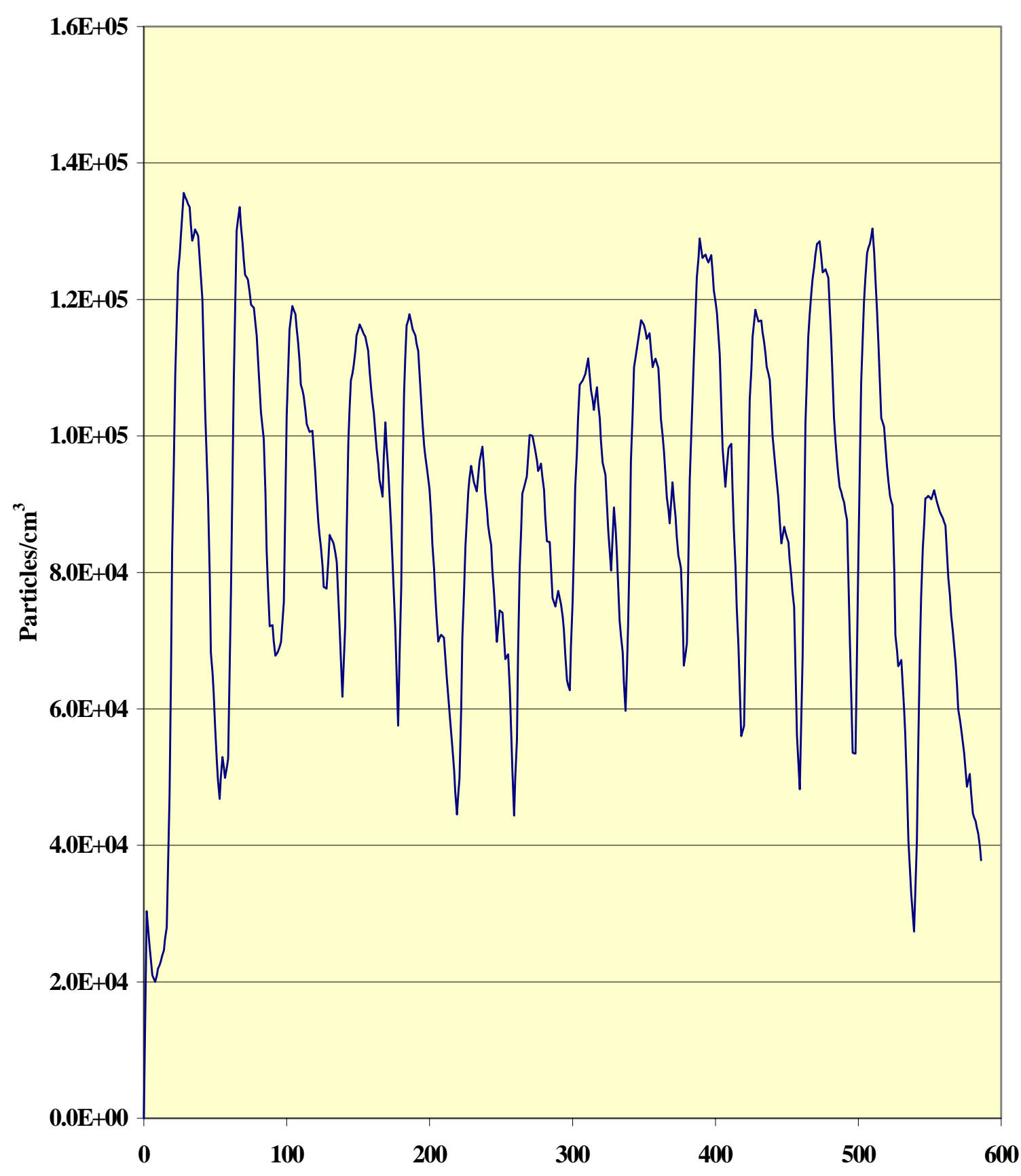

Time (seconds) 
Figure 4.98 - 55 nm SMPS Particle Concentration for Cummins M11 Bus 4221 CBD Run \#4 Fuel: D2

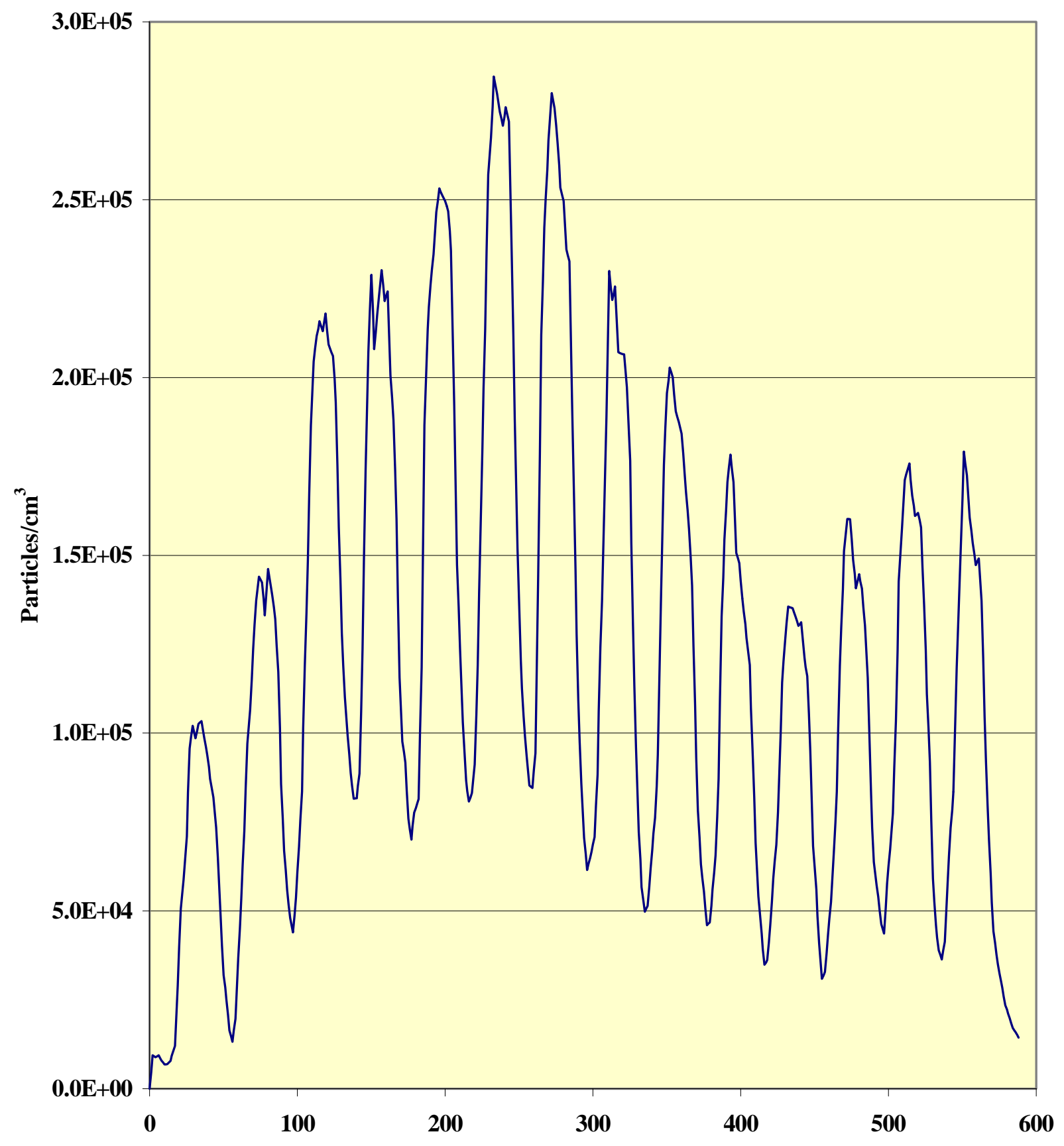

Time (seconds) 
Figure 4.99 - $150 \mathrm{~nm}$ SMPS Particle Concentration for Cummins M11 Bus 4221 CBD Run \#5 Fuel: D2

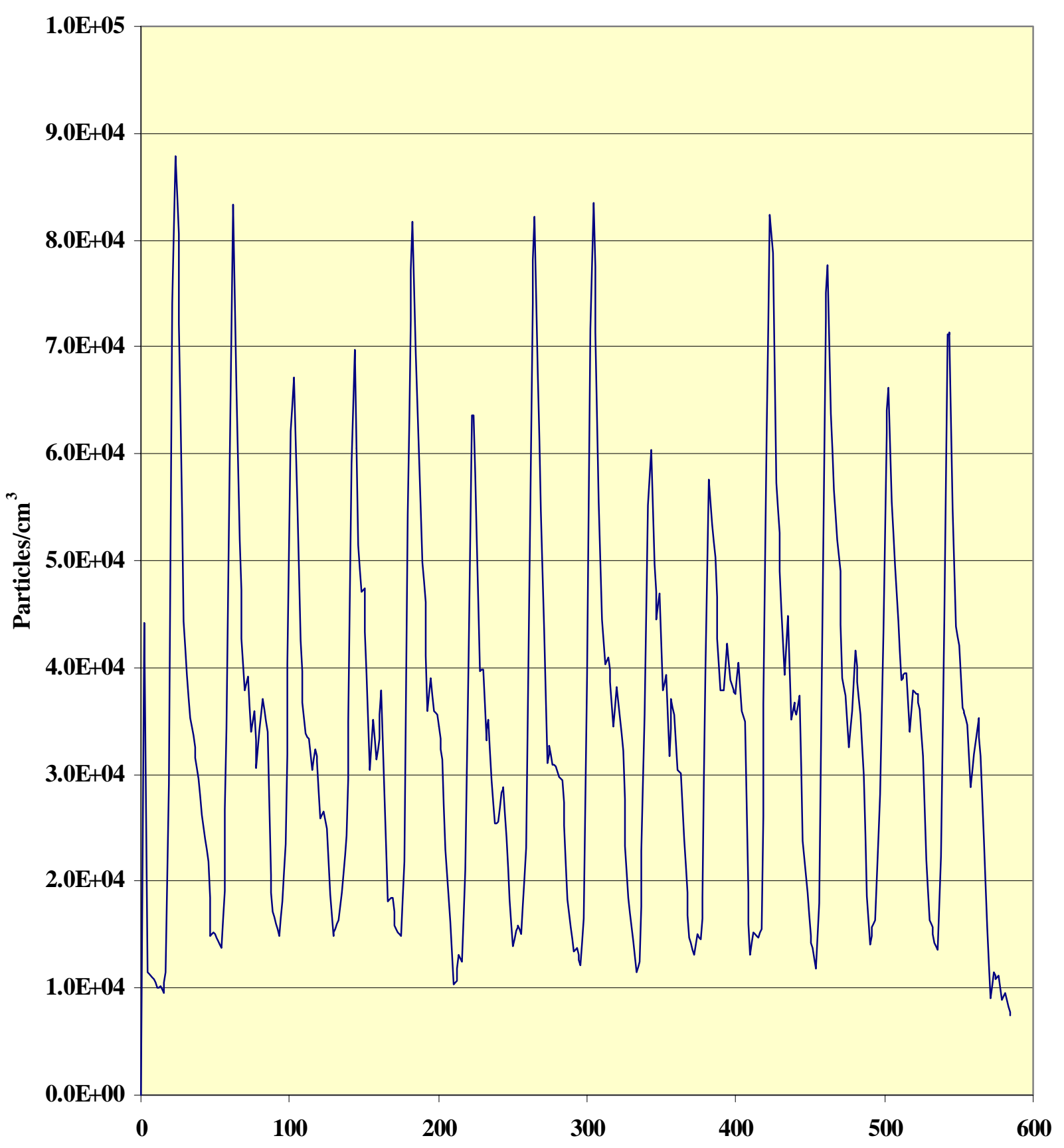

Time (seconds) 
data is possible, these particles will add much more mass than those of $15 \mathrm{~nm}$ in diameter.

The SMPS also adds credence to the hypothesis derived from the cyclones regarding LNG Bus 4321. This was the highest emitter of total particulate mass, but was near or below average compared to the other vehicles with respect to particle number (see Figures 4.100 - 4.104). From the cyclone data, it was shown that for this bus the PM10 cyclone collected considerably more mass than the PM2.5. This suggested, and was supported by the SMPS concentration data, that factors other than fuel combustion were responsible for the high gravimetric, total particle mass.

Testing of Bus 4329 resulted in water condensation at the sampling zone of the cyclones. The SMPS data may also have been affected by this phenomenon. This bus returned very high concentration values (see Figures $4.105-4.109$ ). The concentration at $55 \mathrm{~nm}$ was the largest for all the LNG vehicles. It is possible that many of these particles were water droplets. Water was removed from any filter or substrate through conditioning, so it would not show up in mass collection; however, the SMPS would count it indiscriminately. It is believed that water droplets produced by natural gas engines are much smaller than $55 \mathrm{~nm}$; however, these droplets were likely formed from condensed vapors due to under-dilution rather than natural gas combustion.

From the plots of the particle concentrations from the diesel buses (see Figures 4.90 - 4.99) it can be seen that the concentrations varied throughout the CBD cycle. Particle concentrations from Bus 4220 fluctuated cyclically while those from Bus 4221 fluctuated randomly. No explanation could be determined for these anomalies, though possible sources are the catalyst and temperature fluctuations. 
Figure 4.100 - 15 nm SMPS Particle Concentration for Cummins L10-G Bus 4321 CBD Run \#1 Fuel: LNG

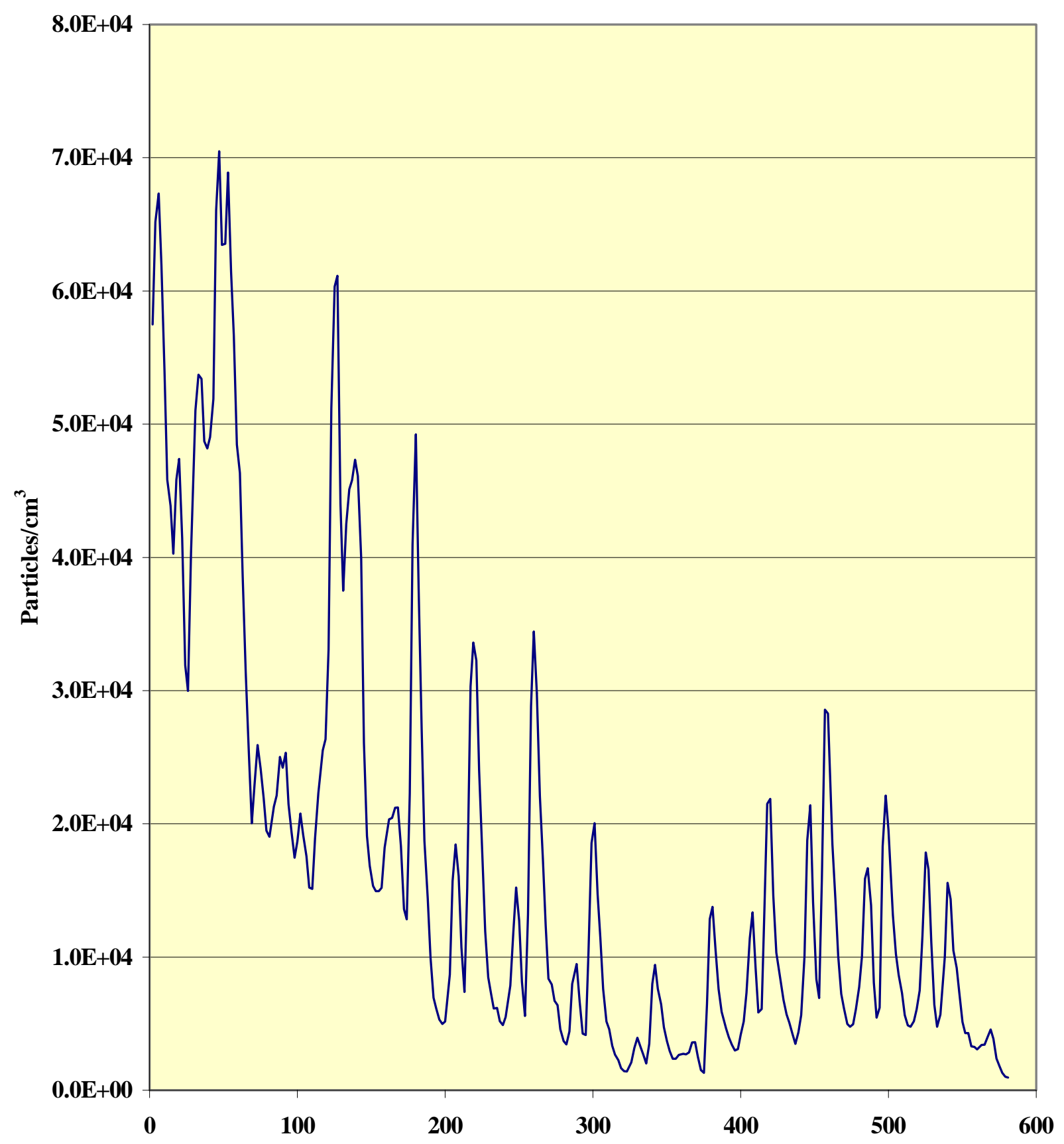

Time (seconds) 
Figure 4.101 - 20 nm SMPS Particle Concentration for Cummins L10-G Bus 4321 CBD Run \#2 Fuel: LNG

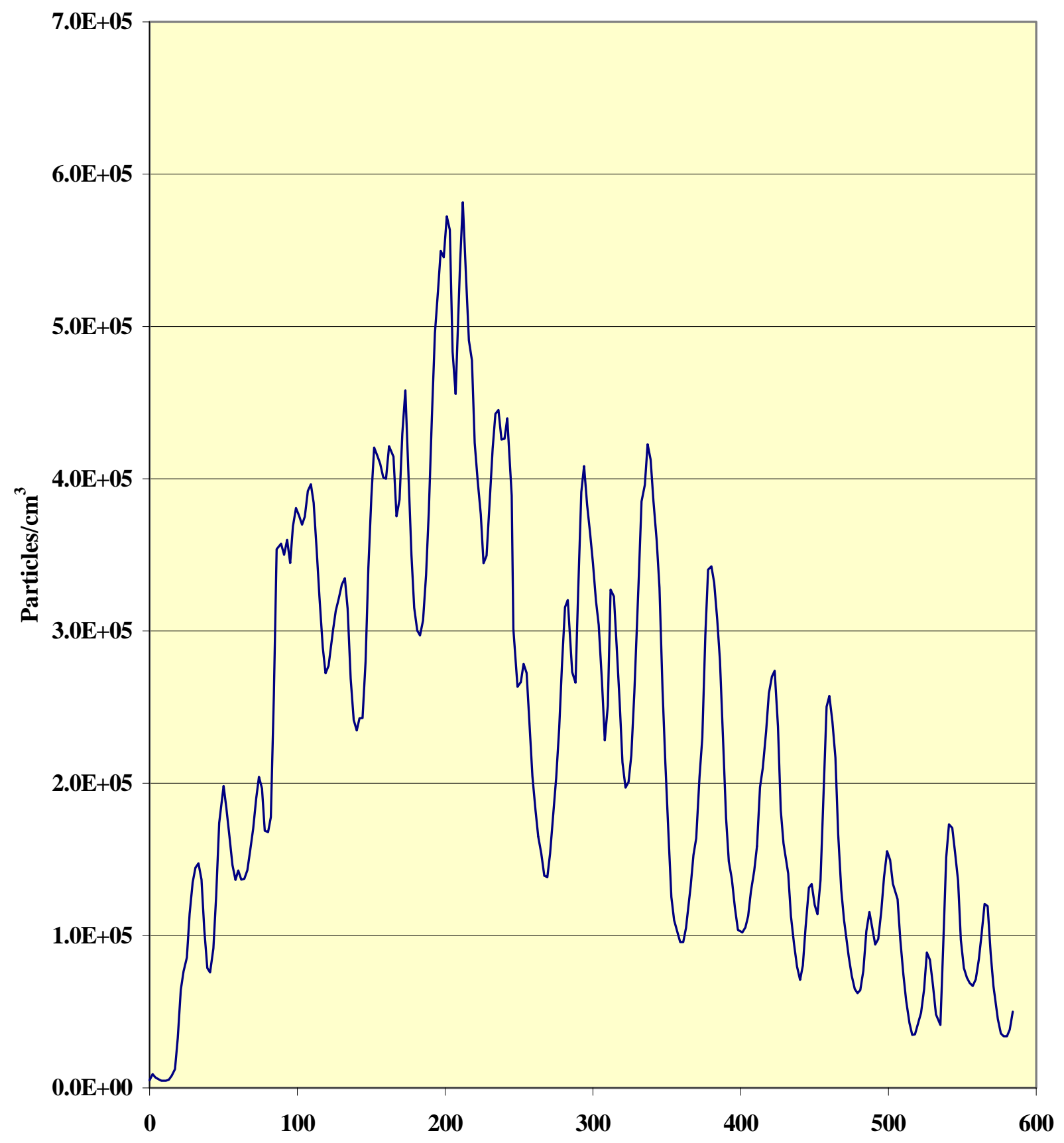

Time (seconds) 
Figure 4.102 - 30 nm SMPS Particle Concentration for Cummins L10-G Bus 4321 CBD Run \#3 Fuel: LNG

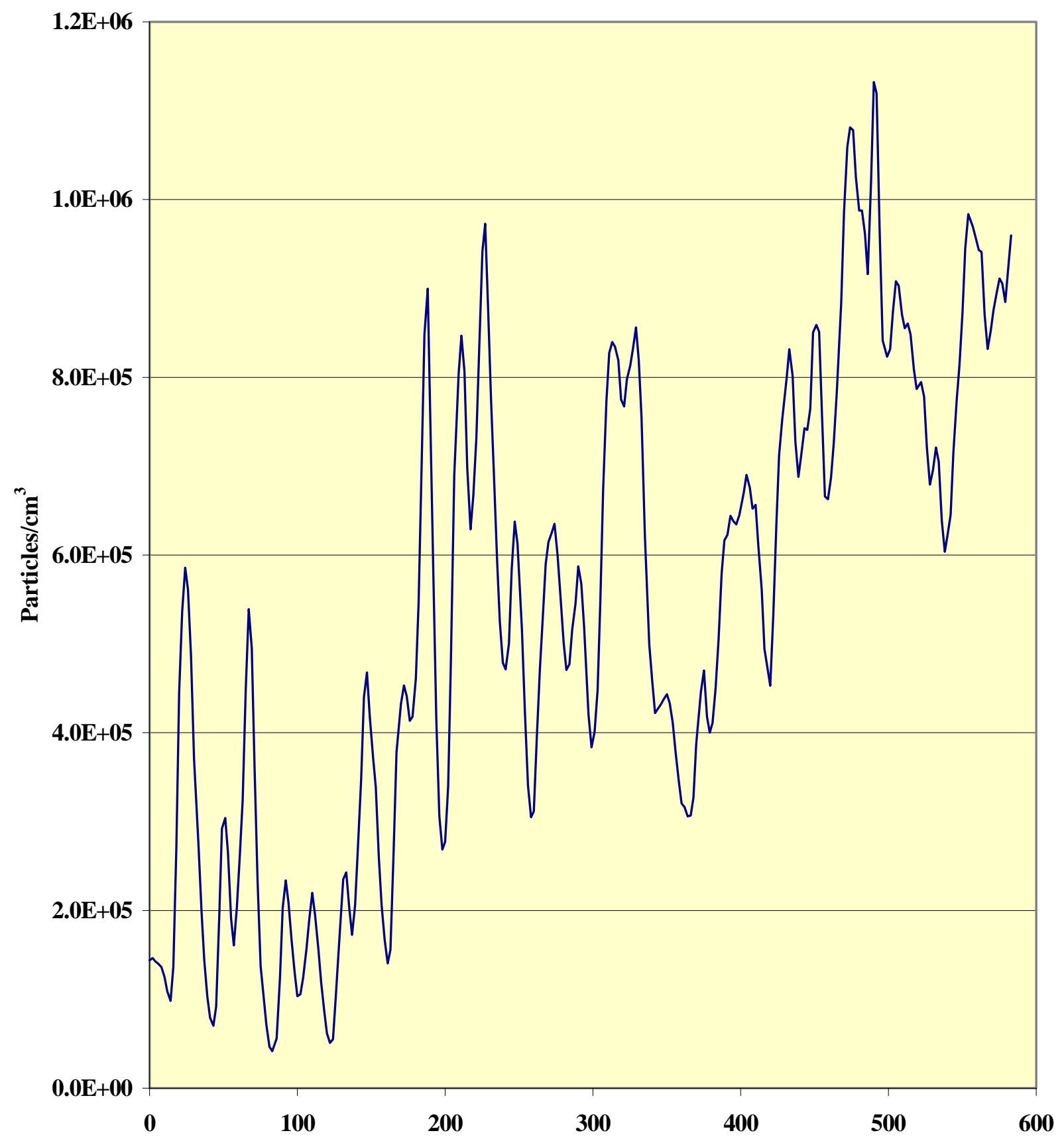

Time (seconds) 
Figure 4.103 - 40 nm SMPS Particle Concentration for Cummins L10-G Bus 4321 CBD Run \#4 Fuel: LNG

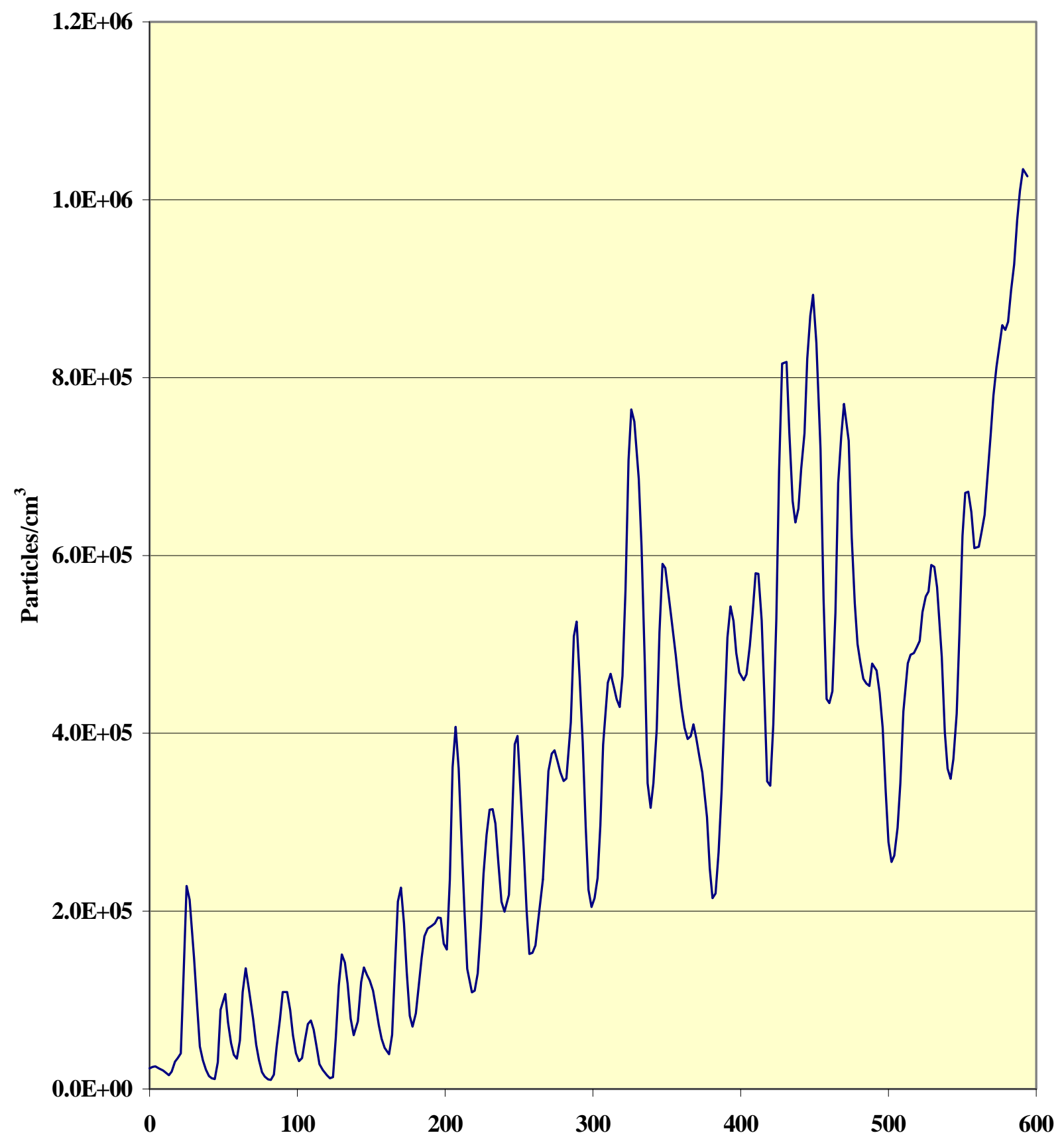

Time (seconds) 
Figure 4.104 - $60 \mathrm{~nm}$ SMPS Particle Concentration for Cummins

L10-G Bus 4321 CBD Run \#5 Fuel: LNG

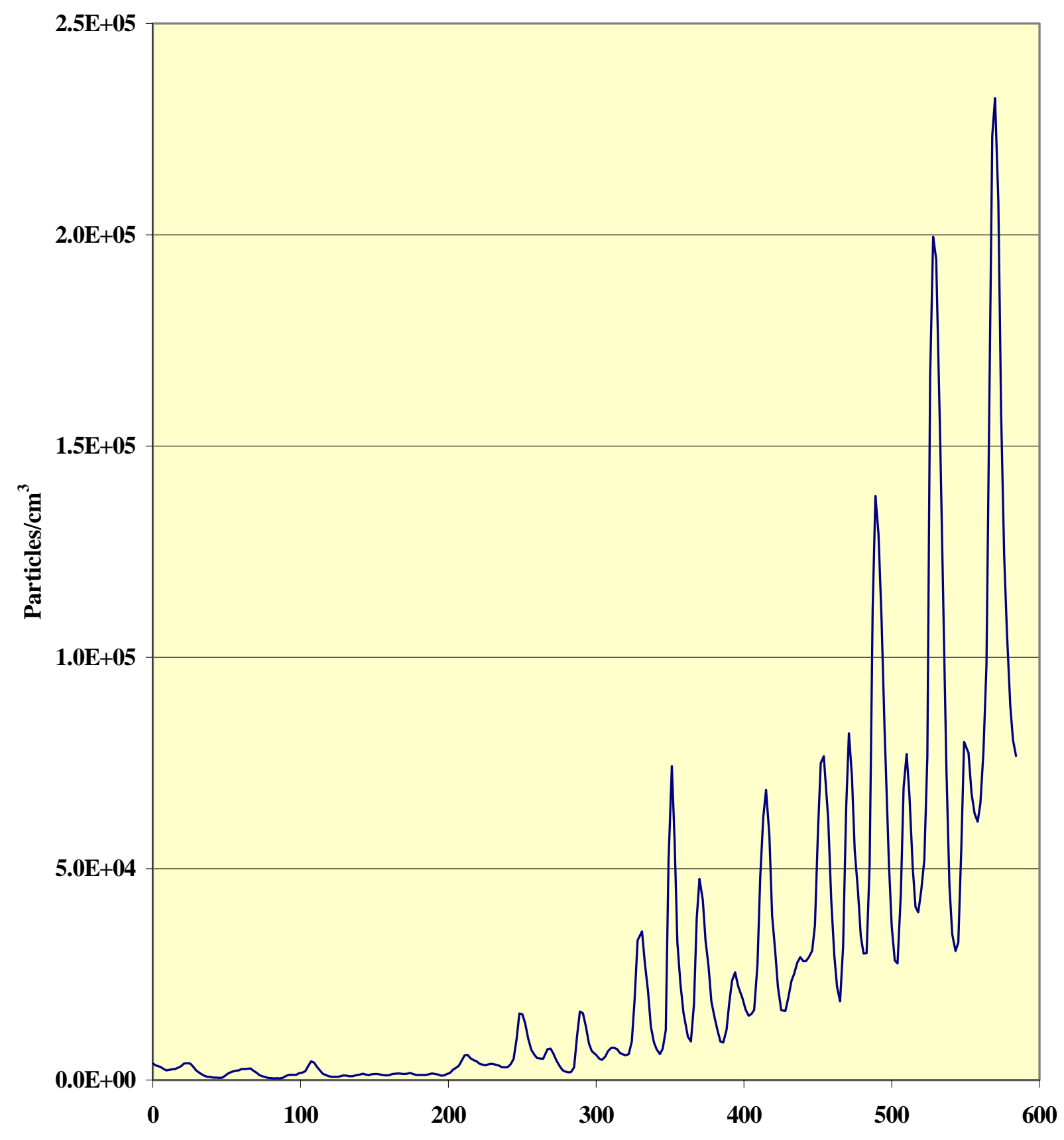

Time (seconds) 
Figure 4.105 - 15 nm SMPS Particle Concentration for Cummins

L10-G Bus 4329 CBD Run \#1 Fuel: LNG

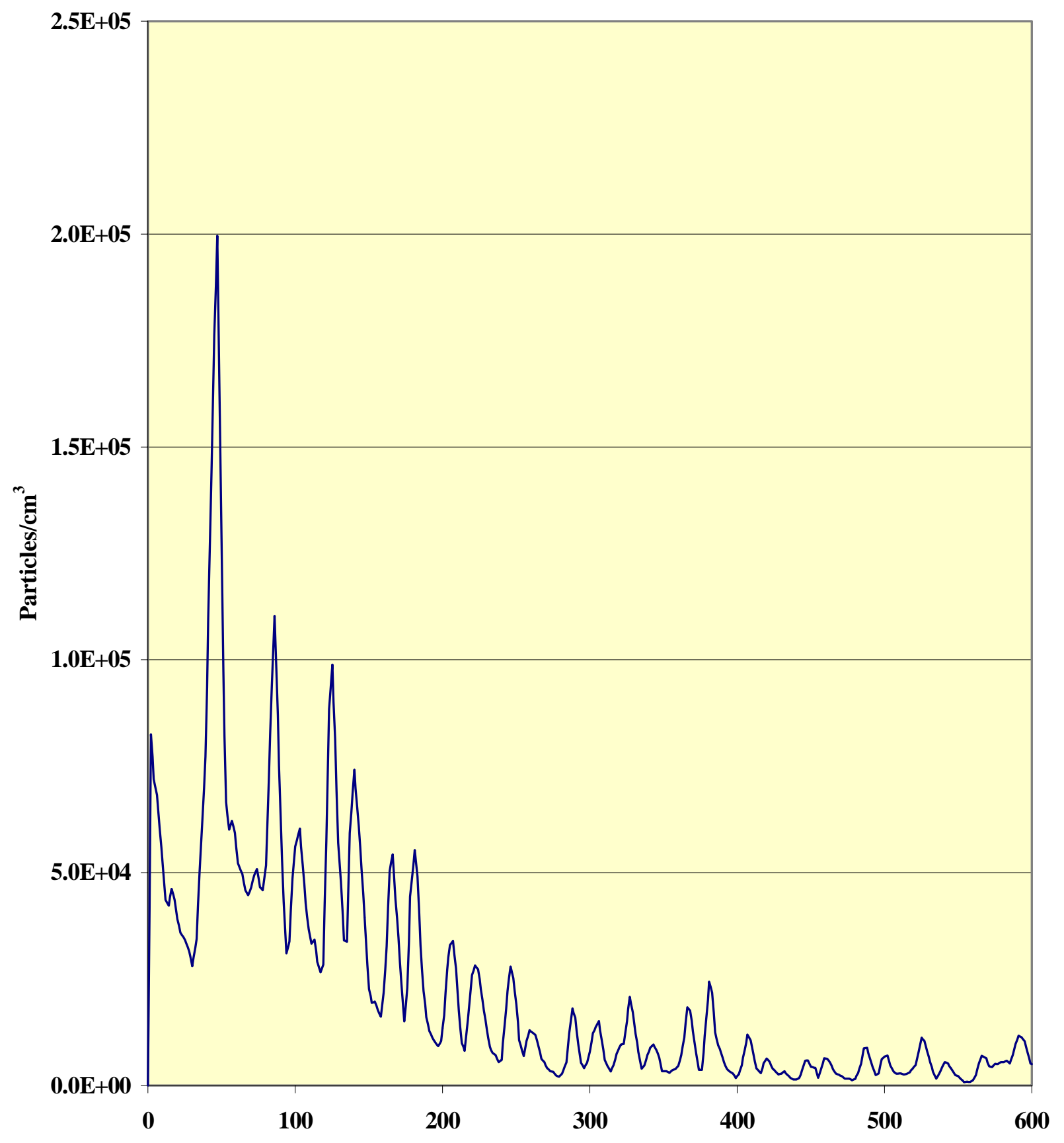

Time (seconds) 
Figure 4.106 - 25 nm SMPS Particle Concentration for Cummins L10-G Bus 4329 CBD Run \#2 Fuel: LNG

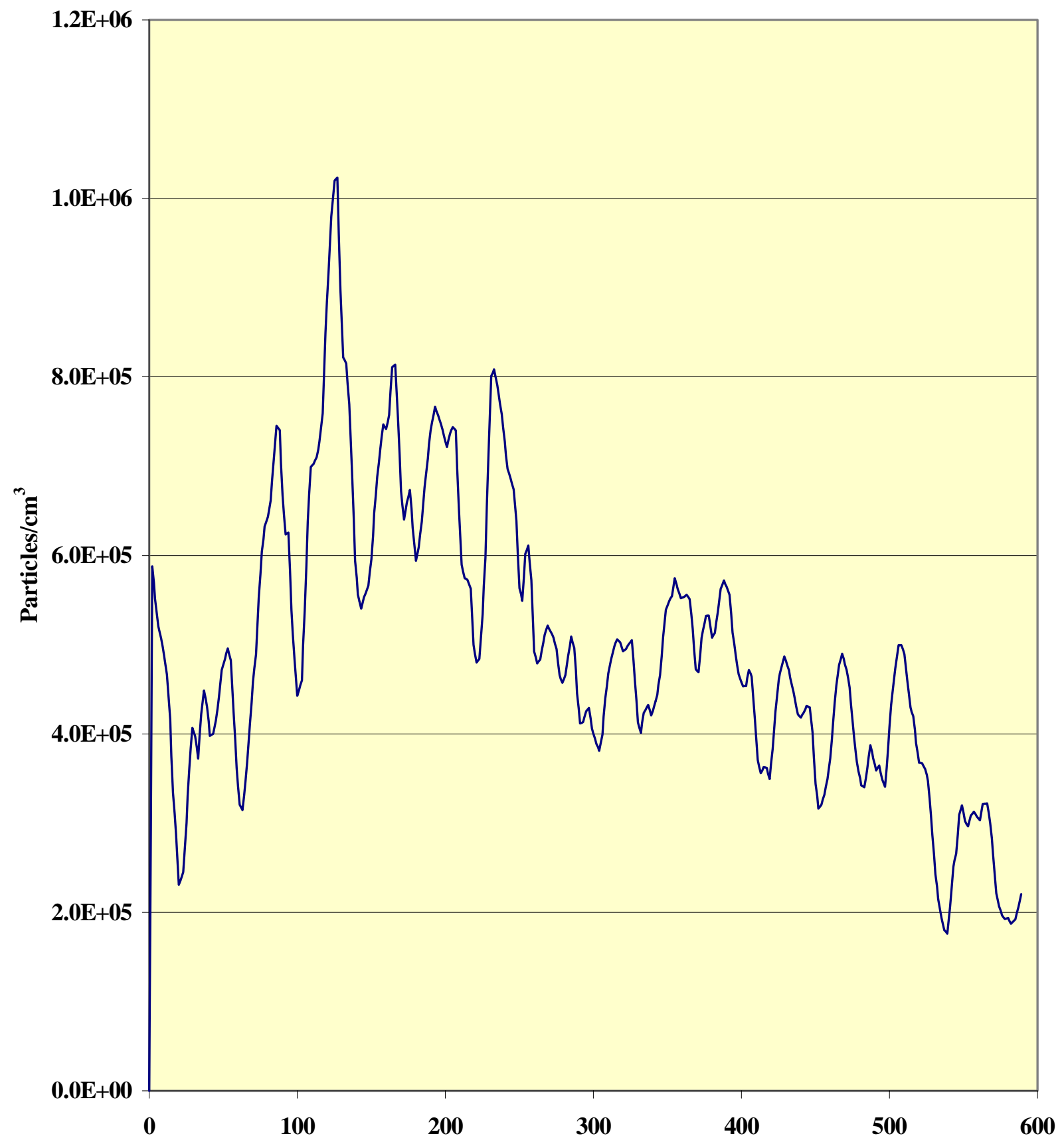

Times (seconds) 
Figure 4.107 - 33 nm SMPS Particle Concentration for Cummins L10-G Bus 4329 CBD Run \#3 Fuel: LNG

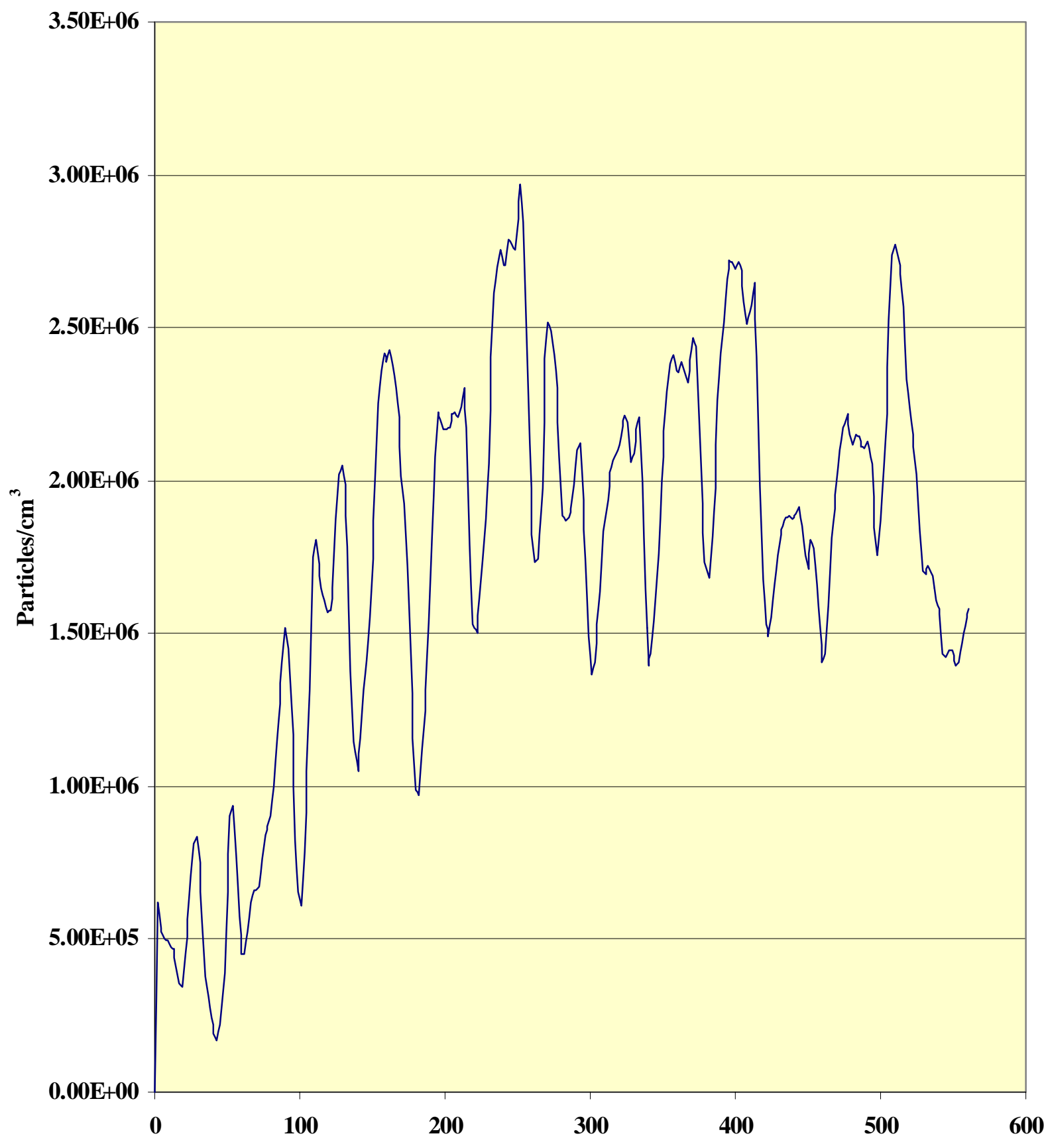

Time (seconds) 
Figure 4.108 - 43 nm SMPS Particle Concentration for Cummins L10-G Bus 4329 CBD Run \#4 Fuel: LNG

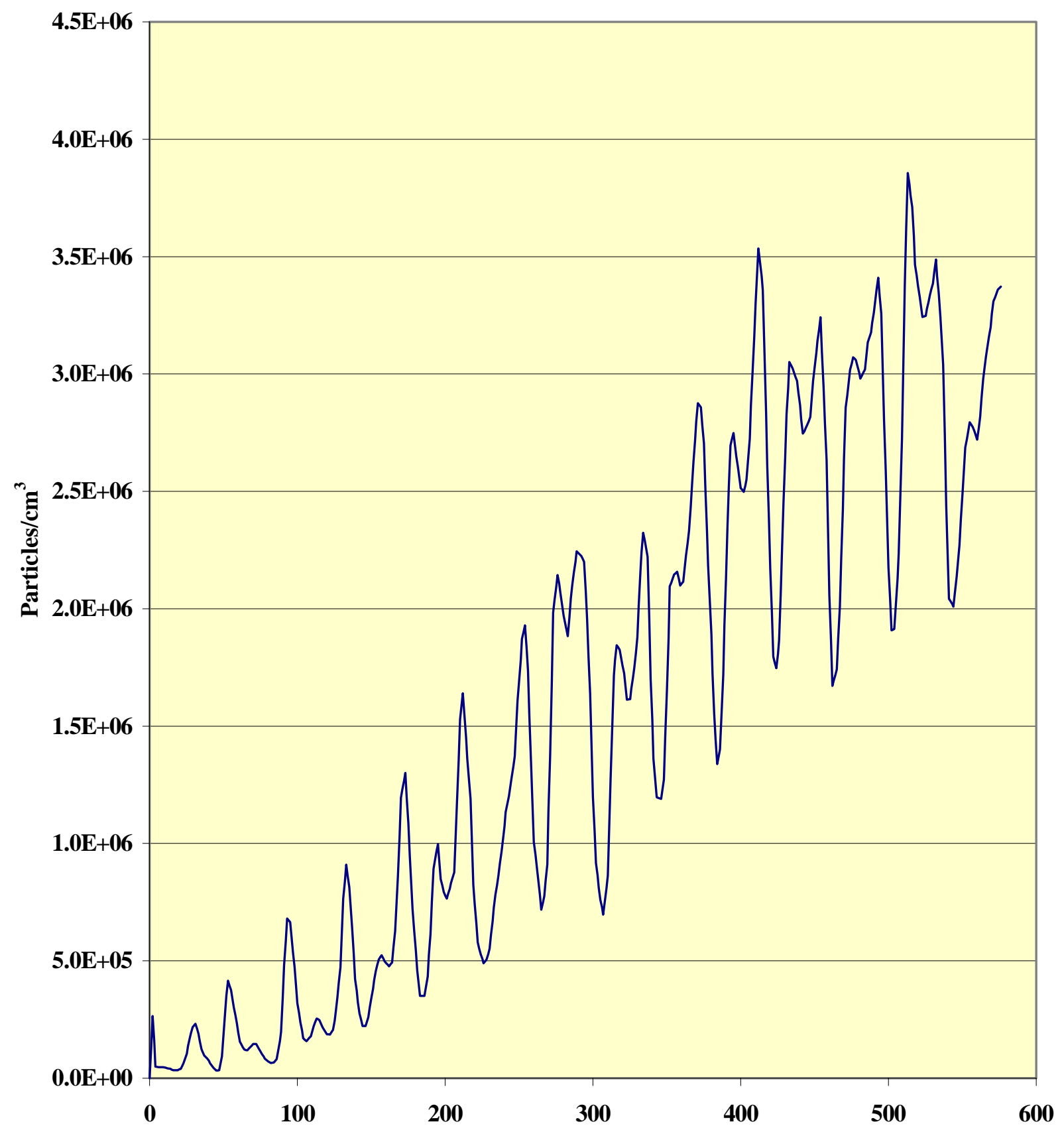

Time (seconds) 
Figure 4.109 - 55 nm SMPS Particle Concentration for Cummins L10-G Bus 4329 CBD Run \#5 Fuel: LNG

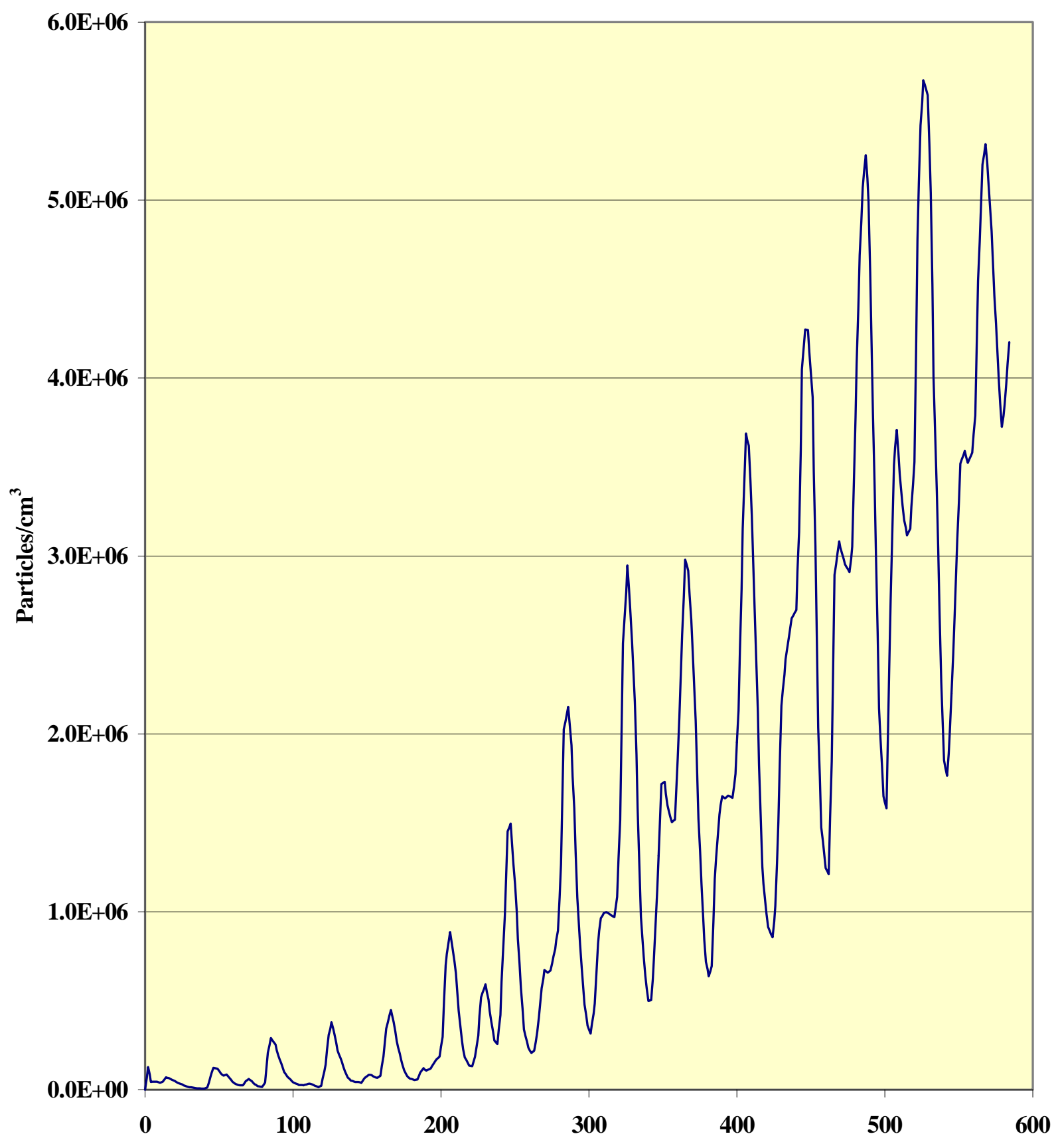

Time (seconds) 
The particle concentrations for the LNG buses also were not constant. However, there was a pattern to the variations (see Figures 4.100 - 4.128). For diameters between 10 and $25 \mathrm{~nm}$, the concentrations either continually decrease or reach a peak maximum then steadily decline. Conversely, for diameters between 30 and $60 \mathrm{~nm}$, the particle concentrations increase throughout the entire test, reaching a maximum at the end of the CBD cycle. Two possible factors may have been the cause of these phenomenon; both are associated with increasing temperatures throughout the duration of the tests. As exhaust temperatures increased during the cycle, the small, near-molecule sized particles may have become excited. The higher temperatures would cause these particles to either vibrate and/or translate. This would increase the rate of coagulation since coagulation is governed by thermal diffusion for particles subject to Brownian motion. As the rate of particle collisions intensified, the concentrations in the smaller diameter region would decrease while the concentrations in the larger diameter region would increase.

It is also possible that the concentration of small particles $(10-25 \mathrm{~nm})$ detected consisted mainly of water droplets. Natural gas has a relatively high water content and may produce fine water droplets during combustion. As exhaust temperatures increased, these droplets would begin to evaporate. The water vapor produced may then condense onto solid cores, thus increasing their diameters. Also, evaporation of the droplets would increase the saturation ratio, thereby enhancing condensation. This, as the coagulation hypothesis, is supported by the concentration data. Evaporation would decrease the concentration of particles in the 10 to $25 \mathrm{~nm}$ range while simultaneously increasing the concentration of the larger diameter particles. The buses that produced the highest peak 
Figure 4.110 - 20 nm SMPS Particle Concentration for Cummins L10-G Bus 4320 CBD Run \#1 Fuel: LNG

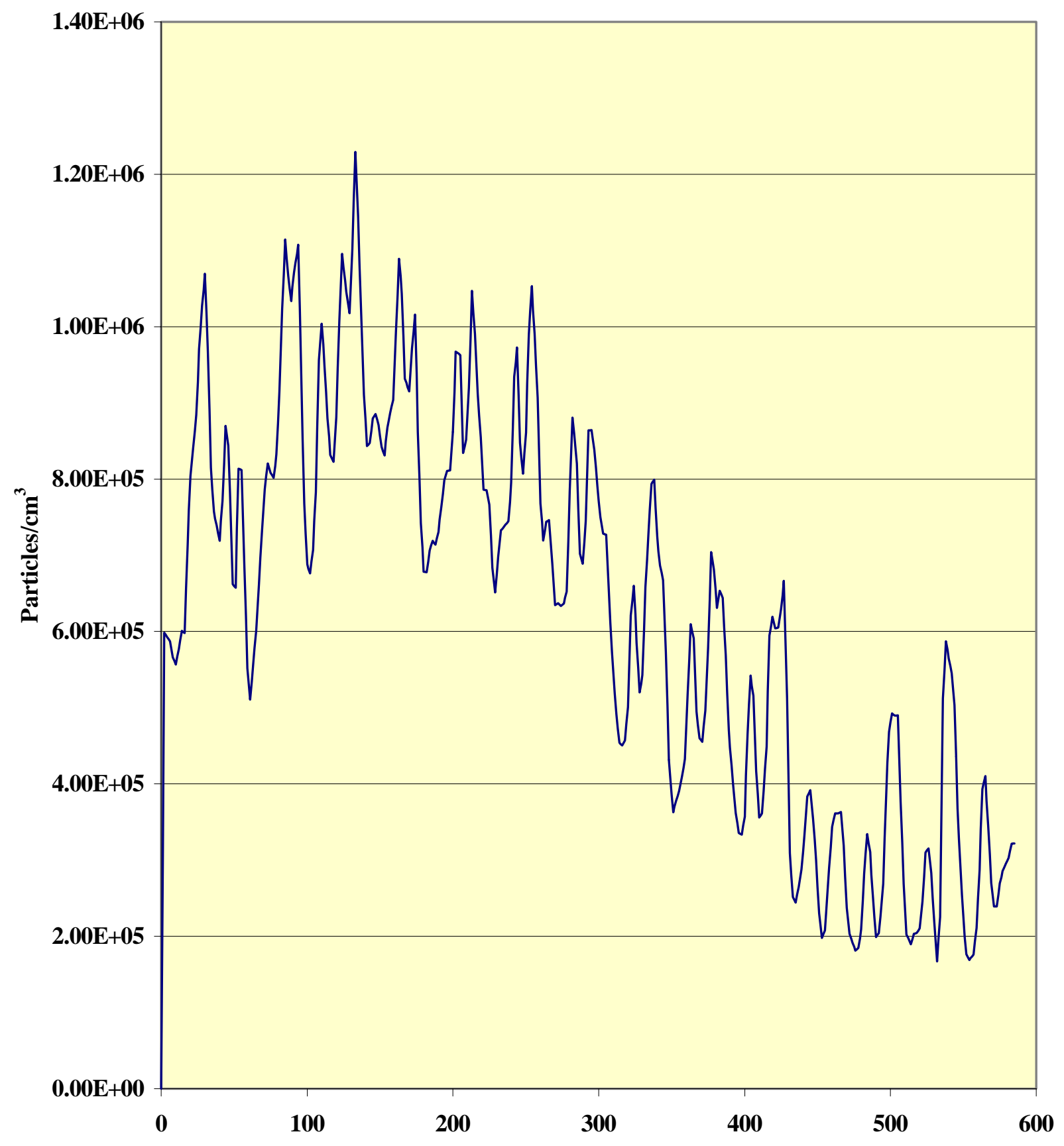

Time (seconds) 
Figure 4.111 - 30 nm SMPS Particle Concentration for Cummins

L10-G Bus 4320 CBD Run \#2 Fuel: LNG

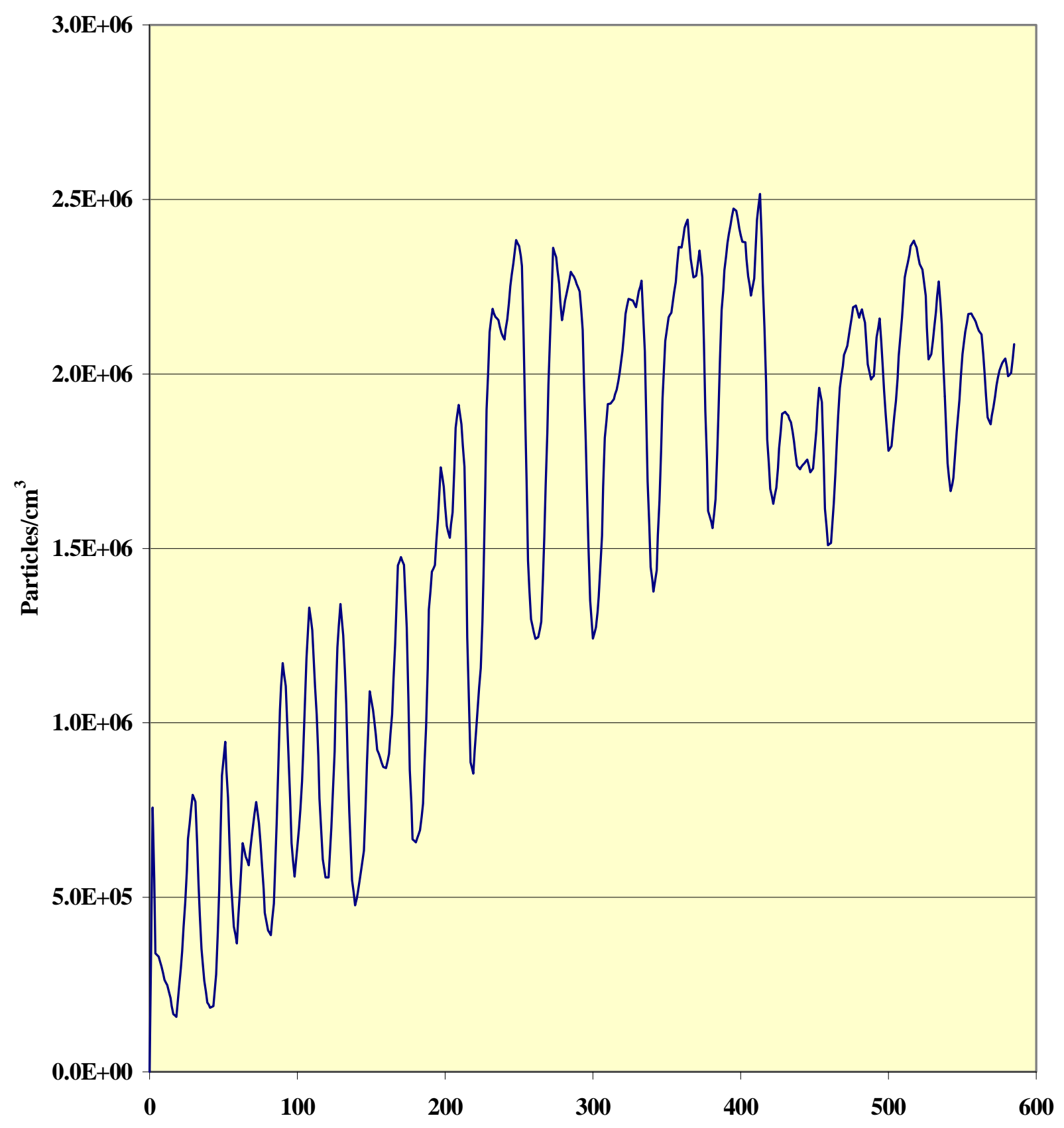

Time (seconds) 
Figure 4.112 - 40 nm SMPS Particle Concentration for Cummins L10-G Bus 4320 CBD Run \#3 Fuel: LNG

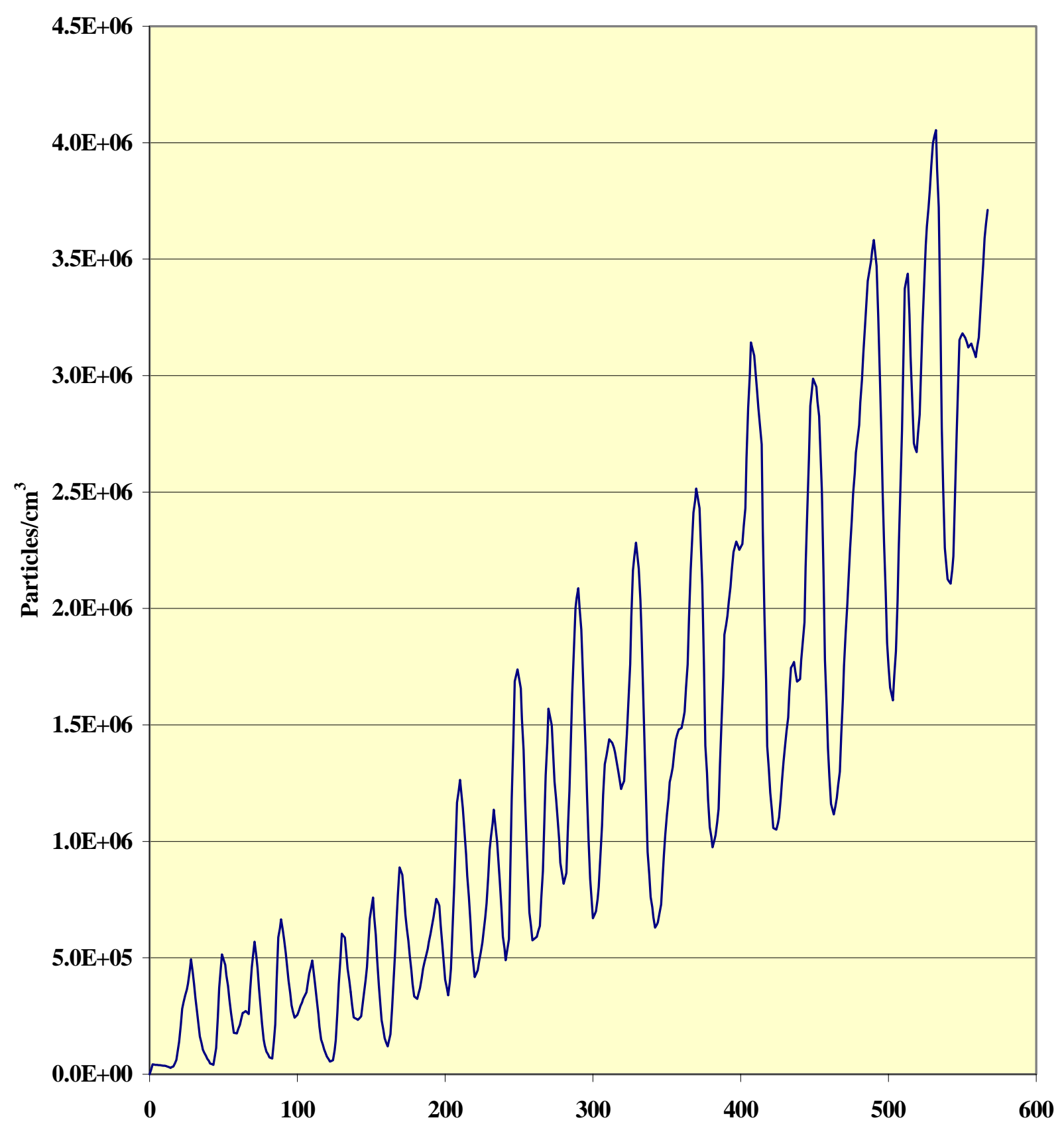

Time (seconds) 
Figure 4.113 - 50 nm SMPS Particle Concentration for Cummins

L10-G Bus 4320 CBD Run \#4 Fuel: LNG

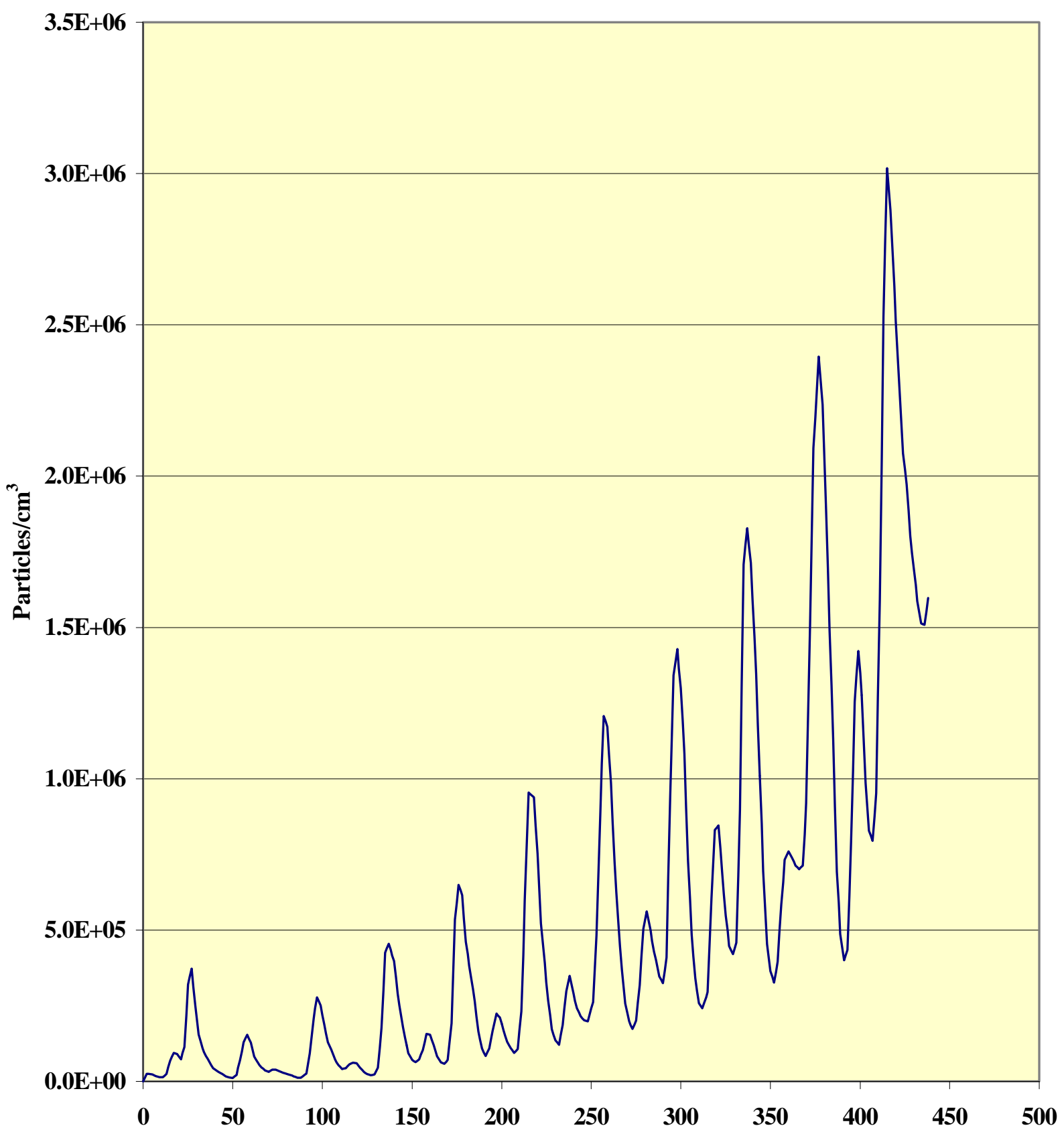

Time (seconds) 
Figure 4.114 - 10 nm SMPS Particle Concentration for Cummins L10-G Bus 4323 CBD Run \#1 Fuel: LNG

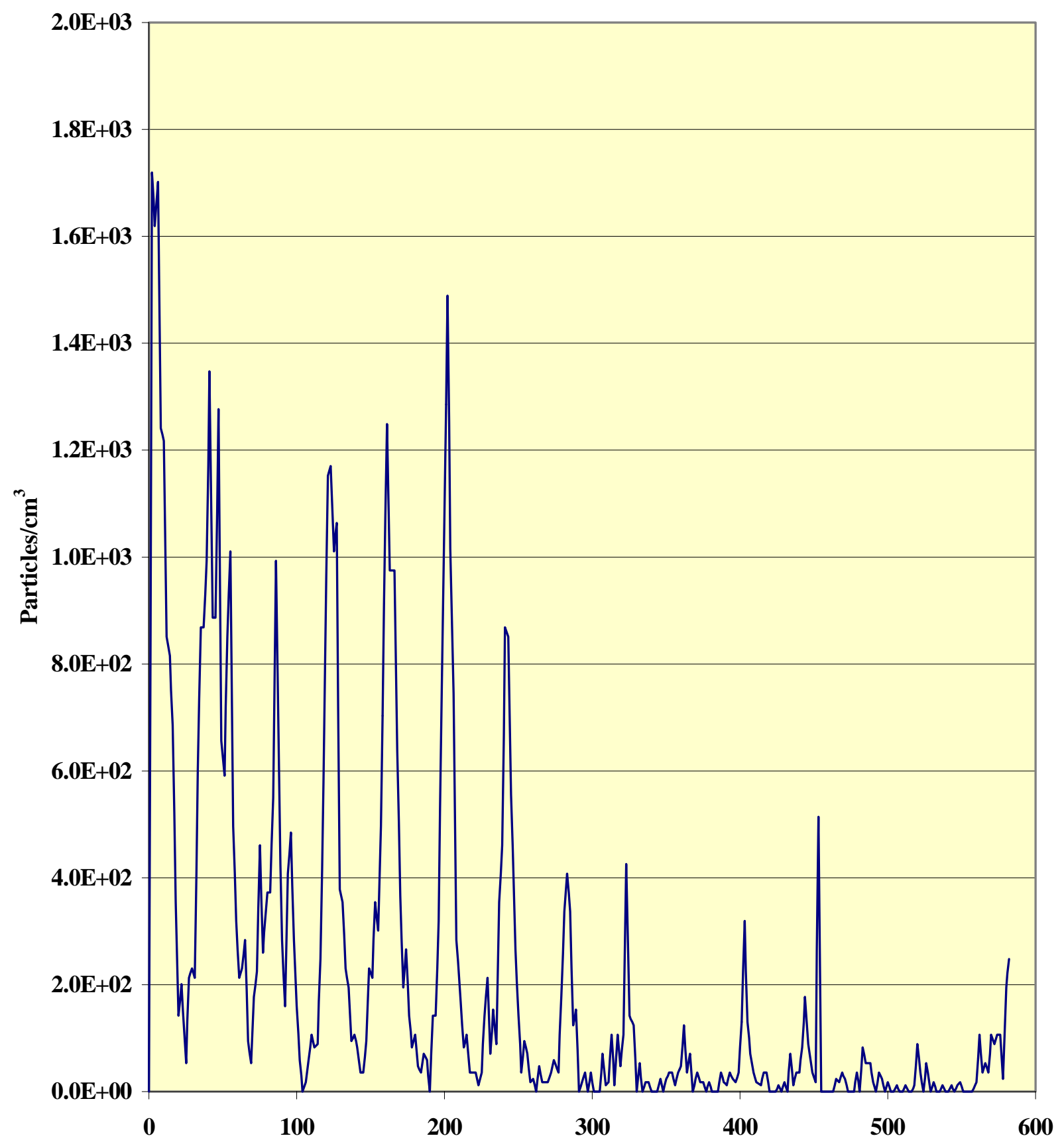

Time (seconds) 
Figure 4.115 - 20 nm SMPS Particle Concentration for Cummins L10-G Bus 4323 CBD Run \#2 Fuel: LNG

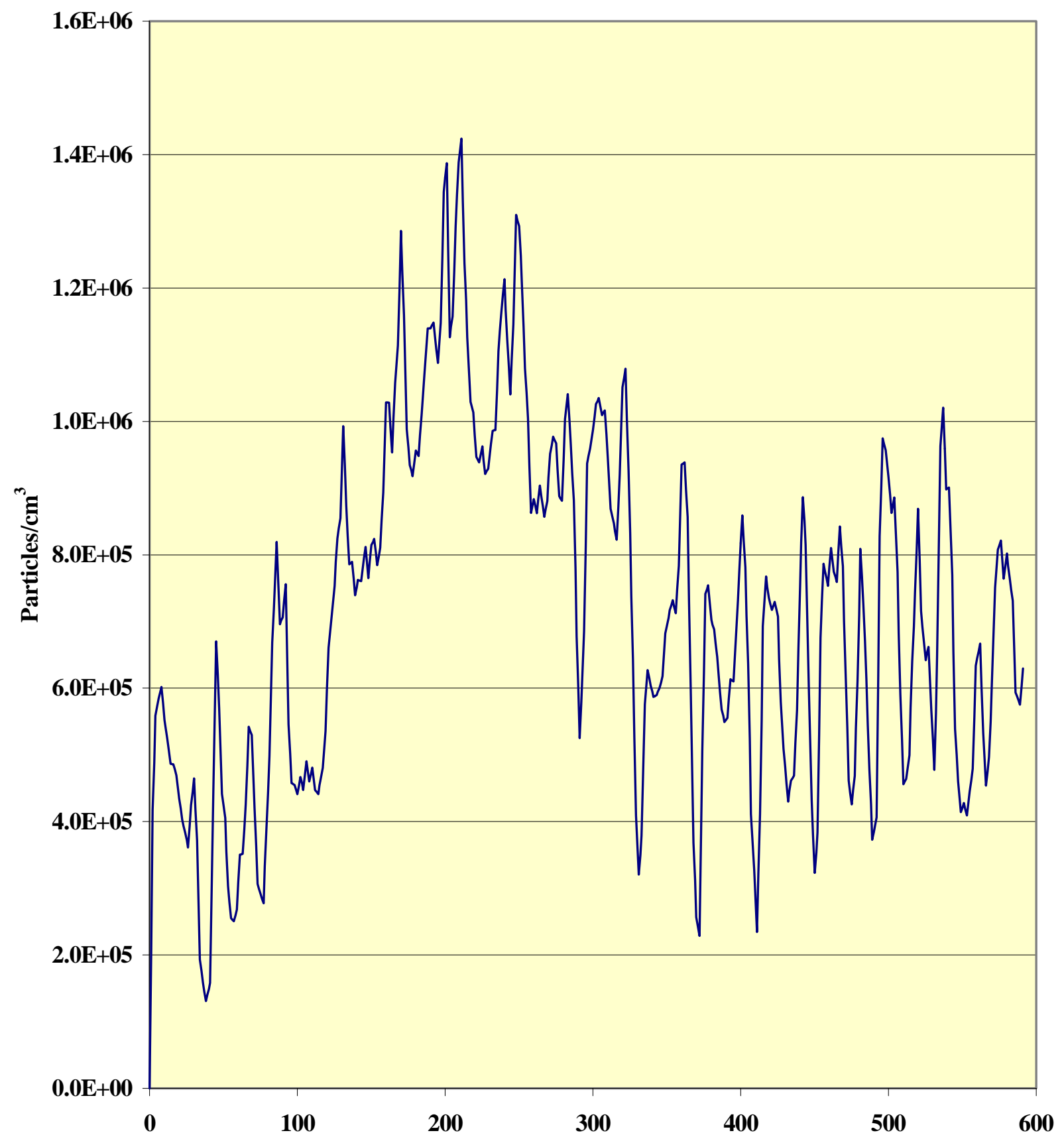

Time (seconds) 
Figure 4.116 - 30 nm SMPS Particle Concentration for Cummins L10-G Bus 4323 CBD Run \#3 Fuel: LNG

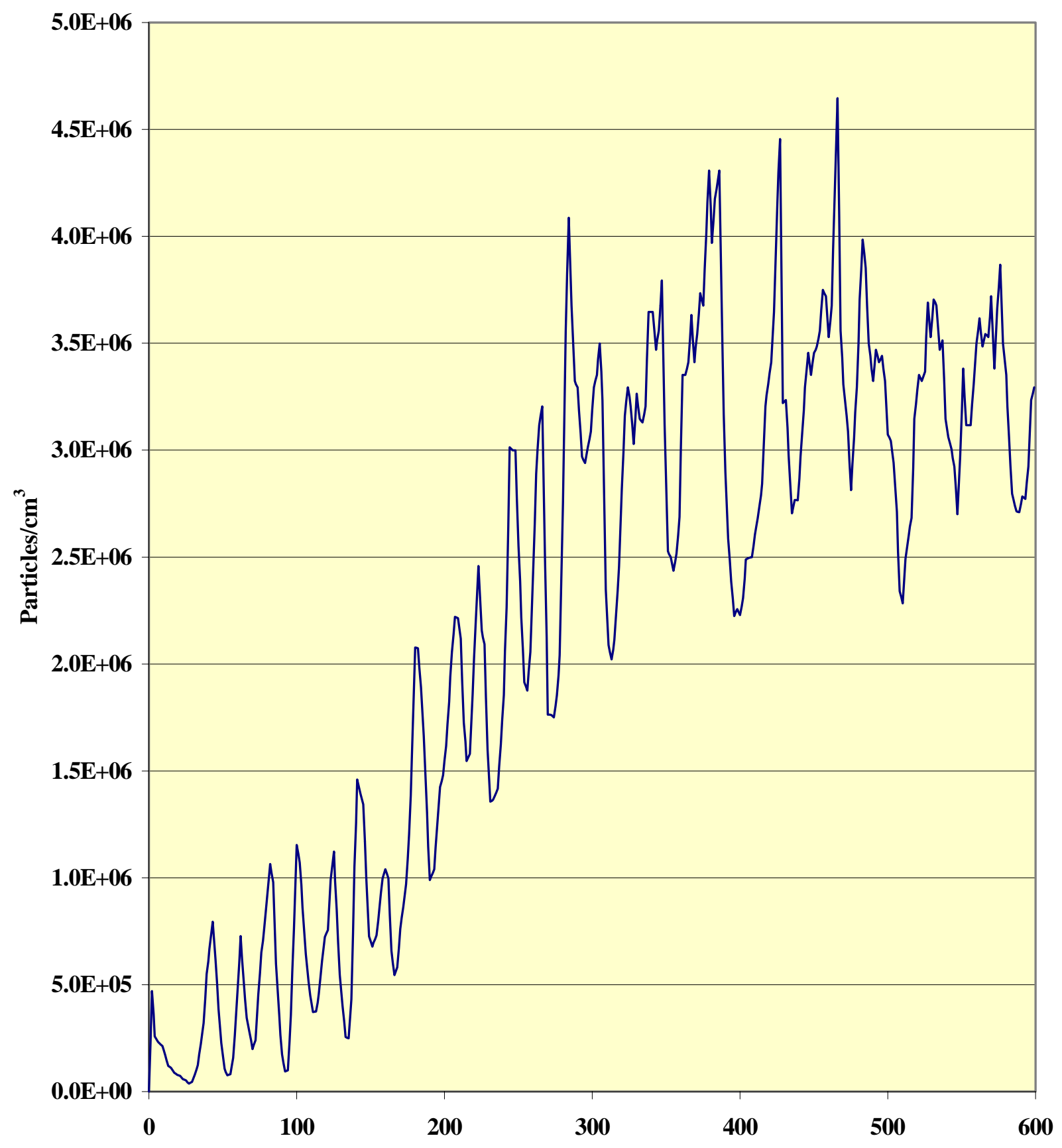

Time (seconds) 
Figure 4.117 - 40 nm SMPS Particle Concentration for Cummins L10-G Bus 4323 CBD Run \#4 Fuel: LNG

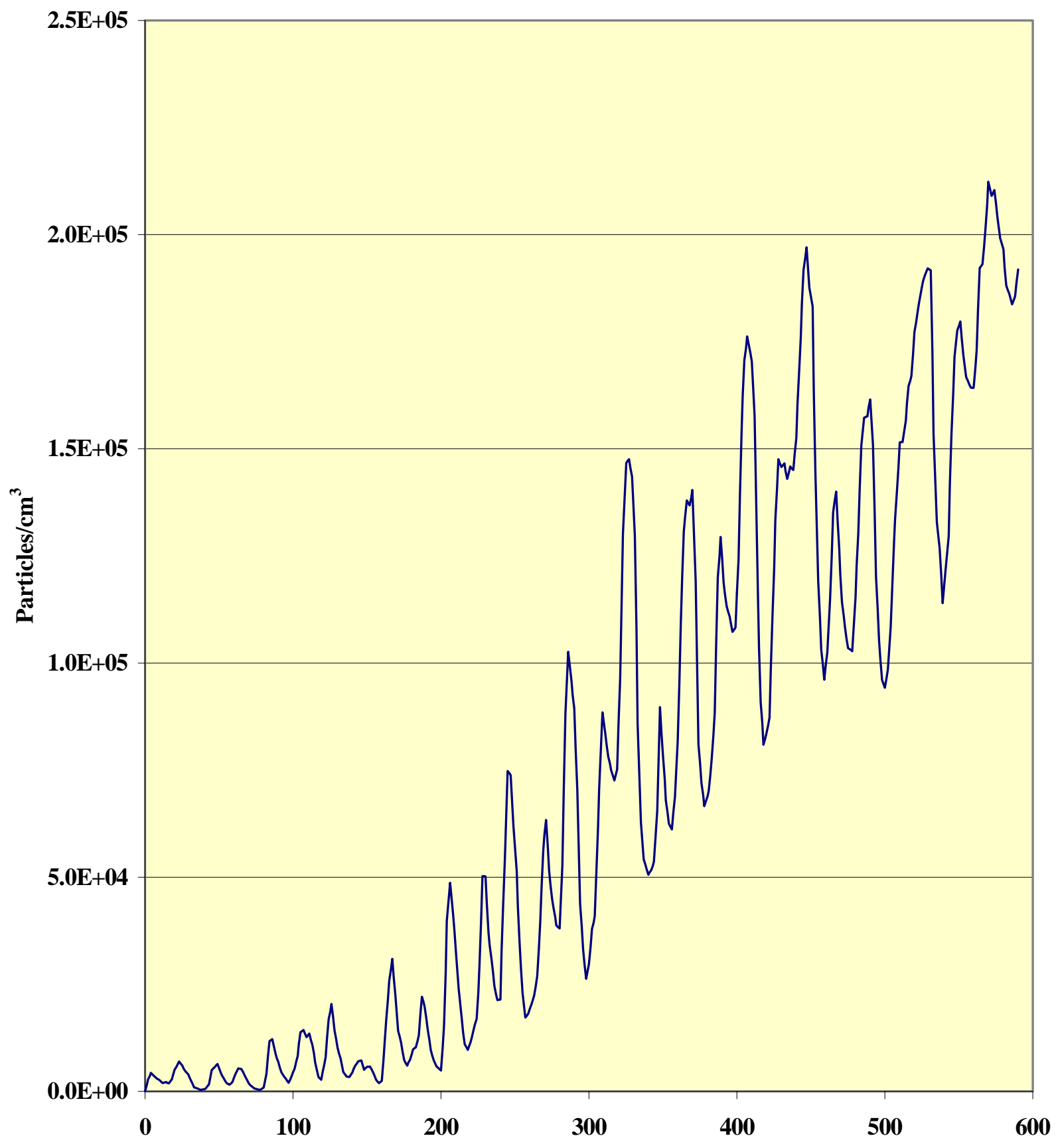

Time (seconds) 
Figure 4.118 - 50 nm SMPS Particle Concentration for Cummins L10-G Bus 4323 CBD Run \#5 Fuel: LNG

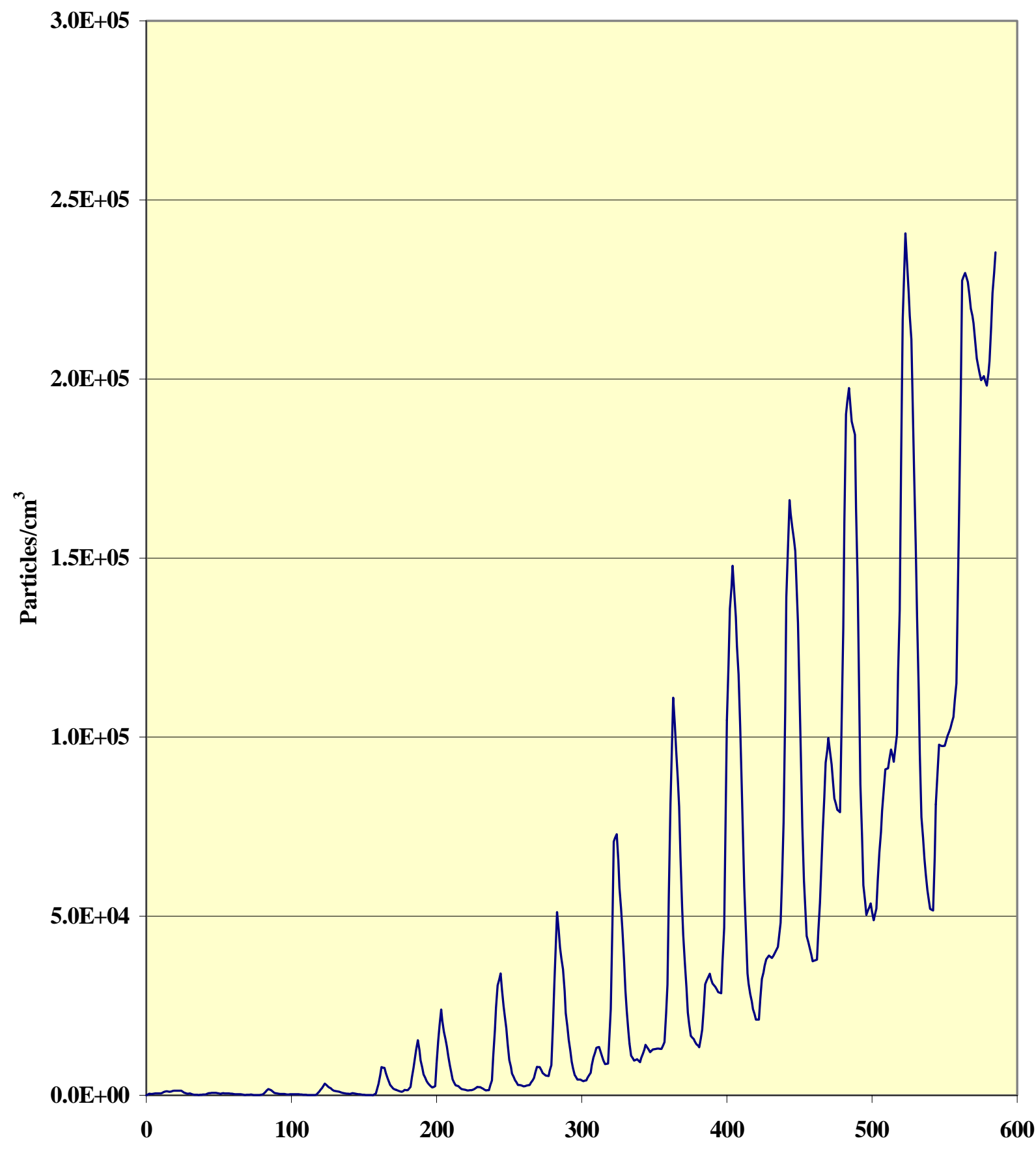

Time (seconds) 
Figure 4.119 - 10 nm SMPS Particle Concentration for Cummins

L10-G Bus 4325 CBD Run \#1 Fuel: LNG

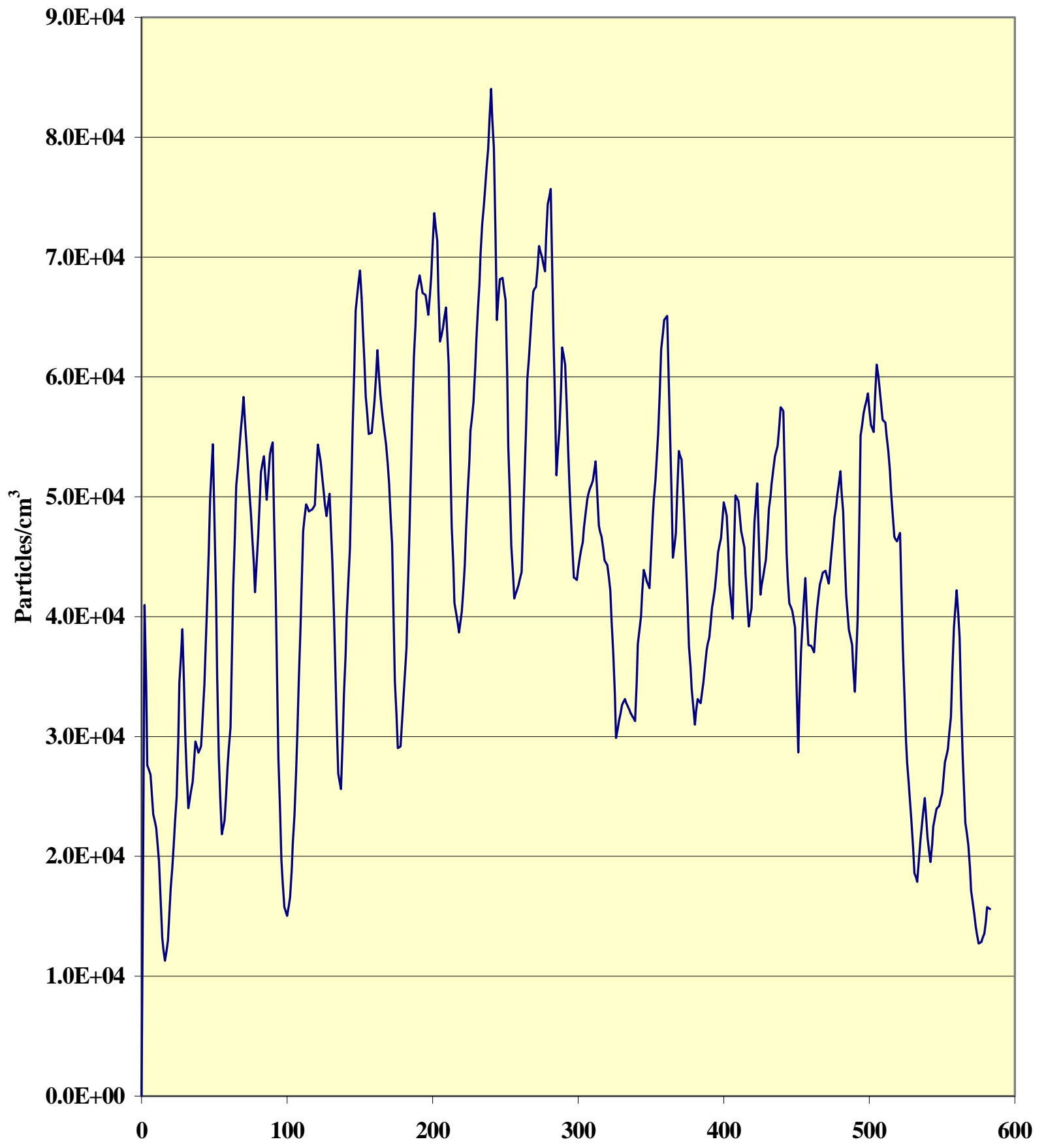

Time (seconds) 
Figure 4.120 -15 nm SMPS Particle Concentration for Cummins L10-G Bus 4325 CBD Run \#2 Fuel: LNG

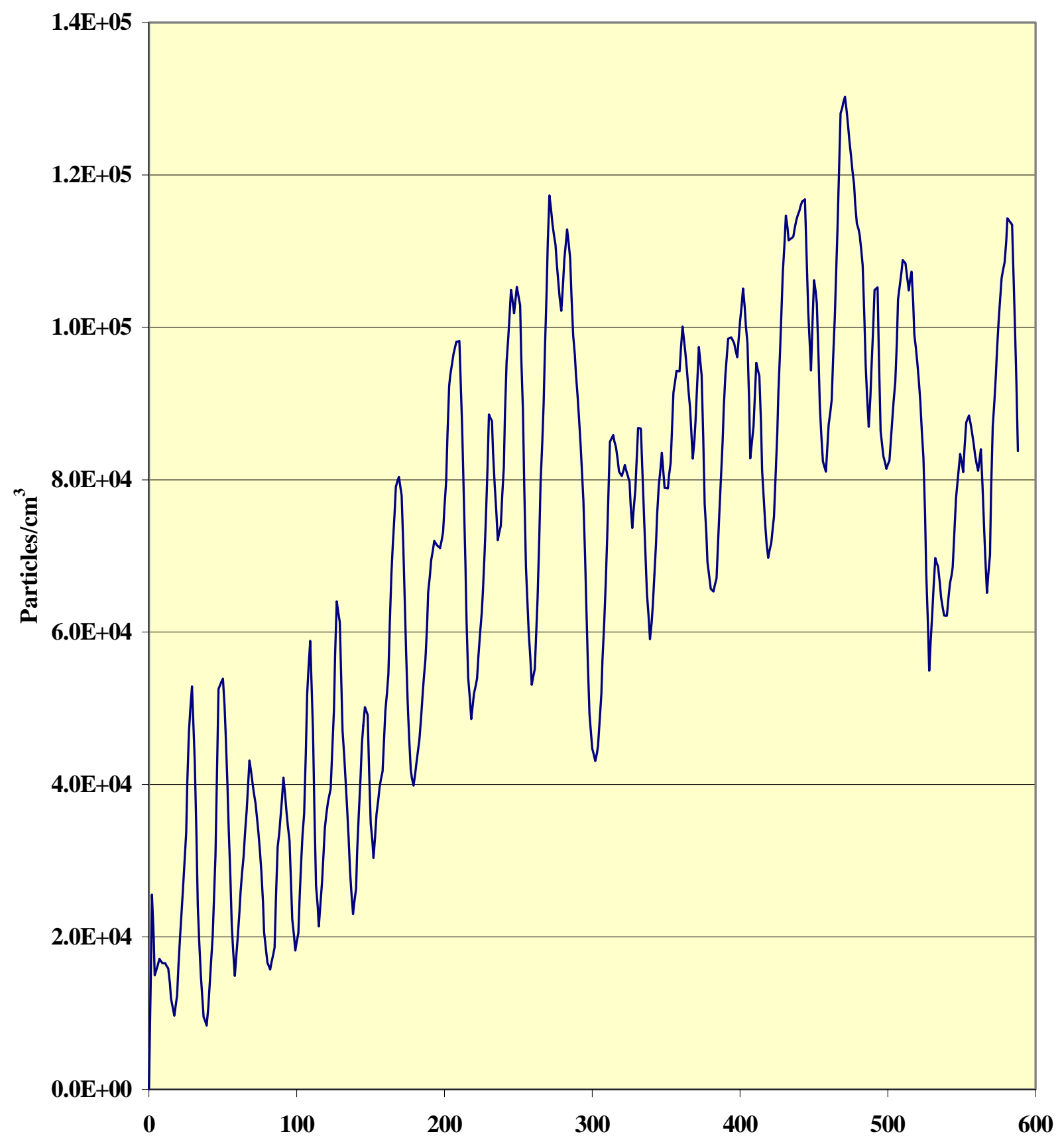

Time (seconds) 
Figure 4.121 - $20 \mathrm{~nm}$ SMPS Particle Concentration for Cummins

L10-G Bus 4325 CBD Run \#3 Fuel: LNG

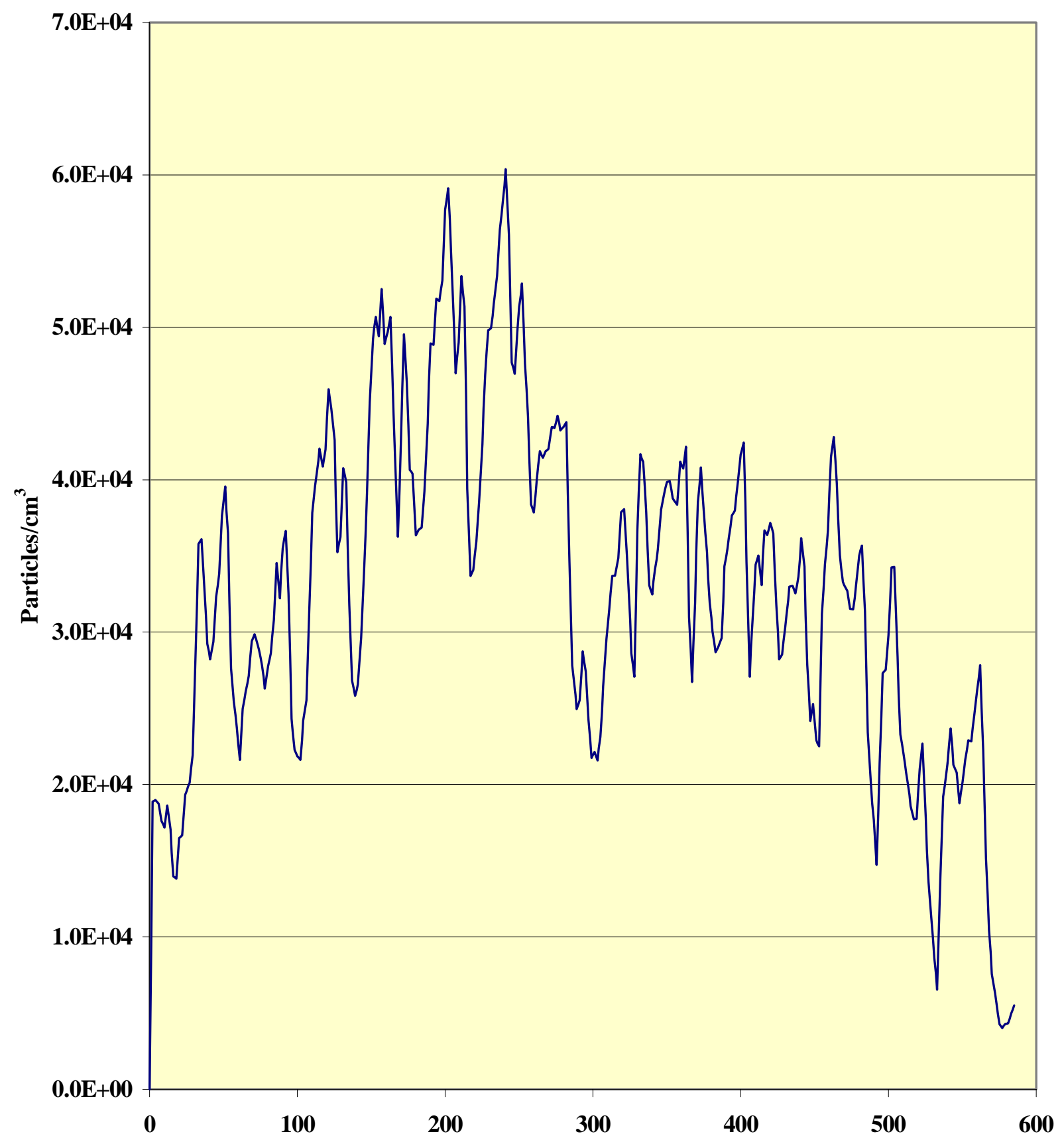

Time (seconds) 
Figure 4.122 - 30 nm SMPS Particle Concentration for Cummins L10-G Bus 4325 CBD Run \#4 Fuel: LNG

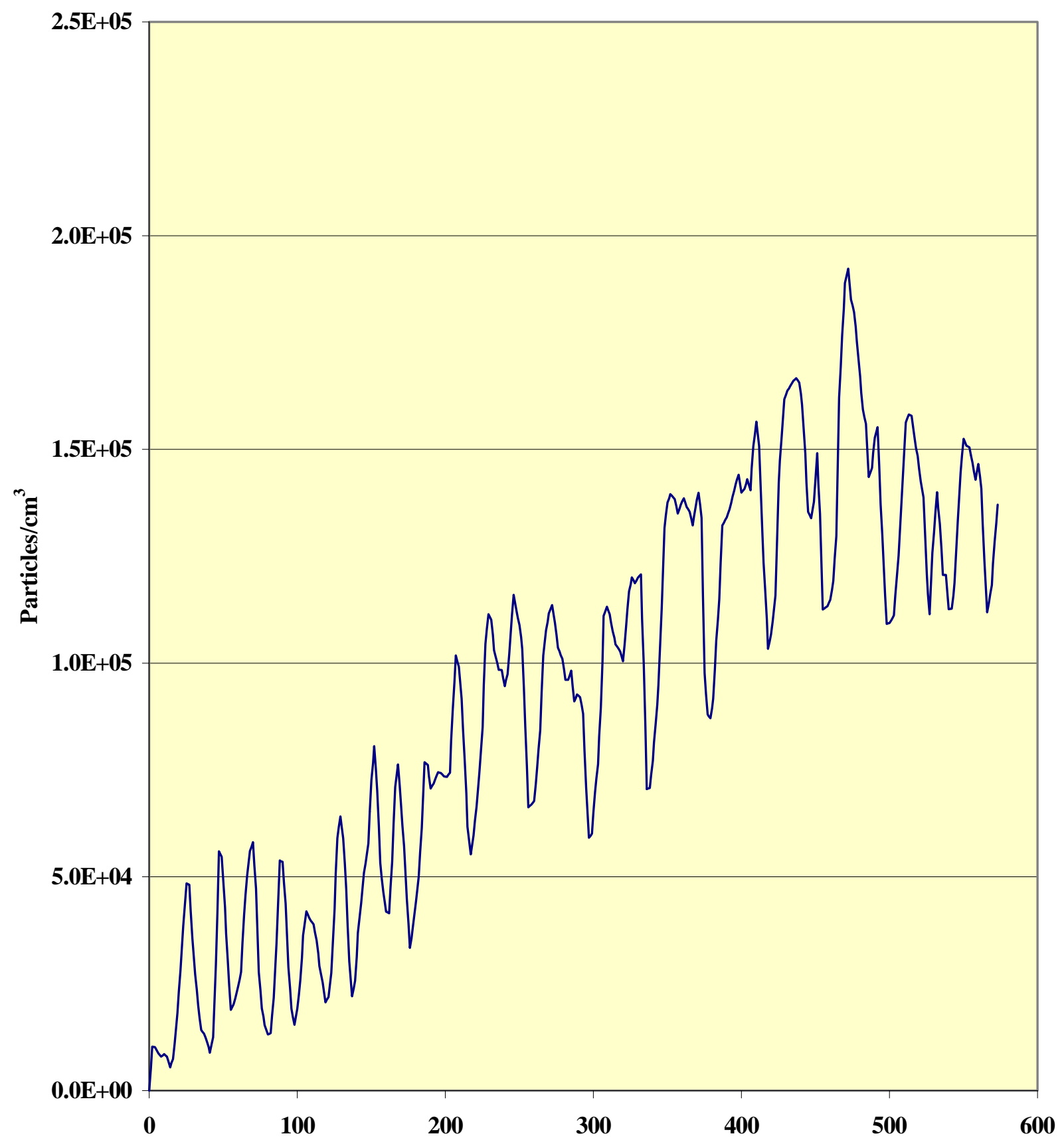

Time (seconds) 
Figure 4.123 - 45 nm SMPS Particle Concentration for Cummins L10-G Bus 4325 CBD Run \#5 Fuel: LNG

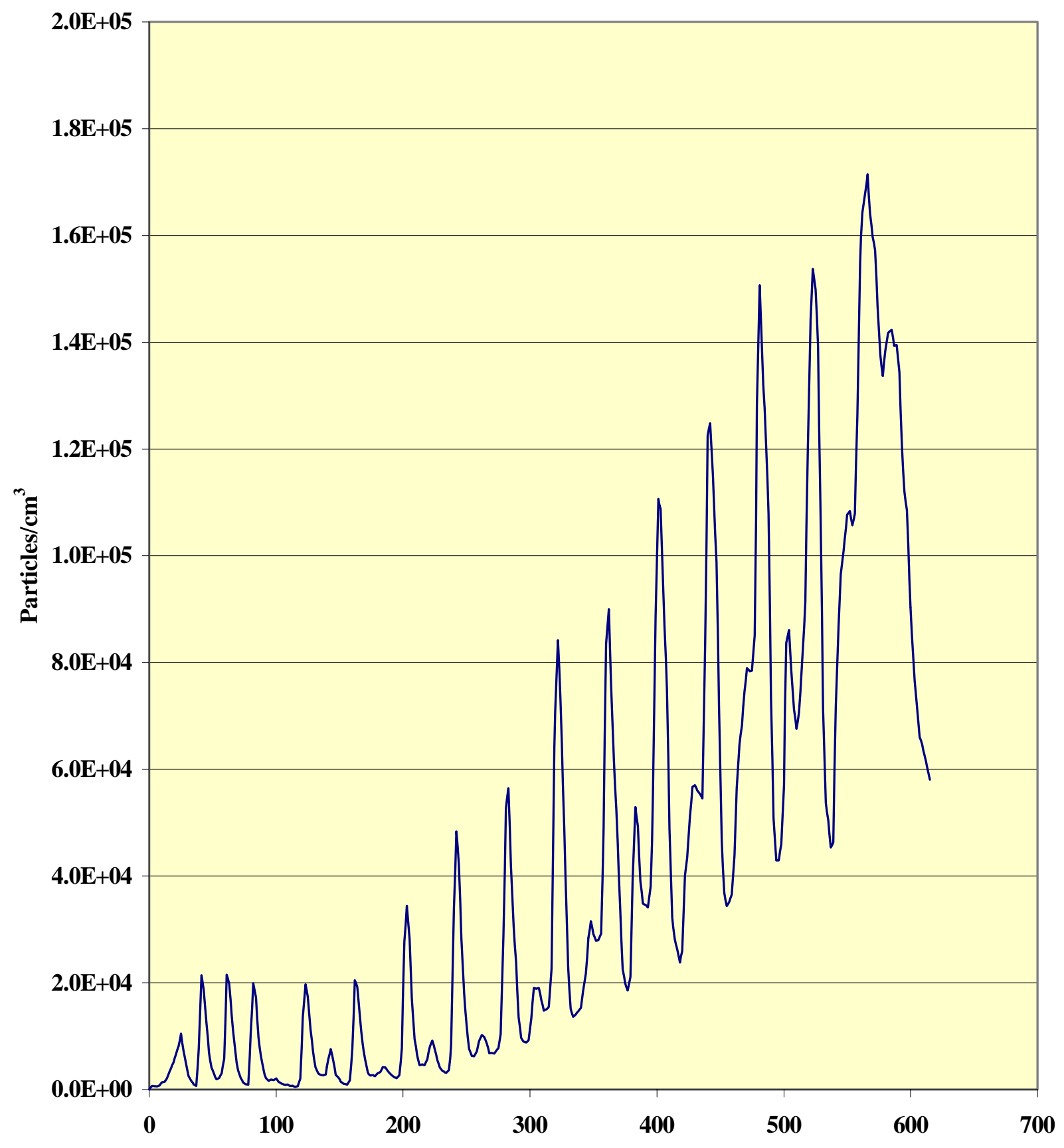

Time (seconds) 
Figure 4.124 - 20 nm SMPS Particle Concentration for Cummins L10-G Bus 4328 CBD Run \#1 Fuel: LNG

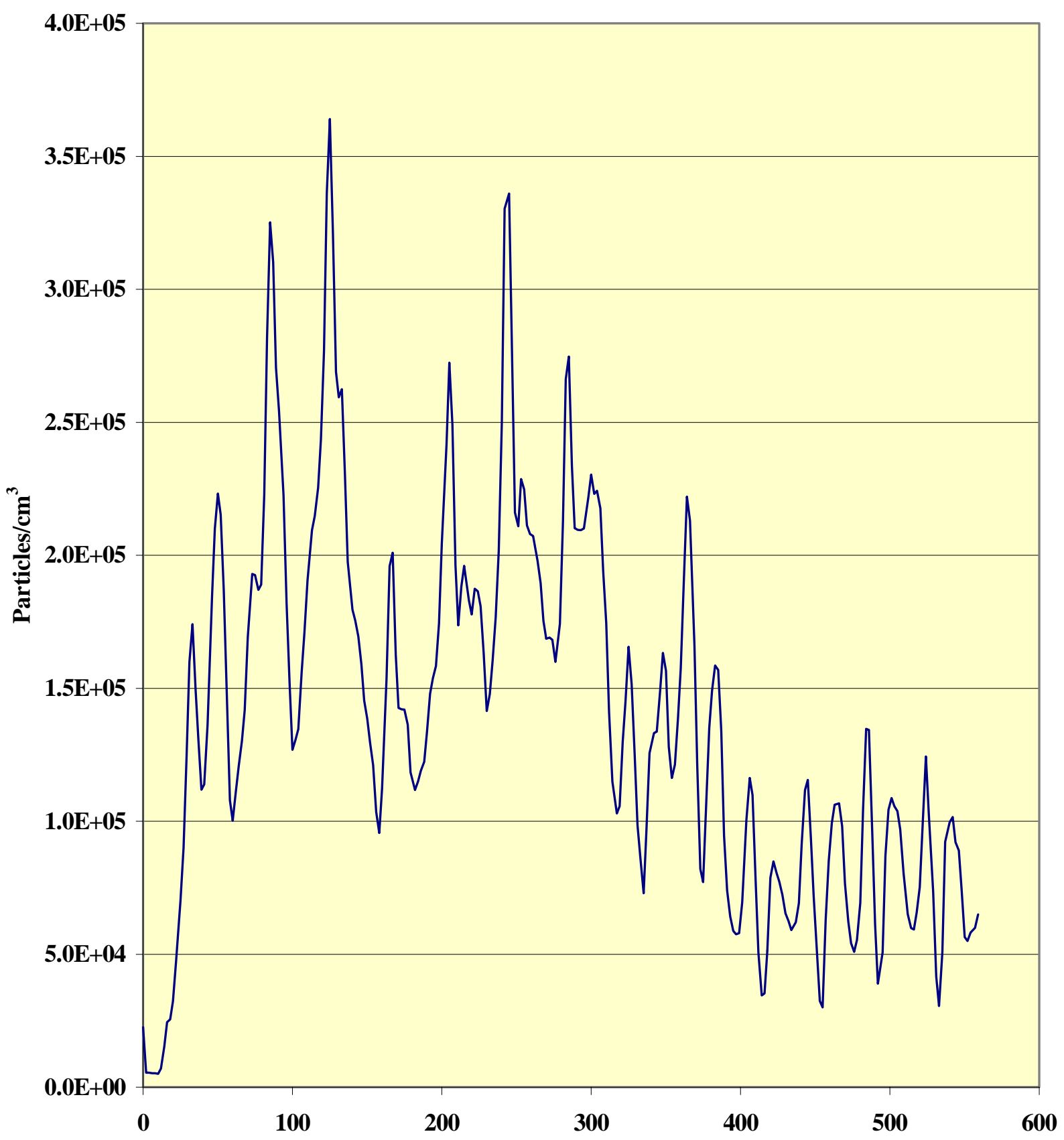

Time (seconds) 
Figure 4.125 - 25 nm SMPS Particle Concentration for Cummins

L10-G Bus 4328 CBD Run \#2 Fuel: LNG

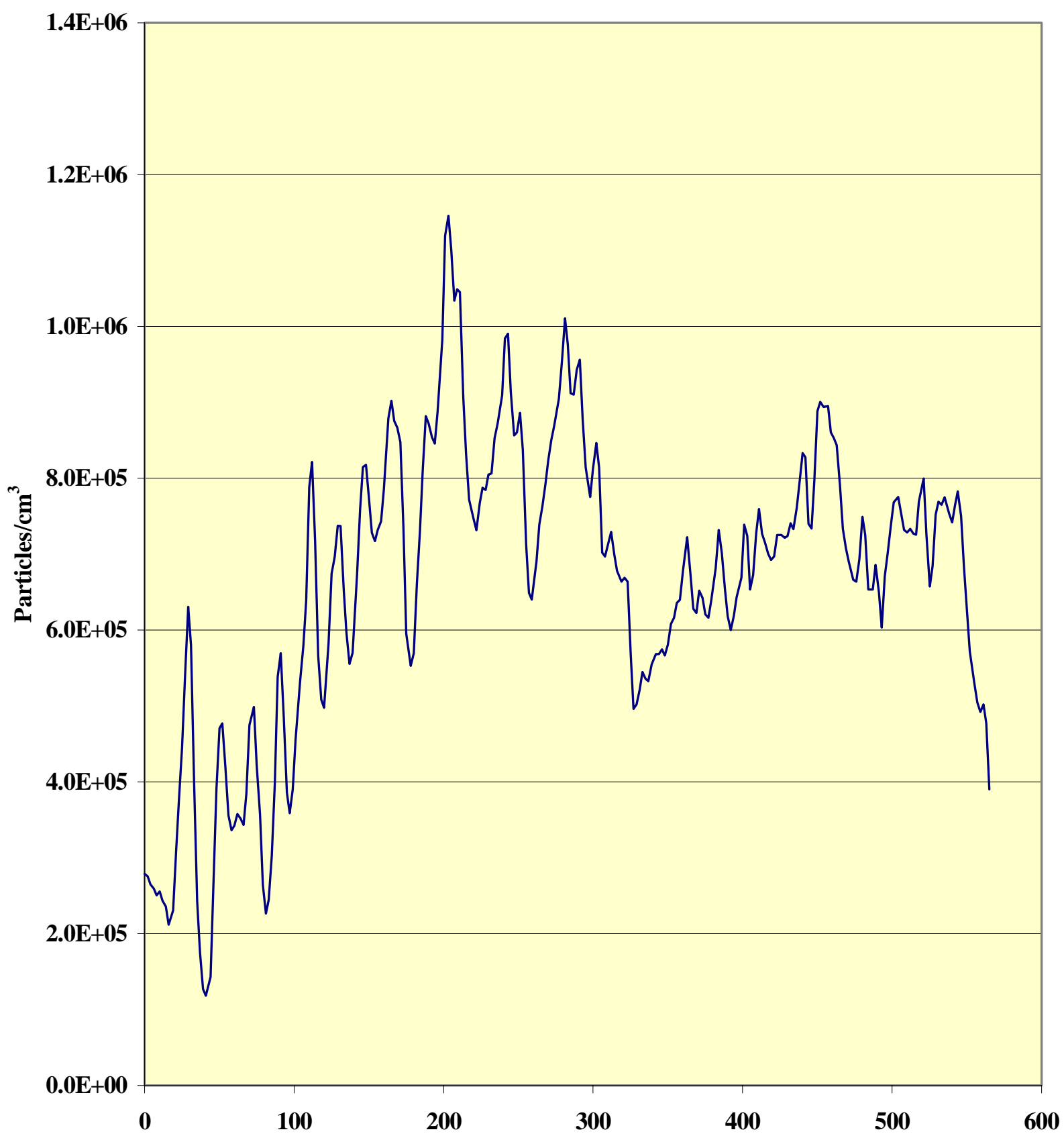

Time (seconds) 
Figure 4.126 - 33 nm SMPS Particle Concentration for Cummins L10-G Bus 4328 CBD Run \#3 Fuel: LNG

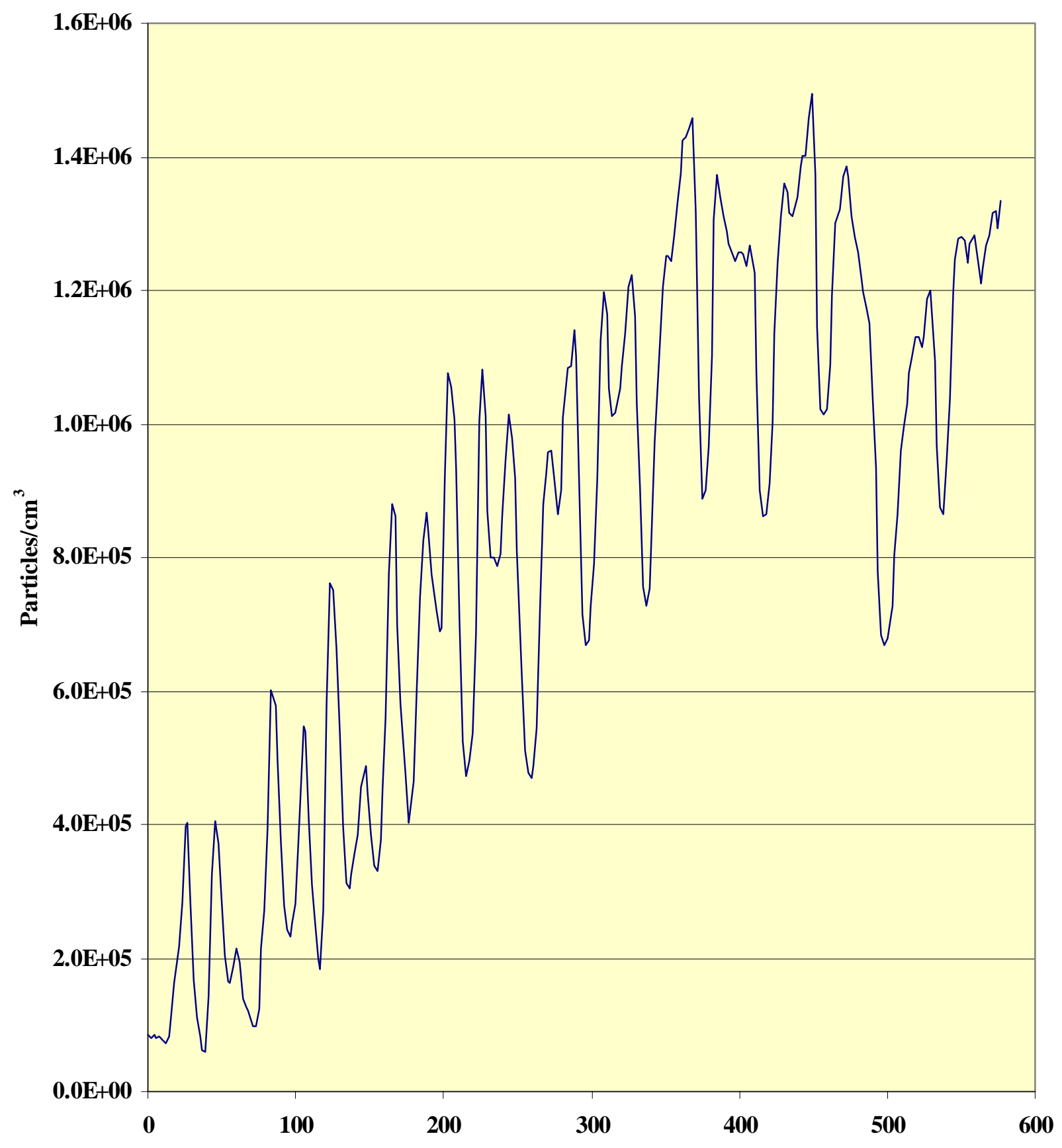

Time (seconds) 
Figure 4.127 - 45 nm SMPS Particle Concentration for Cummins L10-G Bus 4328 CBD Run \#4 Fuel: LNG

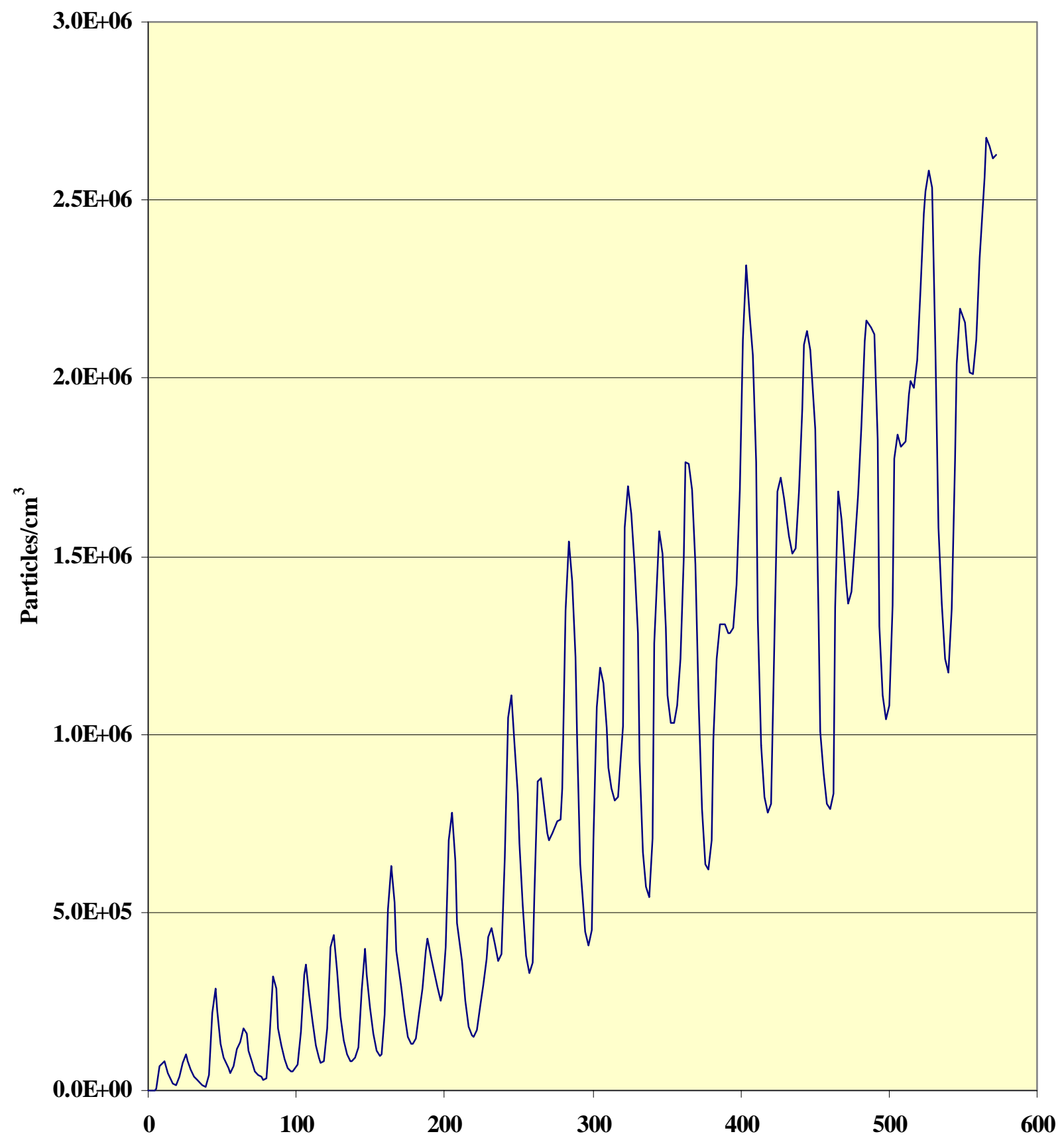

Time (seconds) 
Figure 4.128 - 60 nm SMPS Particle Concentration for Cummins L10-G Bus 4328 CBD Run \#5 Fuel: LNG

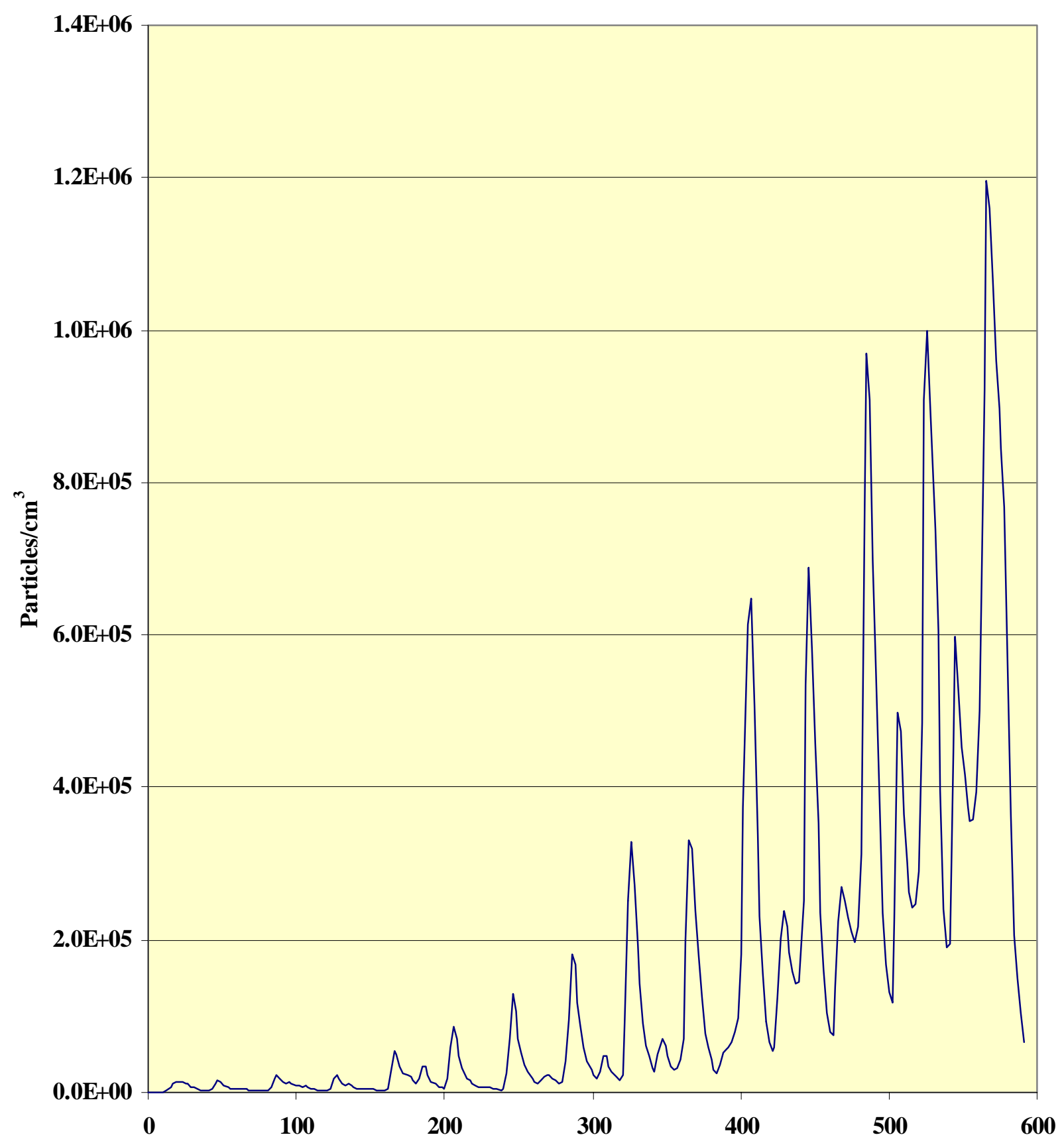

Time (seconds) 
concentrations of particles in the 10 to $25 \mathrm{~nm}$ range were also the buses with the highest concentrations of particles in the 30 to $60 \mathrm{~nm}$ range. This lends support to both theories.

\subsection{Cyclone Error Analysis}

One concern with using cyclones to sample diluted exhaust is the degree of divergence from isokinetic conditions. Since the flow rates are predetermined, the question remains as to whether the cyclones are drawing representative samples from the exhaust stream. Over or under-sampling may result in differences in measured concentration and actual concentration. For anisokinetic sampling conditions, gravitational or inertial deposition of the sample as it flows into the transfer tube can result in the loss of larger particles. Also, deposition or selective collection at the inlet of the sampling probe can yield lesser or greater amounts of larger particles.

If the sampling velocity is greater than that of the exhaust stream, particle inertia may cause particles to diverge from the streamlines and pass collection. This will result in a sampling concentration that is less than the actual. Conversely, for a sampling velocity lower than the stream velocity, concentrations measured may be greater than the actual.

While it is known that for small particles the effects of anisokinetic sampling are often minimal, the accuracy was checked for a series of particle diameters to insure representative concentrations were sampled. The following shows the process used to determine the degree of concentration differences. 


\subsubsection{Equations for Anisokinetic Sampling Error}

The Stokes Number, Stk, is the ratio of the stopping distance to the radius of the inlet tube. This equates the time a particle takes to reach equilibrium once it enters the sampling probe, and is given by

$$
S t k=\frac{\tau u_{o}}{R}
$$

where:

$$
\begin{gathered}
\tau \quad=\text { relaxation time } \\
\mathrm{u}_{\mathrm{o}} \quad=\text { dilution tunnel stream velocity } \\
\mathrm{R} \quad=\text { sampling tube radius } \\
\tau=\frac{d_{p}^{2} \rho C_{c}}{18 \mu}
\end{gathered}
$$

where:

$$
\begin{aligned}
& \mathrm{d}_{\mathrm{p}}=\text { particle diameter } \\
& \rho=\text { particle density } \\
& \mathrm{C}_{\mathrm{c}}=\text { Cunningham Slip Correction Factor } \\
& \mu \quad=\text { viscosity } \\
& \mu \quad=\mu_{o} \sqrt{\frac{T}{T_{o}}}
\end{aligned}
$$

where:

$$
\mathrm{T}=\text { actual average absolute temperature }
$$




$$
\begin{aligned}
& \mathrm{T}_{\mathrm{o}} \quad=\text { standard absolute temperature } \\
& \mu_{\mathrm{o}} \quad=\text { viscosity at standard absolute temperature }
\end{aligned}
$$

$$
C_{c}=1+\frac{\lambda}{d_{p}}\left(2.514+0.8 \exp \left(-0.55 \frac{d_{p}}{\lambda}\right)\right)
$$

where: $\quad \lambda \quad=$ mean free path

$$
\lambda=\lambda_{o}\left(\frac{P_{o}}{P}\right)\left(\frac{T}{T_{o}}\right)
$$

where:

$$
\begin{aligned}
& \lambda_{\mathrm{o}} \quad=\text { mean free path at standard pressure and temperature } \\
& \mathrm{P} \quad=\text { actual average absolute pressure } \\
& \mathrm{P}_{\mathrm{o}} \quad=\text { standard absolute pressure }
\end{aligned}
$$

Some particles may enter the sampling tube due to their inertia although they are outside the streamlines. The fraction that may bypass the streamlines, $\alpha$, varies between 0 and 1 . An exact value of this inertial fraction is not known, but can be estimated by

$$
\alpha=\frac{2 S t k}{1+2 S t k}
$$

From the inertial fraction, the ratio of the aerosol concentration to the sampling concentration can be found. This ratio will determine the degree of error induced by 
anisokinetic sampling by relating the terminal settling velocity of the fraction of particles that will enter the sampling probe to the stream and sampling velocities.

$$
\frac{c}{c_{o}}=1-\alpha+\alpha\left(\frac{u_{o}}{u}\right)
$$

where:

$$
\begin{array}{ll}
\mathrm{c} & =\text { sample concentration } \\
\mathrm{c}_{\mathrm{o}} & =\text { stream concentration } \\
\mathrm{u} & =\text { sampling tube inlet velocity }
\end{array}
$$

From the equations, the anisokinetic errors were calculated for both cyclones for four of the tests that were conducted in Dallas, Texas on the Nova transit buses. These tests were chosen to give all four conditions (mode and fuel) but were randomly selected within each scheme. As can be seen from the tables below, the errors associated with having a sampling velocity lower than the stream velocity are negligible for most particle sizes. The only error that arises is with the PM10 cyclone at particle diameters of $10 \mu \mathrm{m}$. While a noted source of error, the cyclone is only $50 \%$ efficient at this particle size. In short, there are already large inherent problems at this particular particle diameter. With a collection efficiency of only 50\%, any additional errors are inconsequential. More importantly, the smaller size particles are negligibly affected by this anisokinetic sampling. 


\section{Table 4.12 - Cyclone Error Analysis}

Test ID: 4324-1

\section{Anisokinetic Sampling Errors for PM10 Cyclone}

Assumptions

Exhaust viscosity equal to that of air

Unit density particles

Spherical particles

Relative Humidity $=50 \%$

\section{Variables}

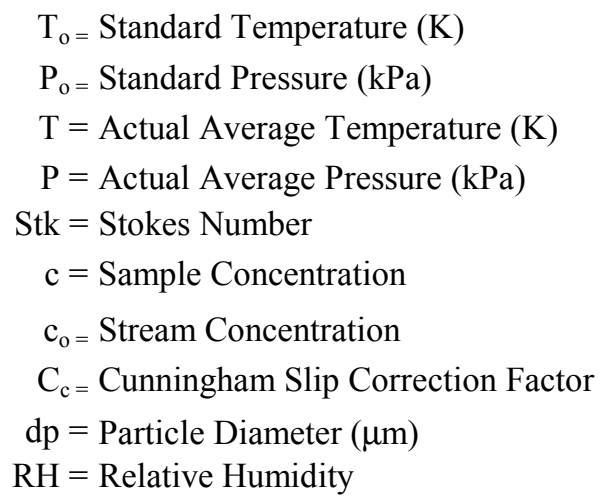

\section{Calculated Values}

$$
\begin{aligned}
\mathrm{C}_{\mathrm{c}, 10 \mu \mathrm{m}} & =1.02 \\
\mathrm{C}_{\mathrm{c}, 2.5 \mu \mathrm{m}} & =1.07 \\
\mathrm{C}_{\mathrm{c}, 1.0 \mu \mathrm{m}} & =1.18 \\
\mathrm{C}_{\mathrm{c}, 0.1 \mu \mathrm{m}} & =3.04 \\
\mathrm{C}_{\mathrm{c}, 0.01 \mu \mathrm{m}} & =24.05 \\
\tau_{10 \mu \mathrm{m}} & =2.98 \mathrm{E}-04 \\
\tau_{2.5 \mu \mathrm{m}} & =1.96 \mathrm{E}-05 \\
\tau_{1.0 \mu \mathrm{m}} & =3.45 \mathrm{E}-06 \\
\tau_{0.1 \mu \mathrm{m}} & =8.92 \mathrm{E}-08 \\
\tau_{0.01 \mu \mathrm{m}} & =7.05 \mathrm{E}-09
\end{aligned}
$$

$$
\begin{array}{rlr}
\text { Constants } & \multicolumn{1}{c}{\text { dp range }} \\
\mathrm{T}_{\mathrm{o}}=293.15 \mathrm{~K} & 10 \mu \mathrm{m} \\
\mathrm{P}_{\mathrm{o}}=101.325 \mathrm{kPa} & 2.5 \mu \mathrm{m} \\
\mu_{\mathrm{o}}=1.82 \mathrm{E}-4 \mathrm{P} & 1 \mu \mathrm{m} \\
\lambda_{\mathrm{o}} & =0.06544 \mu \mathrm{m} & 0.1 \mu \mathrm{m} \\
\mathrm{R} & =0.6625 \mathrm{in} & 0.01 \mu \mathrm{m}
\end{array}
$$

$\alpha=$ Inertial Fraction

$\mu=$ Viscosity $(\mathrm{P})$

$\mu_{\mathrm{o}}=$ Viscosity@STP

$\lambda=$ Mean Free Path $(\mu \mathrm{m})$

$\lambda_{\mathrm{o}}=$ Mean Free Path @ STP, 50\% RH

$\mathrm{r}=$ Particle Density $\left(\mathrm{g} / \mathrm{cm}^{3}\right)$

$\tau=$ Relaxation Time (sec)

$\mathrm{R}=$ Sample Tube Radius $(\mathrm{cm})$

$\mathrm{u}=$ Sampling Tube Velocity $(\mathrm{cm} / \mathrm{s})$

$\mathrm{u}_{\mathrm{o}}=$ Tunnel Velocity $(\mathrm{cm} / \mathrm{s})$

$$
\begin{array}{rlrl}
\mathrm{u}_{\mathrm{o}} & =413.1 \mathrm{~cm} / \mathrm{s} & \mu=1.89 \mathrm{E}-04 \\
\mathrm{R} & =1.68275 \mathrm{~cm} & \lambda=7.08 \mathrm{E}-02 \\
\mathrm{u}_{\mathrm{PM} 10} & =53.2 \mathrm{~cm} / \mathrm{s} & &
\end{array}
$$

$\begin{array}{rlrl}\mathrm{Stk}_{10 \mu \mathrm{m}} & =7.33 \mathrm{E}-02 & \alpha_{10 \mu \mathrm{m}}=1.28 \mathrm{E}-01 \\ \mathrm{Stk}_{2.5 \mu \mathrm{m}}=4.82 \mathrm{E}-03 & \alpha_{2.5 \mu \mathrm{m}}=9.55 \mathrm{E}-03 \\ \mathrm{Stk}_{1.0 \mu \mathrm{m}}=8.48 \mathrm{E}-04 & \alpha_{1.0 \mu \mathrm{m}}=1.69 \mathrm{E}-03 \\ \mathrm{Stk}_{0.1 \mu \mathrm{m}}=2.19 \mathrm{E}-05 & \alpha_{0.1 \mu \mathrm{m}}=4.38 \mathrm{E}-05 \\ \mathrm{Stk}_{0.01 \mu \mathrm{m}}=1.73 \mathrm{E}-06 & \alpha_{0.01 \mu \mathrm{m}}=3.46 \mathrm{E}-06\end{array}$

\begin{tabular}{|l|}
\hline Anisokinetic Error \\
\hline$c / c_{o}(d p=10 \mu m)=1.86$ \\
$c / c_{o}(d p=2.5 \mu m)=1.06$ \\
$c / c_{o}(d p=1.0 \mu m)=1.01$ \\
$c / c_{o}(d p=0.1 \mu m)=1.00$ \\
$c / c_{o}(d p=0.01 \mu m)=1.00$
\end{tabular}




\section{Anisokinetic Sampling Errors for PM2.5 Cyclone}

Test ID: 4324-1

Assumptions

Exhaust viscosity equal to that of air

Unit density particles

Spherical particles

Relative Humidity $=50 \%$

\section{Constants}

$$
\begin{aligned}
\mathrm{T}_{\mathrm{o}} & =293.15 \mathrm{~K} \\
\mathrm{P}_{\mathrm{o}} & =14.696 \mathrm{psi} \\
\mu_{\mathrm{o}} & =1.82 \mathrm{E}-4 \mathrm{P} \\
\lambda_{\mathrm{o}} & =0.06544 \mu \mathrm{m} \\
\mathrm{R} & =0.275 \mathrm{in}
\end{aligned}
$$

$$
\begin{aligned}
\alpha & =\text { Inertial Fraction } \\
\mu & =\text { Viscosity }(\mathrm{P}) \\
\mu_{\mathrm{o}} & =\text { Viscosity } @ \text { STP } \\
\lambda & =\text { Mean Free Path }(\mu \mathrm{m}) \\
\lambda_{\mathrm{o}} & =\text { Mean Free Path } @ \mathrm{STP}, 50 \% \mathrm{RH} \\
\mathrm{r} & =\text { Particle Density }\left(\mathrm{g} / \mathrm{cm}^{3}\right) \\
\tau & =\text { Relaxation Time }(\mathrm{sec}) \\
\mathrm{R} & =\text { Sample Tube Radius }(\mathrm{cm}) \\
\mathrm{u} & =\text { Sampling Tube Velocity }(\mathrm{cm} / \mathrm{s}) \\
\mathrm{u}_{\mathrm{o}} & =\text { Tunnel Velocity }(\mathrm{cm} / \mathrm{s})
\end{aligned}
$$

dp range

$2.5 \mu \mathrm{m}$

$1 \mu \mathrm{m}$

$0.1 \mu \mathrm{m}$

$0.01 \mu \mathrm{m}$

$\mathrm{Stk}=$ Stokes Number

$\mathrm{c}=$ Sample Concentration

$\mathrm{c}_{\mathrm{o}}=$ Stream Concentration

$\mathrm{C}_{\mathrm{c}}=$ Cunningham Slip Correction Factor

$\mathrm{dp}=$ Particle Diameter $(\mu \mathrm{m})$

$\mathrm{RH}=$ Relative Humidity

\section{Calculated Values}

$$
\begin{aligned}
\mathrm{C}_{\mathrm{c}, 2.5 \mu \mathrm{m}} & =1.07 \\
\mathrm{C}_{\mathrm{c}, 1.0 \mu \mathrm{m}} & =1.17 \\
\mathrm{C}_{\mathrm{c}, 0.1 \mu \mathrm{m}} & =3.00 \\
\mathrm{C}_{\mathrm{c}, 0.01 \mu \mathrm{m}} & =23.58 \\
\tau_{2.5 \mu \mathrm{m}} & =1.98 \mathrm{E}-05 \\
\tau_{1.0 \mu \mathrm{m}} & =3.48 \mathrm{E}-06 \\
\tau_{0.1 \mu \mathrm{m}} & =8.87 \mathrm{E}-08 \\
\tau_{0.01 \mu \mathrm{m}} & =6.98 \mathrm{E}-09
\end{aligned}
$$

$$
\begin{aligned}
\mathrm{u}_{\mathrm{o}} & =413.1 \mathrm{~cm} / \mathrm{s} & \mu=1.88 \mathrm{E}-04 \\
\mathrm{R} & =0.6985 \mathrm{~cm} & \lambda=6.94 \mathrm{E}-02 \\
\mathrm{u}_{\mathrm{PM} 2.5}=176.9 \mathrm{~cm} / \mathrm{s} & &
\end{aligned}
$$

$$
\begin{aligned}
\mathrm{Stk}_{2.5 \mu \mathrm{m}} & =1.17 \mathrm{E}-02 & \alpha_{2.5 \mu \mathrm{m}} & =2.29 \mathrm{E}-02 \\
\mathrm{Stk}_{1.0 \mu \mathrm{m}} & =2.06 \mathrm{E}-03 & \alpha_{1.0 \mu \mathrm{m}} & =4.10 \mathrm{E}-03 \\
\mathrm{Stk}_{0.1 \mu \mathrm{m}} & =5.25 \mathrm{E}-05 & \alpha_{0.1 \mu \mathrm{m}} & =1.05 \mathrm{E}-04 \\
\mathrm{Stk}_{0.01 \mu \mathrm{m}} & =4.13 \mathrm{E}-06 & \alpha_{0.01 \mu \mathrm{m}} & =8.26 \mathrm{E}-06
\end{aligned}
$$

\begin{tabular}{|l|}
\hline Anisokinetic Error \\
\hline$c / c_{o}(d p=2.5 \mu m)=1.03$ \\
$c / c_{o}(d p=1.0 \mu m)=1.01$ \\
$c / c_{o}(d p=0.1 \mu m)=1.00$ \\
$c / c_{o}(d p=0.01 \mu m)=1.00$ \\
\hline
\end{tabular}




\section{Anisokinetic Sampling Errors for PM10 Cyclone}

Test ID: 4322-1

\section{Assumptions}

Exhaust viscosity equal to that of air

Unit density particles

Spherical particles

Relative Humidity $=50 \%$

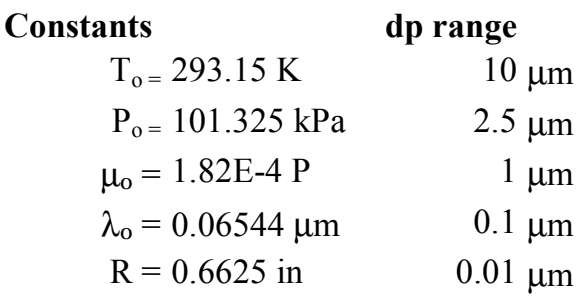

\section{Calculated Values}

$$
\begin{aligned}
\mathrm{C}_{\mathrm{c}, 10 \mu \mathrm{m}} & =1.02 \\
\mathrm{C}_{\mathrm{c}, 2.5 \mu \mathrm{m}} & =1.07 \\
\mathrm{C}_{\mathrm{c}, 1.0 \mu \mathrm{m}} & =1.18 \\
\mathrm{C}_{\mathrm{c}, 0.1 \mu \mathrm{m}} & =3.07 \\
\mathrm{C}_{\mathrm{c}, 0.01 \mu \mathrm{m}} & =24.32 \\
\tau_{10 \mu \mathrm{m}} & =2.99 \mathrm{E}-04 \\
\tau_{2.5 \mu \mathrm{m}} & =1.97 \mathrm{E}-05 \\
\tau_{1.0 \mu \mathrm{m}} & =3.47 \mathrm{E}-06 \\
\tau_{0.1 \mu \mathrm{m}} & =9.02 \mathrm{E}-08 \\
\tau_{0.01 \mu \mathrm{m}} & =7.15 \mathrm{E}-09
\end{aligned}
$$

$$
\begin{aligned}
\mathrm{u}_{\mathrm{o}} & =268.3 \mathrm{~cm} / \mathrm{s} & \mu=1.89 \mathrm{E}-04 \\
\mathrm{R} & =1.68275 \mathrm{~cm} & \lambda=7.16 \mathrm{E}-02 \\
\mathrm{u}_{\mathrm{PM} 10} & =52.8 \mathrm{~cm} / \mathrm{s} &
\end{aligned}
$$

$$
\begin{array}{|l}
\hline \multicolumn{1}{|l}{\text { Anisokinetic Error }} \\
\hline c / c_{o}(d p=10 \mu \mathrm{m})=1.36 \\
c / c_{o}(d p=2.5 \mu \mathrm{m})=1.03 \\
c / c_{o}(d p=1.0 \mu \mathrm{m})=1.00 \\
c / c_{o}(d p=0.1 \mu \mathrm{m})=1.00 \\
c / c_{o}(d p=0.01 \mu \mathrm{m})=1.00
\end{array}
$$

$$
\begin{aligned}
\mathrm{Stk}_{10 \mu \mathrm{m}} & =4.77 \mathrm{E}-02 & \alpha_{10 \mu \mathrm{m}} & =8.71 \mathrm{E}-02 \\
\mathrm{Stk}_{2.5 \mu \mathrm{m}} & =3.14 \mathrm{E}-03 & \alpha_{2.5 \mu \mathrm{m}} & =6.24 \mathrm{E}-03 \\
\mathrm{Stk}_{1.0 \mu \mathrm{m}} & =5.53 \mathrm{E}-04 & \alpha_{1.0 \mu \mathrm{m}} & =1.11 \mathrm{E}-03 \\
\mathrm{Stk}_{0.1 \mu \mathrm{m}} & =1.44 \mathrm{E}-05 & \alpha_{0.1 \mu \mathrm{m}} & =2.88 \mathrm{E}-05 \\
\mathrm{Stk}_{0.01 \mu \mathrm{m}} & =1.14 \mathrm{E}-06 & \alpha_{0.01 \mu \mathrm{m}} & =2.28 \mathrm{E}-06
\end{aligned}
$$




\section{Anisokinetic Sampling Errors for PM2.5 Cyclone}

Test ID: 4322-1

Assumptions

Exhaust viscosity equal to that of air

Unit density particles

Spherical particles

Relative Humidity $=50 \%$

$\begin{array}{rr}\text { Constants } & \text { dp range } \\ \mathrm{T}_{\mathrm{o}}=293.15 \mathrm{~K} & 2.5 \mu \mathrm{m} \\ \mathrm{P}_{\mathrm{o}}=14.696 \mathrm{psi} & 1 \mu \mathrm{m} \\ \mu_{\mathrm{o}}=1.82 \mathrm{E}-4 \mathrm{P} & 0.1 \mu \mathrm{m} \\ \lambda_{\mathrm{o}}=0.06544 \mu \mathrm{m} & 0.01 \mu \mathrm{m} \\ \mathrm{R} & =0.275 \mathrm{in}\end{array}$

Variables

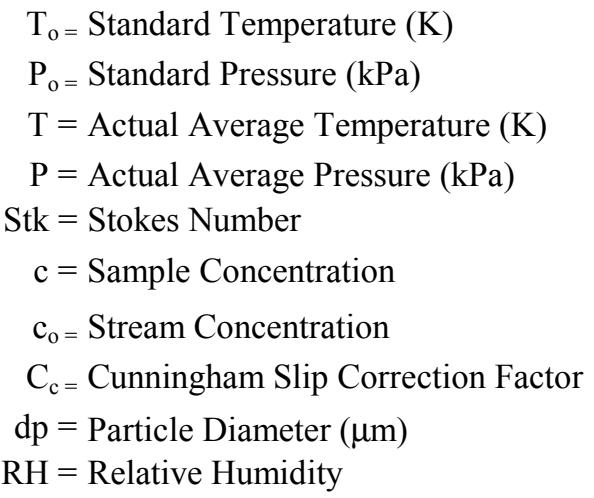

\section{Calculated Values}

$$
\begin{aligned}
\mathrm{C}_{\mathrm{c}, 2.5 \mu \mathrm{m}} & =1.07 \\
\mathrm{C}_{\mathrm{c}, 1.0 \mu \mathrm{m}} & =1.18 \\
\mathrm{C}_{\mathrm{c}, 0.1 \mu \mathrm{m}} & =3.04 \\
\mathrm{C}_{\mathrm{c}, 0.01 \mu \mathrm{m}} & =24.04 \\
\tau_{2.5 \mu \mathrm{m}} & =1.98 \mathrm{E}-05 \\
\tau_{1.0 \mu \mathrm{m}} & =3.48 \mathrm{E}-06 \\
\tau_{0.1 \mu \mathrm{m}} & =8.99 \mathrm{E}-08 \\
\tau_{0.01 \mu \mathrm{m}} & =7.11 \mathrm{E}-09
\end{aligned}
$$

$$
\begin{aligned}
\alpha & =\text { Inertial Fraction } \\
\mu & =\text { Viscosity }(\mathrm{P}) \\
\mu_{\mathrm{o}} & =\text { Viscosity } @ \text { STP } \\
\lambda & =\text { Mean Free Path }(\mu \mathrm{m}) \\
\lambda_{\mathrm{o}} & =\text { Mean Free Path } @ \text { STP, } 50 \% \text { RH } \\
\mathrm{r} & =\text { Particle Density }\left(\mathrm{g} / \mathrm{cm}^{3}\right) \\
\tau & =\text { Relaxation Time }(\mathrm{sec}) \\
\mathrm{R} & =\text { Sample Tube Radius }(\mathrm{cm}) \\
\mathrm{u} & =\text { Sampling Tube Velocity }(\mathrm{cm} / \mathrm{s}) \\
\mathrm{u}_{\mathrm{o}} & =\text { Tunnel Velocity }(\mathrm{cm} / \mathrm{s})
\end{aligned}
$$

\begin{tabular}{|l|}
\hline Anisokinetic Error \\
\hline$c / c_{o}(d p=2.5 \mu m)=1.01$ \\
$c / c_{o}(d p=1.0 \mu m)=1.00$ \\
$c / c_{o}(d p=0.1 \mu m)=1.00$ \\
$c / c_{o}(d p=0.01 \mu m)=1.00$ \\
\hline
\end{tabular}




\section{Anisokinetic Sampling Errors for PM10 Cyclone}

Test ID: 4220-1

\section{Assumptions}

Exhaust viscosity equal to that of air

Unit density particles

Spherical particles

Relative Humidity $=50 \%$

$$
\begin{aligned}
& 10 \mu \mathrm{m} \\
& 2.5 \mu \mathrm{m} \\
& 1 \mu \mathrm{m} \\
& 0.1 \mu \mathrm{m} \\
& 0.01 \mu \mathrm{m}
\end{aligned}
$$

\section{Calculated Values}

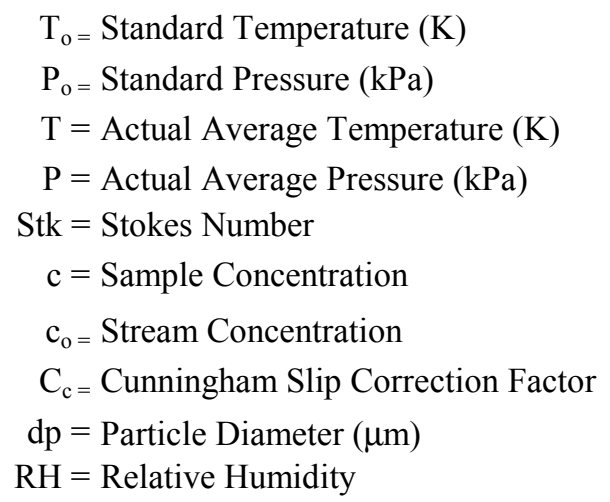

$$
\begin{aligned}
\mathrm{C}_{\mathrm{c}, 10 \mu \mathrm{m}} & =1.02 \\
\mathrm{C}_{\mathrm{c}, 2.5 \mu \mathrm{m}} & =1.07 \\
\mathrm{C}_{\mathrm{c}, 1.0 \mu \mathrm{m}} & =1.18 \\
\mathrm{C}_{\mathrm{c}, 0.1 \mu \mathrm{m}} & =3.03 \\
\mathrm{C}_{\mathrm{c}, 0.01 \mu \mathrm{m}} & =23.94 \\
\tau_{10 \mu \mathrm{m}} & =3.01 \mathrm{E}-04 \\
\tau_{2.5 \mu \mathrm{m}} & =1.98 \mathrm{E}-05 \\
\tau_{1.0 \mu \mathrm{m}} & =3.48 \mathrm{E}-06 \\
\tau_{0.1 \mu \mathrm{m}} & =8.97 \mathrm{E}-08 \\
\tau_{0.01 \mu \mathrm{m}} & =7.08 \mathrm{E}-09
\end{aligned}
$$

$$
\begin{aligned}
\mathrm{u}_{\mathrm{o}} & =409.1 \mathrm{~cm} / \mathrm{s} & \mu=1.88 \mathrm{E}-04 \\
\mathrm{R} & =1.68275 \mathrm{~cm} & \lambda=7.05 \mathrm{E}-02 \\
\mathrm{u}_{\mathrm{PM} 10} & =52.9 \mathrm{~cm} / \mathrm{s} &
\end{aligned}
$$

$$
\begin{aligned}
\mathrm{Stk}_{10 \mu \mathrm{m}} & =7.32 \mathrm{E}-02 & \alpha_{10 \mu \mathrm{m}} & =1.28 \mathrm{E}-01 \\
\mathrm{Stk}_{2.5 \mu \mathrm{m}} & =4.81 \mathrm{E}-03 & \alpha_{2.5 \mu \mathrm{m}} & =9.54 \mathrm{E}-03 \\
\mathrm{Stk}_{1.0 \mu \mathrm{m}} & =8.47 \mathrm{E}-04 & \alpha_{1.0 \mu \mathrm{m}} & =1.69 \mathrm{E}-03 \\
\mathrm{Stk}_{0.1 \mu \mathrm{m}} & =2.18 \mathrm{E}-05 & \alpha_{0.1 \mu \mathrm{m}} & =4.36 \mathrm{E}-05 \\
\mathrm{Stk}_{0.01 \mu \mathrm{m}} & =1.72 \mathrm{E}-06 & \alpha_{0.01 \mu \mathrm{m}} & =3.44 \mathrm{E}-06
\end{aligned}
$$

\begin{tabular}{|l|}
\hline Anisokinetic Error \\
\hline$c / c_{o}(d p=10 \mu \mathrm{m})=1.86$ \\
$c / c_{o}(d p=2.5 \mu \mathrm{m})=1.06$ \\
$c / c_{o}(d p=1.0 \mu \mathrm{m})=1.01$ \\
$c / c_{o}(d p=0.1 \mu \mathrm{m})=1.00$ \\
$c / c_{o}(d p=0.01 \mu \mathrm{m})=1.00$
\end{tabular}




\section{Anisokinetic Sampling Errors for PM2.5 Cyclone}

Test ID: 4220-1

\section{Assumptions}

Unit density particles

Spherical particles

Relative Humidity $=50 \%$
Exhaust viscosity equal to that of air

\section{Constants}

Variables

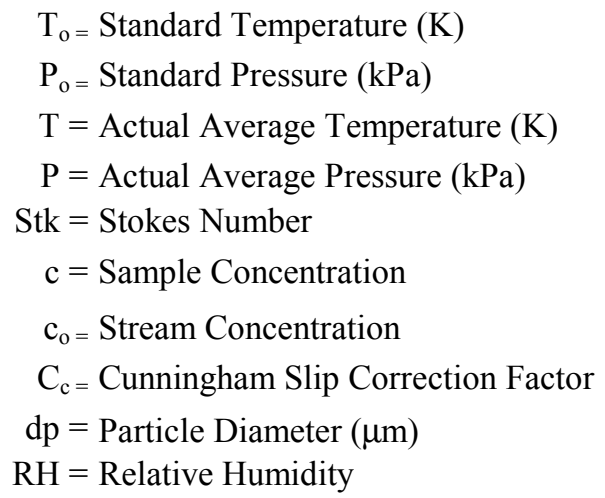

\section{Calculated Values}

$$
\begin{aligned}
\mathrm{C}_{\mathrm{c}, 2.5 \mu \mathrm{m}} & =1.07 \\
\mathrm{C}_{\mathrm{c}, 1.0 \mu \mathrm{m}} & =1.18 \\
\mathrm{C}_{\mathrm{c}, 0.1 \mu \mathrm{m}} & =3.01 \\
\mathrm{C}_{\mathrm{c}, 0.01 \mu \mathrm{m}} & =23.75 \\
\tau_{2.5 \mu \mathrm{m}} & =1.99 \mathrm{E}-05 \\
\tau_{1.0 \mu \mathrm{m}} & =3.49 \mathrm{E}-06 \\
\tau_{0.1 \mu \mathrm{m}} & =8.95 \mathrm{E}-08 \\
\tau_{0.01 \mu \mathrm{m}} & =7.06 \mathrm{E}-09
\end{aligned}
$$

\section{Anisokinetic Error}

$$
\begin{aligned}
& c / c_{o}(d p=2.5 \mu m)=1.03 \\
& c / c_{o}(d p=1.0 \mu m)=1.01 \\
& c / c_{o}(d p=0.1 \mu m)=1.00 \\
& c / c_{o}(d p=0.01 \mu m)=1.00
\end{aligned}
$$

$\mathrm{T}_{\mathrm{o}}=293.15 \mathrm{~K}$

$\mathrm{P}_{\mathrm{o}}=14.696 \mathrm{psi}$

$\mu_{\mathrm{o}}=1.82 \mathrm{E}-4 \mathrm{P}$

$\lambda_{\mathrm{o}}=0.06544 \mu \mathrm{m}$

$\mathrm{R}=0.275$ in

$$
\begin{aligned}
\alpha & =\text { Inertial Fraction } \\
\mu & =\text { Viscosity }(\mathrm{P}) \\
\mu_{\mathrm{o}} & =\text { Viscosity @ STP } \\
\lambda & =\text { Mean Free Path }(\mu \mathrm{m}) \\
\lambda_{\mathrm{o}} & =\text { Mean Free Path @ STP, 50\% RH } \\
\mathrm{r} & =\text { Particle Density }\left(\mathrm{g} / \mathrm{cm}^{3}\right) \\
\tau & =\text { Relaxation Time }(\mathrm{sec}) \\
\mathrm{R} & =\text { Sample Tube Radius }(\mathrm{cm}) \\
\mathrm{u} & =\text { Sampling Tube Velocity }(\mathrm{cm} / \mathrm{s}) \\
\mathrm{u}_{\mathrm{o}} & =\text { Tunnel Velocity }(\mathrm{cm} / \mathrm{s})
\end{aligned}
$$

$2.5 \mu \mathrm{m}$

$1 \mu \mathrm{m}$

$0.1 \mu \mathrm{m}$

$0.01 \mu \mathrm{m}$

$$
\begin{aligned}
\mathrm{u}_{\mathrm{o}} & =409.1 \mathrm{~cm} / \mathrm{s} & \mu=1.87 \mathrm{E}-04 \\
\mathrm{R} & =0.6985 \mathrm{~cm} & \lambda=6.99 \mathrm{E}-02 \\
\mathrm{u}_{\mathrm{PM} 2.5} & =176.3 \mathrm{~cm} / \mathrm{s} &
\end{aligned}
$$

$$
\begin{aligned}
\mathrm{Stk}_{2.5 \mu \mathrm{m}}=1.16 \mathrm{E}-02 & \alpha_{2.5 \mu \mathrm{m}}=2.27 \mathrm{E}-02 \\
\mathrm{Stk}_{1.0 \mu \mathrm{m}}=2.05 \mathrm{E}-03 & \alpha_{1.0 \mu \mathrm{m}}=4.08 \mathrm{E}-03 \\
\mathrm{Stk}_{0.1 \mu \mathrm{m}}=5.24 \mathrm{E}-05 & \alpha_{0.1 \mu \mathrm{m}}=1.05 \mathrm{E}-04 \\
\mathrm{Stk}_{0.01 \mu \mathrm{m}}=4.13 \mathrm{E}-06 & \alpha_{0.01 \mu \mathrm{m}}=8.26 \mathrm{E}-06
\end{aligned}
$$




\section{Anisokinetic Sampling Errors for PM10 Cyclone}

Test ID: $4220-2$

Assumptions

Exhaust viscosity equal to that of air

Unit density particles

Spherical particles

Relative Humidity $=50 \%$

\section{Constants}

$$
\begin{aligned}
\mathrm{T}_{\mathrm{o}} & =293.15 \mathrm{~K} \\
\mathrm{P}_{\mathrm{o}} & =101.325 \mathrm{kPa} \\
\mu_{\mathrm{o}} & =1.82 \mathrm{E}-4 \mathrm{P} \\
\lambda_{\mathrm{o}} & =0.06544 \mu \mathrm{m} \\
\mathrm{R} & =0.6625 \mathrm{in}
\end{aligned}
$$

dp range

$$
\begin{array}{r}
10 \mu \mathrm{m} \\
2.5 \mu \mathrm{m} \\
1 \mu \mathrm{m} \\
0.1 \mu \mathrm{m} \\
0.01 \mu \mathrm{m}
\end{array}
$$

\section{Variables}

$$
\begin{aligned}
\mathrm{T}_{\mathrm{o}} & =\text { Standard Temperature }(\mathrm{K}) \\
\mathrm{P}_{\mathrm{o}} & =\text { Standard Pressure }(\mathrm{kPa}) \\
\mathrm{T} & =\text { Actual Average Temperature }(\mathrm{K}) \\
\mathrm{P} & =\text { Actual Average Pressure }(\mathrm{kPa}) \\
\mathrm{Stk} & =\text { Stokes Number } \\
\mathrm{c} & =\text { Sample Concentration } \\
\mathrm{c}_{\mathrm{o}} & =\text { Stream Concentration } \\
\mathrm{C}_{\mathrm{c}} & =\text { Cunningham Slip Correction Factor } \\
\mathrm{d} p & =\text { Particle Diameter }(\mu \mathrm{m}) \\
\mathrm{RH} & =\text { Relative Humidity }
\end{aligned}
$$

\section{Calculated Values}

$$
\begin{aligned}
\mathrm{C}_{\mathrm{c}, 10 \mu \mathrm{m}} & =1.02 \\
\mathrm{C}_{\mathrm{c}, 2.5 \mu \mathrm{m}} & =1.07 \\
\mathrm{C}_{\mathrm{c}, 1.0 \mu \mathrm{m}} & =1.18 \\
\mathrm{C}_{\mathrm{c}, 0.1 \mu \mathrm{m}} & =3.05 \\
\mathrm{C}_{\mathrm{c}, 0.01 \mu \mathrm{m}} & =24.12 \\
\tau_{10 \mu \mathrm{m}} & =3.01 \mathrm{E}-04 \\
\tau_{2.5 \mu \mathrm{m}} & =1.98 \mathrm{E}-05 \\
\tau_{1.0 \mu \mathrm{m}} & =3.48 \mathrm{E}-06 \\
\tau_{0.1 \mu \mathrm{m}} & =9.01 \mathrm{E}-08 \\
\tau_{0.01 \mu \mathrm{m}} & =7.13 \mathrm{E}-09
\end{aligned}
$$

$$
\begin{array}{rlrl}
\mathrm{u}_{\mathrm{o}} & =405.2 \mathrm{~cm} / \mathrm{s} & \mu & =1.88 \mathrm{E}-04 \\
\mathrm{R} & =1.68275 \mathrm{~cm} & \lambda=7.10 \mathrm{E}-02 \\
\mathrm{u}_{\mathrm{PM} 10} & =53.0 \mathrm{~cm} / \mathrm{s} & &
\end{array}
$$

\begin{aligned} \hline Anisokinetic Error \\ \hline$c / c_{o}(d p=10 \mu m)=1.84 \\ c / c_{o}(d p=2.5 \mu m)=1.06 \\ c / c_{o}(d p=1.0 \mu m)=1.01 \\ c / c_{o}(d p=0.1 \mu m)=1.00 \\ c / c_{o}(d p=0.01 \mu m)=1.00\end{aligned}$

$\begin{aligned} \mathrm{Stk}_{10 \mu \mathrm{m}} & =7.24 \mathrm{E}-02 & \alpha_{10 \mu \mathrm{m}} & =1.27 \mathrm{E}-01 \\ \mathrm{Stk}_{2.5 \mu \mathrm{m}} & =4.77 \mathrm{E}-03 & & \alpha_{2.5 \mu \mathrm{m}}=9.44 \mathrm{E}-03 \\ \mathrm{Stk}_{1.0 \mu \mathrm{m}} & =8.39 \mathrm{E}-04 & \alpha_{1.0 \mu \mathrm{m}} & =1.67 \mathrm{E}-03 \\ \mathrm{Stk}_{0.1 \mu \mathrm{m}} & =2.17 \mathrm{E}-05 & \alpha_{0.1 \mu \mathrm{m}} & =4.34 \mathrm{E}-05 \\ \mathrm{Stk}_{0.01 \mu \mathrm{m}} & =1.72 \mathrm{E}-06 & \alpha_{0.01 \mu \mathrm{m}} & =3.43 \mathrm{E}-06\end{aligned}$




\section{Anisokinetic Sampling Errors for PM2.5 Cyclone}

Test ID: $4220-2$

\section{Assumptions}

Exhaust viscosity equal to that of air

Unit density particles

Spherical particles

Relative Humidity $=50 \%$

\section{Constants}

Variables

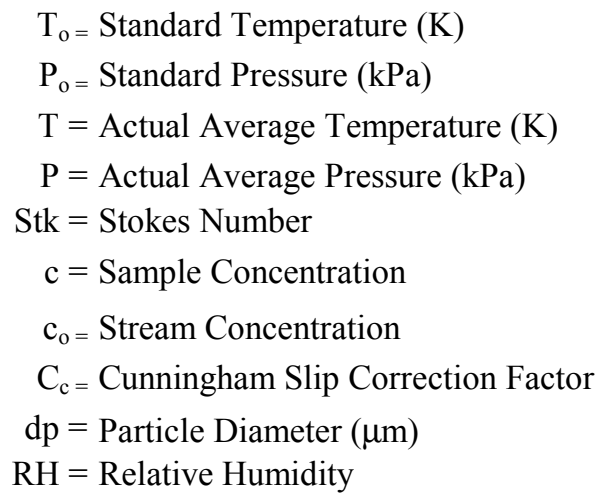

\section{Calculated Values}

$$
\begin{aligned}
\mathrm{C}_{\mathrm{c}, 2.5 \mu \mathrm{m}} & =1.07 \\
\mathrm{C}_{\mathrm{c}, 1.0 \mu \mathrm{m}} & =1.18 \\
\mathrm{C}_{\mathrm{c}, 0.1 \mu \mathrm{m}} & =3.03 \\
\mathrm{C}_{\mathrm{c}, 0.01 \mu \mathrm{m}} & =23.95 \\
\tau_{2.5 \mu \mathrm{m}} & =1.99 \mathrm{E}-05 \\
\tau_{1.0 \mu \mathrm{m}} & =3.49 \mathrm{E}-06 \\
\tau_{0.1 \mu \mathrm{m}} & =8.99 \mathrm{E}-08 \\
\tau_{0.01 \mu \mathrm{m}} & =7.10 \mathrm{E}-09
\end{aligned}
$$

$\mathrm{T}_{\mathrm{o}}=293.15 \mathrm{~K}$

$\mathrm{P}_{\mathrm{o}}=14.696 \mathrm{psi}$

$\mu_{\mathrm{o}}=1.82 \mathrm{E}-4 \mathrm{P}$

$\lambda_{\mathrm{o}}=0.06544 \mu \mathrm{m}$

$\mathrm{R}=0.275$ in

$$
\begin{aligned}
\alpha & =\text { Inertial Fraction } \\
\mu & =\text { Viscosity }(\mathrm{P}) \\
\mu_{\mathrm{o}} & =\text { Viscosity @ STP } \\
\lambda & =\text { Mean Free Path }(\mu \mathrm{m}) \\
\lambda_{\mathrm{o}} & =\text { Mean Free Path } @ \text { STP, } 50 \% \text { RH } \\
\mathrm{r} & =\text { Particle Density }\left(\mathrm{g} / \mathrm{cm}^{3}\right) \\
\tau & =\text { Relaxation Time }(\mathrm{sec}) \\
\mathrm{R} & =\text { Sample Tube Radius }(\mathrm{cm}) \\
\mathrm{u} & =\text { Sampling Tube Velocity }(\mathrm{cm} / \mathrm{s}) \\
\mathrm{u}_{\mathrm{o}} & =\text { Tunnel Velocity }(\mathrm{cm} / \mathrm{s})
\end{aligned}
$$

dp range

$2.5 \mu \mathrm{m}$

$1 \mu \mathrm{m}$

$0.1 \mu \mathrm{m}$

$0.01 \mu \mathrm{m}$

$$
\begin{array}{rlrl}
\mathrm{u}_{\mathrm{o}} & =405.2 \mathrm{~cm} / \mathrm{s} & \mu=1.87 \mathrm{E}-04 \\
\mathrm{R} & =0.6985 \mathrm{~cm} & \lambda=7.05 \mathrm{E}-02 \\
\mathrm{u}_{\mathrm{PM} 2.5}=177.3 \mathrm{~cm} / \mathrm{s} &
\end{array}
$$

$$
\begin{aligned}
\mathrm{Stk}_{2.5 \mu \mathrm{m}} & =1.15 \mathrm{E}-02 & \alpha_{2.5 \mu \mathrm{m}} & =2.25 \mathrm{E}-02 \\
\mathrm{Stk}_{1.0 \mu \mathrm{m}} & =2.03 \mathrm{E}-03 & \alpha_{1.0 \mu \mathrm{m}} & =4.04 \mathrm{E}-03 \\
\mathrm{Stk}_{0.1 \mu \mathrm{m}} & =5.22 \mathrm{E}-05 & \alpha_{0.1 \mu \mathrm{m}} & =1.04 \mathrm{E}-04 \\
\mathrm{Stk}_{0.01 \mu \mathrm{m}} & =4.12 \mathrm{E}-06 & \alpha_{0.01 \mu \mathrm{m}} & =8.24 \mathrm{E}-06
\end{aligned}
$$




\section{ChAPTER 5 - CONCLUSIONS AND RECOMMENDATIONS}

\subsection{Conclusions}

There were two main objectives to this research study. The first was to test four particulate matter sampling systems to evaluate their strengths and weaknesses and more importantly, how they can all be used in conjunction to give an accurate and complete description of particulate mass and size. The hypothesis was that each instrument could be used to build upon the others; from size to mass-based size to size selected mass to total mass. The second objective was to integrate this system into the transportable emissions laboratory in order to study the particle emissions from in-use heavy duty vehicles, associated with diesel, natural gas (compressed and liquefied) and the synthetic diesel, Mossgas. As a result of this study, some new ideas arose as old ones floundered and recommendations for future study are offered.

From the Denver, Colorado transit fleet tests, it became abundantly clear that cyclone flow control must be maintained on a volumetric basis instead of mass. Precise flow rate is a critical parameter for proper operation, and temperature fluctuations in the sample stream can degrade accuracy and efficiency to the point where the data is void. If the flow is maintained at the required, constant rate, the cyclones are an excellent tool for testing the validity of a secondary dilution system, providing a necessary link between total mass and size selective measurements, and also as a preliminary monitor of particle concentrations. 
The cyclones highlighted a possible source of error in the secondary dilution tunnel particulate data. While the negative mass values may have been overlooked as human error, the continual underreporting as compared to the cyclones cannot be. This is a serious problem that may have gone unnoticed without the implementation of the cyclones.

The cyclones can also verify a second mass mode detected by the MOUDI in natural gas vehicle testing. The difference in the mass collected by the PM2.5 and the mass collected by the PM10 cyclones will give an initial indication if a second mode exists. A large difference between the two cyclone mass values would indicate that a large fraction of the total particle mass is greater than $2.5 \mu \mathrm{m}$ but less than $10 \mu \mathrm{m}$ in diameter, which is the range that a second mass mode may appear.

Though very different in measurement techniques and size ranges, the cyclones can also be used as an additional monitor for the SMPS. With the emergence of a second mode as detected by the cyclones, the SMPS particle concentrations can be checked to support the possibility. If very low concentrations of small particles are found even though the total mass values are high, the percent difference between the PM10 and PM2.5 cyclones is likely the result of a second mass mode.

The MOUDI showed itself to be a powerful tool in studying particulate matter; however, it has its limitations. While it performed well for sampling the RTD/SKIP natural gas vehicles, it was unable to do the same for the DART buses. The MOUDI requires more particulate mass than "very clean" vehicles can produce in order to be of value, especially for steady state tests. With that said, the MOUDI was able to sample the buses tested in Denver and Morgantown. 
The largest contributions to this study by the MOUDI were the detection of the second mode in the RTD/SKIP Bus 1013, the portrayal of the different size distribution between the catalyst and non-catalyst version of Bus 1015 and the similar size distribution of diesel \#2 and synthetic diesel. The MOUDI shows its true usefulness as a bridge between size and mass. Since conversion from one to the other is not really valid, the mass-based size distribution is critical for a true understanding of all facets of vehicle particulate matter.

The SMPS allows particle measurement where the MOUDI leaves off. It is able to resolve the mass collected on the afterfilter of the MOUDI. In doing so, particles less than approximately $50 \mathrm{~nm}$ can be described by virtue of their size and number without mathematical conversion.

From these instruments, some conclusions can be made about the effects of fuel on particulate matter emissions. Total mass values aren't quantified, because it was not the intent of this study to rate or compare the cleanliness of the transit fleets tested. It was the goal of this research to determine the associated particle size of the mass not the quantity of particulate. It is known that natural gas vehicles emit less TPM than those fueled with Mossgas, which emit less than diesels.

Diesel and natural gas vehicles produce particulate matter largely in the submicron range. The size distribution data from the MOUDI gave the mass median aerodynamic diameters ranging from approximately 0.11 to $0.12 \mu \mathrm{m}$ for diesel and less than $50 \mathrm{~nm}$ for natural gas. The SMPS gives the particle concentration peak for the natural gas vehicles to be approximately $30 \mathrm{~nm}$. While it has been suggested that these particles are only water droplets rather than particulates, this is clearly untrue. The mass 
collected/measured with the MOUDI is devoid of water (with the exception of sulfate bound water) as a result of temperature and humidity conditioning. Also, Bus 4329 demonstrated what water droplets will do to the instruments; the MOUDI and cyclone data is corrupted and the SMPS particle concentrations at $\sim 55 \mathrm{~nm}$ are double or triple the average value. Water droplets smaller than this readily grow until they reach their Kelvin diameter. That is why the concentration levels for this bus peak at larger diameters than any other test. All the water droplets will grow or shrink to approximately the same size (the Kelvin diameter) based on the local saturation ratio. For these droplets to exist, that is, reach equilibrium at very small diameters, the saturation ratios would need to be extremely high. Since millions of particles are detected in this region, they cannot be water droplets.

The second mode detected in Bus 1013 and possibly again in Buses 4321 and 4324 was an anomaly that was engine specific. The actual cause of the appearance of this mode is unclear, though it is assumed that its source is the engine cylinder.

From the tests run on the DDC 6V92TA transit fleet buses it was concluded that the synthetic diesel fuel, Mossgas, lowerd TPM but, contrary to expectations, did not affect the mass median particle diameter, as compared to diesel \#2. No difference with respect to the mass weighted size distribution was detected, with the MMAD approximately 0.11 to $0.12 \mu \mathrm{m}$ for Mossgas, diesel $\# 2$ and the blend of the two fuels.

This study was broad in scope. The research was conducted in three cities across the country on four fuels with five instruments. However, the objectives were met, as the system proved able to paint a picture of particulate matter in terms of size and mass accordingly for each fuel. Though the goals of this study were completed, there is still 
considerable work to be done based upon the results and conclusions of the research presented here.

\subsection{Recommendations}

There is a great deal of work that can be done in the area of particle size and mass measurement. Based upon the findings from this study, recommendations are made to better current testing procedures, streamline ones performed here and highlight areas for future research.

Two problems associated with current procedures should be addressed and remedied. Most importantly, the method of monitoring the background particle concentrations needs to be modified. The obvious solution is to filter the intake dilution air, thus negating the necessity for the background measurement. This does lead to other problems, namely large increases in the backpressure. This also may run counter to the concept of dilution tunnels, which are designed to simulate the diluting effects of the atmosphere. Without the ambient particles, sites for adsorption/condensation are lost that would otherwise be present in the actual atmosphere. Another approach could be to sample the background conditions after each transient test is completed. It should be noted that this was a standard practice with the transportable laboratory several years ago. It is not known why it was discontinued. There is a twenty minute period between these tests in which the sample could be taken. This would require ducting the vehicle exhaust away from the tunnel. If this is done, the tunnel air would have less time to cool and the associated background could be subtracted from each individual test. 
The other problem found during this work was the presence of water condensation during natural gas vehicle testing. Hood and Silvis (1998) describe a method to insure condensation does not take place. To prevent condensation in the dilution tunnel, the concentration of water in the diluted exhaust mixture must be less than the saturation concentration of water at the lowest temperature encountered by the mixture. To determine the minimum flow rate required to prevent condensation, $\mathrm{Q}_{\mathrm{CVS}}$, Hood and Silvis (1998) give the following equation:

$$
Q_{C V S} \geq Q_{t p}\left(\frac{\frac{\left[\mathrm{H}_{2} \mathrm{O}\right]_{t p}}{\left[\mathrm{H}_{2} \mathrm{O}\right]_{s a t}}-\Phi_{d i l}}{1-\Phi_{d i l}}\right)
$$

where the subscript $t p$ denotes the tailpipe and $\Phi_{\text {dil }}$ is the humidity of the dilution air. A system to solve for these variables should be devised to determine the necessary dilution tunnel flow rate and implemented into the procedure for testing natural gas vehicles because of the severe sampling disturbances caused by water condensation.

If the work presented here is to continue there are ways to increase the efficiency. Steady state tests should be sampled by the MOUDI only if the run times are considerably increased. Since each time the MOUDI is operated approximately two hours are required, any useless data adds up to hours of wasted time and effort. Also along those lines, a maximum of three transient tests needs to be run for the MOUDI. It has proven itself effective and accurate, so five runs are overkill. With this extra time, a reliable, accurate system can be developed to grease the substrates. While the benefits may be few in terms of size distribution, the MOUDI could be used as a total particulate 
matter mass measurement device if particle bounce on the upper stages is avoided. This also enables a more direct comparison between the MOUDI and the cyclones.

The cyclones should be a standard for the transportable emissions testing laboratory. They have been shown to be effective tools for particulate sampling and require little maintenance.

The final two recommendations arose from unexpected results during the course of this study. The first is to investigate particle size below $100 \mathrm{~nm}$ for natural gas vehicles with and without a catalyst. The average total mass for the lower two stages of the MOUDI was very similar for the two versions of Bus 1015, but the fraction of mass was not. This could be of great interest if the catalyst is increasing the total number of 50 - $100 \mathrm{~nm}$ particles while decreasing those below approximately $50 \mathrm{~nm}$ while lowering TPM. The last recommendation is to study the second mode found in some of the natural gas vehicles for elemental carbon and metals to determine its source and nature. 


\section{REFERENCES}

Abbass, M., Andrews, G., and Williams, P., "Diesel Particulate Composition Changes Along an Air Cooled Exhaust Pipe and Dilution Tunnel." SAE Paper No. 890789.

Baranescu, R., "Influence of Fuel Sulfur of Diesel Particulate Emissions." SAE Paper No. 881174.

Baron, P., and Willeke, K., Aerosol Measurement: Principles, Techniques, and Applications. Van Nostrand Reinhold, New York, New York, 1993.

Braun, C., Ackermann, U., Schwartz, J., Gnehm, H., Rutishauser, M., and Wanner, H., "Air Pollution and Respiratory Symptoms in Preschool Children." American Review of Respiratory Disease, Vol. 145, pgs. 42-47, 1992.

Bugarski, A., "Characterization of Particulate Matter and Hydrocarbon Emissions from In-Use Heavy Duty Diesel Engine." West Virginia University, 1999. Publication pending.

Cadle, R., The Measurement of Airborne Particles. John Wiley \& Sons, New York, New York, 1975.

Clark, N., Gautam, M., Rapp, B., Lyons, D., Graboski, M., McCormick, R., Alleman, T., and Norton, P., "Diesel and CNG Transit Bus Emissions Characterization by Two Chassis Dynamometer Laboratories: Results and Issues." SAE Paper No. 991469.

Clark, N., Lyons, D., Gautam, M., Norton, P., Vertin, K., Goguen, S., and Eberhardt, J., "Emissions from Buses with DDC 6V92 Engines using Synthetic Diesel Fuel." SAE Paper No. 991512.

Code of Federal Regulations, Title 40, Part 86, Subpart N - Emission Regulations for New Otto-Cycle and Diesel Heavy-Duty Engines; Gaseous and Particulate Exhaust Test Procedures, 1991.

CONCAWE, "A Study of the Number, Size \& Mass of Exhaust Particles Emitted from European Diesel and Gasoline Vehicles Under Steady-State and European Driving Cycle Conditions." Prepared for CONCAWE Automotive Emissions Management Group by Special task Force AE/STF-10: D. E. Hall, Chairman, Brussels, Belgium, 1998. 
Dockery, D., Pope, C., Xu, X., Spengler, J., Ware, J., Fay, M., Ferris, B., and Speizer, F., "An Association Between Air Pollution and Mortality in Six U.S. Cities." New England Journal of Medicine, Vol. 329, No. 24, pgs. 1753-1759, 1993.

Ferguson, C., Internal Combustion Engines: Applied Thermosciences. John Wiley \& Sons, New York, New York, 1986.

Ferguson, D., Design, Fabrication and Testing of an Emissions Measurement System for a Transportable Heavy Duty Vehicle Emissions Testing Laboratory. MSME Thesis, Department of Mechanical and Aerospace Engineering, West Virginia University, 1993.

Gautam, M., Clark, N., Lyons, D., Long, Jr., T., Howell, A., Loth, J., Palmer, G., and Bata, R., "Design Overview of a Heavy-Duty Mobile Vehicle Emissions Testing Laboratory." ASME DE-Vol. 40, Advanced Automotive Technologies, 1991.

Greenwood, S.J., Coxon, J.E., Biddulph, T., and Bennet, J., “An Investigation to Determine the Exhaust Particulate Size Distributions for Diesel, Petrol, and Compressed Natural Gas Fuelled Vehicles." SAE Paper No. 961085.

Health Effects Institute, "A Critical Analysis of Emissions, Exposure, and Health Effects: A Special Report on the Institute's Diesel Working Group." Health Effects Institute, 1995.

Hering, S., "Impactors, Cyclones, and Other Inertial Gravitational Collectors." Air Sampling Instruments for Evaluation of Atmospheric Contaminants, $8^{\text {th }}$ Edition, Edited by Beverly S. Cohen and Suzanne V. Hering, ACGIH, pgs. 279-321, Cincinnati, Ohio, 1995.

Heywood, J., Internal Combustion Engine Fundamentals. McGraw-Hill Publishing Company, New York, New York, 1988.

Hinds, W., Aerosol Technology. John Wiley \& Sons, New York, New York, 1982.

Hood, J., and Silvis, W., "Predicting and Preventing Water Condensation in Sampled Vehicle Exhaust for Optimal CVS Dilution." SAE Paper No. 980404.

International Agency Research on Cancer, "Diesel and Gasoline Engine Exhausts and Some Nitroarenes." IARC Monograph Vol. 46, 1989.

Johnson, J., Bagley, S., Gratz, L., and Leddy, D., "A Review of Diesel Particulate Control Technology and Emissions Effects - 1992 Horning Memorial Award Lecture." SAE Paper No. 940233.

Kittelson, D., Ambs, J., and Hadjkacem, H., "Particulate Emissions from Diesel Engines: Influence of In-Cylinder Surface." SAE Paper No. 900645. 
Kittelson, D., Watts Jr., W., and Arnold, M., "Review of Diesel Particulate Sampling Methods: Supplemental Report No. 2 Aerosol Dynamics, Laboratory and OnRoad Studies." University of Minnesota, 1998.

Kelly, W., and McMurry, P., "Measurement of Particle Density by Inertial Classification of Differential Mobility Analyzer - Generated Monodisperse Aerosols." Aerosol Science and Technology, Vol. 17, No. 3, pgs. 199-212, 1992.

Kreso, A., Johnson, J., Gratz, L., Bagley, S., and Leddy, D., "A Study of the Effects of Exhaust Gas Recirculation on Heavy-Duty Diesel Engine Emissions." SAE Paper No. 981422.

Knutson, E., and Lioy, P., "Measurement and Presentation of Aerosol Size Distributions." Air Sampling Instruments for Evaluation of Atmospheric Contaminants, $8^{\text {th }}$ Edition, Edited by Beverly S. Cohen and Suzanne V. Hering, ACGIH, pgs. 121-136, Cincinnati, Ohio, 1995.

Lippmann, M., Gurman, J., and Schlesinger, R., "Role of Particle Deposition in Occupational Lung Disease." Aerosols in the Mining and Industrial Work Environment Volume 1: Fundamentals and Status, Edited by Virgil A. Marple and Benjamin Y. H. Liu, Ann Arbor Science Publishers, Ann Arbor, Michigan, Vol. 1, pgs. 119-138, 1983.

Lee, K. and Mukund, R., "Filter Collection." Aerosol Measurement: Principles, Techniques, and Applications. Edited by P. Baron and K. Willeke. Van Nostrand Reinhold, New York, New York, 1993.

Maricq, M., Chase, R., Podsiadlik, D., and Vogt, R., "Vehicle Exhaust Particle Size Distributions: A Comparison of Tailpipe and Dilution Tunnel Measurements," SAE Paper No. 991461.

Marple, V., and Liu, B., Environmental Science and Technology. Vol. 8, 1974.

Marple, V., Rubow, K., Behm, S., "A Microorifice Uniform Deposit Impactor (MOUDI): Description, Calibration, and Use." Aerosol Science and Technology, Vol. 14, No. 4, pgs. 434-446, 1991.

Marple, V., and Willeke, K., "Inertial Impactors." Aerosol Measurement, University Press of Florida, pgs. 90-107, 1979.

Martonen, T., and Lowe, J., "Assessment of Aerosol Deposition Patterns in Human Respiratory Tract Casts." Aerosols in the Mining and Industrial Work Environment Volume 1: Fundamentals and Status, Edited by Virgil A. Marple and Benjamin Y. H. Liu, Ann Arbor Science Publishers, Ann Arbor, Michigan, Vol. 1, pgs. 151-164, 1983. 
Mauderly, J., Schlesinger, R., and Neas, L., "Measurement Needs Related to Health Effects." Prepared for EPA by Health Effects Institute: Correspondence by Maria G. Costantini, 1998.

Monn, C., Braendli, O., Schaeppi, G., Schindler, C., Ackermann - Liebrich, U., Leuenberger, P., and SAPALDIA Team, "Particulate Matter $<10 \mu \mathrm{m}$ (PM10) and Total Suspended Particulates (TSP) in Urban, Rural and Alpine Air in Switzerland." Atmospheric Environment, Vol. 29, No. 19, pgs. 2565-2573, 1995.

National Institute for Occupational Safety and Health, "Carcinogenic Effects of Exposure to Diesel Exhaust." CIB, 1988.

National Research Council, Airborne Particles. Subcommittee on Medical and Biologic Effects of Environmental Pollutants, University Park Press, Baltimore, Maryland, 1979.

Pope, A., and Dockery, D., "Acute Health Effects of PM10 Pollution on Symptomatic and Asymptotic Children." American Review of Respiratory Disease, Vol. 145, pgs. 1123-1128, 1992.

Reist, P., Aerosol Science and Technology. Second Edition, McGraw-Hill, Inc., New York, New York, 1993.

Smith, R., and Gautam, M., an inter-office correspondence, West Virginia University, 1992.

Stern, A., Wohlers, H., Boubel, R., and Lowry, W., Fundamentals of Air Pollution. $3^{\text {rd }}$ Edition, Academic Press, Orlando, Florida, 1973.

Swift, D., "Aerosol Sampling Requirements to Assess the Health Risk of Special Particulates." Aerosols in the Mining and Industrial Work Environment Volume 1: Fundamentals and Status, Edited by Virgil A. Marple and Benjamin Y. H. Liu, Ann Arbor Science Publishers, Ann Arbor, Michigan, Vol. 1, pgs. 219-234, 1983.

Young, J., Guide to the Microorifice Uniform Depsoit Impactor. November, 1994.

Yu, C., Hu, J., "Diffusional Deposition of Ultrafine Particles in the Human Lung." Aerosols in the Mining and Industrial Work Environment Volume 1: Fundamentals and Status, Edited by Virgil A. Marple and Benjamin Y. H. Liu, Ann Arbor Science Publishers, Ann Arbor, Michigan, Vol. 1, pgs. 139-149, 1983. 


\section{ApPendix A}

Cyclone Qbasic Reduction Program 
' This program calculates cyclone comparisons and also compares PM10 and PM2.5 with 70mm filter data

DECLARE SUB ID (TestID\$, testsite\$, vehicle\$, fuel\$, Mode\$, testdate\$)

DECLARE SUB FILTERS (Secwt, PM10input, PM2.5input, PM10time, Answer1\$, PM2.5time, sectime) DECLARE SUB CYCLONEFLOW (PM10flow, PM2.5flow)

DECLARE SUB MAINTUNNEL (Vmix, Vsample, DF)

DECLARE SUB BACKGROUND (Backwt70, Backflw70, BackwtPM10, BackflwPM10, BacktimePM10, BackwtPM2.5, BackflwPM2.5, BacktimePM2.5)

20

CLS

SCREEN 0

CALL ID(TestID\$, testsite\$, vehicle\$, fuel\$, Mode\$, testdate\$)

$\mathrm{a}=\mathrm{a}+1$

IF a > 1 THEN

GOTO 65

ELSE PRINT

END IF

30

CLS

CALL FILTERS(Secwt, PM10input, PM2.5input, PM10time, Answer1\$, PM2.5time, sectime)

$\mathrm{b}=\mathrm{b}+1$

IF $b>1$ THEN

GOTO 65

ELSE PRINT

END IF

40

CLS

CALL CYCLONEFLOW(PM10flow, PM2.5flow)

$\mathrm{c}=\mathrm{c}+1$

IF c > 1 THEN

GOTO 65

ELSE PRINT

END IF

50

CALL MAINTUNNEL(Vmix, Vsample, DF)

$\mathrm{d}=\mathrm{d}+1$

IF $d>1$ THEN

GOTO 65

ELSE PRINT

END IF 
CLS

60

CALL BACKGROUND(Backwt70, Backflw70mm, BackwtPM10, BackflwPM10, BacktimePM10, BackwtPM2.5, BackflwPM2.5, BacktimePM2.5)

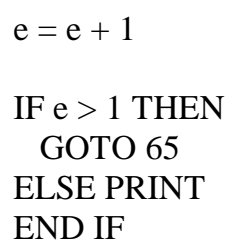

ELSE

END IF 
LET TOTAL70mm $=((($ Secwt $/ 1000) /$ Vsample70mm $)-(((B a c k w t 70 / 1000) /$ Vback $) *(1-(1 /$ DF $)))) *$ Vmixin

LET TOTALPM10 = $((($ PM10input $/ 1000) /$ VcyclonePM10 $)-(((B a c k w t P M 10 / 1000) /$ Vback10 $) *(1-$ $(1 / \mathrm{DF})))) *($ Vmixin * 1000)

LET TOTALPM2.5 $=(((\mathrm{PM} 2.5$ input $/ 1000) /$ VcyclonePM2.5 $)-((($ BackwtPM2.5 / 1000) $/$ Vback2.5 $) *(1$ $-(1 / \mathrm{DF})))) *($ Vmixin $* 1000)$

LET PM10and70mm $=($ TOTAL70mm - TOTALPM10 $) /(($ TOTAL70mm + TOTALPM10 $) / 2)$

LET diffPM10and70mm = PM10and70 $\mathrm{mm} * 100$

LET PM2.5andPM10 = $($ TOTALPM10 - TOTALPM2.5 $) /(($ TOTALPM10 + TOTALPM2.5 $) / 2)$

LET diffPM2.5andPM10 $=$ PM2.5andPM10 $* 100$

LET PM2.5and70mm $=($ TOTAL70mm - TOTALPM2.5 $) /(($ TOTAL70mm + TOTALPM2.5 $) / 2)$

LET diffPM2.5and70mm $=$ PM2.5and70 $\mathrm{mm} * 100$

INPUT "Enter Path and Filename: "; n\$

OPEN $n \$$ FOR OUTPUT AS \#1

PRINT \#1, "Test ID: "; TestID\$

PRINT \#1, "Test Site: "; testsite\$

PRINT \#1, "Vehicle ID: "; vehicle\$

PRINT \#1, "Date of Test: "; testdate\$

PRINT \#1, "Test Mode: "; Mode\$

PRINT \#1, "Fuel Type: "; fuel\$

PRINT \#1,

PRINT \#1, "70mm Filter Weight"; SPC(1.5); "="; SPC(5); TOTAL70mm; " grams" PRINT \#1, "PM10 Filter Weight"; SPC(2.25); "="; SPC(5); TOTALPM10; " grams" PRINT \#1, "PM2.5 Filter Weight"; SPC(1.25); "="; SPC(5); TOTALPM2.5; " grams" PRINT \#1, "Percent Difference"; SPC(3.5); "="; SPC(5); diffPM10and70mm; "\% PM10 and 70mm" PRINT \#1, "Percent Difference"; SPC(3.5); "="; SPC(5); diffPM2.5andPM10; "\% PM10 and PM2.5" PRINT \#1, "Percent Difference"; SPC(3.5); "="; SPC(5); diffPM2.5and70mm; "\% PM2.5 and 70mm"

\section{CLOSE}

SUB BACKGROUND (Backwt70, Backflw70mm, BackwtPM10, BackflwPM10, BacktimePM10, BackwtPM2.5, BackflwPM2.5, BacktimePM2.5)

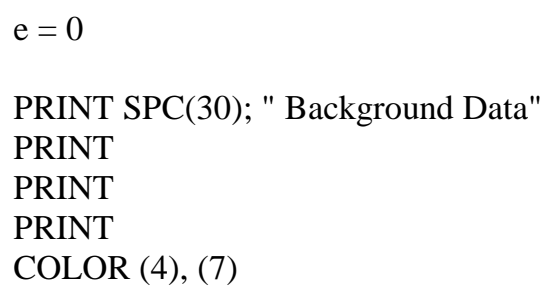


INPUT "70mm background filter weight [milligrams]: ", Backwt70

INPUT "Background volume for secondary tunnel [cubic feet]: ", Backflw70mm

INPUT "PM10 cyclone background filter weight [milligrams]: ", BackwtPM10

INPUT "Background flow rate for PM10 cyclone [lpm]: ", BackflwPM10

INPUT "PM10 cyclone background sample time [seconds]: ", BacktimePM10

INPUT "PM2.5 cyclone background filter weight [milligrams]: ", BackwtPM2.5

INPUT "Background flow rate for PM2.5 cyclone [lpm]: ", BackflwPM2.5

INPUT "PM2.5 cyclone background sample time [seconds]: ", BacktimePM2.5

END SUB

SUB CYCLONEFLOW (PM10flow, PM2.5flow)

$\mathrm{c}=0$

PRINT SPC(30); "Cyclone flow rates"

COLOR (4), (7)

PRINT

PRINT

PRINT

INPUT "PM10 [lpm]: ", PM10flow

INPUT "PM2.5 [lpm]: ", PM2.5flow

END SUB

SUB FILTERS (Secwt, PM10input, PM2.5input, PM10time, Answer1\$, PM2.5time, sectime)

$\mathrm{b}=0$

PRINT SPC(20); " Cyclone and 70 mm Filter Weight Data"

COLOR (4), (7)

PRINT

PRINT

INPUT " 70mm filter weight [milligrams]: ", Secwt

INPUT " PM10 cyclone filter weight [milligrams]: ", PM10input

INPUT " PM2.5 cyclone filter weight [milligrams]: ", PM2.5input

INPUT " PM10 cyclone test time [seconds]: ", PM10time

PRINT

10 INPUT " Is PM2.5 cylcone time the same? y or n "; Answer1\$

IF Answer1 $\$=$ "y" THEN

LET PM2.5time $=$ PM10time

ELSEIF Answer1\$ = "n" THEN

INPUT " PM2.5 cyclone time [seconds]: ", PM2.5time

ELSE GOTO 10

END IF

INPUT " Secondary dilution test time [seconds]: ", sectime

END SUB 
SUB ID (TestID\$, testsite\$, vehicle\$, fuel\$, Mode\$, testdate\$)

$\mathrm{a}=0$

INPUT " $\quad$ Enter the Test Sequence and ID: ", TestID\$

PRINT

INPUT "Test Site: ", testsite\$

INPUT "Vehicle ID: ", vehicle\$

INPUT "Fuel: ", fuel\$

INPUT "Mode: ", Mode\$

INPUT "Date: ", testdate\$

PRINT

END SUB

SUB MAINTUNNEL (Vmix, Vsample, DF)

$\mathrm{d}=0$

PRINT SPC(25); " Main Tunnel Information"

PRINT

PRINT

INPUT "Mixing volume in main tunnel [cubic feet]: ", Vmix

INPUT "Sampling volume in secondary tunnel [cubic feet]: ", Vsample

INPUT "Dilution factor: ", DF

PRINT

END SUB 
APPENDIX B

CyClone COMParison for Nova DART FleEt CumMins L10 AND M11 BuSES 


\section{Cyclone Comparison Data Sheet for Bus 4220 - SS 20mph}

Test ID: TX_3156-1

Test Site: Dallas

Vehicle ID: Nova Bus 4221

Date of Test: 3/10/99

Test Mode: SS-20mph

Fuel Type: D2

70mm Filter Weight $=.480183$ grams

PM10 Filter Weight $=.7567326$ grams

$\mathrm{PM} 2.5$ Filter Weight $=\quad .7518315$ grams

Percent Difference $=-44.71601 \%$ PM10 and 70mm

Percent Difference $=.6497796 \%$ PM10 and PM2.5

Percent Difference $=-44.09826 \%$ PM2.5 and $70 \mathrm{~mm}$

Test ID: TX_3156-2

Test Site: Dallas

Vehicle ID: Nova Bus 4221

Date of Test: $3 / 10 / 99$

Test Mode: SS-20mph

Fuel Type: D2

$70 \mathrm{~mm}$ Filter Weight $=.779292$ grams

PM10 Filter Weight $=\quad .922635$ grams

$\mathrm{PM} 2.5$ Filter Weight $=0$ grams

Percent Difference $=-16.84478 \%$ PM10 and $70 \mathrm{~mm}$

Percent Difference $=200 \%$ PM10 and PM2.5

Percent Difference $=200 \%$ PM 2.5 and $70 \mathrm{~mm}$

Test ID: TX_3156-3

Test Site: Dallas

Vehicle ID: Nova Bus 4221

Date of Test: 3/10/99

Test Mode: SS-20mph

Fuel Type: D2

70mm Filter Weight $=.9812233$ grams

PM10 Filter Weight $=1.178739$ grams

$\mathrm{PM} 2.5$ Filter Weight $=1.173465$ grams

Percent Difference $=-18.28877 \%$ PM10 and 70mm

Percent Difference $=.4484355 \%$ PM10 and PM2.5

Percent Difference $=-17.84399 \%$ PM2.5 and $70 \mathrm{~mm}$ 


\section{Cyclone Comparison Data Sheet for Bus 4220 - CBD}

Test ID: TX_3157-2

Test Site: Dallas

Vehicle ID: Nova Bus 4220

Date of Test: $3 / 10 / 99$

Test Mode: CBD

Fuel Type: D2

70mm Filter Weight $=1.048094$ grams

PM10 Filter Weight $=1.386438$ grams

$\mathrm{PM} 2.5$ Filter Weight $=1.354076$ grams

Percent Difference $=-27.79539 \%$ PM10 and $70 \mathrm{~mm}$

Percent Difference $=2.361789 \%$ PM10 and PM2.5

Test ID: $\mathbf{T X \_ 3 1 5 7 - 3}$

Test Site: Dallas

Vehicle ID: Nova Bus 4220

Date of Test: 3/10/99

Test Mode: CBD

Fuel Type: D2

70mm Filter Weight $=.9951113$ grams

PM10 Filter Weight $=1.364771$ grams

$\mathrm{PM}$ 2.5 Filter Weight $=1.311772$ grams

Percent Difference $=-31.32863 \%$ PM10 and 70mm

Percent Difference $=3.960206 \%$ PM10 and PM2.5

Test ID: TX_3157-4

Test Site: Dallas

Vehicle ID: Nova Bus 4220

Date of Test: 3/10/99

Test Mode: CBD

Fuel Type: D2

$70 \mathrm{~mm}$ Filter Weight $=1.148019$ grams

PM10 Filter Weight $=1.519381$ grams

$\mathrm{PM} 2.5$ Filter Weight $=1.37959$ grams

Percent Difference $=-27.8445 \%$ PM10 and 70mm

Percent Difference $=9.644231 \%$ PM10 and PM2.5 


\section{Cyclone Comparison Data Sheet for Bus 4221 - SS 20mph}

Test ID: TX_3163-1

Test Site: Dallas

Vehicle ID: Nova Bus 4221

Date of Test: 3/11/99

Test Mode: SS-20mph

Fuel Type: D2

70mm Filter Weight $=.3510512$ grams

PM10 Filter Weight $=.5468455$ grams

$\mathrm{PM} 2.5$ Filter Weight $=.5272833$ grams

Percent Difference $=-43.61175 \%$ PM10 and 70mm

Percent Difference $=3.642439 \%$ PM10 and PM2.5

Percent Difference $=-40.12868 \%$ PM2.5 and $70 \mathrm{~mm}$

Test ID: TX_3163-2

Test Site: Dallas

Vehicle ID: Nova Bus 4221

Date of Test: 3/11/99

Test Mode: SS-20mph

Fuel Type: D2

$70 \mathrm{~mm}$ Filter Weight $=.4060701$ grams

PM10 Filter Weight $=.8085012$ grams

PM2.5 Filter Weight $=\quad .6266986$ grams

Percent Difference $=-66.26719 \%$ PM10 and $70 \mathrm{~mm}$

Percent Difference $=25.33481 \%$ PM10 and PM2.5

Percent Difference $=-42.72565 \%$ PM2.5 and $70 \mathrm{~mm}$ 


\title{
Cyclone Comparison Data Sheet for Bus 4221 - CBD
}

\author{
Test ID: TX_3164-2 \\ Test Site: Dallas \\ Vehicle ID: Nova Bus 4221 \\ Date of Test: $3 / 11 / 99$ \\ Test Mode: CBD \\ Fuel Type: D2 \\ $70 \mathrm{~mm}$ Filter Weight $=.6111265$ grams \\ PM10 Filter Weight $=\quad .87065$ grams \\ PM2.5 Filter Weight $=.7733744$ grams \\ Percent Difference $=-35.02869 \%$ PM10 and $70 \mathrm{~mm}$ \\ Percent Difference $=11.83383 \%$ PM10 and PM2.5
}

Test ID: TX_3164-3

Test Site: Dallas

Vehicle ID: Nova Bus 4221

Date of Test: 3/11/99

Test Mode: CBD

Fuel Type: D2

$70 \mathrm{~mm}$ Filter Weight $=.6212296$ grams

PM10 Filter Weight $=.9063028$ grams

$\mathrm{PM} 2.5$ Filter Weight $=\quad .8364013$ grams

Percent Difference $=-37.32466 \% \mathrm{PM} 10$ and $70 \mathrm{~mm}$

Percent Difference $=8.022184 \%$ PM10 and PM2.5

Test ID: TX_3164-4

Test Site: Dallas

Vehicle ID: Nova Bus 4221

Date of Test: 3/11/99

Test Mode: CBD

Fuel Type: D2

70mm Filter Weight $=.6010873$ grams

PM10 Filter Weight $=1.052898$ grams

PM2.5 Filter Weight $=\quad .8131041$ grams

Percent Difference $=-54.63301 \%$ PM10 and $70 \mathrm{~mm}$

Percent Difference $=25.70139 \%$ PM10 and PM2.5 


\section{Cyclone Comparison Data Sheet for Bus 4320 - SS 20mph}

Test ID: TX_3129-1

Test Site: Dallas

Vehicle ID: Nova Bus 4320

Date of Test: 2/18/99

Test Mode: SS-20mph

Fuel Type: LNG

$70 \mathrm{~mm}$ Filter Weight $=0$ grams

PM10 Filter Weight $=.1303047$ grams

PM2.5 Filter Weight $=.1208016$ grams

Percent Difference $=-200 \%$ PM10 and $70 \mathrm{~mm}$

Percent Difference $=7.568958 \%$ PM10 and PM2.5

Percent Difference $=-200 \%$ PM2.5 and $70 \mathrm{~mm}$ 


\section{Cyclone Comparison Data Sheet for Bus 4320 - CBD}

Test ID: TX_3130-1

Test Site: Dallas

Vehicle ID: Nova Bus 4320

Date of Test: 2/18/99

Fuel Type: LNG

$70 \mathrm{~mm}$ Filter Weight $=0$ grams

PM10 Filter Weight $=8.744861 \mathrm{E}-02$ grams

$\mathrm{PM} 2.5$ Filter Weight $=\quad .1063982$ grams

Percent Difference $=-200 \%$ PM10 and 70mm

Percent Difference $=-19.55114 \%$ PM10 and PM2.5

Test ID: TX_3130-2

Test Site: Dallas

Vehicle ID: Nova Bus 4320

Date of Test: 2/18/99

Fuel Type: LNG

$70 \mathrm{~mm}$ Filter Weight $=0$ grams

PM10 Filter Weight $=\quad .0911497$ grams

PM2.5 Filter Weight $=7.675907 \mathrm{E}-02$ grams

Percent Difference $=-200 \%$ PM10 and 70mm

Percent Difference $=17.14101 \%$ PM10 and PM2.5

Test ID: TX_3130-3

Test Site: Dallas

Vehicle ID: Nova Bus 4320

Date of Test: $2 / 18 / 99$

Fuel Type: LNG

$70 \mathrm{~mm}$ Filter Weight $=0$ grams

PM10 Filter Weight $=8.269566 \mathrm{E}-02$ grams

PM2.5 Filter Weight $=.0890444$ grams

Percent Difference $=-200 \%$ PM10 and 70mm

Percent Difference $=-7.393425 \%$ PM10 and PM2.5

Test ID: TX_3130-4

Test Site: Dallas

Vehicle ID: Nova Bus 4320

Date of Test: 2/18/99

Fuel Type: LNG

$70 \mathrm{~mm}$ Filter Weight $=0$ grams

PM10 Filter Weight $=9.203298 \mathrm{E}-02$ grams

PM2.5 Filter Weight $=8.287035 \mathrm{E}-02$ grams

Percent Difference $=-200 \% \mathrm{PM} 10$ and $70 \mathrm{~mm}$

Percent Difference $=10.47736 \%$ PM10 and PM2.5

Test ID: TX_3130-5

Test Site: Dallas

Vehicle ID: Nova Bus 4320

Date of Test: 2/18/99

Fuel Type: LNG

$70 \mathrm{~mm}$ Filter Weight $=0$ grams

PM10 Filter Weight $=8.933651 \mathrm{E}-02$ grams

PM2.5 Filter Weight $=6.415546 \mathrm{E}-02$ grams

Percent Difference $=-200 \%$ PM10 and $70 \mathrm{~mm}$ 


\section{Cyclone Comparison Data Sheet for Bus 4321 - SS 20mph}

Test ID: TX_3120-1

Test Site: Dallas

Vehicle ID: Nova Bus 4321

Date of Test: 2/15/99

Test Mode: SS-20mph

Fuel Type: LNG

$70 \mathrm{~mm}$ Filter Weight $=-3.667691 \mathrm{E}-02$ grams

PM10 Filter Weight $=.1284709$ grams

PM2.5 Filter Weight $=9.294499 \mathrm{E}-02$ grams

Percent Difference $=-359.8227 \%$ PM10 and $70 \mathrm{~mm}$

Percent Difference $=32.08976 \%$ PM10 and PM2.5

Percent Difference $=-460.7298 \%$ PM2.5 and $70 \mathrm{~mm}$

Test ID: TX_3120-2

Test Site: Dallas

Vehicle ID: Nova Bus 4321

Date of Test: 2/15/99

Test Mode: SS-20mph

Fuel Type: LNG

$70 \mathrm{~mm}$ Filter Weight $=-2.083771 \mathrm{E}-02$ grams

PM10 Filter Weight $=8.641606 \mathrm{E}-02$ grams

$\mathrm{PM} 2.5$ Filter Weight $=\quad .0180898$ grams

Percent Difference $=-327.1012 \%$ PM10 and $70 \mathrm{~mm}$

Percent Difference $=130.7606 \%$ PM10 and PM2.5

Percent Difference $=2833.243 \%$ PM2.5 and $70 \mathrm{~mm}$ 


\title{
Cyclone Comparison Data Sheet for Bus 4321 - CBD
}

\author{
Test ID: TX_3121-1 \\ Test Site: Dallas \\ Vehicle ID: Nova Bus 4321 \\ Date of Test: 2/15/99 \\ $70 \mathrm{~mm}$ Filter Weight $=9.985918 \mathrm{E}-03$ grams \\ PM10 Filter Weight $=.257928$ grams \\ PM2.5 Filter Weight $=\quad .0867375$ grams \\ Percent Difference $=-185.0909 \% \mathrm{PM} 10$ and $70 \mathrm{~mm}$ \\ Percent Difference $=99.3372 \%$ PM10 and PM2.5
}

Test ID: TX_3121-2

Test Site: Dallas

Vehicle ID: Nova Bus 4321

Date of Test: $2 / 15 / 99$

$70 \mathrm{~mm}$ Filter Weight $=2.104415 \mathrm{E}-02$ grams

PM10 Filter Weight $=.2516149$ grams

$\mathrm{PM} 2.5$ Filter Weight $=\quad .1083273$ grams

Percent Difference $=-169.1275 \%$ PM10 and $70 \mathrm{~mm}$

Percent Difference $=79.61697 \%$ PM10 and PM2.5

Test ID: TX_3121-3

Test Site: Dallas

Vehicle ID: Nova Bus 4321

Date of Test: 2/15/99

70mm Filter Weight $=9.963271 \mathrm{E}-03$ grams

PM10 Filter Weight $=.2216841$ grams

PM2.5 Filter Weight $=\quad .1202062$ grams

Percent Difference $=-182.7958 \%$ PM10 and 70mm

Percent Difference $=59.36285 \%$ PM10 and PM2.5

Test ID: TX_3121-4

Test Site: Dallas

Vehicle ID: Nova Bus 4321

Date of Test: 2/15/99

$70 \mathrm{~mm}$ Filter Weight $=2.088684 \mathrm{E}-02$ grams

PM10 Filter Weight $=.1720386$ grams

PM2.5 Filter Weight $=8.226548 \mathrm{E}-02$ grams

Percent Difference $=-156.6945 \% \mathrm{PM} 10$ and $70 \mathrm{~mm}$

Percent Difference $=70.60299 \%$ PM10 and PM2.5

Test ID: TX_3121-5

Test Site: Dallas

Vehicle ID: Nova Bus 4321

Date of Test: 2/15/99

$70 \mathrm{~mm}$ Filter Weight $=1.507728 \mathrm{E}-02$ grams

PM10 Filter Weight $=.1590698$ grams

PM2.5 Filter Weight $=6.619752 \mathrm{E}-02$ grams

Percent Difference $=-165.3689 \%$ PM10 and $70 \mathrm{~mm}$

Percent Difference $=82.45518 \%$ PM10 and PM2.5 


\section{Cyclone Comparison Data Sheet for Bus 4322 - SS 20mph}

Test ID: TX_3132-1

Test Site: Dallas

Vehicle ID: Nova Bus 4322

Date of Test: $3 / 1 / 99$

Test Mode: SS-20mph

Fuel Type: LNG

$70 \mathrm{~mm}$ Filter Weight $=5.557664 \mathrm{E}-02$ grams

PM10 Filter Weight $=8.025198 \mathrm{E}-02$ grams

PM2.5 Filter Weight $=6.467639 \mathrm{E}-02$ grams

Percent Difference $=-36.33304 \%$ PM10 and $70 \mathrm{~mm}$

Percent Difference $=21.49419 \%$ PM10 and PM2.5

Percent Difference $=-15.13433 \%$ PM2.5 and $70 \mathrm{~mm}$ 


\section{Cyclone Comparison Data Sheet for Bus 4322 - CBD}

Test ID: TX_3133-2

Test Site: Dallas

Vehicle ID: Nova Bus 4322

Date of Test: 3/1/99

Test Mode: CBD

Fuel Type: LNG

70mm Filter Weight $=\quad 6.797972 \mathrm{E}-02$ grams

PM10 Filter Weight $=.1101423$ grams

$\mathrm{PM} 2.5$ Filter Weight $=\quad .1043405$ grams

Percent Difference $=-47.3412 \%$ PM10 and 70mm

Percent Difference $=5.409965 \%$ PM10 and PM2.5

Test ID: TX_3133-3

Test Site: Dallas

Vehicle ID: Nova Bus 4322

Date of Test: 3/1/99

Test Mode: CBD

Fuel Type: LNG

70mm Filter Weight $=\quad 5.551767 \mathrm{E}-02$ grams

PM10 Filter Weight $=8.227418 \mathrm{E}-02$ grams

PM2.5 Filter Weight $=7.919853 \mathrm{E}-02$ grams

Percent Difference $=-38.83613 \%$ PM10 and $70 \mathrm{~mm}$

Percent Difference $=3.809491 \%$ PM10 and PM2.5

Test ID: TX_3133-4

Test Site: Dallas

Vehicle ID: Nova Bus 4322

Date of Test: 3/1/99

Test Mode: CBD

Fuel Type: LNG

70mm Filter Weight $=\quad 5.466477 \mathrm{E}-02$ grams

PM10 Filter Weight $=8.666347 \mathrm{E}-02$ grams

$\mathrm{PM} 2.5$ Filter Weight $=8.663701 \mathrm{E}-02$ grams

Percent Difference $=-45.28281 \%$ PM10 and $70 \mathrm{~mm}$

Percent Difference $=0 \%$ PM10 and PM2.5 


\section{Cyclone Comparison Data Sheet for Bus 4323 - SS 20mph}

Test ID: TX_3135-1

Test Site: Dallas

Vehicle ID: Nova Bus 4324

Date of Test: 3/1/99

Test Mode: SS-20mph

Fuel Type: LNG

$70 \mathrm{~mm}$ Filter Weight $=-6.375285 \mathrm{E}-02$ grams

PM10 Filter Weight $=5.160714 \mathrm{E}-02$ grams

$\mathrm{PM} 2.5$ Filter Weight $=4.700992 \mathrm{E}-02$ grams

Percent Difference $=1899.6 \%$ PM10 and 70mm

Percent Difference $=9.323378 \%$ PM10 and PM2.5

Percent Difference $=1323.099 \%$ PM2.5 and $70 \mathrm{~mm}$

Test ID: TX_3135-2

Test Site: Dallas

Vehicle ID: Nova Bus 4323

Date of Test: 3/2/99

Test Mode: SS-20mph

Fuel Type: LNG

$70 \mathrm{~mm}$ Filter Weight $=-7.272349 \mathrm{E}-02$ grams

PM10 Filter Weight $=7.189088 \mathrm{E}-02$ grams

PM2.5 Filter Weight $=6.891637 \mathrm{E}-02$ grams

Percent Difference $=34737.3 \%$ PM10 and $70 \mathrm{~mm}$

Percent Difference $=4.224939 \%$ PM10 and PM2.5

Percent Difference $=7440.773 \%$ PM2.5 and $70 \mathrm{~mm}$ 


\section{Cyclone Comparison Data Sheet for Bus 4323 - CBD}

Test ID: TX_3136-3

Test Site: Dallas

Vehicle ID: Nova Bus 4323

Date of Test: 3/2/99

Test Mode: CBD

Fuel Type: LNG

$70 \mathrm{~mm}$ Filter Weight $=-.0462024$ grams

PM10 Filter Weight $=5.717885 \mathrm{E}-02$ grams

PM2.5 Filter Weight $=4.599312 \mathrm{E}-02$ grams

Percent Difference $=-1883.691 \%$ PM10 and $70 \mathrm{~mm}$

Percent Difference $=21.68366 \%$ PM10 and PM 2.5

Percent Difference $=88107.6 \%$ PM2.5 and 70mm

Test ID: TX_3136-4

Test Site: Dallas

Vehicle ID: Nova Bus 4323

Date of Test: 3/2/99

Test Mode: CBD

Fuel Type: LNG

$70 \mathrm{~mm}$ Filter Weight $=-3.293376 \mathrm{E}-02$ grams

PM10 Filter Weight $=6.613584 \mathrm{E}-02$ grams

PM2.5 Filter Weight $=5.047006 \mathrm{E}-02$ grams

Percent Difference $=-596.7675 \%$ PM10 and 70mm

Percent Difference $=26.86961 \%$ PM10 and PM2.5

Percent Difference $=-951.2134 \%$ PM2.5 and 70mm 


\section{Cyclone Comparison Data Sheet for Bus 4324 - SS 20mph}

Test ID: TX_3118-2

Test Site: Dallas

Vehicle ID: Nova Bus 4324

Date of Test: 2/13/99

Test Mode: SS-20mph

Fuel Type: LNG

$70 \mathrm{~mm}$ Filter Weight $=0$ grams

PM10 Filter Weight $=.2010005$ grams

PM2.5 Filter Weight $=\quad .1598679$ grams

Percent Difference $=-200 \%$ PM10 and 70mm

Percent Difference $=22.79647 \%$ PM10 and PM2.5

Percent Difference $=-200 \%$ PM2.5 and $70 \mathrm{~mm}$ 


\section{Cyclone Comparison Data Sheet for Bus 4324 - CBD}

Test ID: TX_3119-1

Test Site: Dallas

Vehicle ID: Nova Bus 4324

Date of Test: 2/13/99

$70 \mathrm{~mm}$ Filter Weight $=.2327923$ grams

PM10 Filter Weight $=.4195415$ grams

PM2.5 Filter Weight $=.3240868$ grams

Percent Difference $=-57.25573 \%$ PM10 and $70 \mathrm{~mm}$

Percent Difference $=25.6727 \%$ PM10 and PM2.5

Test ID: TX_3119-2

Test Site: Dallas

Vehicle ID: Nova Bus 4324

Date of Test: 2/13/99

70mm Filter Weight $=.1477126$ grams

PM10 Filter Weight $=.2639361$ grams

$\mathrm{PM} 2.5$ Filter Weight $=.211658$ grams

Percent Difference $=-56.46729 \%$ PM10 and $70 \mathrm{~mm}$

Percent Difference $=21.98432 \%$ PM10 and PM2.5

Test ID: TX_3119-3

Test Site: Dallas

Vehicle ID: Nova Bus 4324

Date of Test: $2 / 13 / 99$

$70 \mathrm{~mm}$ Filter Weight $=.1327052$ grams

PM10 Filter Weight $=.2468226$ grams

PM2.5 Filter Weight $=.229065$ grams

Percent Difference $=-60.13651 \%$ PM10 and $70 \mathrm{~mm}$

Percent Difference $=7.462956 \%$ PM10 and PM2.5

Test ID: TX_3119-4

Test Site: Dallas

Vehicle ID: Nova Bus 4324

Date of Test: 2/13/99

$70 \mathrm{~mm}$ Filter Weight $=.1543977$ grams

PM10 Filter Weight $=.2247383$ grams

PM2.5 Filter Weight $=.1647273$ grams

Percent Difference $=-37.10572 \%$ PM10 and $70 \mathrm{~mm}$

Percent Difference $=30.81709 \%$ PM10 and PM2.5

Test ID: TX_3119-5

Test Site: Dallas

Vehicle ID: Nova Bus 4324

Date of Test: 2/13/99

$70 \mathrm{~mm}$ Filter Weight $=.1364215$ grams

PM10 Filter Weight $=.2547871$ grams

PM2.5 Filter Weight $=.1286548$ grams

Percent Difference $=-60.51276 \%$ PM10 and $70 \mathrm{~mm}$

Percent Difference $=65.78951 \%$ PM10 and PM2.5 


\section{Cyclone Comparison Data Sheet for Bus 4325 - SS 20mph}

Test ID: TX_3138-2

Test Site: Dallas

Vehicle ID: Nova Bus 4325

Date of Test: 3/3/99

Test Mode: SS-20mph

Fuel Type: LNG

$70 \mathrm{~mm}$ Filter Weight $=-1.578954 \mathrm{E}-02$ grams

PM10 Filter Weight $=3.970791 \mathrm{E}-02$ grams

$\mathrm{PM} 2.5$ Filter Weight $=3.236452 \mathrm{E}-02$ grams

Percent Difference $=-464.0573 \%$ PM10 and $70 \mathrm{~mm}$

Percent Difference $=20.37778 \%$ PM10 and PM2.5

Percent Difference $=-581.0452 \%$ PM2.5 and $70 \mathrm{~mm}$ 


\section{Cyclone Comparison Data Sheet for Bus 4325 - CBD}

Test ID: TX_3139-2

Test Site: Dallas

Vehicle ID: Nova Bus 4325

Date of Test: 3/3/99

Test Mode: CBD

Fuel Type: LNG

$70 \mathrm{~mm}$ Filter Weight $=3.238144 \mathrm{E}-02$ grams

PM10 Filter Weight $=6.631608 \mathrm{E}-02$ grams

$\mathrm{PM} 2.5$ Filter Weight $=3.752926 \mathrm{E}-02$ grams

Percent Difference $=-68.76493 \%$ PM10 and 70mm

Percent Difference $=55.44173 \%$ PM10 and PM2.5

Test ID: TX_3139-3

Test Site: Dallas

Vehicle ID: Nova Bus 4325

Date of Test: 3/3/99

Test Mode: CBD

Fuel Type: LNG

$70 \mathrm{~mm}$ Filter Weight $=0$ grams

PM10 Filter Weight $=6.133489 \mathrm{E}-02$ grams

PM2.5 Filter Weight $=.0242902$ grams

Percent Difference $=-200 \%$ PM10 and $70 \mathrm{~mm}$

Percent Difference $=86.52765 \%$ PM10 and PM2.5

Test ID: TX_3139-4

Test Site: Dallas

Vehicle ID: Nova Bus 4325

Date of Test: 3/3/99

Test Mode: CBD

Fuel Type: LNG

70mm Filter Weight $=3.057593 \mathrm{E}-02$ grams

PM10 Filter Weight $=8.527575 \mathrm{E}-02$ grams

PM2.5 Filter Weight $=7.347159 \mathrm{E}-02$ grams

Percent Difference $=-94.43079 \%$ PM10 and 70mm

Percent Difference $=14.87164 \%$ PM10 and PM2.5 


\section{Cyclone Comparison Data Sheet for Bus 4328 - SS 20mph}

Test ID: TX_3126-3

Test Site: Dallas

Vehicle ID: Nova Bus 4328

Date of Test: $2 / 17 / 99$

Test Mode: SS-20mph

Fuel Type: LNG

$70 \mathrm{~mm}$ Filter Weight $=0$ grams

PM10 Filter Weight $=.1156933$ grams

PM2.5 Filter Weight $=9.705552 \mathrm{E}-02$ grams

Percent Difference $=-200 \%$ PM10 and 70mm

Percent Difference $=17.5209 \%$ PM10 and PM2.5

Percent Difference $=-200 \%$ PM2.5 and 70mm 


\section{Cyclone Comparison Data Sheet for Bus 4328 - CBD}

Test ID: TX_3127-1

Test Site: Dallas

Vehicle ID: Nova Bus 4328

Date of Test: 2/17/99

$70 \mathrm{~mm}$ Filter Weight $=0$ grams

PM10 Filter Weight $=.1289327$ grams

PM2.5 Filter Weight $=.1051225$ grams

Percent Difference $=-200 \%$ PM10 and 70mm

Percent Difference $=20.34577 \%$ PM10 and PM2.5

Test ID: TX_3127-2

Test Site: Dallas

Vehicle ID: Nova Bus 4328

Date of Test: $2 / 17 / 99$

$70 \mathrm{~mm}$ Filter Weight $=0$ grams

PM10 Filter Weight $=9.348227 \mathrm{E}-02$ grams

PM2.5 Filter Weight $=6.618633 \mathrm{E}-02$ grams

Percent Difference $=-200 \%$ PM10 and 70mm

Percent Difference $=34.19074 \%$ PM10 and PM2.5

Test ID: TX_3127-3

Test Site: Dallas

Vehicle ID: Nova Bus 4328

Date of Test: 2/17/99

$70 \mathrm{~mm}$ Filter Weight $=0$ grams

PM10 Filter Weight $=9.229887 \mathrm{E}-02$ grams

PM2.5 Filter Weight $=\quad .0867708$ grams

Percent Difference $=-200 \%$ PM10 and $70 \mathrm{~mm}$

Percent Difference $=6.174212 \%$ PM10 and PM2.5

Test ID: TX_3127-4

Test Site: Dallas

Vehicle ID: Nova Bus 4328

Date of Test: 2/17/99

$70 \mathrm{~mm}$ Filter Weight $=0$ grams

PM10 Filter Weight $=9.307744 \mathrm{E}-02$ grams

PM2.5 Filter Weight $=8.984243 \mathrm{E}-02$ grams

Percent Difference $=-200 \%$ PM10 and 70mm

Percent Difference $=3.537081 \%$ PM10 and PM2.5

Test ID: TX_3127-5

Test Site: Dallas

Vehicle ID: Nova Bus 4328

Date of Test: 2/17/99

$70 \mathrm{~mm}$ Filter Weight $=0$ grams

PM10 Filter Weight $=\quad .1022967$ grams

PM2.5 Filter Weight $=8.962727 \mathrm{E}-02$ grams

Percent Difference $=-200 \%$ PM10 and $70 \mathrm{~mm}$

Percent Difference $=13.20256 \%$ PM10 and PM2.5 


\section{Cyclone Comparison Data Sheet for Bus 4329 - SS 20mph}

Test ID: TX-3123-1

Test Site: Dallas

Vehicle ID: Nova Bus 4329

Date of Test: 2/16/99

Test Mode: SS-20mph

Fuel Type: LNG

$70 \mathrm{~mm}$ Filter Weight $=-4.953168 \mathrm{E}-02$ grams

PM10 Filter Weight $=8.488767 \mathrm{E}-02$ grams

PM2.5 Filter Weight $=3.318183 \mathrm{E}-02$ grams

Percent Difference $=-760.3767 \%$ PM10 and $70 \mathrm{~mm}$

Percent Difference $=87.58542 \%$ PM10 and PM2.5

Percent Difference $=1011.796 \%$ PM2.5 and 70mm 


\title{
Cyclone Comparison Data Sheet for Bus 4329 - CBD
}

\author{
Test ID: TX_3124-1 \\ Test Site: Dallas \\ Vehicle ID: Nova Bus 4329 \\ Date of Test: 2/16/99 \\ $70 \mathrm{~mm}$ Filter Weight $=6.105543 \mathrm{E}-02$ grams \\ PM10 Filter Weight $=.1781862$ grams \\ PM2.5 Filter Weight $=\quad .1066683$ grams \\ Percent Difference $=-97.91837 \%$ PM10 and $70 \mathrm{~mm}$ \\ Percent Difference $=50.21359 \%$ PM10 and PM2.5
}

Test ID: TX_3124-2

Test Site: Dallas

Vehicle ID: Nova Bus 4329

Date of Test: $2 / 16 / 99$

$70 \mathrm{~mm}$ Filter Weight $=4.210318 \mathrm{E}-02$ grams

PM10 Filter Weight $=.1216387$ grams

PM2.5 Filter Weight $=9.280431 \mathrm{E}-02$ grams

Percent Difference $=-97.14745 \%$ PM10 and 70mm

Percent Difference $=26.89238 \%$ PM10 and PM2.5

Test ID: TX_3124-3

Test Site: Dallas

Vehicle ID: Nova Bus 4329

Date of Test: 2/16/99

70mm Filter Weight $=\quad .0437047$ grams

PM10 Filter Weight $=.1342117$ grams

PM2.5 Filter Weight $=\quad .1389265$ grams

Percent Difference $=-101.7411 \%$ PM10 and $70 \mathrm{~mm}$

Percent Difference $=-3.45232 \%$ PM10 and PM2.5

Test ID: TX_3124-5

Test Site: Dallas

Vehicle ID: Nova Bus 4329

Date of Test: 2/16/99

$70 \mathrm{~mm}$ Filter Weight $=4.100756 \mathrm{E}-02$ grams

PM10 Filter Weight $=.1178178$ grams

PM2.5 Filter Weight $=\quad .1279395$ grams

Percent Difference $=-96.72287 \%$ PM10 and $70 \mathrm{~mm}$

Percent Difference $=-8.237197 \%$ PM10 and PM2.5

Test ID: TX_3124-6

Test Site: Dallas

Vehicle ID: Nova Bus 4329

Date of Test: 2/16/99

70mm Filter Weight $=3.740744 \mathrm{E}-02$ grams

PM10 Filter Weight $=9.393422 \mathrm{E}-02$ grams

PM2.5 Filter Weight $=\quad .1214609$ grams

Percent Difference $=-86.07594 \%$ PM10 and 70mm

Percent Difference $=-25.55928 \%$ PM10 and PM2.5

Percent Difference $=32.81091 \%$ PM10 and PM2.5 